\title{
Estructura y propiedades físicas de envolturas circunestelares en estrellas Be
}

TESIS PRESENTADA PARA OPTAR POR EL GRADO ACADÉMICO DE DOCTORA EN ASTRONOMÍA

YANINA ROXANA CochetTI

DireCTORA: DRA. Lydia CidALE

CODIRECTORA: Dra. María LAURA ARIAS

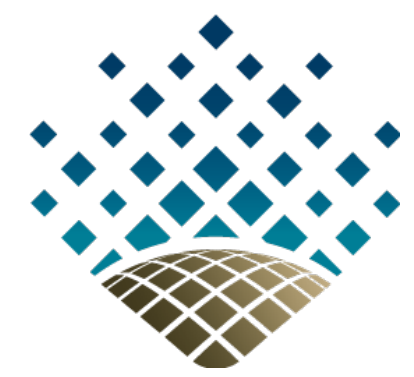

Facultad de Ciencias Astronómicas y Geofísicas

Universidad Nacional de La Plata

La Plata - Argentina

Marzo de 2019 



\section{Prefacio}

Esta Tesis es presentada como parte de los requisitos para obtener el grado académico de Doctora en Astronomía de la Universidad Nacional de La Plata. La misma contiene los resultados de la investigación desarrollada bajo la dirección de las Dras. Lydia Cidale y María Laura Arias, entre los años 2014 y 2019.

Yanina Roxana Cochetti

e-mail: cochetti@fcaglp.unlp.edu.ar

La Plata, Marzo de 2019

\section{Directora:}

Dra. Lydia Cidale

Facultad de Ciencias Astronómicas y Geofísicas

Instituto de Astrofísica de La Plata

Universidad Nacional de La Plata, La Plata, Argentina

\section{Codirectora:}

Dra. María Laura Arias

Facultad de Ciencias Astronómicas y Geofísicas

Instituto de Astrofísica de La Plata

Universidad Nacional de La Plata, La Plata, Argentina

\section{Jurado:}

Dr. Roberto Gamen

Facultad de Ciencias Astronómicas y Geofísicas

Instituto de Astrofísica de La Plata

Universidad Nacional de La Plata, La Plata, Argentina

Dra. Andrea Costa

Observatorio Astronómico de Córdoba

Universidad Nacional de Córdoba

Córdoba, Argentina

Dr. Marcelo Borges Fernandes

Observatorio Nacional

Río de Janeiro, Brasil 

"Cuando pienso

hacia arriba

me transformo en estrellas,

hacia abajo,

en raíces.

Cuando miro

hacia adentro

me compongo en silencios,

hacia afuera,

en canciones."

(Liliana Bodoc, Relatos de los confines)

A mi mamá y mi abuela. 



\section{Agradecimientos}

A la Universidad Nacional de La Plata y la Facultad de Ciencias Astronómicas y Geofísicas, por haberme formado a lo largo de estos años.

Al CONICET, por la beca otorgada para realizar este Doctorado.

A la Educación Pública y todes les que la defienden.

A Lydia y María, por la confianza, la paciencia y las largas horas compartidas.

Al jurado, por su dedicación en la lectura de esta tesis y por sus sugerencias para mejorarla.

$\mathrm{Al}$ Grupo MEP, por la compañía diaria, los consejos y las palabras de ánimo.

A quienes colaboraron de alguna manera en el desarrollo de este trabajo, ya sea en los turnos de observación o en la discusión de algunos resultados.

A mis amigues del Obser, por las charlas de mates dentro y fuera de las oficinas, por los recreos de almuerzo entre risas y pasto. A Inti Puca, por los debates e ideas compartidos, y por saber comprender los momentos de ausencias.

A Tiara e Iri, por acompañarme y abrazarme en la lucha.

A mi mamá, mi papá, mi hermano y mi abuela, por estar presentes en cada momento de mi vida y alegrarse infinitamente con mis logros. A la familia de Fran, por ser mi familia también.

A Fran, por todo su apoyo y amor, por ser mi gran compañero.

¡Gracias! 



\section{RESUMEN}

$\mathrm{L}$

os objetos que manifiestan el fenómeno Be son estrellas de tipo espectral B no supergigantes rodeadas de una envoltura circunestelar gaseosa extendida. Estos objetos son rotadores rápidos y presentan (o han presentado) en su espectro líneas en emisión de hidrógeno y de metales una vez ionizados. La presencia de una envoltura extendida no sólo se revela a través de las líneas en emisión, sino que también se manifiesta mediante numerosas peculiaridades espectroscópicas, fotométricas y polarimétricas que caracterizan al fenómeno Be. Los modelos propuestos para explicar la formación y estructura de los discos y/o envolturas circunestelares gaseosas en las estrellas Be se basan en diferentes mecanismos, entre los cuales se destacan: la rotación, los vientos estelares, las pulsaciones, las interacciones gravitatorias y los campos magnéticos. Una de las hipótesis más aceptada es la de un disco Kepleriano producido por la alta rotación de la estrella. Sin embargo, el origen y la evolución de las envolturas es un tema controversial desde hace muchas décadas.

El objetivo principal de esta Tesis es investigar la geometría, estructura física y evolución de las envolturas circunestelares que se desarrollan alrededor de las estrellas con fenómeno Be, con el fin de aportar elementos relevantes para el estudio de los posibles mecanismos que dan lugar a este fenómeno. Para ello analizamos un grupo de objetos Be a partir de diferentes técnicas (espectroscopía e interferometría) y modelamos la geometría y dinámica de sus envolturas.

La región del infrarrojo (IR) cercano provee una herramienta valiosa para el análisis de las propiedades y variabilidad de las envolturas circunestelares, dado que en ella aparecen numerosas líneas de recombinación del hidrógeno que casi no poseen contribución fotosférica y cuyas intensidades relativas están directamente relacionadas con las propiedades físicas de sus regiones de formación. Utilizando espectros IR de una muestra de 23 estrellas Be estudiamos las líneas de recombinación de las series de Paschen, Brackett, Pfund y Humphreys, realizando mediciones directas sobre sus perfiles (anchos equivalentes, flujos y anchos a mitad de altura). Para 8 de los objetos estudiados, nuestras observaciones son las primeras reportadas en este rango espectral. Clasificamos los espectros obtenidos en base a la intensidad relativa de las líneas de las series de Pfund, Brackett y Humphreys en tres grupos que representan envolturas con propiedades físicas diferentes (Mennickent et al., 2009; Granada et al., 2010). Obtuvimos además diagramas de cocientes de flujos que están directamente relacionados con el régimen de opacidad de las líneas y sus posibles cambios, tales como el diagrama de Lenorzer et al. (2002a) o diagramas equivalentes que proponemos en este trabajo de tesis. En base a modelos simples, pudimos obtener densidades columnares de átomos, extensiones relativas de las regiones de formación de las diferentes líneas y radios internos de dichas regiones en unidades de radios estelares. A su vez, hemos podido estudiar la evolución de las propiedades físicas de las envolturas a partir del seguimiento temporal de 8 objetos de la muestra observada, donde encontramos evidencia de procesos de formación o disipación del disco.

Por otra parte, utilizamos otra técnica útil para analizar la geometría y cinemática de las 
envolturas de las estrellas Be: la interferometría de larga base con alta resolución espectral. Analizamos observaciones espectrointerferométricas en la región de la línea $\mathrm{Br} \gamma$ de una muestra de 26 estrellas Be. Ajustando los datos mediante un modelo cinemático 2D para un disco ecuatorial en rotación (Delaa et al., 2011), logramos determinar parámetros físicos y cinemáticos del disco de 18 estrellas que presentaban la línea $\mathrm{Br} \gamma$ en emisión. De esos objetos, 10 fueron estudiados también mediante espectros IR. Los tamaños encontrados para los discos en la línea $\mathrm{Br} \gamma$ se encuentran entre 2 y $13 R_{\star}$. Para 5 de los objetos de la muestra, los tamaños determinados en este trabajo son los primeros reportados. En casi todos los casos, encontramos que el disco sigue una rotación Kepleriana, a excepción de un objeto (228 Eri) en el que fue necesario agregarle al disco una sobredensidad con forma de brazo espiral. Asimismo, estos resultados fueron analizados en forma conjunta con los obtenidos para otros 7 objetos por Meilland et al. (2012). Del análisis total de 25 estrellas Be con emisión en $\mathrm{Br} \gamma$ no encontramos correlación entre la velocidad de rotación respecto de la velocidad crítica $V / V_{\text {crit }}$ y el tipo espectral de la estrella central, y encontramos que el eje de rotación de los discos está uniformemente distribuido en el plano del cielo. Además, estimamos un valor medio de $\overline{V / V_{\text {crit }}}=0.75 \pm 0.08$ y una tasa de rotación media de $\overline{\Omega / \Omega_{\text {crit }}}=0.90 \pm 0.05$. Este resultado concuerda con los valores propuestos por Cranmer (2005), Frémat et al. (2005) y Zorec et al. (2016), quienes sostienen que las estrellas Be no rotan a la velocidad crítica. Este resultado sugiere la necesidad de considerar un mecanismo adicional a la rotación que contribuya a la formación de la envoltura circunestelar.

La importancia de la rotación se manifiesta a través de la correlación encontrada entre la cota máxima para el tamaño del disco en la línea $\operatorname{Br} \gamma$ y la velocidad de rotación en la base del mismo. Este resultado obtenido a partir del modelado de datos interferométricos está en buen acuerdo con los radios internos de las regiones de formación de la línea $\mathrm{Br} \gamma$ obtenidos mediante el análisis de los espectros IR para estrellas con diferentes velocidades de rotación.

El seguimiento temporal de los objetos y la utilización de dos técnicas complementarias para determinar parámetros de los discos circunestelares de las estrellas Be nos permitió encontrar evidencia de variaciones relacionadas con procesos de formación/disipación del disco en escalas de tiempo relativamente cortas (de algunos meses a unos pocos años). 
PÁGINA

ÍNDICE GENERAL $\quad$ ix

ÍNDICE DE TABLAS

ÍNDICE DE FIGURAS $\quad$ xvii

1 Introducción 1

2 Las estrellas Be $\quad 5$

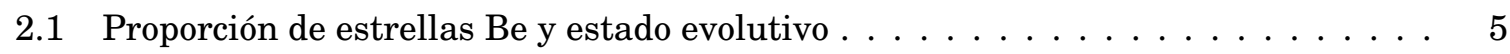

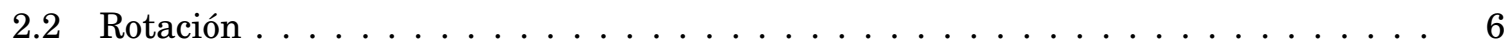

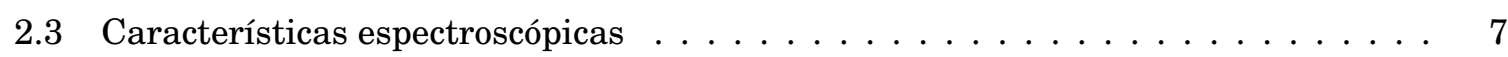

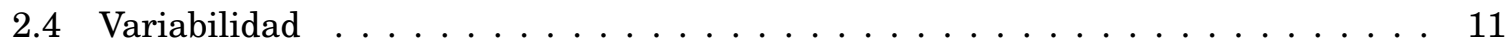

2.4.1 Transiciones B $\leftrightarrow \mathrm{Be} \leftrightarrow$ Be-shell . . . . . . . . . . . . . . . . . . . . 11

2.4 .2 Variaciones V/R . . . . . . . . . . . . . . . . . . . 11

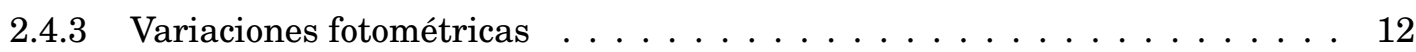

2.4 .4 Erupciones . . . . . . . . . . . . . . . . . . . . 12

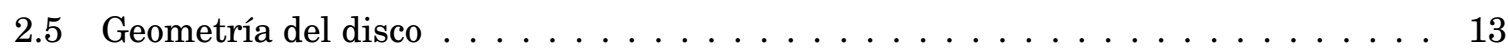

2.5.1 Altura del disco y ángulo de apertura . . . . . . . . . . . . . . . . . 13

2.5.2 Densidad del disco . . . . . . . . . . . . . . . . . . . . . . . 14

2.5.3 Tamaño del disco . . . . . . . . . . . . . . . . . . . . . . . . 14

2.5 .4 Ángulo de posición . . . . . . . . . . . . . . . . . . . . 15

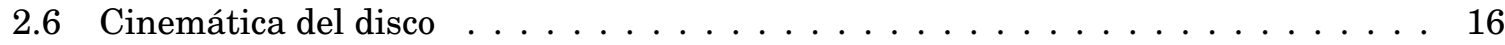

3 Observaciones en el IR cercano $\quad 17$

3.1 El espectro IR de las estrellas B . . . . . . . . . . . . . . 18

3.1 .1 Estrellas normales . . . . . . . . . . . . . . . . . . 18

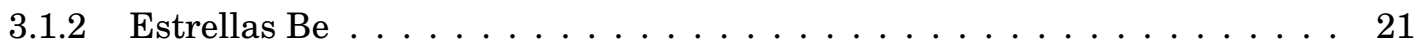

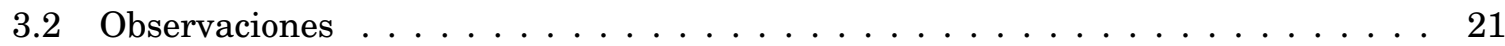

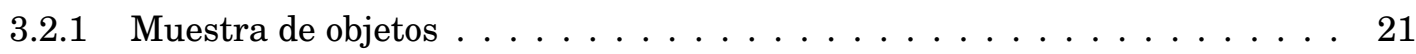

3.2.2 Observaciones realizadas en Gemini . . . . . . . . . . . . . . . . . . 22 
3.2.3 Observaciones realizadas en Las Campanas . . . . . . . . . . . . . . . . . . 27

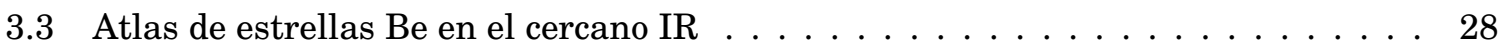

4 Metodología aplicada al análisis de las observaciones infrarrojas 49

4.1 Diagramas de cocientes de flujos como diagnóstico de las propiedades de la envoltura . . . . . . . . . . . . . . . . . . . . . . . . . 49

4.2 Propiedades de las envolturas según la intensidad de las líneas . . . . . . . . . 51

4.2 .1 Clasificación en grupos $\ldots \ldots \ldots \ldots \ldots \ldots \ldots$. . . . . . . . . . 51

4.2 .2 Decrementos de líneas de una misma serie . . . . . . . . . . . . . . . 52

4.3 Evidencias de la evolución de las envolturas . . . . . . . . . . . . . . . 54

4.4 Densidad columnar y tamaño de la envoltura . . . . . . . . . . . . . . . 54

4.5 Correcciones aplicadas y estimación de errores $\ldots \ldots \ldots \ldots \ldots$

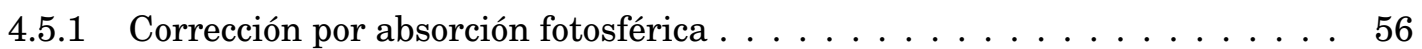

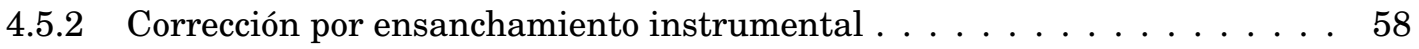

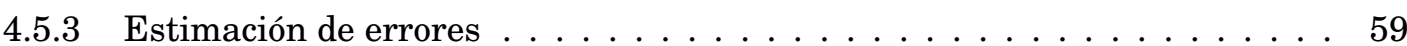

5 Resultados individuales $\quad 61$

5.1 Mediciones y resultados para cada objeto estudiado . . . . . . . . . . . . . . . . 61

$5.1 .148 \mathrm{Per}-\mathrm{HD} 25940$. . . . . . . . . . . . . . . . . . . . . . 62

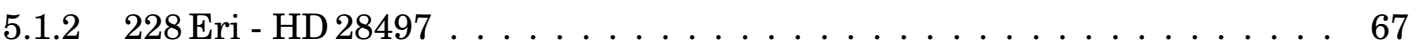

$5.1 .3105 \mathrm{Tau}-\mathrm{HD} 32991 \ldots \ldots \ldots \ldots \ldots \ldots \ldots$

$5.1 .4 \quad \Psi$ Ori - HD $35439 \ldots \ldots \ldots \ldots \ldots \ldots$

$5.1 .5120 \mathrm{Tau}-\mathrm{HD} 36576 \ldots \ldots \ldots \ldots \ldots$

$5.1 .6 \omega$ Ori - HD $37490 \ldots \ldots \ldots \ldots \ldots \ldots$

5.1 .7 V696 Mon - HD $41335 \ldots \ldots \ldots \ldots \ldots$

5.1 .8 MXPup $-\mathrm{HD} 68980 \ldots \ldots \ldots \ldots \ldots \ldots \ldots$

$5.1 .948 \mathrm{Lib}-\mathrm{HD} 142983 \ldots \ldots \ldots$. . . . . . . . . . . . . . . . . 97

5.1 .10 V1075 Sco - HD $155806 \ldots \ldots \ldots \ldots 10 \ldots \ldots$

5.1 .1188 Her - HD $162732 \ldots \ldots \ldots$. . . . . . . . . . . . . . . . . . . . . . . 104

5.1 .12 HD 171623 - HR $6977 \ldots \ldots$. . . . . . . . . . . . . . . . . . 107

5.1 .13 V4024 Sgr - HD $178175 \ldots \ldots \ldots$. . . . . . . . . . . . . . . . . . 108

5.1 .14 o Aqr - HD $209409 \ldots \ldots \ldots \ldots$. . . . . . . . . . . . . . 111

$5.1 .15 \pi \mathrm{Aqr}-\mathrm{HD} 212571 \ldots \ldots \ldots$

5.2 Resultados generales . . . . . . . . . . . . . . . . . . . . . 119

5.2 .1 Ubicación en el diagrama de Lenorzer . . . . . . . . . . . . . . . . . . . 119

5.2.2 Comportamiento de las series de hidrógeno . . . . . . . . . . . . . . . 120

6 Análisis de la variabilidad $\quad 125$

6.1 Mediciones y análisis de cada objeto . . . . . . . . . . . . . . . . 125 
6.1.1 BK Cam - HD $20336 \ldots \ldots \ldots \ldots \ldots$

$6.1 .228 \mathrm{Tau}-\mathrm{HD} 23862 \ldots \ldots \ldots \ldots \ldots \ldots \ldots$

6.1.3 V1150 Tau - HD $29441 \ldots \ldots \ldots \ldots \ldots$. . . . . . . . . . . . . . . . . . . 144

6.1 .466 Oph - HD $164284 \ldots \ldots \ldots \ldots \ldots \ldots$

6.1 .5 V923 Aql - HD $183656 \ldots \ldots \ldots \ldots \ldots$. . . . . . . . . . . . 154

$6.1 .612 \mathrm{Vul}-\mathrm{HD} 187811 \ldots \ldots \ldots \ldots \ldots$

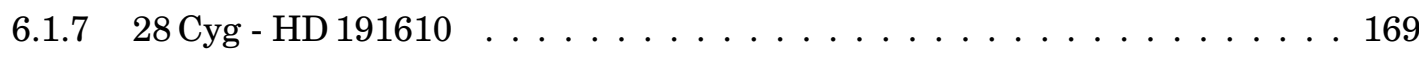

6.1 .8 EW Lac - HD $217050 \ldots \ldots \ldots \ldots \ldots$

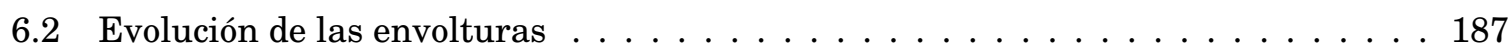

6.2.1 Ley de velocidad en el disco . . . . . . . . . . . . . . . . . . . . . . 187

6.2 .2 Evolución de las envolturas . . . . . . . . . . . . . . . . . . . . . . 190

7 Resultados generales del IR $\quad 193$

7.1 Evolución temporal de las envolturas . . . . . . . . . . . . . . . . 193

7.2 El diagrama de Lenorzer . . . . . . . . . . . . . . . . . . . . . . . . . . . . . 194

7.3 Diferentes diagramas de cocientes de flujos . . . . . . . . . . . . . . . 195

7.4 Cocientes de flujos y anchos equivalentes para $\operatorname{Br} \alpha-\operatorname{Br} \gamma$ y $\operatorname{Pa} \alpha-\operatorname{Pa} \beta \quad \ldots \ldots$

8 Observaciones interferométricas $\quad 201$

8.1 Antecedentes de observaciones interferométricas en estrellas Be . . . . . . . . . . . 201

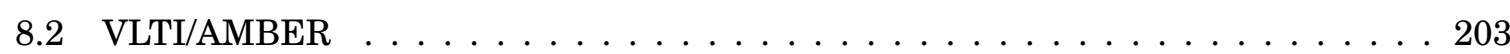

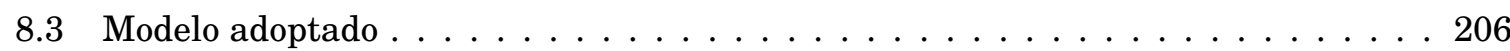

8.3.1 Extensión de la región de emisión en $\operatorname{Br} \gamma \ldots \ldots$. . . . . . . . . . . 206

8.3.2 Extensión del disco en el continuo . . . . . . . . . . . . . . . . . . . . . . 207

8.3 .3 Modelo cinemático . . . . . . . . . . . . . . . . . . . . . . . . . 208

8.4 Observaciones y reducción de datos f . . . . . . . . . . . . . . . . . 211

8.4.1 Muestra de estrellas Be . . . . . . . . . . . . . . . . . . . . 212

8.5 Ajustes realizados . . . . . . . . . . . . . . . . . . . . . . . . 214

8.5.1 Parámetros estelares a partir del ajuste de la SED . . . . . . . . . . . . 214

8.5.2 Parámetros del disco a partir del ajuste de datos interferométricos . . . . . 215

8.6 Modelos obtenidos . . . . . . . . . . . . . . . . . . . . . . . 217

8.7 Comentarios y resultados individuales de cada objeto . . . . . . . . . . . . 219

8.8 Comentarios y resultados generales . . . . . . . . . . . . . . . . . . 237

8.8.1 Ángulo de polarización . . . . . . . . . . . . . . . . . . . 238

8.8.2 Distribución de la extensión del disco en $\operatorname{Br} \gamma\left(a_{\mathrm{Br} \gamma}\right)$ y comparación con $\mathrm{H} \alpha 238$

8.8.3 Distribución del ángulo de inclinación $(i) \ldots \ldots \ldots$. . . . . . . 240

8.8.4 Distribución de la velocidad de rotación en la base del disco $\left(V_{\text {rot }}\right) \ldots$. . . 240

8.8.5 Distribución de velocidad de rotación lineal $(V)$ y angular $(\Omega) \ldots \ldots . .241$

8.9 Discusión de los resultados del capítulo . . . . . . . . . . . . . . . . . . . 242 
8.9 .1 Tasas de rotación . . . . . . . . . . . . . . . . . . . . . . . 244

8.9 .2 Orientación de los ejes de rotación . . . . . . . . . . . . . . . . . 244

9 Análisis conjunto de los datos IR e interferométricos 249

9.1 Correlación entre el tamaño del disco y la velocidad de rotación . . . . . . . . . . 249

9.2 Comentarios sobre objetos particulares estudiados con ambas técnicas . . . . . . 253

10 Conclusiones $\quad 257$

A Mediciones correspondientes al Capítulo 5 261

B Mediciones correspondientes al Capítulo 6 275

C Conceptos básicos de interferometría $\quad 301$

C.1 La experiencia de Young . . . . . . . . . . . . . . . . . . . . . . . . . 301

C.2 Interferencia y difracción . . . . . . . . . . . . . . . . . . . . . . 302

C.3 Interferometría estelar . . . . . . . . . . . . . . . . . . . . . . . . . . 302

C.3.1 El interferómetro de Michelson . . . . . . . . . . . . . . . . . . . 303

C.3.2 El patrón de difracción de Fraunhofer . . . . . . . . . . . . . . . . . . . . 304

C.3.3 La función de dispersión de punto (PSF) . . . . . . . . . . . . . . . . . . . 305

C.3.4 Relación entre la función de coherencia y el patrón de interferencia . . . . 305

C.3.5 Teorema de Van Cittert-Zernike . . . . . . . . . . . . . . . . . . . . . 307

C.3.6 Modelos geométricos . . . . . . . . . . . . . . . . . . . . . . . . . . . . 308

C.3.7 Síntesis de apertura . . . . . . . . . . . . . . . . . . . . . 310

C.3.8 Closure phase . . . . . . . . . . . . . . . . . . . . . . . . . . 312

$\begin{array}{ll}\text { Bibliografía } & 313\end{array}$ 


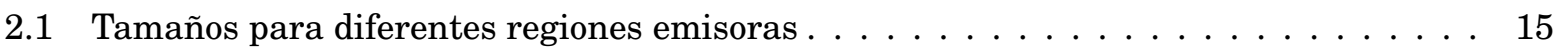

3.1 Líneas de hidrógeno presentes en las bandas J, H, K y L . . . . . . . . . . . . . 19

3.2 Líneas de helio y metales presentes en las bandas J, H, K y L . . . . . . . . . . . 20

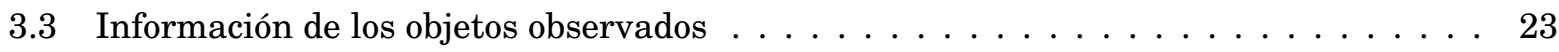

3.4 Configuraciones instrumentales utilizadas para la obtención de espectros IR . . . . 24

3.5 Información de los turnos de observación . . . . . . . . . . . . . . . . . . . 24

3.6 Observaciones realizadas en el Observatorio Gemini . . . . . . . . . . . . . . 26

3.7 Observaciones realizadas en el Observatorio Las Campanas . . . . . . . . . . . . . 28

6.1 BK Cam - Apartamientos del caso ópticamente delgado . . . . . . . . . . . . . . . . 131

$6.228 \mathrm{Tau}$ - Apartamientos del caso ópticamente delgado . . . . . . . . . . . . . . . . . 140

6.3 V1150 Tau - Apartamientos del caso ópticamente delgado . . . . . . . . . . . . . . . 148

6.4 V923 Aql - Apartamientos del caso ópticamente delgado . . . . . . . . . . . . . . 158

6.528 Cyg - Apartamientos del caso ópticamente delgado . . . . . . . . . . . . . . 173

6.6 EW Lac - Apartamientos del caso ópticamente delgado . . . . . . . . . . . . . . . . . . 184

7.1 Clasificación en grupos y estados evolutivos de los discos f . . . . . . . . . . 194

8.1 Observaciones interferométricas en $\mathrm{Br} \gamma$ realizadas con VLTI/AMBER . . . . . . . . . . 211

8.2 Parámetros estelares de los objetos de la muestra . . . . . . . . . . . . . . . . 215

8.3 Parámetros de los modelos de mejor ajuste . . . . . . . . . . . . . . . . . . 220

$8.4 V / V_{\text {crit }}$ y $\Omega / \Omega_{\text {crit }}$ para nuestra muestra de estrellas Be . . . . . . . . . . . . . 243

8.5 Parámetros de los modelos de mejor ajuste obtenidos por Meilland et al. (2012) . . . 243

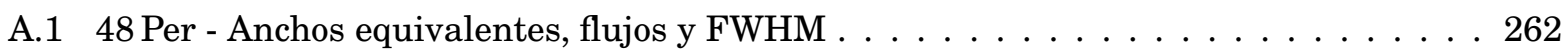

A.2 48 Per - Tamaños de las regiones emisoras . . . . . . . . . . . . . . . . . . . . . 262

A.3 228 Eri - Anchos equivalentes, flujos y FWHM _ . . . . . . . . . . . . . . . . . 263

A.4 228 Eri - Tamaños de las regiones emisoras . . . . . . . . . . . . . . . . . 263

A.5 105 Tau - Anchos equivalentes, flujos y FWHM . . . . . . . . . . . . . . . . 264

A.6 105 Tau - Tamaños de las regiones emisoras . . . . . . . . . . . . . . . . . . . . . 264 


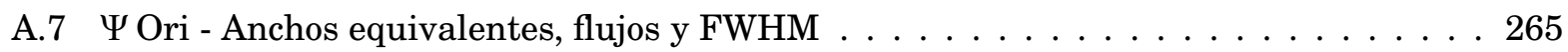

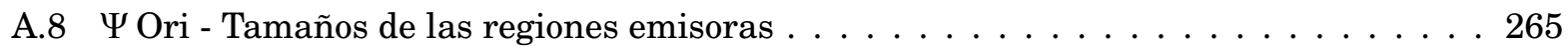

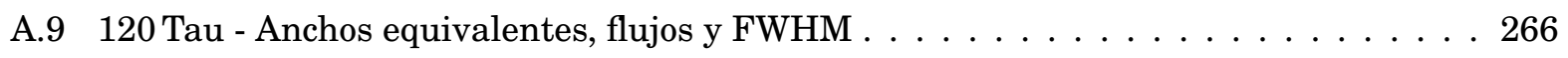

A.10 120 Tau - Tamaños de las regiones emisoras . . . . . . . . . . . . . . . 266

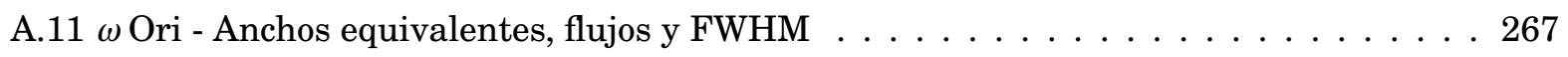

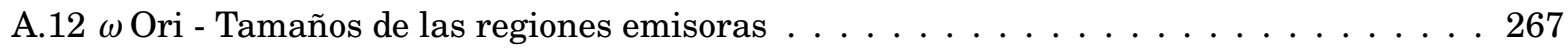

A.13 V696 Mon - Anchos equivalentes, flujos y FWHM . . . . . . . . . . . . . 268

A.14 V696 Mon - Tamaños de las regiones emisoras . . . . . . . . . . . . . . 268

A.15 MX Pup - Anchos equivalentes, flujos y FWHM . . . . . . . . . . . . . . . . 269

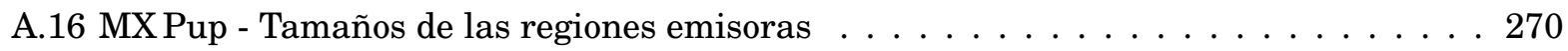

A.17 $48 \mathrm{Lib}$ - Anchos equivalentes, flujos y FWHM . . . . . . . . . . . . . . . . 270

A.18 48 Lib - Tamaños de las regiones emisoras . . . . . . . . . . . . . . . . . 271

A.19 V1075 Sco - Anchos equivalentes, flujos y FWHM _ . . . . . . . . . . . . 271

A.20 V1075 Sco - Tamaños de las regiones emisoras . . . . . . . . . . . . . . . . . 272

A.21 88 Her - Anchos equivalentes, flujos y FWHM _ . . . . . . . . . . . . . 272

A.22 88 Her - Tamaños de las regiones emisoras . . . . . . . . . . . . . . . . . 272

A.23 V4024 Sgr - Anchos equivalentes, flujos y FWHM _ . . . . . . . . . . . . . 273

A.24 V4024 Sgr - Tamaños de las regiones emisoras . . . . . . . . . . . . . . . . . 273

A.25 o Aqr - Anchos equivalentes, flujos y FWHM _ . . . . . . . . . . . . . . . 273

A.26 o Aqr - Tamaños de las regiones emisoras . . . . . . . . . . . . . . . . . 273

A.27 $\pi$ Aqr - Anchos equivalentes, flujos y FWHM . . . . . . . . . . . . . . . . . 274

A.28 $\pi$ Aqr - Tamaños de las regiones emisoras . . . . . . . . . . . . . . . . . 274

B.1 BK Cam - Banda J - Anchos equivalentes, flujos y FWHM . . . . . . . . . . . . . 275

B.2 BK Cam - Banda H - Anchos equivalentes, flujos y FWHM . . . . . . . . . . . . . 276

B.3 BK Cam - Banda K - Anchos equivalentes, flujos y FWHM . . . . . . . . . . . . 276

B.4 BK Cam - Banda L - Anchos equivalentes, flujos y FWHM . . . . . . . . . . . . . 277

B.5 BK Cam - Serie de Humphreys - Tamaños de las regiones emisoras . . . . . . . . . 277

B.6 BK Cam - Serie de Pfund - Tamaños de las regiones emisoras . . . . . . . . . . . . . 278

B.7 BK Cam - Serie de Brackett - Tamaños de las regiones emisoras . . . . . . . . . . 278

B.8 BK Cam - Serie de Paschen - Tamaños de las regiones emisoras . . . . . . . . . . 278

B.9 $28 \mathrm{Tau}$ - Banda J - Anchos equivalentes, flujos y FWHM . . . . . . . . . . . . . 279

B.10 28 Tau - Banda H - Anchos equivalentes, flujos y FWHM . . . . . . . . . . . . . . . 279

B.11 28 Tau - Banda K - Anchos equivalentes, flujos y FWHM . . . . . . . . . . . . . 279

B.12 28 Tau - Banda L - Anchos equivalentes, flujos y FWHM . . . . . . . . . . . . . 280

B.13 28 Tau - Serie de Humphreys - Tamaños de las regiones emisoras . . . . . . . . . 280

B.14 28 Tau - Serie de Humphreys - Tamaños de las regiones emisoras . . . . . . . . . . . 281

B.15 28 Tau - Serie de Pfund - Tamaños de las regiones emisoras . . . . . . . . . . . . 281

B.16 28 Tau - Serie de Pfund - Tamaños de las regiones emisoras . . . . . . . . . . . . . . 282 
B.17 28 Tau - Serie de Brackett - Tamaños de las regiones emisoras . . . . . . . . . . . . . 282

B.18 28 Tau - Serie de Paschen - Tamaños de las regiones emisoras . . . . . . . . . . . . 282

B.19 V1150 Tau - Banda J - Anchos equivalentes, flujos y FWHM . . . . . . . . . . . . . 283

B.20 V1150 Tau - Banda H - Anchos equivalentes, flujos y FWHM . . . . . . . . . . . 283

B.21 V1150 Tau - Banda K - Anchos equivalentes, flujos y FWHM . . . . . . . . . . . . 284

B.22 V1150 Tau - Banda L - Anchos equivalentes, flujos y FWHM . . . . . . . . . . . . 284

B.23 V1150 Tau - Serie de Humphreys - Tamaños de las regiones emisoras . . . . . . . . . 285

B.24 V1150 Tau - Serie de Pfund - Tamaños de las regiones emisoras . . . . . . . . . . . 285

B.25 V1150 Tau - Serie de Brackett - Tamaños de las regiones emisoras . . . . . . . . . . . 286

B.26 V1150 Tau - Serie de Paschen - Tamaños de las regiones emisoras . . . . . . . . . . 286

B.27 66 Oph - Bandas K y L - Anchos equivalentes, flujos y FWHM . . . . . . . . . . . 286

B.28 66 Oph - Series de Brackett y Pfund - Tamaños de las regiones emisoras . . . . . . . 287

B.29 V923 Aql - Banda J - Anchos equivalentes, flujos y FWHM . . . . . . . . . . . . . . . 287

B.30 V923 Aql - Banda H - Anchos equivalentes, flujos y FWHM . . . . . . . . . . . . 287

B.31 V923 Aql - Banda K - Anchos equivalentes, flujos y FWHM . . . . . . . . . . . . . . 287

B.32 V923 Aql - Banda L - Anchos equivalentes, flujos y FWHM . . . . . . . . . . . . 288

B.33 V923 Aql - Serie de Humphreys - Tamaños de las regiones emisoras . . . . . . . . . . 288

B.34 V923 Aql - Serie de Pfund - Tamaños de las regiones emisoras . . . . . . . . . . . . . . 289

B.35 V923 Aql - Serie de Brackett - Tamaños de las regiones emisoras . . . . . . . . . . . . 289

B.36 V923 Aql - Serie de Paschen - Tamaños de las regiones emisoras . . . . . . . . . . . . . 289

B.37 12 Vul - Banda J - Anchos equivalentes, flujos y FWHM . . . . . . . . . . . . . . 289

B.38 12 Vul - Banda H - Anchos equivalentes, flujos y FWHM . . . . . . . . . . . . . . . . 290

B.39 12 Vul - Banda K - Anchos equivalentes, flujos y FWHM . . . . . . . . . . . . . . 290

B.40 12 Vul - Banda L - Anchos equivalentes, flujos y FWHM . . . . . . . . . . . . . . 291

B.41 12 Vul - Serie de Humphreys - Tamaños de las regiones emisoras . . . . . . . . . . . . . 291

B.42 12 Vul - Serie de Pfund - Tamaños de las regiones emisoras . . . . . . . . . . . . . 292

B.43 12 Vul - Serie de Brackett - Tamaños de las regiones emisoras . . . . . . . . . . . . 292

B.44 28 Cyg - Banda J - Anchos equivalentes, flujos y FWHM . . . . . . . . . . . . . . 292

B.45 28 Cyg - Banda H - Anchos equivalentes, flujos y FWHM . . . . . . . . . . . . . . . . 293

B.46 28 Cyg - Banda K - Anchos equivalentes, flujos y FWHM . . . . . . . . . . . . . . 293

B.47 28 Cyg - Banda L - Anchos equivalentes, flujos y FWHM . . . . . . . . . . . . . . . . . 294

B.48 28 Cyg - Serie de Humphreys - Tamaños de las regiones emisoras . . . . . . . . . . 294

B.49 28 Cyg - Serie de Pfund - Tamaños de las regiones emisoras . . . . . . . . . . . . 295

B.50 28 Cyg - Serie de Brackett - Tamaños de las regiones emisoras . . . . . . . . . . . . 295

B.51 28 Cyg - Serie de Paschen - Tamaños de las regiones emisoras . . . . . . . . . . 296

B.52 EW Lac - Banda J - Anchos equivalentes, flujos y FWHM _ . . . . . . . . . . . . . 296

B.53 EW Lac - Banda H - Anchos equivalentes, flujos y FWHM . . . . . . . . . . . . . . . . . . . . . . . 296

B.54 EW Lac - Banda K - Anchos equivalentes, flujos y FWHM . . . . . . . . . . . . . 297 
B.55 EW Lac - Banda L - Anchos equivalentes, flujos y FWHM _ . . . . . . . . . . . . 297

B.56 EW Lac - Serie de Humphreys - Tamaños de las regiones emisoras . . . . . . . . . . 298

B.57 EW Lac - Serie de Humphreys - Tamaños de las regiones emisoras . . . . . . . . . . 298

B.58 EW Lac - Serie de Pfund - Tamaños de las regiones emisoras . . . . . . . . . . . . . 299

B.59 EW Lac - Serie de Pfund - Tamaños de las regiones emisoras . . . . . . . . . . . . . 299

B.60 EW Lac - Serie de Brackett - Tamaños de las regiones emisoras . . . . . . . . . . . . . 300

B.61 EW Lac - Serie de Paschen - Tamaños de las regiones emisoras . . . . . . . . . . . . . 300 


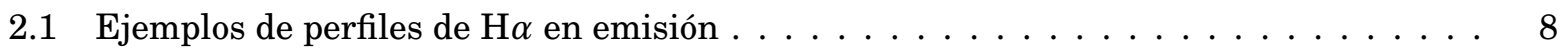

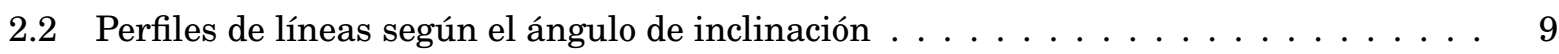

2.3 Perfiles P Cygni y componentes discretas de C IV para una estrella Be y una B . . . 10

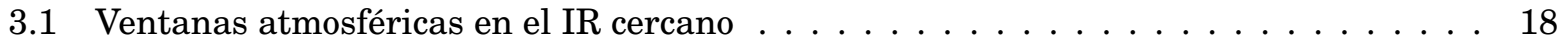

3.2 Espectros obtenidos en la banda J utilizando GNIRS o FIRE . . . . . . . . . . . . . . 31

3.3 Espectros obtenidos en la banda J utilizando GNIRS o FIRE . . . . . . . . . . . . . . . 32

3.4 Espectros obtenidos en la banda J utilizando GNIRS o FIRE . . . . . . . . . . . . . 33

3.5 Espectros obtenidos en la banda $\mathrm{H}$ utilizando GNIRS o FIRE . . . . . . . . . . . . . . 34

3.6 Espectros obtenidos en la banda $\mathrm{H}$ utilizando GNIRS o FIRE . . . . . . . . . . . . . . 35

3.7 Espectros obtenidos en la banda $\mathrm{H}$ utilizando GNIRS o FIRE . . . . . . . . . . . . . . 36

3.8 Espectros obtenidos en la banda $\mathrm{H}$ utilizando GNIRS o FIRE . . . . . . . . . . . . . 37

3.9 Espectros obtenidos en la banda K utilizando NIRI, GNIRS o FIRE . . . . . . . . . . 38

3.10 Espectros obtenidos en la banda K utilizando NIRI, GNIRS o FIRE . . . . . . . . . . 39

3.11 Espectros obtenidos en la banda K utilizando NIRI, GNIRS o FIRE . . . . . . . . . 40

3.12 Espectros obtenidos en la banda K utilizando NIRI, GNIRS o FIRE . . . . . . . . . . 41

3.13 Espectros obtenidos en la banda K utilizando NIRI, GNIRS o FIRE . . . . . . . . . . 42

3.14 Espectros obtenidos en la banda L utilizando NIRI o GNIRS . . . . . . . . . . . . 43

3.15 Espectros obtenidos en la banda L utilizando NIRI o GNIRS . . . . . . . . . . . . . 44

3.16 Espectros obtenidos en la banda L utilizando NIRI o GNIRS . . . . . . . . . . . . 45

3.17 Espectros obtenidos en la banda L utilizando NIRI o GNIRS . . . . . . . . . . . . 46

3.18 Perfiles de línea de $\mathrm{Pa} \alpha \ldots \ldots \ldots \ldots \ldots \ldots \ldots$

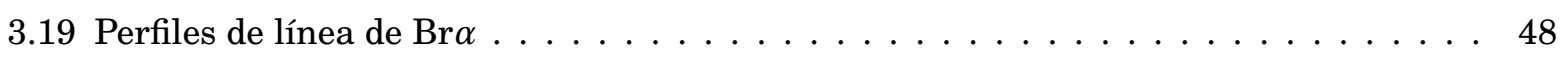

4.1 Diagrama de Lenorzer et al. (2002a) . . . . . . . . . . . . . . . . . . 51

4.2 Ejemplos de los grupos definidos por Mennickent et al. (2009) _ . . . . . . . . . . 52

4.3 Ubicación de los grupos de Mennickent en diferentes diagramas . . . . . . . . . . . 53

4.4 Ajuste de anchos equivalentes de $\mathrm{Pa} \beta \ldots \ldots \ldots \ldots \ldots \ldots \ldots$

4.5 Ajuste de anchos equivalentes de $\mathrm{Br}_{11} \ldots \ldots \ldots \ldots \ldots \ldots \ldots$

4.6 Ajuste de anchos equivalentes de $\mathrm{Br} \gamma \ldots \ldots \ldots \ldots \ldots \ldots$ 
$4.7 \quad$ Ajuste de anchos equivalentes de $\operatorname{Br} \alpha, \operatorname{Pf} \gamma$ y $\operatorname{Pf} \delta \ldots \ldots \ldots \ldots \ldots$

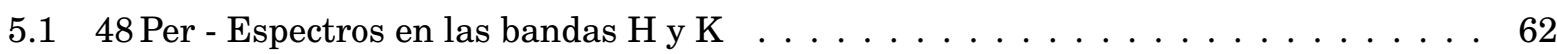

5.248 Per - Espectro en la banda L . . . . . . . . . . . . . . . . . . . . . . 63

$5.348 \mathrm{Per}-\mathrm{EW} / \lambda$ y FWHM vs. $\lambda \ldots \ldots \ldots \ldots \ldots \ldots$

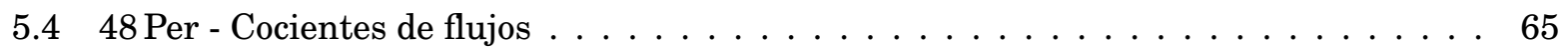

5.548 Per - Radios internos de las regiones emisoras . . . . . . . . . . . . . . 65

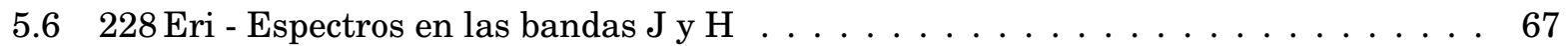

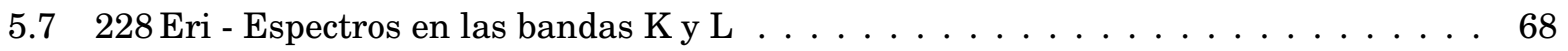

5.8228 Eri - EW/ $\lambda$ y FWHM vs. $\lambda \ldots \ldots \ldots \ldots \ldots \ldots \ldots \ldots$

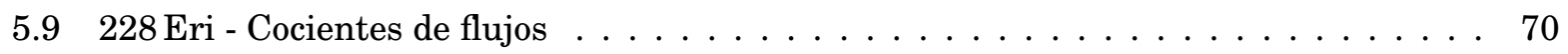

5.10228 Eri - Radios internos de las regiones emisoras . . . . . . . . . . . . . . . . 70

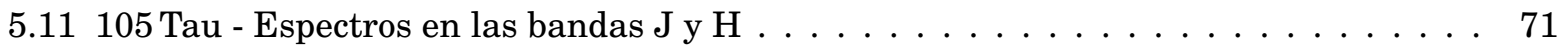

$5.12105 \mathrm{Tau}$ - Espectros en las bandas K y L . . . . . . . . . . . . . . . . . . . . . . 72

$5.13105 \mathrm{Tau}-\mathrm{EW} / \lambda$ y FWHM vs. $\lambda \ldots \ldots \ldots \ldots \ldots \ldots \ldots \ldots$

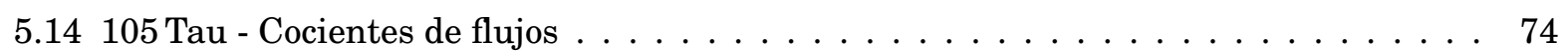

5.15105 Tau - Radios internos de las regiones emisoras . . . . . . . . . . . . 74

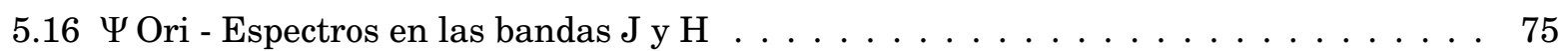

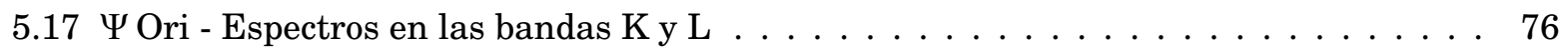

$5.18 \Psi$ Ori $-\mathrm{EW} / \lambda$ y FWHM vs. $\lambda \ldots \ldots \ldots \ldots \ldots \ldots$

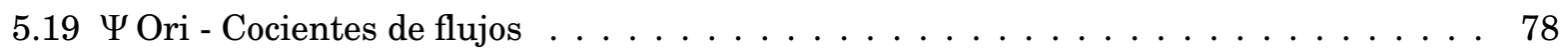

$5.20 \Psi$ Ori - Radios internos de las regiones emisoras . . . . . . . . . . . . . . . . 78

$5.21120 \mathrm{Tau}$ - Espectros en las bandas J y H . . . . . . . . . . . . . . . . . . 79

$5.22120 \mathrm{Tau}$ - Espectros en las bandas K y L . . . . . . . . . . . . . . . . . . . . 80

$5.23120 \mathrm{Tau}-\mathrm{EW} / \lambda$ y FWHM vs. $\lambda \ldots \ldots \ldots \ldots \ldots \ldots \ldots$

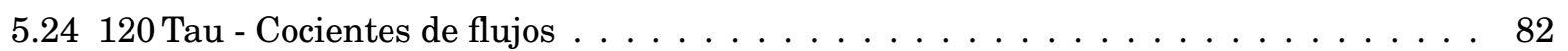

$5.25120 \mathrm{Tau}$ - Radios internos de las regiones emisoras . . . . . . . . . . . . . . 82

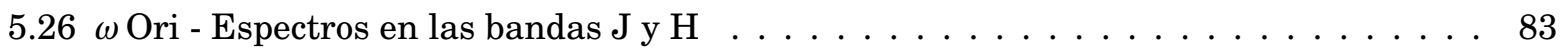

$5.27 \omega$ Ori - Espectros en las bandas K y L . . . . . . . . . . . . . . . . . . . . 84

$5.28 \omega$ Ori $-\mathrm{EW} / \lambda$ y FWHM vs. $\lambda \ldots \ldots \ldots \ldots \ldots \ldots$

$5.29 \omega$ Ori - Cocientes de flujos $\ldots \ldots \ldots \ldots \ldots$

$5.30 \omega$ Ori - Radios internos de las regiones emisoras . . . . . . . . . . . . . . 86

5.31 V696 Mon - Espectros en las bandas J y H f . . . . . . . . . . . . . . . . . 88

5.32 V696 Mon - Espectros en las bandas K y L . . . . . . . . . . . . . . . . . 89

5.33 V696 Mon - EW/ $\lambda$ y FWHM vs. $\lambda \ldots \ldots \ldots \ldots \ldots \ldots$

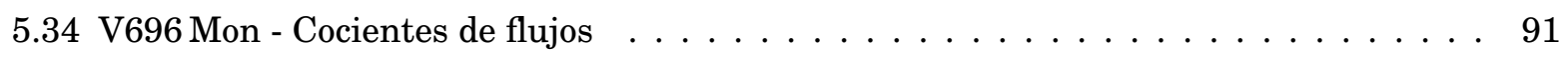

5.35 V696 Mon - Radios internos de las regiones emisoras . . . . . . . . . . . . . . . . . 91

5.36 MXPup - Espectro en la banda $J \ldots \ldots \ldots \ldots$

5.37 MXPup - Espectros banda $\mathrm{H} \mathrm{y} \mathrm{K} \mathrm{\ldots} \mathrm{.} \mathrm{.} \mathrm{.} \mathrm{.} \mathrm{.} \mathrm{.} \mathrm{.} \mathrm{.} \mathrm{.} \mathrm{.} \mathrm{.} \mathrm{.} \mathrm{.} \mathrm{.} \mathrm{.} \mathrm{.} 94$ 
5.38 MXPup $-\mathrm{EW} / \lambda$ y FWHM vs. $\lambda \ldots \ldots \ldots \ldots \ldots \ldots \ldots \ldots$

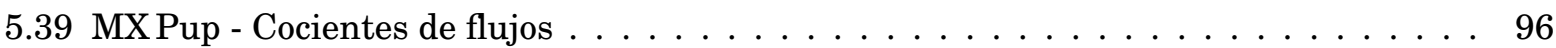

5.40 MX Pup - Radios internos de las regiones emisoras _ . . . . . . . . . . . . 96

$5.4148 \mathrm{Lib}$ - Espectros en las bandas J y H . . . . . . . . . . . . . . . . . . . 97

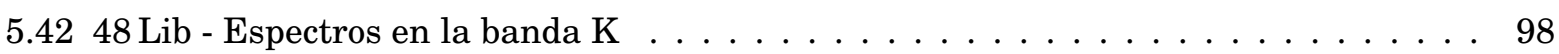

$5.4348 \mathrm{Lib}-\mathrm{EW} / \lambda$ y FWHM vs. $\lambda \ldots \ldots \ldots \ldots \ldots \ldots$

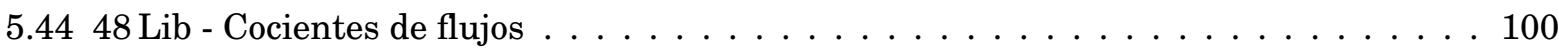

$5.4548 \mathrm{Lib}$ - Radios internos de las regiones emisoras . . . . . . . . . . . . . 100

5.46 V1075 Sco - Espectros en las bandas J, H y K . . . . . . . . . . . . . . . . 101

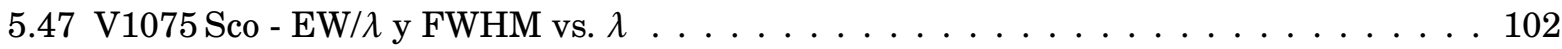

5.48 V1075 Sco - Cocientes de flujos . . . . . . . . . . . . . . . . 103

5.49 V1075 Sco - Radios internos de las regiones emisoras . . . . . . . . . . . . . . 103

$5.5088 \mathrm{Her}$ - Espectros en las bandas K y L . . . . . . . . . . . . . . . . . 104

$5.5188 \mathrm{Her}-\mathrm{EW} / \lambda$ y FWHM vs. $\lambda \ldots \ldots \ldots \ldots \ldots \ldots \ldots$

$5.5288 \mathrm{Her}$ - Radios internos de las regiones emisoras . . . . . . . . . . . . 106

5.53 HD 171623 - Espectros en las bandas J, H y K . . . . . . . . . . . . . . . . . . . . 107

5.54 V4024 Sgr - Espectros en las bandas J, H y K . . . . . . . . . . . . . . . . 108

$5.55 \mathrm{~V} 4024 \mathrm{Sgr}-\mathrm{EW} / \lambda$ vs. $\lambda \ldots \ldots \ldots$. . . . . . . . . . . . . . . 109

5.56 V4024 Sgr - Cocientes de flujos . . . . . . . . . . . . . . . . . . . . 109

5.57 V4024 Sgr - Radios internos de las regiones emisoras . . . . . . . . . . . . . . 110

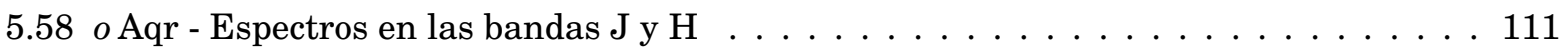

5.59 o Aqr - Espectro en la banda K . . . . . . . . . . . . . . . . . . 112

5.60 o Aqr - EW/ $\lambda$ y FWHM vs. $\lambda \ldots \ldots \ldots \ldots \ldots \ldots \ldots \ldots \ldots$

5.61 o Aqr - Cocientes de flujos . . . . . . . . . . . . . . . . . . . . . . 114

5.62 o Aqr - Radios internos de las regiones emisoras . . . . . . . . . . . . . . . . . . 114

$5.63 \pi$ Aqr - Espectros en las bandas J, H y K . . . . . . . . . . . . . . . . . 115

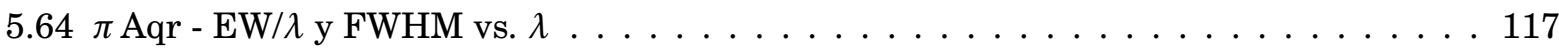

$5.65 \pi \mathrm{Aqr}-$ Cocientes de flujos $\ldots \ldots \ldots \ldots \ldots \ldots \ldots \ldots \ldots$

$5.66 \pi$ Aqr - Radios internos de las regiones emisoras . . . . . . . . . . . . . . . . 118

5.67 Ubicación en el diagrama de Lenorzer de las estrellas Be estudiadas . . . . . . . . 119

5.68 Comportamientos de los cocientes de flujos . . . . . . . . . . . . . . . . . 121

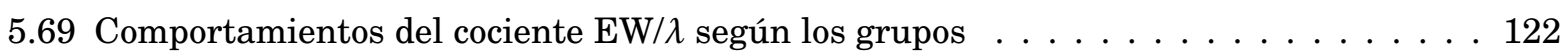

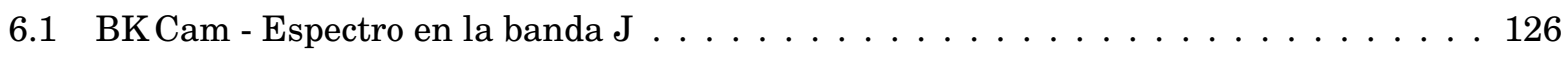

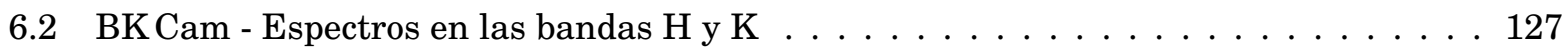

6.3 BK Cam - Espectros en la banda L y principales líneas . . . . . . . . . . . . . . 128

6.4 BK Cam - Ubicación en el diagrama de Lenorzer . . . . . . . . . . . . . . . . . . . 129

6.5 BKCam $-\mathrm{EW} / \lambda$ vs. $\lambda \ldots \ldots \ldots \ldots \ldots \ldots \ldots \ldots$

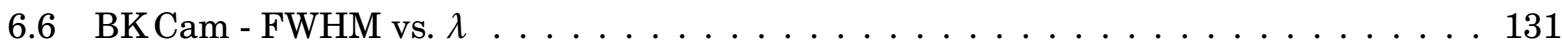


6.7 BK Cam - Cocientes de flujos . . . . . . . . . . . . . . . . . . . . . . . . 132

6.8 BK Cam - Radios internos de las regiones emisoras . . . . . . . . . . . . . . . . 134

$6.928 \mathrm{Tau}$ - Espectro en la banda J . . . . . . . . . . . . . . . . . . . 136

$6.1028 \mathrm{Tau}$ - Espectros en las bandas K y L . . . . . . . . . . . . . . . . . . 137

$6.1128 \mathrm{Tau}$ - Espectros en la banda L y principales líneas . . . . . . . . . . . . . . . 138

6.1228 Tau - Ubicación en el diagrama de Lenorzer . . . . . . . . . . . . . . . . . . . . . 139

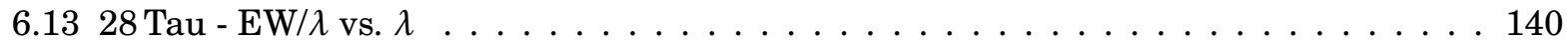

$6.1428 \mathrm{Tau}-\mathrm{FWHM}$ vs. $\lambda \ldots \ldots \ldots \ldots \ldots \ldots$

$6.1528 \mathrm{Tau}$ - Cocientes de flujos . . . . . . . . . . . . . . . . . . . . 142

$6.1628 \mathrm{Tau}$ - Radios internos de las regiones emisoras . . . . . . . . . . . . . . . 143

6.17 V1150 Tau - Espectro en la banda J . . . . . . . . . . . . . . . . . . . . . 144

6.18 V1150 Tau - Espectros en las bandas H y K . . . . . . . . . . . . . . . 145

6.19 V1150 Tau - Espectro en la banda L . . . . . . . . . . . . . . . . . . . . . 146

6.20 V1150 Tau - Ubicación en el diagrama de Lenorzer . . . . . . . . . . . . . . 146

$6.21 \mathrm{~V} 1150 \mathrm{Tau}-\mathrm{EW} / \lambda$ vs. $\lambda \ldots \ldots \ldots \ldots \ldots \ldots$

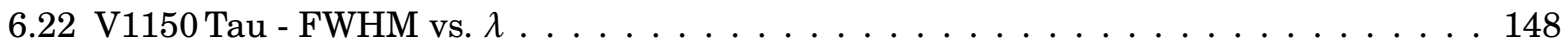

6.23 V1150 Tau - Cocientes de flujos . . . . . . . . . . . . . . . . . . . . . 149

6.24 V1150 Tau - Radios internos de las regiones emisoras . . . . . . . . . . . . . . . . . 149

$6.2566 \mathrm{Oph}$ - Espectros en las bandas J y H . . . . . . . . . . . . . . . . . 151

$6.2666 \mathrm{Oph}$ - Espectros en las bandas K y L . . . . . . . . . . . . . . . . . 152

6.2766 Oph - Radios internos de las regiones emisoras . . . . . . . . . . . . . . 153

6.28 V923 Aql - Espectros en la banda J . . . . . . . . . . . . . . . . . . . . . . . . . 154

6.29 V923 Aql - Espectros en las bandas H y K . . . . . . . . . . . . . . . . . . . 155

6.30 V923 Aql - Espectros en la banda L y principales líneas . . . . . . . . . . . . . . 156

6.31 V923 Aql - Ubicación en el diagrama de Lenorzer . . . . . . . . . . . . . . . . . 157

$6.32 \mathrm{~V} 923 \mathrm{Aql}-\mathrm{EW} / \lambda$ vs. $\lambda \ldots \ldots \ldots \ldots \ldots \ldots \ldots \ldots$

6.33 V923 Aql - FWHM vs. $\lambda \ldots \ldots \ldots \ldots \ldots \ldots$

6.34 V923 Aql - Cocientes de flujos . . . . . . . . . . . . . . . . . 160

6.35 V923 Aql - Radios internos de las regiones emisoras . . . . . . . . . . . . . . . . 161

6.3612 Vul - Espectros en la banda J . . . . . . . . . . . . . . . . . . . . . . . . 162

$6.3712 \mathrm{Vul}$ - Espectros en las bandas H y K . . . . . . . . . . . . . . . . . 163

$6.3812 \mathrm{Vul}$ - Espectros en la banda L y principales líneas . . . . . . . . . . . . . . . . 164

$6.3912 \mathrm{Vul}$ - Ubicación en el diagrama de Lenorzer . . . . . . . . . . . . . . . . . . . 165

$6.4012 \mathrm{Vul}-\mathrm{EW} / \lambda$ y FWHM vs. $\lambda \ldots \ldots \ldots \ldots \ldots \ldots$

$6.4112 \mathrm{Vul}-$ Cocientes de flujos $\ldots \ldots \ldots \ldots \ldots \ldots \ldots$

6.4212 Vul - Radios internos de las regiones emisoras . . . . . . . . . . . . . . 168

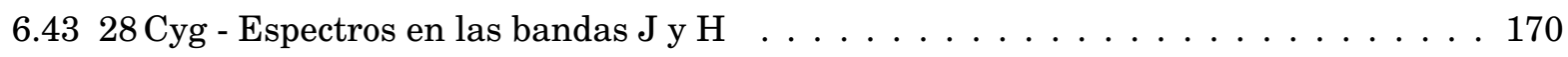

6.4428 Cyg - Espectros en las bandas K y L . . . . . . . . . . . . . . . . . . . 171 
6.4528 Cyg - Principales líneas . . . . . . . . . . . . . . . . . . . . . . . 172

6.4628 Cyg - Ubicación en el diagrama de Lenorzer . . . . . . . . . . . . . . . . . . . . . 172

$6.4728 \mathrm{Cyg}-\mathrm{EW} / \lambda$ vs. $\lambda \ldots \ldots \ldots \ldots \ldots \ldots \ldots \ldots \ldots$

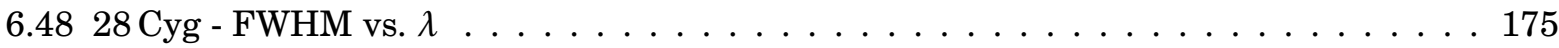

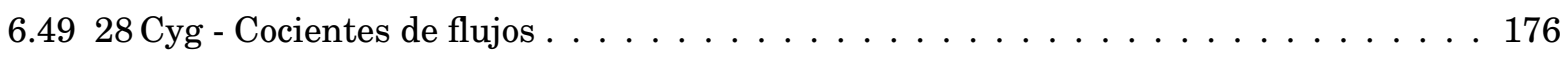

6.5028 Cyg - Radios internos de las regiones emisoras . . . . . . . . . . . . . . . 177

$6.51 \mathrm{EW} \mathrm{Lac}$ - Espectros en las bandas J y H . . . . . . . . . . . . . . . . . . 179

6.52 EW Lac - Espectros en las bandas K y L . . . . . . . . . . . . . . . . . . . . . . . 180

6.53 EW Lac - Principales líneas . . . . . . . . . . . . . . . . . . . . . . . 181

6.54 EW Lac - Ubicación en el diagrama de Lenorzer . . . . . . . . . . . . . . . . . . . . 181

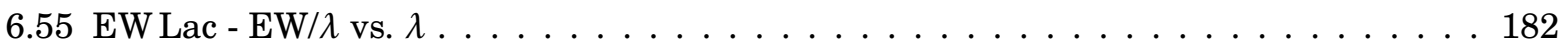

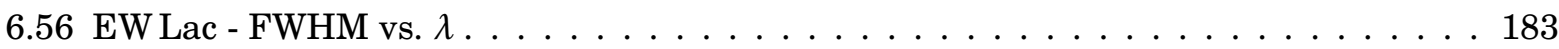

6.57 EW Lac - Cocientes de flujos . . . . . . . . . . . . . . . . . . . . . . . . 184

$6.58 \mathrm{EW}$ Lac - Radios internos de las regiones emisoras $\ldots \ldots \ldots$

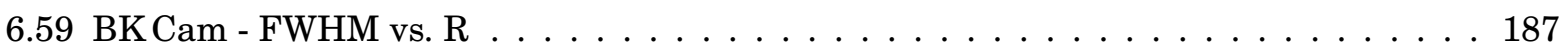

$6.6028 \mathrm{Tau}, \mathrm{V} 923 \mathrm{Aql}$ y $12 \mathrm{Vul}$ - FWHM vs. R . . . . . . . . . . . . . . . . . 188

6.6128 Cyg y EW Lac - FWHM vs. R . . . . . . . . . . . . . . . . . . . . . . . . 189

7.1 Ubicación en el diagrama de Lenorzer de todas las estrellas de nuestra muestra . . . 195

7.2 Ubicación en distintos diagramas según la pertenencia a los diferentes grupos . . . 196

7.3 Ubicación en distintos diagramas según la pertenencia a los diferentes grupos . . . . 196

7.4 Flujos y anchos equivalentes para $\operatorname{Br} \alpha-\operatorname{Br} \gamma \ldots \ldots \ldots \ldots 7 . \ldots \ldots$

7.5 Flujos y anchos equivalentes para $\mathrm{Pa} \alpha-\mathrm{Pa} \beta \ldots \ldots \ldots \ldots \ldots$

8.1 VLTI - Posiciones de los UTs y bases para los ATs . . . . . . . . . . . . . . . . . . 204

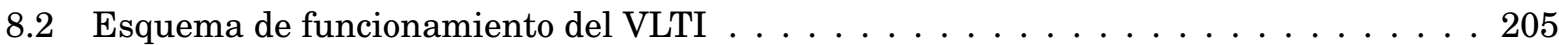

8.3 Ejemplo de mapa de velocidades . . . . . . . . . . . . . . . . . . 210

8.4 Ejemplo de mapa de intensidades . . . . . . . . . . . . . . . . . . . . . . . 210

8.5 Espectros en la región de $\mathrm{Br} \gamma$ de los objetos observados $\ldots \ldots \ldots \ldots \ldots \ldots$

8.6 Distribución por TE y CL . . . . . . . . . . . . . . . . . . . . . . . . . . . 214

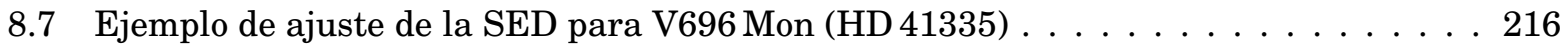

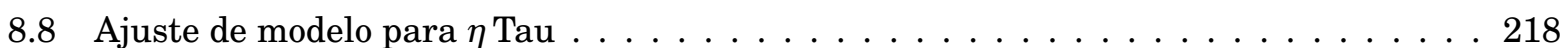

8.9 Observación de HD $23408 \ldots \ldots \ldots$. . . . . . . . . . . . . . . . 219

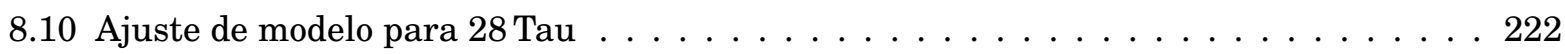

8.11 Ajuste de modelo para 228 Eri . . . . . . . . . . . . . . . . . . . . . . . 222

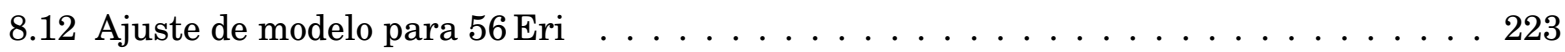

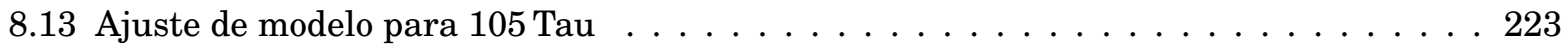

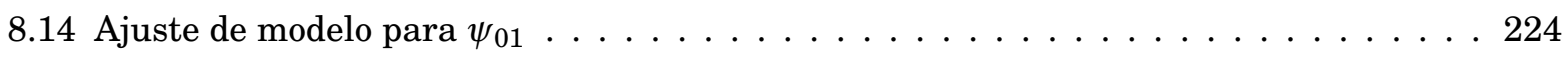

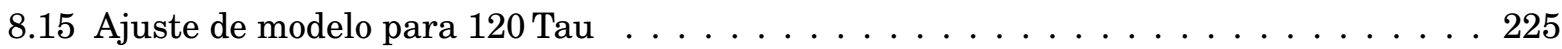


8.16 Ajuste de modelo para $\zeta \mathrm{Tau} \ldots \ldots \ldots \ldots \ldots \ldots \ldots \ldots \ldots$

8.17 Ajuste de modelo para $\omega$ Ori . . . . . . . . . . . . . . . . . . . . . 228

8.18 Ajuste de modelo para V696 Mon . . . . . . . . . . . . . . . . . . . . . 228

8.19 Ajuste de modelo para $\beta_{01}$ Mon . . . . . . . . . . . . . . . . . . . . 229

8.20 Ajuste de modelo para OW Pup . . . . . . . . . . . . . . . . . . 230

8.21 Ajuste de modelo para V374 Car . . . . . . . . . . . . . . . . . . . 231

8.22 Ajuste de modelo para MX Pup . . . . . . . . . . . . . . . . . . . 232

8.23 Ajuste de modelo para $o$ Aqr . . . . . . . . . . . . . . . . . . . . . 233

8.24 Ajuste de modelo para $o$ Aqr (continuación) . . . . . . . . . . . . . . . . . . . 234

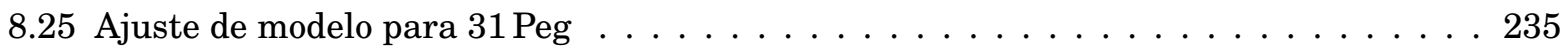

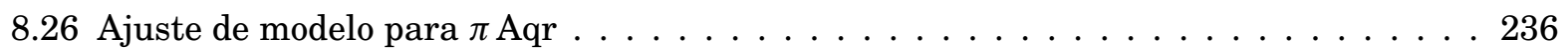

8.27 Ajuste de modelo para $\epsilon$ PsA . . . . . . . . . . . . . . . . . . . 237

8.28 Ángulo de posición del disco PA vs. ángulo de polarización $P_{\star} \ldots \ldots \ldots \ldots$. . . . . 239

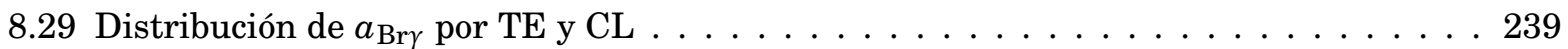

8.30 Distribución de inclinaciones por TE y CL . . . . . . . . . . . . . 240

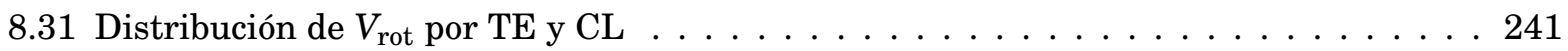

8.32 Relación entre $V_{\text {rot }} \mathrm{y}$ la extensión del disco en $\mathrm{Br} \gamma \ldots \ldots \ldots \ldots$. . . . . . . . 241

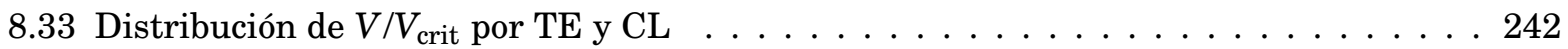

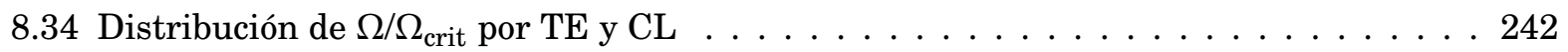

8.35 Distribución de estrellas Be por tipo espectral y CL para la muestra combinada . . . . 244

8.36 Distribución de $i, a_{\mathrm{Br} \gamma}, V_{\text {rot }}, V / V_{\text {crit }}$ y $\Omega / \Omega_{\text {crit }}$ para la muestra combinada . . . . . . 245

8.37 Distribuciones de $V / V_{\text {crit }} \mathrm{y} \Omega / \Omega_{\text {crit }}$ con la temperatura efectiva $\ldots \ldots \ldots \ldots$

8.38 Distribución de los ángulos de inclinación . . . . . . . . . . . . . . . . 246

9.1 Extensión del disco en $\operatorname{Br} \gamma$ vs. $V_{\text {rot }} \ldots \ldots \ldots \ldots \ldots \ldots \ldots$

9.2 Extensión del disco en $\mathrm{Br} \gamma$ vs. $V_{\text {rot }}$; estrellas con disco estable . . . . . . . . . . . . 250

9.3 Extensión del disco en $\mathrm{Br} \gamma$ vs. $V_{\text {rot }} / V_{\text {crit }}$; muestra combinada . . . . . . . . . . . 251

9.4 Radios internos de las regiones de formación de $\operatorname{Br} \gamma \operatorname{según} V=V \sin i / \sin i \ldots \ldots 253$

C.1 Configuración de la experiencia de doble rendija de Young . . . . . . . . . . . . . . 302

C.2 Interferómetro de Michelson . . . . . . . . . . . . . . . . . . . . . . . . . . . 303

C.3 Patrón de difracción de Fraunhofer . . . . . . . . . . . . . . . . . . . . . . 305

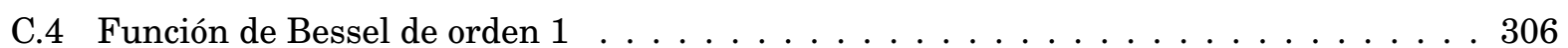

C.5 Visibilidad para diferentes modelos geométricos . . . . . . . . . . . . . . . 310

C.6 Separación de los receptores $\mathbf{B}$ y su proyección $\mathbf{r}$ en el plano $(u, v) \ldots \ldots$. . . . . . 311 


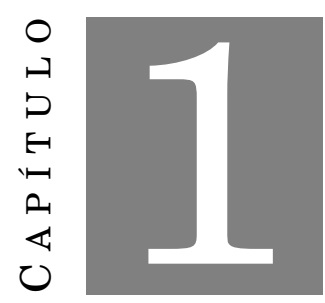

\section{INTRODUCCIÓN}

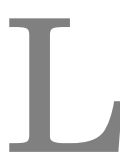

as estrellas Be son objetos tempranos no supergigantes que presentan o han presentado alguna vez líneas en emisión de hidrógeno, en especial $\mathrm{H} \alpha$, y de metales una vez ionizados (Jaschek et al., 1981; Collins, 1987; Rivinius et al., 2013). Esta definición abarca más de un tipo de estrellas B con líneas en emisión, por lo que se habla de estrellas Be clásicas para diferenciarlas de grupos tales como las estrellas Herbig Ae-Be (objetos de presecuencia principal), o sistemas binarios tipo Algol. La definición de estrellas Be clásicas incluye no solo estrellas de tipo espectral $\mathrm{B}$, sino que se extiende desde los tipos espectrales $\mathrm{O}$ tardíos hasta $\mathrm{A}$ tempranos. En esta Tesis, al hablar de estrellas Be nos estaremos refiriendo a las estrellas Be clásicas.

La formación de las líneas en emisión observadas en las estrellas Be es atribuida a la presencia de una envoltura circunestelar en forma de disco rodeando a la estrella central. Este disco sería además responsable del exceso infrarrojo (IR, 1.0 - $2.5 \mu \mathrm{m}$ ) observado en la distribución espectral de energía debido a la emisión libre-libre del mismo material circunestelar ionizado. Las líneas en emisión pueden formarse en una región muy cercana a la estrella (líneas de helio y de metales dos veces ionizados), en una región extendida del disco (líneas de hidrógeno), o relativamente lejos de la estrella (líneas de metales una vez ionizados; Rivinius et al., 2013). Por otra parte, el seguimiento temporal de las estrellas Be proporciona una clara evidencia de que estas estrellas presentan variabilidad fotométrica y espectroscópica en escalas de tiempo que van desde minutos hasta años (Porter \& Rivinius, 2003). Estos cambios pueden ser debidos a variaciones en la estructura física y al tamaño de la envoltura, o a la creación de un nuevo disco en eventos de expulsión de masa desde la estrella central.

Una característica común de las estrellas Be es que son rotadores rápidos. Struve (1931) fue el primero en proponer a la rotación como el principal mecanismo responsable de la formación del disco, debido a la pérdida de masa al disminuir la gravedad efectiva en el ecuador de la estrella. 
Para entender los procesos que dan lugar a la formación del disco es fundamental conocer cuan cerca se encuentra la velocidad de rotación de la estrella $(V)$ de la velocidad de rotación crítica $\left(V_{\text {crit }}{ }^{1}\right)$. Si la velocidad de rotación es cercana a la crítica, existen muchos procesos débiles que podrían entregar fácilmente material a la órbita. En cambio, si está por debajo, cualquier modelo teórico que trate de explicar el origen de los discos debe requerir de un incremento sustancial en el momento angular entre la fotosfera y el borde interior del disco (Cranmer, 2005). Muchos investigadores han obtenido valores de las velocidades de rotación proyectadas y han arribado a diferentes conclusiones (Slettebak, 1979; Fukuda, 1982; Grady et al., 1989; Balona, 1990; Porter, 1996; Steele et al., 1999; Yudin, 2001; Townsend et al., 2004; Cranmer, 2005; Frémat et al., 2005; Zorec et al., 2016). Algunos sostienen que las estrellas Be rotan con velocidades cercanas a la crítica, mientras que otros proponen que la probabilidad de que las estrellas Be sean rotadoras críticas es extremadamente baja y sugieren velocidades de rotación del orden de $0.7 V_{\text {crit }}$.

Las observaciones interferométricas han revelado más información acerca de las envolturas estelares, encontrando en algunos casos asimetrías a gran escala a lo largo de las direcciones polares que sugieren un viento polar (Kervella \& Domiciano de Souza, 2006; Meilland et al., 2007b), en contraste con el flujo de masa en el ecuador, mucho más lento y más denso, que no está de acuerdo con la teoría estándar del viento impulsado por radiación (Castor et al., 1975; Pauldrach et al., 1986; Friend \& Abbott, 1986). Las investigaciones hechas en una pocas estrellas han confirmado que sus discos están en rotación cuasi-Kepleriana (Meilland et al., 2007b; Delaa et al., 2011; Kraus et al., 2012; Wheelwright et al., 2012; Meilland et al., 2012; Cochetti et al., 2019).

Frecuentemente, los discos de las estrellas Be se modelan considerando una distribución de densidad con forma de ley de potencia simple en la dirección radial (Waters, 1986; Cote \& Waters, 1987; Waters et al., 1987; Jones et al., 2008; Silaj et al., 2014). Por lo general, los valores para el índice de la ley de potencia radial se encuentran en el rango 2.0-3.5, según los ajustes de $\mathrm{H} \alpha$ o el continuo IR (Waters, 1986; Silaj et al., 2014; Vieira et al., 2017). Vieira et al. (2017) han definido intervalos para el valor del exponente de la ley de potencia, relacionados con el estado evolutivo del disco (formación, disipación o estabilidad).

El modelo que ha logrado explicar muchas de las observaciones en las estrellas Be, y por lo tanto el más aceptado (Carciofi et al., 2012), es el de un "Disco de decreción viscoso" propuesto originalmente por Lee et al. (1991, VDD por sus siglas en inglés). Este modelo sugiere que la formación del disco en rotación cuasi-Kepleriana se debe a la transferencia de impulso angular desde el interior a la superficie de la estrella mediante algún mecanismo, inyectándose desde allí material al disco. Posteriormente, Krtička et al. (2011) examinaron los mecanismos de pérdida de masa y de momento angular a través de un disco de decreción ecuatorial asociado con la rotación casi crítica. Estos autores enfatizaron el papel del acoplamiento viscoso en el transporte del momento angular en la región externa del disco de decreción y la ablación por radiación de la

\footnotetext{
${ }^{1}$ La velocidad de rotación critica es la velocidad de rotación de una estrella en la cual se iguala la fuerza centrífuga y la fuerza gravitatoria en el ecuador.
} 
región más cercana a la estrella. En este contexto, se ha demostrado que los modelos de ablación de disco impulsados por radiación pueden llevar a la destrucción de un disco ópticamente delgado (como los observados alrededor de una estrella $\mathrm{Be}$ ) en una escala de tiempo dinámica del orden de meses a años (Kee et al., 2016), sin la necesidad de considerar una fuerte difusión viscosa anómala.

El mecanismo preciso para explicar la formación de los discos de las estrellas Be, así como sus propiedades físicas todavía son tema de debate (para una revisión completa del fenómeno, ver Rivinius et al., 2013). Además, la mayor parte de los estudios mencionados anteriormente se basan en el modelado de la distribución espectral de energía y en el ajuste de líneas espectrales en el rango óptico. La línea más usada para modelar es $\mathrm{H} \alpha$, la cual nos permite obtener información de las propiedades promedio del disco ya que se forma en una extensa región del mismo.

A fin de investigar la estructura y geometría de la parte más interna del disco de las estrellas Be, que podría darnos información sobre su formación y posterior evolución, proponemos utilizar datos espectroscópicos e interferométricos en el IR. Esta región espectral se encuentra menos estudiada que el rango óptico, ya que recién en las últimas décadas se ha logrado contar con una instrumentación adecuada que brinde datos con alta resolución espectral (espectrógrafos del IR cercano y medio en grandes telescopios) y espacial (interferometría óptica). Esta región espectral ha demostrado ser de gran utilidad para los fines que buscamos (Lenorzer et al., 2002b; Mennickent et al., 2009; Granada et al., 2010; Sabogal et al., 2017).

El objetivo principal de esta Tesis se centra en investigar la geometría, estructura física y evolución de las envolturas circunestelares que se desarrollan alrededor de las estrellas con fenómeno Be, con el fin de aportar elementos relevantes para el estudio de los posibles mecanismos que dan lugar a este fenómeno. Para eso realizamos un estudio exhaustivo de datos IR (espectroscópicos e interferométricos) de una muestra de estrellas Be. La Tesis está organizada de la siguiente manera:

- En el Capítulo 2 presentaremos las características más relevantes de las estrellas Be.

- En el Capítulo 3 realizaremos una descripción del rango espectral IR y de las principales características espectroscópicas encontradas en esta región espectral en las estrellas tempranas. Presentaremos además la muestra seleccionada para ser observada en el IR y las observaciones realizadas. Al finalizar el capítulo mostraremos, a modo de atlas, los espectros obtenidos, junto con una breve descripción de los mismos. La metodología utilizada para el análisis cuantitativo de las líneas espectrales observadas en cada uno de los objetos será descripta en el Capítulo 4. En el Capítulo 5 presentaremos el análisis realizado para los objetos de la muestra que han sido observados en una sola oportunidad, mientras que los que han sido observados en varias oportunidades serán presentados en el Capítulo 6. Los resultados generales obtenidos a partir del análisis de la muestra completa de estrellas Be observadas en el IR cercano se presentarán en el Capítulo 7. 
- En el Capítulo 8 presentaremos un resumen de resultados obtenidos a partir de observaciones interferométricas de estrellas Be disponibles en la literatura, y describiremos la instrumentación utilizada para nuestras observaciones junto al modelo adoptado para nuestro estudio. Analizaremos los resultados individuales para cada objeto de la muestra, y finalizaremos el capítulo con los resultados generales a partir de una muestra más amplia de objetos.

- En el Capítulo 9 realizaremos un análisis conjunto de los resultados alcanzados a partir de las dos técnicas utilizadas.

- Las conclusiones más importantes de este trabajo, junto con las perspectivas a futuro, serán presentadas en el Capítulo 10.

Además, presentaremos 3 apéndices con material complementario. En los Apéndices A y B mostraremos las tablas correspondientes a los capítulos 5 y 6 , mientras que en el Apéndice $\mathbf{C}$ presentaremos una introducción a los conceptos de interferometría utilizados en el capítulo 8 . 


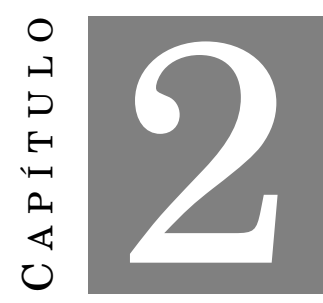

\section{LAS ESTRELLAS Be}

$\mathrm{E}$

n este capítulo presentaremos una descripción general de las estrellas con fenómeno Be, principal objeto de estudio de esta tesis. Detallaremos el conocimiento actual acerca de su proporción, estado evolutivo y rotación, las principales características espectroscópicas observadas en distintos rangos espectrales, su variabilidad y distintos modelos propuestos para explicar el origen y la estructura de sus discos.

\subsection{Proporción de estrellas Be y estado evolutivo}

En la Vía Láctea, las estrellas Be representan aproximadamente el 17\% de las estrellas B, con una mayor fracción (34 $\pm 1 \%$ ) en los tipos espectrales B1-B2 (Zorec \& Briot, 1997). Zorec et al. (2005) estudiaron el estado evolutivo de las estrellas Be, obteniendo la edad en unidades de tiempo de vida en la secuencia principal. Sus resultados indican que dependiendo del tipo espectral, el fenómeno Be se manifiesta en distintos estados evolutivos: para las estrellas B tardías, la fase Be aparece en la segunda mitad de su tiempo en la secuencia principal; para las estrellas $\mathrm{B}$ intermedias, el fenómeno Be pueden aparecer en toda la secuencia principal y permanecer hasta finalizar la misma; en cambio, para las estrellas B más tempranas, la fase Be se observa solo en la primera parte de la secuencia principal. Este resultado concuerda con lo encontrado por Aidelman et al. (2018) mediante el análisis de estrellas Be en cúmulos abiertos: en cúmulos jóvenes y de edad intermedia, el máximo según el tipo espectral se encuentra en estrellas B2-B4, mientras que en los cúmulos de mayor edad el máximo se desplaza hasta los tipos espectrales B6-B8.

La metalicidad es un factor que afecta la aparición del fenómeno Be, debido a que modifica la efectividad de la pérdida de momento angular a través de los vientos y la efectividad de los procesos de disipación. Maeder et al. (1999) encontraron que la fracción media de estrellas Be en 
cúmulos abiertos aumenta cuando disminuye la metalicidad, probablemente como consecuencia de la mayor velocidad de rotación. Estos resultados fueron confirmados para una muestra más grande por Wisniewski \& Bjorkman (2006), Martayan et al. (2006) y Martayan et al. (2007). Posteriormente Martayan et al. (2010) determinaron que para las estrellas B0-B3 la frecuencia de estrellas Be es 3 a 5 veces más grande en la Pequeña Nube de Magallanes (PNM) que en la Vía Láctea (VL). Los altos valores para la tasa de rotación en la secuencia principal de edad cero (ZAMS por sus siglas en inglés) para baja metalicidad, especialmente en la PNM, indican que algunas estrellas Be pueden nacer como Be, y debido a la baja metalicidad, mantener esa peculiaridad a lo largo de toda la secuencia principal.

Respecto a la distribución por tipos espectrales según la metalicidad, Martayan et al. (2006, 2007) encontraron que para bajas metalicidades la distribución es similar a la de la VL, indicando que la distribución no depende directamente de la metalicidad.

\subsection{Rotación}

A partir del ensanchamiento de las líneas fotosféricas se puede obtener la velocidad de rotación de una estrella, proyectada en la dirección de la línea de la visual, $V \sin i$. En el caso de las estrellas Be se obtienen valores de varios cientos de km/s (Slettebak, 1982; Uesugi \& Fukuda, 1981).

Existe un consenso general sobre la importancia de la rotación en los procesos de eyección de masa necesarios para formar los discos de las estrellas Be. Chauville et al. (2001) y Zorec (2004) encontraron que estas estrellas tienen velocidades de rotación de entre un $70 \%$ y $80 \%$ de su velocidad de rotación crítica. Esta proximidad a la velocidad de rotación crítica disminuye la gravedad efectiva en el ecuador y favorece la creación de una envoltura. Por otro lado, Frémat et al. (2005) obtuvieron un valor medio para la velocidad de rotación de las estrellas Be del orden de $88 \%$ de su $V_{\text {crit }}$. Cranmer (2005) mostró que las estrellas Be más calientes tienen una distribución uniforme para la velocidad de rotación entre $40 \%$ y $100 \%$ de sus $V_{\text {crit }}$, mientras que las más frías son todas rotadores casi críticos.

Meilland et al. (2012) encontraron un valor medio de $\overline{V / V_{\text {crit }}}=0.82 \pm 0.08$ que corresponde con una tasa de rotación de $\overline{\Omega / \Omega_{\text {crit }}}=0.95 \pm 0.02{ }^{1}$. Esto significa que solo una pequeña cantidad de energía es necesaria para expulsar material material de la superficie estelar.

Es importante tener en cuenta que en las estrellas que tienen velocidades de rotación cercanas al $80 \%$ de su $V_{\text {crit }}$ las determinaciones de $V \sin i$ pueden estar subestimadas por oscurecimiento gravitatorio. Este oscurecimiento hace que la distribución de temperatura y flujo de la estrella dependa de la latitud (Stoeckley, 1968; Collins \& Truax, 1995; Owocki, 2004; Zorec, 2004; Townsend et al., 2004; Rivinius et al., 2013). Este resultado fue corroborado por observaciones interferométricas por van Belle (2012), quien notó que los valores de achatamiento obtenidos siempre exceden los predichos a partir de $V \sin i$.

\footnotetext{
${ }^{1} \mathrm{La}$ tasa de rotación $\overline{\Omega / \Omega_{\text {crit }}}$ es el cociente entre la velocidad angular de rotación y la velocidad angular de rotación crítica.
} 
Zorec et al. (2016) estudiaron una muestra de 233 estrellas y transformaron la distribución de velocidades de rotación proyectada en una distribución de velocidad de rotación verdadera. Como el valor de $V / V_{\text {crit }}$ se hace más grande a medida que la estrella evoluciona desde la secuencia principal a fases más evolucionadas, la distribución de las velocidades de rotación verdaderas dependerá del estado evolutivo de la muestra analizada. Estos autores concluyeron que una vez que la moda de la distribución de $V / V_{\text {crit }}$ de la estrellas Be llega a 0.77 en la secuencia principal, se mantiene estable hasta el final de la misma. Las correcciones estadísticas por sobrestimaciones de $V \sin i$, causadas por macroturbulencia y binaridad, generan un corrimiento en la distribución hacia valores más bajos cuando todas las etapas evolutivas de la secuencia principal se consideran juntas. Finalmente, la distribución final obtenida tiene una moda en $V / V_{\text {crit }}=0.65$ con una distribución casi simétrica, mostrando que el fenómeno Be está presente en un amplio rango de velocidades de rotación $\left(0.3<V / V_{\text {crit }}<0.95\right)$. Con este resultado, la probabilidad de que las Be sean rotadores críticos es muy baja.

Modelos teóricos de Puls et al. (2008) predicen que para bajas metalicidades los vientos estelares impulsados por radiación son menos eficientes. En acuerdo con ese resultado, Bouret et al. (2003) y Mokiem et al. (2007) encontraron un gradiente en la tasa de pérdida de masa con la metalicidad, comparando valores en la VL, la Gran Nube de Magallanes (GNM) y la PNM ( $\mathrm{Z}=0.020,0.008$ y 0.004 respectivamente; Westerlund, 1997). Debido a los vientos más débiles y la pérdida de masa menor, las estrellas pierden menor momento angular y rotan más rápido (Ekström et al., 2008), lo que fue confirmado observacionalmente para estrellas O, B y Be en la VL, GNM y PNM (Keller, 2004; Hunter et al., 2008; Martayan et al., 2006, 2007).

Martayan et al. (2007) determinaron la distribución de la velocidad de rotación en la ZAMS para las estrellas Be en la VL, GNM y PNM. Estos autores observaron que la velocidad de rotación en la ZAMS depende de la masa, siendo el gradiente de esta distribución independiente de la metalicidad. Encontraron además que la velocidad de rotación aumenta para menores metalicidades.

\subsection{Características espectroscópicas}

En la región del visible (4000 - 8000 Å) la característica más destacada es la presencia de líneas de la serie de Balmer en emisión. El carácter de la emisión difiere de una estrella a la otra (ver Fig. 2.1) y puede también ser variable en el tiempo para una misma estrella. Esta variabilidad temporal se observa no solo en el cambio de la cantidad de líneas observadas, sino también en la modificación de la forma de los perfiles y la intensidad de las líneas, manteniéndose la intensidad decreciente desde $\mathrm{H} \alpha$ hacia los miembros superiores de la serie.

En las estrellas Be se observan dos tipos diferentes de espectro: el espectro Be y el espectro Be de envoltura o shell. Las líneas en emisión del espectro Be presentan típicamente uno o dos máximos intensos. En este último caso la parte central de la línea tiene una inversión en la 
Figura 2.1: Ejemplos de perfiles de H $\alpha$ en emisión, extraído de Gray \& Corbally (2009).

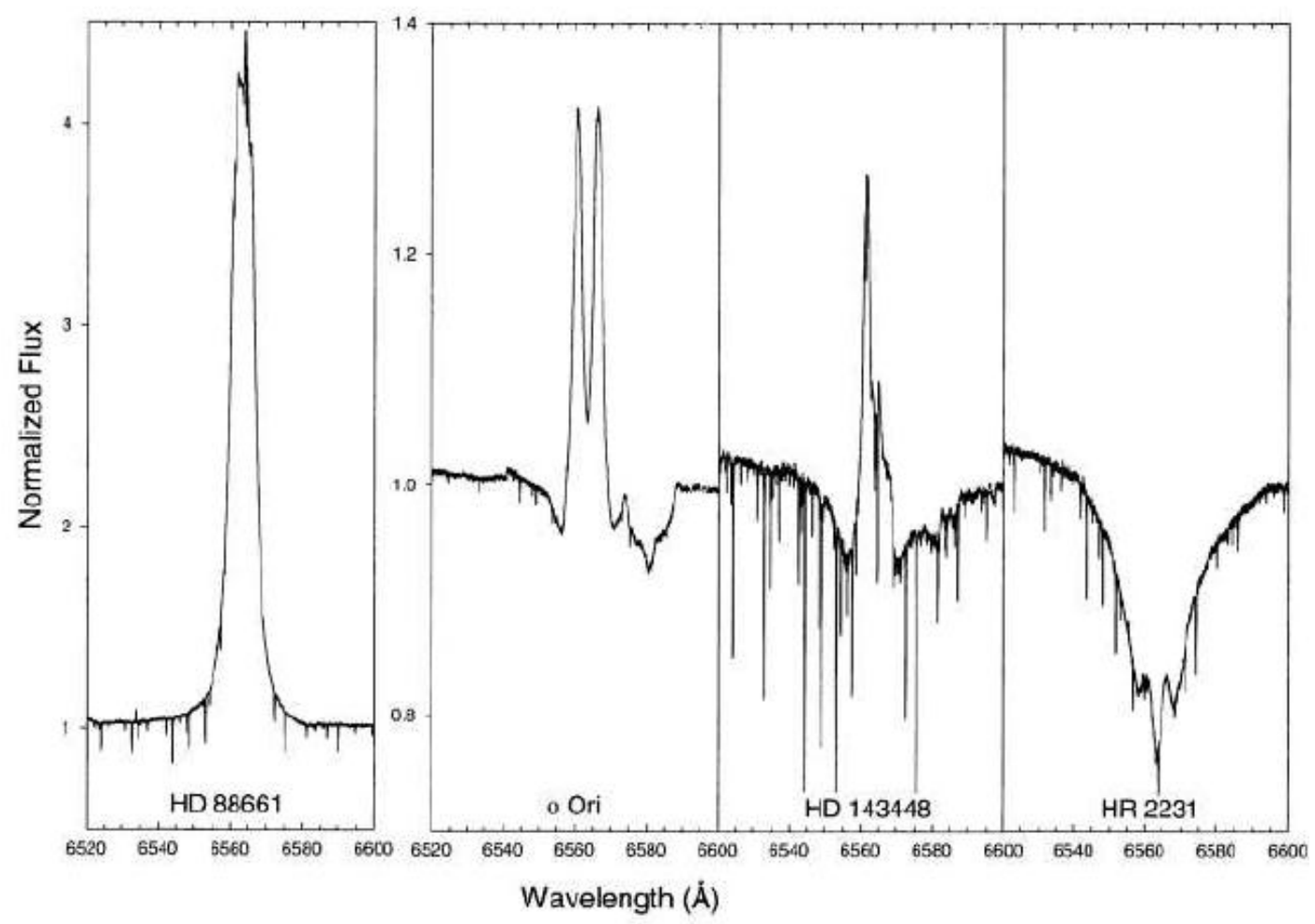

intensidad que no alcanza a cruzar el nivel del flujo continuo emergente de la fotosfera estelar. En cambio, en el espectro Be-shell los núcleos centrales de las líneas son delgados y en absorción, atravesando el nivel del continuo, pudiendo presentar o no las alas en emisión. Ejemplos de estos perfiles se muestran en la Fig. 2.2, donde se observan perfiles con un pico (por ejemplo $\mathrm{H} \alpha$ en el caso A), perfiles con dos picos (como $\mathrm{H} \alpha$ en el caso B) y perfiles tipo shell (por ejemplo $\mathrm{H} \beta$ en el caso D). En los espectros de las estrellas Be se observan además numerosas líneas angostas en absorción de metales una vez ionizados. En el caso de las líneas con dos picos en emisión, la separación de éstos correlaciona con el ancho a mitad de altura (FWHM - full width at half maximum) medido en las líneas fotosféricas (Struve, 1931), ya que correlaciona con el valor de $V \sin i$, y con el tamaño de la región emisora (Hanuschik, 1996). Sin embargo, se debe tener en cuenta los efectos de la profundidad óptica, especialmente en la línea $\mathrm{H} \alpha$, ya que los mismos afectan los parámetros de la correlación (Hummel, 1994). Tanto la separación de los picos como las velocidades radiales medidas en las líneas en emisión son mayores en las estrellas con espectros de envoltura que en las estrellas con espectro Be. La diferencia entre los espectros Be y Be-shell fue originalmente atribuida a un efecto geométrico relacionado con el ángulo de inclinación del eje de rotación de la estrella respecto a la visual, como pueden observarse en la Fig. 2.2. Vemos entonces que los espectros tipo Be con un pico corresponden con estrellas vistas de polo (A), los Be-shell con estrellas vistas desde el plano del ecuador (D), y los Be con dos picos 
Figura 2.2: Forma esquemática de los perfiles de líneas según el ángulo de inclinación, extraída de Rivinius et al. (2013).
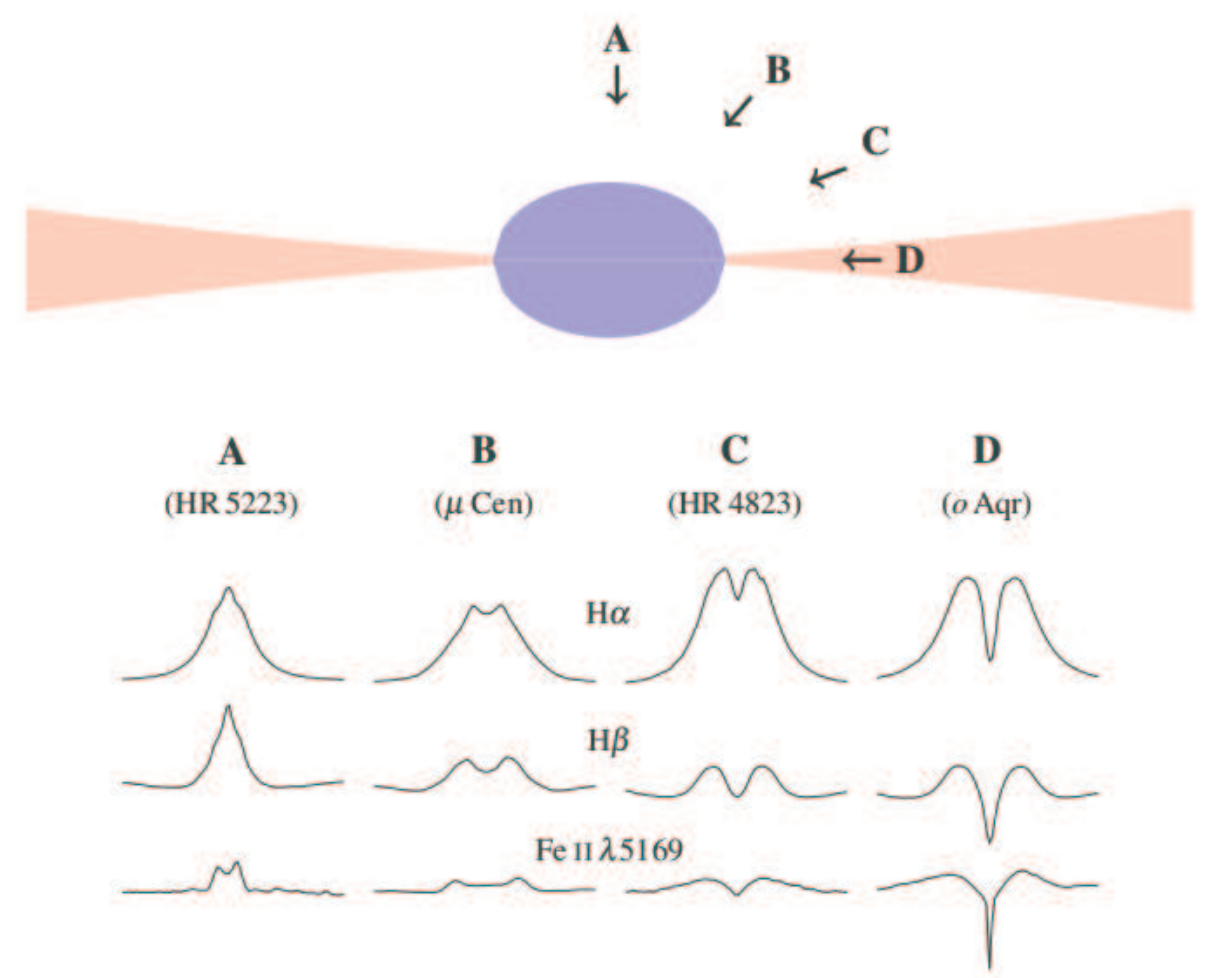

con ángulos de inclinación intermedios (B-C). Este modelo fue propuesto por Struve (1931).

Las líneas de hidrógeno son ópticamente gruesas y el mecanismo de formación dominante es la recombinación, mientras que para muchos metales las líneas son ópticamente delgadas. A diferencia de las líneas de hidrógeno, que se forman en una gran parte del disco, las líneas de helio se forman en una región muy cercana a la estrella (Rivinius et al., 2013).

Además, dependiendo de la orientación en que se observa la estrella (polar o ecuatorial) otros efectos son observados. Para líneas ópticamente gruesas, el ensanchamiento por scattering no coherente desplaza el pico cuando se observa desde el polo y crea un perfil de tipo "pico de botella" (Hummel, 1994), como en el caso A de la Fig. 2.2. Si se observa desde el ecuador, la emisión del disco no solo es autoabsorbida, sino que además, tapa la emisión de la estrella central, por lo que se observan líneas en absorción estrechas y profundas. Esto sucede generalmente para las líneas de $\mathrm{H}$ y metales una vez ionizados, pero en estrellas Be tempranas el He I también puede mostrar esta característica.

Los parámetros observados de las líneas en emisión incluyen el ancho equivalente (EW equivalent width), la altura del pico respecto a la intensidad del continuo $(E / C)$, el ancho a mitad de altura (FWHM - full width at half maximum) y el cociente entre la intensidad de los dos picos en emisión (relación V/R). 
Figura 2.3: Ejemplos de ajuste de perfiles P Cygni de C IV en 1548.2-1550.8 Å (línea punteada), para una estrella Be (izquierda) y una B (derecha). Para HD 28497 (Be) se modelaron también múltiples componentes discretas en absorción. Extraído de Prinja (1989).

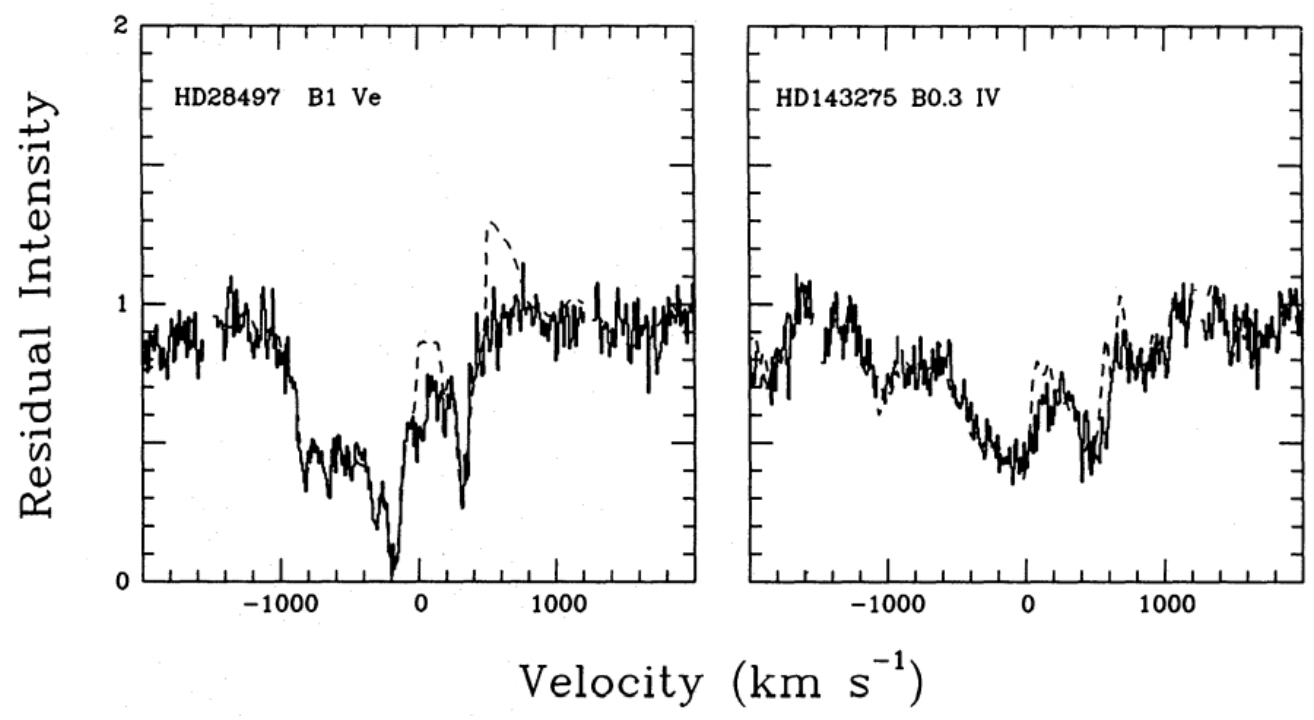

Las estrellas Be presentan también un exceso en el continuo IR, asociado con emisión librelibre y libre-ligado proveniente del disco. La estructura en temperatura es difícil de determinar a partir del exceso en IR, pero a partir de los modelos de Waters (1986) y Poeckert \& Marlborough (1978) se obtiene que la temperatura en el plano del disco es aproximadamente constante con el radio. En configuraciones más complejas, incluyendo metales, se obtiene que la temperatura disminuye con el radio.

En la región UV del espectro (100 - 4000 Å), las estrellas Be presentan líneas en absorción de metales de baja ionización. También se observan intensas líneas de resonancia de elementos altamente ionizados como O VI, N V, Si IV y C IV, que pueden ser altamente asimétricas con perfiles P Cygni (indicando la presencia de vientos) o presentar componentes múltiples en absorción desplazadas hacia el violeta (que son interpretadas como evidencia de un flujo de materia alejándose de la fotosfera, Snow, 1979). La existencia de estas líneas de elementos muy ionizados con abundancias anómalas se conoce como fenómeno de superionización. En las estrellas Be dicho fenómeno aparece hasta tipos espectrales tan tardíos como B8. Este fenómeno es atribuido a la existencia de una fuente de energía no radiativa, ya que el campo de radiación de una estrella de tipo B tardío no alcanzaría para producir esos iones. Superpuestos a las líneas de alta ionización se distinguen componentes de absorción discretas como las que se muestran en la Fig. 2.3. Las mismas se observan desplazadas hacia longitudes de onda más cortas, desde velocidades bajas hasta velocidades de aproximadamente $1000 \mathrm{~km} / \mathrm{s}$, no son observadas en estrellas B normales y se deberían a la propagación de perturbaciones de densidad en el plasma circunestelar (Underhill \& Doazan, 1982; Baade, 2000).

En la región de los rayos X, las estrellas Be aisladas son levemente más activas que las 
estrellas B normales (Cohen et al., 1997; Cohen, 2000). Sin embargo, en algunas estrellas Be en sistemas binarios la radiación en rayos $\mathrm{X}$ es muy intensa y variable, en escalas de tiempo de semanas a años (Coe, 2000). Esto podría suceder si el acompañante de la estrella Be fuera un objeto compacto en una órbita excéntrica, acretando material de la estrella Be (Coe et al., 2000).

\subsection{Variabilidad}

Una de las características más notorias de las estrellas Be es su variabilidad, explícita desde la definición de estrella Be. Esta variabilidad, tanto espectroscópica como fotosférica, se presenta en varias escalas de tiempo y difiere de una estrella a otra.

\subsubsection{Transiciones $B \leftrightarrow B e \leftrightarrow B e-s h e l l$}

Las transiciones $\mathrm{B} \leftrightarrow \mathrm{Be} \leftrightarrow \mathrm{Be}$-shell son las transformaciones de un espectro Be en uno Be-shell o en uno B normal, con intervalos de tiempo variables entre estrellas. Por ejemplo, $\gamma$ Cas exhibió dos fases Be-shell separadas por cuatro años en un siglo (Baldwin, 1939, 1940, 1941); para 59 Cyg el intervalo entre dos fases Be-shell fue de un año (Barker, 1982); y para 28 Tau fue de 35 años (Gulliver, 1977).

La apariencia de los espectros Be y Be-shell fue originalmente atribuida a un efecto geométrico, donde la apariencia dependía del ángulo de inclinación del eje de rotación de la estrella respecto al observador, por lo cual transiciones entre un tipo y otro no era esperables. Hummel (1998) propuso que las variaciones $\mathrm{Be} \leftrightarrow$ Be-shell podían explicarse suponiendo que el plano ecuatorial de la estrella y el plano orbital de la envoltura, supuesta en forma de disco, están inclinados uno respecto del otro y, por lo tanto, el disco precesa presentando distintas orientaciones hacia el observador. La dificultad más importante de este modelo reside en el hecho de que la precesión generaría una variación periódica, cuando los cambios de fase $\mathrm{Be} \leftrightarrow \mathrm{Be}$-shell observados no siempre lo son. El modelo de disco en precesión también ha sido ideado para explicar las variaciones fotométricas extraordinarias de $\gamma$ Cas. Zorec et al. (2000) explicaron con mejor fidelidad la curva de luz observada en esta estrella suponiendo que hubo una eyección discreta de masa en forma de protuberancia gigante. Esta masa quedó luego rotando en torno de la estrella por un tiempo dado, disipándose posteriormente. Este escenario puede explicar, de manera más razonable, las variaciones fotométricas irregulares observadas.

Las variaciones $\mathrm{Be} \leftrightarrow \mathrm{B}$ podrían indicar un fuerte cambio en la distribución del material circunestelar, implicando en algunos casos, la pérdida completa del disco y su posterior reconstrucción. Por ejemplo, $\theta$ CrB mostró un intenso espectro shell hasta 1980, cuando su espectro se transformó en B normal (Doazan et al., 1986). Se observaron fenómenos similares en o And (Gulliver et al., 1980) y en $v$ Pup (Rivinius et al., 1999). El estudio de este fenómeno puede aportar información útil sobre el mecanismo que da origen a la formación y disipación del disco (Gayley et al., 1999; Bjorkman, 2000; Rivinius et al., 2013). 


\subsubsection{Variaciones $\mathrm{V} / \mathrm{R}$}

Para las típicas líneas con dos picos en emisión, llamamos $\mathrm{V}$ y $\mathrm{R}$ a las alturas con respecto al continuo de los picos azul y rojo, respectivamente. Se han observado variaciones de largo período (de un par de semanas a décadas) en el cociente V/R, especialmente en la línea H $\alpha$. Estas variaciones son independientes del tipo espectral de la estrella central.

Las variaciones con períodos de algunas semanas usualmente están asociadas a sistemas binarios, ya que la acción de la estrella secundaria más la influencia del campo de velocidades genera deformaciones en el disco (Arias et al., 2004). Por otro lado, las variaciones de períodos más largas se asocian a la evolución de una onda de densidad o brazo espiral en el disco (Hesselbach, 2009). Para explicar sucesivas variaciones en necesario que esa onda de densidad dé varias vueltas al disco antes de disiparse, por lo que la velocidad de rotación debe ser mucho mayor que la velocidad radial de expansión.

Para explicar otras variaciones se sugirió la presencia de un anillo ecuatorial elíptico circunestelar rotando alrededor de la línea de las ápsides, en el cual los átomos siguen órbitas Keplerianas. Este modelo logra buen acuerdo con las observaciones a partir del ajuste de parámetros geométricos (Huang, 1972, 1973; Albert \& Huang, 1974), pero no logra explicar un cociente $V / R<1$ para los miembros bajos de la serie de Balmer y $V / R>1$ para los miembros altos.

\subsubsection{Variaciones fotométricas}

El espectro continuo de las estrellas Be también sufre variaciones, generalmente irregulares, con intervalos de tiempo desde fracciones de días hasta décadas.

Las variaciones fotométricas periódicas o multiperiódicas de corto período (Balona, 1990, 1995; Oudmaijer \& Drew, 1997) pueden estar acompañadas de variaciones espectroscópicas, y no se encuentran en tipos espectrales más tardíos que B5 (Baade, 1989). Los cortos períodos indican que estas variaciones se originarían en regiones cercanas a la fotosfera estelar. Esas variaciones darían información de los mecanismos adicionales que permitirían a una estrella B convertirse en Be (Slettebak \& Snow, 1987), ya que como mencionamos anteriormente, no hay certeza de que la alta rotación sea suficiente para dar origen a la envoltura. El origen de estas variaciones es atribuido a pulsaciones no radiales (Baade, 1982; Rivinius et al., 2003; Smith, 2001), aunque también se ha propuesto que esos períodos son mejor explicados por la rotación estelar, con una modulación mantenida por un campo magnético (Sareyan et al., 2002).

Las variaciones fotométricas cuasi-cíclicas o irregulares en escalas de tiempo largas, también relacionadas con variaciones espectroscópicas, estarían asociadas con cambios en la estructura de densidad y temperatura del disco en la región próxima a la estrella central, causando variaciones en la opacidad y/o modificando el flujo de masa. 


\subsubsection{Erupciones}

A partir de observaciones del Hipparcos, Hubert \& Floquet (1998) y Hubert et al. (2000) detectaron aumentos de brillo en estrellas Be, en períodos que van desde los días a años. Estos aumentos de brillo (outbursts) pueden deberse a variaciones en la profundidad óptica de capas eyectadas por la estrella (Hubert et al., 2000) o eyecciones locales de masa (Zorec et al., 2000).

En las estrellas Be, la presencia de varios modos de pulsación podría contribuir a la eyección de masa, ya que la interferencia constructiva de distintos modos aumentaría localmente la velocidad de la capa superficial, ayudando a que se alcance la velocidad de ruptura de la estrella y se expulse material (Neiner, 2010). Neiner et al. (2013) sugirieron que eso se produjo en la estrella HD 49 330, en la cual se observó una activación transitoria de los modos g de pulsación a la vez que disminuían los modos $p$ durante un outbursts.

Estos eventos pueden ser una fuente de abastecimiento de material para el disco, además de contribuir a una eventual pérdida del mismo.

\subsection{Geometría del disco}

Los discos de las estrellas Be son discos en decreción ${ }^{2}$, en los que el material fluye desde la estrella. Las técnicas interferométricas, espectroscópicas y polarimétricas desarrolladas en los últimos años revelaron la estructura de las envolturas circunestelares, descartando una distribución de masa con simetría esférica (Domiciano de Souza et al., 2003; Kervella \& Domiciano de Souza, 2006; Stee, 2011). La idea de una envoltura achatada fue confirmada por Quirrenbach et al. (1994) por medio de observaciones con interferometría óptica de larga base. Sin embargo, la técnica no permite determinaciones independientes de la inclinación y el grosor del disco, ya que ambos parámetros afectan a como se observa el disco en el plano del cielo.

\subsubsection{Altura del disco y ángulo de apertura}

Si suponemos un disco delgado formado por un gas isotermo, la distribución vertical de densidad en equilibrio hidrostático será Gaussiana. En este caso, la altura de escala $H(r)$ depende del gradiente de presión del gas y la fuerza de gravedad de la estrella, adoptando la expresión (Bjorkman, 1997):

$$
H(r)=\frac{c_{s}}{V_{\text {orb }}} \frac{r^{3 / 2}}{R_{\star}^{1 / 2}} .
$$

La altura de escala es proporcional al cociente entre la velocidad del sonido $c_{s}$ y la velocidad orbital $V_{\text {orb }}$. Además, el ángulo de apertura aumenta con la distancia a la estrella.

\footnotetext{
${ }^{2}$ Rivinius et al. (2013) indica que el término de "disco de decreción" (decretion disk) se origina a partir de la palabra decretion, que originalmente significa "disminución". Un disco en decreción representa lo contrario a un disco de acreción, al menos en términos de la dirección del transporte de masa, siendo la propia estrella la que eyecta masa y origina el disco.
} 
Quirrenbach et al. (1997) encontraron un límite superior de $20^{\circ}$ para el ángulo de apertura de $\zeta$ Tau y otras estrellas Be, mientras que observaciones espectropolarimétricas de $\zeta$ Tau realizadas por Wood et al. (1997) solo podían ser explicadas en términos de una envoltura muy delgada con un ángulo de apertura de $2.5^{\circ}$ o una muy gruesa de $52^{\circ}$. Considerando los dos resultados anteriores, el disco de $\zeta$ Tau debía tener un ángulo de apertura pequeño. La hipótesis del disco delgado fue verificada por Carciofi et al. (2006) a partir del análisis de la polarización en el continuo de $\delta$ Sco.

Por otro lado, Hanuschik (1996) analizó una muestra de estrellas Be y Be-shell, y utilizando una distribución de inclinaciones al azar encontró un ángulo de apertura de $13^{\circ}$. Por otro lado, Porter (1996) encontró un ángulo de apertura característico de $5^{\circ}$ a partir del análisis de la distribución de la velocidad de rotación. Estos dos resultados no contradicen los resultados obtenidos a partir de la polarización, ya que brindan el ángulo de apertura a diferentes distancias de la estrella.

Otra señal que indica la presencia de un ángulo de apertura pequeño viene del hecho de que para casi todas las observaciones realizadas con interferometría óptica de larga base, el ángulo de polarización es perpendicular al disco. Esto está de acuerdo con los modelos de scattering para un disco ecuatorial delgado con poca contribución de material en la región polar (Quirrenbach et al., 1997). Si el disco fuera geométrica y ópticamente grueso, el ángulo de polarización debería ser paralelo al disco (Wood et al., 1996).

A partir de observaciones de las líneas de emisión de Fe II, Zorec et al. (2007) encontraron un valor más grande para el ángulo de apertura del disco en la región cercana a la estrella $\left(H \gtrsim 0.5 R_{\star}\right)$. Esto podría ser el resultado de la interacción entre el viento estelar y el disco, una distribución de temperatura fuera del equilibrio radiativo o la presencia de campos magnéticos de intensidad $\sim 10^{2} G$.

\subsubsection{Densidad del disco}

Históricamente, la mejor fuente de información sobre la densidad del disco fue la distribución espectral de energía (SED, por su siglas en inglés spectral energy distribution), ya que el exceso de flujo en el continuo depende de las propiedades físicas de la región emisora. El tamaño de estas regiones puede vincularse con las propiedades globales del disco a partir de la implementación de diferentes modelos. Por ejemplo, la aplicación del modelos de disco viscoso contribuyó a explicar el exceso IR encontrado en las estrellas Be con discos delgados (Porter, 1997).

Además de la distribución vertical Gaussiana, la densidad del disco cae radialmente según

$$
\rho(r)=\rho_{0}\left(\frac{r}{R_{\star}}\right)^{-n},
$$

donde $\rho_{0}$ es la densidad en la base del disco y $n$ es el exponente de la ley de potencia (Waters, 1986; Jones et al., 2008). Los valores obtenidos para $\rho_{0}$ y $n$ varían entre $10^{-9}-10^{-12} \mathrm{~g} \mathrm{~cm}^{-3}$ y 
Tabla 2.1: Tamaños máximos y mínimos para las regiones emisoras en diferente líneas/bandas recopilados por Rivinius et al. (2013).

\begin{tabular}{lcccc}
\hline \hline Línea / Banda & Mínimo $\left(R_{\star}\right)$ & Fuente & Máximo $\left(R_{\star}\right)$ & Fuente \\
\hline $\mathrm{H} \alpha$ & $3.24 \pm 0.23$ & Tycner et al. $(2005)$ & $16.36 \pm 0.82$ & Delaa et al. (2011) \\
\hline Banda H & $1.60 \pm 0.2$ & Che et al. (2012) & $5.64 \pm 0.66$ & Stefl et al. (2012) \\
\hline Banda K & $1.19 \pm 0.27$ & Touhami et al. (2013) & $13.40 \pm 3.7$ & Meilland et al. (2009) \\
\hline $8 \mu \mathrm{m}$ & $7.30 \pm 2.8$ & Chesneau et al. (2005) & $10.30 \pm 3.8$ & Meilland et al. (2009) \\
\hline $12 \mu \mathrm{m}$ & $10.90 \pm 4.2$ & Meilland et al. (2009) & $14.90 \pm 1.1$ & Meilland et al. (2009) \\
\hline \hline
\end{tabular}

1.5 - 3.5, respectivamente (Cote \& Waters, 1987; Waters et al., 1987; Silaj et al., 2010; Arcos et al., 2017).

Vieira et al. (2017) analizaron la la evolución de los discos utilizando el modelo VDD y encontraron que la escala de tiempo en la que el disco se desarrolla es menor que la escala de tiempo de disipación del mismo. Además, encontraron una correlación entre los valores de $\rho_{0}$ y $n$, y determinaron diferentes regiones en el plano $n-\log \rho_{0}$. En este diagrama, la región $n \lesssim 3$ está asociada con un disco en proceso de disipación, la franja entre $n \simeq 3$ y $n \simeq 3.5$ corresponde a una zona de estabilidad del disco, y la región donde $n \gtrsim 3.5$ se relaciona con un disco en formación.

Usando un código simple de transferencia radiativa, Touhami et al. (2011) encontraron que una ley de potencia para la densidad radial y un modelo Gaussiano para la vertical pueden reproducir el exceso de color de una muestra de 130 estrellas Be. Este resultado fue confirmado por Silaj et al. (2010) y Arcos et al. (2018) modelando el perfil de la línea H $\alpha$ en emisión.

\subsubsection{Tamaño del disco}

A partir de observaciones de una línea espectral determinada o de una banda en el continuo podemos obtener el tamaño de la región emisora correspondiente. Sin embargo, a menos que el disco este truncado por algún mecanismo y la región emisora coincida con la extensión total del mismo (como por ejemplo debido a la presencia de un sistema binario), el tamaño del disco de las estrellas Be es algo difícil de determinar observacionalmente.

El tamaño de la región emisora refleja las propiedades físicas del disco. La interferometría óptica de larga base es la única técnica que permite obtener mediciones directas del tamaño de la región emisora para el rango óptico. Los tamaños estimados provienen de ajustar los datos interferométricos con un modelo geométrico o físico, y los resultados dependerán del modelo adoptado.

La Tabla 2.1 lista los tamaños máximos y mínimos encontrados para las regiones emisoras en diferentes líneas y bandas. En la longitud de onda de $2 \mathrm{~cm}$ la única estimación realizada corresponde a $\psi$ Per, con una extensión de $450 R_{\star}$ (Dougherty \& Taylor, 1992). 


\subsection{4 Ángulo de posición}

La primera confirmación de que el ángulo de polarización es perpendicular al eje mayor del disco, como predicen los modelos de scattering, fue dada por Quirrenbach et al. (1997). El único caso reportado donde el ángulo de polarización no parece ser perpendicular al eje mayor es 48 Per (Delaa et al., 2011). Sin embargo, como 48 Per es una estrella vista casi de polo, la polarización intrínseca observada es muy débil y la determinación del ángulo de posición del disco a partir de la interferometría es poco precisa, ya que la envoltura se aparta poco de una forma circular. Además, la presencia de una onda de densidad complicaría aún más las determinaciones.

El ángulo de polarización parece no variar en muchas estrellas, pero existen excepciones. Las variaciones más extrañas están asociadas a transiciones $\mathrm{Be} \leftrightarrow$ Be-shell observadas, por ejemplo, en $\gamma$ Cas, 28 Tau y 59 Cyg. En los casos de $\gamma$ Cas y 59 Cyg las transiciones no se observan actualmente, pero si continúan observándose en 28 Tau. En esta estrella, se observó una transición Be-shell $\rightarrow$ Be durante 1988, y posteriormente una transición Be $\rightarrow$ Be-shell en 2006 (Tanaka et al., 2007). Durante ese tiempo, el sistema fue monitoreado polarimétricamente (Hirata, 2007) y el ángulo de polarización cambió de aproximadamente $60^{\circ}$ en la fase Be-shell a alrededor de $130^{\circ}$ en 2003 . Hummel (1998) sugirió que esas variaciones se deben a la presencia de un disco en precesión, debido a la influencia de la órbita de la binaria.

Cambios menores en el ángulo de posición podrían estar relacionados con transferencia de masa al disco y los ciclos V/R.

\subsection{Cinemática del disco}

Determinar la cinemática del disco es de gran importancia, debido a que la manera en la que el disco rota depende del mecanismo de formación que haya intervenido. Inicialmente, para los discos de las estrellas Be fueron propuestos tres modos de rotación:

- En un viento impulsado por las líneas, la fuerza dominante es la presión de radiación, que no ejerce torque. Por lo tanto, el momento angular del material se conserva, y la velocidad azimutal $V_{\phi}$ cae con $r^{-2}$.

- En un campo de velocidad Kepleriano, $V_{\phi} \propto r^{-1 / 2}$, el momento angular crece con $r^{1 / 2}$. Eso requiere un torque, como el que otorga un disco viscoso. En un disco Kepleriano, la velocidad radial en la estrella es nula $(0 \mathrm{~km} / \mathrm{s})$.

- En el caso de un plasma atrapado por un campo magnético fuerte y forzado a corotar con la estrella, $V_{\phi} \propto r$.

Observando la forma de las líneas en emisión y shell, esta última opción fue descartada. Sin embargo, para los dos primeros casos, las líneas generadas son muy similares. 
Evidencias teóricas de un disco Kepleriano provienen de las variaciones V/R, atribuidas a la presencia de una onda de densidad en forma de brazo espiral (Okazaki, 1991; Papaloizou et al., 1992). El hecho de que los períodos de precesión sean dos órdenes de magnitud más largos que los períodos orbitales de las partículas impone que el movimiento radial debe ser pequeño, ya que un movimiento no Kepleriano volvería el disco inestable.

Por otro lado, las pequeñas emisiones centrales observadas en estrellas Be-shell solo puede ocurrir si la velocidad radial es menor a unos cuantos km/s (Rivinius et al., 1999; Hanuschik et al., 1995). La misma conclusión se obtiene para las líneas metálicas en absorción (Rivinius et al., 2006). Esos resultados proveen evidencia de rotación cercana a la Kepleriana.

Finalmente, a partir de observaciones interferométricas con resolución espectral (espectrointerferometría) de líneas en emisión se comprobó la rotación Kepleriana (Meilland et al., 2007b; Delaa et al., 2011; Kraus et al., 2012; Wheelwright et al., 2012; Meilland et al., 2012; Cochetti et al., 2019). 



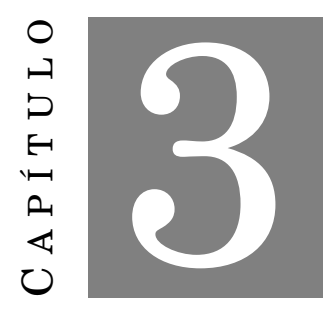

OBSERVACIONES EN EL IR CERCANO

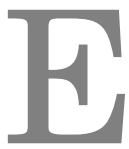

l espectro de las estrellas Be está formado por el espectro de la estrella central (mayormente una estrella de tipo espectral B), sumado a la emisión generada por la presencia de una envoltura circunestelar en forma de disco. En particular, en la región del infrarrojo (IR), las estrellas Be presentan un espectro poblado de numerosas líneas de recombinación de hidrógeno $(\mathrm{H})$ que, debido a la densidad necesaria para su formación, provienen de una región más interna del disco que las observadas en el rango óptico. Además, en muchas de ellas la contribución fotosférica resulta prácticamente despreciable. En consecuencia, estas líneas aportan información valiosa sobre las propiedades físicas, morfológicas y dinámicas de las envolturas de estas estrellas (Hony et al., 2000; Steele \& Clark, 2001; Lenorzer et al., 2002a; Mennickent et al., 2009; Granada et al., 2010; Sabogal et al., 2017).

A diferencia del rango óptico, la región del infrarrojo cercano $(0.8-5 \mu \mathrm{m})$ no ha sido aún suficientemente explorada. Existe un número limitado de trabajos en los cuales se describen las características infrarrojas de las estrellas Be, muchos de los cuales presentan espectros de baja resolución mientras que en otros las muestras de objetos son pequeñas. En general, el rango espectral observado es muy acotado, y en muchos casos abarca una sola de las bandas definidas en esta región. No obstante, se han logrado avances que han permitido extender los criterios de clasificación espectral, basados originalmente en características del rango óptico, a la región infrarroja del espectro. Esto ha permitido clasificar estrellas fuertemente enrojecidas como las presentes en regiones de formación estelar, donde no pueden aplicarse los criterios clásicos de clasificación.

Comenzaremos este capítulo con una descripción de la región espectral IR y de las principales características encontradas en el espectro de IR de las estrellas tempranas. Posteriormente, presentaremos la muestra seleccionada y las observaciones realizadas. Al finalizar el capítulo 
Figura 3.1: Ventanas atmosféricas en el IR cercano. Gráfico realizado a partir de información provista por el Observatorio Gemini (Fuente: Página web GEMINI). La transmisión corresponde a una masa de aire de 1 y una columna de vapor de agua de $1 \mathrm{~mm}$, para Mauna Kea (Hawai).

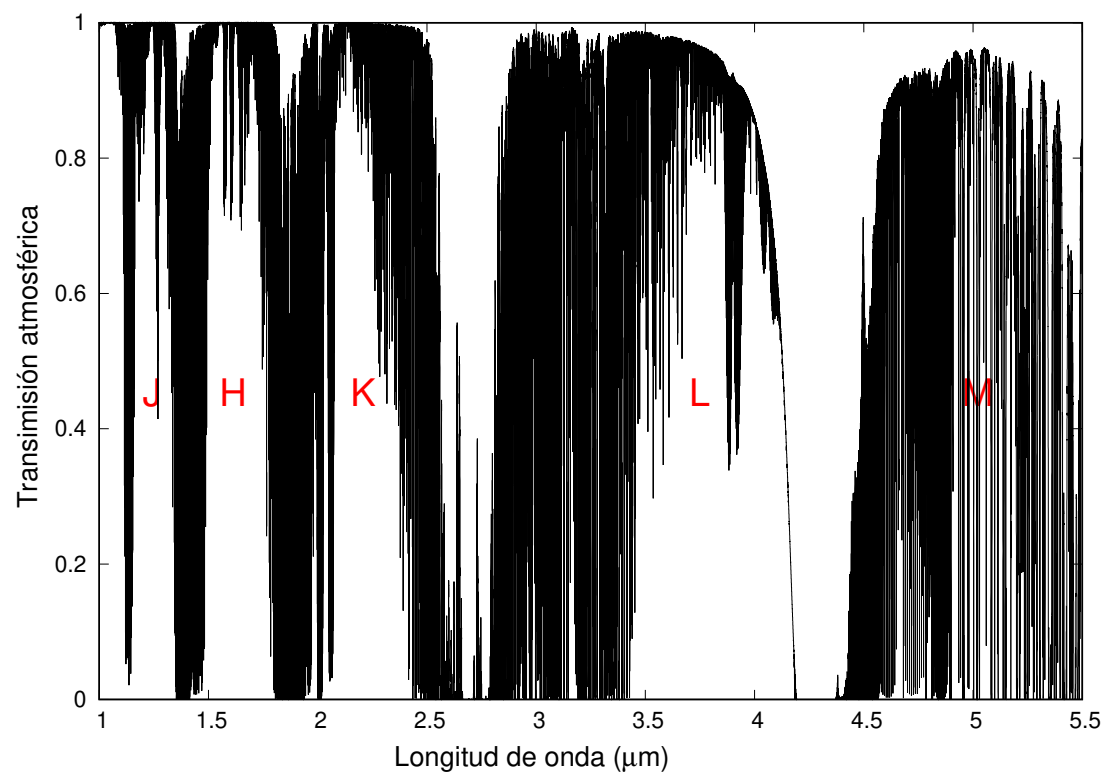

mostraremos, a modo de atlas, los espectros obtenidos junto con una breve descripción de los mismos.

\subsection{El espectro IR de las estrellas B}

Presentaremos a continuación las principales características del espectro en las bandas J, $\mathrm{H}, \mathrm{K}$ y $\mathrm{L}$ de las estrellas B y Be, y los criterios de clasificación en el IR disponibles en la literatura para estos objetos. La Fig. 3.1 ilustra la ubicación de las ventanas atmosféricas en el IR cercano, donde pueden observarse las bandas mencionadas. En la Tabla 3.1 se listan las líneas de H presentes en cada banda, junto con los niveles de energía involucrados en la transición y la longitud de onda correspondiente. En la Tabla 3.2 se detallan las líneas correspondientes a otros elementos.

\subsubsection{Estrellas normales}

Wallace et al. (2000) realizaron un catálogo con espectros en la banda J de 88 estrellas estándares de tipos espectrales O7-M6 y clases de luminosidades V-I. Estos autores encontraron que la línea de $\mathrm{H} \mathrm{Pa} \beta$ domina el espectro de las estrellas tempranas, con una intensidad máxima cerca del tipo espectral A0, y reportaron también la presencia de líneas de helio neutro (He I).

En la banda $\mathrm{H}$, el espectro está dominado por la presencia de líneas de helio neutro e ionizado (He I, He II) y de la serie de Brackett del H, desde la línea $\mathrm{Br}_{10}$ hasta el límite de la serie. A partir de observaciones de 34 estrellas estándares, Hanson et al. (1998) sugirieron un método de clasificación utilizando estas líneas. La línea de He II $(1.6930 \mu \mathrm{m})$ solo es detectada en estrellas 
Tabla 3.1: Líneas de hidrógeno presentes en las bandas J, H, K y L. Las longitudes de onda corresponden al vacío y fueron obtenidas a partir de la expresión $\frac{1}{\lambda}=R_{H}\left(\frac{1}{n_{1}^{2}}-\frac{1}{n_{2}^{2}}\right)$, donde consideramos la constante de Rydberg para el hidrógeno con valor $R_{H}=10.967758 \mu \mathrm{m}^{-1}$.

\begin{tabular}{|c|c|c|c|c|c|c|c|}
\hline \multicolumn{2}{|c|}{$\begin{array}{c}\text { Banda J } \\
(1.1-1.4 \mu \mathrm{m})\end{array}$} & \multicolumn{2}{|c|}{$\begin{array}{c}\text { Banda } \mathrm{H} \\
(1.45-1.85 \mu \mathrm{m})\end{array}$} & \multicolumn{2}{|c|}{$\begin{array}{c}\text { Banda } \mathrm{K} \\
(1.85-2.55 \mu \mathrm{m})\end{array}$} & \multicolumn{2}{|c|}{$\begin{array}{c}\text { Banda L } \\
(3.2-4.2 \mu \mathrm{m})\end{array}$} \\
\hline Línea & $\lambda$ & Línea & $\lambda$ & Línea & $\lambda$ & Línea & $\lambda$ \\
\hline \multirow{25}{*}{\multicolumn{2}{|c|}{$\mathrm{Pa} \beta(5-3) \quad 1.2822$}} & $\mathrm{Br}_{\text {lím }}(\infty-4)$ & 1.4588 & $\operatorname{Pa} \alpha(4-3)$ & 1.8756 & $\operatorname{Pf} \delta(9-5)$ & 3.2970 \\
\hline & & & & $\operatorname{Br} \delta(8-4)$ & 1.9451 & $\mathrm{Hu}_{\text {lím }}(\infty-6)$ & 3.2823 \\
\hline & & $\mathrm{Br}_{27}(27-4)$ & 1.4916 & $\operatorname{Br} \gamma(7-4)$ & 2.1661 & $\ldots$ & \\
\hline & & $\mathrm{Br}_{26}(26-4)$ & 1.4942 & $\mathrm{Pf}_{\text {lím }}(\infty-5)$ & 2.2794 & $\mathrm{Hu}_{31}(31-6)$ & 3.4101 \\
\hline & & $\mathrm{Br}_{25}(25-4)$ & 1.4971 & $\ldots$ & & $\mathrm{Hu}_{30}(30-6)$ & 3.4191 \\
\hline & & $\mathrm{Br}_{24}(24-4)$ & 1.5005 & $\mathrm{Pf}_{35}(35-5)$ & 2.3269 & $\mathrm{Hu}_{29}(29-6)$ & 3.4291 \\
\hline & & $\mathrm{Br}_{23}(23-4)$ & 1.5043 & $\mathrm{Pf}_{34}(34-5)$ & 2.3298 & $\mathrm{Hu}_{28}(28-6)$ & 3.4403 \\
\hline & & $\mathrm{Br}_{22}(22-4)$ & 1.5087 & $\mathrm{Pf}_{33}(33-5)$ & 2.3330 & $\mathrm{Hu}_{27}(27-6)$ & 3.4529 \\
\hline & & $\mathrm{Br}_{21}(21-4)$ & 1.5137 & $\mathrm{Pf}_{32}(32-5)$ & 2.3365 & $\mathrm{Hu}_{26}(26-6)$ & 3.4670 \\
\hline & & $\mathrm{Br}_{20}(20-4)$ & 1.5196 & $\mathrm{Pf}_{31}(31-5)$ & 2.3403 & $\mathrm{Hu}_{25}(25-6)$ & 3.4830 \\
\hline & & $\mathrm{Br}_{19}(19-4)$ & 1.5265 & $\mathrm{Pf}_{30}(30-5)$ & 2.3445 & $\mathrm{Hu}_{24}(24-6)$ & 3.5012 \\
\hline & & $\mathrm{Br}_{18}(18-4)$ & 1.5346 & $\mathrm{Pf}_{29}(29-5)$ & 2.3492 & $\mathrm{Hu}_{23}(23-6)$ & 3.5220 \\
\hline & & $\mathrm{Br}_{17}(17-4)$ & 1.5443 & $\mathrm{Pf}_{28}(28-5)$ & 2.3545 & $\mathrm{Hu}_{22}(22-6)$ & 3.5461 \\
\hline & & $\mathrm{Br}_{16}(16-4)$ & 1.5561 & $\mathrm{Pf}_{27}(27-5)$ & 2.3604 & $\mathrm{Hu}_{21}(21-6)$ & 3.5741 \\
\hline & & $\mathrm{Br}_{15}(15-4)$ & 1.5705 & $\mathrm{Pf}_{26}(26-5)$ & 2.3669 & $\mathrm{Hu}_{20}(20-6)$ & 3.6070 \\
\hline & & $\mathrm{Br}_{14}(14-4)$ & 1.5885 & $\mathrm{Pf}_{25}(25-5)$ & 2.3744 & $\mathrm{Hu}_{19}(19-6)$ & 3.6459 \\
\hline & & $\mathrm{Br}_{13}(13-4)$ & 1.6114 & $\mathrm{Pf}_{24}(24-5)$ & 2.3828 & $\mathrm{Hu}_{18}(18-6)$ & 3.6926 \\
\hline & & $\mathrm{Br}_{12}(12-4)$ & 1.6412 & $\mathrm{Pf}_{23}(23-5)$ & 2.3925 & $\operatorname{Pf} \gamma(8-5)$ & 3.7406 \\
\hline & & $\mathrm{Br}_{11}(11-4)$ & 1.6811 & $\mathrm{Pf}_{22}(22-5)$ & 2.4036 & $\mathrm{Hu}_{17}(17-6)$ & 3.7494 \\
\hline & & $\mathrm{Br}_{10}(10-4)$ & 1.7367 & $\mathrm{Pf}_{21}(21-5)$ & 2.4164 & $\mathrm{Hu}_{16}(16-6)$ & 3.8195 \\
\hline & & $\mathrm{Br}_{9}(9-4)$ & 1.8179 & $\mathrm{Pf}_{20}(20-5)$ & 2.4314 & $\mathrm{Hu}_{15}(15-6)$ & 3.9076 \\
\hline & & & & $\mathrm{Pf}_{19}(19-5)$ & 2.4490 & $\mathrm{Hu}_{14}(14-6)$ & 4.0209 \\
\hline & & & & $\mathrm{Pf}_{18}(18-5)$ & 2.4700 & $\operatorname{Br} \alpha(5-4)$ & 4.0523 \\
\hline & & & & $\mathrm{Pf}_{17}(17-5)$ & 2.4953 & $\mathrm{Hu}_{13}(13-6)$ & 4.1708 \\
\hline & & & & $\mathrm{Pf}_{16}(16-5)$ & 2.5261 & & \\
\hline
\end{tabular}

$\mathrm{O}$, siendo más intensa en las supergigantes $\mathrm{O}$ tardías que en las enanas del mismo tipo espectral. Para las estrellas $\mathrm{OB}$, la absorción en la línea $\mathrm{Br}_{11}$ es mayor en las estrellas más frías, siendo las líneas más anchas en las enanas que en las supergigantes. Para el caso de una estrella enana, si la misma presenta las líneas de He I $\lambda 1.7007 \mu \mathrm{m}$, He II $\lambda 1.6930 \mu \mathrm{m}$ y Br $\mathrm{Br}_{11}$ se corresponde con una estrella de tipo $\mathrm{O}$ tardío o más temprana. Si presenta débil $\mathrm{Br}_{11}$, He I con moderada intensidad y no presenta He II, la estrella tiene un tipo espectral entre O9 y B1. En cambio, si presenta $\mathrm{Br}_{11}$ con He I detectable, es de tipo espectral B2-B3. Las más tardías no presentan líneas de He I, pero presentan $\mathrm{Br}_{11}$ intensa. Por otro lado, Meyer et al. (1998) observaron una muestra de 85 estrellas estándares con tipos espectrales O7-M5 y clases de luminosidad V-I. Ellos reportaron, en acuerdo con Hanson et al. (1998), que la línea de He I es más intensa que las de la serie de Brackett para 
Tabla 3.2: Líneas de helio y metales presentes en las bandas J, H, K y L. Las longitudes de onda corresponden al vacío y fueron obtenidas en la base de datos NIST Atomic Spectra Database.

\begin{tabular}{|c|c|c|c|c|c|c|c|}
\hline \multicolumn{2}{|c|}{$\begin{array}{c}\text { Banda J } \\
(1.1-1.4 \mu \mathrm{m})\end{array}$} & \multicolumn{2}{|c|}{$\begin{array}{c}\text { Banda H } \\
(1.45-1.85 \mu \mathrm{m})\end{array}$} & \multicolumn{2}{|c|}{$\begin{array}{c}\text { Banda K } \\
(1.85-2.55 \mu \mathrm{m})\end{array}$} & \multicolumn{2}{|c|}{$\begin{array}{c}\text { Banda L } \\
(3.2-4.2 \mu \mathrm{m})\end{array}$} \\
\hline Elemento & $\lambda$ & Elemento & $\lambda$ & Elemento & $\lambda$ & Elemento & $\lambda$ \\
\hline $\mathrm{O} \mathrm{I}$ & 1.1290 & Fe I & 1.5299 & He I & 2.0587 & O I & 3.6620 \\
\hline C I & 1.1663 & $\mathrm{~N}_{\mathrm{I}}$ & 1.5586 & $\mathrm{Fe}$ II & 2.0892 & $\mathrm{He} \mathrm{I}$ & 3.7037 \\
\hline C I & 1.1673 & $\mathrm{Fe} \mathrm{I}$ & 1.5626 & $\mathrm{He} \mathrm{I}$ & 2.1126 & C I & 3.7223 \\
\hline C I & 1.1756 & $\mathrm{Fe}$ II & 1.5761 & Mg II & 2.1375 & $\mathrm{Fe}$ II & 3.8662 \\
\hline $\mathrm{Fe} I$ & 1.1786 & $\mathrm{Si}$ I & 1.5964 & Mg II & 2.1438 & $\mathrm{Fe}$ II & 3.9380 \\
\hline$?$ & 1.1838 & C I & 1.6009 & $\mathrm{He} \mathrm{I}$ & 2.1614 & C I & 3.9886 \\
\hline C I & 1.1899 & $\mathrm{C}_{\mathrm{I}}$ & 1.6026 & $\mathrm{Na} \mathrm{I}$ & 2.2062 & C I & 3.9994 \\
\hline He I & 1.1972 & Si I & 1.6064 & $\mathrm{Na} \mathrm{I}$ & 2.2090 & $?$ & 4.0360 \\
\hline O I & 1.2467 & $\mathrm{Fe} \mathrm{I}$ & 1.6236 & Mg II & 2.4048 & & \\
\hline $\mathrm{He} \mathrm{I}$ & 1.2530 & $\mathrm{Fe} \mathrm{I}$ & 1.6297 & Mg II & 2.4131 & & \\
\hline C I & 1.2565 & Si I & 1.6685 & $\mathrm{He} \mathrm{I}$ & 2.4730 & & \\
\hline C I & 1.2617 & Mg II & 1.6765 & & & & \\
\hline$?$ & 1.2689 & Fe II & 1.6787 & & & & \\
\hline $\mathrm{He} \mathrm{I}$ & 1.2788 & $\mathrm{Fe}$ II & 1.6878 & & & & \\
\hline He I & 1.2972 & C I & 1.6895 & & & & \\
\hline O I & 1.3168 & $\mathrm{He} \mathrm{I}$ & 1.7007 & & & & \\
\hline & & $\mathrm{Fe} I$ & 1.7119 & & & & \\
\hline & & $\mathrm{O}$ I & 1.8026 & & & & \\
\hline & & O I & 1.8247 & & & & \\
\hline
\end{tabular}

las estrellas más tempranas que B8, y se vuelve indetectable para las estrellas más tardías. En las estrellas B tardías y A tempranas dominan las líneas de la serie de Brackett.

En la banda K, se observan líneas de H, He, nitrógeno (N) y carbono (C) (Hanson et al., 1996). De las líneas de H, Br $\gamma$ es la más destacada. Las estrellas O3-O4 son las que no presentan el triplete de He I en $\lambda \sim 2.1126 \mu \mathrm{m}$, presentan He II $\lambda 2.1885 \mu \mathrm{m}$ en absorción, moderada absorción en $\mathrm{Br} \gamma$ y N III $\lambda 2.1155 \mu \mathrm{m}$ en emisión. Las estrellas O5-O6 presentan las mismas características que las O3-O4, junto con la línea de C IV $\lambda 2.0780 \mu \mathrm{m}$. Las estrellas O7-O8 presentan simultáneamente $\mathrm{N}$ III en emisión y He I en absorción, moderada absorción en $\mathrm{Br} \gamma$ y He II en absorción. El grupo O9-B1 no presenta líneas de He II, C IV ni N III, pero presenta He I y Br $\gamma$ con intensidad moderada. Las estrellas B2-B3 presentan He I en absorción y Br $\gamma$ intensa. Una vez que el triplete del He I en $\lambda \sim 2.1126 \mu$ m se vuelve imperceptible, la intensidad de $\mathrm{Br} \gamma$ nos permite distinguir entre las estrellas de tipo B intermedio B4-B7 (línea intensa) y tardío B8-A3 (línea muy intensa).

En la banda L se encuentran las líneas de las series de Humphreys, Brackett y Pfund, junto con líneas de He I y He II . Lenorzer et al. (2002b) realizaron un atlas con observaciones espectroscópicas de estrellas tempranas, abarcando el rango 2.4 - $4.1 \mu \mathrm{m}$. Dentro de su muestra de 
objetos, separaron a las estrellas según si presentaban o no emisión. Para las estrellas sin emisión de la secuencia principal, el ancho de las líneas está dominado por efectos de la temperatura. En las supergigantes, en cambio, el ancho de las primeras líneas de cada serie estaría relacionado con la tasa de pérdida de masa de la estrella, $\dot{M}$. Además, las líneas de He se encuentran en objetos más tempranos que B3, mientras que la línea de He I (3.0736 $\mu \mathrm{m})$ pareciera ser más intensa en las estrellas supergigantes que en las enanas.

\subsubsection{Estrellas Be}

El estudio IR de las estrellas Be aún se encuentra en desarrollo. Producto de eso, no todas las bandas se encuentran igualmente estudiadas para una muestra grande de estrellas Be.

En la banda J no encontramos trabajos previos disponibles que reporten un análisis completo para varios objetos. Entre los pocos trabajos encontrados, se destacan el estudio de la fluorescencia de la línea $\mathrm{Ly} \beta$ en la intensidad de las líneas del O I (Mathew et al., 2012b) y el estudio espectroscópico de la estrella X Per (Mathew et al., 2012a).

En la banda H, se observa en emisión la serie de Brackett, junto con líneas de N I, C I, hierro (Fe I, Fe II), magnesio (Mg I, Mg II), silicio (Si I, Si II), calcio (Ca II) y algunas transiciones aún no identificadas (Steele \& Clark, 2001; Lenorzer et al., 2002b; Chojnowski et al., 2015). Con respecto a la clasificación espectral, Steele \& Clark (2001) encontraron que a partir del análisis de las líneas observadas en las estrellas Be, esta banda solo permite distinguir estrellas tempranas de tardías, sin diferenciar clases de luminosidades, debido a que no hay líneas fotosféricas sin contaminación por la emisión del disco circunestelar.

En la banda $\mathrm{K}$, se encuentra frecuentemente $\operatorname{Br} \gamma, \operatorname{Br} \delta$ y miembros de la serie de Pfund en emisión, junto con líneas de He I, Mg II, Fe II y Na I (Hanson et al., 1996; Clark \& Steele, 2000; Granada et al., 2010). Clark \& Steele (2000) indicaron que si se encuentran líneas de He I (en emisión o absorción) la estrella es de tipo espectral B3 o más temprano; si tiene líneas de Mg II en emisión, pero no tiene líneas de He I es una estrella B2-B4; mientras que si presenta Bry en emisión, sin He I ni Mg II es B5 o más tardía.

En la banda L, Mennickent et al. (2009) y Granada et al. (2010) reportaron emisiones en $\mathrm{Br} \alpha$, Pf $\gamma$, líneas de la serie de Humphreys y He I $\lambda 4.0377 \mu \mathrm{m}$ y He I $\lambda 4.0409 \mu \mathrm{m}$. Sabogal et al. (2017) observaron también una muestra de estrellas Be en la banda L, y correlacionaron el comportamiento de las líneas en emisión en esta banda con lo observado en la línea H $\alpha$.

\subsection{Observaciones}

En esta sección describiremos la muestra de objetos estudiada y las observaciones espectroscópicas obtenidas en forma simultánea en diferentes bandas del infrarrojo cercano. 


\subsubsection{Muestra de objetos}

La muestra observada incluye un total de 23 estrellas Be pertenecientes a la Vía Láctea. De los 23 objetos, 21 presentan subtipos espectrales dentro del tipo espectral B, mientras que los dos restantes son de tipo espectral O7.5 y A0. Las clases de luminosidad de los objetos de la muestra se encuentran en el rango V-III. En la Tabla 3.3 se lista el nombre, el tipo espectral, la clase de luminosidad, la velocidad de rotación proyectada, la temperatura efectiva y el logaritmo de la gravedad superficial de los objetos de la muestra.

De la muestra de objetos, 10 habían sido observados previamente por miembros del grupo $\mathrm{MEP}^{1}$, mientras que los 13 restantes fueron incluidos durante el desarrollo de la presente tesis. Varios de estos objetos fueron especialmente seleccionados por presentar características variables que permitirán estudiar la evolución de sus envolturas extendidas y/o por contar con observaciones espectrointerferométricas en $\mathrm{Br} \gamma$ (ver Capítulo 8) y espectros ópticos que permiten un análisis más completo con diferentes técnicas y en distintos rangos espectrales. Las observaciones espectroscópicas en el rango IR fueron llevadas a cabo en los observatorios Gemini Norte (Mauna Kea, Hawai) y Las Campanas (Atacama, Chile).

\subsubsection{Observaciones realizadas en Gemini}

El Observatorio Gemini fue construido y es operado desde 1993 por un consorcio de países que incluye, actualmente, a Estados Unidos, Canadá, Brasil, Argentina, Chile y Corea del Sur. El tiempo de observación es repartido, según la participación financiera de cada socio, entre los países miembros del consorcio y la Universidad de Hawai.

El Observatorio consta de dos telescopios gemelos de 8.1 metros de diámetro, que permiten realizar observaciones en los rangos espectrales óptico e infrarrojo. Desde sus ubicaciones, en Mauna Kea (Hawai) y Cerro Pachón (Chile), se puede observar el cielo entero desde dos de los mejores lugares de observación del planeta.

Para realizar observaciones en el IR, se utilizaron los siguientes instrumentos:

- NIRI (Near InfraRed Imager and Spectrometer): instalado en Gemini Norte, permite realizar observaciones en el IR en el rango de 1 a $5 \mu \mathrm{m}$. Actualmente solo permite la obtención de imágenes, y no está disponible en la modalidad de espectroscopía. Para la obtención de espectros, NIRI poseía 8 grismas ${ }^{2}$ y ranuras, cuyas combinaciones permitían obtener baja a moderada resolución. Cinco de esos grismas eran utilizados con la cámara f/6 y permitían cubrir todo el rango 1-5 $\mu \mathrm{m}$, con resoluciones que llegaban a $\mathrm{R}=1300$ en la banda $\mathrm{K}$ y $\mathrm{R}=1100$ en la banda $\mathrm{L}$.

\footnotetext{
${ }^{1} \mathrm{El}$ grupo MEP (Modelos de estrellas peculiares) es un grupo de investigación de la FCAG que tiene por objetivo estudiar y modelar estrellas tempranas con líneas en emisión o anomalías químicas. El presente trabajo de tesis fue realizado en el marco de las investigaciones desarrolladas en ese grupo.

${ }^{2}$ Adaptación del inglés grisms, prisma con una red en una de sus superficies.
} 
Tabla 3.3: Información de los objetos observados.

\begin{tabular}{llccccc}
\hline \hline HD & Nombre & TE $^{(a)}$ & CL $^{(a)}$ & $V \sin i^{(b)}$ & $T_{\text {eff }}^{(b)}$ & $\log g^{(b)}$ \\
\hline HD 20336 & BK Cam & B2.5 & V & $341 \pm 23$ & $18684 \pm 517$ & $3.865 \pm 0.072$ \\
\hline HD 23862 & 28 Tau & B8 & V & $290 \pm 15$ & $12106 \pm 272$ & $3.937 \pm 0.052$ \\
\hline HD 25940 & 48 Per & B3 & V & $220 \pm 13$ & $16258 \pm 582$ & $3.572 \pm 0.084$ \\
\hline HD 28497 & 228 Eri & B2 & V & $342 \pm 24$ & $26724 \pm 427$ & $4.200 \pm 0.046$ \\
\hline HD 29441 & V1150 Tau & B2.5 & V & - & $18000^{(f)}$ & $3.8^{(f)}$ \\
\hline HD 32991 & 105 Tau & B2 & V & $175 \pm 18^{(c)}$ & $20900^{(g)}$ & $3.77^{(g)}$ \\
\hline HD 35439 & $\psi_{01}$ Ori & B1 & V & $266 \pm 13$ & $22134 \pm 665$ & $3.920 \pm 0.087$ \\
\hline HD 36576 & 120 Tau & B2 & IV-V & $266 \pm 13$ & $22618 \pm 508$ & $3.804 \pm 0.062$ \\
\hline HD 37490 & $\omega$ Ori & B3 & V & $155 \pm 5^{(d)}$ & $14492^{(g)}$ & $3.86^{(g)}$ \\
\hline HD 41335 & V696 Mon & B3-5 & V & $376 \pm 26$ & $20902 \pm 610$ & $3.886 \pm 0.081$ \\
\hline HD 68980 & MX Pup & B1.5 & IV & $152 \pm 08$ & $25126 \pm 642$ & $3.951 \pm 0.077$ \\
\hline HD 142983 & 48 Lib & B3 ${ }^{(e)}$ & V $(e)$ & $407 \pm 22$ & $17642 \pm 554$ & $3.845 \pm 0.080$ \\
\hline HD 155806 & V1075 Sco & O7.5 & V & - & $34000^{(h)}$ & $4.0^{(h)}$ \\
\hline HD 162732 & 88 Her & B6 & III & $321 \pm 23$ & $13523 \pm 243$ & $3.754 \pm 0.043$ \\
\hline HD 164284 & 66 Oph & B2 & V & $287 \pm 21$ & $21609 \pm 523$ & $3.943 \pm 0.068$ \\
\hline HD 171623 & HR 6977 & A0 & V & - & $9853 \pm 2911^{(i)}$ & $3.91 \pm 0.23$ \\
\hline HD 178175 & V4024 Sgr & B2 & V & $111 \pm 07$ & $18939 \pm 286$ & $3.489 \pm 0.035$ \\
\hline HD 183656 & V923 Aql & B7 & III & $284 \pm 16$ & $12626 \pm 524$ & $3.295 \pm 0.081$ \\
\hline HD 187811 & 12 Vul & B2.5 & V & $258 \pm 13$ & $18086 \pm 583$ & $3.810 \pm 0.083$ \\
\hline HD 191610 & 28 Cyg & B2.5 & V & $318 \pm 22$ & $18353 \pm 516$ & $3.718 \pm 0.072$ \\
\hline HD 209409 & $o$ Aqr & B7 & IV & $282 \pm 20$ & $12942 \pm 402$ & $3.701 \pm 0.067$ \\
\hline HD 212571 & $\pi$ Aqr & B1 & III-IV & $233 \pm 15$ & $26061 \pm 736$ & $3.915 \pm 0.088$ \\
\hline HD 217050 & EW Lac & B4 & III & $355 \pm 25$ & $17893 \pm 509$ & $3.571 \pm 0.070$ \\
\hline \hline
\end{tabular}

${ }^{(a)}$ tomados de la base de datos CDS, excepto que se indique otra fuente;

(b) tomados de Frémat et al. (2005), excepto que se indique otra fuente;

(c) tomados de Abt et al. (2002);

(d) tomados de Chauville et al. (2001);

(e) tomados de Zorec et al. (2005);

(f) según $T_{\text {eff y }} \log g$ de otras estrellas con mismo TE y CL;

(g) según ajuste de la SED (ver Capítulo 8);

(h) según valores típicos para el TE y CL obtenidos de Cox \& Pilachowski (2000);

(i) según ajuste de la discontinuidad de Balmer (Cochetti et al., 2020).

- GNIRS (Gemini Near-Infrared Spectrograph): inicialmente instalado en Gemini Sur, en 2007 sufrió daños y luego de ser reparado fue instalado en Gemini Norte. Ofrece una amplia variedad de capacidades. Por un lado, permite hacer espectroscopía de ranura larga (LS por sus siglas en inglés) en el rango 1.0-5.4 $\mu \mathrm{m}$ con $R$ entre 1800 y 18000 , utilizando distintas combinaciones de distintas redes con las cámaras corta (0.15"/pix) o larga (0.05"/pix). Por el otro, dispone de un dispersor cruzado (XD) cubriendo completamente el rango 0.9-2.5 $\mu \mathrm{m}$ con $R \sim 1800$ o una cobertura parcial con $R \sim 5000$. Es posible, además, elegir entre una 
Tabla 3.4: Configuraciones instrumentales utilizadas para la obtención de espectros IR.

\begin{tabular}{|c|c|c|c|c|c|c|}
\hline$\overline{\text { Año }}$ & Instrumento & Cámara & Red & Ranura & Bandas & Resolución \\
\hline \multirow[t]{2}{*}{$2006 / 2008$} & NIRI-LS & $f / 6$ & $166.7 \mathrm{l} / \mathrm{mm}$ & 2 pix & $\mathrm{K}$ & 1300 \\
\hline & & & $104.1 \mathrm{l} / \mathrm{mm}$ & 2 pix & $\mathrm{L}$ & 1100 \\
\hline \multirow[t]{2}{*}{2010} & GNIRS-LS & corta azul $(0.15 " /$ pix $)$ & $32 \mathrm{l} / \mathrm{mm}$ & $0.3 "$ & H-K & 1800 \\
\hline & & corta roja $(0.15 \% / p i x)$ & $32 \mathrm{l} / \mathrm{mm}$ & $0.3 ”$ & $\mathrm{~L}$ & 1800 \\
\hline \multirow[t]{2}{*}{2012} & GNIRS-LS & corta azul $(0.15 \% /$ pix $)$ & $32 \mathrm{l} / \mathrm{mm}$ & $0.3 ”$ & H-K & 1800 \\
\hline & & larga roja $(0.05 \% /$ pix $)$ & $10 \mathrm{l} / \mathrm{mm}$ & $0.1 "$ & $\mathrm{~L}$ & 1800 \\
\hline \multirow[t]{2}{*}{$2016 / 2017$} & GNIRS-XD & corta azul $(0.15 " /$ pix $)$ & $32 \mathrm{l} / \mathrm{mm}$ & $0.3 ”$ & J-H-K & 1800 \\
\hline & GNIRS-LS & larga roja $(0.05 " / p i x)$ & $10 \mathrm{l} / \mathrm{mm}$ & $0.1 ”$ & $\mathrm{~L}$ & 1800 \\
\hline
\end{tabular}

Tabla 3.5: Información de los turnos de observación.

\begin{tabular}{lllll}
\hline \hline Turno & $\begin{array}{l}\text { Investigadora } \\
\text { principal }\end{array}$ & $\begin{array}{l}\text { Condiciones } \\
\text { de tiempo }\end{array}$ & $\begin{array}{l}\text { Tiempo } \\
\text { asignado }\end{array}$ & $\begin{array}{l}\text { Porcentaje } \\
\text { observado }\end{array}$ \\
\hline GN-2006B-Q-28 & Lydia Cidale & Banda 2 & 1.3 horas & $70 \%$ \\
\hline GN-2008B-Q-100 & Anahí Granada & Banda 3 & 2.5 horas & $100 \%$ \\
\hline GN-2010B-Q-2 & María Laura Arias & Banda 1 & 2.5 horas & $100 \%$ \\
\hline GN-2012B-Q-56 & María Laura Arias & Banda 2 & 2 horas & $100 \%$ \\
\hline GN-2016A-Q-96 & Yanina Cochetti & Poor Weather & 4.3 horas & $62 \%$ \\
\hline GN-2016B-Q-83 & Yanina Cochetti & Poor Weather & 2.6 horas & $100 \%$ \\
\hline GN-2017A-Q-84 & Yanina Cochetti & Poor Weather & 3.9 horas & $100 \%$ \\
\hline GN-2017A-Q-89 & Yanina Cochetti & Poor Weather & 3.2 horas & $13 \%$ \\
\hline GN-2017B-Q-81 & Yanina Cochetti & Poor Weather & 7.84 horas & $100 \%$ \\
\hline GN-2017B-Q-86 & María Laura Arias & Poor Weather & 0.7 horas & $100 \%$ \\
\hline \hline
\end{tabular}

variedad de ranuras con anchos entre 0.10 y 1.0 arcsec.

Los datos fueron obtenidos entre los años 2006 y 2017. El tiempo total de observación fue de 26 horas, de las cuales 18 fueron tomadas en condiciones meteorológicas con alta nubosidad (Poor weather conditions). En la Tabla 3.4 se describen las configuraciones instrumentales utilizadas, y en la Tabla 3.5 se brinda información complementaria de cada turno. Los objetos observados en cada turno, junto con la fecha de observación correspondiente y las bandas espectrales observadas se presentan en la Tabla 3.6. Las observaciones fueron obtenidas orientando la ranura según el ángulo paraláctico, a fin de evitar pérdidas de flujo por refracción diferencial. Cabe destacar que las observaciones realizadas en 2006 y 2008, obtenidas por otros miembros del grupo MEP, se obtuvieron con el espectrógrafo NIRI y solo registran las bandas K y L. El resto de las observaciones fueron obtenidas con GNIRS, dado que NIRI dejó de estar disponible en modo de espectroscopía. En los espectros de GNIRS se incorporó la banda H, no observada previamente. Por otra parte, a partir de 2012 fue necesario cambiar la configuración en la banda L, ya que la cámara roja corta dejó de funcionar. Finalmente, incorporamos el modo de observación en dispersión cruzada, lo que nos permitió observar simultáneamente las bandas J, H y K, 
prácticamente con el mismo tiempo de exposición que una sola banda, mientras que continuamos observando en la banda L empleando la cámara roja larga. La diversidad de instrumentos y configuraciones utilizadas hizo que el conjunto de observaciones no sea homogéneo.

Los datos fueron reducidos utilizando los paquetes y tareas del código IRAF ${ }^{3}$. Este software permite que se añadan paquetes externos, como los desarrollados por cada observatorio para el procesamiento de sus datos. En este caso, se utilizaron las tareas incluidas en el paquete del Observatorio Gemini, y los paquetes específicos para cada instrumento.

El proceso de reducción incluye:

- Preprocesamiento de los espectros: para normalizar la respuesta no uniforme del CCD, se dividen las imágenes obtenidas por las provenientes de un espectro uniformemente iluminado. Además, algunos píxeles pueden no funcionar correctamente o haber sido alcanzados por rayos cósmicos, por lo que deben ser descartados utilizando una máscara de píxeles malos.

- Extracción de los espectros: para los objetos de ciencia y las estándares telúricas, se identifica la traza del espectro y se ajusta un polinomio para convertir la imagen bidimensional en una relación píxel vs. señal. Utilizando las mismas trazas se extraen los espectros de las lámparas de comparación (si los hay).

- Calibración en longitud de onda: en los espectros de las lámparas de comparación se identifican líneas y se ajusta un polinomio para obtener una solución píxel vs. longitud de onda. En la banda L no se utiliza lámpara de comparación, si no que se utilizan las líneas generadas en la atmósfera. La solución píxel vs. longitud de onda generada es aplicada a los espectros de los objetos de ciencia y las estándares telúricas.

- Corrección de líneas telúricas: utilizamos como estándares telúricas estrellas de tipo espectral $\mathrm{A} 0 \mathrm{~V}$ o similar, ya que solamente presentan líneas de hidrógeno fácilmente identificables. $\mathrm{Al}$ remover las líneas de hidrógeno, obtenemos el espectro de absorción producido por la atmósfera. Eso nos permite corregir los espectros de ciencia dividiendo por el espectro telúrico.

- Calibración en flujo: como estándar de flujo utilizamos las mismas estándares telúricas, para las que realizamos la corrección telúrica de la misma manera que en los espectros de ciencia. Se obtiene la sensibilidad del CCD para cada longitud de onda, y a partir de esa sensibilidad se calcula el flujo en cada longitud de onda de los espectros de ciencia. Para

\footnotetext{
${ }^{3}$ IRAF es la sigla de Reducción de Imágenes y Facilidades de Análisis (Image Reduction and Analysis Facility), un sistema de software de utilidad general para el procesamiento de datos astronómicos. IRAF fue escrito y avalado por el Grupo de Programación IRAF (IRAF Programming Group) en los Observatorios Nacionales de Astronomía Ópticos (NOAO) en Tucson, Arizona. NOAO es dirigido por la Asociación de Universidades para la Investigación en la Astronomía S.A (AURA), con el acuerdo cooperativo de la Fundación de Ciencia Nacional.
} 
los objetos observados en varias bandas, verificamos que al graficar el espectro completo la transición entre las distintas bandas sea suave.

Algunos de los espectros obtenidos en los años 2006 y 2008 fueron presentados y analizados previamente por Granada et al. (2010). En este trabajo, con el objetivo de tener una muestra de espectros lo más homogénea posible, los espectros fueron reducidos nuevamente según los pasos aquí descriptos. Esto genera pequeñas diferencias entre los valores obtenidos previamente, pero siempre dentro del margen de error de los mismos.

Tabla 3.6: Observaciones IR de estrellas Be realizadas en el Observatorio Gemini.

\begin{tabular}{|c|c|c|c|c|c|c|c|c|}
\hline $\mathrm{HD}$ & Nombre & Programa & Instrumento & Fecha de obs. & \multicolumn{4}{|c|}{ Banda } \\
\hline \multirow[t]{4}{*}{ HD 20336} & BK Cam & GN-2008B-Q-100 & NIRI & $2008-11-25$ & & & $\mathrm{~K}$ & $\mathrm{~L}$ \\
\hline & & GN-2010B-Q-02 & GNIRS & 2011-01-01 & & $\mathrm{H}$ & $\mathrm{K}$ & $\mathrm{L}$ \\
\hline & & GN-2012B-Q-56 & GNIRS & $2012-12-24$ & & $\mathrm{H}$ & $\mathrm{K}$ & $\mathrm{L}$ \\
\hline & & GN-2016B-Q-83 & GNIRS & 2016-11-16 & $\mathrm{J}$ & $\mathrm{H}$ & $\mathrm{K}$ & $\mathrm{L}$ \\
\hline \multirow[t]{5}{*}{ HD 23862} & $28 \mathrm{Tau}$ & GN-2006B-Q-28 & NIRI & $2006-08-20$ & & & $\mathrm{~K}$ & $\mathrm{~L}$ \\
\hline & & GN-2010B-Q-02 & GNIRS & $2010-12-03$ & & $\mathrm{H}$ & $\mathrm{K}$ & $\mathrm{L}$ \\
\hline & & GN-2012B-Q-56 & GNIRS & 2012-12-12 & & $\mathrm{H}$ & $\mathrm{K}$ & $\mathrm{L}$ \\
\hline & & GN-2016B-Q-83 & GNIRS & 2016-11-16 & & & & $\mathrm{L}$ \\
\hline & & & & 2016-11-18 & $\mathrm{J}$ & $\mathrm{H}$ & $\mathrm{K}$ & \\
\hline HD 25940 & 48 Per & GN-2012B-Q-56 & GNIRS & 2013-01-01 & & $\mathrm{H}$ & $\mathrm{K}$ & $\mathrm{L}$ \\
\hline \multirow[t]{2}{*}{ HD 28497} & 228 Eri & GN-2017B-Q-81 & GNIRS & $2017-12-10$ & $\mathrm{~J}$ & $\mathrm{H}$ & $\mathrm{K}$ & \\
\hline & & & & $2017-12-14$ & & & & $\mathrm{~L}$ \\
\hline \multirow[t]{2}{*}{ HD 29441} & V1150 Tau & GN-2012B-Q-56 & GNIRS & $2012-12-23$ & & $\mathrm{H}$ & $\mathrm{K}$ & $\mathrm{L}$ \\
\hline & & GN-2016B-Q-83 & GNIRS & $2016-11-17$ & $\mathrm{~J}$ & $\mathrm{H}$ & $\mathrm{K}$ & \\
\hline HD 32991 & $105 \mathrm{Tau}$ & GN-2017B-Q-81 & GNIRS & 2017-01-04 & $\mathrm{J}$ & $\mathrm{H}$ & $\mathrm{K}$ & $\mathrm{L}$ \\
\hline \multirow[t]{2}{*}{ HD 35439} & $\psi_{01}$ Ori & GN-2017B-Q-81 & GNIRS & $2017-12-14$ & & & & $\mathrm{~L}$ \\
\hline & & & GNIRS & 2018-01-04 & $\mathrm{J}$ & $\mathrm{H}$ & $\mathrm{K}$ & \\
\hline \multirow[t]{2}{*}{ HD 36576} & $120 \mathrm{Tau}$ & GN-2017B-Q-81 & GNIRS & $2017-12-29$ & $\mathrm{~J}$ & $\mathrm{H}$ & $\mathrm{K}$ & \\
\hline & & & GNIRS & 2018-01-04 & & & & $\mathrm{L}$ \\
\hline HD 37490 & $\omega$ Ori & GN-2017B-Q-81 & GNIRS & 2018-01-04 & $\mathrm{J}$ & $\mathrm{H}$ & $\mathrm{K}$ & $\mathrm{L}$ \\
\hline \multirow[t]{2}{*}{ HD 41335} & V696 Mon & GN-2017B-Q-81 & GNIRS & $2017-12-29$ & $\mathrm{~J}$ & $\mathrm{H}$ & $\mathrm{K}$ & \\
\hline & & & & 2018-01-14 & & & & $\mathrm{L}$ \\
\hline HD 142983 & $48 \mathrm{Lib}$ & GN-2017A-Q-89 & GNIRS & 2017-07-09 & $\mathrm{J}$ & $\mathrm{H}$ & $\mathrm{K}$ & \\
\hline HD 162732 & $88 \mathrm{Her}$ & GN-2006B-Q-28 & NIRI & $2006-08-21$ & & & $\mathrm{~K}$ & $\mathrm{~L}$ \\
\hline \multirow[t]{3}{*}{ HD 164284} & $66 \mathrm{Oph}$ & GN-2008B-Q-100 & NIRI & $2008-08-25$ & & & $\mathrm{~K}$ & $\mathrm{~L}$ \\
\hline & & GN-2010B-Q-02 & GNIRS & 2010-09-15 & & $\mathrm{H}$ & $\mathrm{K}$ & $\mathrm{L}$ \\
\hline & & GN-2016A-Q-96 & GNIRS & 2016-07-06 & $\mathrm{J}$ & $\mathrm{H}$ & $\mathrm{K}$ & $\mathrm{L}$ \\
\hline
\end{tabular}


Tabla 3.6 (continuación)

\begin{tabular}{|c|c|c|c|c|c|c|c|c|}
\hline & & GN-2017A-Q-84 & GNIRS & 2017-07-03 & $\mathrm{J}$ & $\mathrm{H}$ & $\mathrm{K}$ & $\mathrm{L}$ \\
\hline \multirow[t]{4}{*}{ HD 183656} & V923 Aql & GN-2006B-Q-28 & NIRI & 2006-09-17 & & & $\mathrm{K}$ & $\mathrm{L}$ \\
\hline & & GN-2016A-Q-96 & GNIRS & 2016-06-24 & $\mathrm{J}$ & $\mathrm{H}$ & $\mathrm{K}$ & $\mathrm{L}$ \\
\hline & & GN-2017A-Q-84 & GNIRS & $2017-05-29$ & & & & $\mathrm{~L}$ \\
\hline & & & & 2017-07-08 & $\mathrm{J}$ & $\mathrm{H}$ & $\mathrm{K}$ & \\
\hline \multirow[t]{3}{*}{ HD 187811} & $12 \mathrm{Vul}$ & GN-2010B-Q-02 & GNIRS & 2010-09-15 & & $\mathrm{H}$ & $\mathrm{K}$ & $\mathrm{L}$ \\
\hline & & GN-2016A-Q-96 & GNIRS & 2016-06-25 & $\mathrm{J}$ & $\mathrm{H}$ & $\mathrm{K}$ & \\
\hline & & GN-2017A-Q-84 & GNIRS & 2017-07-08 & $\mathrm{J}$ & $\mathrm{H}$ & $\mathrm{K}$ & $\mathrm{L}$ \\
\hline \multirow[t]{5}{*}{ HD 191610} & $28 \mathrm{Cyg}$ & GN-2008B-Q-100 & NIRI & $2008-08-25$ & & & $\mathrm{~K}$ & $\mathrm{~L}$ \\
\hline & & GN-2010B-Q-02 & GNIRS & 2010-09-15 & & $\mathrm{H}$ & $\mathrm{K}$ & $\mathrm{L}$ \\
\hline & & GN-2016A-Q-96 & GNIRS & 2016-07-14 & $\mathrm{J}$ & $\mathrm{H}$ & $\mathrm{K}$ & \\
\hline & & & & 2016-07-20 & & & & $\mathrm{L}$ \\
\hline & & GN-2017A-Q-84 & GNIRS & 2017-07-09 & $\mathrm{J}$ & $\mathrm{H}$ & $\mathrm{K}$ & $\mathrm{L}$ \\
\hline HD 209409 & $o$ Aqr & GN-2017B-Q-81 & GNIRS & $2017-07-10$ & $\mathrm{~J}$ & $\mathrm{H}$ & $\mathrm{K}$ & \\
\hline \multirow[t]{7}{*}{ HD 217050} & EW Lac & GN-2006B-Q-28 & NIRI & $2006-08-20$ & & & $\mathrm{~K}$ & $\mathrm{~L}$ \\
\hline & & GN-2008B-Q-100 & NIRI & 2008-09-09 & & & $\mathrm{K}$ & $\mathrm{L}$ \\
\hline & & GN-2010B-Q-02 & GNIRS & 2010-09-15 & & $\mathrm{H}$ & $\mathrm{K}$ & $\mathrm{L}$ \\
\hline & & GN-2016B-Q-83 & GNIRS & 2016-08-03 & & & & $\mathrm{L}$ \\
\hline & & & & 2016-08-09 & $\mathrm{J}$ & $\mathrm{H}$ & $\mathrm{K}$ & \\
\hline & & GN-2017B-Q-86 & GNIRS & $2017-12-27$ & & & & $\mathrm{~L}$ \\
\hline & & & & 2018-01-11 & $\mathrm{J}$ & $\mathrm{H}$ & $\mathrm{K}$ & \\
\hline
\end{tabular}

\subsubsection{Observaciones realizadas en Las Campanas}

El Observatorio Las Campanas (Chile) está operado por el Carnegie Institution for Science (Washington-Estados Unidos). Está localizado en la cordillera de Los Andes, Región de Atacama, y cuenta con los siguientes telescopios: Magallanes (dos telescopios gemelos de 6.5 metros, Walter Baade y Landon Clay), du Pont (telescopio de 2.5 metros) y Swope (telescopio de 1 metro).

Dentro de los instrumentos IR disponibles para ser utilizados en el telescopio Baade, se encuentra FIRE (Folded port InfraRed Echellette). Su configuración primaria incluye una combinación de red y prisma de difracción que permiten obtener un espectro en dispersión cruzada cubriendo el cercano IR en el rango 0.8-2.5 $\mu \mathrm{m}$ en una sola exposición, con resolución $R \sim 6000$. FIRE también ofrece un modo de baja dispersión $(R \sim 1200)$ donde la red de difracción es reemplazada por un espejo.

Las observaciones fueron realizadas utilizando el espectrógrafo FIRE, en el marco de la beca para astrónomos argentinos otorgada por el Carnegie Institution for Science. Esta beca me permitió realizar una estadía en el Observatorio, durante la cual participé de un turno de 
Tabla 3.7: Observaciones IR de estrellas Be realizadas en el Observatorio Las Campanas, en el rango 0.8-2.5 $\mu \mathrm{m}$, con resolución $R \sim 6000$.

\begin{tabular}{llcccc}
\hline \hline HD & Nombre & Fecha de observación & \multicolumn{3}{c}{ Banda } \\
\hline HD 68980 & MX Pup & $2017-06-03$ & J & H & K \\
\hline HD 155806 & V1075 Sco & $2017-06-04$ & J & H & K \\
\hline HD 171623 & HR 6977 & $2017-06-04$ & J & H & K \\
\hline HD 178175 & V4024 Sgr & $2017-06-04$ & J & H & K \\
\hline HD 187811 & 12 Vul & $2017-06-04$ & J & H & K \\
\hline HD 212571 & $\pi$ Aqr & $2017-06-04$ & J & H & K \\
\hline \hline
\end{tabular}

observación del Dr. Gabriel Ferrero y la Dra. Nidia Morrell, quienes permitieron la adquisición de espectros IR para ser utilizados en el desarrollo de esta tesis. En la Tabla 3.7 se listan los objetos, junto con la fecha de observación y las bandas observadas.

La reducción de los datos fue realizada junto al Dr. Gabriel Ferrero y la Dra. Nidia Morrell, utilizando la pipeline provista por el Observatorio. Este software está escrito en formato IDL, y se basa en varios paquetes externos, incluyendo SDSS idlutils, idlspec2d, el paquete xidl de Jason Prochaska y Spextool de Mike Cushing (Fuente: Página web FIRE). Luego, el formato de los espectros fue modificado para poder ser analizados utilizando IRAF.

\subsection{Atlas de estrellas Be en el cercano IR}

El objetivo principal de esta Tesis se centra en investigar la geometría, estructura física y evolución de las envolturas circunestelares que se desarrollan alrededor de las estrellas con fenómeno Be. Para esto es necesario realizar una descripción amplia del espectro IR cercano de las estrellas Be, abarcando en forma simultánea el mayor rango espectral posible con una resolución aceptable. En esta sección presentaremos, a modo de atlas, la muestra completa de las observaciones infrarrojas realizadas y describiremos sus principales características.

Contamos actualmente con espectros IR de 23 estrellas Be, 8 de las cuales fueron observadas más de una vez. Como mencionamos anteriormente, los atlas de espectros IR de estrellas Be disponibles en la literatura se centran en el análisis de una o dos bandas, por lo que nuestras observaciones permiten una mejor caracterización de los discos. Además, el seguimiento temporal de un grupo de objetos a partir de observaciones tomadas en diferentes años, permite estudiar la variabilidad de las envolturas desarrolladas alrededor de estos objetos.

En las Figs. 3.2 a 3.17 mostramos los espectros obtenidos en las bandas J, H, K y L, junto con las principales líneas identificadas. Los mismos están normalizados y desplazados sobre el eje $y$ para una mejor visualización. Además, en las Figs. 3.18 a 3.19 se muestran las líneas de hidrógeno más intensas que quedan superpuestas en los gráficos anteriores. Todos los espectros serán mostrados nuevamente en los próximos capítulos, dentro del análisis individual realizado para cada objeto. 
La mayor dificultad en la reducción de espectros en el infrarrojo radica en la corrección por las líneas producidas por la atmósfera terrestre. En algunos casos, la corrección telúrica pudo mejorarse manualmente, comparando cuidadosamente los espectros del objeto de ciencia y la estándar telúrica, y eliminando señales espurias. En otros casos, los residuos de las líneas telúricas no han podido eliminarse, y se marcaron en las figuras con el símbolo $\oplus$. Esto ocurre, generalmente, en los extremos de cada banda y en la región alrededor de los $2 \mu \mathrm{m}$ (banda $\mathrm{K}$ ). Por otra parte, algunos espectros tomados con GNIRS presentan un continuo con aspecto de "serrucho" (ver por ejemplo 66 Oph en la observación del 2016 y 12 Vul en la observación del 2017) que surge a causa de efectos instrumentales que no han podido ser corregidos de forma óptima. No obstante, este problema no afecta de forma significativa los perfiles de las líneas observadas, aunque desmejora en cierta medida la señal-ruido. En los espectros obtenidos con FIRE se observan también saltos en las longitudes de onda ubicadas entre dos órdenes, por ejemplo alrededor de $2.28 \mu \mathrm{m}$. Finalmente, algunos espectros de la banda L presentan una calibración en longitud de onda poco precisa, lo que causa el desplazamiento de algunas líneas. Esto se debe a que en esta banda infrarroja no existen espectros de lámparas de comparación, por lo que suelen tomarse las escasas líneas telúricas presentes en esta banda como referencia. Esto hace que, principalmente en el caso de los espectros con menor señal ruido, haya solo dos o tres líneas de referencia para realizar la calibración en longitud de onda, dando como resultado una calibración pobre. Sin embargo, como nuestro objetivo no necesita de una calibración en longitud de onda sumamente precisa, esto no afecta los resultados de nuestro trabajo.

La mayor parte de las estrellas observadas muestran emisión de las líneas de hidrógeno por sobre el nivel del continuo (por ejemplo BK Cam), y solo en algunos casos la emisión no logra rellenar la absorción fotosférica (como HD 171623, donde solo se ve una pequeña emisión central en $\mathrm{Pa} \beta$ y $\mathrm{Br} \gamma$ ). Algunas estrellas muestran perfiles tipo shell (por ejemplo las líneas de la serie de Brackett observadas en la banda $\mathrm{H}$ en $28 \mathrm{Tau}$ ), y otras muestran dos picos en emisión (como en $\pi$ Aqr).

Las estrellas más tempranas presentan líneas de He I. Las mismas se observan en absorción, como por ejemplo la línea en $\lambda 1.1972 \mu \mathrm{m}$, o en emisión como en $\lambda 2.0587 \mu \mathrm{m}$. La estrella EW Lac, de tipo espectral B4, es la más tardía que presenta la línea de He I $\lambda 2.0587 \mu \mathrm{m}$, y la misma presenta un perfil tipo shell. Las líneas de He II suelen ser difíciles de distinguir sobre el nivel del continuo. Las estrellas que presentan emisión de He I, también suelen presentar emisión en las líneas de Mg II.

Los espectros de 14 objetos muestran líneas de Fe II en emisión, generalmente de manera simultánea en todas las bandas. Se observan también líneas de C I en emisión en 18 de los objetos de la muestra. Los que no presentan C I en emisión, tampoco presentan emisión de Fe II. La emisión de estas líneas no parece estar relacionada con el tipo espectral, ya que se observa a lo largo de todos los tipos espectrales comprendidos en nuestra muestra. La emisión de $\mathrm{Na}$ I es menos frecuente, y se encuentra en 5 objetos de la muestra (todos de tipo espectral B3 o más 
tardíos).

De los 21 objetos observados en la banda J, 16 presentan emisión en las líneas de O I. La emisión de $\mathrm{O}$ I también puede observarse en las bandas $\mathrm{H}$ y L.

Todas las líneas observadas en nuestros espectros se listan en la Tabla 3.2. En la misma se encuentran algunas líneas sin identificar, debido a que no logramos establecer correlaciones con las líneas de distintos elementos presentes en los espectros.

Hemos presentado en este capítulo una descripción cualitativa de las características observadas en la región IR en el espectro de las estrellas B y Be. Presentamos además la muestra de objetos, las observaciones realizadas, y los espectros obtenidos en el IR para nuestra muestra de estrellas $\mathrm{Be}$, junto con la identificación de las líneas espectrales presentes en los mismos. Encontramos también correlaciones entre la presencia de determinadas líneas y el tipo espectral de la estrella.

Presentaremos a continuación la metodología que utilizaremos para analizar los espectros IR. $\mathrm{El}$ análisis cuantitativo detallado de las características espectrales para cada objeto individual y el estudio de la variabilidad de las líneas en aquellos objetos para los que contamos con más de una observación, serán desarrollados en dos capítulos aparte (Capítulos 5 y 6, respectivamente). 
Figura 3.2: Espectros obtenidos en la banda J utilizando GNIRS o FIRE.

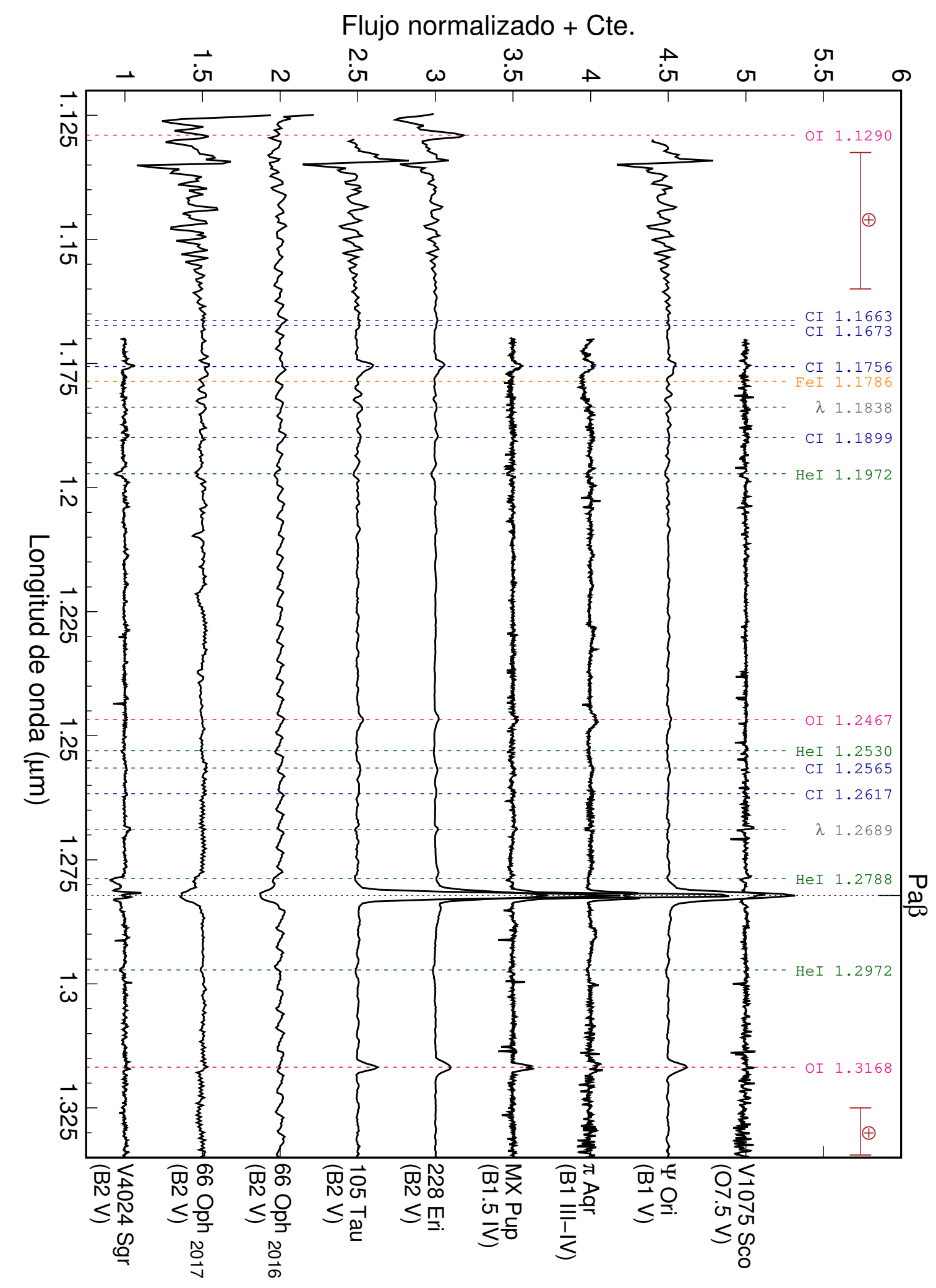


Figura 3.3: Espectros obtenidos en la banda J utilizando GNIRS o FIRE.

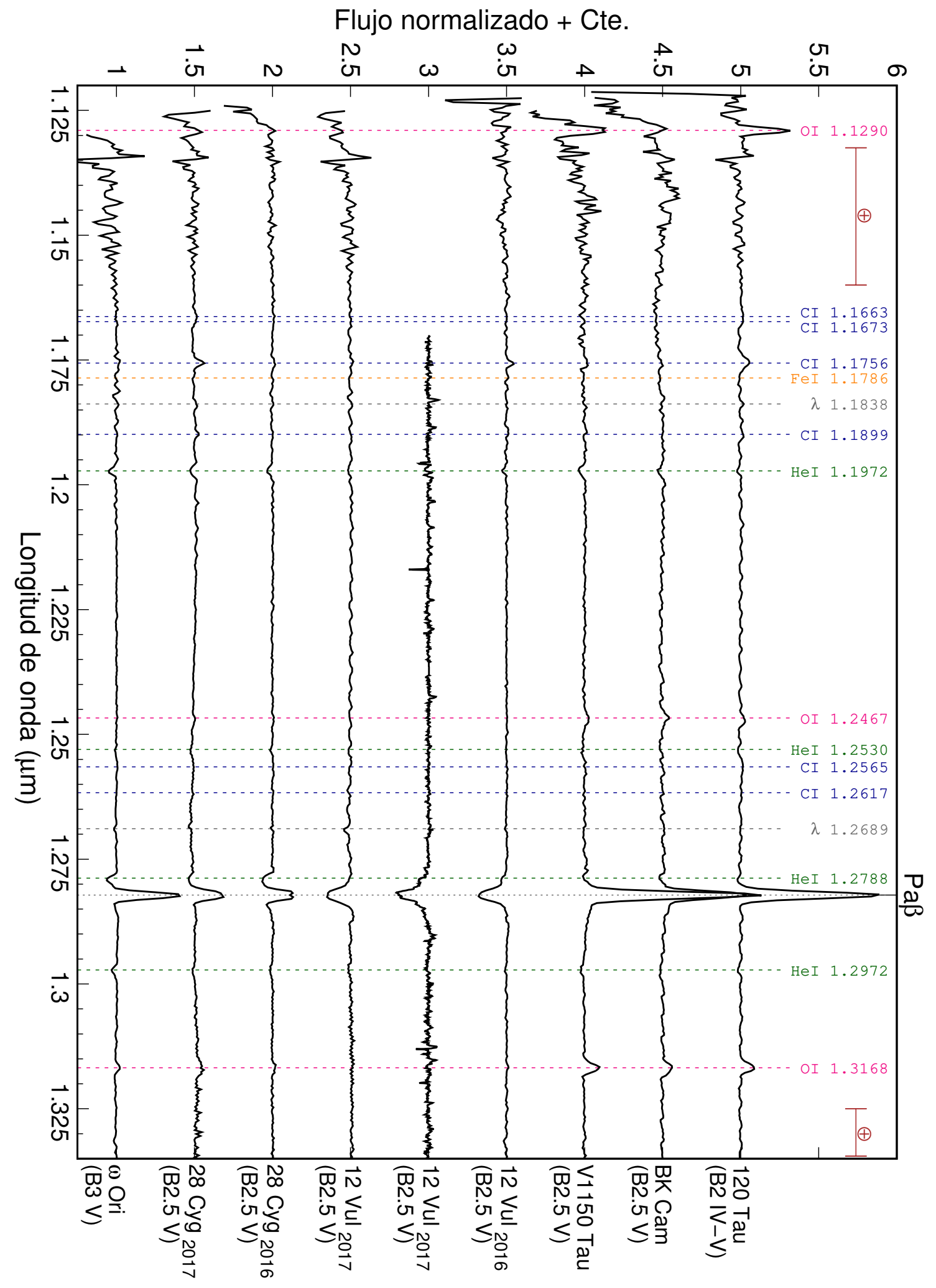


Figura 3.4: Espectros obtenidos en la banda J utilizando GNIRS o FIRE.

Flujo normalizado + Cte.

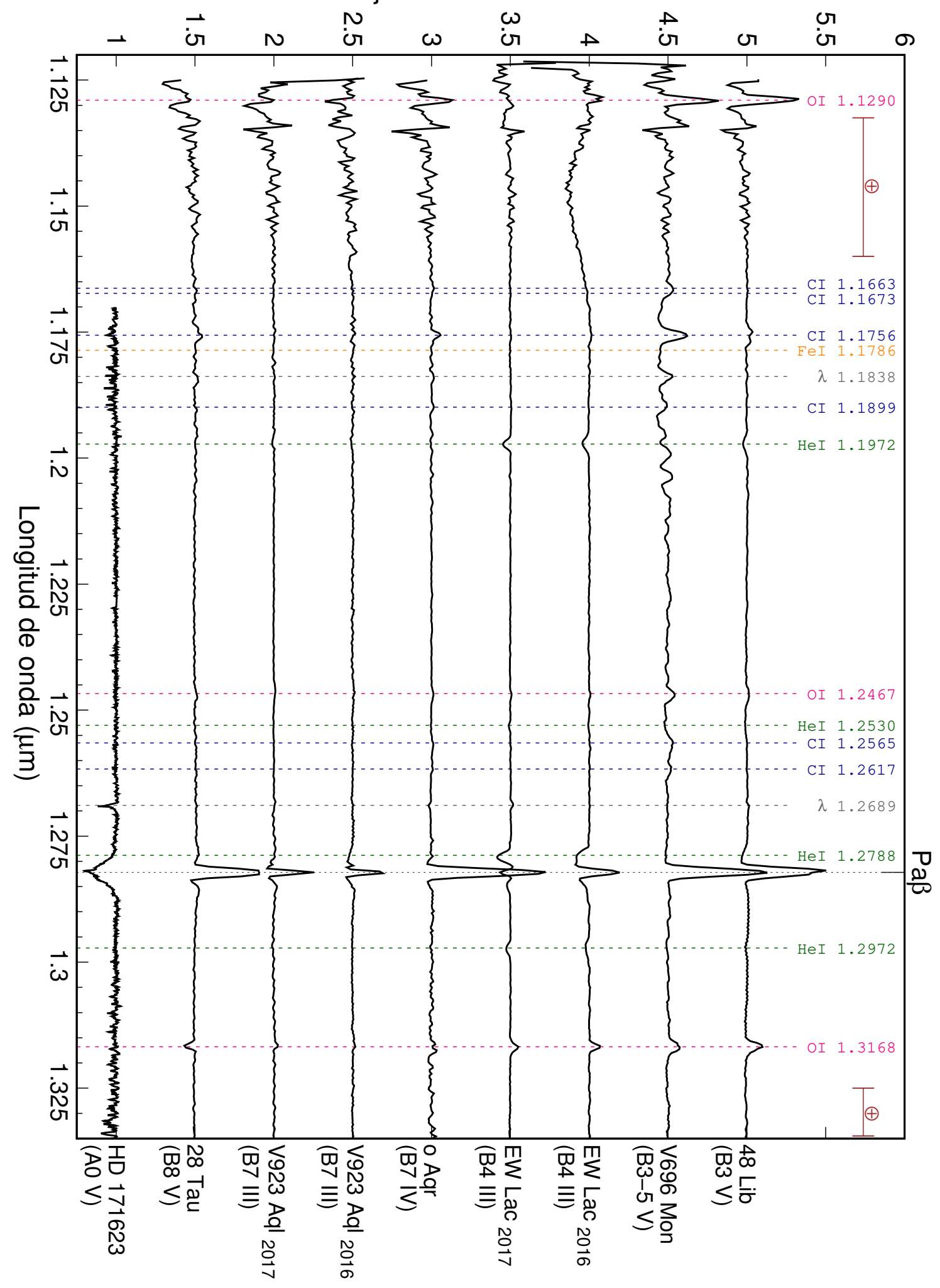


Figura 3.5: Espectros obtenidos en la banda H utilizando GNIRS o FIRE.

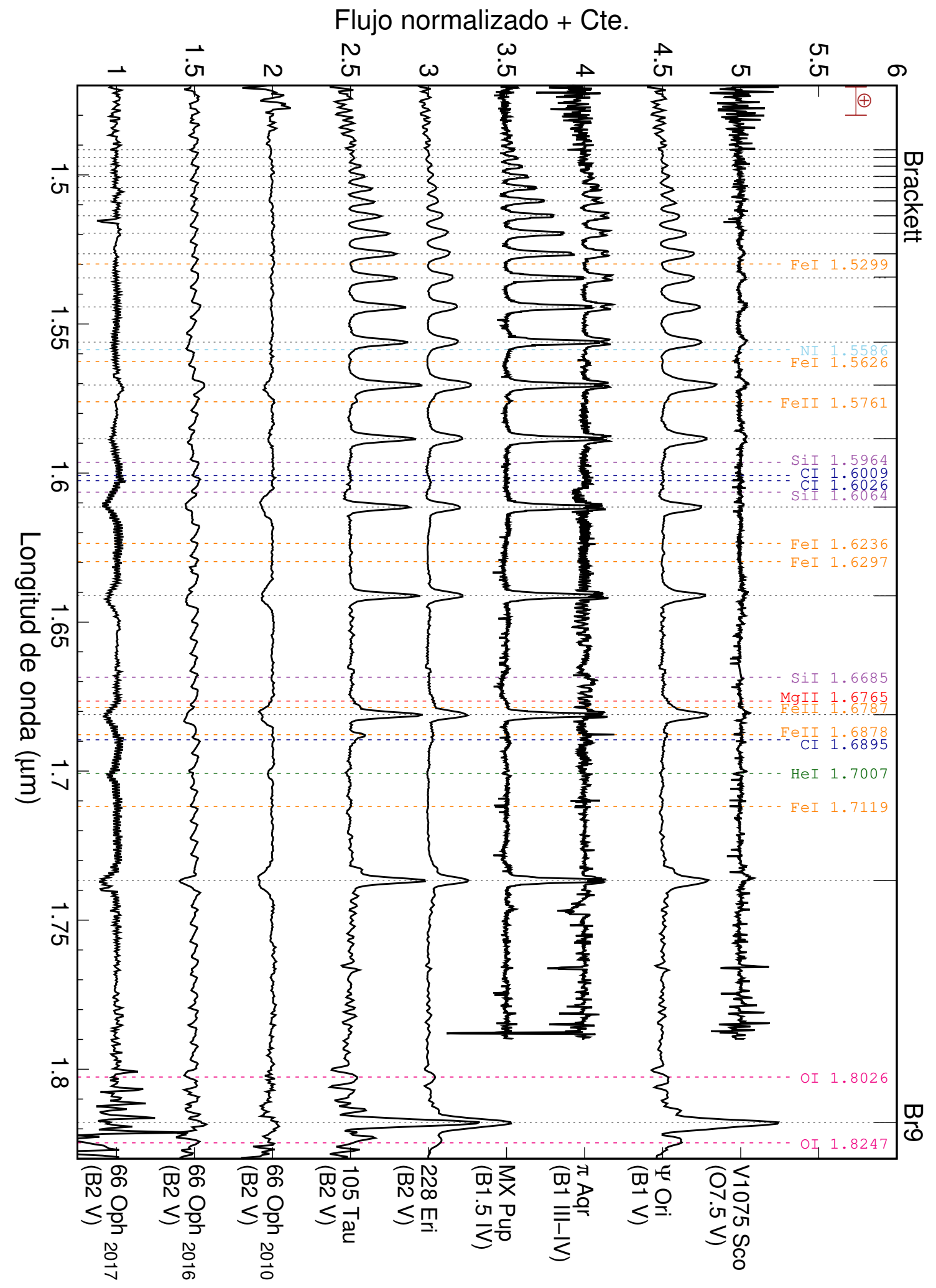


Figura 3.6: Espectros obtenidos en la banda H utilizando GNIRS o FIRE.

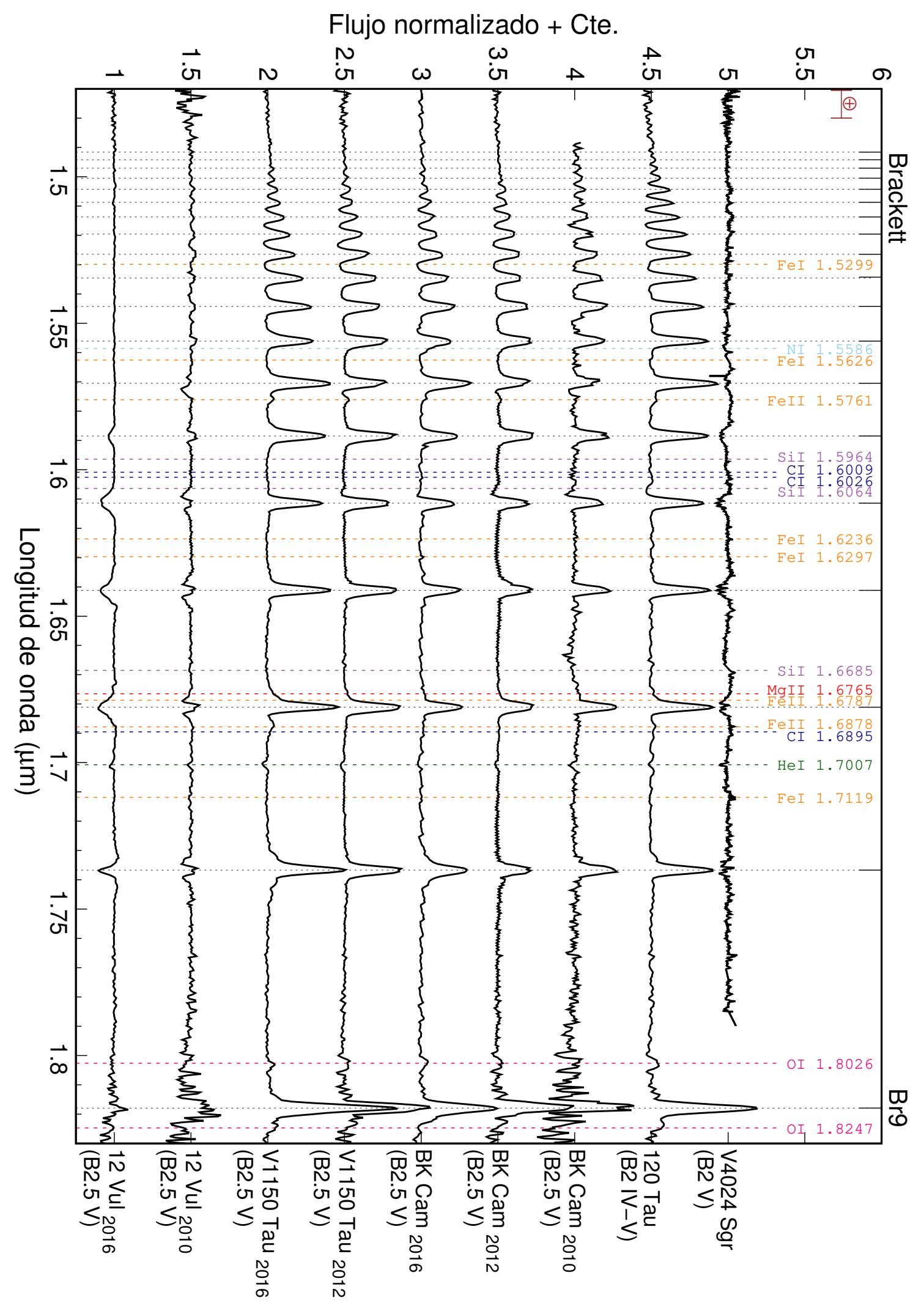


Figura 3.7: Espectros obtenidos en la banda H utilizando GNIRS o FIRE.

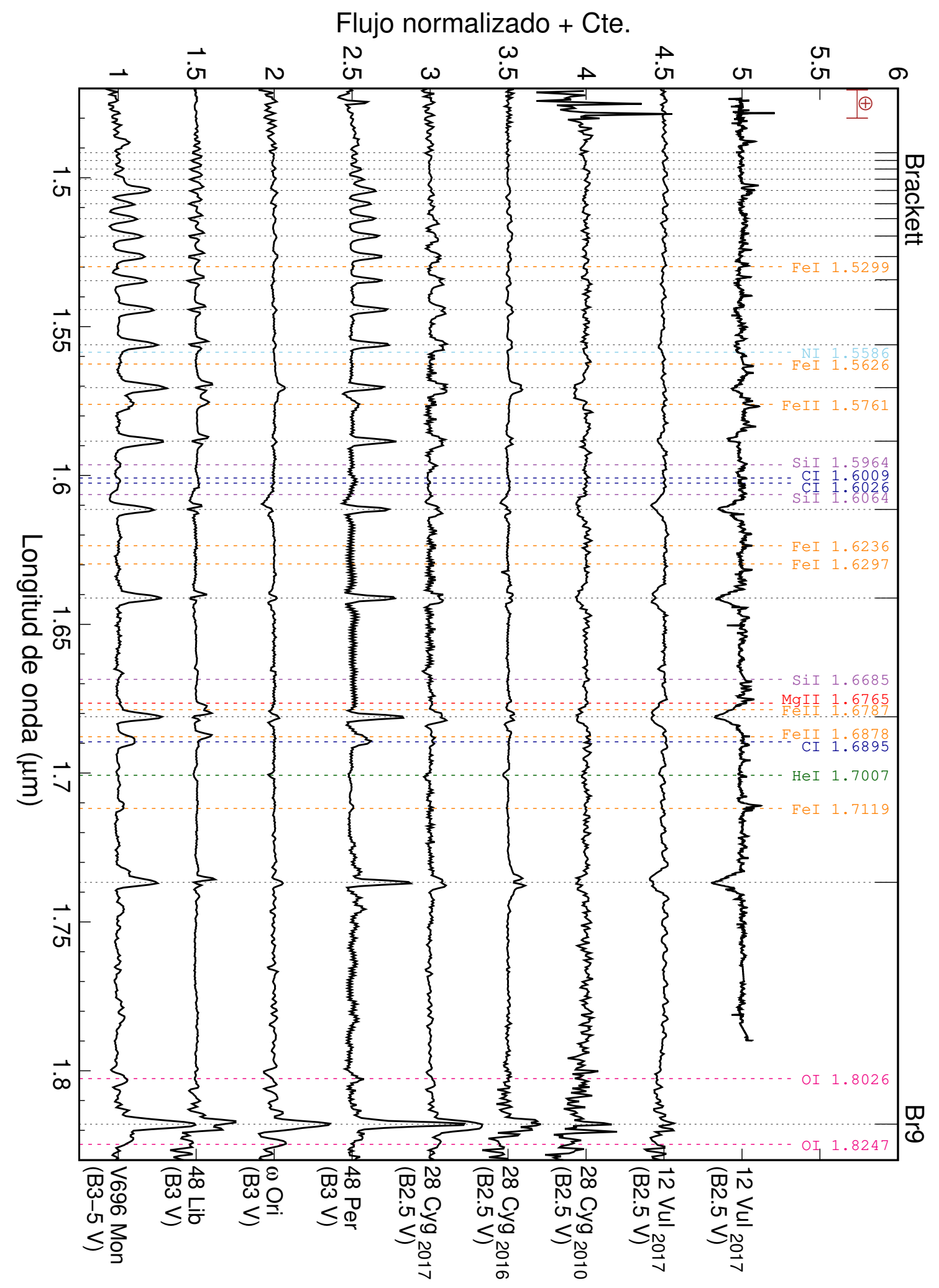


Figura 3.8: Espectros obtenidos en la banda H utilizando GNIRS o FIRE.

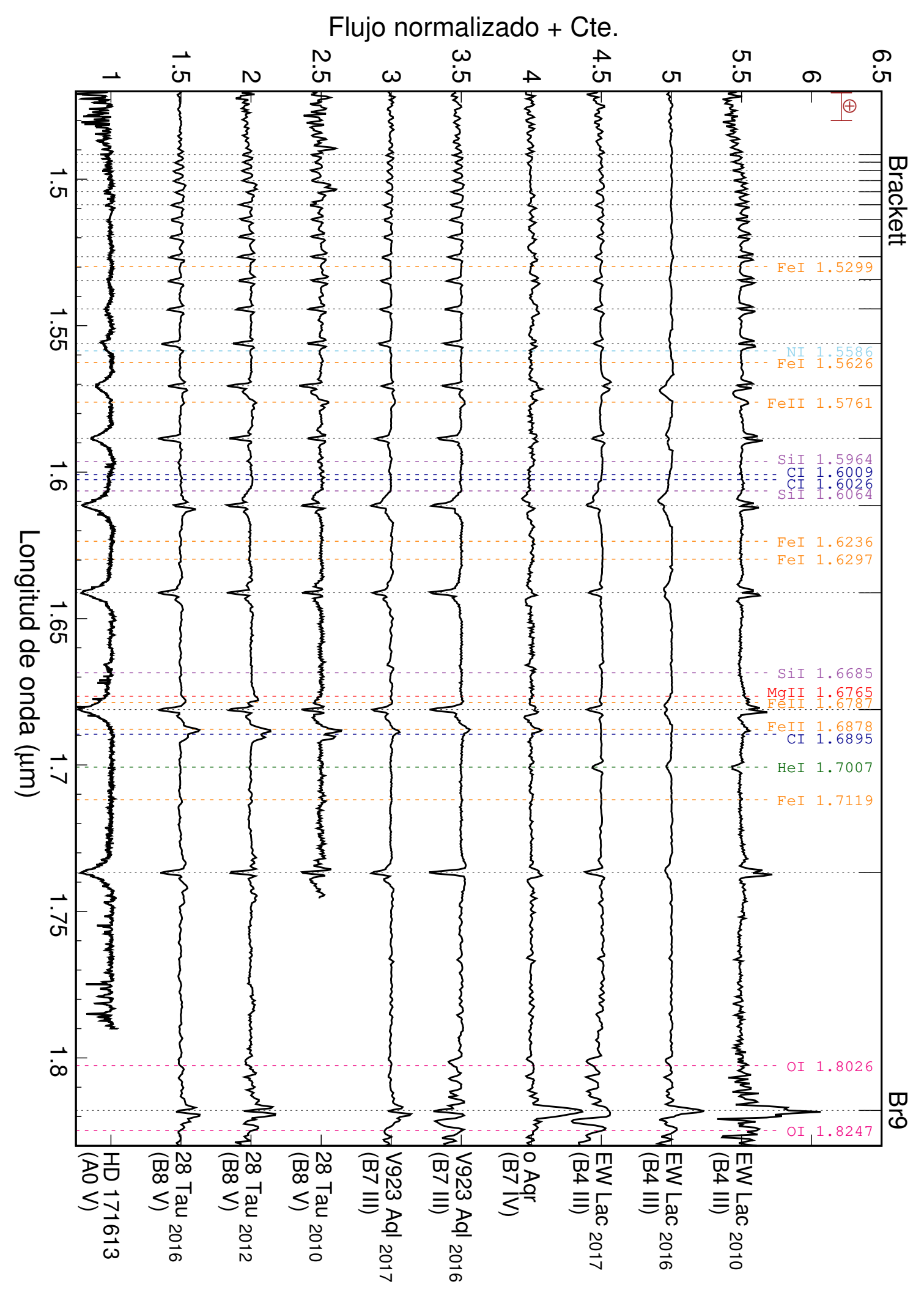


Figura 3.9: Espectros obtenidos en la banda K utilizando NIRI, GNIRS o FIRE.

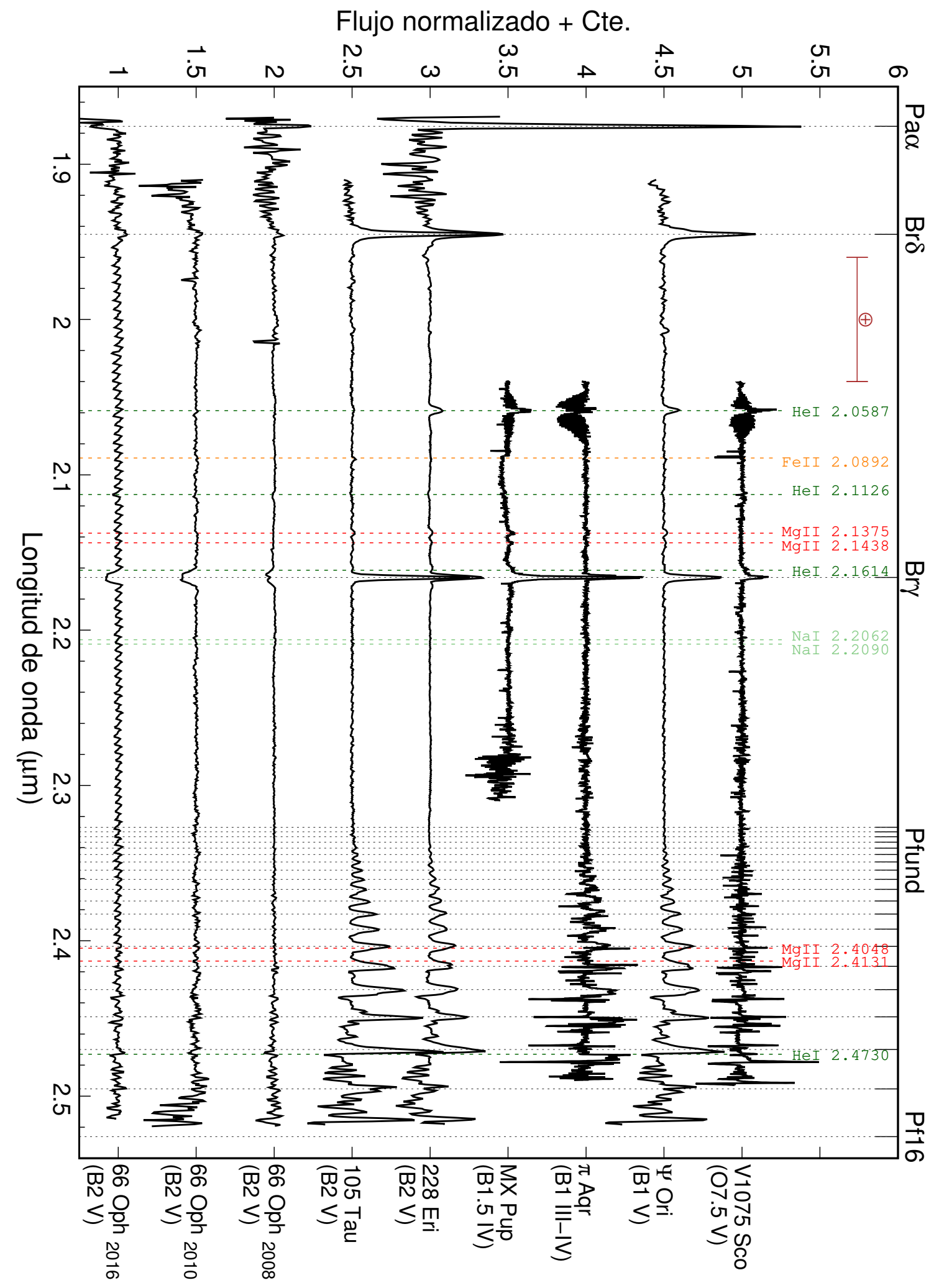


Figura 3.10: Espectros obtenidos en la banda K utilizando NIRI, GNIRS o FIRE.

Flujo normalizado + Cte.

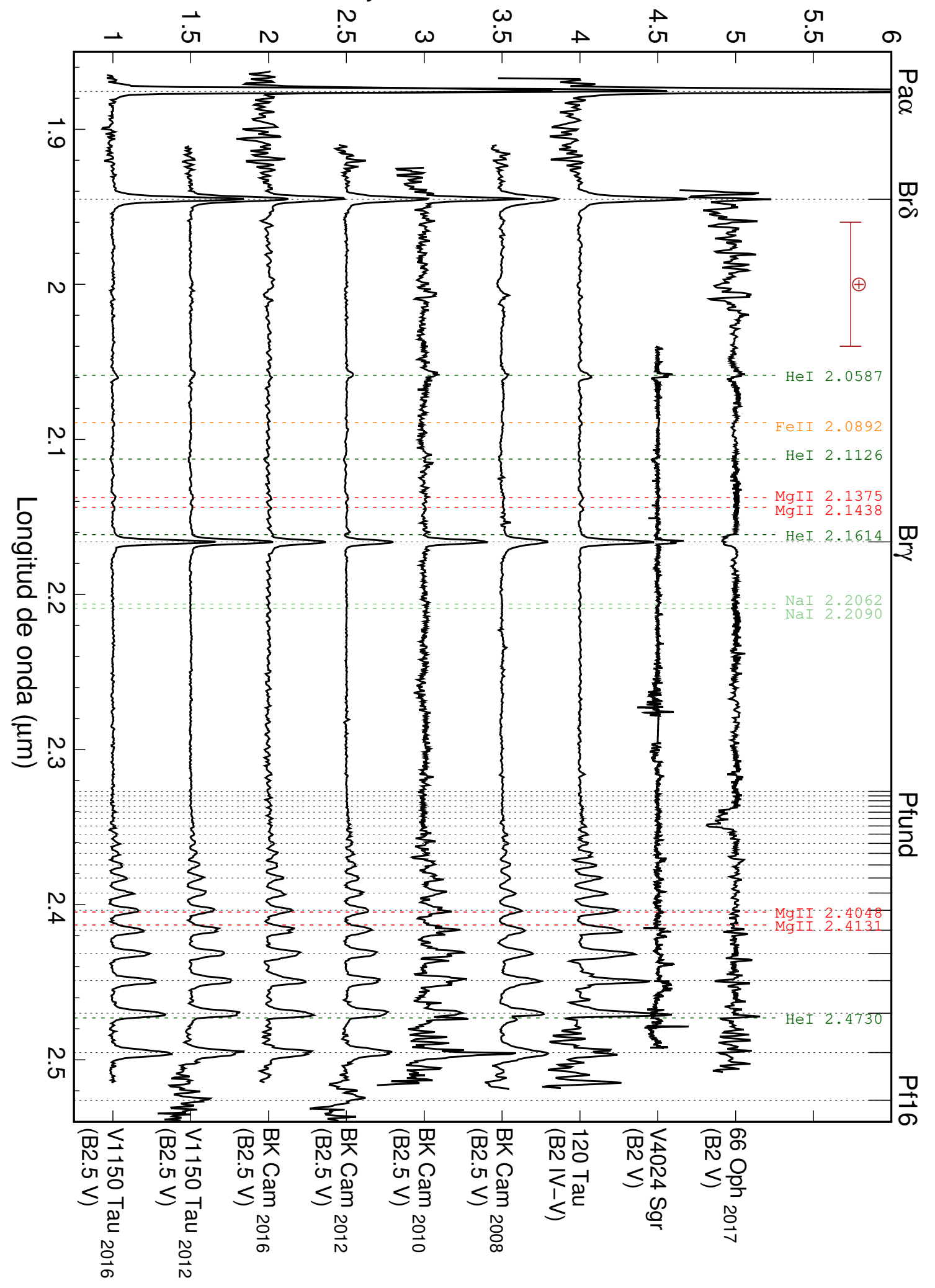


Figura 3.11: Espectros obtenidos en la banda K utilizando NIRI, GNIRS o FIRE.

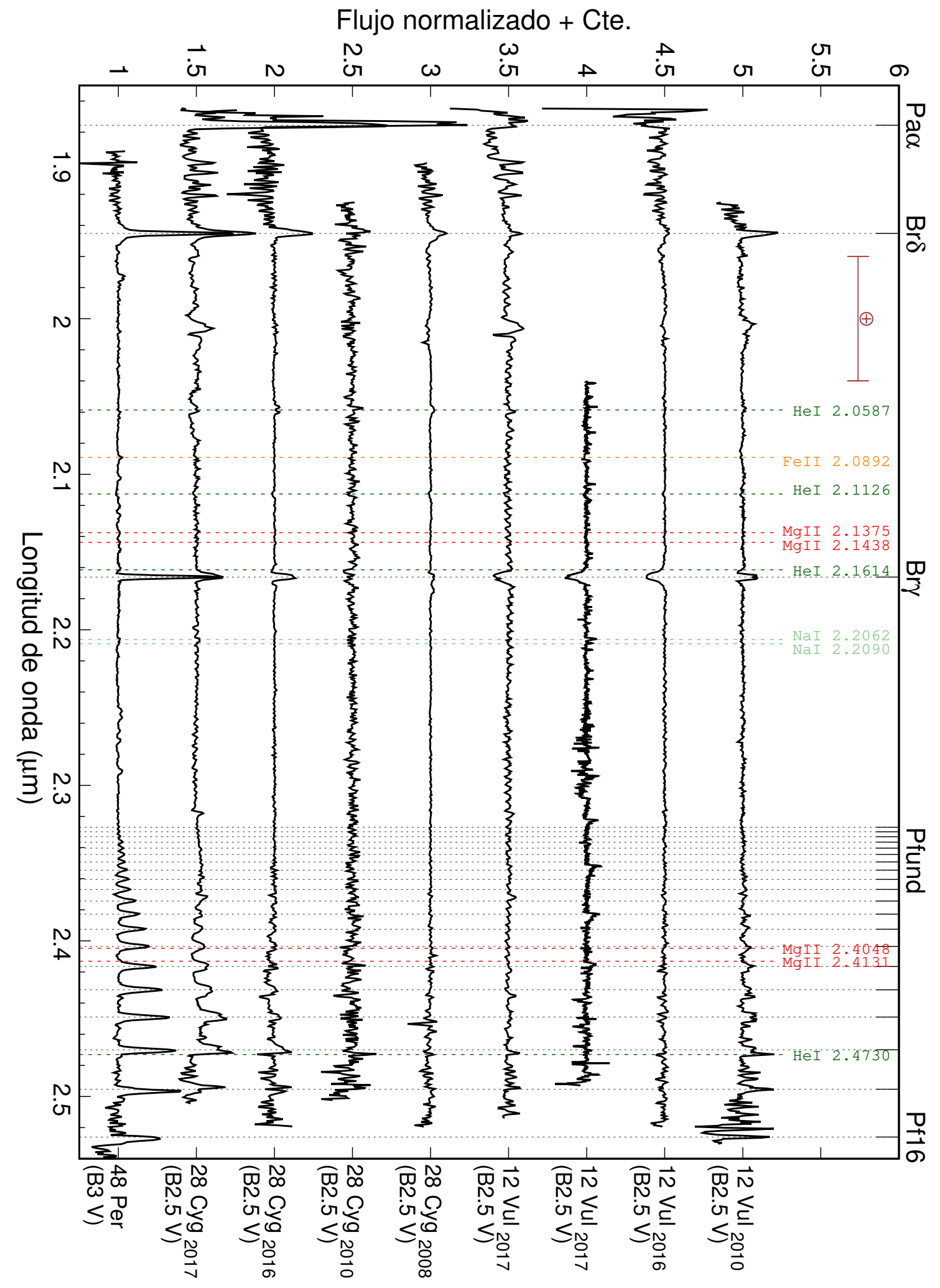


Figura 3.12: Espectros obtenidos en la banda K utilizando NIRI, GNIRS o FIRE.

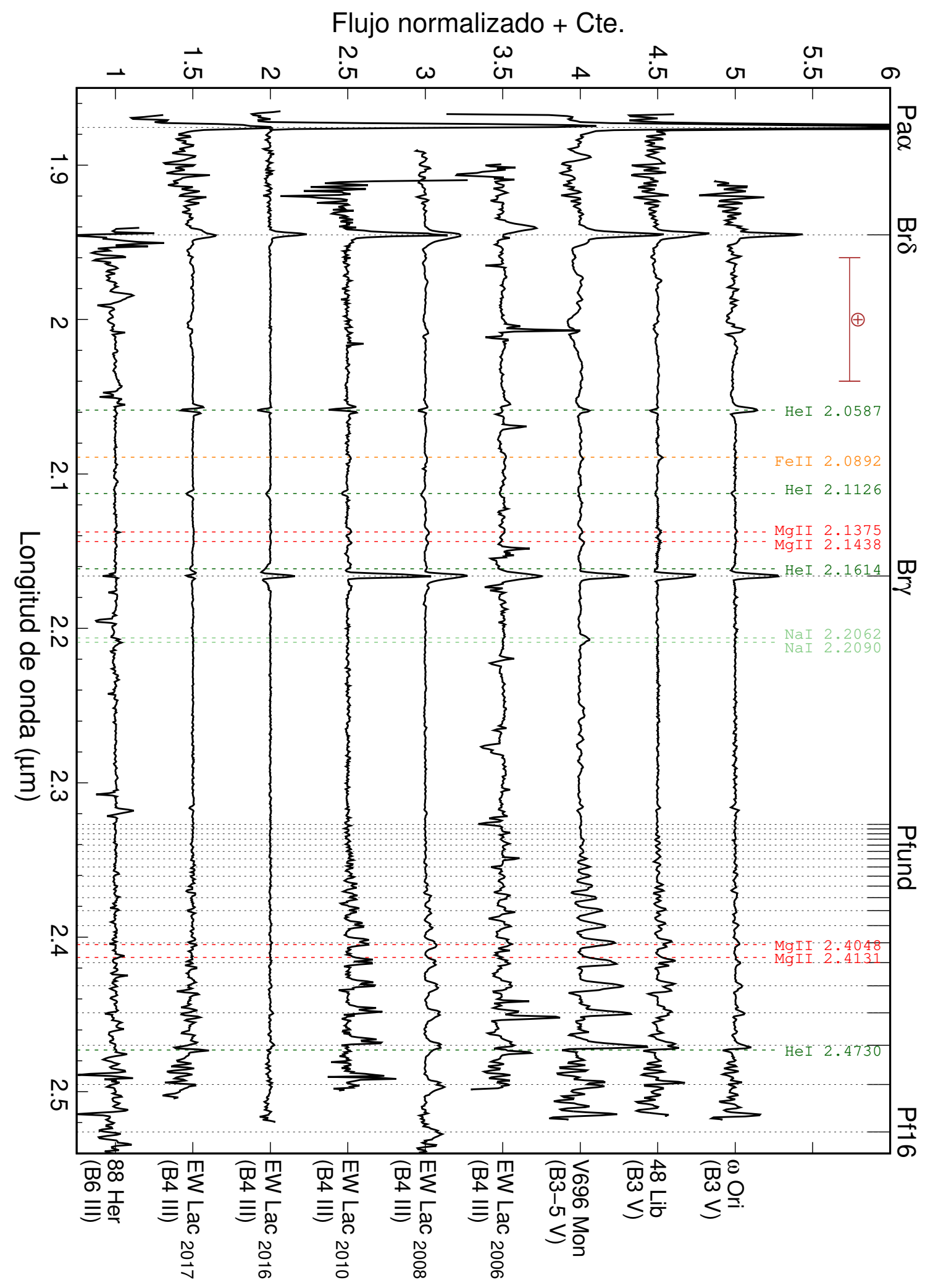


Figura 3.13: Espectros obtenidos en la banda K utilizando NIRI, GNIRS o FIRE.

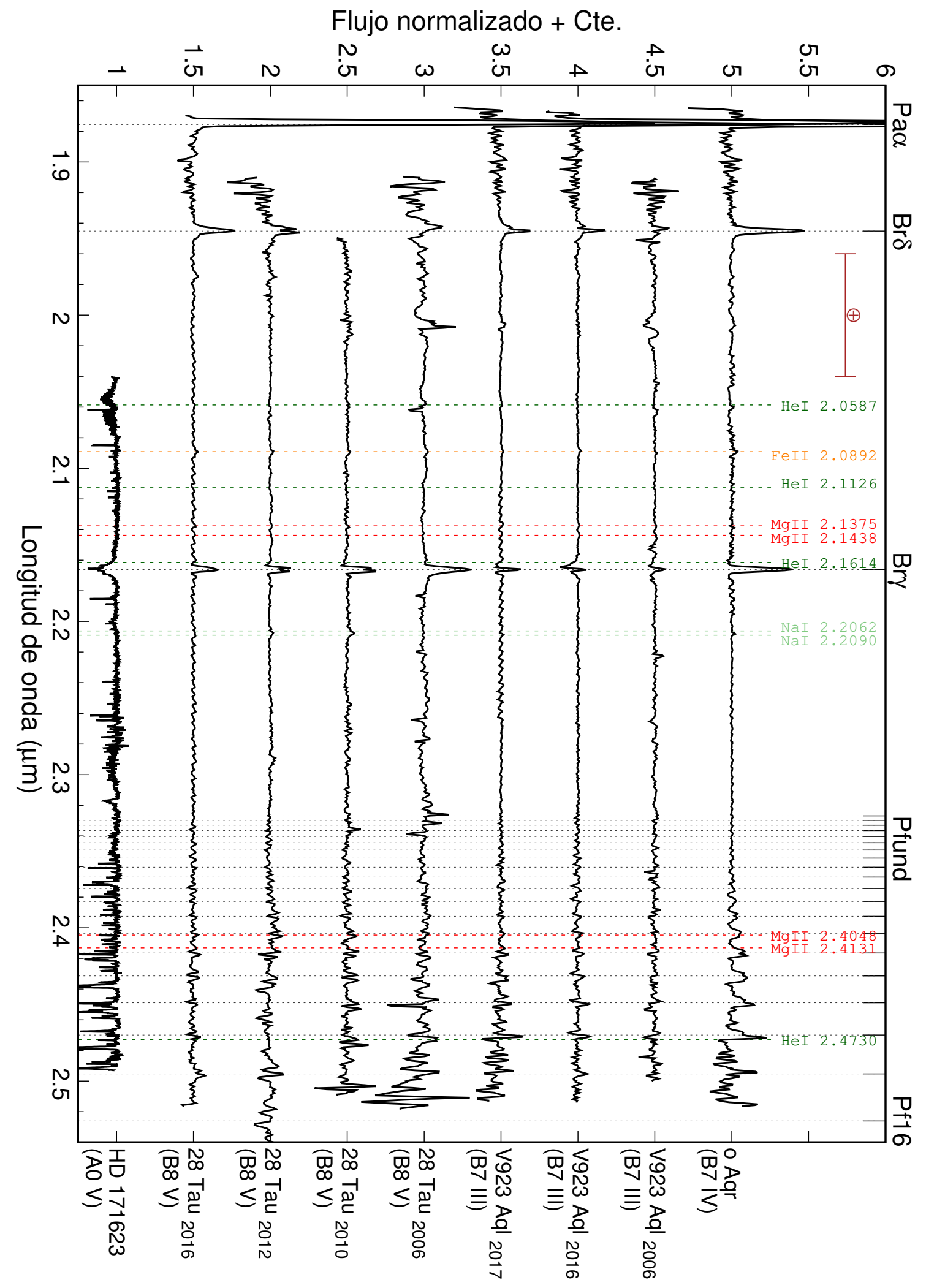


Figura 3.14: Espectros obtenidos en la banda L utilizando NIRI o GNIRS.

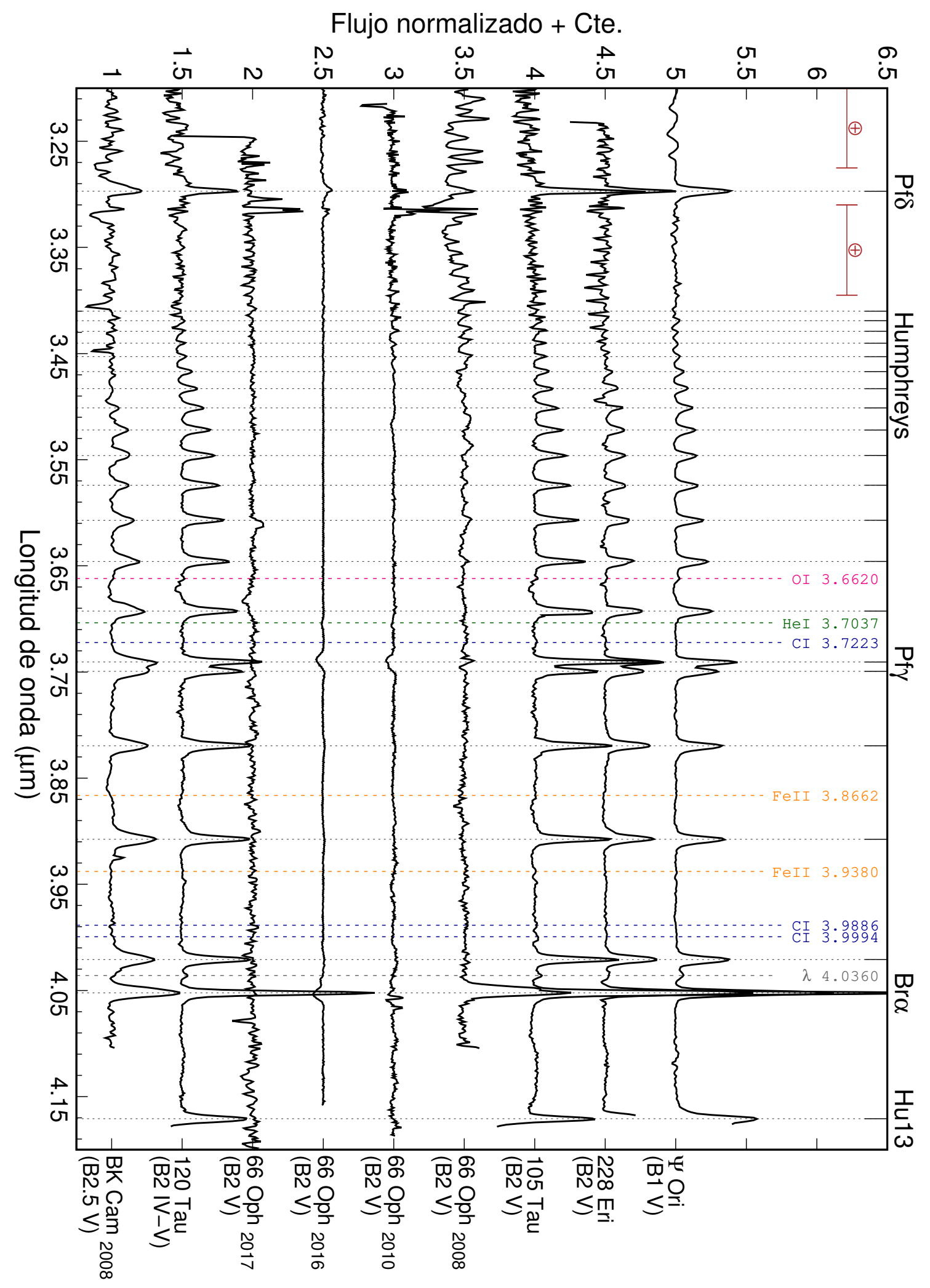


Figura 3.15: Espectros obtenidos en la banda L utilizando NIRI o GNIRS.

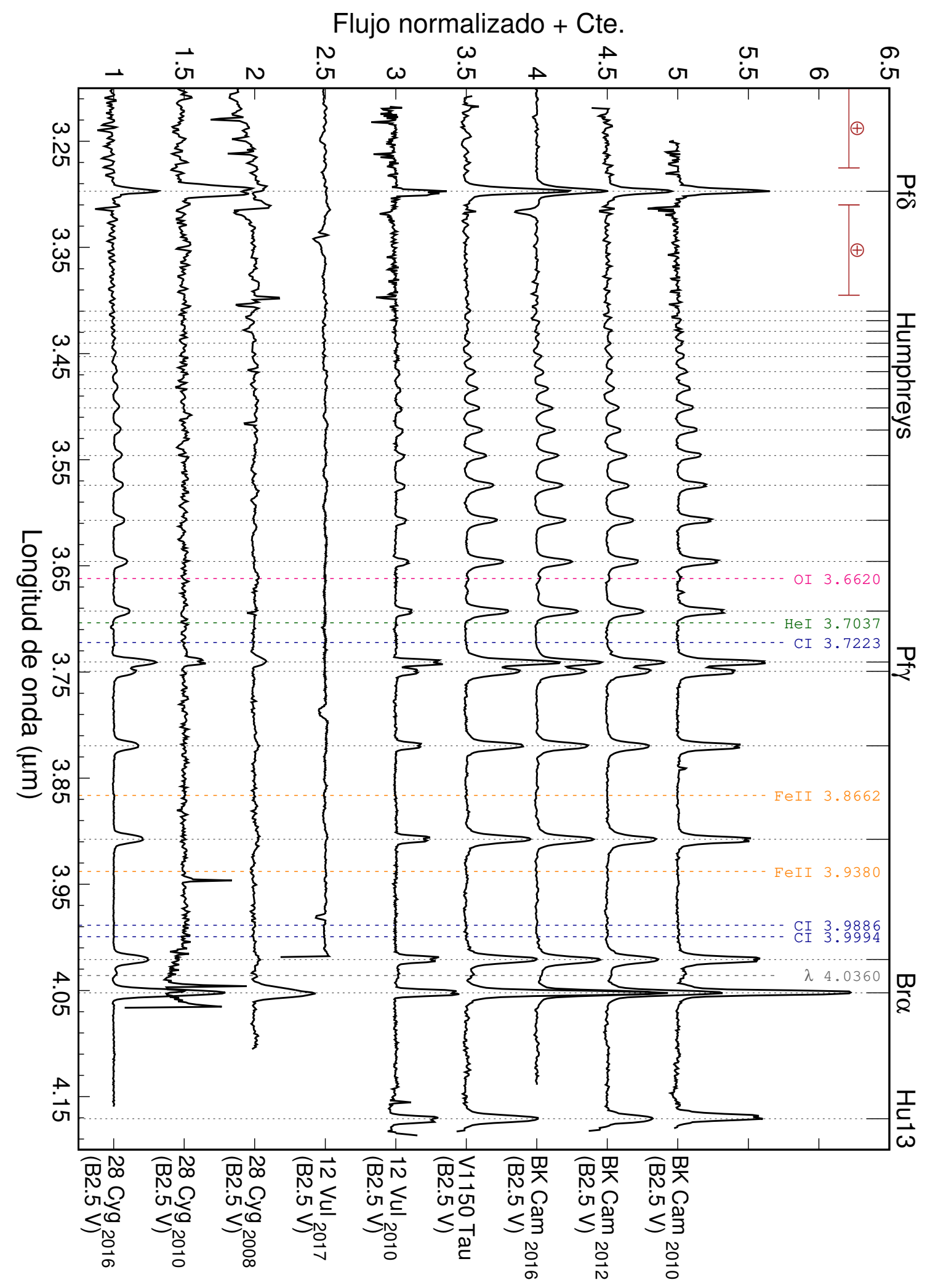


Figura 3.16: Espectros obtenidos en la banda L utilizando NIRI o GNIRS.

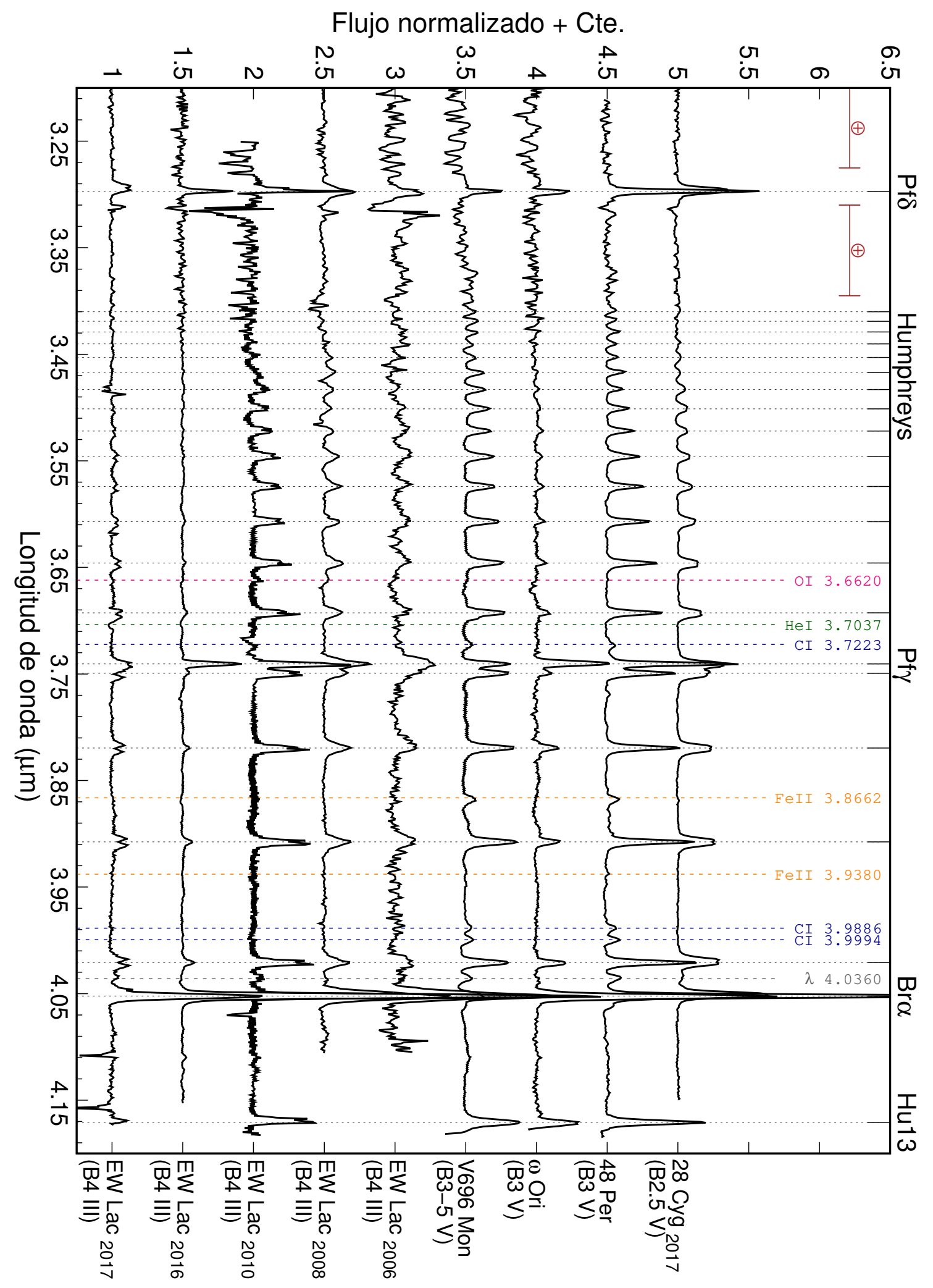


Figura 3.17: Espectros obtenidos en la banda L utilizando NIRI o GNIRS.

Flujo normalizado + Cte.

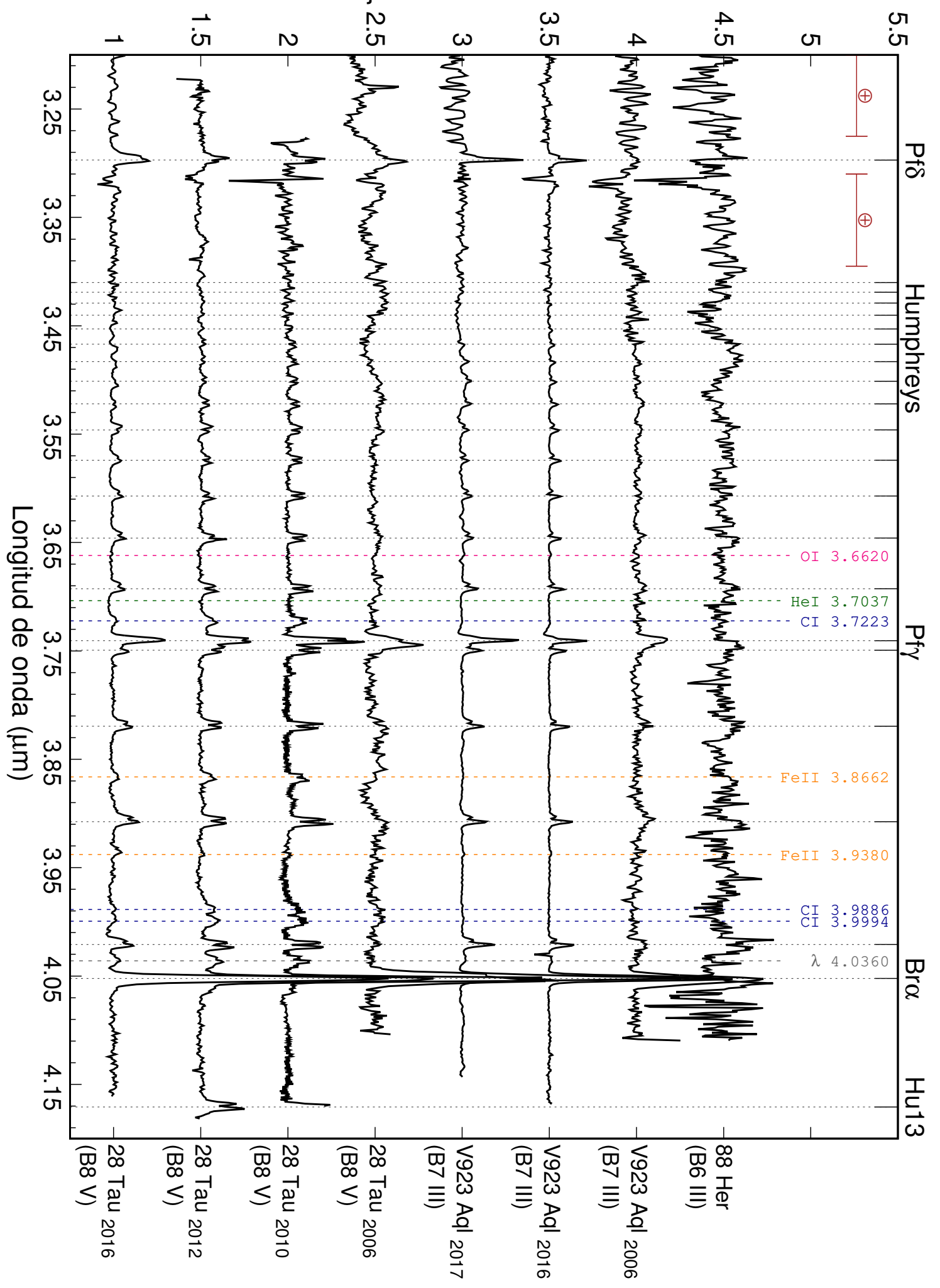


Figura 3.18: Perfiles de línea de $\operatorname{Pa} \alpha$.

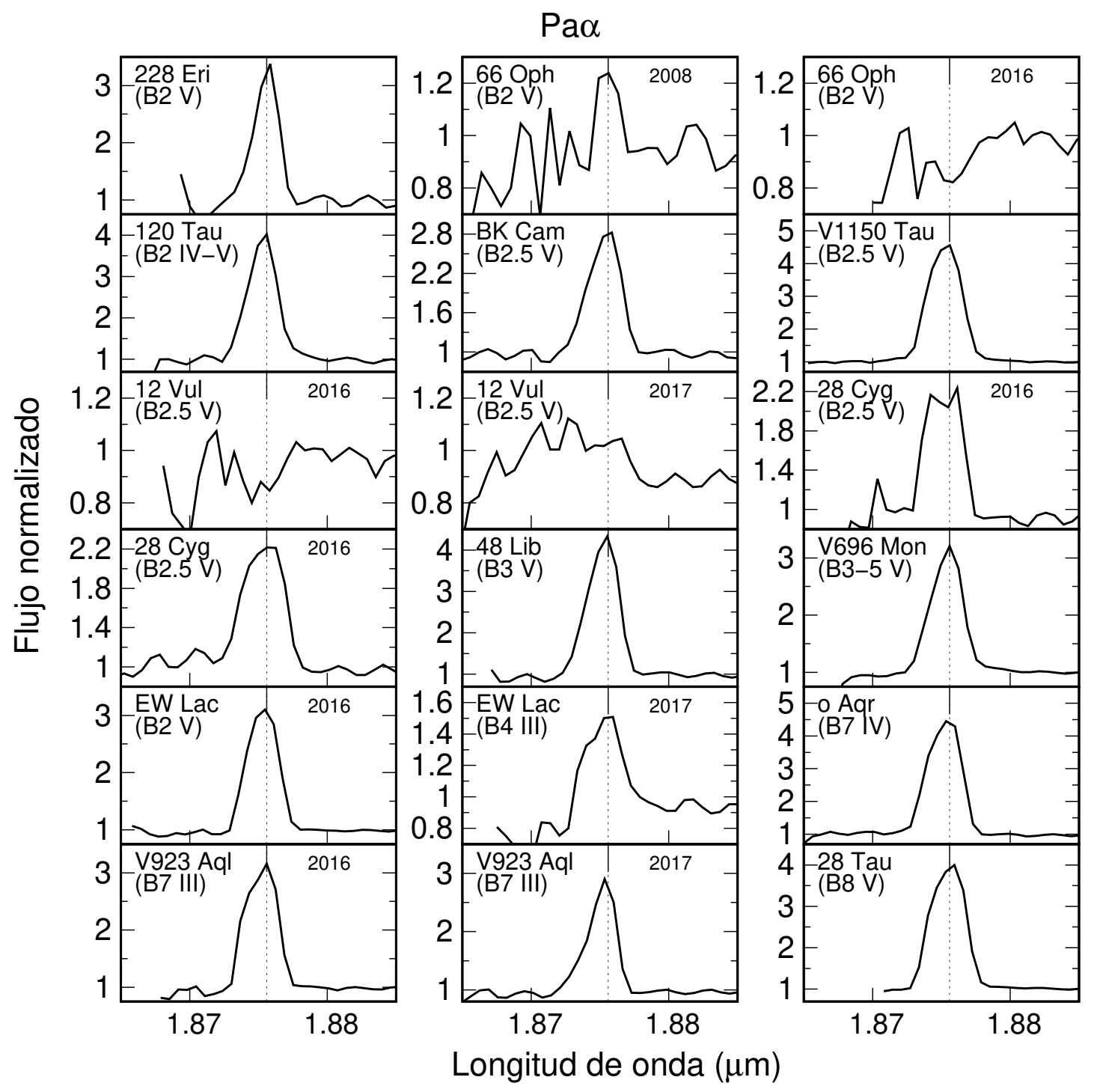


Figura 3.19: Perfiles de línea de $\operatorname{Br} \alpha$.

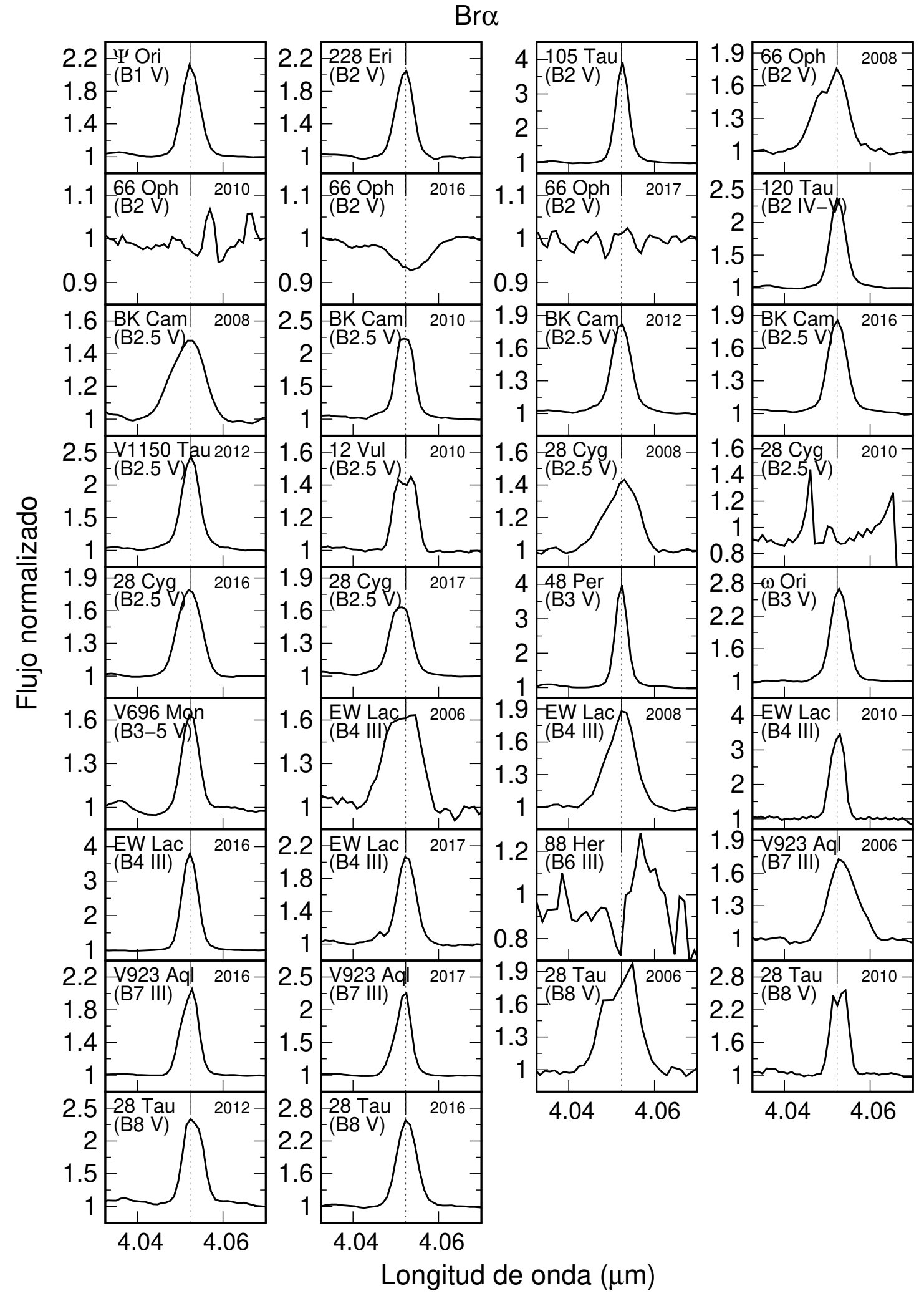




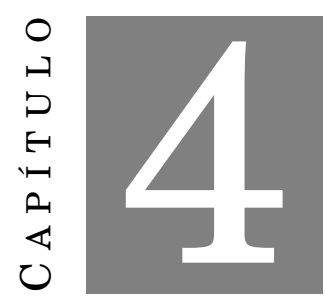

\section{METOdología APLICADA AL ANÁLISIS DE LAS OBSERVACIONES}

INFRARROJAS

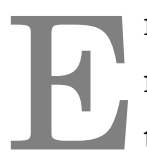

$\mathrm{n}$ el capítulo anterior hemos presentado los espectros en el infrarrojo cercano de nuestra muestra de estrellas Be. Como hemos mencionado anteriormente, estos espectros presentan numerosas líneas en emisión de hidrógeno que revelan información valiosa acerca de las características de las envolturas extendidas. Describiremos a continuación la metodología utilizada para el análisis cuantitativo de las líneas espectrales observadas en cada uno de los objetos, que luego darán lugar a la obtención de las propiedades físicas de las envolturas.

\subsection{Diagramas de cocientes de flujos como diagnóstico de las propiedades de la envoltura}

Simon et al. (1983) fueron los primeros en proponer que el cociente de los flujos de las líneas $\mathrm{Br} \gamma$, Pf y Pa $\alpha$ podían ser interpretados en términos de las profundidades ópticas de las regiones de formación de las mismas. Estos autores indicaron que para un medio ópticamente delgado, se espera que los cocientes de los flujos sigan el caso B de la teoría de recombinación de Menzel (Baker \& Menzel, 1938), donde la envoltura es opaca a las líneas de la serie de Lyman, y transparente a las líneas de las otras series. Por otra parte, en un caso ópticamente grueso, el cociente de los flujos es independiente del coeficiente de absorción másico, y el flujo de cada línea depende del tamaño de la región emisora. Suponiendo un medio isotermo en equilibrio termodinámico local, el cociente de los flujos entre dos líneas puede escribirse como

$$
\frac{I_{1}}{I_{2}}=\frac{B\left(v_{1}, T\right) S_{\mathrm{eff}, 1}}{B\left(v_{2}, T\right) S_{\mathrm{eff}, 2}}
$$


donde $B(v, T)$ es la función de Planck en la frecuencia de la línea y $S_{\text {eff }}$ es la superficie efectiva que emite en la línea. Si $S_{\text {eff }}$ es similar para ambas líneas, y usamos la aproximación de RayleighJeans, la expresión se reduce a

$$
\frac{I_{1}}{I_{2}}=\left(\frac{v_{1}}{v_{2}}\right)^{2}
$$

En base a esta propuesta, Persson \& McGregor (1985) construyeron un diagrama utilizando los cocientes de $\mathrm{Br} \alpha$, Pf $\gamma$ y $\mathrm{Hu}_{14}$, donde las estrellas Be observadas se ubican entre las posiciones correspondientes al caso $\mathrm{B}$ y al límite ópticamente grueso.

Posteriormente, Lenorzer et al. (2002a) estudiaron el espectro infrarrojo cercano para una muestra de estrellas masivas y propusieron el diagrama con ejes $\log \left(\mathrm{Hu}_{14} / \mathrm{Pf} \gamma\right)-\log \left(\mathrm{Hu}_{14} / \mathrm{Br} \alpha\right)$ como una herramienta de diagnóstico sobre las propiedades físicas del material circunestelar (ver Fig. 4.1). En este diagrama, el símbolo $\star$ ubicado en la parte superior derecha representa el caso extremo para un medio ópticamente grueso, mientras que el área rayada de la parte inferior izquierda representa un medio ópticamente delgado, calculado empleando el Caso B de la teoría de recombinación de Menzel para temperaturas mayores que $10^{4} \mathrm{~K}$ (Hummer \& Storey, 1987; Storey \& Hummer, 1995). Lenorzer et al. incluyeron en su muestra objetos en cuyas envolturas circunestelares domina un viento estelar, tales como las LBV (Luminous Blue Variables) y otros en los cuales existe una geometría de tipo disco (estrellas Be y B[e]). Todos los objetos se ubican a lo largo de la diagonal que une los casos ópticamente delgado y ópticamente grueso, separados en distintas regiones según el tipo de objeto: en la parte superior se ubican las estrellas Be con discos opacos, en la parte inferior las LBV, mientras que las $\mathrm{B}[\mathrm{e}]$ se ubican en una región intermedia. Lenorzer et al. (2002a) propusieron que la ubicación de las estrellas LBVs sugiere que la emisión en las líneas proviene principalmente de un gas ópticamente delgado, mientras que el apartamiento de la ubicación de las estrellas Be respecto del caso ópticamente grueso sugiere que existe una contribución a la emisión en las líneas proveniente de un gas ópticamente delgado. Además, debido a la naturaleza transitoria de los discos en torno a las estrellas Be, es de esperarse que se produzca una variación en la ubicación de estos objetos en el diagrama a lo largo de la diagonal.

Estos autores advirtieron que debe tenerse cuidado al realizar interpretaciones a partir de este tipo de diagrama, dado que se han reportado estrellas que se ubican en la región correspondiente al caso B de Menzel, sugiriendo que las líneas se forman en una región ópticamente delgada, pero que al analizarlas con otras técnicas presentan evidencia de la presencia de un disco de polvo. Un ejemplo de esto es MWC349, donde la emisión observada no proviene del disco. De manera similar, para las estrellas que se encuentran cerca del caso ópticamente grueso, se puede afirmar que las líneas son ópticamente opacas aunque no se pueden excluir geometrías diferentes a la de un disco para las regiones emisoras.

A partir de las líneas más intensas observadas en las bandas K y L, Granada et al. (2010) construyeron un diagrama utilizando los cocientes de flujos $\log (\operatorname{Br} \alpha / \operatorname{Pf} \gamma)-\log (\operatorname{Br} \alpha / \operatorname{Br} \gamma)$ y encon- 
Figura 4.1: Diagrama de Lenorzer et al. (2002a). Los diferentes tipos de objetos (Be con círculos vacíos, $\mathrm{B}[\mathrm{e}]$ con círculos llenos, LBV con cuadrados) quedan separados según los cocientes de líneas $\log \left(\mathrm{Hu}_{14} / \mathrm{Pf} \gamma\right)-\log \left(\mathrm{Hu}_{14} / \mathrm{Br} \alpha\right)$. El símbolo $\star$ representa la posición de una fuente ópticamente gruesa. La región rayada muestra el rango de cocientes de flujos para una región ópticamente delgada.

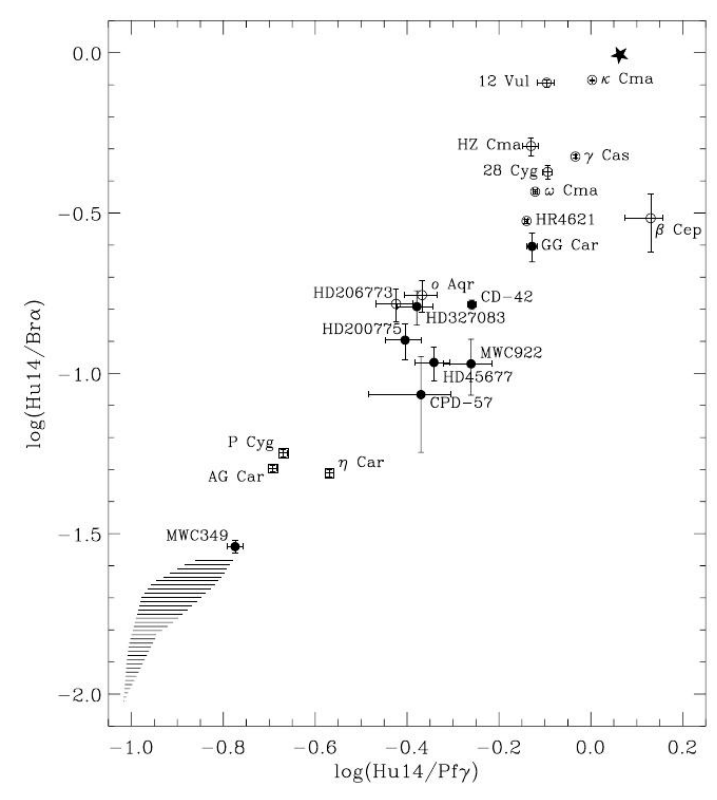

traron que estas líneas también son útiles para discriminar entre envolturas ópticamente gruesas y delgadas (ver Fig. 4.3).

El diagrama de Lenorzer resulta una herramienta valiosa para el diagnóstico de las propiedades físicas y geométricas del material circunestelar alrededor de objetos tempranos peculiares, dado que contribuyen a estudiar la evolución de las envolturas en el caso de estrellas Be variables y a poner restricciones a los modelos teóricos de estrellas con líneas en emisión.

\subsection{Propiedades de las envolturas según la intensidad de las líneas}

\subsubsection{Clasificación en grupos}

Mennickent et al. (2009) estudiaron un conjunto de 13 estrellas Be en la banda L y las separaron en tres grupos, según la relación entre las intensidades de $\operatorname{Br} \alpha$, $\operatorname{Pf} \gamma$ y las líneas de la serie de Humphreys. Las estrellas donde las líneas de la serie de Humphreys tienen intensidades comparables a $\operatorname{Br} \alpha$ y $\operatorname{Pf} \gamma$ forman el Grupo I, las estrellas donde $\operatorname{Br} \alpha$ y $\operatorname{Pf} \gamma$ son más intensas que las líneas de la serie de Humphreys forman el Grupo II, y las estrellas que no presentan emisión forman el Grupo III. Ejemplos de cada grupo pueden observarse en la Fig. 4.2. Ubicando a las estrellas pertenecientes a los diferentes grupos en el diagrama de Lenorzer, estos autores 
Figura 4.2: Ejemplos de los grupos definidos por Mennickent et al. (2009).

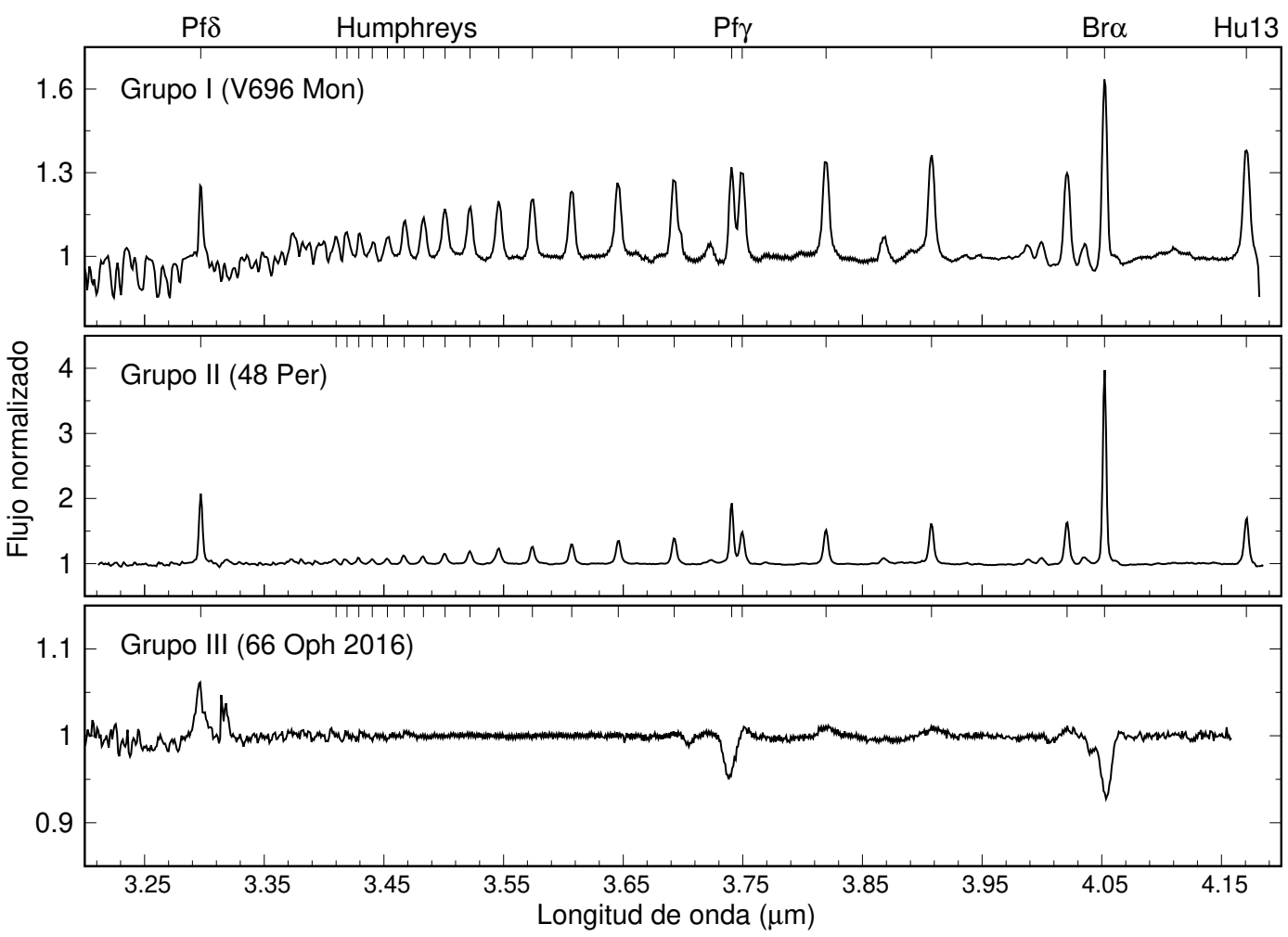

encontraron que las estrellas pertenecientes al Grupo I se ubican en la parte superior derecha, indicando la presencia de envolturas ópticamente gruesas, mientras que las estrellas del Grupo II quedan distribuidas en una región de moderada a pequeña profundidad óptica. Por otra parte, sugirieron que las estrellas del grupo III, que no presentan líneas en emisión, serían objetos que han perdido la mayor parte de su envoltura. De esta manera, los grupos propuestos dan cuenta de la profundidad óptica del material circunestelar. En la Fig. 4.3 pueden verse los objetos estudiados por Granada et al. (2010), donde se observan las ubicaciones de distintos grupos en diagramas que utilizan cocientes de distintas líneas.

\subsubsection{Decrementos de líneas de una misma serie}

En diversos trabajos se reportaron evidencias de una fuerte correlación entre el cociente entre el ancho equivalente EW y la longitud de onda $(\mathrm{EW} / \lambda)$ vs. la longitud de onda para las líneas de hidrógeno. Hony et al. (2000) observaron el espectro de $\gamma$ Cas y obtuvieron que el cociente EW/ $\lambda$ se comportaba de manera similar en todas las series. Para los miembros intermedios de cada serie el cociente se mantenía independiente de la longitud de onda con un valor $\sim 5 \cdot 10^{-4}$. Además, los primeros miembros de cada serie se desviaban de ese comportamiento hacia valores mayores, y hacia los miembros más altos los valores de $\mathrm{EW} / \lambda$ decrecían. Esto fue interpretados por los autores en términos del decrecimiento de la función fuente de la línea en las partes externas 
Figura 4.3: Ubicación de las estrellas observadas por Granada et al. (2010) en el diagrama de Lenorzer et al. (2002a, izquierda) y en el diagrama que utiliza los cocientes $\log (\operatorname{Br} \alpha / \mathrm{Pf} \gamma)-\log (\operatorname{Br} \alpha / \operatorname{Br} \gamma)($ derecha). Las estrellas quedan ubicadas según los grupos definidos por Mennickent et al. (2009).
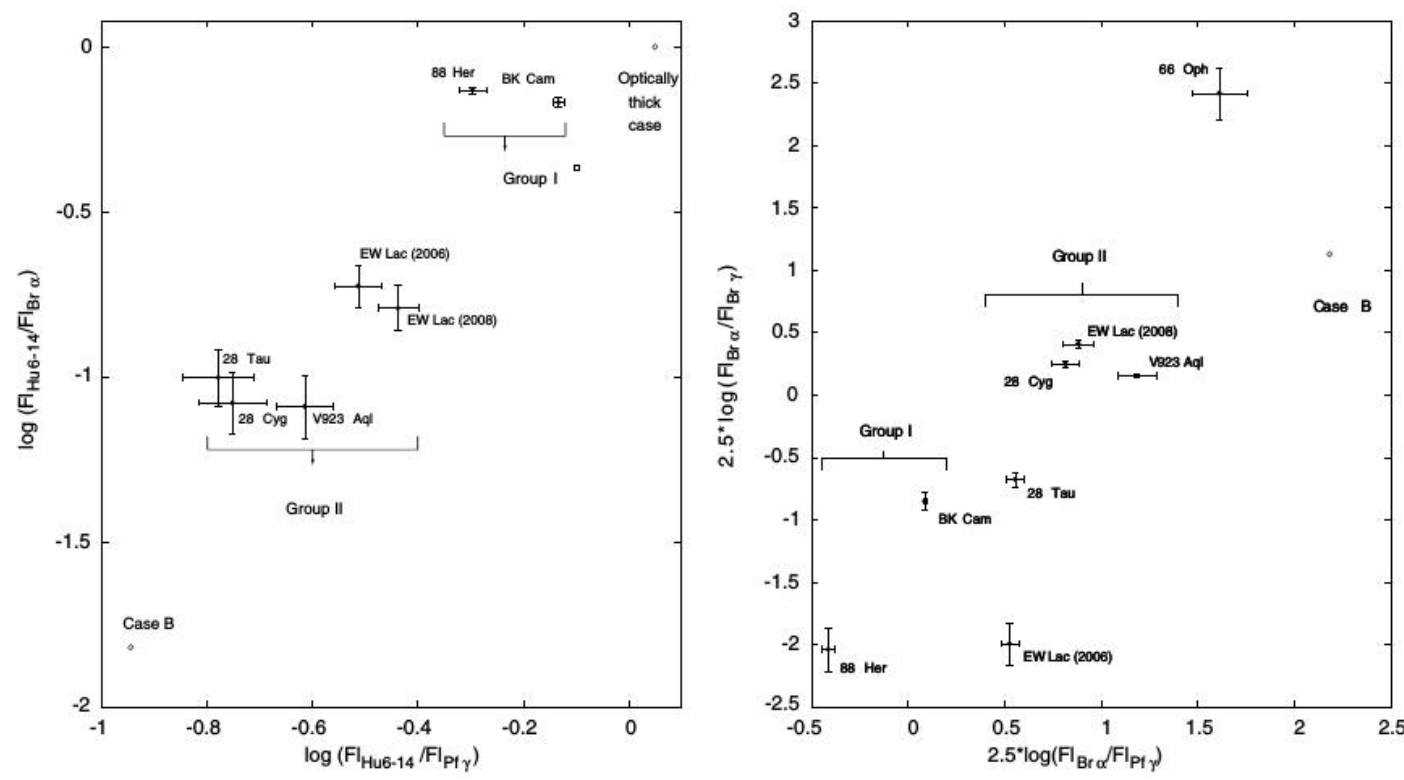

de la envoltura circunestelar. Más adelante, Mennickent et al. (2009) encontraron que EW/ $\lambda$ aumentaba con $\lambda$ hasta saturar en $\mathrm{EW} / \lambda \sim 6 \cdot 10^{-4}$ para todas las líneas observadas en la banda $\mathrm{L}$ en las estrellas del grupo I, mientras que en las del grupo II Br $\alpha$ y $\mathrm{Pf} \gamma$ se apartan de la tendencia definida por las líneas de la serie de Humphreys.

Por otro lado, Mennickent et al. (2009) estudiaron la relación entre los flujos $\mathrm{Fl}\left(\mathrm{Hu} \mathrm{n}_{\mathrm{n}}\right) / \mathrm{Fl}\left(\mathrm{Hu}_{19}\right)$ (donde $\mathrm{n}$ es el numero cuántico del nivel superior de la transición) vs. $\lambda$ para todas las estrellas que presentaban líneas en emisión. Estos autores encontraron que para las estrellas del grupo II el cociente crece con $\lambda$, indicando condiciones ópticamente delgadas, mientras que para las estrellas del grupo I, los decrementos cambian su comportamiento a partir de cierta transición pasando de condiciones ópticamente delgadas para las longitudes de onda más cortas a ópticamente gruesas para las longitudes de onda mayores. Esta transición podría reflejar un cambio en las propiedades de las envolturas a partir de cierta distancia de la estrella. Cabe destacar que aún para estrellas Be con envolturas ópticamente gruesas, los miembros altos de la serie de Humphreys se formarían en regiones ópticamente delgadas.

Granada et al. (2010) continuaron con el análisis de los decrementos a partir de observaciones de un grupo de ocho estrellas Be, encontrando resultados similares a los de Mennickent et al. (2009) para los decrementos de la serie de Humphreys. Además, analizaron las series de Pfund y Brackett y encontraron que, en el caso de la serie de Pfund, todas las líneas siguen la relación lineal correspondiente al caso ópticamente delgado, excepto $\operatorname{Pf} \gamma$ y $\operatorname{Pf} \delta$. Este apartamiento del caso ópticamente delgado sugiere cambios en la profundidad óptica de la envoltura, aunque la 
presencia de un disco truncado o de geometrías diferentes a las de un disco podrían influir en este apartamiento. Con respecto a la serie de Brackett, encontraron que para las estrellas del grupo I el cociente $\mathrm{EW}(\mathrm{Br} \alpha) / \mathrm{EW}(\mathrm{Br} \gamma)$ tomaba valores entre 2 y 3 , mientras que las del grupo II presentaban valores en $\mathrm{EW}(\mathrm{Br} \alpha)>5 \mathrm{EW}(\mathrm{Br} \gamma)$. Por otra parte, el cociente entre $\mathrm{EW}(\mathrm{Br} \delta)$ y $\mathrm{EW}(\mathrm{Br} \gamma)$ permanece casi constante, sugiriendo que esta cantidad no cambia mucho entre un grupo y el otro.

\subsection{Evidencias de la evolución de las envolturas}

Debido al carácter variable de las estrellas Be, es esperable que las propiedades de las envolturas varíen a lo largo del tiempo y, por consiguiente, varíen las posiciones en los diferentes diagramas.

Cambios en la posición en el diagrama de Lenorzer fueron reportados para 12 Vul por Mennickent et al. (2009) y para 28 Cyg por Granada et al. (2010). En ambos casos, los objetos eran estrellas pertenecientes al grupo I en la observación realizada por Lenorzer et al. (2002a), y posteriormente mostraron características que las ubicaban en el grupo II. Además, Granada et al. (2010) reportaron que la ubicación de EW Lac en el diagrama de Lenorzer parece ser una transición entre ambos grupos. Estos cambios fueron interpretados en términos de la eyección de una envoltura ópticamente gruesa, que se expande y se convierte en una envoltura ópticamente delgada.

Sabogal et al. (2017) presentaron observaciones en la banda L de 7 estrellas Be, 2 de las cuales no habían sido observadas previamente, y correlacionaron la intensidad de las líneas en emisión presentes en esa banda con lo observado en la línea H $\alpha$. Además, ubicaron a las estrellas observadas por Lenorzer et al. (2002a), Mennickent et al. (2009), Granada et al. (2010) y Touhami et al. (2010), junto a las de su trabajo, en el diagrama de Lenorzer. En este diagrama, destacaron que cinco objetos ( $28 \mathrm{Cyg}, 28 \mathrm{Tau}, \gamma \mathrm{Cas}, \phi$ Per y $12 \mathrm{Vul}$ ) fueron observados en diferentes épocas y presentaron desplazamientos en el diagrama. A raíz de eso, estos autores proponen una clasificación basada en los desplazamientos de los objetos en el diagrama de Lenorzer y en la variabilidad de $\mathrm{H} \alpha$, separando a las estrellas en estrellas con discos estables (cuya posición en el diagrama no cambia notablemente y $\mathrm{H} \alpha$ no presenta grandes cambios a lo largo del tiempo), con discos en disipación (decaying stars, que presentan grandes cambios en sus espectros que indican cambios de un disco ópticamente grueso a uno delgado), y con discos en desarrollo (building-up, que comienzan a desarrollar una envoltura luego de una fase como estrella B normal, o dónde la envoltura se vuelve ópticamente gruesa). Espectros de discos en desarrollo no fueron observados en la banda L, pero los espectros obtenidos en la línea $\mathrm{H} \alpha$, por ejemplo para la estrellas $12 \mathrm{Vul}$, indican transferencia de masa hacia el disco.

Estos resultados constituyen un paso inicial para el estudio sistemático de las líneas infrarrojas de estrellas Be variables y su relación con la evolución de sus envolturas. 


\subsection{Densidad columnar y tamaño de la envoltura}

A partir del apartamiento de los cocientes de los flujos $\mathrm{Fl}\left(\mathrm{Hu}_{\mathrm{n}}\right) / \mathrm{Fl}\left(\mathrm{Hu}_{19}\right)$ respecto a lo esperado para un caso ópticamente delgado, se puede realizar una estimación de la densidad columnar de átomos y de la extensión de la región de formación de la línea. Por ejemplo, para la serie de Humphreys, el flujo emitido por la n-ésima línea de la serie puede escribirse como $\mathrm{Fl}\left(\mathrm{Hu}_{\mathrm{n}}\right)=\mathrm{Fl}\left(\mathrm{Hu}_{\mathrm{n} \text {-del }}\right) \cdot e^{-\tau}$, donde $\mathrm{Fl}\left(\mathrm{Hu}_{\mathrm{n}}\right)$ es el flujo observado y $\mathrm{Fl}\left(\mathrm{Hu}_{\mathrm{n} \text {-del }}\right)$ es el flujo esperado si la transición fuera ópticamente delgada. La profundidad óptica de la línea, $\tau$, se define como

$$
\tau=\frac{\sqrt{\pi} e^{2} f_{\mathrm{n}}}{m_{\mathrm{e}} c \Delta v_{\mathrm{Dn}}} N
$$

donde $m_{\mathrm{e}}$ es la masa del electrón, $c$ es la velocidad de la luz, $\Delta v_{\mathrm{Dn}}$ es el ensanchamiento Doppler en frecuencia, $e$ es la carga del electrón, $f_{\mathrm{n}}$ es la intensidad de oscilador de la línea y $N$ es la densidad columnar de átomos absorbentes. La densidad columnar puede calcularse entonces como

$$
N=\ln \left(\frac{\mathrm{Fl}\left(\mathrm{Hu}_{\mathrm{n}-\mathrm{del}}\right)}{\mathrm{Fl}\left(\mathrm{Hu}_{\mathrm{n}}\right)}\right) \frac{m_{\mathrm{e}} c \Delta v_{\mathrm{D} n}}{\sqrt{\pi} e^{2} f_{n}} .
$$

Según Zorec et al. (2007), la variación de la densidad con la distancia en las regiones interiores del disco es de la forma

$$
\rho(r)=\rho_{o}\left(\frac{R}{R_{\star}}\right)^{-0.5}
$$

por lo que la densidad columnar de átomos puede reescribirse como

$$
N=\int_{R_{\text {int }}}^{R_{\text {ext }}} n(R) d R=\bar{n} \Delta R
$$

Esta aproximación supone que las líneas de Humphreys se forman en una región cercana a la estrella central donde podemos aproximar la densidad numérica $n(R)$ por un valor medio en la región $\bar{n}$, y $\Delta R=R_{\text {ext }}-R_{\text {int }}$ es la extensión de la región emisora de la línea. Como $\bar{n}$ es el mismo para todas las líneas de la serie, es posible estimar la extensión de la región de formación de cada línea respecto a una línea de referencia, por ejemplo $\mathrm{Hu}_{14}$, a partir de la siguiente relación

$$
\frac{N\left(\mathrm{Hu}_{\mathrm{n}}\right)}{N\left(\mathrm{Hu}_{14}\right)}=\frac{\Delta R\left(\mathrm{Hu}_{\mathrm{n}}\right)}{\Delta R\left(\mathrm{Hu}_{14}\right)}=\frac{\ln \left(\frac{\mathrm{Fl}\left(\mathrm{Hu}_{\mathrm{n}-\mathrm{del}}\right)}{\mathrm{Fl}\left(\mathrm{Hu}_{\mathrm{n}}\right)}\right)}{\ln \left(\frac{\mathrm{Fl}\left(\mathrm{Hu}_{14-\mathrm{del}}\right)}{\mathrm{Fl}\left(\mathrm{Hu}_{14}\right)}\right)} \cdot \frac{\Delta v_{\mathrm{Dn}}}{\Delta v_{\mathrm{D} 14}} \cdot \frac{f_{14}}{f_{\mathrm{n}}} .
$$

Este método para determinar densidades columnares y extensiones de las regiones de formación lo llamaremos "Método $N$ ".

Por otro lado, si suponemos una ley de rotación Kepleriana de la forma

$$
v(R)=v_{\star}\left(\frac{R}{R_{\star}}\right)^{j},
$$


donde $j=-0.5$ (como se verifica en el Cap. 8) el radio interno de la región de formación de la línea n-ésima de la serie de Humphreys se obtiene haciendo

$$
\frac{R\left(\mathrm{Hu}_{\mathrm{n}}\right)}{R_{\star}}=\left(\frac{v\left(\mathrm{Hu}_{\mathrm{n}}\right)}{v_{\star}}\right)^{-2} .
$$

Esto nos permite obtener los cocientes de radios internos de las regiones de formación de las diferentes líneas relativos a una de referencia, lo que llamaremos "Método FWHM", a partir de la expresión

$$
\frac{R\left(\mathrm{Hu}_{\mathrm{n}}\right)}{R\left(\mathrm{Hu}_{14}\right)}=\left(\frac{v\left(\mathrm{Hu}_{\mathrm{n}}\right)}{v\left(\mathrm{Hu}_{14}\right)}\right)^{-2}=\left(\frac{F W H M\left(\mathrm{Hu}_{\mathrm{n}}\right)}{F W H M\left(\mathrm{Hu}_{14}\right)}\right)^{-2},
$$

donde consideramos una relación lineal entre $v\left(\mathrm{Hu}_{\mathrm{n}}\right)$ y $F W H M\left(\mathrm{Hu}_{\mathrm{n}}\right)$ (Hanuschik, 1996).

Algo similar puede hacerse a partir de las líneas con doble pico en emisión, lo que denominaremos "Método $\Delta V$ ". A partir de la separación de los mismos $\left(\Delta V_{\mathrm{n}}\right)$, podemos obtener los radios internos de las regiones emisoras en términos del radio estelar. Si consideramos que la separación de los picos es debido al desplazamiento Doppler por la velocidad de rotación de la región emisora, y que la misma sigue una ley Kepleriana, podemos obtener que

$$
R\left(\mathrm{Hu}_{\mathrm{n}}\right)=\left(\frac{2 V \sin (i)}{\Delta V_{\mathrm{n}}}\right)^{2} R \star .
$$

Estos métodos para determinar tamaños y extensiones de las regiones emisoras pueden aplicarse también a las líneas de las otras series.

\subsection{Correcciones aplicadas y estimación de errores}

Para realizar diagramas de cocientes de flujos, analizar el decremento de las líneas de una misma serie o estimar las extensiones y radios de las regiones de formación, necesitamos medir los flujos en las líneas (Fl), anchos equivalentes (EW), anchos a mitad de altura (FWHM) y separación de los picos en emisión $(\Delta V)$. Las medidas obtenidas a partir de mediciones directas sobre los espectros deben ser corregidas considerando la absorción fotosférica de la estrella central y el ensanchamiento instrumental.

\subsubsection{Corrección por absorción fotosférica}

La presencia de una envoltura circunestelar se evidencia a partir de la observación de las líneas de hidrógeno en emisión. Sin embargo, muchas veces esa emisión no supera el nivel del continuo de la estrella, sino que solamente alcanza a rellenar parcialmente la absorción fotosférica en esa línea. Para poder obtener la emisión neta en cada línea generada por la envoltura, es necesario conocer cuál es la absorción fotosférica correspondiente a cada línea.

En la literatura se encuentran ajustes para los anchos equivalentes de las líneas fotosféricas de hidrógeno según el TE de la estrella solamente para líneas específicas en el rango 2.4-4.1 $\mu \mathrm{m}$ 
(Lenorzer et al., 2002b). De esas líneas, en este trabajo utilizaremos $\operatorname{Br} \alpha, \operatorname{Pf} \gamma$ y $\operatorname{Pf} \delta$. Para algunas líneas en las otras bandas, hay reportes de mediciones de anchos equivalentes para estrellas $\mathrm{O}$ y B estándares, pero no hay ajustes realizados que permitan obtener el ancho equivalente de la línea fotosférica a partir del TE de la estrella.

Para poder obtener entonces los anchos equivalentes fotosféricos de todas las líneas observadas, utilizamos el programa synspec (Hubeny, 1988; Hubeny \& Lanz, 1995, 2011, 2017). El mismo permite obtener espectros sintéticos a partir de modelos de atmósferas calculados previamente u obtenidos de la literatura (por ejemplo, los modelos de Kurucz, 1979). Para las líneas con ajustes o medidas reportadas en la literatura, comparamos los valores reportados con los anchos equivalentes obtenidos a partir de los espectros sintéticos para diferentes valores de $T$ y $\log g$, a fin de evaluar la precisión de la corrección a partir de espectros sintéticos.

Para la línea $\mathrm{Pa} \beta$, comparamos los anchos equivalentes obtenidos a partir de los espectros sintéticos con un ajuste realizado a partir de anchos equivalentes reportados por Wallace et al. (2000). El ajuste obtenidos a partir de los anchos reportados para estrellas de clase de luminosidad V-III, y la comparación de ese ajuste con los valores obtenidos a partir de espectros sintéticos se muestra en la Fig. 4.4.

Por otro lado, a partir de los valores reportados por Meyer et al. (1998) para la línea $\mathrm{Br}_{11}$, pudimos realizar un ajuste a partir de mediciones de 10 estrellas con TE O-B y CL V-III. Ese ajuste lo comparamos con los anchos obtenidos a partir de los espectros sintéticos, como se muestra en la Fig. 4.5. En el caso de Bry, es posible comparar con un ajuste lineal obtenido a partir de las mediciones de anchos equivalentes realizadas por Hanson et al. (1996). El ajuste obtenido a partir de las mediciones y la comparación entre el ajuste y los anchos equivalentes obtenidos de los espectros sintéticos se pueden ver en la Fig. 4.6.

Los anchos equivalentes obtenidos para $\operatorname{Br} \alpha$, $\operatorname{Pf} \gamma$ y $\operatorname{Pf} \delta$ fueron comparados con los ajustes realizados por Lenorzer et al. (2002b) para las estrellas con clases de luminosidad V-III. Las comparaciones pueden verse en las Fig. 4.7.

En general, puede observarse que los ajustes obtenidos para $\operatorname{Pa} \beta, \operatorname{Br}_{11}, \operatorname{Br} \gamma, \operatorname{Br} \alpha$, $\operatorname{Pf} \gamma$ y $\operatorname{Pf} \delta$ están en buen acuerdo con los anchos equivalentes medidos a partir de los espectros sintéticos, especialmente en los intervalos de temperatura y log g más comunes en las estrellas Be ( $\mathrm{T} \sim$ $20000-30000 \mathrm{~K}, \log g \sim 3.5-4.5$ ). Por lo tanto, utilizamos los anchos equivalentes obtenidos a partir de los espectros sintéticos para corregir la absorción fotosférica de todas las líneas observadas en nuestra muestra.

\subsubsection{Corrección por ensanchamiento instrumental}

Se realizaron correcciones a los FWHM observados $\left(\mathrm{FWHM}_{\mathrm{obs}}\right)$ según el FWHM instrumental $\left(\mathrm{FWHM}_{\text {inst }}\right)$. El $\mathrm{FWHM}_{\text {inst }}$ fue obtenido para cada configuración utilizada, según la resolución obtenida. Para las observaciones obtenidas con NIRI en los años 2006 y 2008, el FWHM en la banda K es de $230 \mathrm{~km} / \mathrm{s}$ y para la banda L de $270 \mathrm{~km} / \mathrm{s}$. Para las observaciones obtenidas del 2010 
Figura 4.4: Comparación entre el ajuste lineal para los anchos equivalentes de $\mathrm{Pa} \beta$ obtenido a partir de las mediciones de Wallace et al. (2000, izquierda) y los anchos equivalentes medidos a partir de los espectros sintéticos (derecha).
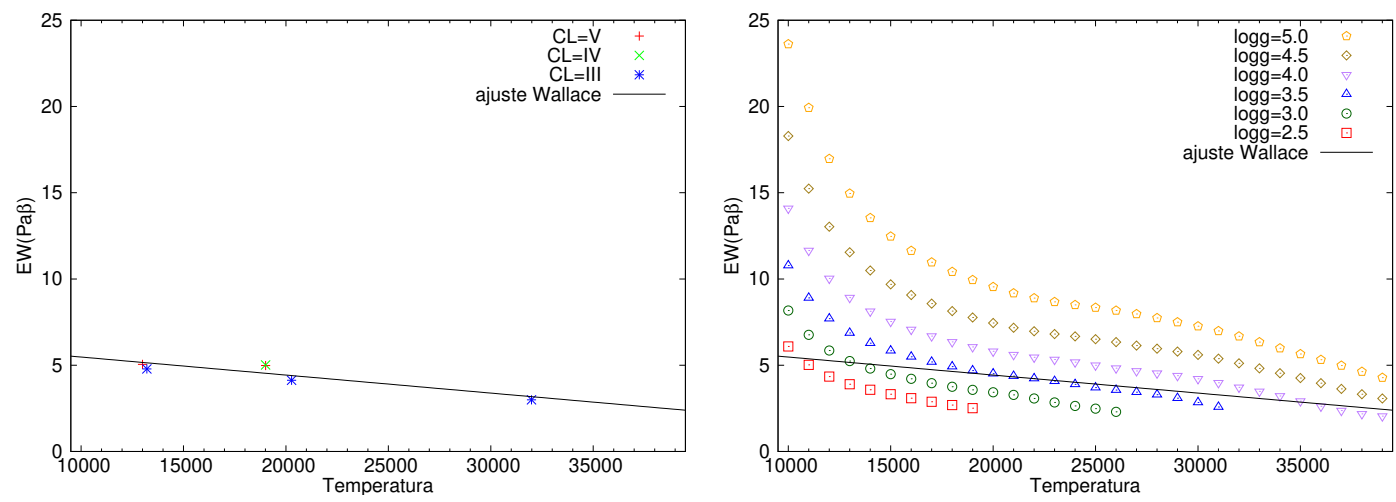

Figura 4.5: Comparación entre el ajuste lineal para los anchos equivalentes de $\mathrm{Br}_{11}$ obtenido a partir de las mediciones de Meyer et al. (1998, izquierda) y los anchos equivalentes medidos a partir de los espectros sintéticos (derecha).
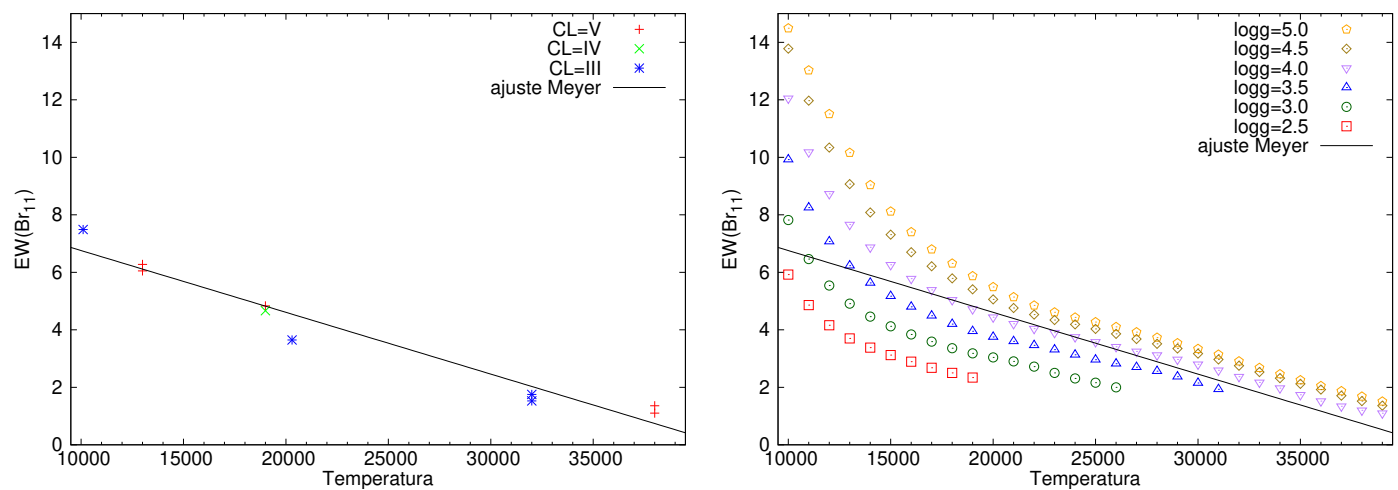

Figura 4.6: Comparación entre el ajuste lineal para los anchos equivalentes de $\mathrm{Br} \gamma$ obtenido a partir de las mediciones de Hanson et al. (1996, izquierda) y los anchos equivalentes medidos a partir de los espectros sintéticos (derecha).
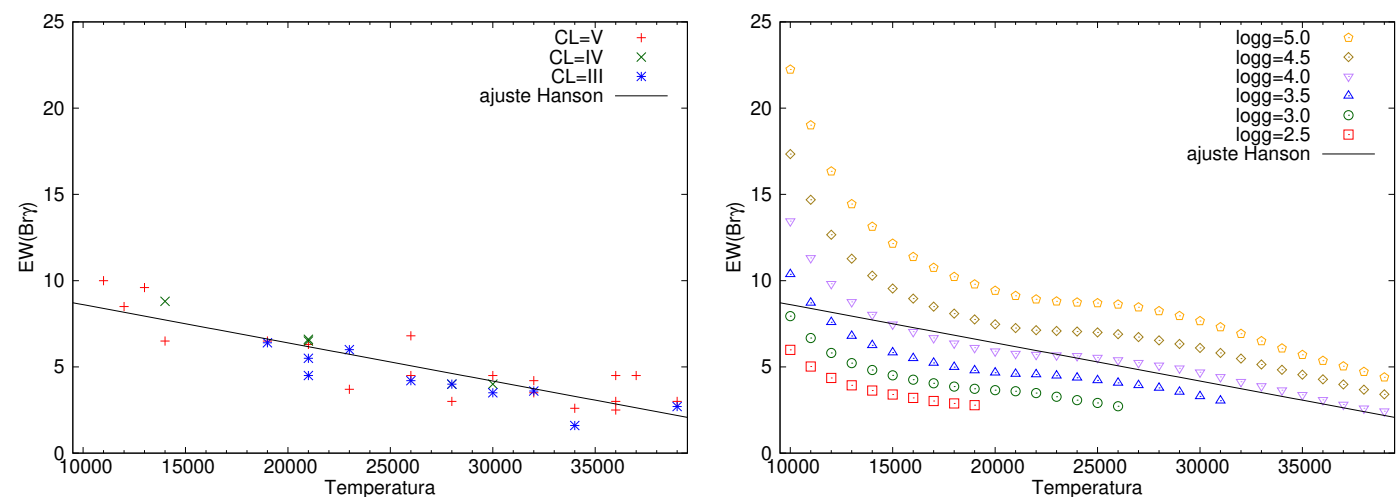
Figura 4.7: Comparación entre el ajuste lineal de Lenorzer para los anchos equivalentes de $\operatorname{Br} \alpha$ (superior izquierda), Pf $\gamma$ (superior derecha) y Pf $\delta$ (inferior) en las estrellas enanas con clase de luminosidad V-III y los anchos equivalentes medidos a partir de los espectros sintéticos.

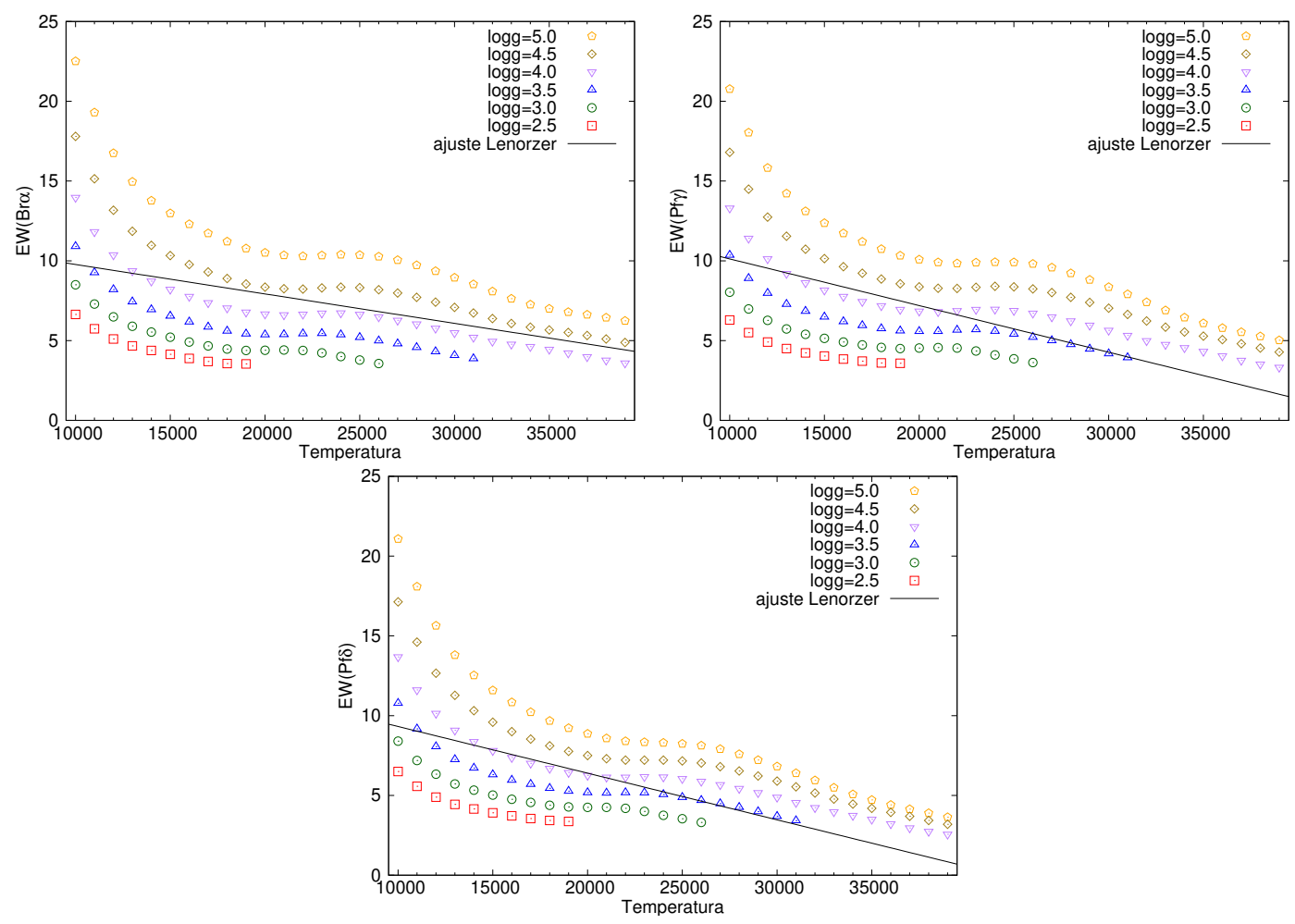

en adelante, el FWHM instrumental es de $170 \mathrm{~km} / \mathrm{s}$ en todas las bandas. $\mathrm{El} \mathrm{FWHM}_{\text {inst }}$ para las observaciones realizadas con FIRE es de $50 \mathrm{~km} / \mathrm{s}$. Con el FWHM observado y el FWHM instrumental se obtuvo el FWHM corregido $\left(\mathrm{FWHM}_{\mathrm{cor}}\right)$ según $\mathrm{FWHM}_{\mathrm{cor}}=\sqrt{\mathrm{FWHM}_{\mathrm{obs}}^{2}-\mathrm{FWHM}_{\text {inst }}^{2}}$ (Fekel, 1997).

\subsubsection{Estimación de errores}

En las tablas correspondientes a cada estrella se listan los anchos equivalentes (en $\AA$ ), flujos (en unidades de $10^{-13} \frac{\mathrm{erg}}{\mathrm{cm}^{2} \mathrm{~s} \AA}$ ) y FWHM (en $\mathrm{km} / \mathrm{s}$ ) de las líneas observadas en cada banda. Los errores estimados para los anchos equivalentes y flujos son del 10\% para las líneas más intensas, debido al error introducido en la determinación del continuo. Para determinar los errores en los FWHM realizamos 10 medidas sobre la misma línea, a fin de estimar el error introducido al realizar el ajuste. Obtuvimos que en las observaciones del 2006 y 2008, el error no supera los $100 \mathrm{~km} / \mathrm{s}$ en la banda $\mathrm{K}$ y los $150 \mathrm{~km} / \mathrm{s}$ en la banda L, mientras que en las observaciones del 2010 en adelante se puede acotar a $75 \mathrm{~km} / \mathrm{s}$ en todas las bandas. De la misma manera estimamos el error en las mediciones de la separación de los picos, encontrando que el mismo es de $25 \mathrm{~km} / \mathrm{s}$.

El método $N$ para determinar densidades columnares y extensión de las regiones de formación lo aplicamos a las líneas de las series de Humphreys y Pfund, y a los miembros más altos de 
la serie de Brackett, donde podemos suponer que las regiones de formación de las líneas son similares. Los métodos FWHM o $\Delta V$ los aplicamos a todos las líneas en las que pudimos realizar las mediciones necesarias, ya que no es necesario que las líneas provengan de regiones de formación similares. A partir de los errores obtenidos para los flujos, los ensanchamientos y la separación de los picos, realizamos la propagación de errores para determinar las incertezas en las densidades columnares, extensiones y radios internos de las regiones de formación. Para las densidades columnares obtuvimos errores de alrededor del $50 \%$ del valor determinado, mientras que para las extensiones de las regiones de formación alcanzan el 60\%. Para los radios de las regiones de formación determinados a partir del FWHM, los errores son del 60\%, y para los obtenidos a partir de $\Delta V$ son del $30 \%$.

En el siguiente capítulo mostraremos los resultados obtenidos para los objetos observados una sola vez, dejando el análisis de la variabilidad de los objetos observados en varias oportunidades para un capítulo aparte. 


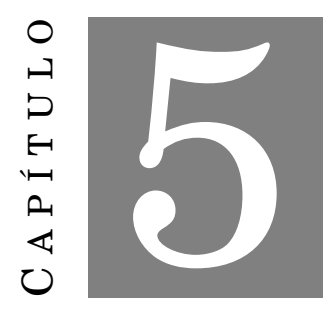

\section{RESULTADOS INDIVIDUALES}

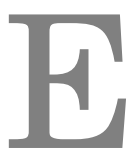

n los Capítulos 3 y 4 describimos las principales características observadas en los espectros IR de las estrellas Be y la metodología utilizada para obtener propiedades físicas de los discos de estos objetos. En este capítulo haremos un análisis detallado de 15 de los objetos de nuestra muestra, los cuales fueron observados en una sola oportunidad. El análisis de los 8 objetos restantes, para los cuales tenemos más de una observación, será discutido en el próximo capítulo.

En la Sección 5.1 presentaremos los resultados individuales para cada objeto, y en la Sección 5.2 las características generales que nos permiten separar a los objetos según características comunes.

\subsection{Mediciones y resultados para cada objeto estudiado}

Para cada estrella presentaremos un breve reseña con información relevante de la literatura, describiremos los espectros IR y presentaremos los resultados obtenidos. Los valores de los anchos equivalentes (EW) en $\AA$, los flujos (Fl) en unidades de $10^{-13} \frac{\mathrm{erg}}{\mathrm{cm}^{2} \mathrm{~s} \AA}$ y los anchos a mitad de altura (FWHM) en km/s de las líneas observadas en cada banda se muestran en el Apéndice A. Para los anchos equivalentes consideramos valores positivos para representar las líneas en emisión (contrario a lo estándar). Los valores obtenidos para las densidades columnares, extensiones relativas y radios internos de las regiones de formación calculados utilizando la metodología descripta en el capítulo anterior (métodos $N$, FWHM y $\Delta V$ ) se muestran también en el Apéndice A. 


\subsubsection{Per - HD 25940}

48 Per es una estrella de tipo espectral B3 V, identificada como Be por Merrill et al. (1925). Más adelante, Slettebak (1951) reportó la presencia las líneas $\mathrm{H} \alpha, \mathrm{H} \beta$ y O I $\lambda 8446$ A en emisión. Woolf et al. (1970) indicaron que las líneas en emisión observadas en la región de $11 \mu \mathrm{m}$ presentaban variabilidad.

Quirrenbach et al. (1997) obtuvieron un ángulo de inclinación del disco para $48 \mathrm{Per}$ de $i=27^{\circ}$, modelando datos interferométricos en $\mathrm{H} \alpha$ utilizando un ajuste Gaussiano. Delaa et al. (2011), a partir un modelo cinemático y observaciones interferométricas en $\mathrm{H} \alpha$, obtuvieron un valor similar $\left(i=30 \pm 10^{\circ}\right)$ y determinaron un ángulo de posición para el disco de $\mathrm{PA}=108 \pm 1^{\circ}$, cercano al valor determinado previamente por Yudin (2001) utilizando datos polarimétricos $\left(\mathrm{PA}=110^{\circ}\right)$.

Persson \& McGregor (1985) reportaron valores de flujos obtenidos a partir de observaciones realizadas en 1983, y la ubicaron en una región central de su diagrama. Posteriormente, a partir de observaciones obtenidas en los años 2006/2008, Touhami et al. (2010) la ubicaron en la región central del diagrama de Lenorzer, entre los casos ópticamente grueso y delgado.

Clark \& Steele (2000) realizaron observaciones de 48 Per en la banda K, donde reportaron la presencia de la línea Br $\gamma$ y líneas de Mg II en emisión, sin evidencia de He I. En la banda H también se observaron líneas de la serie de Brackett en emisión (Steele \& Clark, 2001).

Figura 5.1: Espectros de 48 Per en las bandas H (superior) y K (inferior).

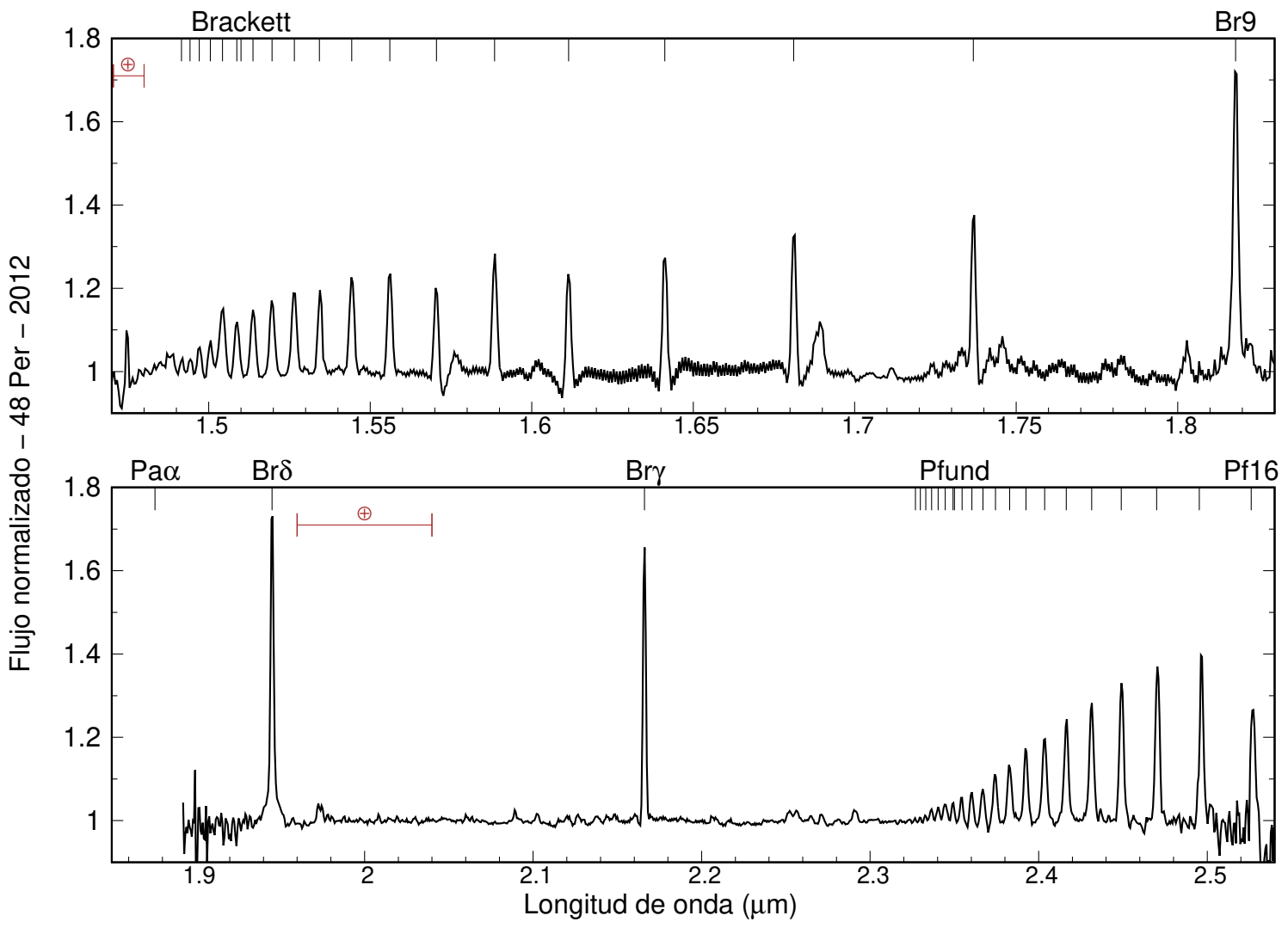


Figura 5.2: Espectro de 48 Per en la banda L.

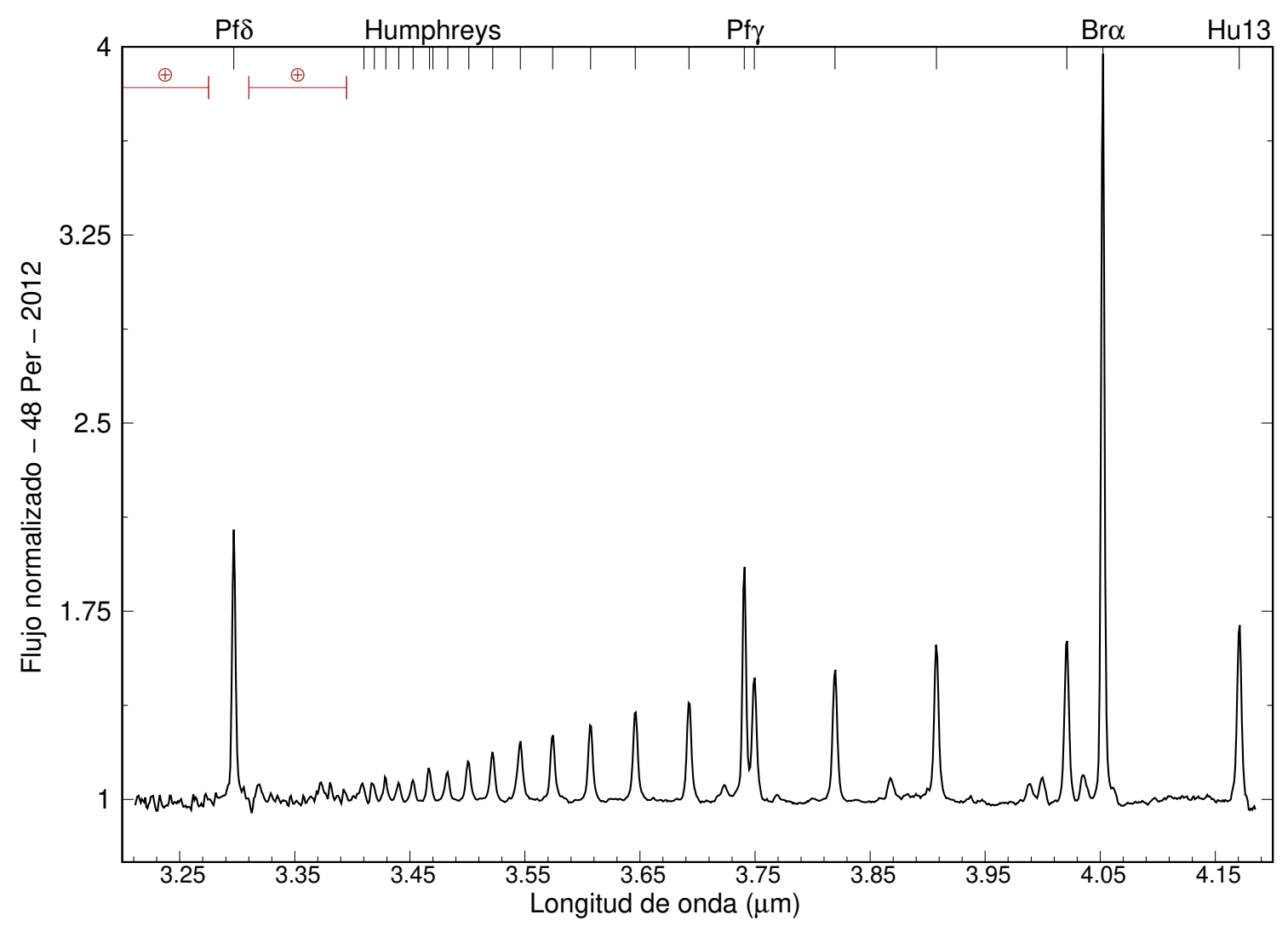

Vieira et al. (2017) obtuvieron a partir del modelado del continuo IR, valores para $\log \rho_{0}$ y $n$ (ver Ecuación 2.2) utilizando observaciones de 3 años diferentes. Con observaciones del año 1983 obtuvieron $\log \rho_{0}=-11.4 \pm 0.2$ y $n=2.9 \pm 0.2$, con las obtenidas entre 2006 у 2007 $\log \rho_{0}=-11.4 \pm 0.1$ y $n=2.8 \pm 0.1$, y en el $2010 \log \rho_{0}=-11.5 \pm 0.1$ y $n=2.7 \pm 0.1$. Considerando estos valores de $n$, los autores concluyeron que 48 Per presentaba un disco en disipación al momento de las observaciones.

\section{Descripción de los espectros y mediciones realizadas}

En las Figs. 5.1 y 5.2 se muestran los espectros obtenidos en el 2012 en las bandas H, K y L para 48 Per utilizando el espectrógrafo GNIRS. En todas las bandas las líneas de hidrógeno se observan en emisión, y según el criterio de clasificación dado por Mennickent et al. (2009) la estrella pertenece al grupo II. Los detalles de los EW, Fl y FWHM de las líneas observadas se muestran en la Tabla A.1. Para realizar la corrección por absorción fotosférica se utilizó el espectro sintético correspondiente a una estrella con $T_{\text {eff }}=16000 \mathrm{~K}$ y $\log g=3.5$ (ver 3.3).

A partir de los cocientes de flujos entre $\mathrm{Hu}_{14}, \operatorname{Pf} \gamma$ y $\mathrm{Br} \alpha$ ubicamos a $48 \mathrm{Per}$ en el diagrama de Lenorzer (ver Fig. 5.67). La misma se ubica en una región central del diagrama, donde se encuentran las estrellas del Grupo II, y cerca del límite con la región correspondiente al Grupo 
Figura 5.3: 48 Per - EW/ $\lambda$ y FWHM vs. $\lambda$ de las líneas observadas en las diferentes series. La línea punteada corresponde a un valor de $2 V \sin (i)=440 \mathrm{~km} / \mathrm{s}$, y la región sombreada al error correspondiente (Tabla 3.3).
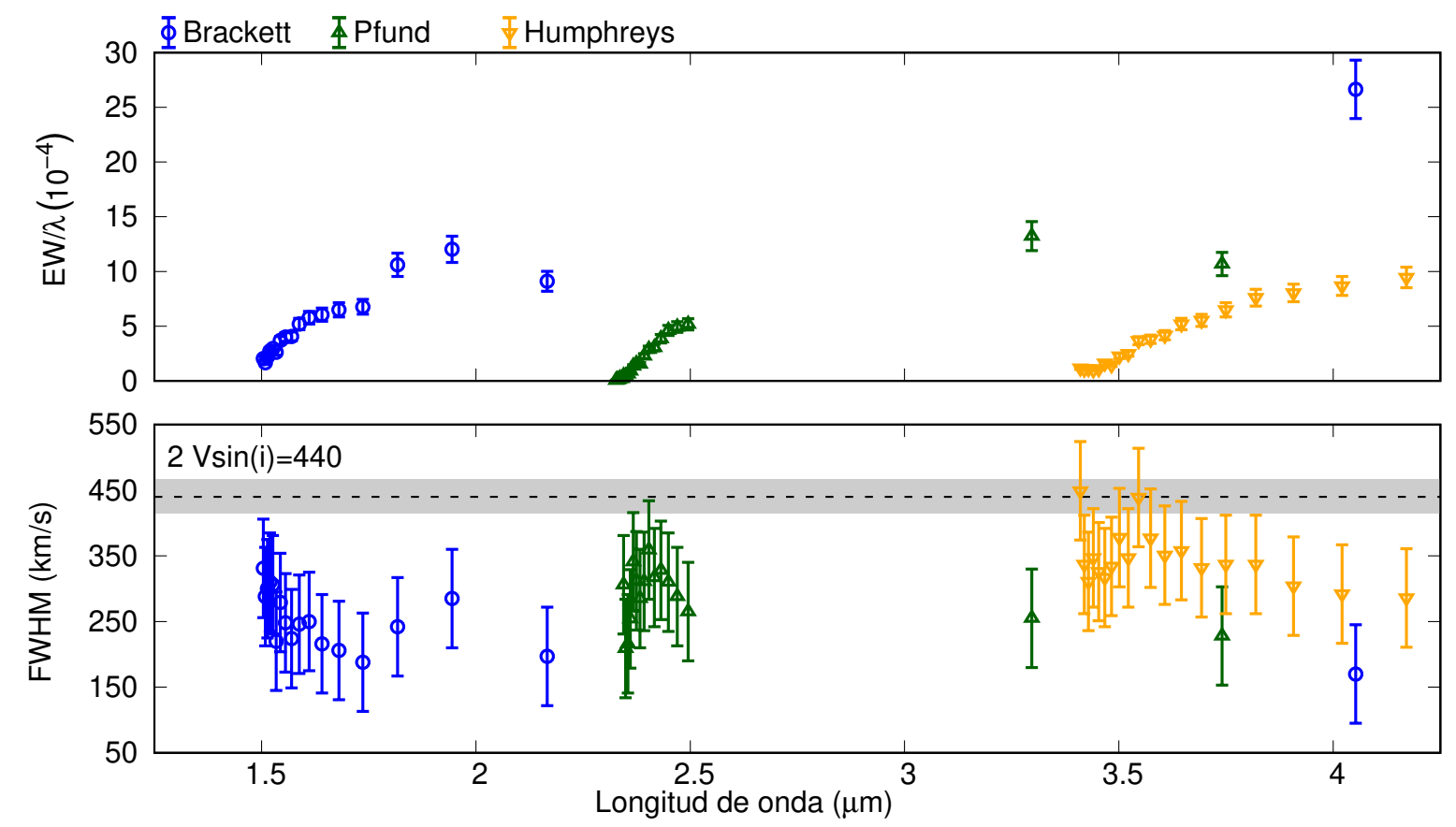

I. Esta ubicación en el diagrama es similar a la reportada por Touhami et al. (2010) utilizando un espectro obtenido en el 2006, y coincide con la ubicación derivada utilizando los flujos que muestran Persson \& McGregor (1985).

\section{Comportamiento de las series de hidrógeno}

En la Fig. 5.3 se muestran los gráficos de EW/ $/ \lambda$ y FWHM en función de la longitud de onda. Observamos que EW/ $\lambda$ converge al valor $10 \cdot 10^{-4}$ en todas las series observadas. En las series de Brackett y Humphreys los valores del FWHM crecen hacia los miembros más altos, mientras que para la serie de Pfund, se observa un comportamiento no monótono, donde los FWHM aumentan hasta $\mathrm{Pf}_{23}$ y luego disminuyen. El alto valor encontrado para $\mathrm{Pf}_{16}$ puede deberse a errores de calibración en el nivel del continuo al borde del espectro de la banda $\mathrm{K}$.

Los cocientes de los flujos y su comparación con los cocientes esperados para los casos ópticamente grueso y delgado se muestran en la Fig. 5.4. Los miembros más bajos de cada serie (hasta $\mathrm{Br}_{16}, \mathrm{Pf}_{18} \mathrm{y} \mathrm{Hu} \mathrm{Hu}_{18}$ ) se apartan del caso ópticamente delgado. Para los cocientes de anchos equivalentes relativos a $\mathrm{EW}(\mathrm{Br} \gamma)$ se obtiene que $\mathrm{EW}(\mathrm{Br} \alpha) / \mathrm{EW}(\mathrm{Br} \gamma) \sim 5$, valor compatible con lo esperado para una estrella del Grupo II. 
Figura 5.4: 48 Per - Cocientes de flujos para las líneas de la serie de Humphreys, Pfund y Brackett. En la parte superior se identifican la líneas correspondientes. Los símbolos utilizados son los mismos que en la Fig. 5.3.

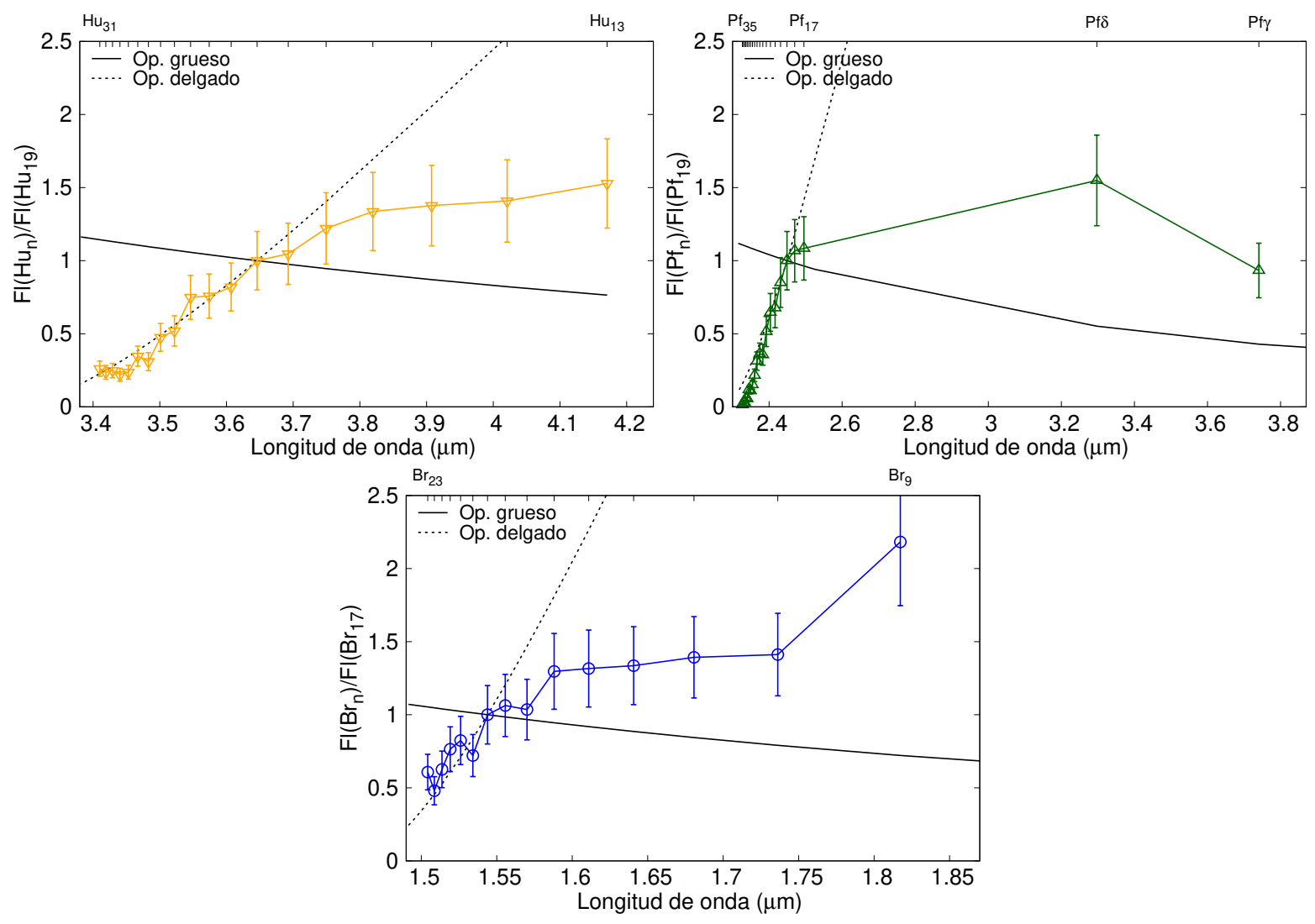

Figura 5.5: 48 Per - Radios internos de las regiones emisoras de las diferentes líneas relativos a la región emisora de Pf $\delta$. Los símbolos utilizados son los mismos que en la Fig. 5.3.

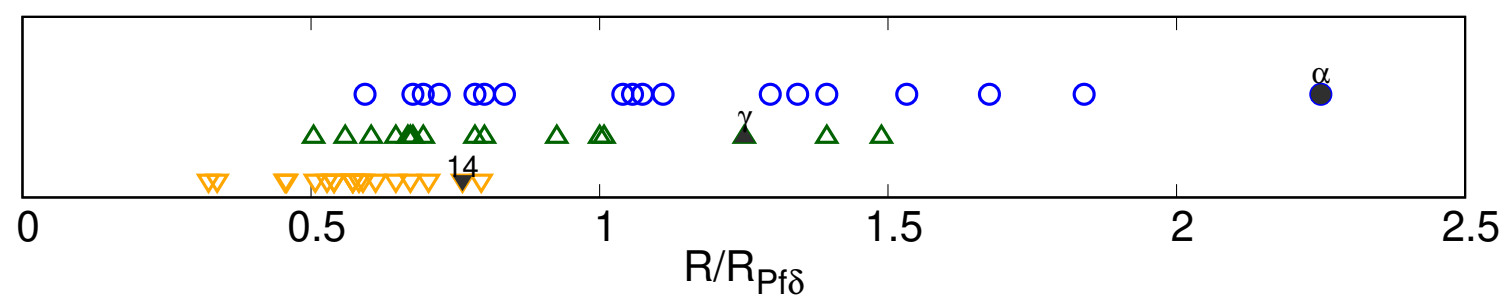

\section{Determinación de densidades columnares y extensiones de las regiones de formación}

Utilizando los métodos $N$ y FWHM obtuvimos las densidades columnares, extensiones relativas de las regiones de formación y los radios internos de las mismas. Los valores se muestran en la Tabla A.2.

Para la serie de Humphreys obtuvimos que los miembros más altos de la serie presentan 
menores densidades columnares y extensiones de las regiones de formación, contrario a lo obtenido para las series de Pfund y Brackett, donde los menores valores corresponden a los primeros miembros de la serie.

Los radios internos de las regiones de formación de las series de Humphreys y Brackett disminuyen a medida que avanzamos hacia los miembros mas altos. En la serie de Pfund se alcanza un valor mínimo en $\mathrm{Pf}_{22}$ y luego los valores vuelven a aumentar. En la Fig. 5.5 se muestran estos valores, relativos a $\mathrm{Pf} \delta$ a fin de comparar los valores para las distintas series. Las líneas de la serie de Humphreys y los miembros más altos de la serie de Pfund se ubican en la región más interna, mientras que los primeros miembros de las series de Brackett y Pfund se forman en una región más alejada de la estrella central. 


\subsubsection{Eri - HD 28497}

228 Eri es una estrella con clasificación espectral B2 V, que muestra emisión variable y episodios tipo shell. Andrillat et al. (1990) determinaron un tamaño para la región emisora en $\mathrm{H} \alpha$ entre $1.79 R_{\star}$ y $4.79 R_{\star}$. Por otra parte, Silaj et al. (2010) ajustaron la línea de $\mathrm{H} \alpha$ con un doble pico en emisión y obtuvieron como parámetros del mejor ajuste un tipo espectral B0 para la estrella central, $i=45^{\circ}, \rho_{0}=10^{-10} \mathrm{~g} \mathrm{~cm}^{-3}$ y $n=4$. A partir del continuo IR, Vieira et al. (2017) obtuvieron valores de $\log \rho_{0}=-10.3 \pm 0.8$ y $n=4 \pm 1$ utilizando observaciones realizadas en 1983, $\log \rho_{0}=-10.5 \pm 0.9$ y $n=4 \pm 0.8$ entre mayo del 2006 y agosto del $2007, \mathrm{y} \log \rho_{0}=-10.7 \pm 0.3 \mathrm{y}$ $n=3.4 \pm 0.1$ con observaciones de 2010. Según estos autores, 228 Eri presentaba una envoltura en formación en 1983 y 2006/2007, que alcanzó la estabilidad en 2010.

Hanson et al. (1996) reportaron que en el año 1994 se observaban las líneas $\mathrm{Br} \gamma$ y He I $\lambda 2.0587 \mu \mathrm{m}$ con dos picos en emisión.

\section{Descripción de los espectros y mediciones realizadas}

228 Eri fue observada en el 2017 en las bandas J, H, K y L utilizando el espectrógrafo GNIRS. Los espectros obtenidos se muestran en las Figs. 5.6 y 5.7. Las líneas de hidrógeno observadas en

Figura 5.6: Espectros de 228 Eri en las bandas J (superior) y H (inferior).

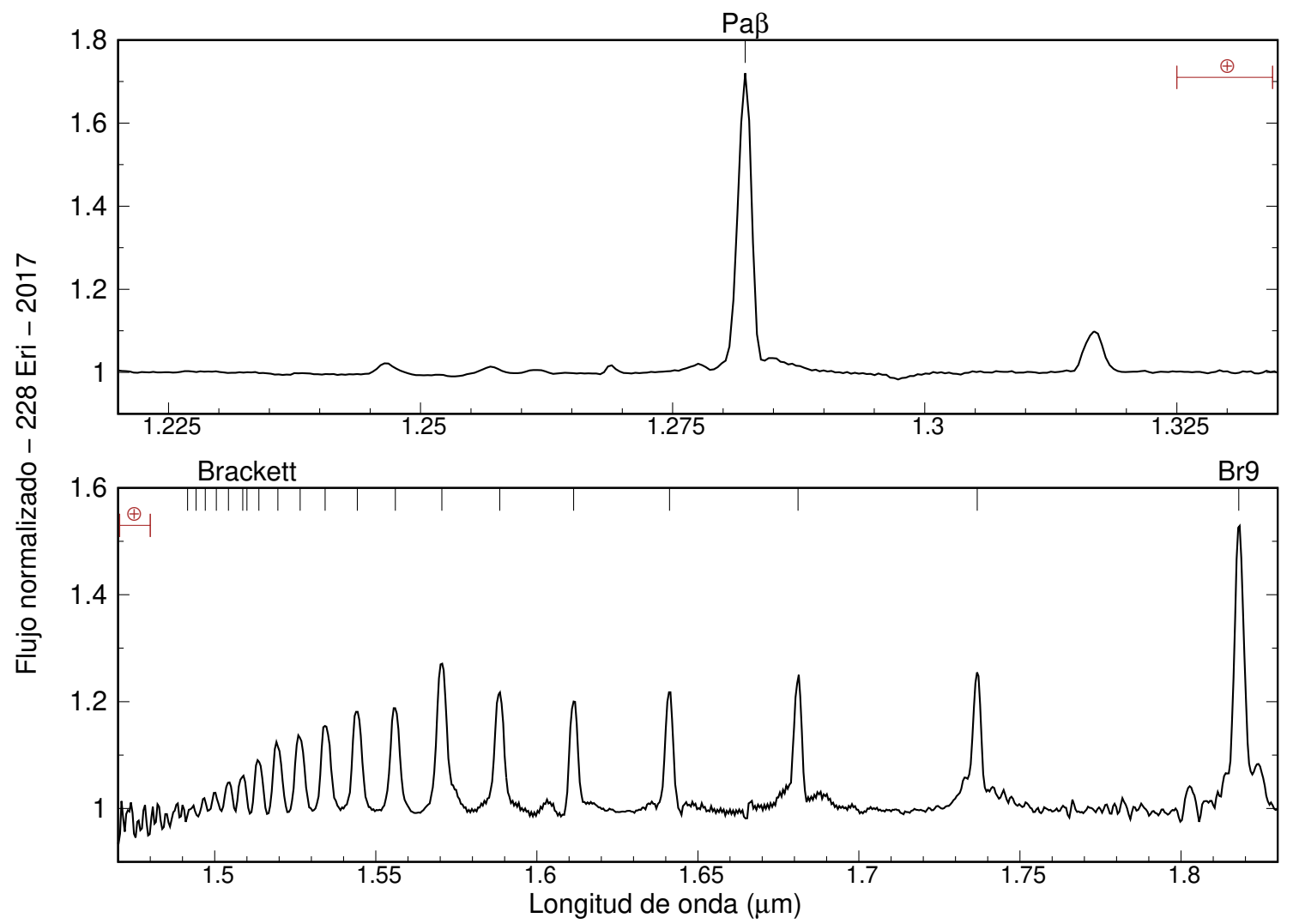


Figura 5.7: Espectros de 228 Eri en las bandas K (superior) y L (inferior).

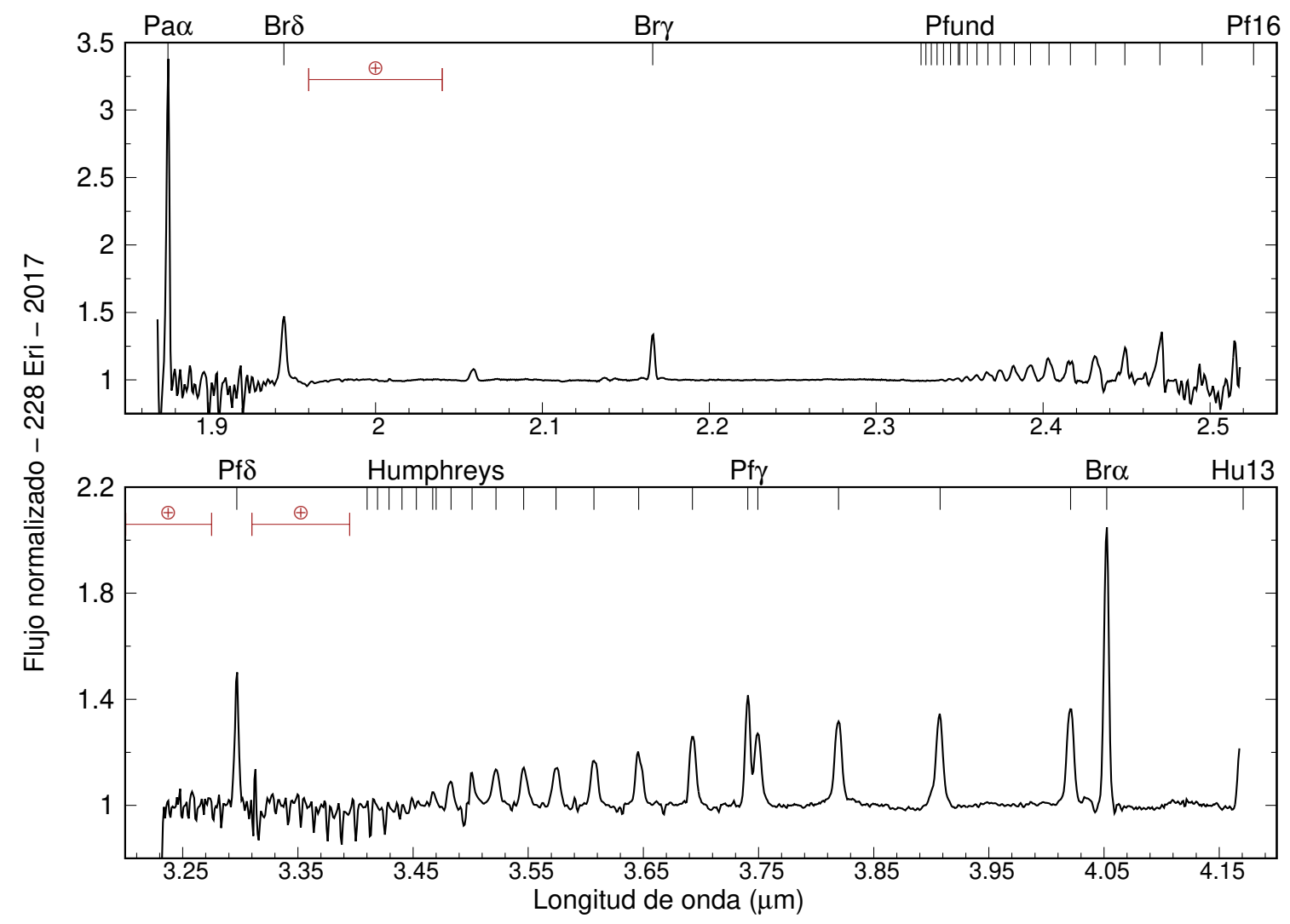

todas las bandas se encuentran en emisión, y si bien las líneas de la serie de Brackett son bastante intensas, no alcanzan a ser comparables a las líneas de $\operatorname{Br} \alpha$ y Pf $\gamma$, por lo que clasificamos a 228 Eri como perteneciente al Grupo II. En la Tabla A.3 se muestran los valor obtenidos de EW, Fl y FWHM, corregidas por absorción fotosférica considerando un espectro sintético de $T_{\text {eff }}=27000 \mathrm{~K}$ y $\log g=4.0$ según los valores de la Tabla 3.3.

En la Fig. 5.67 se muestra la ubicación de 228 Eri en el diagrama de Lenorzer. La misma se encuentra próxima al límite que separa las regiones de los grupos I y II.

\section{Comportamiento de las series de hidrógeno}

En la Fig. 5.3 se muestran los gráficos de EW/ y FWHM en función de la longitud de onda. Observamos que los valores de EW/ $\lambda$ alcanzan un valor aproximadamente estable de $7 \cdot 10^{-4}$ en todas las series. Para los valores de FWHM, observamos que en las series de Humphreys y Brackett los anchos aumentan desde los miembros más bajos hacia los más altos, y decaen nuevamente a partir de $\mathrm{Br}_{15}$ y $\mathrm{Hu}_{19}$. Hay líneas que presentan valores de $\mathrm{FWHM} \sim 2 \mathrm{~V} \sin (i)$, indicando regiones de formación cercanas a la fotosfera estelar. Los FWHM más bajos que $2 \mathrm{~V} \sin (i)$ indican regiones de formación más alejadas de la estrella central. Los miembros de la serie de Pfund presentan una gran dispersión en los valores medidos. 
Figura 5.8: 228 Eri - EW/ $\lambda$ y FWHM vs. $\lambda$ de las líneas observadas en las diferentes series. La línea punteada corresponde a un valor de $2 V \sin (i)=684 \mathrm{~km} / \mathrm{s}$, y la región sombreada al error correspondiente (Tabla 3.3).
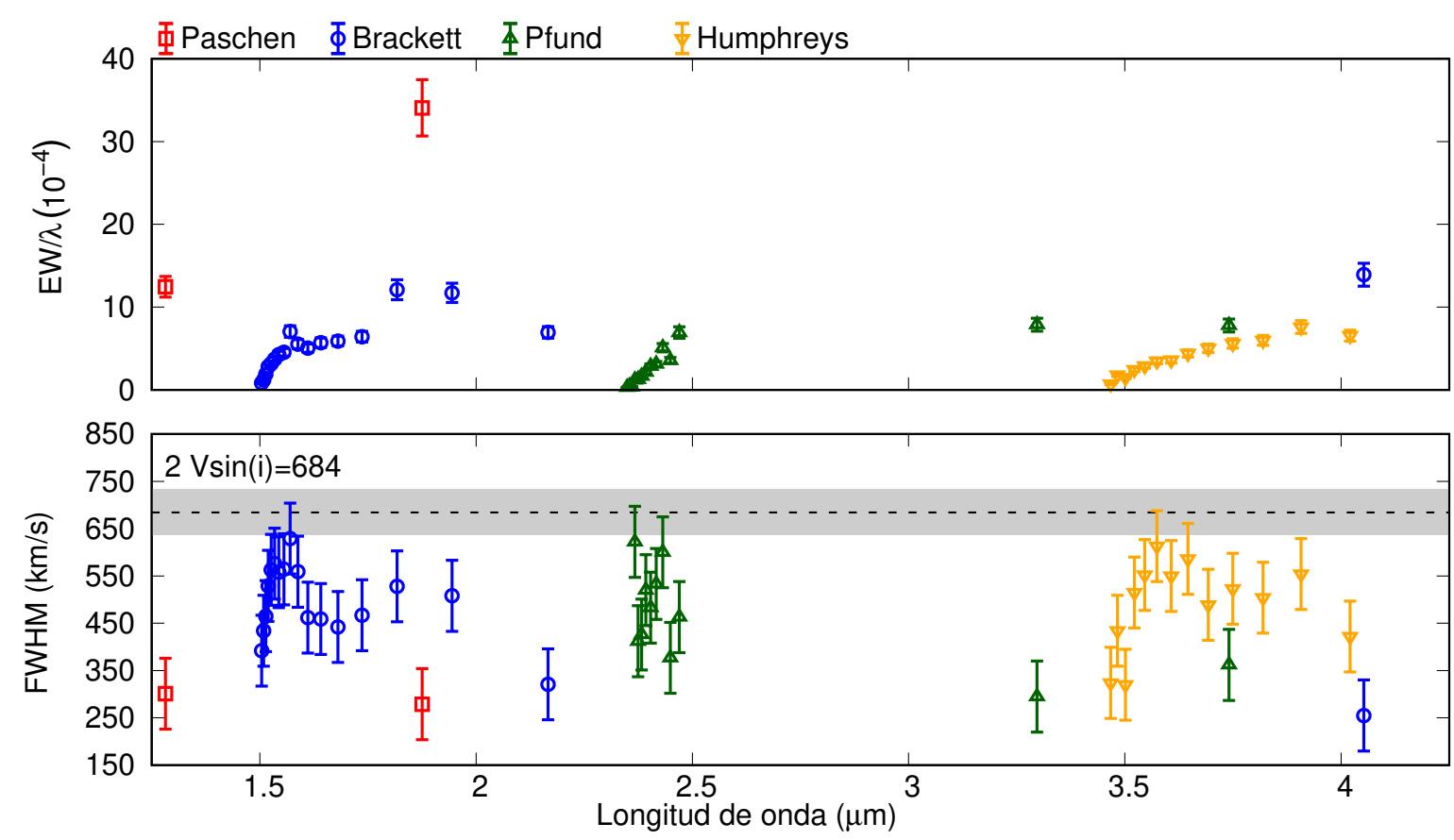

Los cocientes de los flujos y su comparación con los casos ópticamente grueso y delgado se muestran en la Fig. 5.9. En todos los casos los miembros más altos de la serie siguen los valores esperados para un caso ópticamente delgado, mientras que hasta $\mathrm{Br}_{14}$, $\mathrm{Pf} \delta$ y $\mathrm{Hu}_{17}$ se apartan hacia el caso ópticamente grueso. Determinamos además que $\mathrm{EW}(\mathrm{Br} \alpha) / \mathrm{EW}(\mathrm{Br} \gamma) \sim 3.7$ y $E W_{\mathrm{Pa} \alpha} / E W_{\mathrm{Pa} \beta} \sim 5.8$. El valor obtenido para $\mathrm{EW}(\mathrm{Br} \alpha) / \mathrm{EW}(\mathrm{Br} \gamma)$ se encuentra entre los valores esperados para objetos del grupo I y objetos del grupo II.

\section{Determinación de densidades columnares y extensiones de las regiones de formación}

Utilizando los métodos $N$ y FWHM obtuvimos las densidades columnares, extensiones relativas de las regiones de formación y los radios internos de las mismas. Los parámetros obtenidos se muestran en la Tabla A.4.

Con respecto a la serie de Humphreys no pudimos definir un comportamiento general en cuanto a las densidades columnares, mientras que en las series de Pfund y Brackett los miembros más altos de las series presentan mayores densidades columnares y extensiones de las regiones de formación.

Los radios internos de las regiones de formación de las líneas de la serie de Humphreys son similares entre si. Para las líneas de las series de Pfund y Brackett los tamaños son menores a medida que observamos miembros más altos de la serie, tendencia que también se observa en los 
Figura 5.9: 228 Eri - Cocientes de flujos para las líneas de la serie de Humphreys, Pfund y Brackett. En la parte superior se identifican la líneas correspondientes. Los símbolos utilizados son los mismos que en la Fig. 5.8.
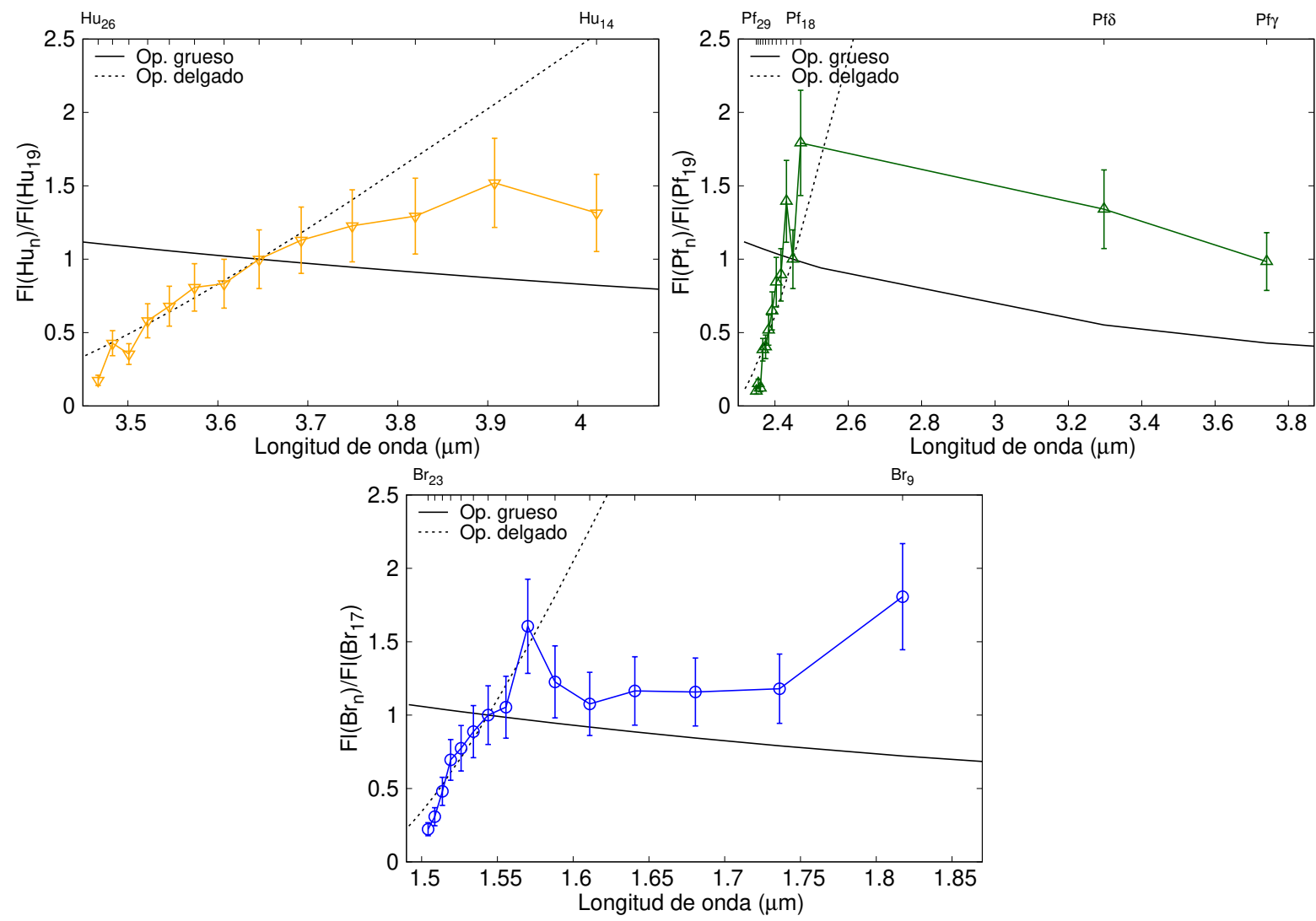

Figura 5.10: 228 Eri - Radios internos de las regiones emisoras de las diferentes líneas relativos a la región emisora de $\operatorname{Br} \delta$. Los símbolos utilizados son los mismos que en la Fig. 5.8.

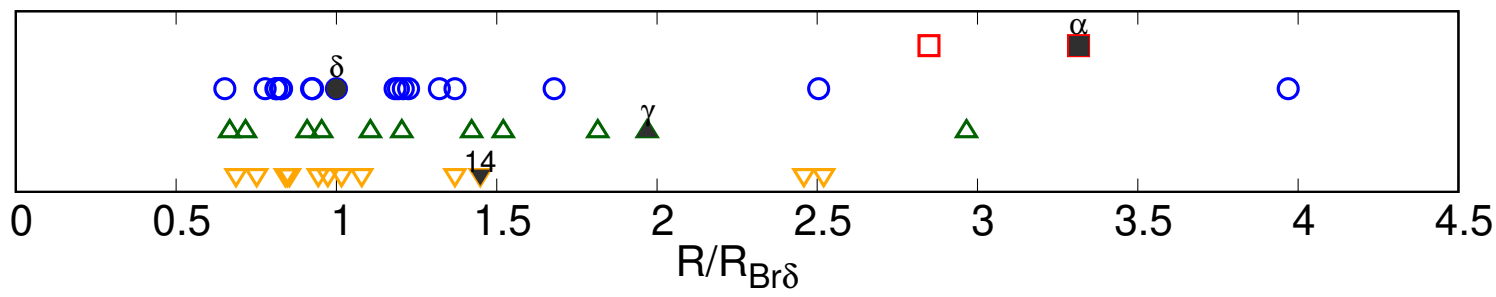

dos miembros observados de la serie de Paschen. Utilizando los FWHM obtenidos para todas las líneas, graficamos los radios internos de las regiones de formación, relativos al correspondiente a $\operatorname{Br} \delta$. Los mismos pueden verse en la Fig. 5.10. Se observa que las líneas formadas en las regiones más externas son los primeros miembros de las series de Brackett, Pfund y Paschen, mientras que los miembros altos de todas las series se ubican en regiones más cercanas a la estrella central que $\sim 2 R_{\mathrm{Br} \delta}$. 


\subsubsection{Tau - HD 32991}

105 Tau es una estrella de tipo espectral B2 V que ha presentado un espectro tipo shell y variaciones fotométricas del tipo $\gamma$ Cas (Lefèvre et al., 2009).

$\mathrm{El}$ ancho equivalente de la línea $\mathrm{H} \alpha$ fue estudiado por Jones et al. (2011), quienes encontraron que la misma es variable con un nivel de confianza de $\sim 50 \%$. Silaj et al. (2010) ajustaron la línea de $\mathrm{H} \alpha$ con un pico en emisión y obtuvieron como parámetros del mejor ajuste un tipo espectral B2 para la estrella central, $i=20^{\circ}, \rho_{0}=5 \cdot 10^{-10} \mathrm{~g} \mathrm{~cm}^{-3}$ y $n=3.5$.

No encontramos reportes en la literatura de espectros IR obtenidos para este objeto, ni determinaciones previas del tamaño del disco.

\section{Descripción de los espectros y mediciones realizadas}

En las Figs. 5.11 y 5.12 se muestran los espectros obtenidos en 2017 en las bandas J, H, K y L utilizando el espectrógrafo GNIRS. Las líneas de hidrógeno se encuentran en emisión en todas las bandas, y según el criterio de clasificación de Mennickent et al. (2009) la estrella pertenece al grupo II. En la Tabla A.5 se muestran los valor de EW, Fl y FWHM de las líneas de hidrógeno, corregidos utilizando el espectro sintético correspondiente a una estrella con $T_{\text {eff }}=21000 \mathrm{~K} \mathrm{y}$

Figura 5.11: Espectros de 105 Tau en las bandas J (superior) y H (inferior).

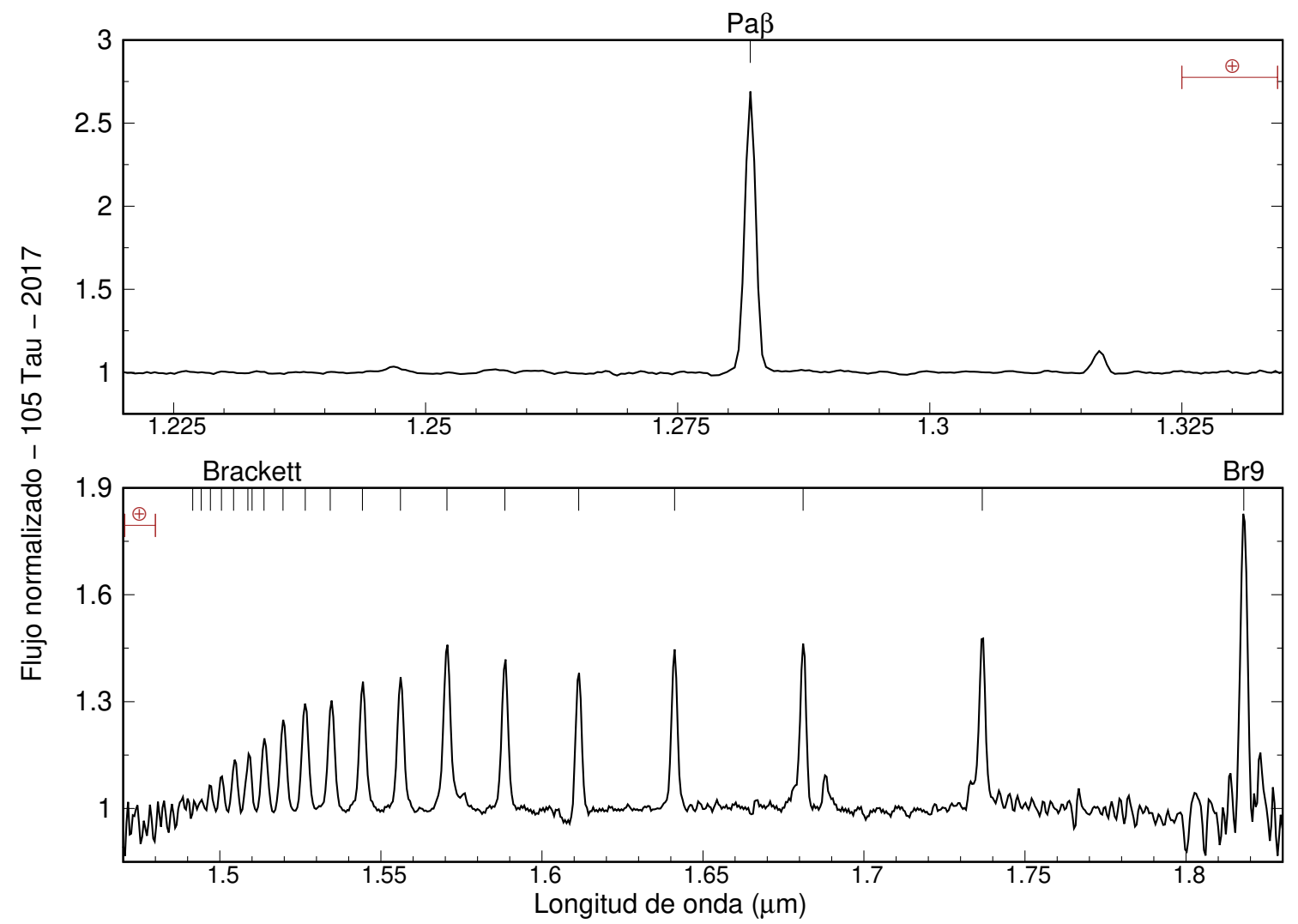


Figura 5.12: Espectros de 105 Tau en las bandas K (superior) y L (inferior).

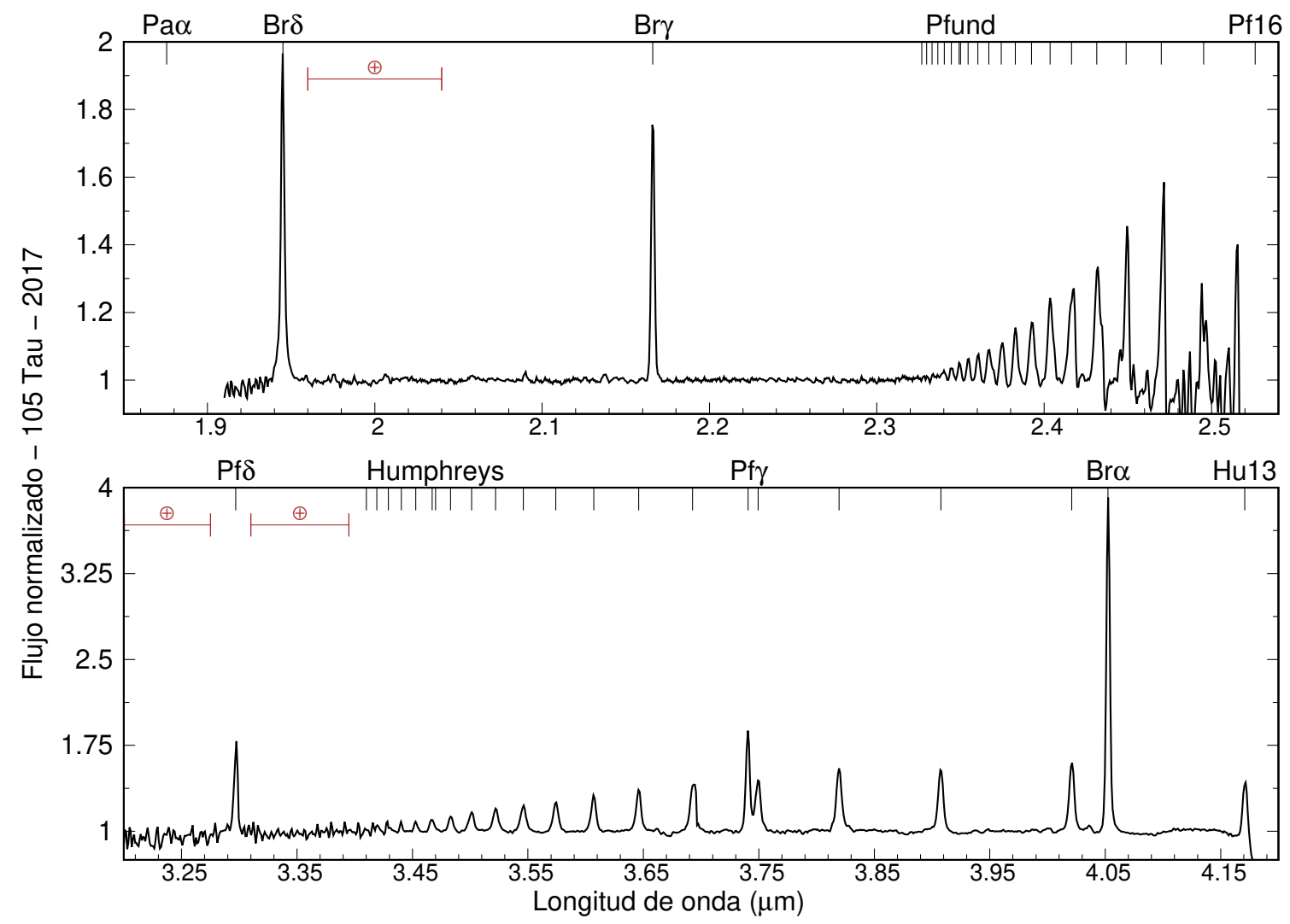

$\log g=4.0$ según los parámetros estelares presentados en la Tabla 3.3.

La ubicación de 105 Tau en el diagrama de Lenorzer se muestra en la Fig. 5.67. La misma se encuentra en una región central del diagrama, más cercana a la región ópticamente gruesa, como otros objetos del grupo II.

\section{Comportamiento de las series de hidrógeno}

En la Fig. 5.13 vemos que los valores de EW/ $\lambda$ llegan a un valor estable en $\sim 9 \cdot 10^{-4}$ (ver Fig. 5.13) en todas las series. Los valores de FWHM crecen hacia los miembros más altos de cada serie, decreciendo nuevamente desde $\mathrm{Pf}_{26} \mathrm{y} \mathrm{Br}_{15}$. Los miembros más bajos de las series de Pfund, Brackett y Paschen muestran valores moderadamente más bajos que $2 V \sin (i)$. Algunas líneas muestran ensanchamientos mayores a $2 V \sin (i)$, indicando regiones de formación muy cercanas a la estrella central, o algún mecanismo de ensanchamiento adicional.

En la Fig. 5.14 se muestran los cocientes de los flujos para las series de Humphreys, Pfund y Brackett, junto con la comparación con los casos ópticamente grueso y delgado. Observamos que los miembros más bajos de cada serie (hasta $\mathrm{Br}_{14}$, $\mathrm{Pf} \delta$ y $\mathrm{Hu}_{15}$ ) se apartan de los cocientes esperados para el caso ópticamente delgado. Además, $\mathrm{EW}(\mathrm{Br} \alpha) / \mathrm{EW}(\mathrm{Br} \gamma) \sim 4.75$, valor consistente con la clasificación de la estrella en el grupo II. 
Figura 5.13: 105 Tau - EW/ $\lambda$ y FWHM vs. $\lambda$ de las líneas observadas en las diferentes series. La línea punteada corresponde a un valor de $2 V \sin (i)=350 \mathrm{~km} / \mathrm{s}$, y la región sombreada al error correspondiente (Tabla 3.3).

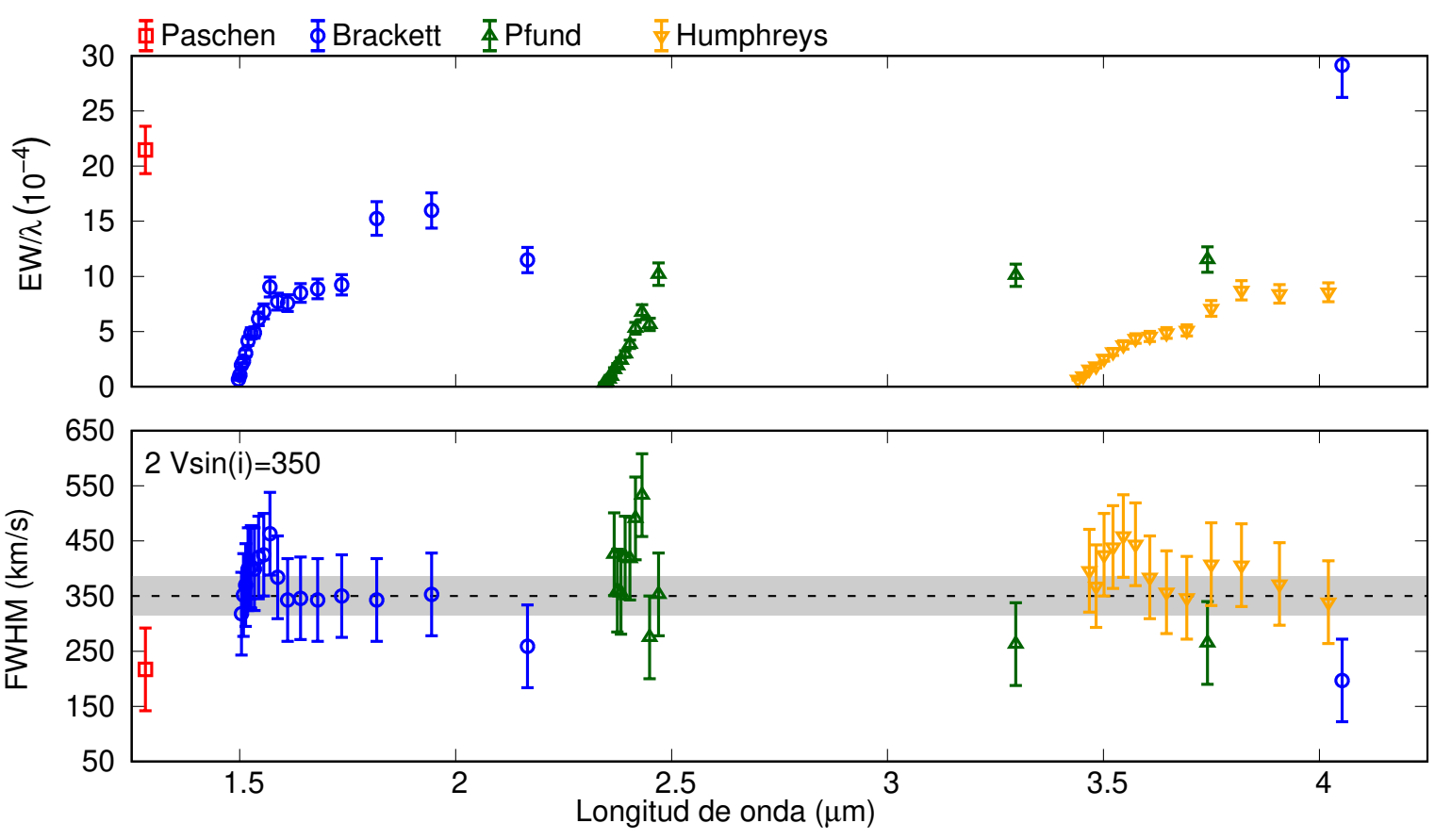

\section{Determinación de densidades columnares y extensiones de las regiones de formación}

A partir de los métodos $N$ y FWHM obtuvimos las densidades columnares, extensiones relativas y radios internos de las regiones de formación. Los mismos se muestran en la Tabla A.6.

Las líneas de las series de Pfund y Brackett muestran mayores densidades columnares y extensiones de las regiones de formación hacia los miembros más altos de la serie, contrario a lo observado para las líneas de la serie de Humphreys.

En la serie de Humphreys observamos una tendencia a valores menores de los radios internos para los miembros más altos de la serie. En las series de Brackett y Pfund, los radios disminuyen hasta alcanzar valores mínimos en $\mathrm{Pf}_{20}$ y $\mathrm{Br}_{15}$, para luego crecer nuevamente. En la Fig. 5.15 se muestran los radios internos de las regiones de formación relativos a $\operatorname{Br} \delta$ obtenidos a partir de todos los FWHM medidos. Las líneas que se forman en una región más interna a $\mathrm{Br} \delta$ son los miembros altos de las distintas series, mientras que los miembros más bajos son los que se forman en las regiones más externas de la envoltura. 
Figura 5.14: 105 Tau - Cocientes de flujos para las líneas de la serie de Humphreys, Pfund y Brackett. En la parte superior se identifican la líneas correspondientes. Los símbolos utilizados son los mismos que en la Fig. 5.13.
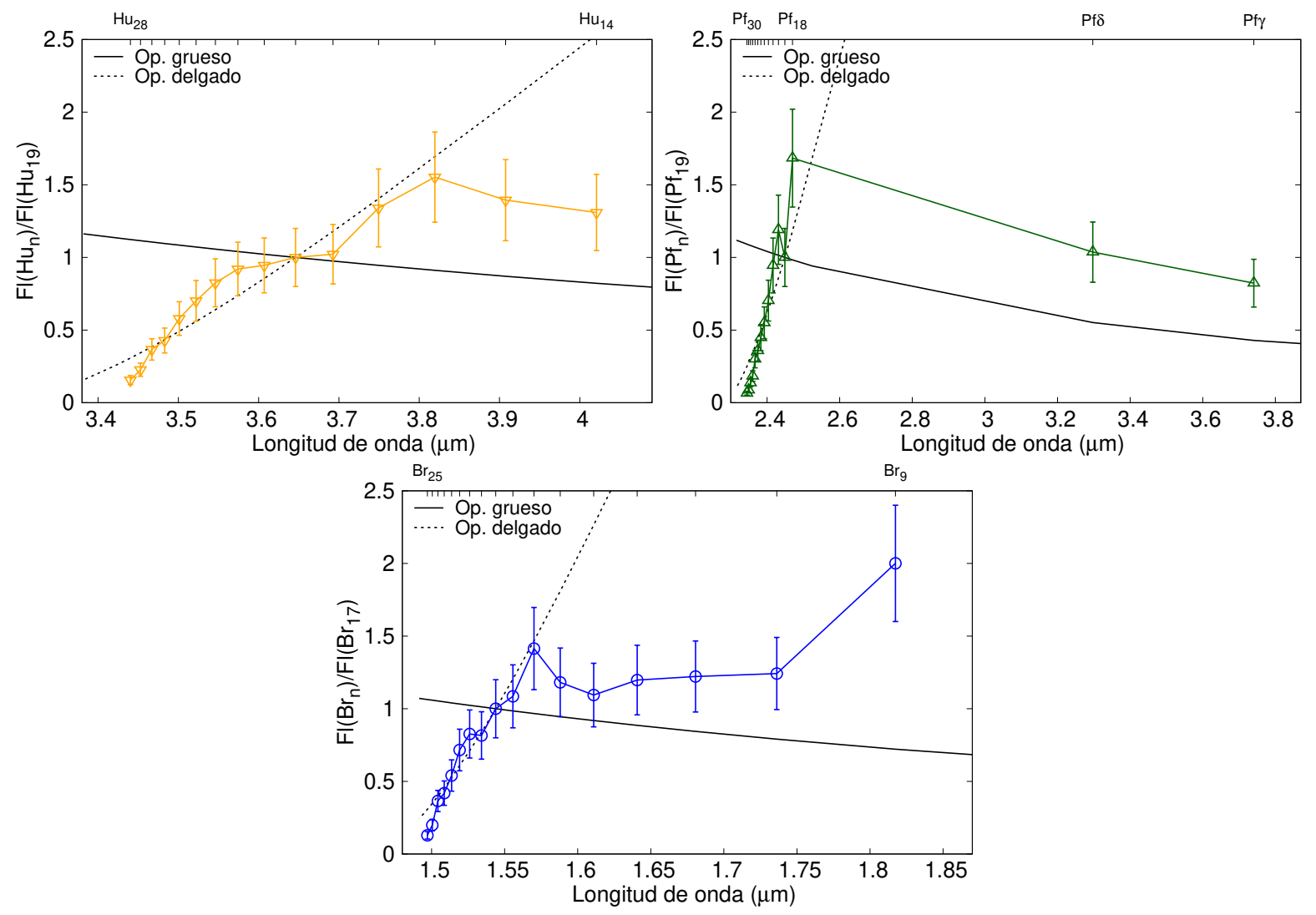

Figura 5.15: 105 Tau - Radios internos de las regiones emisoras de las diferentes líneas relativos a la región emisora de $\operatorname{Br} \delta$. Los símbolos utilizados son los mismos que en la Fig. 5.13.

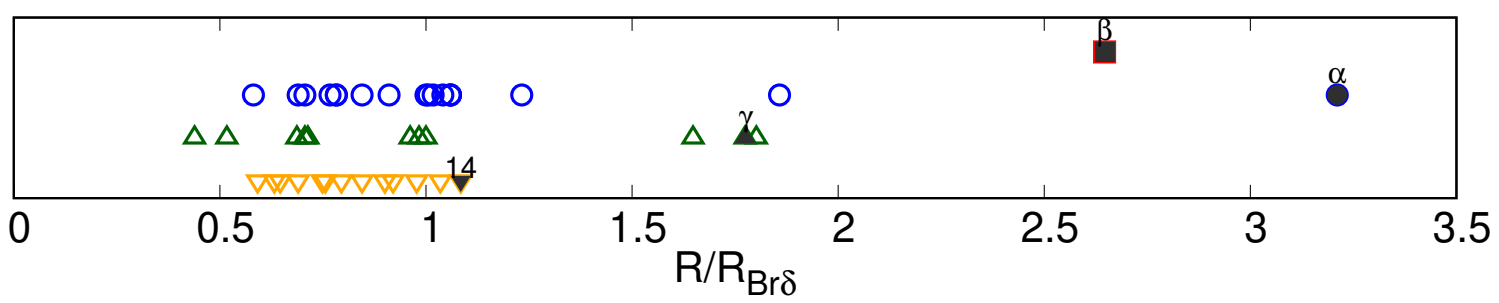




\subsection{4 $\Psi$ Ori - HD 35439}

$\Psi$ Ori es una estrella de tipo espectral B1 V, que presenta variaciones $\mathrm{Be} \rightarrow \mathrm{B} \rightarrow \mathrm{Be}$. Andrillat et al. (1990) obtuvieron que la región emisora de $\mathrm{H} \alpha$ se extiende entre $1.31 \mathrm{R}_{\star}$ y $3.66 \mathrm{R}_{\star}$, mientras que Jones et al. (2011) reportaron variabilidad en el ancho equivalente de la línea $\mathrm{H} \alpha$.

Silaj et al. (2010) ajustaron la línea de $\mathrm{H} \alpha$ con un doble pico en emisión y obtuvieron como parámetros del mejor ajuste un tipo espectral B0 para la estrella central, $i=45^{\circ}, \rho_{0}=5$. $10^{-11} \mathrm{~g} \mathrm{~cm}^{-3}$ y $n=3.5$. Por otro lado, Arcos et al. (2017) encontraron dos conjuntos de parámetros: $i=50^{\circ}, \rho_{0}=2.5 \cdot 10^{-11} \mathrm{~g} \mathrm{~cm}^{-3}, n=2.5$ modelando observaciones de $\mathrm{H} \alpha$ tomadas en 2012 e $i=50^{\circ}$, $\rho_{0}=5 \cdot 10^{-12} \mathrm{~g} \mathrm{~cm}^{-3}, \mathrm{y} n=2$ a partir de observaciones tomadas en 2015 .

A partir del modelado del continuo IR, Vieira et al. (2017) obtuvieron $\log \rho_{0}=-10 \pm 1$ y $n=6 \pm 2$ para el año $1983, \log \rho_{0}=-10.6 \pm 0.4$ y $n=4.2 \pm 0.7$ entre 2006 y 2007 , y $\log \rho_{0}=-11 \pm 0.3$ y $n=3.4 \pm 0.1$ en el 2010. Según el criterio definido por estos autores, los valores de $n$ indican que la estrella presentaba un disco en formación, que se estabilizó hacia el 2010.

No encontramos en la literatura reportes de observaciones espectroscópicas en el IR cercano.

Figura 5.16: Espectros de $\Psi$ Ori en las bandas J (superior) y H (inferior).

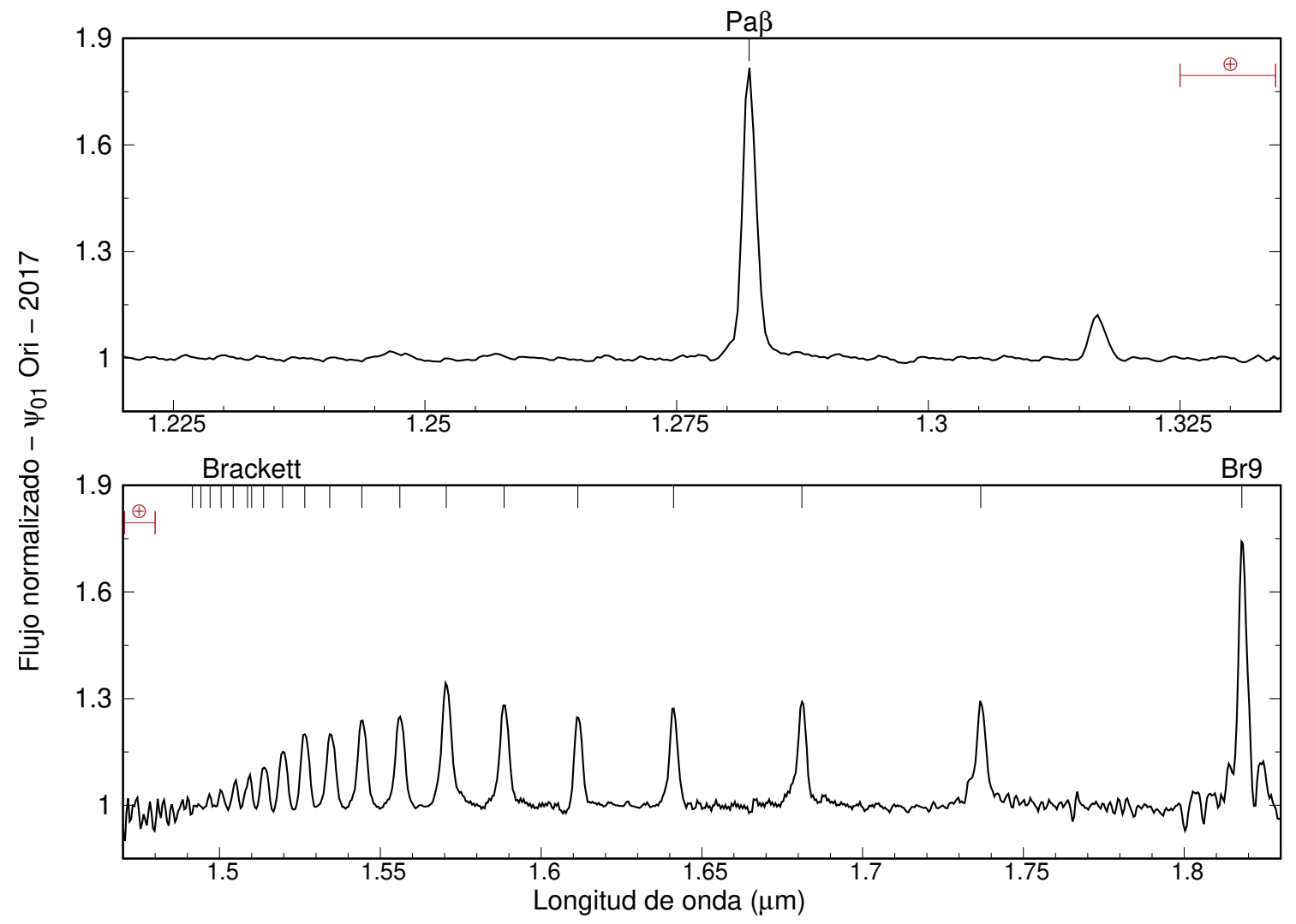


Figura 5.17: Espectros de $\Psi$ Ori en las bandas K (superior) y L (inferior).

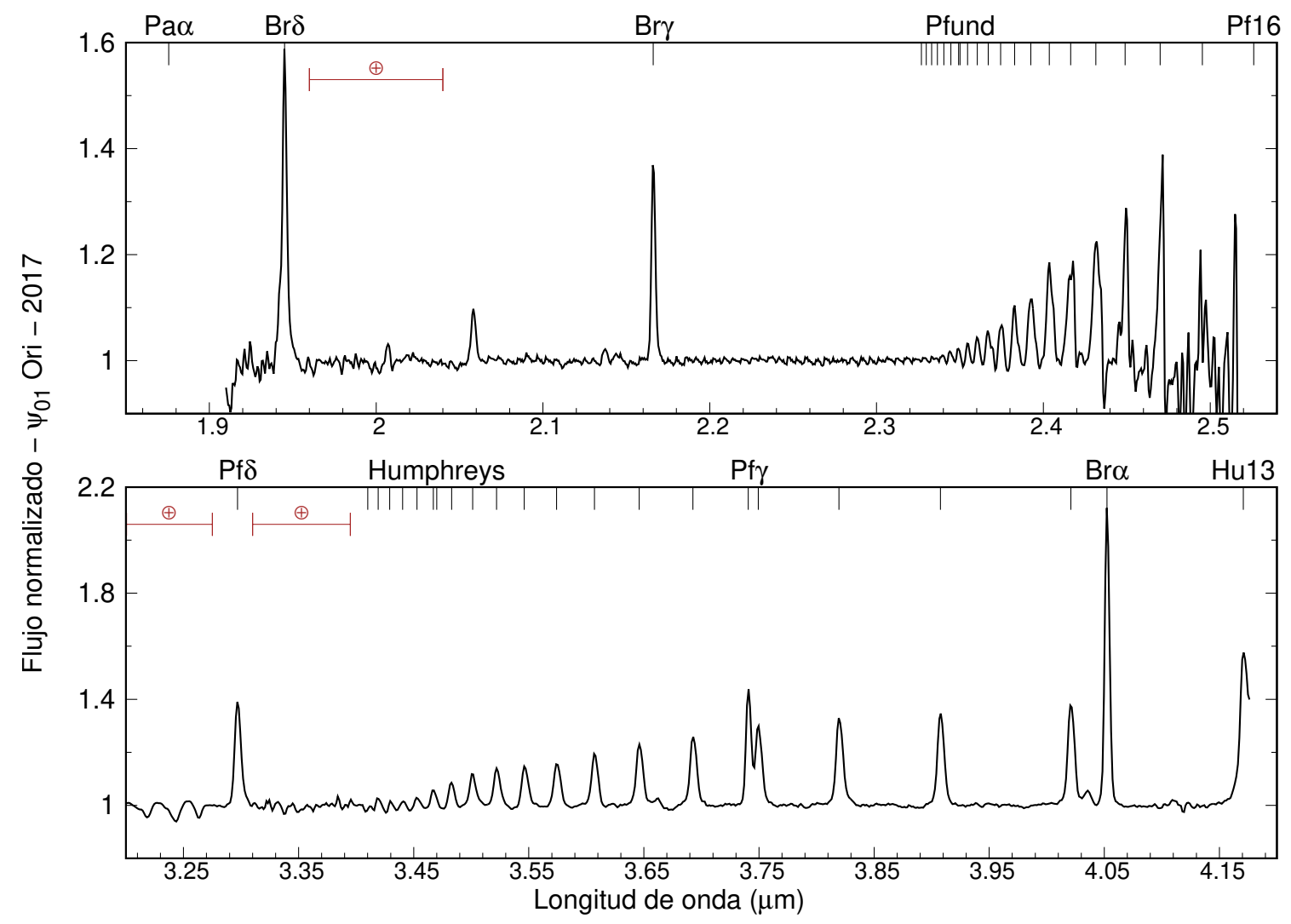

\section{Descripción de los espectros y mediciones realizadas}

En las Figs. 5.16 y 5.17 se muestran los espectros obtenidos en las bandas J, H, K y L en el 2017 utilizando el espectrógrafo GNIRS. En los mismos, se observan numerosas líneas de hidrógeno en emisión, cuyos valores de EW, Fl y FWHM se muestran en la Tabla A.7. Según los valores de la Tabla 3.3, los valores de EW y Fl obtenidos en las líneas fueron corregidas teniendo en cuenta la absorción fotosférica correspondiente a una estrella con $T_{\text {eff }}=22000 \mathrm{~K}$ y $\log g=4.0$. La estrella pertenece al grupo II de la clasificación de Mennickent et al. (2009), aunque la intensidad de las líneas de la serie de Humphreys no es despreciable respecto a la intensidad de $\operatorname{Br} \alpha$ y $\operatorname{Pf} \gamma$.

La Fig. 5.67 muestra la posición de $\Psi$ Ori en el diagrama de Lenorzer. La misma se ubica en una región cercana a la región superior derecha, correspondiente a los objetos del grupo I.

\section{Comportamiento de las series de hidrógeno}

En el gráfico de EW/ $\lambda$ en función de la longitud de onda, vemos que los primeros miembros de cada series presentan valores estables $\sim 7 \cdot 10^{-4}$ (ver Fig. 5.18). Los FWHM de las líneas de la serie de Humphreys son similares a $2 V \sin (i)$, indicando regiones de formación cercanas a la fotosfera estelar. Los miembros de las series de Pfund y Brackett presentan una tendencia 
Figura 5.18: $\Psi$ Ori - EW/ $\lambda$ y FWHM vs. $\lambda$ de las líneas observadas en las diferentes series. La línea punteada corresponde a un valor de $2 V \sin (i)=532 \mathrm{~km} / \mathrm{s}$, y la región sombreada al error correspondiente (Tabla 3.3).
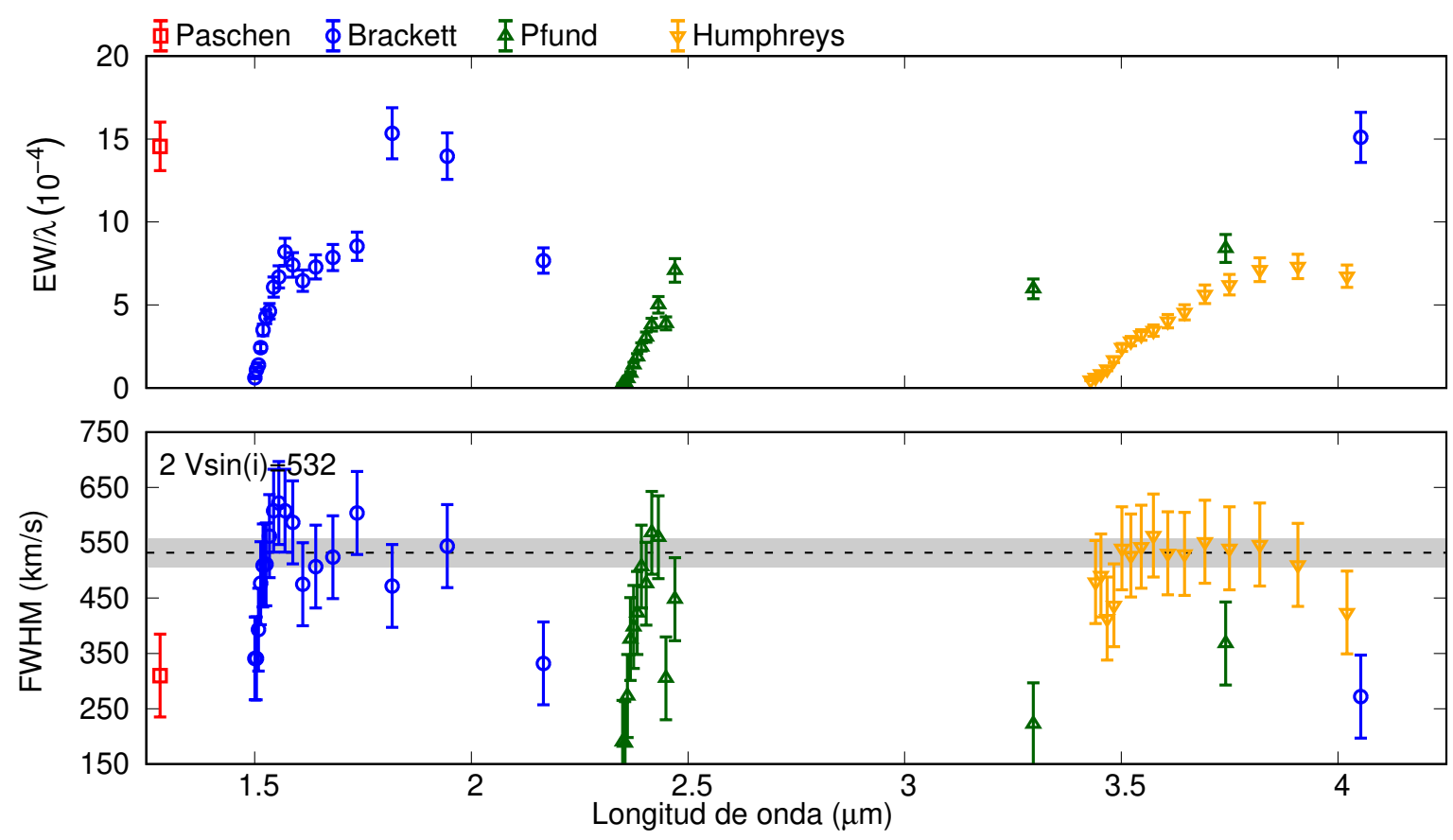

decreciente para los FWHM desde $\mathrm{Pf}_{21}$ y $\mathrm{Br}_{16}$ hacia los miembros más altos de cada serie.

En la Fig. 5.19 mostramos los cocientes de los flujos de las líneas de cada serie, junto con los valores esperados para los casos ópticamente grueso y delgado. Los miembros más bajos de cada serie se apartan de lo esperado para un caso ópticamente delgado, hasta $\mathrm{Br}_{14}, \operatorname{Pf} \delta$ y $\mathrm{Hu}_{17}$. Además, encontramos que $\mathrm{EW}(\mathrm{Br} \alpha) / \mathrm{EW}(\mathrm{Br} \gamma) \sim 3.7$, valor que se encuentra entre los esperados para estrellas del grupo I y II, más cercano a los esperados para el grupo I.

\section{Determinación de densidades columnares y extensiones de las regiones de formación}

Utilizando los métodos $N$ y FWHM obtuvimos las densidades columnares, extensiones de las regiones de formación y radios internos de las mismas que se muestran en la Tabla A.8.

Para la serie de Humphreys obtuvimos densidades columnares y extensiones menores para los miembros más altos, comportamiento opuesto a lo observado en la serie de Brackett. Para la serie de Pfund, los valores obtenidos en las dos líneas son similares.

Los valores de los radios internos de las regiones de formación son similares para las líneas de la serie de Humphreys, indicando una región de formación compacta. Por otro lado, para las series de Pfund y Brackett, los valores se hacen menores para luego volver a aumentar a medida que observamos miembros más altos de la serie, con mínimos en $\mathrm{Pf}_{21}$ y $\mathrm{Br}_{16}$. A partir de los FWHM obtenidos para las distintas líneas observadas, obtuvimos los radios relativos comparados 
Figura 5.19: $\Psi$ Ori - Cocientes de flujos para las líneas de la serie de Humphreys, Pfund y Brackett. En la parte superior se identifican la líneas correspondientes. Los símbolos utilizados son los mismos que en la Fig. 5.18.
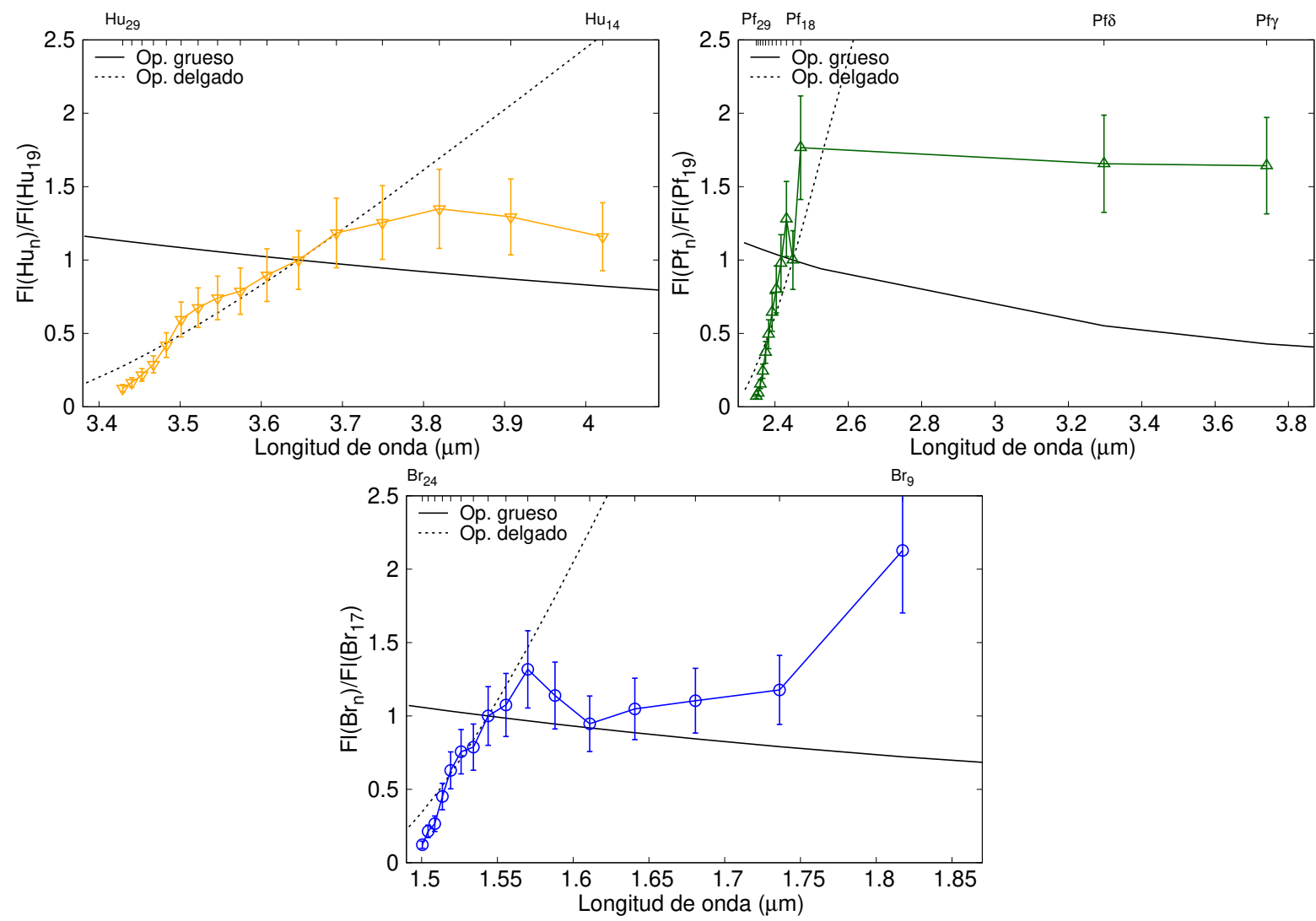

Figura 5.20: $\Psi$ Ori - Radios internos de las regiones emisoras de las diferentes líneas relativos a la región emisora de $\mathrm{Br} \delta$. Los símbolos utilizados son los mismos que en la Fig. 5.18.

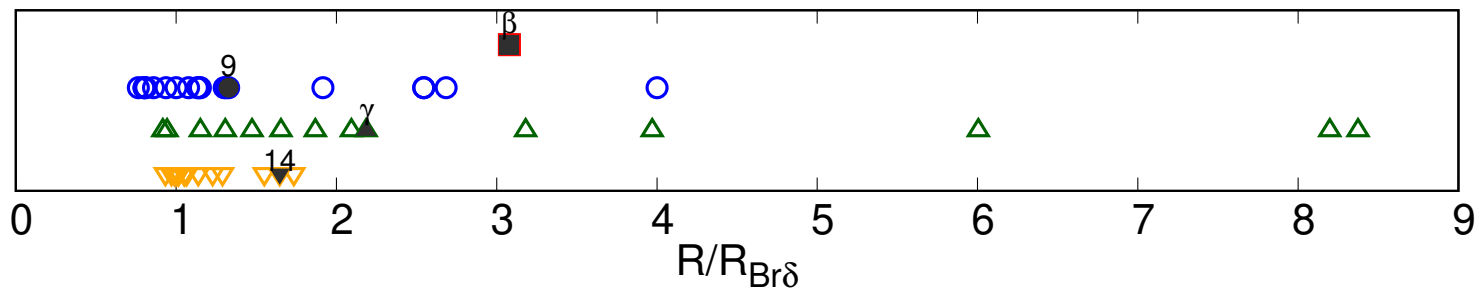

con el radio correspondiente a $\mathrm{Br} \delta$. Los mismos se muestran en la Fig. 5.20. Los miembros más altos de cada serie se forman en una región compacta cercana a la estrella central, mientras que los más bajos se forman en regiones más separadas. 


\subsubsection{Tau - HD 36576}

120 Tau es una estrella con clasificación espectral B2 IV-V, identificada como Be por Merrill et al. (1925). Estos autores reportaron que las líneas $\mathrm{H} \alpha$ y $\mathrm{H} \beta$ se encontraban en emisión, mientras que $\mathrm{H} \gamma$ presentaba emisión rellenando la absorción fotosférica, sin llegar a sobrepasar el nivel del continuo.

Esta estrella presenta variaciones multiperiódicas consistentes con pulsaciones no radiales multimodales (Bossi et al., 1989). El tamaño de la región emisora de $\mathrm{H} \alpha$ fue estimado entre $1.28 R_{\star}$ y $3.59 R_{\star}$ por Andrillat et al. (1990). Silaj et al. (2010) modelaron la línea de $\mathrm{H} \alpha$ con un pico en emisión usando una estrella central B2 con un disco con $i=45^{\circ}, \rho_{0}=5 \cdot 10^{-10} \mathrm{~g} \mathrm{~cm}^{-3}$, y $n=3.5$. Por otro lado, a partir del modelado del continuo IR, Vieira et al. (2017) obtuvieron valores $\log \rho_{0}=-12 \pm 0.2$ y $n=1.7 \pm 0.1$ durante $1983, \log \rho_{0}=-11.4 \pm 0.1$ y $n=2.3 \pm 0.1$ en los años 2006/2007, y $\log \rho_{0}=-11.4 \pm 0.1$ y $n=2.36 \pm 0.4$ en 2010. Según estos valores, estos autores indicaron que el disco se estaba disipando en las tres observaciones.

No encontramos reportes de observaciones espectroscópicas en el IR cercano en la literatura.

Figura 5.21: Espectros de 120 Tau en las bandas J (superior) y H (inferior).

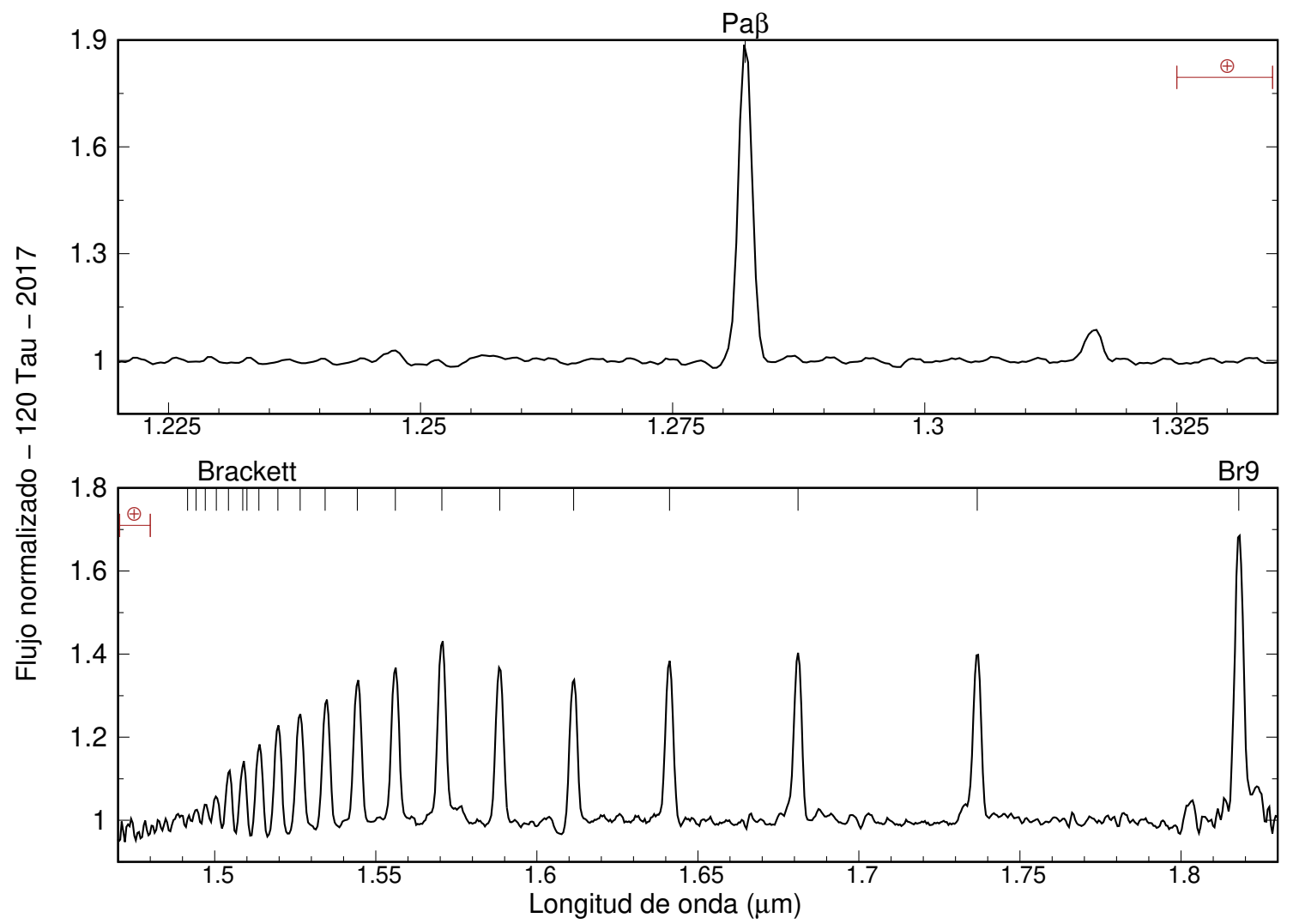


Figura 5.22: Espectros de 120 Tau en las bandas K (superior) y L (inferior).

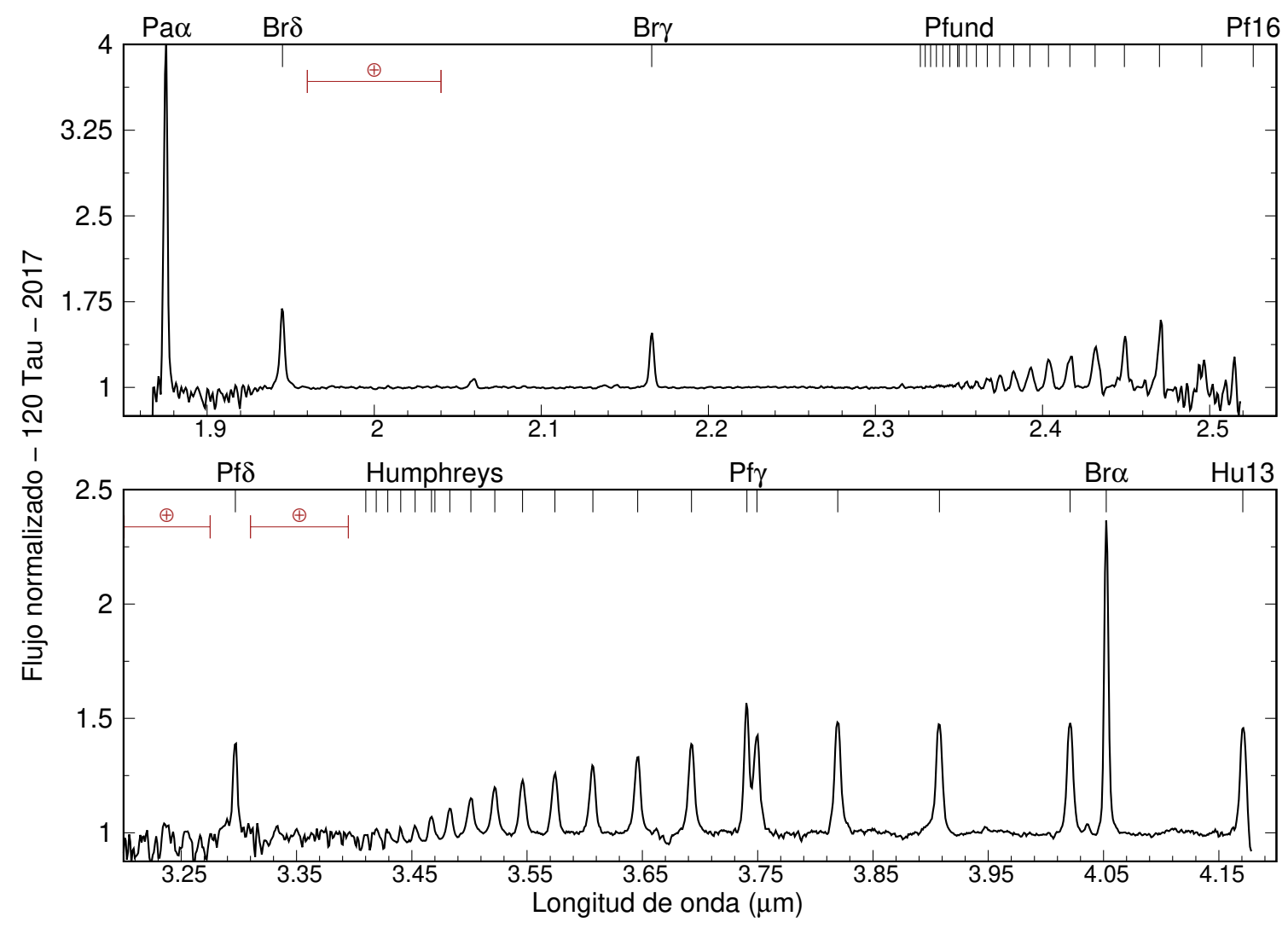

\section{Descripción de los espectros y mediciones realizadas}

Las Figs. 5.21 y 5.22 muestran los espectros en las bandas J, H, K y L obtenidos en el 2017 con el espectrógrafo GNIRS. Las líneas de hidrógeno se observan en emisión en todas las bandas. La intensidad de las líneas de la serie de Humphreys es menor que la de Br $\alpha$ y $\operatorname{Pf} \gamma$, por lo que la estrella pertenece al grupo II de la clasificación de Mennickent et al. (2009). Para realizar la corrección por absorción fotosférica se utilizó el espectro sintético correspondiente a una estrella con $T_{\text {eff }}=23000 \mathrm{~K}$ y $\log g=4.0$ (ver Tabla 3.3), y los detalles de los EW, Fl y FWHM obtenidos se muestran en la Tabla A.9.

La ubicación de 120 Tau en el diagrama de Lenorzer se muestra en la Fig. 5.67. La misma se ubica en la región superior derecha, cercana al caso ópticamente grueso esperado para los objetos del grupo I.

\section{Comportamiento de las series de hidrógeno}

La Fig.5.23 muestra los gráficos de EW/ $\lambda$ y FWHM en función de la longitud de onda. Los valores de EW/ $\lambda$ alcanzan valores estables $\sim 9 \cdot 10^{-4}$ en cada serie. Los valores de FWHM aumentan y luego disminuyen a medida que observamos miembros más altos de las series, alcanzando los val- 
Figura 5.23: 120 Tau - EW/ $\lambda$ y FWHM vs. $\lambda$ de las líneas observadas en las diferentes series. La línea punteada corresponde a un valor de $2 V \sin (i)=532 \mathrm{~km} / \mathrm{s}$, y la región sombreada al error correspondiente (Tabla 3.3).
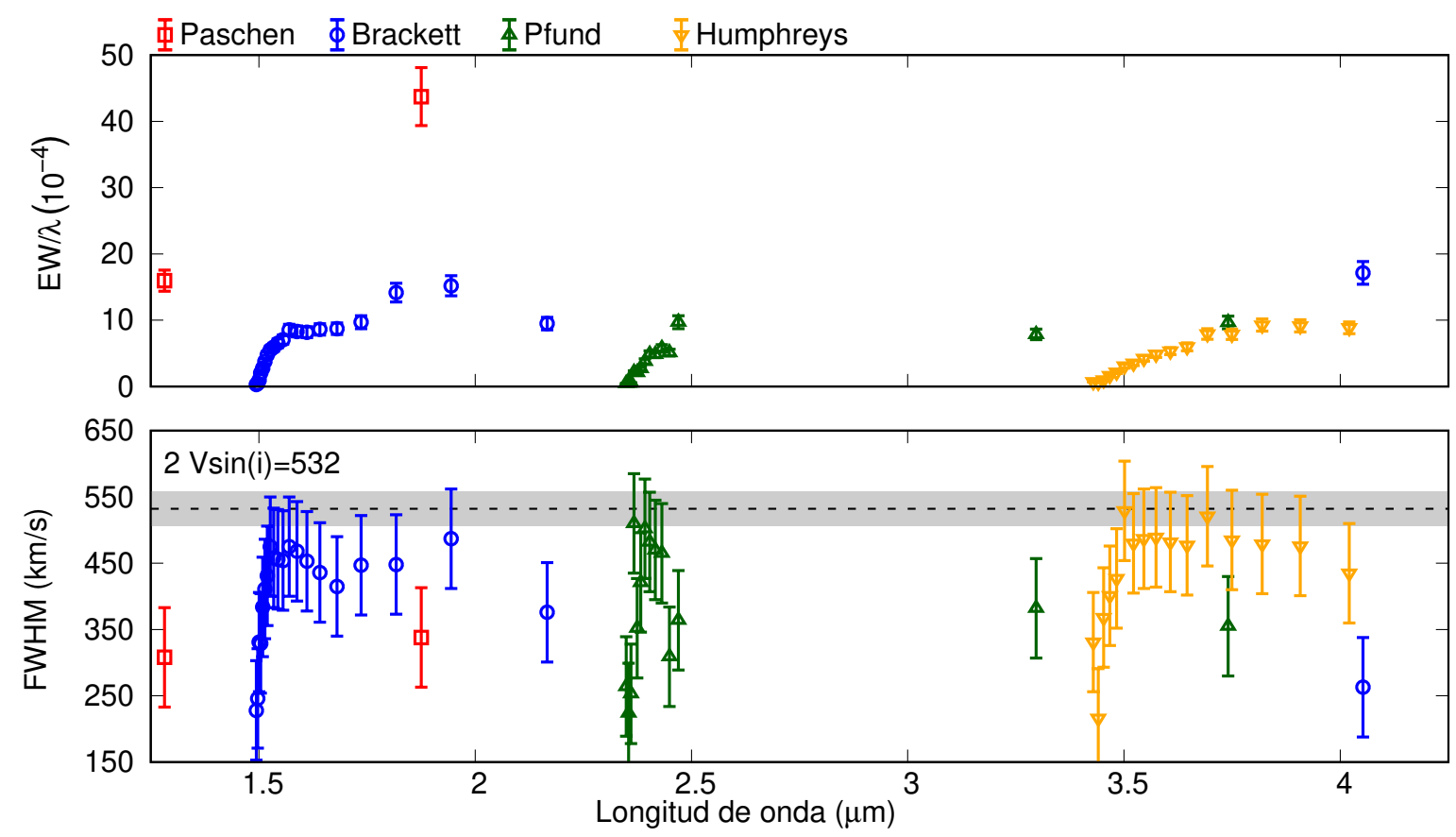

ores máximos en $\mathrm{Br}_{15}, \mathrm{Pf}_{26}$ y $\mathrm{Hu}_{24}$. Los ensanchamientos más grandes observados corresponden a un valor similar a $2 \mathrm{~V} \sin (i)$, lo que indicaría regiones de formación cercanas a la fotosfera.

Los cocientes de los flujos y la comparación con los casos ópticamente delgado y grueso se muestran en la Fig. 5.24. En las tres series se observa que primeros miembros se alejan de los cocientes esperados para un caso ópticamente delgado. Además determinamos que $\mathrm{EW}(\mathrm{Br} \alpha) / \mathrm{EW}(\mathrm{Br} \gamma) \sim 3.5$ y $\mathrm{EW}(\mathrm{Pa} \alpha) / \mathrm{EW}(\mathrm{Pa} \beta) \sim 4$. El valor de $\mathrm{EW}(\mathrm{Br} \alpha) / \mathrm{EW}(\mathrm{Br} \gamma)$ se encuentra entre los esperados para estrellas del grupo I y II.

\section{Determinación de densidades columnares y extensiones de las regiones de formación}

Las densidades columnares, extensiones relativas y radios internos de las regiones de formación obtenidas con los métodos $N$ y FWHM se muestran en la Tabla A.10.

Los miembros de la serie de Humphreys presentan menores densidades columnares y extensiones de las regiones de formación hacia los miembros más altos, contrario a lo observado en las series de Pfund y Brackett.

La serie de Humphreys presenta radios internos similares en los primeros miembros observados y que aumentan hacia los más altos. En las series de Pfund y Brackett los radios disminuyen a medida que observamos miembros más altos y luego vuelven a aumentar, con valores mínimos en $\mathrm{Br}_{15}$ y $\mathrm{Pf}_{26}$. Utilizando los FWHM de todas las líneas observadas, podemos 
Figura 5.24: 120 Tau - Cocientes de flujos para las líneas de la serie de Humphreys, Pfund y Brackett. En la parte superior se identifican la líneas correspondientes. Los símbolos utilizados son los mismos que en la Fig. 5.23.

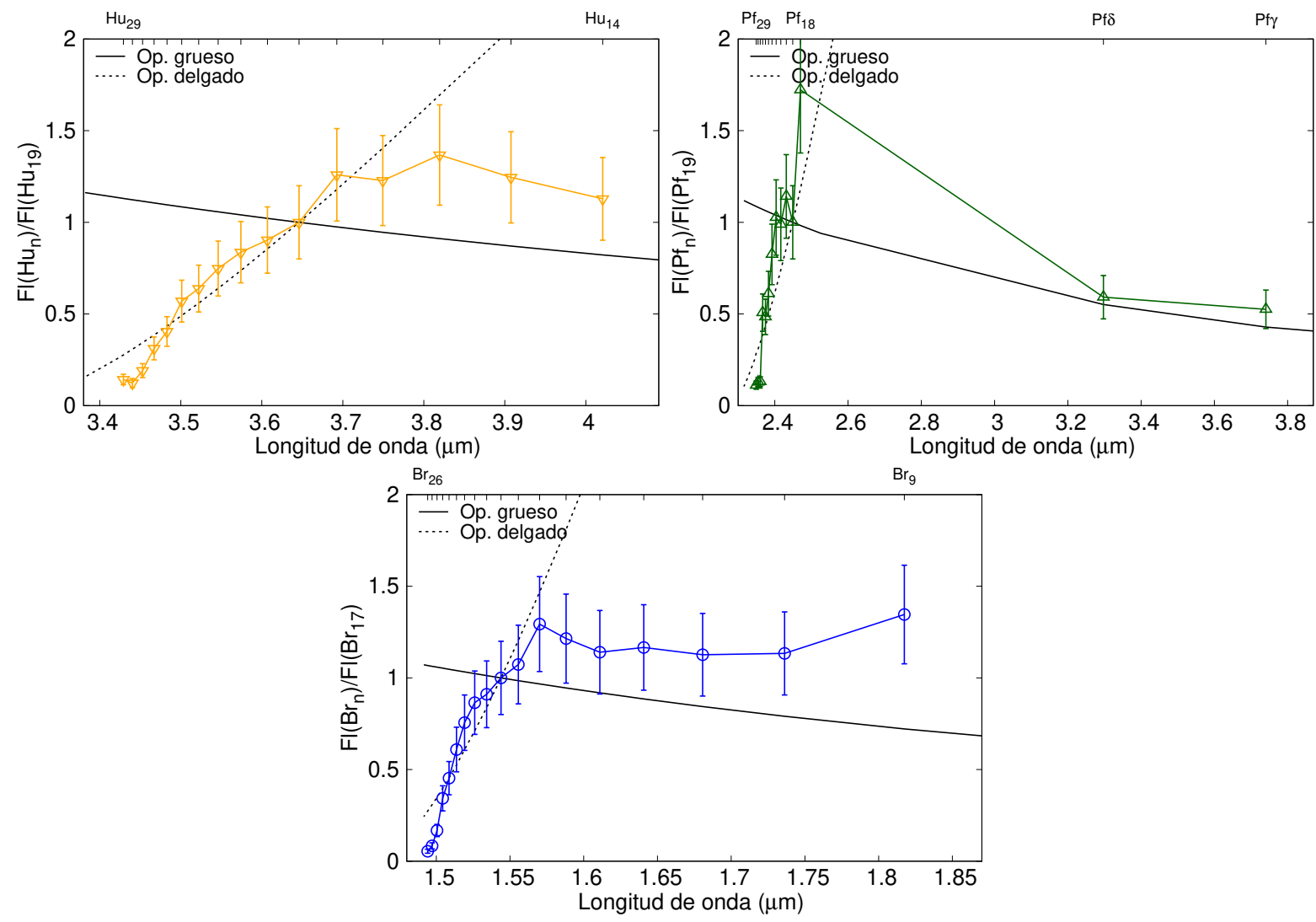

Figura 5.25: 120 Tau - Radios internos de las regiones emisoras de las diferentes líneas relativos a la región emisora de $\operatorname{Br} \delta$. Los símbolos utilizados son los mismos que en la Fig. 5.23.

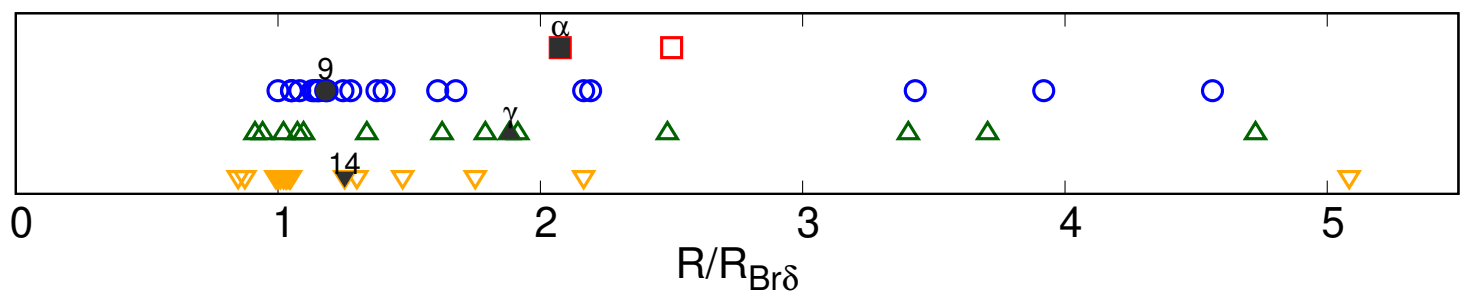

obtener los tamaños relativos para los radios internos de todas las regiones de formación respecto a $\operatorname{Br} \delta$ que se muestran en la Fig. 5.25. Los tamaños más grandes corresponden a los primeros miembros de cada serie. 


\subsection{6 $\omega$ Ori - HD 37490}

$\omega$ Ori es una estrella de tipo espectral B3 V, que ha sido estudiada por muchos años. Ha presentado variaciones de corto y medio período (Balona et al., 1987; Bergin et al., 1989; Balona et al., 1992; McDavid et al., 1996). Las variaciones de corto período ( 1 día) estarían asociadas a pulsaciones no radiales, mientras que las de medio período ( $\sim 11$ meses) se relacionarían al crecimiento/decrecimiento del disco.

Andrillat et al. (1990) estimaron un tamaño para la región emisora de $\mathrm{H} \alpha$ entre $1.07 \mathrm{R}_{\star}$ y $2.27 \mathrm{R}_{\star}$. Neiner et al. (2002) determinaron, a partir de los parámetros determinados con el método BCD, $\log T_{\text {eff }}=4.306 \pm 0.016$ y $\log g=3.48 \pm 0.03$, determinaron $\omega=0.83 \omega_{\mathrm{c}}, i=32 \pm 15^{\circ}$, $R_{\star}=6.84 \pm 0.25 R_{\odot}, M_{\star}=8.02 \pm 0.25 M_{\odot}$ utilizando los tracks evolutivos de Schaller et al. (1992).

Balona et al. (2002) encontraron un valor $V \sin i=173 \pm 2 \mathrm{~km} \mathrm{~s}^{-1}$ para la velocidad de rotación proyectada utilizando líneas de He en absorción, y un valor de $V \sin i=226 \pm 7 \mathrm{~km} \mathrm{~s}^{-1}$ a partir de las líneas metálicas. Este resultado fue atribuido a que probablemente las líneas de $\mathrm{He}$ también estén afectadas por la emisión del disco. Neiner et al. (2003) encontraron evidencia de un campo magnético débil $\left(B_{p o l}=530 \pm 230 \mathrm{G}\right.$ ), que varía sinusoidalmente con un período de 1.29 días. Sin embargo, nuevas observaciones no pudieron confirmar la presencia de ese campo magnético (Neiner et al., 2012).

Figura 5.26: Espectros de $\omega$ Ori en las bandas $\mathrm{J}$ (superior) y H (inferior).

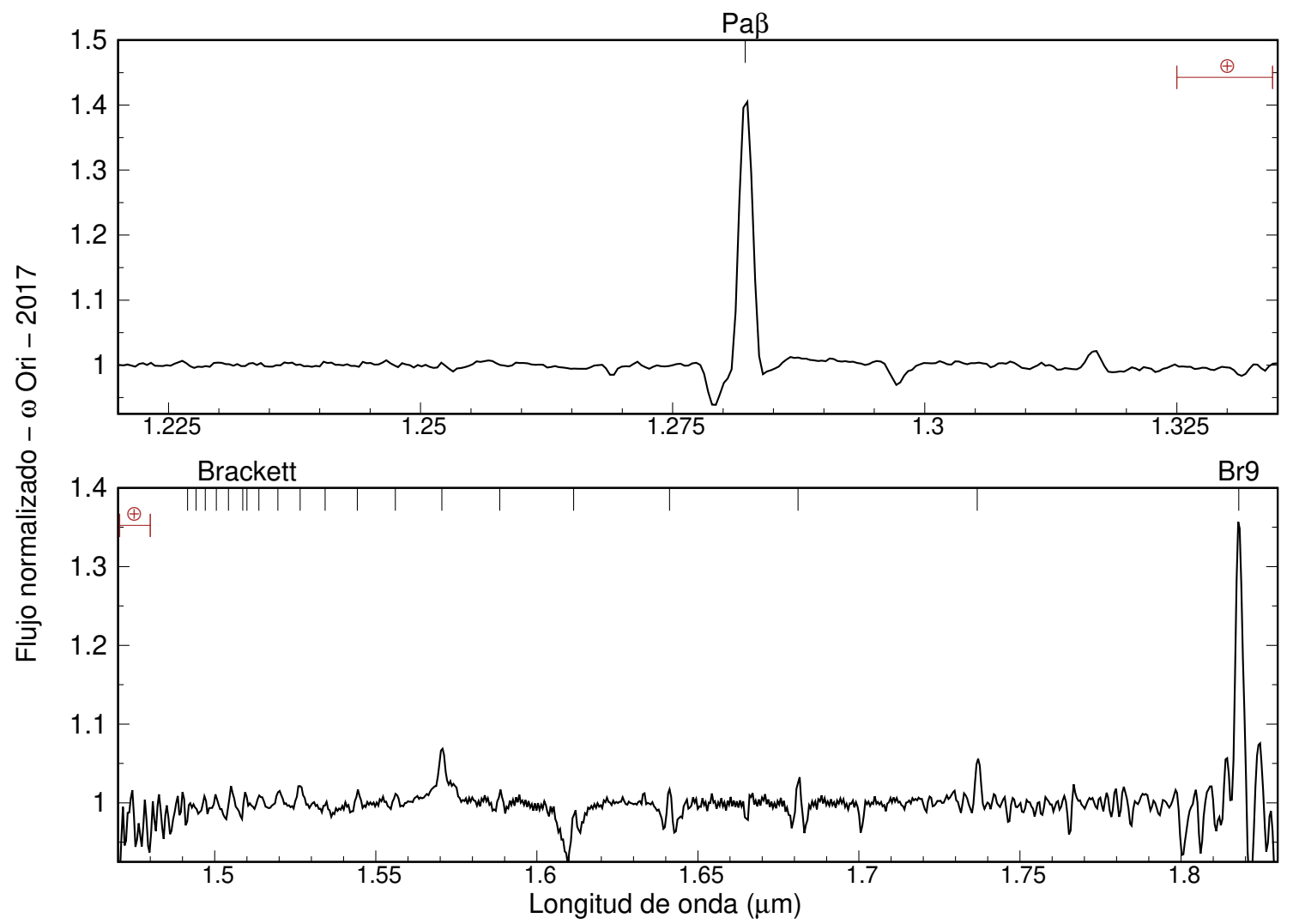


Figura 5.27: Espectros de $\omega$ Ori en las bandas K (superior) y L (inferior).

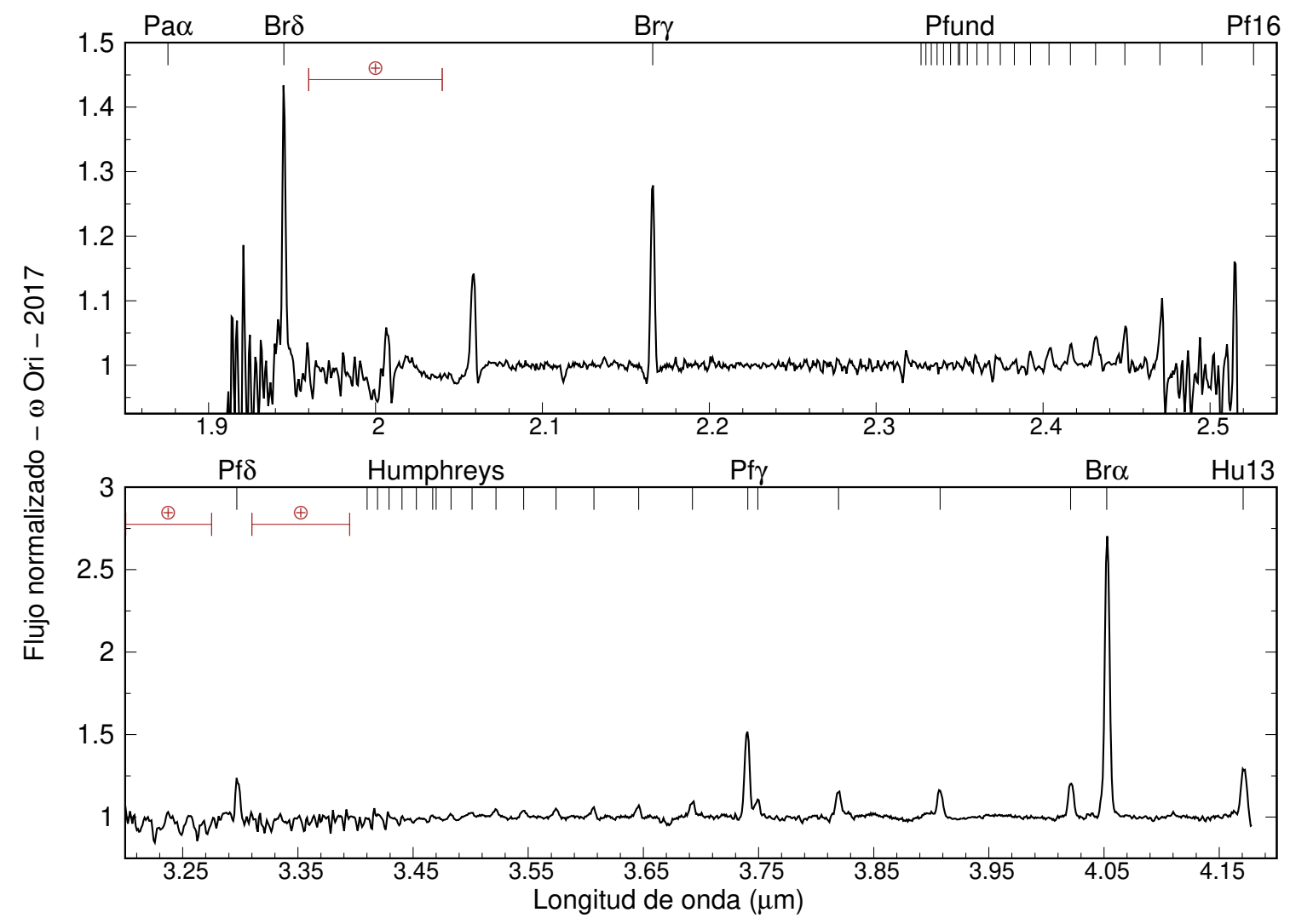

Silaj et al. (2010) ajustaron la línea de $\mathrm{H} \alpha$ con un perfil sintético de doble pico y obtuvieron como parámetros del mejor ajuste un tipo espectral B2 para la estrella central, $i=20^{\circ}$, $\rho_{0}=10^{-11} \mathrm{~g} \mathrm{~cm}^{-3}, \mathrm{y} n=3.5$. Además, Jones et al. (2011) reportaron variabilidad en el ancho equivalente de la línea $\mathrm{H} \alpha$.

No encontramos observaciones espectroscópicas en el IR reportadas en la literatura.

\section{Descripción de los espectros y mediciones realizadas}

En las Figs. 5.26 y 5.27 se muestran los espectros obtenidos en el año 2017 con el espectrógrafo GNIRS. Los valores de EW, Fl y FWHM, corregidos utilizando un espectro sintético correspondiente a una estrella con $T_{\text {eff }}=14000 \mathrm{~K}$ y $\log g=4.0$ (ver Tabla 3.3), se presentan en la Tabla A.11. Todas las líneas de hidrógenos observadas se encuentran en emisión, y en el caso de los miembros más altos de la serie de Brackett la emisión es pequeña y no llega a rellenar completamente la absorción fotosférica. Según el criterio de clasificación de Mennickent et al. (2009) la estrella pertenece al grupo II.

En la Fig. 5.67 se muestra la ubicación de $\omega$ Ori en el diagrama de Lenorzer. La misma se ubica en la región central del diagrama, entre los casos ópticamente grueso y delgado, consistente con su pertenencia al grupo II. 
Figura 5.28: $\omega$ Ori - EW/ $\lambda$ y FWHM vs. $\lambda$ de las líneas observadas en las diferentes series. La línea punteada corresponde a un valor de $2 V \sin (i)=310 \mathrm{~km} / \mathrm{s}$, y la región sombreada al error correspondiente (Tabla 3.3).

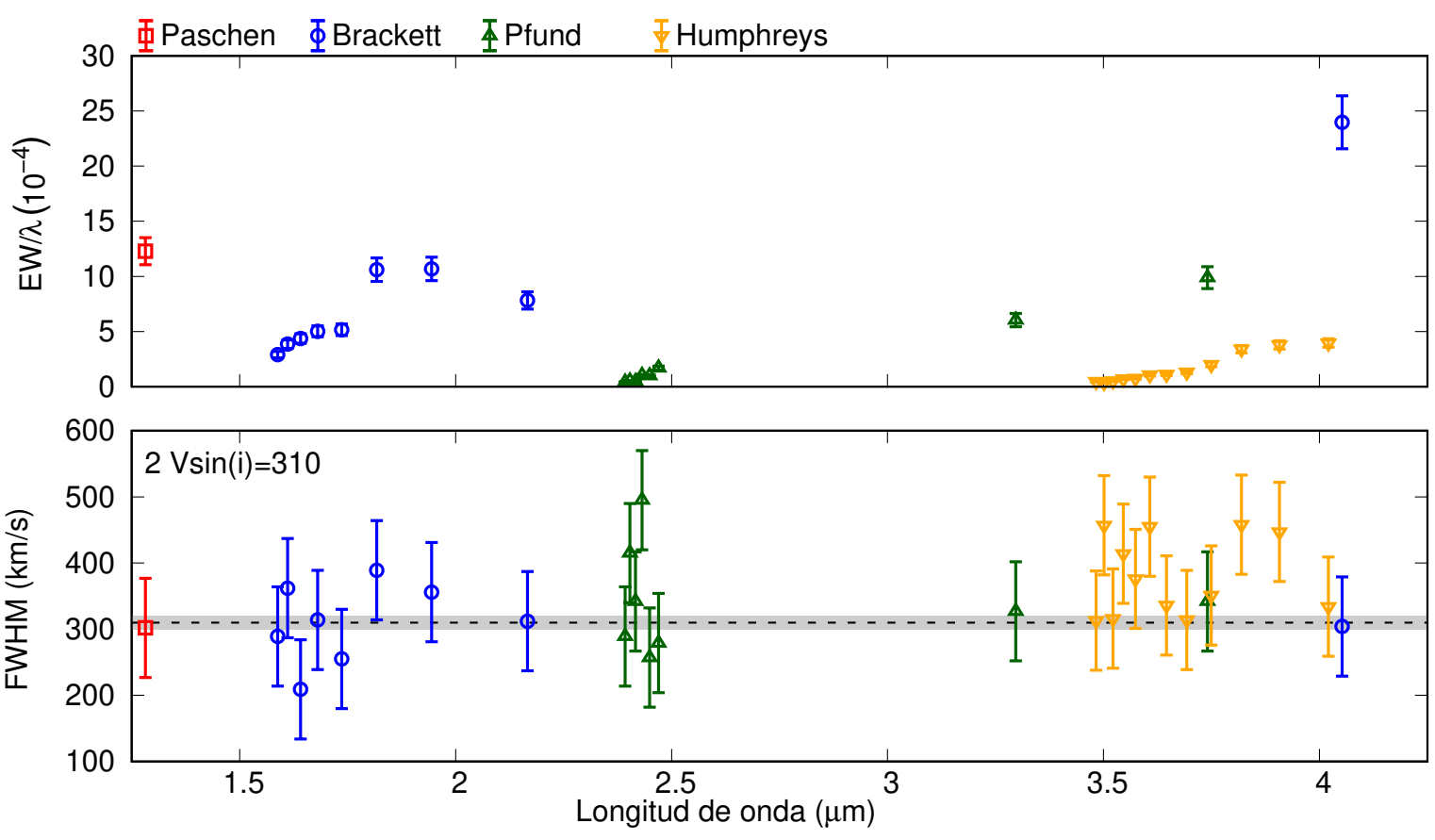

\section{Comportamiento de las series de hidrógeno}

En la Fig. 5.28 vemos que las medidas de EW/ $\lambda$ en función de la longitud de onda no alcanzan valores estables. Por otra parte los FWHM presentan una gran dispersión en los valores obtenidos en las series de Pfund, Brackett y Humphreys. Varias líneas presentan ensanchamientos mayores a $2 \mathrm{~V} \sin (i)$, indicando regiones de formación cercana a la fotosfera estelar o la contribución de otros factores aparte de la rotación al ensanchamiento de las líneas.

Los cocientes de los flujos y la comparación con los casos ópticamente grueso y delgado se muestran en la Fig. 5.29. En la serie de Humphreys, los valores obtenidos oscilan alrededor de los cocientes esperados para el caso ópticamente delgado, tomando valores prácticamente constantes en los miembros más bajos observados. En la serie de Brackett, los cocientes de los flujos se alejan del caso ópticamente delgado en $\mathrm{Br}_{11-10}$ y vuelven a acercarse en $\mathrm{Br}_{9}$. Este comportamiento podría estar relacionado con la presencia de zonas con densidades muy distintas, producto de la formación de un nuevo disco sobre uno anteriormente disipado. Para la serie de Pfund, observamos que $\operatorname{Pf} \gamma$ y $\operatorname{Pf} \delta$ se apartan de los valores de cocientes de flujos esperados para un caso ópticamente delgado. Obtuvimos además que $\mathrm{EW}(\mathrm{Br} \alpha) / \mathrm{EW}(\mathrm{Br} \gamma) \sim 5.7$, consistente con lo esperado para una estrella del grupo II. 
Figura 5.29: $\omega$ Ori - Cocientes de flujos para las líneas de la serie de Humphreys, Pfund y Brackett. En la parte superior se identifican la líneas correspondientes. Los símbolos utilizados son los mismos que en la Fig. 5.28.
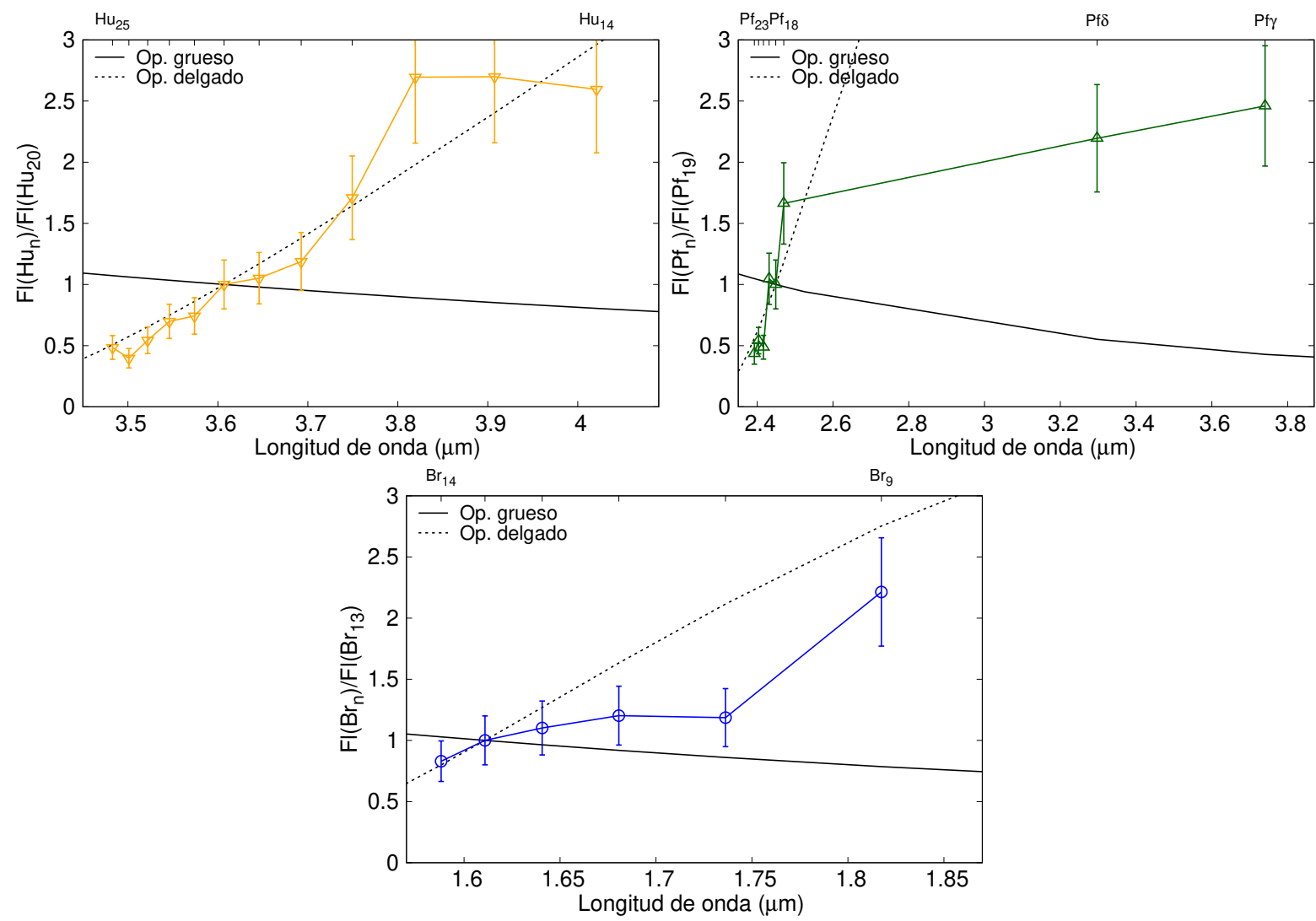

Figura 5.30: $\omega$ Ori - Radios internos de las regiones emisoras de las diferentes líneas relativos a la región emisora de $\mathrm{Br} \delta$. Los símbolos utilizados son los mismos que en la Fig. 5.28.

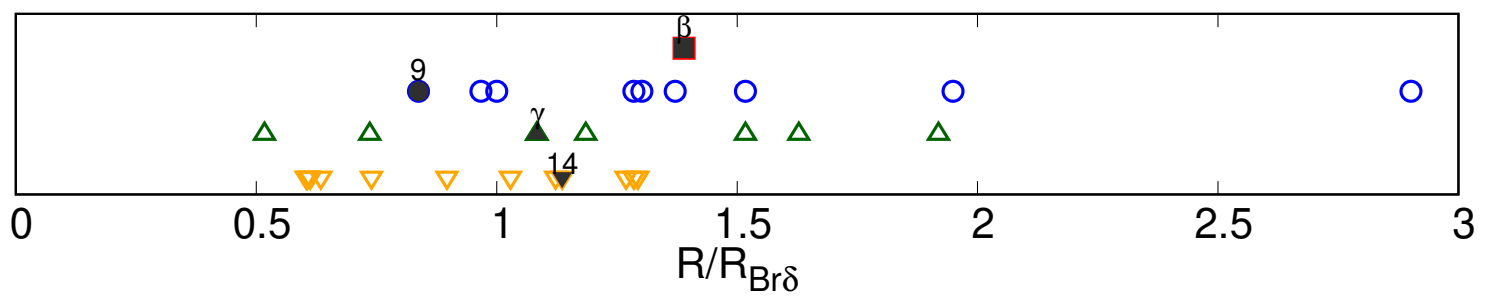

\section{Determinación de densidades columnares y extensiones de las regiones de formación}

Obtuvimos los tamaños relativos de los radios internos de las regiones emisoras utilizando el método FWHM, y las densidades columnares y extensiones de las regiones de formación para Pf $\gamma$ y Pf $\delta$ (ver Tabla A.12).

Debido a la gran dispersión de los valores obtenidos para los FWHM, no se observa una 
tendencia clara para los tamaños a lo largo de las series. Por otro lado, los valores de densidad columnar y extensiones de las regiones de formación son mayores para $\operatorname{Pf} \delta$ que para $\operatorname{Pf} \gamma$. En los radios internos de las regiones de formación relativos a $\operatorname{Br} \delta$ que se muestran en la Fig. 5.30, la mayor parte de las líneas muestran regiones de formación muy similares, entre 0.5 y $1.5 R_{\mathrm{Br} \delta}$, indicando una envoltura pequeña cercana a la estrella central. 


\subsubsection{V696 Mon - HD 41335}

V696 Mon es una estrella con clasificación espectral B3-5 V, con emisión reportada en la línea $\mathrm{H} \alpha$ por Merrill et al. (1925). Slettebak (1951) reportaron en emisión las líneas $\mathrm{H} \alpha, \mathrm{H} \beta$ y O I $\lambda 7774 \AA$ y $\lambda 8446 \AA$, junto con emisión en líneas de la serie de Paschen. Esta estrella forma parte de un sistema binario interactuante, al que Peters (1983) le determinó un período de 80.86 \pm 0.005 días, con una estrella primaria B1.5IV-V ( 8 a $11 M_{\odot}$ ) y una secundaria de $1 M_{\odot}$, a partir del análisis de las velocidades radiales y suponiendo una órbita circular. Esto implica una separación entre las componentes de $0.84 \mathrm{UA}\left(180 R_{\odot}\right)$.

A partir del análisis de la SED, Waters et al. (1991) encontraron que la compañera no puede ser una enana fría, y propusieron en su lugar una subenana caliente (Sd). Posteriormente, Peters et al. (2016) estudiaron observaciones en el UV y en $\mathrm{H} \alpha$, y detectaron una señal débil de líneas espectrales correspondientes a una Sd, proponiendo que la masa para la primaria es de $9 M_{\odot}$ y para la estrella secundaria es de $0.7 M_{\odot}$. Además, concluyeron que la presencia de la compañera crea un truncamiento en el disco inferior al radio de Roche de la estrella $\mathrm{Be}$, generando una estructura tipo brazo espiral en el disco, similar al sistema Be+Sd0 o tipo $\phi$ Per reportado por Thaller et al. (1995).

Andrillat et al. (1990) encontraron que la región emisora en $\mathrm{H} \alpha$ abarca entre $1.66 \mathrm{R}_{\star} \mathrm{y}$

Figura 5.31: Espectros de V696 Mon en las bandas J (superior) y H (inferior).

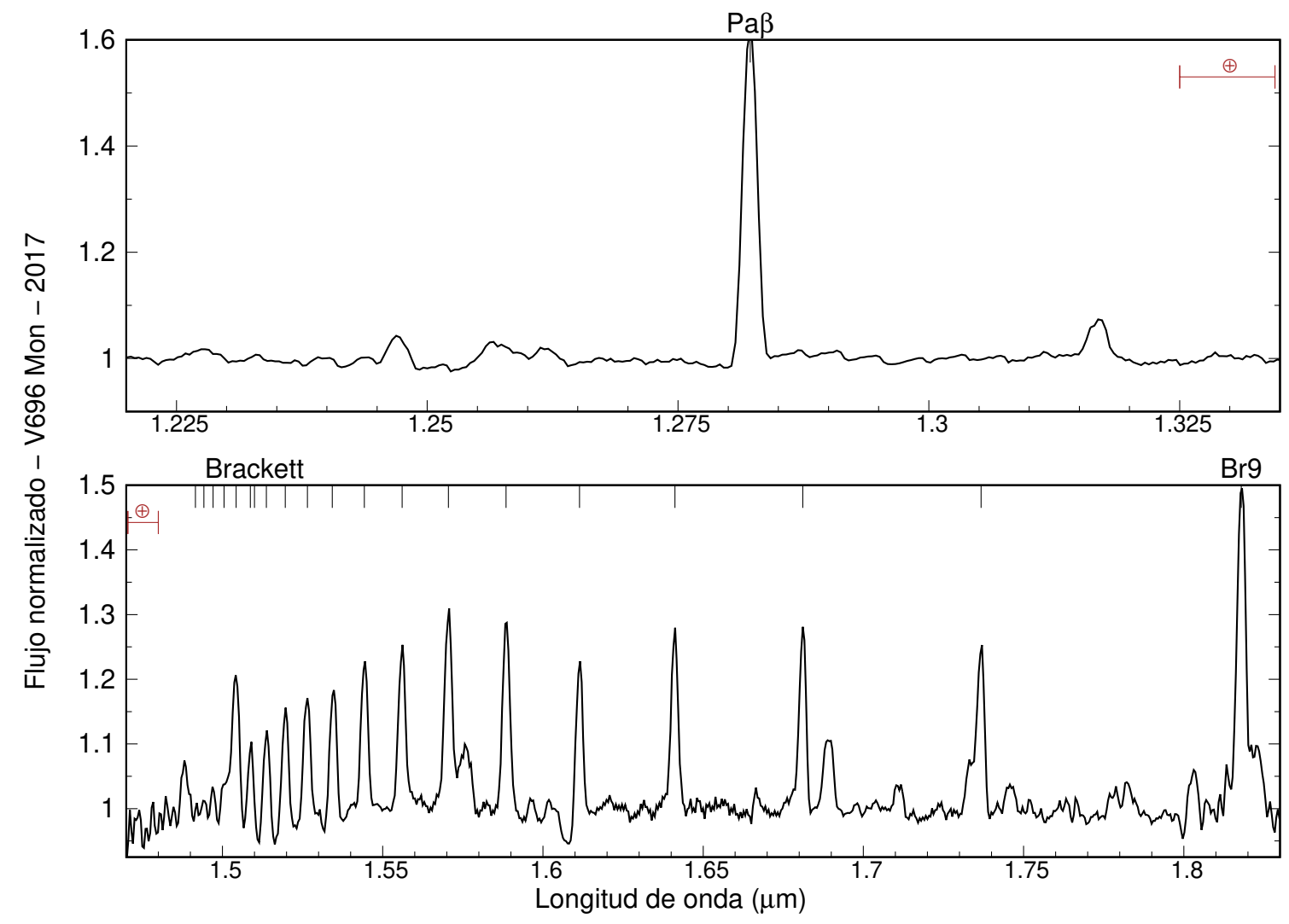


Figura 5.32: Espectros de V696 Mon en las bandas K (superior) y L (inferior).

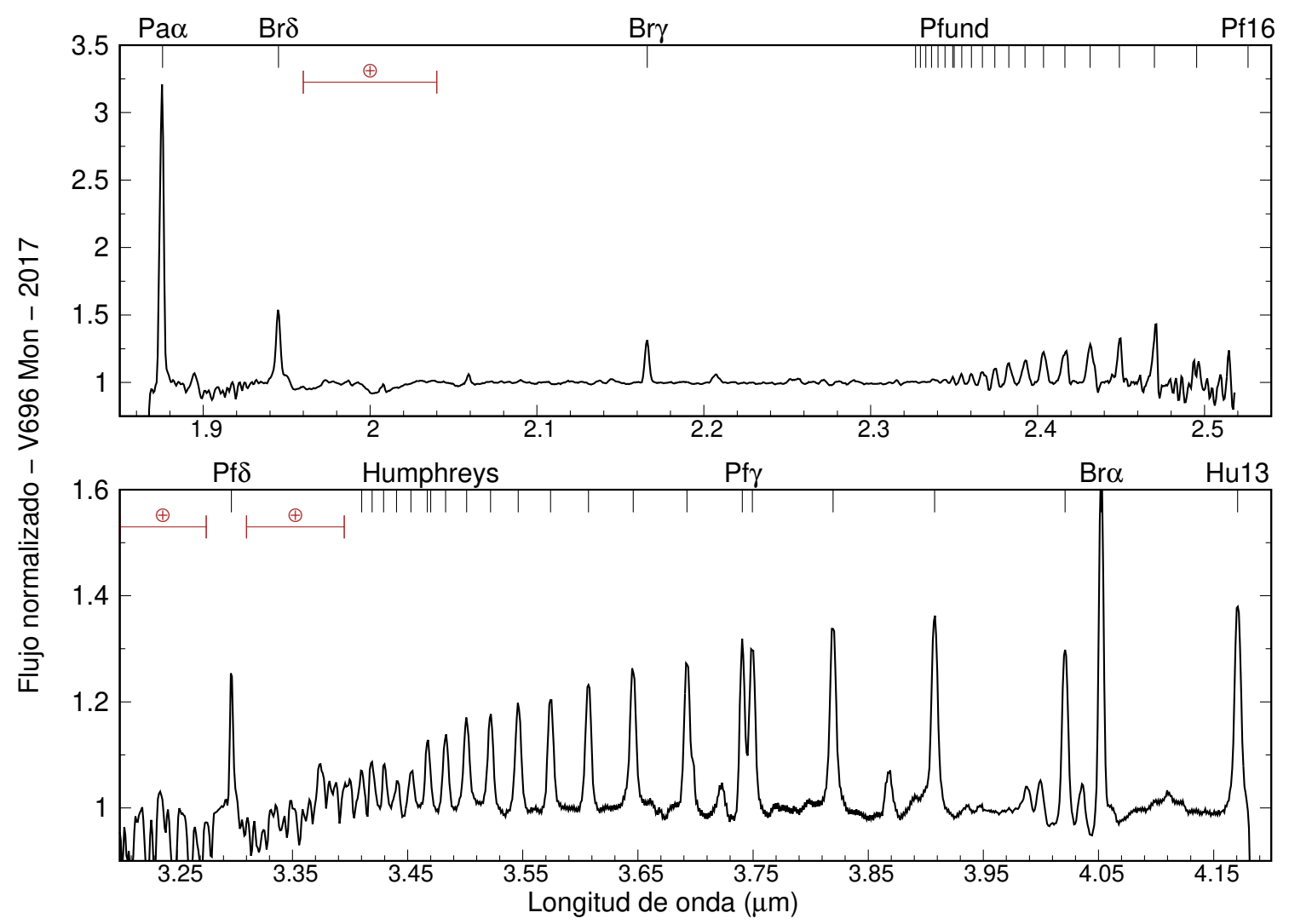

$3.43 \mathrm{R}_{\star}$. Arcos et al. (2017) obtuvieron valores de $\rho_{0}=5 \cdot 10^{-12} \mathrm{~g} \mathrm{~cm}^{-3}, n=2.0$ e $i=80^{\circ}$, a partir de modelos de $\mathrm{H} \alpha$. Por otra parte, a partir del modelado del continuo IR, Vieira et al. (2017) obtuvieron $\log \rho_{0}=-9.8 \pm 0.7$ y $n=3.4 \pm 0.6$ en 1983 , y $\log \rho_{0}=-10.3 \pm 0.3$ y $n=3 \pm 0.1$ en el 2010. Según el criterio de estos autores, esto indica un disco estable en la primera observación, y disipándose en la segunda.

No encontramos en la literatura determinaciones previas del tamaño del disco, ni espectros obtenidos en el IR.

\section{Descripción de los espectros y mediciones realizadas}

Los espectros obtenidos en el año 2017 utilizando el espectrógrafo GNIRS, se muestran en la Figs. 5.31 y 5.32. Todas las líneas de hidrógenos observadas se encuentran en emisión, y según el criterio de Mennickent et al. (2009) V696 Mon es miembro del grupo I. Los valores de los EW, Fl y FWHM de las líneas de hidrógeno que se muestran en la Tabla A.13 fueron corregidos por la absorción fotosférica considerando un espectro sintético correspondiente a una estrella con $T_{\text {eff }}=21000 \mathrm{~K}$ y $\log g=4.0$, ya que a pesar de la ambigüedad en su tipo espectral (ver Tabla 3.3) el espectro presenta líneas de He presentes en tipos espectrales B3 o más tempranos.

En la Fig. 5.67 se muestra la ubicación de V696 Mon en el diagrama de Lenorzer. La misma 
Figura 5.33: V696 Mon - EW/ $\lambda$ y FWHM vs. $\lambda$ de las líneas observadas en las diferentes series. La línea punteada corresponde a un valor de $2 V \sin (i)=752 \mathrm{~km} / \mathrm{s}$, y la región sombreada al error correspondiente (Tabla 3.3).
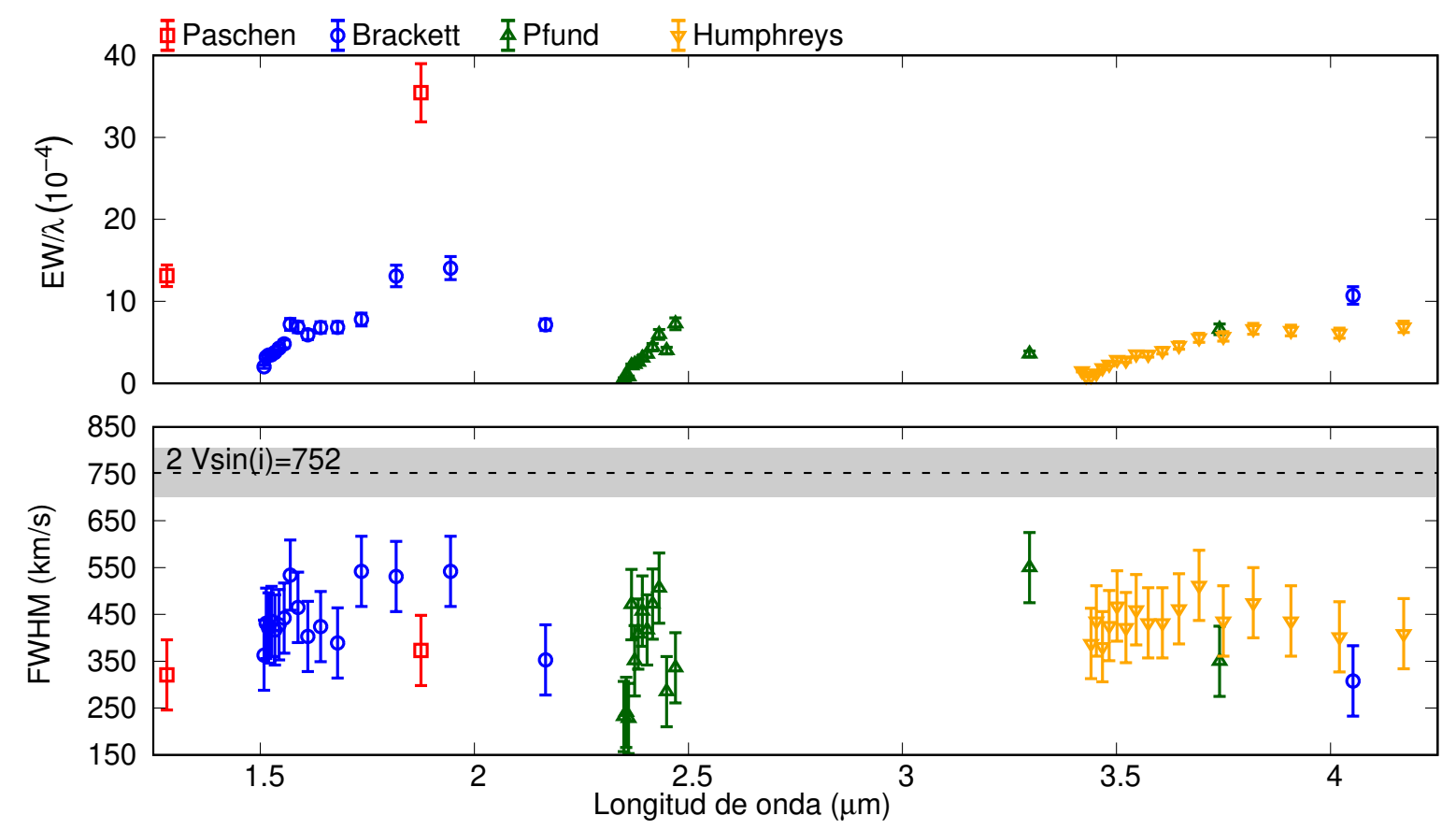

se ubica en la zona cercana al caso ópticamente grueso en la región superior del diagrama, como se espera para un objeto del grupo I.

\section{Comportamiento de las series de hidrógeno}

La Fig. 5.33 muestra los gráficos de EW/ $\lambda$ y FWHM en función de la longitud de onda, donde vemos que el cociente $\mathrm{EW} / \lambda$ se estabilizan en valores $\sim 7 \cdot 10^{-4}$. Los FWHM de las líneas de la series de Humphreys y Brackett muestran valores similares menores a $2 V \sin (i)$, lo que indica regiones de formación alejadas de la estrella central. Para la serie de Pfund se observa que los FWHM decaen a partir de $\mathrm{Pf}_{20}$. Las líneas $\mathrm{Br} \alpha, \operatorname{Br} \gamma, \operatorname{Pa} \alpha$ y $\operatorname{Pa} \beta$ son las que presentan valores de FWHM más bajos, indicando regiones de formación más alejadas de la estrella central.

La comparación de los cocientes de los flujos con los casos ópticamente grueso y delgado se muestra en la Fig. 5.34. Observamos que en las tres series, los miembros más altos (a partir de $\mathrm{Br}_{15}, \mathrm{Pf}_{18} \mathrm{y} \mathrm{Hu}_{18}$ ) se mantienen cerca de la recta correspondiente al caso ópticamente delgado, mientras que los más bajos se apartan. Obtuvimos además que $\mathrm{EW}(\mathrm{Br} \alpha) / \mathrm{EW}(\mathrm{Br} \gamma) \sim 2.8$ (valor dentro del rango esperado para un objeto del grupo I) y $\mathrm{EW}(\mathrm{Pa} \alpha) / \mathrm{EW}(\mathrm{Pa} \beta) \sim 4$. 
Figura 5.34: V696 Mon - Cocientes de flujos para las líneas de la serie de Humphreys, Pfund y Brackett. En la parte superior se identifican la líneas correspondientes. Los símbolos utilizados son los mismos que en la Fig. 5.33.
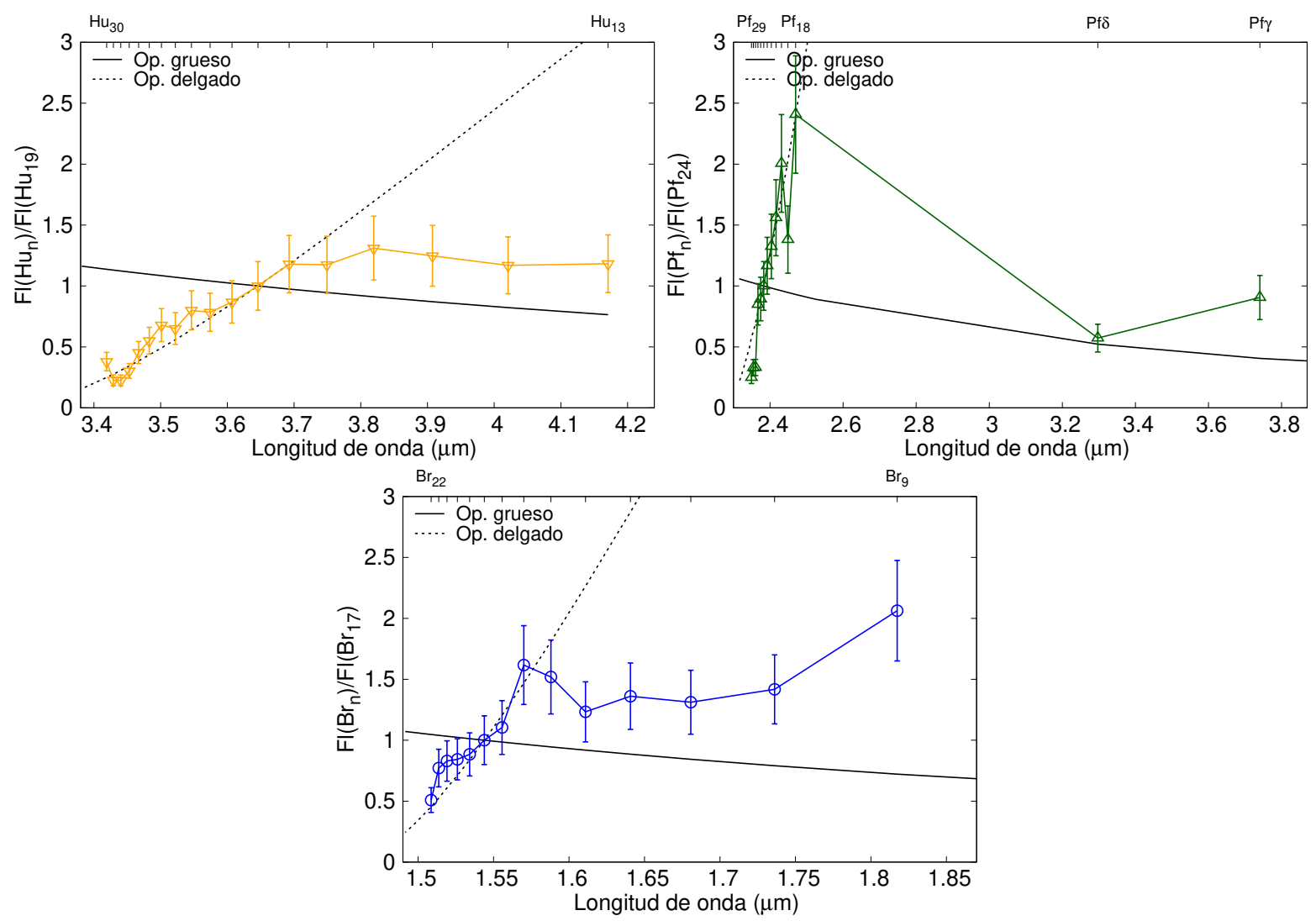

Figura 5.35: V696 Mon - Radios internos de las regiones emisoras de las diferentes líneas relativos a la región emisora de $\mathrm{Br} \delta$. Los símbolos utilizados son los mismos que en la Fig. 5.33.

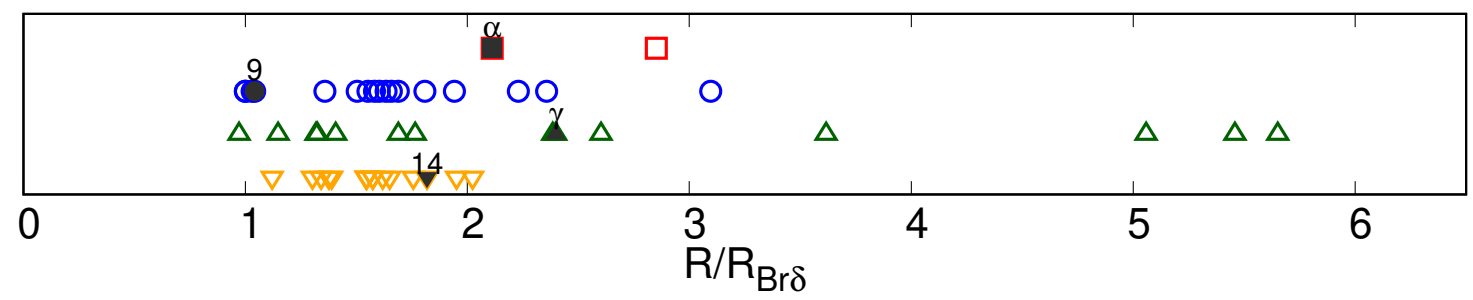

\section{Determinación de densidades columnares y extensiones de las regiones de formación}

Utilizando el los métodos $N$ y FWHM obtuvimos las densidades columnares, extensiones de las regiones de formación y los radios internos de las mismas relativos a un región de referencia. Los valores se muestran en la Tabla A.14.

No observamos una tendencia clara en los valores de las densidades columnares y extensiones 
relativas hacia los miembros más altos de las series.

Para las líneas de la serie de Humphreys los radios obtenidos son similares, excepto para los miembros más altos donde son mayores. En la serie de Pfund los radios alcanzan un valor mínimo en $\mathrm{Pf}_{20}$ y son mayores para los miembros más altos, mientras que para la serie de Brackett el mayor tamaño corresponde a la línea Br $\alpha$. A partir de los FWHM obtenidos para las líneas de todas las series, obtuvimos los radios internos de las regiones de formación relativos al correspondiente a $\operatorname{Br} \delta$. Los mismos se muestran en la Fig. 5.35 y evidencian una envoltura extendida, donde los radios alcanzan $\sim 6 R_{\mathrm{Br} \delta}$. 


\subsubsection{Pup - HD 68980}

MX Pup fue identificada como estrella Be por Merrill et al. (1925). Es una binaria espectroscópica de tipo espectral B1.5 IVe (Slettebak, 1982), con un período de 5.1526 días y una órbita muy excéntrica (Carrier et al., 2002). La curva de luz presenta variabilidad de largo período con una escala de tiempo de 9 años, acompañada de variaciones V/R (Mennickent et al., 1997; Hanuschik et al., 1995).

Silaj et al. (2010) ajustaron la línea de $\mathrm{H} \alpha$ mediante un modelo simple de ley de potencia, obteniendo como parámetros del mejor ajuste un tipo espectral B2 para la estrella central, $i=20^{\circ}$, $\rho_{0}=2 \cdot 10^{-10} \mathrm{~g} \mathrm{~cm}^{-3}$ y $n=3.5$. Arcos et al. (2017) obtuvieron dos conjuntos de parámetros para el disco, $\rho_{0}=5 \cdot 10^{-12} \mathrm{~g} \mathrm{~cm}^{-3}, n=2$ e $i=50^{\circ}$ usando observaciones de $\mathrm{H} \alpha$ tomadas en 2013 , y $\rho_{0}=2.5 \cdot 10^{-11} \mathrm{~g} \mathrm{~cm}^{-3}, n=2.5$ e $i=50^{\circ}$ con datos adquiridos en 2015 .

Por otro lado, a partir del modelado del continuo IR Vieira et al. (2017) obtuvieron valores de $\log \rho_{0}=-11.8 \pm 0.1$ y $n=2.1 \pm 0.1$ en $1983, \log \rho_{0}=-11.5 \pm 0.2$ y $n=2.8 \pm 0.1$ en $2006 / 2007$, y $\log \rho_{0}=-11.5 \pm 0.1$ y $n=2.8 \pm 0.1$ en el 2010 , indicando en todos los casos un disco en disipación.

No encontramos reportes de observaciones espectroscópicas en el IR cercano para este objeto.

Figura 5.36: Espectro de MXPup en la banda J.

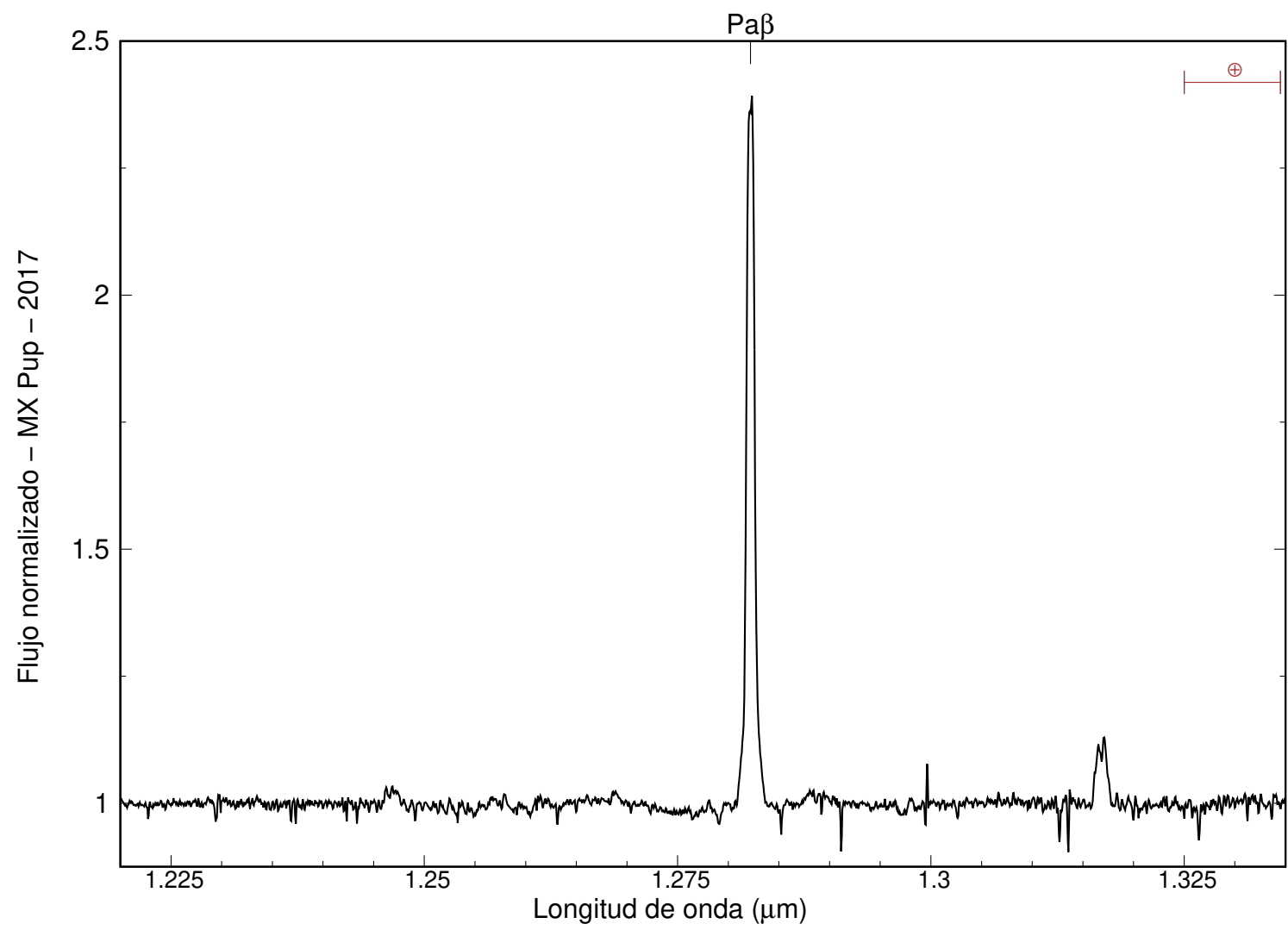


Figura 5.37: Espectros de MX Pup en las bandas H (superior) y K (inferior).

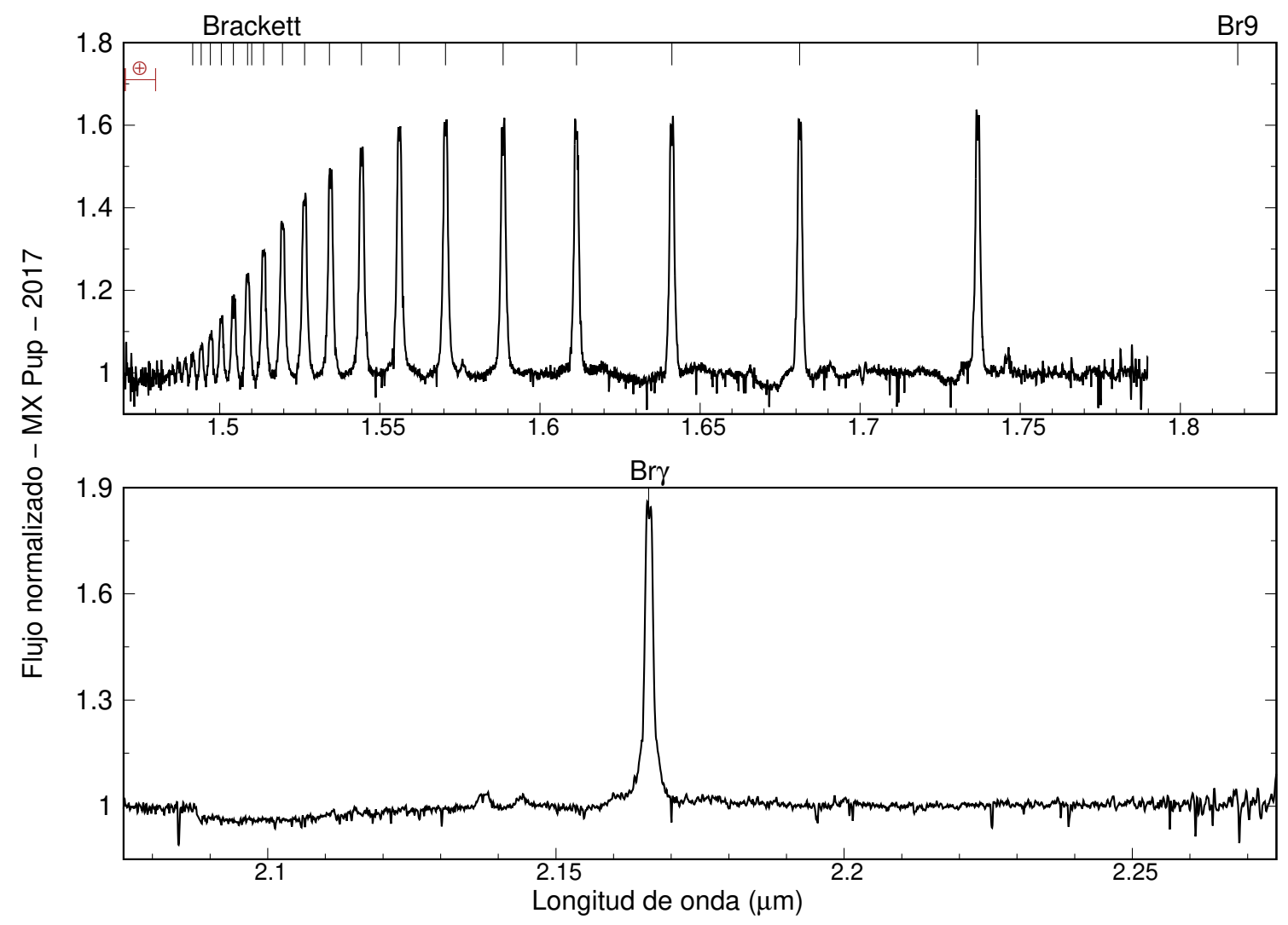

\section{Descripción de los espectros y mediciones realizadas}

En las Figs. 5.36 y 5.37 se muestran los espectros obtenidos en 2017, utilizando el espectrógrafo FIRE. Las líneas de hidrógeno observadas se encuentran en emisión, y en la mayor parte de ellas se distingue un perfil con dos picos. En la Tabla A.15 se listan los valores correspondientes a los EW, Fl y FWHM. Para realizar la corrección por absorción fotosférica se utilizó el espectro sintético correspondiente a una estrella con $T_{\text {eff }}=25000 \mathrm{~K}$ y $\log g=4.0$, según los parámetros estelares de la Tabla 3.3. Como no obtuvimos observaciones de la banda L, no pudimos ubicar a MX Pup en el diagrama de Lenorzer.

\section{Comportamiento de las series de hidrógeno}

En los gráficos de EW/ $\lambda$ y FWHM en función de la longitud de onda vemos que los valores de $\mathrm{EW} / \lambda$ alcanzan un valor aproximadamente estable $\sim 9 \cdot 10^{-4}$ para las líneas de la serie de Brackett. Los FWHM obtenidos para las líneas de la serie de Brackett presentan un máximo en $\mathrm{Br}_{17}$.

En la Fig. 5.39 se muestran los cocientes de los flujos de las líneas de la serie de Brackett, y las comparaciones con los casos ópticamente grueso y delgado. Hasta $\mathrm{Br}_{16}$ las líneas son ópticamente opacas, mientras que los miembros más altos de la serie se mantienen sobre la recta 
Figura 5.38: MX Pup - EW/ $\lambda$ y FWHM vs. $\lambda$ de las líneas observadas en las diferentes series. La línea punteada corresponde a un valor de $2 V \sin (i)=304 \mathrm{~km} / \mathrm{s}$, y la región sombreada al error correspondiente (Tabla 3.3).

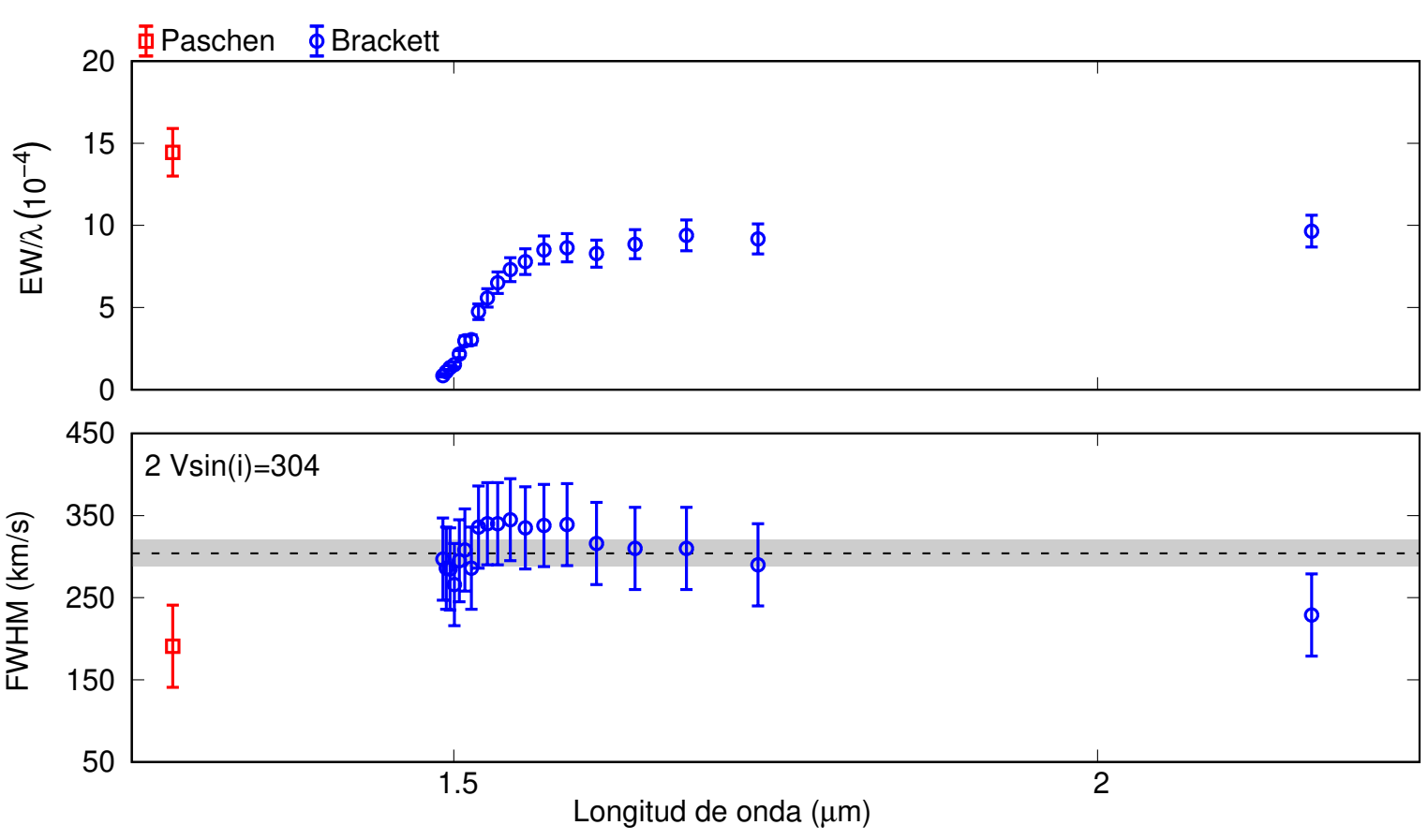

correspondiente al caso ópticamente delgado.

\section{Determinación de densidades columnares y extensiones de las regiones de formación}

Utilizando los métodos $N$, FWHM y $\Delta V$ obtuvimos las densidades columnares y extensiones de las regiones de formación, los radios internos de las regiones de formación relativos al correspondiente a $\mathrm{Br}_{10}$ y en radios estelares. Los valores se muestran en la Tabla A.16.

Las densidades columnares muestran valores crecientes hacia los miembros más altos de la serie de Brackett y luego disminuyen.

Los miembros más altos de la serie se Brackett presentan radios menores. La región de formación de $\mathrm{Pa} \beta$ comienza en una región más externa del disco que la correspondiente a $\mathrm{Br} \gamma$ (ver Fig. 5.40 superior). En radios estelares, los valores obtenidos para las regiones de formación de las líneas de la serie de Brackett se encuentran en el rango 3.5-9.0 $R_{\star}$, mientras que para $\mathrm{Pa} \beta$ obtuvimos un radio de $9.6 R_{\star}$. Las líneas más externas corresponden a los primeros miembros observados de cada serie, y todas las líneas se forman en una región interior a los $10 R_{\star}$. Los valores se muestran en la Fig. 5.40 (inferior), y evidencian la presencia de un disco denso hasta $\operatorname{los} 10 R_{\star}$. 
Figura 5.39: MX Pup - Cocientes de flujos para las líneas de la serie de Brackett. En la parte superior se identifican la líneas correspondientes. Los símbolos utilizados son los mismos que en la Fig. 5.38.

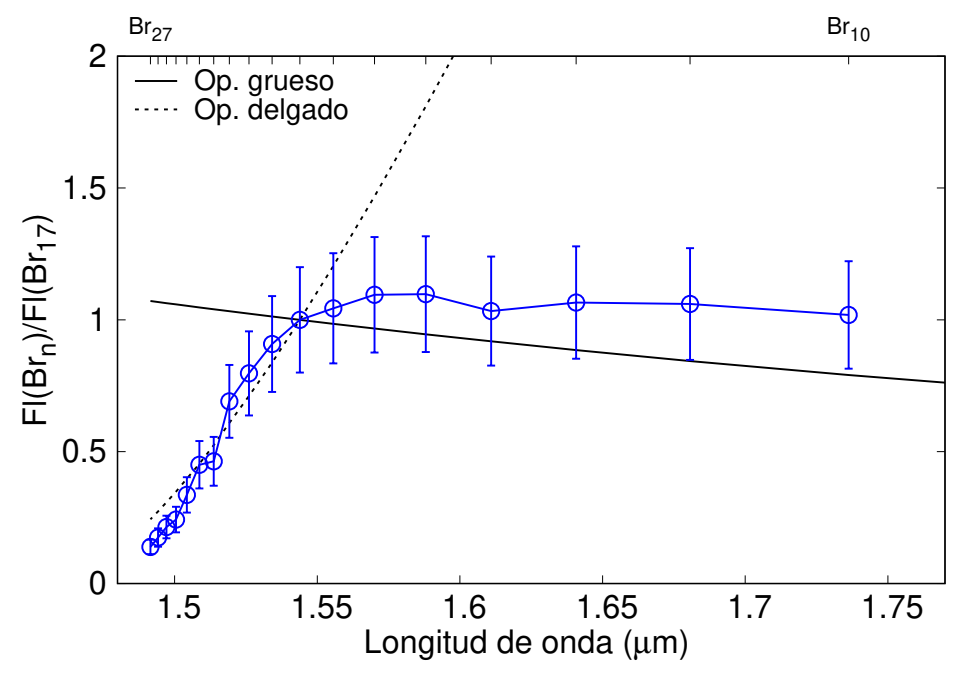

Figura 5.40: MX Pup - Radios internos de las regiones emisoras de las diferentes líneas relativos a la región emisora de $\mathrm{Br} \gamma$ y en radios estelares. Los símbolos utilizados son los mismos que en la Fig. 5.38.
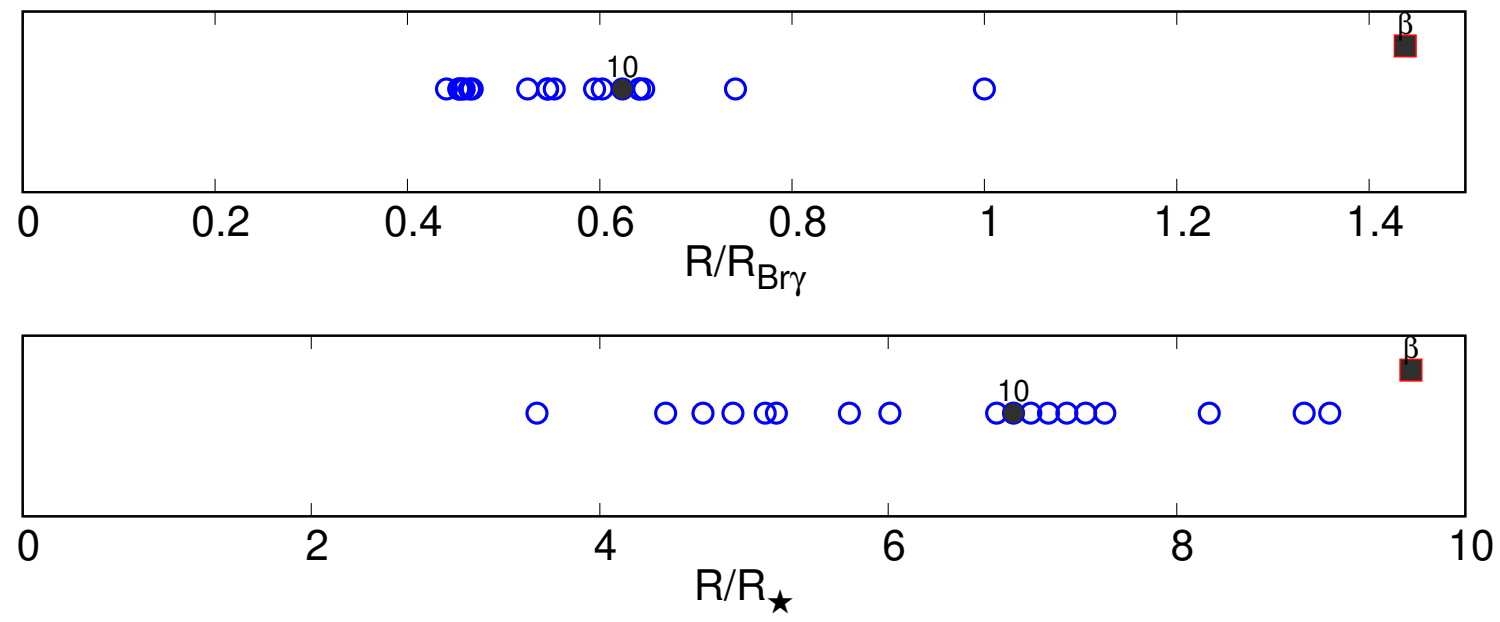


\subsubsection{Lib - HD 142983}

$48 \mathrm{Lib}$ es una estrella de clasificación espectral B3 V, que ha presentado perfiles tipo shell. Inicialmente esta estrella fue mal clasificada como una supergigante con un perfil $\mathrm{P}$ Cygni, debido a la gran asimetría observada en el perfil de $\mathrm{H} \alpha$. Según lo reportado por Mennickent \& Vogt (1988), presenta variaciones en el cociente V/R, con un período de $\sim 10$ años en las líneas en emisión de la serie de Balmer. Sin embargo, Štefl et al. (2012) reportaron un período de 16 años para las variaciones $\mathrm{V} / \mathrm{R}$ junto con variaciones en la intensidad del máximo de $\mathrm{V} / \mathrm{R}$ en cada ciclo (Štefl et al., 2012).

Slettebak (1951) reportaron la presencia de las líneas $\mathrm{H} \alpha$ y $\mathrm{H} \beta$ en emisión, mientras que Persson \& McGregor (1985) reportaron la presencia de Br $\alpha$ en emisión. El perfil de la línea $\mathrm{H} \alpha$ es variable, según reportaron Jones et al. (2011).

Silaj et al. (2016) implementaron un modelo que incluía una estrella central deformada por rotación, con oscurecimiento al limbo, rodeada de un disco según el modelo VDD, para intentar reproducir las características observadas en esta estrella. Consideraron también un modelo bidimensional para incluir perturbaciones al disco. Obtuvieron un tipo espectral y clase de luminosidad B3 V para la estrella central y una inclinación $i=85^{\circ}$ para el disco. Encontraron también una perturbación en el disco con un período de 12 años, que podría explicar las variaciones

Figura 5.41: Espectros de 48 Lib en las bandas J (superior) y H (inferior).

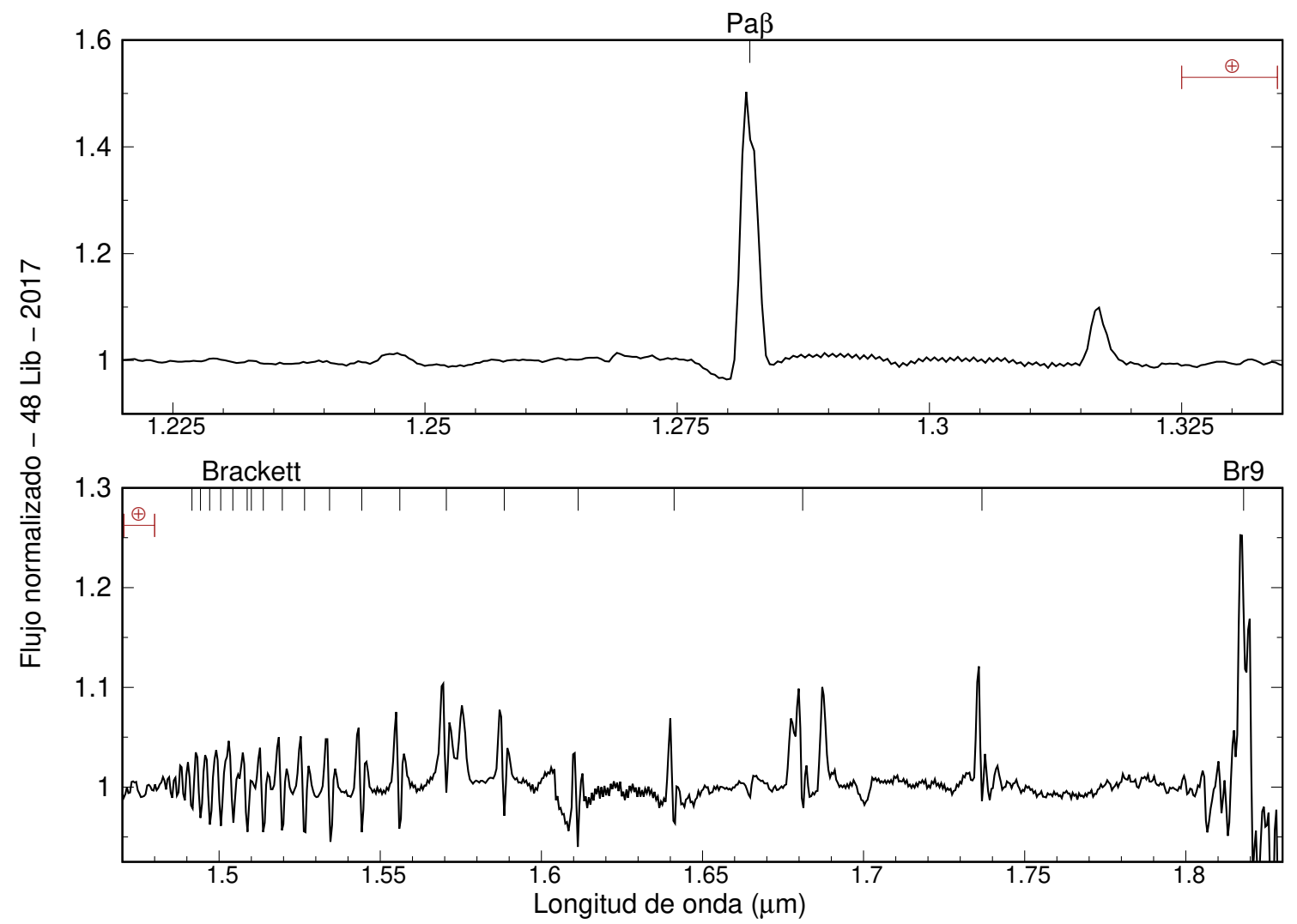


Figura 5.42: Espectro de $48 \mathrm{Lib}$ en la banda K.

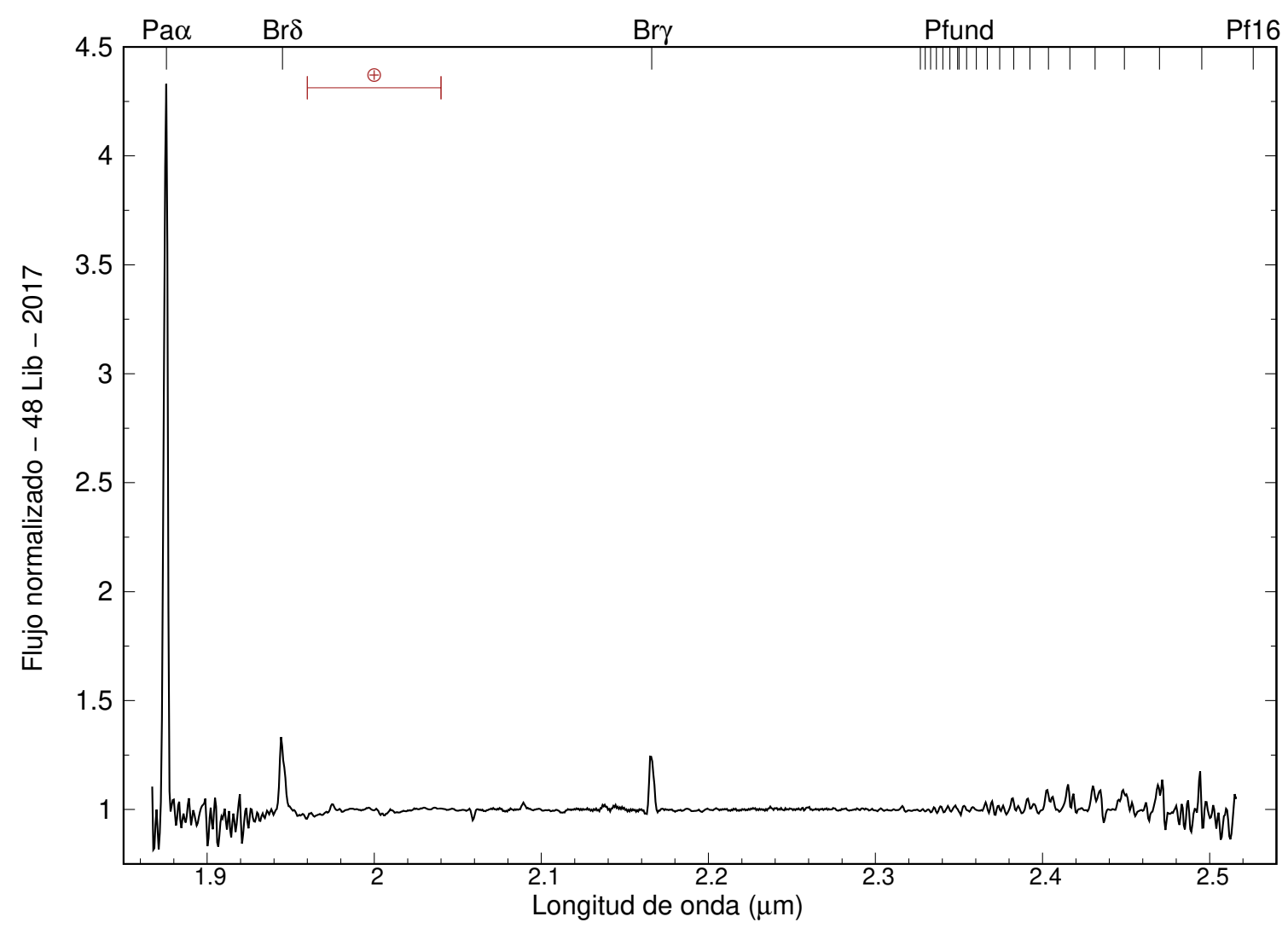

V/R observadas. Los anchos equivalentes de la línea $\mathrm{H} \alpha$ obtenidos a partir del modelo también se encuentran en buen acuerdo con las observaciones, mientras que las formas de los perfiles no logran reproducirse completamente.

No encontramos reportes de observaciones espectroscópicas obtenidas en el IR cercano.

\section{Descripción de los espectros y mediciones realizadas}

En las Figs. 5.41 y 5.42 se muestran los espectros obtenidos en el año 2017 utilizando el espectrógrafo GNIRS, y en la Tabla A.17 los valores de EW, Fl y FWHM teniendo en cuenta la corrección por absorción fotosférica mediante un espectro sintético correspondiente a una estrella con $T_{\text {eff }}=18000 \mathrm{~K}$ y $\log g=4.0$ (ver Tabla 3.3). Las líneas de hidrógeno observadas se encuentran en emisión, con dos picos en el caso de los miembros más altos de las series de Brackett y Pfund. En los miembros más altos de la serie de Brackett, la absorción central alcanza a superar el nivel del continuo. Como no tenemos observaciones en la banda L, no podemos ubicarla en el diagrama de Lenorzer ni clasificar a la estrella según el criterio dado por Mennickent et al. (2009) 
Figura 5.43: $48 \mathrm{Lib}-\mathrm{EW} / \lambda$ y FWHM vs. $\lambda$ de las líneas observadas en las diferentes series. La línea punteada corresponde a un valor de $2 V \sin (i)=814 \mathrm{~km} / \mathrm{s}$, y la región sombreada al error correspondiente (Tabla 3.3).

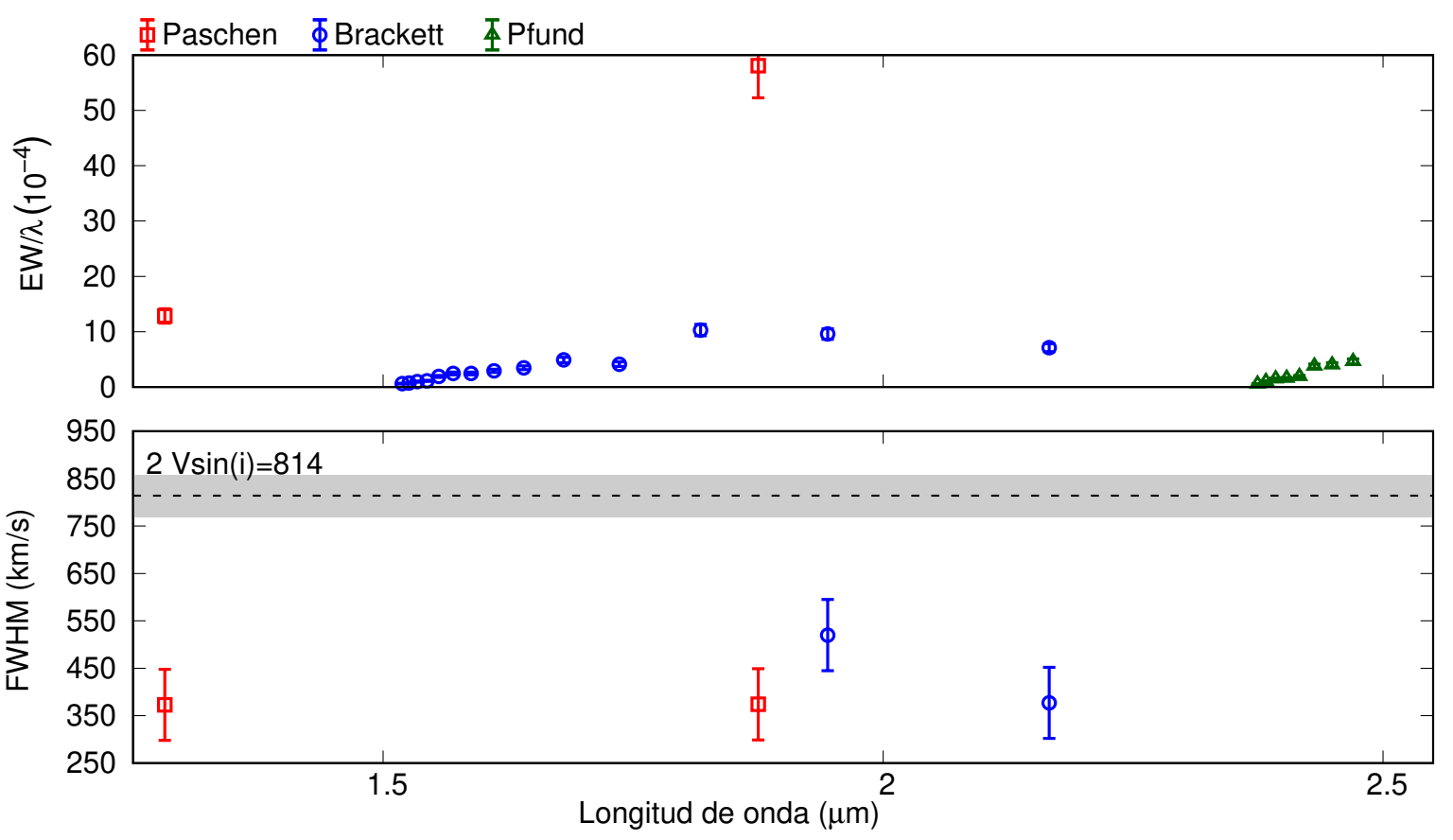

\section{Comportamiento de las series de hidrógeno}

La Fig. 5.43 muestra los gráficos de EW/ $\lambda$ y FWHM en función de la longitud de onda. Debido a las pocas líneas observadas y a la dificultad de obtener valores de FWHM en las líneas tipo shell, no pudimos concluir sobre la estabilidad de los valores de EW/ $\lambda$ en las distintas series o sobre el comportamiento de los FWHM. Podemos destacar que los ensanchamientos obtenidos son menores a lo esperado para líneas formadas en una región cercana a la fotosfera, indicando regiones de formación alejadas de la estrella central.

Los cocientes de los flujos de las líneas de cada serie se muestran en la Fig. 5.44. Para la serie de Pfund, todas las líneas observadas se mantienen en la recta esperada para un caso ópticamente delgado, mientras que los miembros más bajos de la serie de Brackett (hasta $\mathrm{Br}_{14}$ ) se apartan del caso ópticamente delgado, pero no significativamente. Por otro lado, obtuvimos que $\operatorname{EW}(\operatorname{Pa} \alpha) / \mathrm{EW}(\mathrm{Pa} \beta) \sim 6.5$.

\section{Determinación de densidades columnares y extensiones de las regiones de formación}

Debido a que no pudimos determinar los FWHM de las líneas que se apartan del caso ópticamente delgado, no pudimos obtener densidades columnares y extensiones de las regiones de formación mediante el método $N$. Para las líneas en las que pudimos determinar los FWHM se muestran 
Figura 5.44: $48 \mathrm{Lib}$ - Cocientes de flujos para las líneas de la serie de Pfund y Brackett. En la parte superior se identifican la líneas correspondientes. Los símbolos utilizados son los mismos que en la Fig. 5.43.
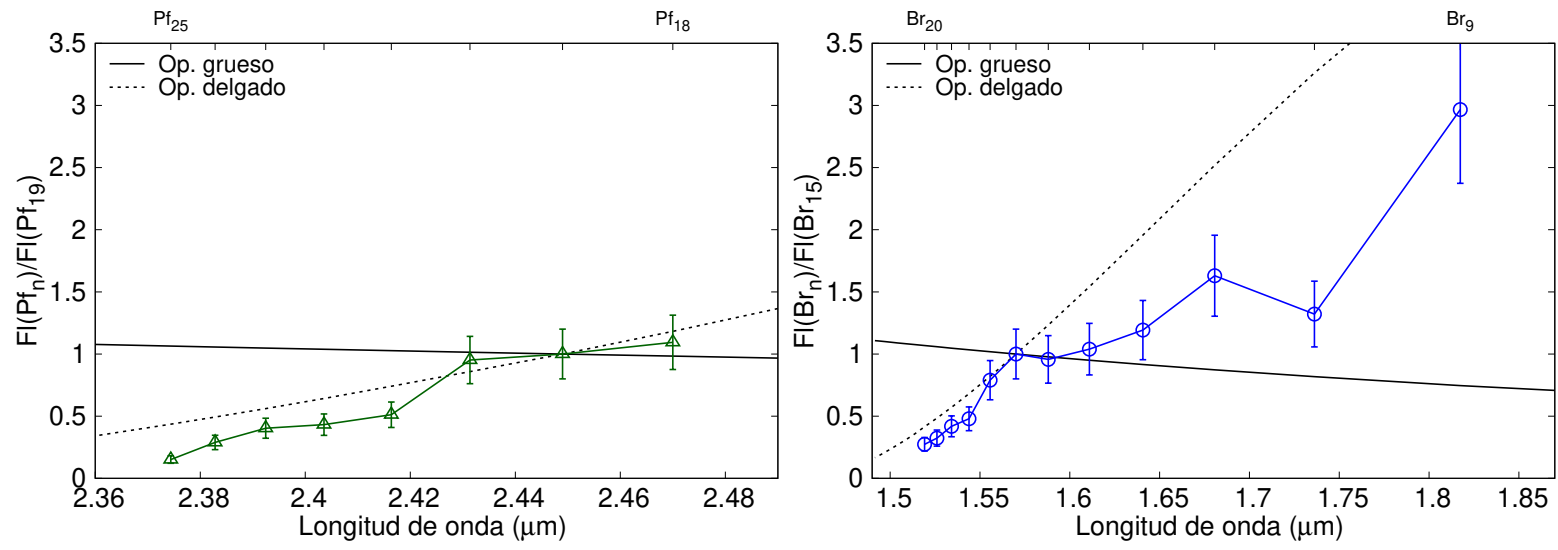

Figura 5.45: $48 \mathrm{Lib}$ - Radios internos de las regiones emisoras de las diferentes líneas relativos a la región emisora de $\operatorname{Br} \delta$ y en radios estelares. Los símbolos utilizados son los mismos que en la Fig. 5.43.
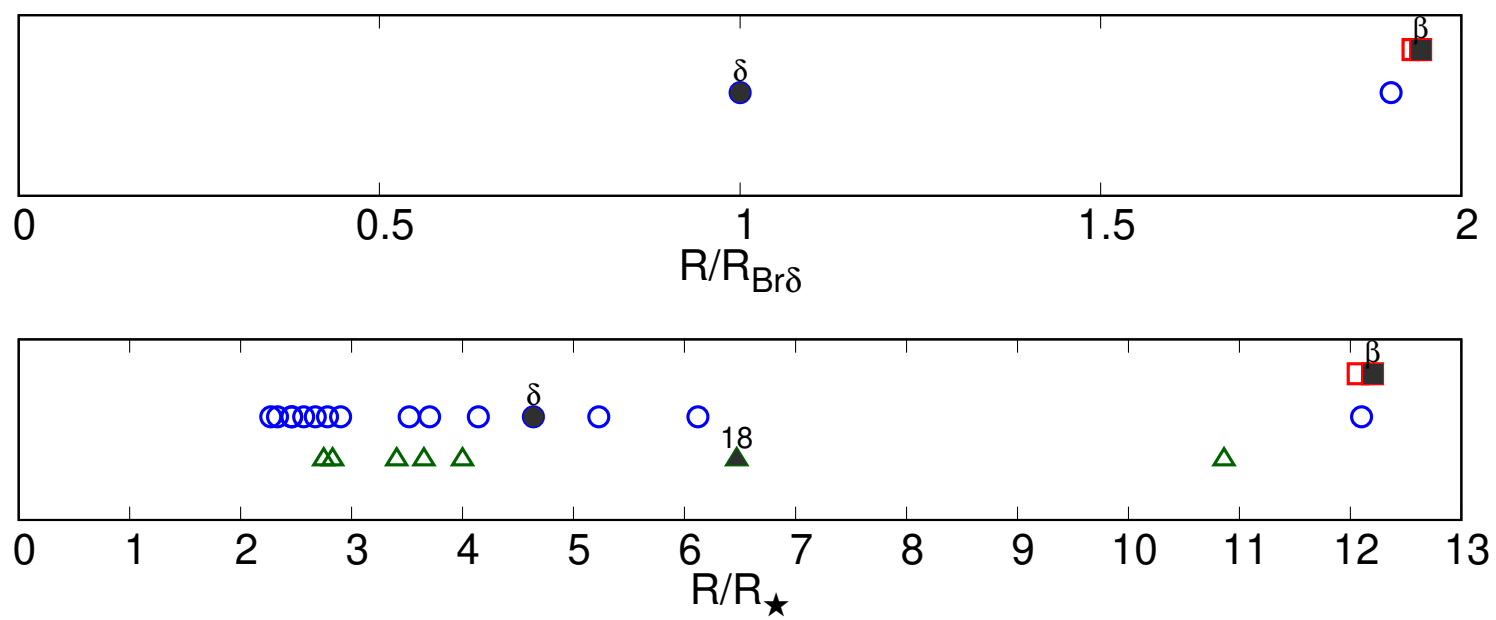

los tamaños relativos en la Tabla A.18, calculados mediante el método FWHM. La Tabla muestra también los valores en radios estelares obtenidos mediante el método $\Delta V$. Para Pa $\alpha$ no pudimos obtener $\Delta V$, pero calculamos un valor aproximado para el radio interno de la región de formación en radios estelares a partir del tamaño relativo a $\mathrm{Pa} \beta$ obtenido con el método FWHM.

Para la serie de Pfund, los tamaños obtenidos se encuentran entre $2.75 R_{\star}$ y $9.65 R_{\star}$ mientras que para las líneas $\mathrm{Br} \gamma$ y $\mathrm{Pa} \beta$ los radios alcanzan los $12 R_{\star}$ (ver Fig. 5.45). 


\subsubsection{V1075 Sco - HD 155806}

V1075 Sco es una estrella con clasificación espectral O7.5 V, identificada como Be por Merrill et al. (1925). Hanson et al. (1996) reportaron la presencia de $\operatorname{Br} \gamma$ y He I $\lambda 20581 \AA$ a partir de espectros obtenidos en la banda $\mathrm{K}$ en el intervalo 2.04-2.2 $\mu \mathrm{m}$.

A partir de un estudio espectropolarimétrico en la línea $\mathrm{H} \alpha$ mediante observaciones obtenidas en agosto del 2003, Vink et al. (2009) no detectaron una polarización significativa en V1075 Sco. Por otra parte, Silaj et al. (2010) ajustaron la línea de $\mathrm{H} \alpha$ con un doble pico en emisión, y obtuvieron como parámetros del mejor ajuste un tipo espectral B0 para la estrella central, $i=20^{\circ}$, $\rho_{0}=1^{-11} \mathrm{~g} \mathrm{~cm}^{-3}$ y $n=3.5$. Jones et al. (2011) reportaron variabilidad en el ancho equivalente de la línea $\mathrm{H} \alpha$.

\section{Descripción de los espectros y mediciones realizadas}

Los espectros obtenidos en 2017 en las bandas J, H y K utilizando el espectrógrafo FIRE se muestran en la Fig. 5.46. Las líneas de hidrógeno se observan en emisión, en algunos casos superpuestas al perfil de absorción fotosférica. Los valores de EW, Fl y FWHM obtenidos se muestran en la Tabla A.19, y para realizar la corrección por absorción fotosférica se utilizó el

Figura 5.46: Espectros de V1075 Sco en as bandas J (superior), H (centro) y K (inferior).

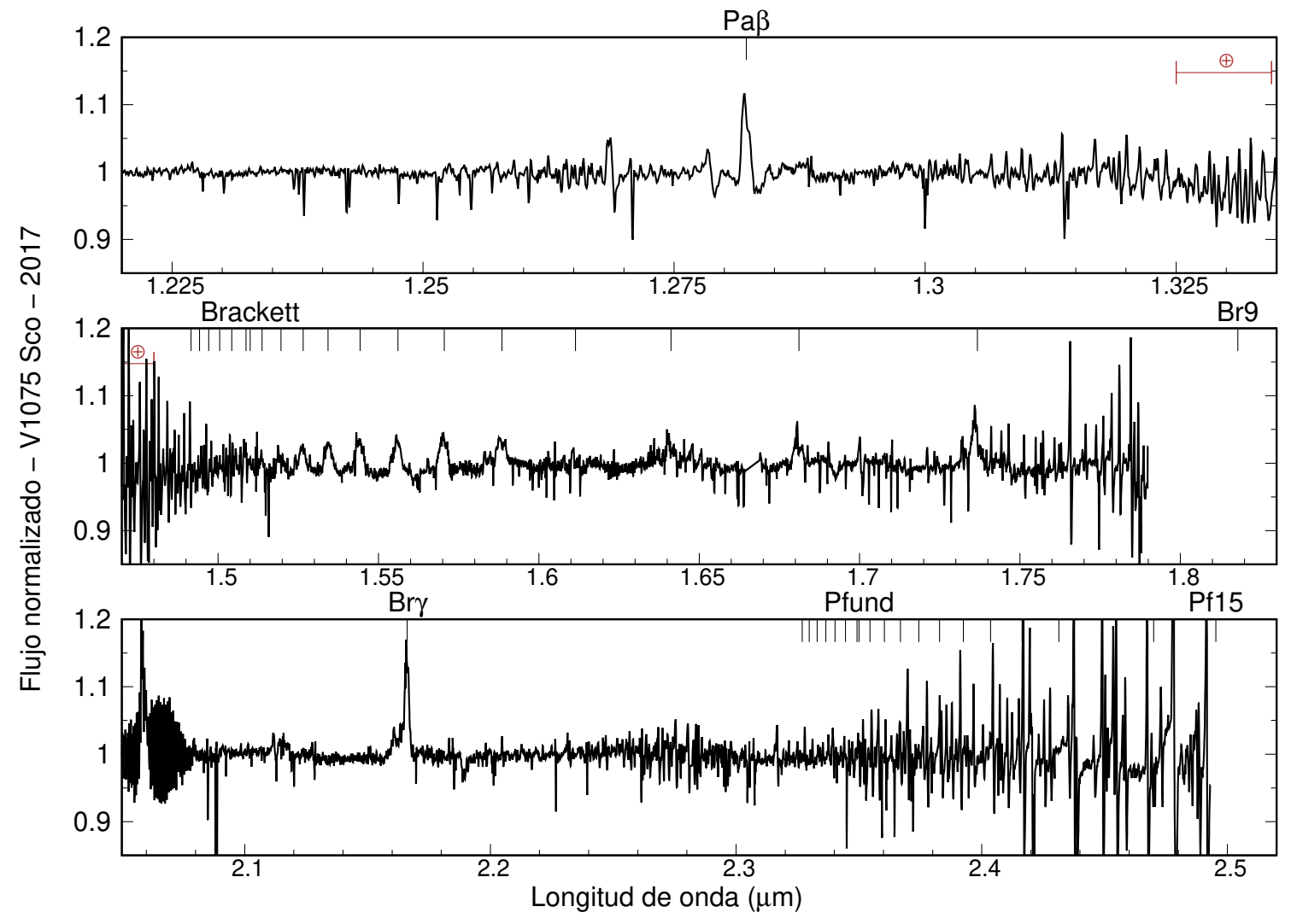


Figura 5.47: V1075 Sco - EW/ $\lambda$ y FWHM vs. $\lambda$ de las líneas observadas en las diferentes series.
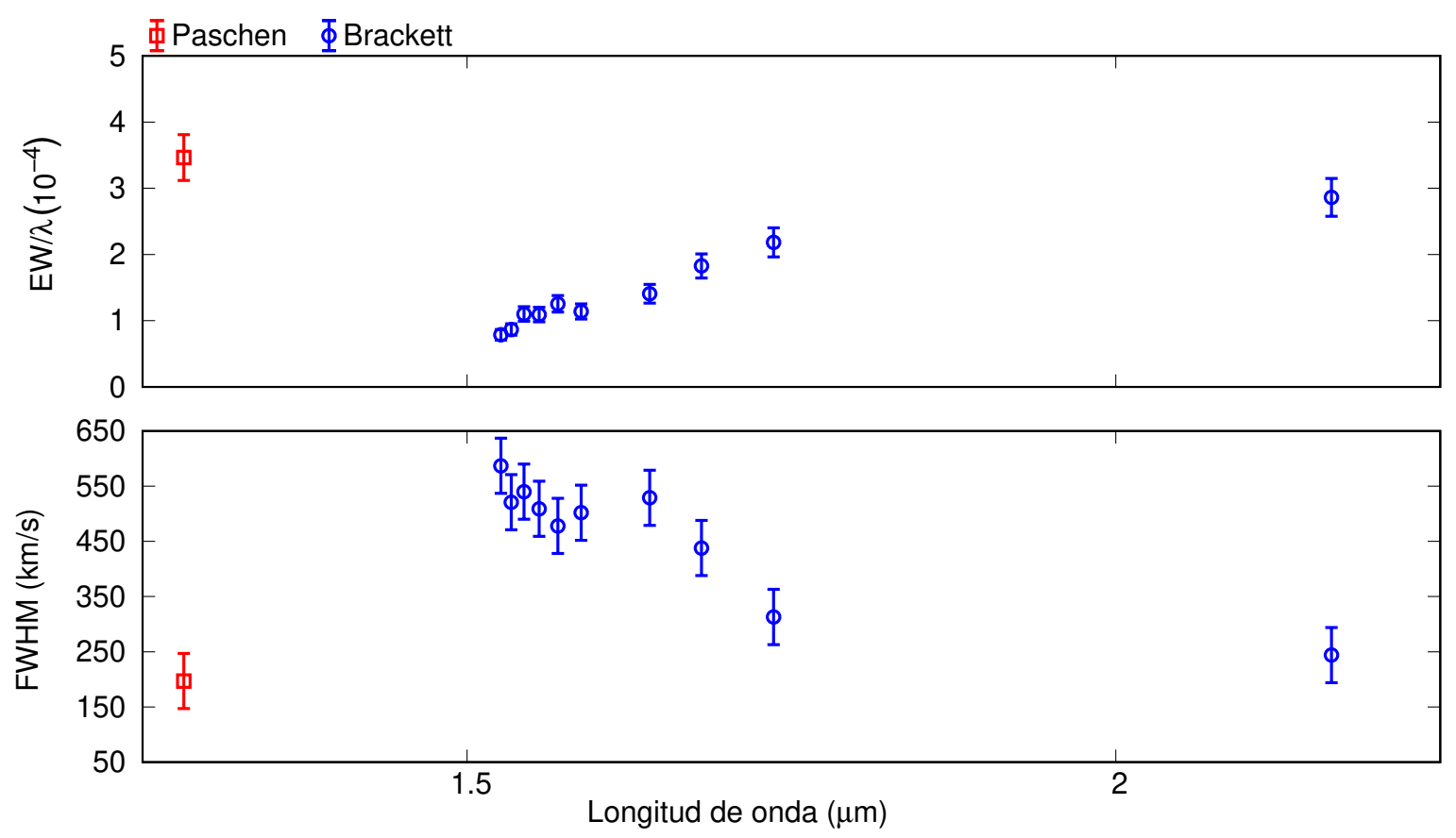

espectro sintético correspondiente a una estrella con $T_{\text {eff }}=34000 \mathrm{~K}$ y $\log g=4.0$ (ver Tabla 3.3). Como no obtuvimos observaciones de la banda L, no pudimos ubicar a V1075 Sco en el diagrama de Lenorzer.

\section{Comportamiento de las series de hidrógeno}

Según se observa en la Fig. 5.47, los valores de EW/ $\lambda$ vs. $\lambda$ no presenta el comportamiento asintótico esperado. Los FWHM de la serie de Brackett aumentan hacia los miembros más altos de la serie, comportamiento esperado si los miembros más altos se forman en regiones más internas de la envoltura.

Los cocientes de los flujos de las líneas de la serie de Brackett y su comparación con los casos ópticamente grueso y delgado se muestran en la Fig. 5.48. Los miembros más bajos de la serie (hasta $\mathrm{Br}_{16}$ ) se separan del caso ópticamente delgado.

\section{Determinación de densidades columnares y extensiones de las regiones de formación}

Obtuvimos densidades columnares, extensiones de las regiones de formación y radios internos de las mismas mediante los métodos $N$ y FWHM. Los valores se muestran en la Tabla A.20.

En la serie de Brackett, los miembros más altos tienden a presentar densidades columnares y extensiones mayores que los más bajos, además de radios internos menores. Si bien $\mathrm{Br} \gamma$ presenta 
Figura 5.48: V1075 Sco - Cocientes de flujos para las líneas de la serie de Brackett. En la parte superior se identifican la líneas correspondientes. Los símbolos utilizados son los mismos que en la Fig. 5.47.

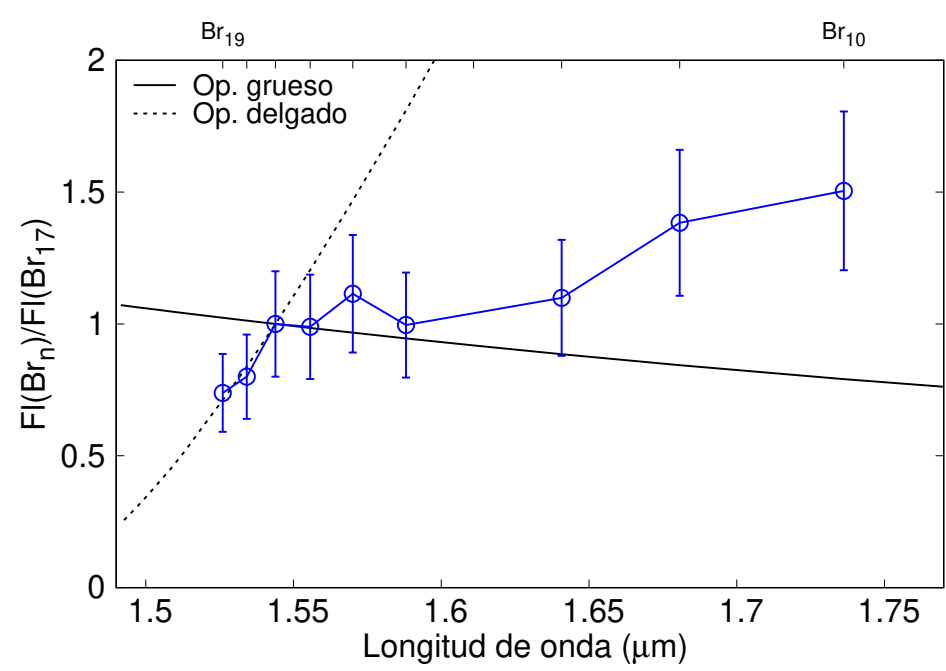

Figura 5.49: V1075 Sco - Radios internos de las regiones emisoras de las diferentes líneas relativos a la región emisora de Br $\gamma$. Los símbolos utilizados son los mismos que en la Fig. 5.47.

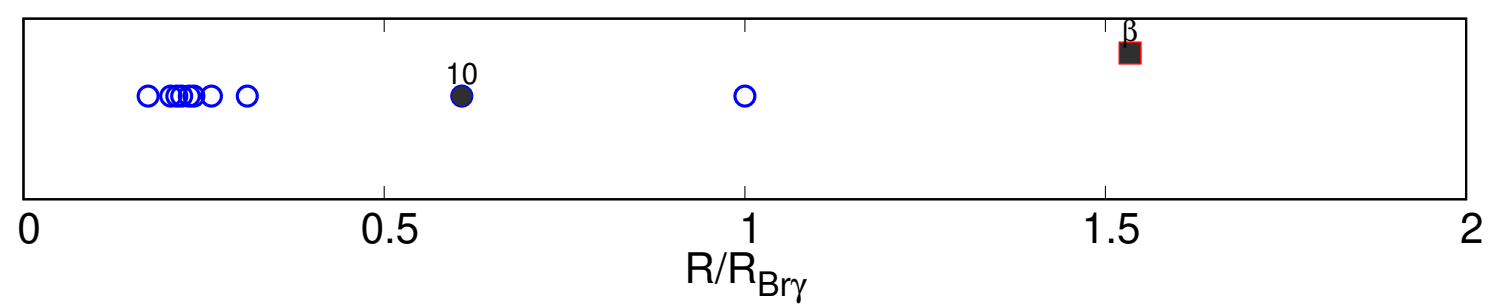

dos picos en emisión separados, no pudimos determinar el radio interno de la región de formación ya que no contamos con medidas de $V \sin (i)$.

En la Fig. 5.49 se muestran los radios internos de las regiones de formación relativos al correspondiente a $\mathrm{Br} \gamma$. Las líneas formadas en una región más externa son $\mathrm{Br} \gamma$ y $\mathrm{Pa} \beta$, mientras que los miembros altos de la serie de Brackett se forman en una región más interna. 


\subsubsection{Her - HD 162732}

88 Her es una estrella de clasificación espectral B6 III, que ha mostrado transiciones entre fases Be shell $\leftrightarrow$ B normal. Harmanec et al. (1972) reportaron variaciones periódicas en la velocidad radial con un período de 86.7 días, las cuales fueron atribuidas a la presencia de un sistema binario. Las variaciones reportadas en el cociente V/R siguen el período de la binaria, y se superponen a la variabilidad de largo período observada en la intensidad de las líneas en emisión (Doazan et al., 1982).

Utilizando observaciones tomadas en 2009, Bhat et al. (2016) determinaron un tamaño para la región emisora de la línea $\mathrm{H} \alpha$ de $3.4-4.2 R_{\star}$ a partir de la separación de los picos y atribuyeron a la órbita de la binaria las variaciones $\mathrm{V} / \mathrm{R}$.

Granada et al. (2010) analizaron espectros en las bandas K y L obtenidos en el 2006 y reportaron que la estrella pertenecía al grupo I y se ubicaba en la región superior del diagrama de Lenorzer.

Figura 5.50: Espectros de 88 Her en las bandas K (superior) y L (inferior).

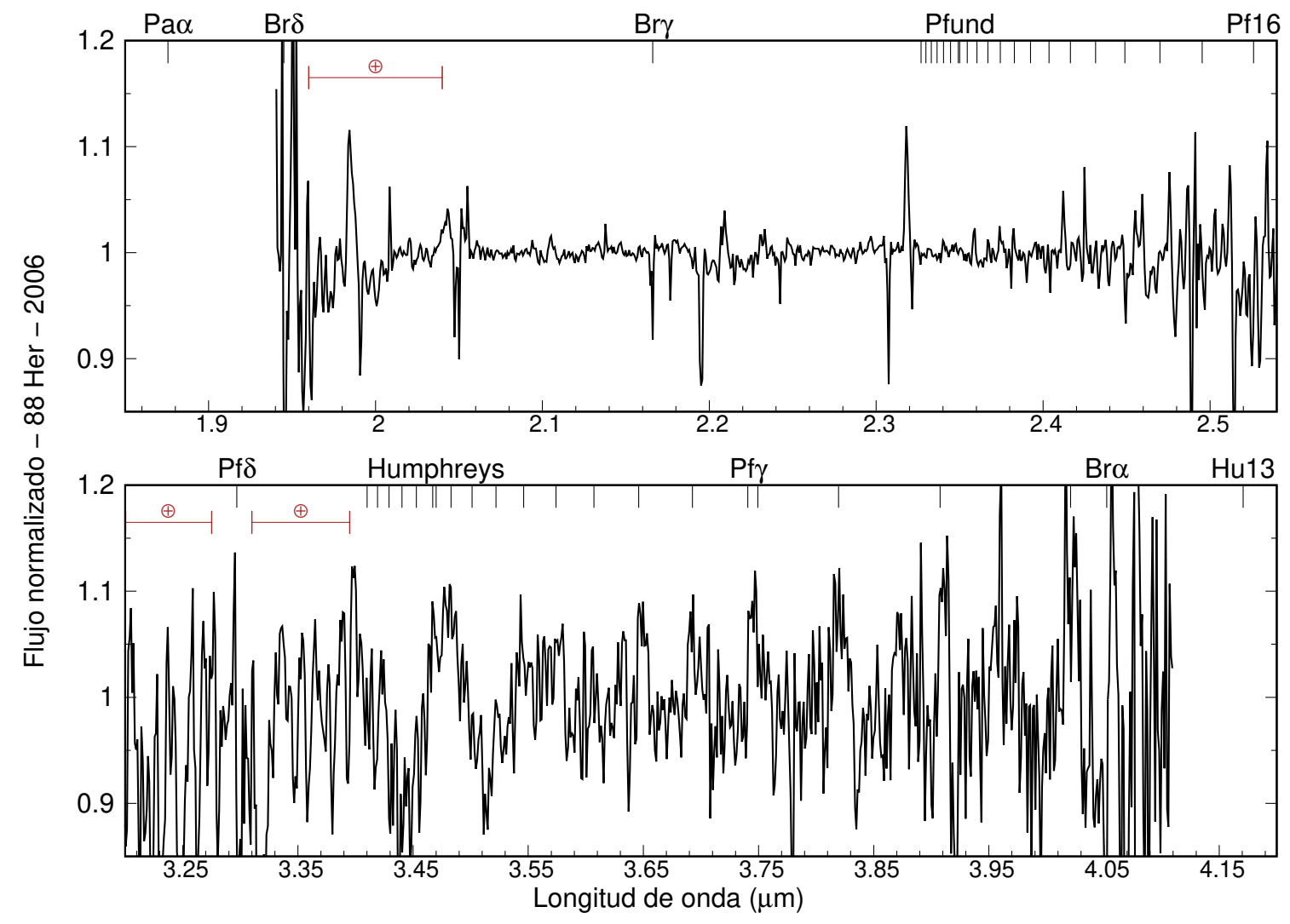




\section{Descripción de los espectros y mediciones realizadas}

En la Fig. 5.50 se muestran los espectros obtenidos en el año 2006 utilizando el espectrógrafo NIRI en las bandas K y L. En la banda K se observa que la línea Bry rellena la absorción fotosférica y $\operatorname{Br} \delta$ se encuentra en emisión. En la banda L, las líneas de hidrógeno se observan en emisión. Según la intensidad de los miembros de la serie de Humphreys, clasificamos a 88 Her como perteneciente al grupo I, según el criterio de clasificación de Mennickent et al. (2009). Los valores de los EW, Fl y FWHM se muestran en la Tabla A.21. Para realizar la corrección por absorción fotosférica se utilizó el espectro sintético correspondiente a una estrella con $T_{\text {eff }}=14000 \mathrm{~K} \mathrm{y}$ $\log g=3.5$, según los parámetros presentados en la Tabla 3.3.

La ubicación de 88 Her en el diagrama de Lenorzer se muestra en la Fig. 5.67. La misma se ubica en la región superior derecha, cercana al caso ópticamente grueso, como los objetos del grupo I.

\section{Comportamiento de las series de hidrógeno}

En la Fig. 5.51 se muestran los gráficos de EW/ $\lambda$ y FWHM en función de la longitud de onda. Los valores de EW/ $\lambda$ no muestra el comportamiento asintótico esperado debido a los pocos miembros observados de cada serie. Los anchos en los FWHM medidos están próximos a $2 \mathrm{~V} \sin (i)$, esperado para una región de formación cercana a la fotosfera. Por otro lado, debido que no observamos miembros altos de las series, no podemos comparar los cocientes de flujos con los casos ópticamente grueso y delgado.

\section{Determinación de densidades columnares y extensiones de las regiones de formación}

A partir de los FWHM obtuvimos los tamaños relativos de las regiones de formación respecto a Pf $\gamma$. Los mismos se muestran en la Fig. 5.52 y la Tabla A.22. Las regiones de formación de las líneas de la serie de Humphreys observadas se ubican entre las correspondientes a $\operatorname{Pf} \gamma$ y $\operatorname{Br} \alpha$. 
Figura 5.51: 88 Her - EW/ $\lambda$ y FWHM vs. $\lambda$ de las líneas observadas en las diferentes series. La línea punteada corresponde a un valor de $2 V \sin (i)=642 \mathrm{~km} / \mathrm{s}$, y la región sombreada al error correspondiente (Tabla 3.3).

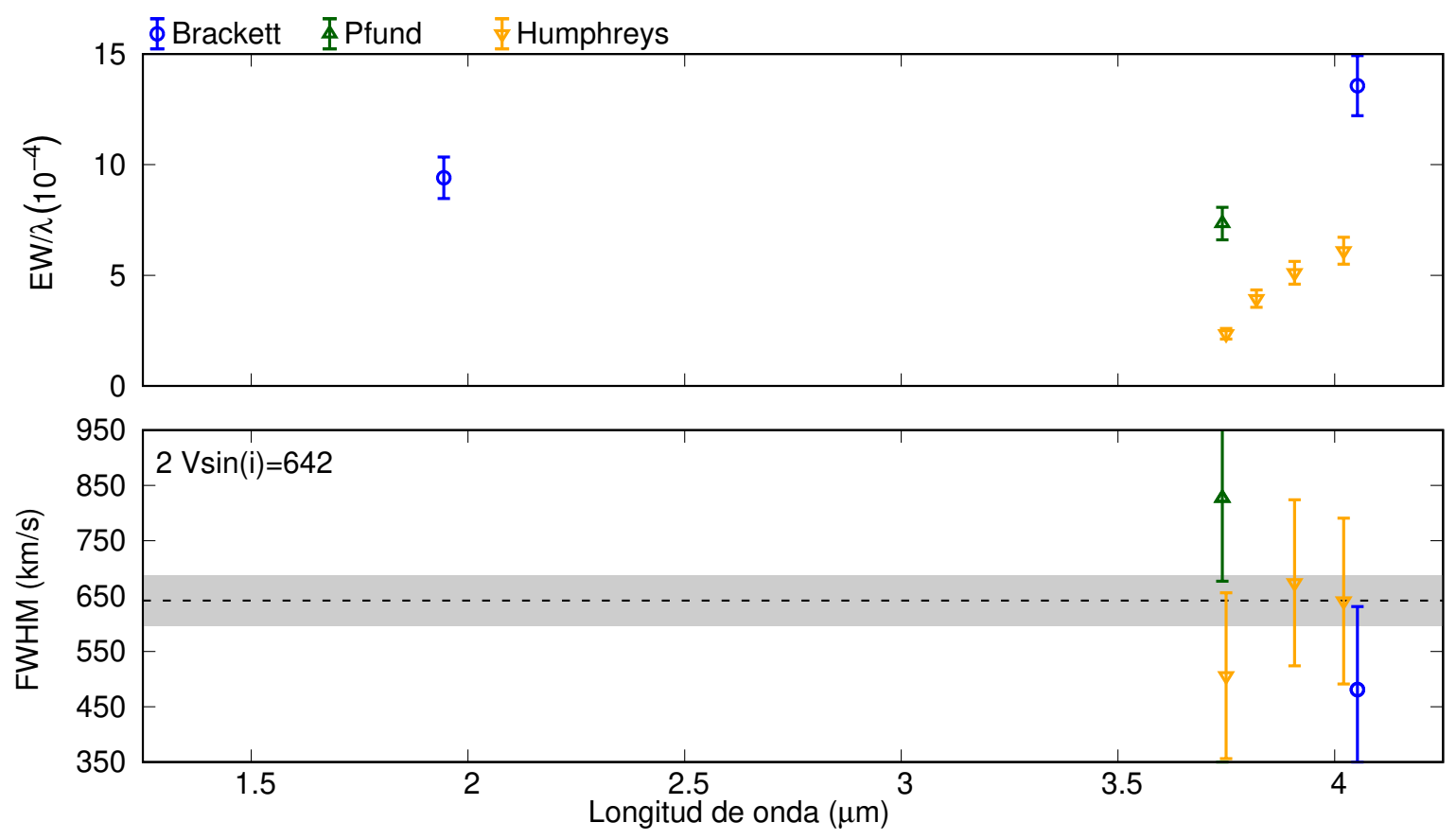

Figura 5.52: 88 Her - Radios internos de las regiones emisoras de las diferentes líneas relativos a la región emisora de Pf $\gamma$. Los símbolos utilizados son los mismos que en la Fig. 5.51.

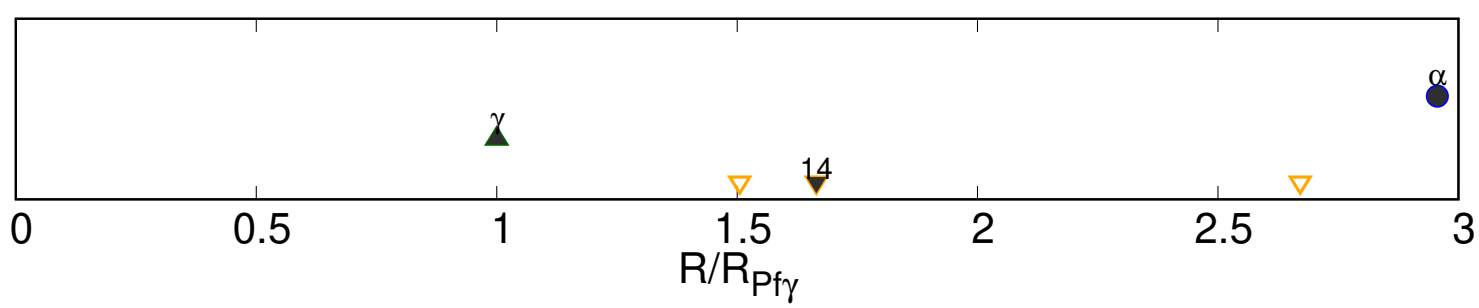




\subsubsection{HD 171623 - HR 6977}

HD 171623 es una estrella de tipo espectral A0 V, inicialmente catalogada como Bn (Cowley et al., 1969). En mayo del 2017 detectamos una componente en emisión en $\mathrm{H} \alpha$, por lo que la catalogamos como una estrella Be (Cochetti et al., 2020).

No hay reportes de observaciones espectroscópicas obtenidas en el IR cercano.

\section{Descripción de los espectros y mediciones realizadas}

En la Fig. 5.53 se muestran las observaciones realizadas en junio de 2017 con el espectrógrafo FIRE. Las líneas $\mathrm{Pa} \beta$ y $\mathrm{Br} \gamma$ presentan una estructura tipo shell superpuesta a la absorción fotosférica, mientras que los miembros más altos de la serie de Brackett se encuentran en absorción. A partir de las componentes shell observadas, concluimos que HD 171623 presentaba un disco muy difuso visto de canto. No obtuvimos valores para los EW, Fl y FWHM de las componentes en emisión de las líneas.

Figura 5.53: Espectros de HD 171623 en las bandas J (superior), H (centro) y K (inferior).

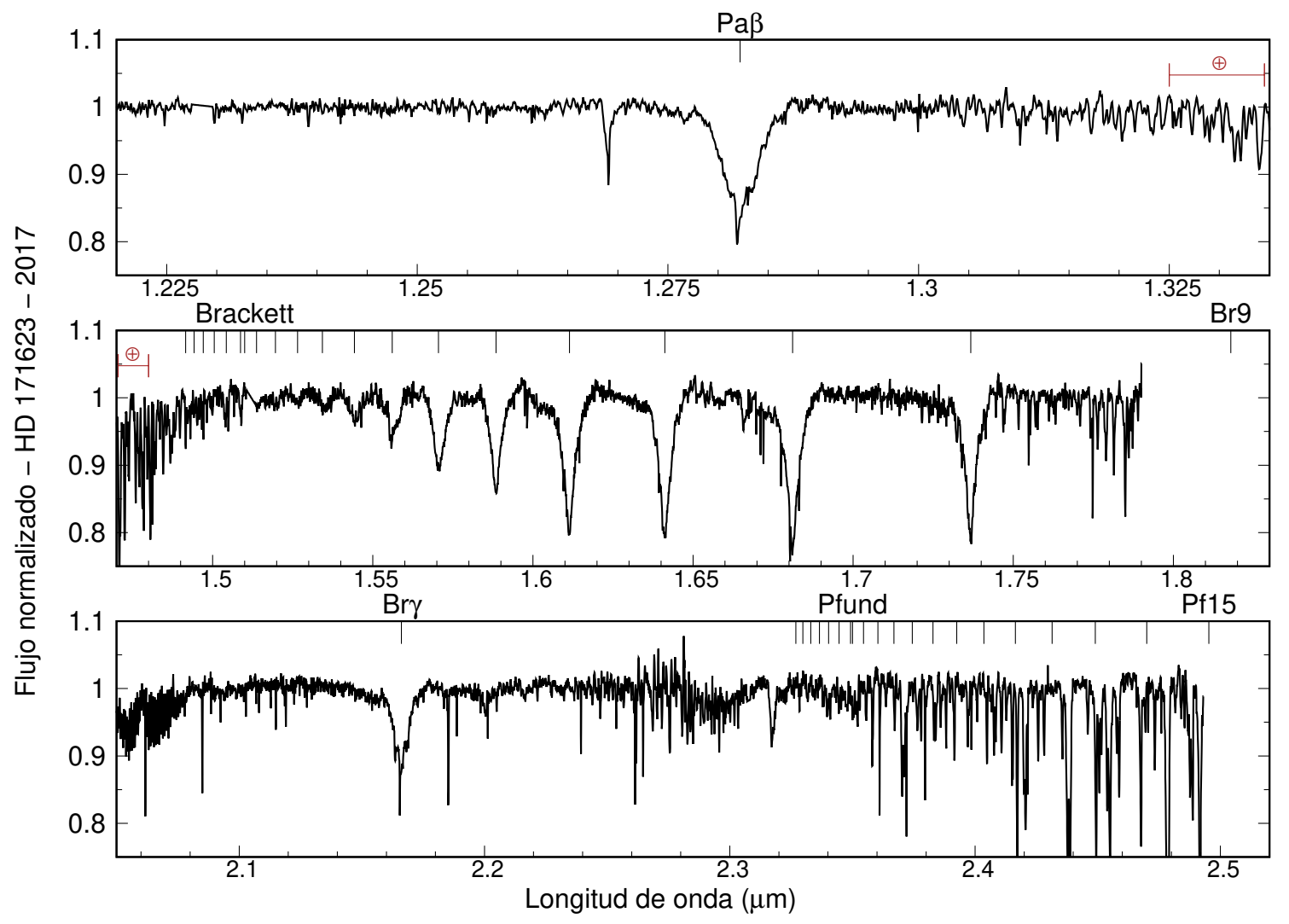




\subsubsection{V4024 Sgr - HD 178175}

V4024 Sgr es una estrella de tipo espectral y clase de luminosidad B2 V (Merrill \& Burwell, 1933).

Mennickent et al. (2009) obtuvieron espectros en el IR y, a partir de ellos, la catalogaron como perteneciente al grupo I y la ubicaron en la región superior derecha del diagrama de Lenorzer, cerca del límite ópticamente grueso. Estos autores indicaron además que la observación IR fue coincidente con un momento de intensidad máxima en la línea $\mathrm{H} \alpha$.

\section{Descripción de los espectros y mediciones realizadas}

En la Fig. 5.54 se muestran los espectros obtenidos en el 2017 utilizando el espectrógrafo FIRE. Las líneas de hidrógeno observadas se encuentran en emisión. Para la línea $\mathrm{Pa} \beta$ y algunos miembros de la serie de Brackett la emisión se superpone al perfil de absorción fotosférica, sin llegar a superar el nivel del continuo en el caso de las líneas de la serie de Brackett. Los valores de EW, Fl y FWHM se muestran en la Tabla A.23. Según los parámetros de la Tabla 3.3, se utilizó el espectro sintético correspondiente a una estrella con $T_{\text {eff }}=19000 \mathrm{~K}$ y $\log g=3.5$ para realizar la corrección por absorción fotosférica. Como no obtuvimos observaciones de la banda L, no pudimos ubicar a V4024 Sgr en el diagrama de Lenorzer.

Figura 5.54: Espectros de V4024 Sgr en as bandas J (superior), H (centro) y K (inferior).

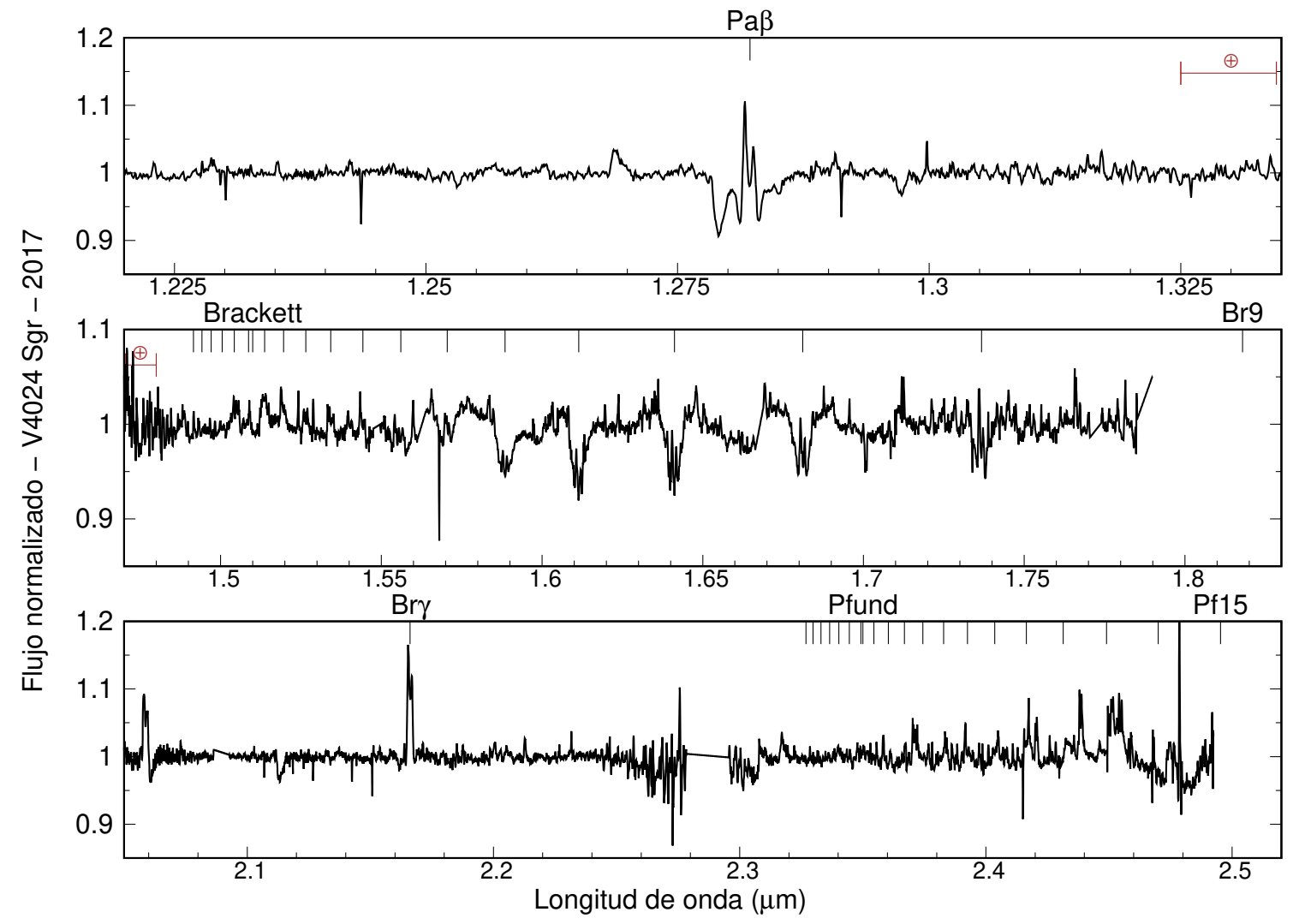


Figura 5.55: V4024 Sgr - EW/ $\lambda$ vs. $\lambda$ de las líneas observadas en las diferentes series.

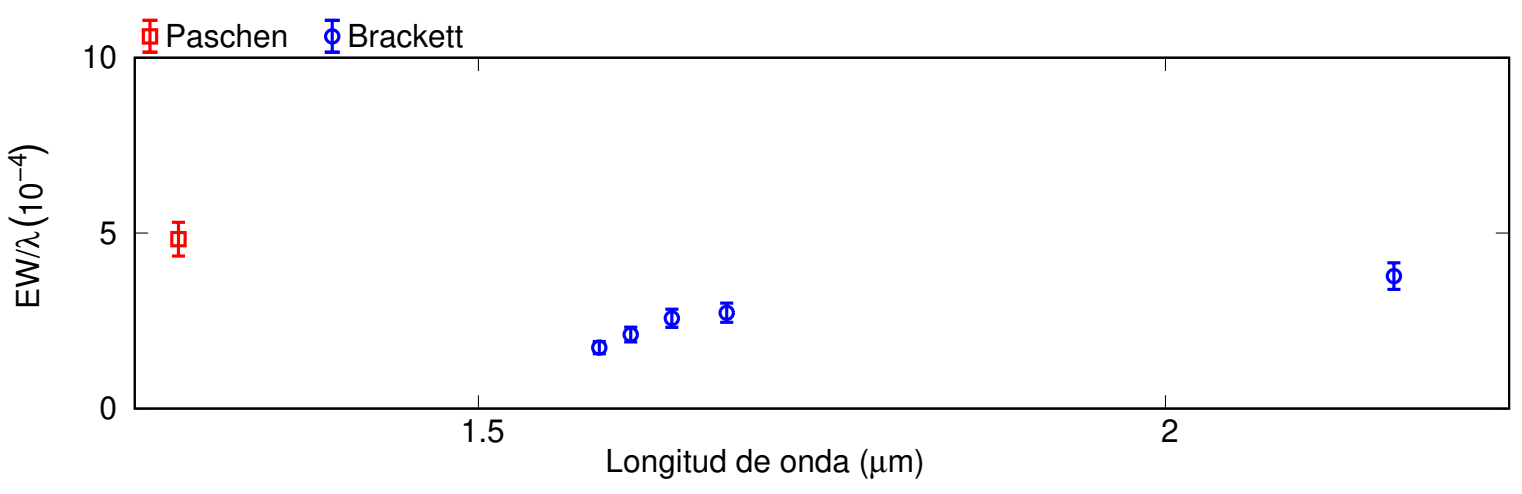

Figura 5.56: V4024 Sgr - Cocientes de flujos para las líneas de la serie de Brackett. En la parte superior se identifican la líneas correspondientes. Los símbolos utilizados son los mismos que en la Fig. 5.55.

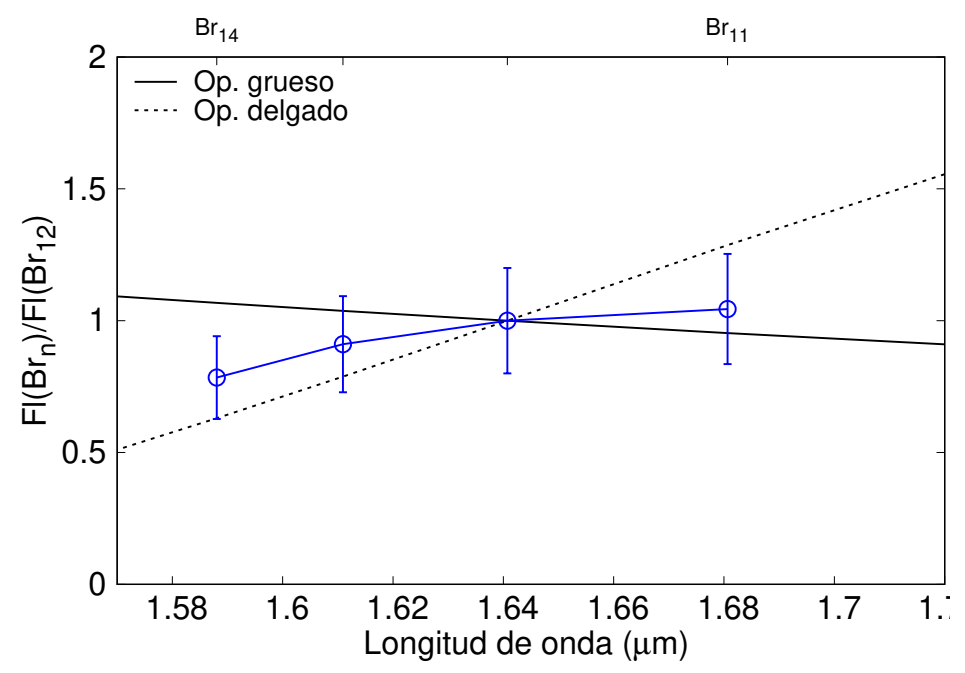

\section{Comportamiento de las series de hidrógeno}

En el gráfico de EW/ $\lambda$ vs. $\lambda$ no pudimos graficar suficientes miembros de cada serie como para obtener resultados concluyentes (ver Fig. 5.55). Para la única línea con FWHM determinado, el mismo es mayor que $2 V \sin (i)=350 \mathrm{~km} / \mathrm{s}$, indicando una región de formación cercana a la estrella central o algún mecanismo de ensanchamiento adicional.

En la Fig. 5.56 se muestran los cocientes de flujos y las comparaciones con los casos ópticamente grueso y delgado para la serie de Brackett. Debido a las pocas líneas observadas no podemos distinguir cuáles se mantienen sobre la recta esperada para el caso ópticamente delgado. 
Figura 5.57: V4024 Sgr - Radios internos de las regiones emisoras de las diferentes líneas en radios estelares. Los símbolos utilizados son los mismos que en la Fig. 5.55.

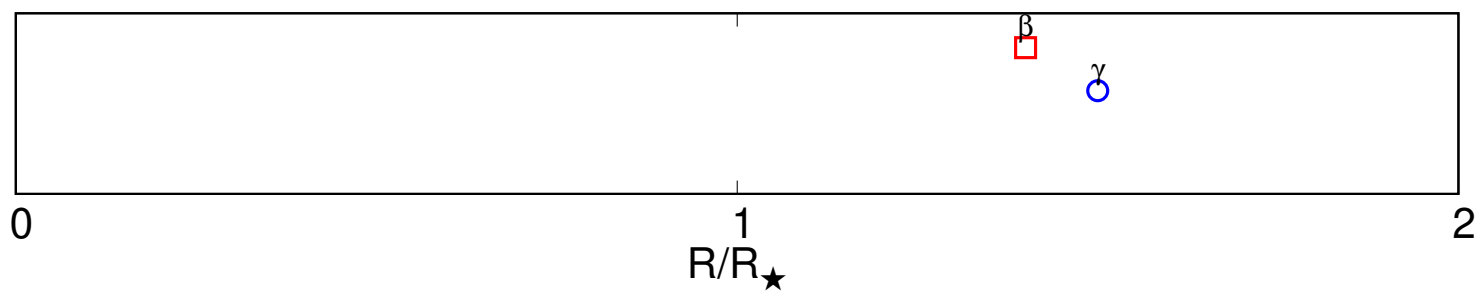

Determinación de densidades columnares y extensiones de las regiones de formación

Utilizando en método $\Delta V$ obtuvimos los radios internos de las regiones de formación de $\mathrm{Br} \gamma \mathrm{y}$ $\mathrm{Pa} \beta$. Los mismos se muestran en la Tabla A.24 y la Fig. $5.57 \mathrm{y}$ son $\sim 1.5 R_{\star}$. Al ser los primeros miembros observados de cada serie, suelen indicar las regiones de formación más externas, por lo que V4024 Sgr presenta una envoltura delgada y compacta. 


\subsubsection{4 o Aqr - HD 209409}

o Aqr es una estrella de tipo espectral B7 IV, reportada como Be por Merrill et al. (1925). Ha presentado un perfil de $\mathrm{H} \alpha$ en emisión estable sin grandes variaciones en $V / R$ (Rivinius et al., 2006), pero con variabilidad en el ancho equivalente (Jones et al., 2011).

Silaj et al. (2010) ajustaron la línea de $\mathrm{H} \alpha$ con un doble pico, y obtuvieron como parámetros del mejor ajuste un tipo espectral B8 para la estrella central, $i=45^{\circ}, \rho_{0}=5 \cdot 10^{-11} \mathrm{~g} \mathrm{~cm}^{-3}$ y $n=3.5$. Por otro lado, Arcos et al. (2017) obtuvieron densidades e índice menores $\left(\rho_{0}=5 \cdot 10^{-10} \mathrm{~g} \mathrm{~cm}^{-3}\right.$, $n=2.0$ ), y dos diferentes inclinaciones: $i=25^{\circ}$ (en 2013) e $i=50^{\circ}$ (en 2015).

Touhami et al. (2010) obtuvieron espectros IR de o Aqr en el año 2008 y reportaron la presencia de $\mathrm{Br} \gamma$ en emisión.

La estrella fue resuelta a partir de observaciones interferométricas. Touhami et al. (2013) encontraron una relación disco-estrella $R_{\mathrm{d}} / R_{\star}=5.776$ y $\mathrm{PA}=107.5^{\circ}$, mientras que Cyr et al. (2015) encontraron un cociente entre los ejes de 0.25 en la Banda K. Sigut et al. (2015), combinando observaciones interferométricas y en $\mathrm{H} \alpha$ durante un período de 7 años, concluyó que las observaciones son consistentes con un disco circunestelar visto con una inclinación $i=75^{\circ}$ y $\mathrm{PA}=110^{\circ}$ con $\rho_{0}=6.6 \cdot 10^{-11} \mathrm{~g} \mathrm{~cm}^{-3}$ y $n=2.7$. Meilland et al. (2012) obtuvo los siguientes parámetros para el disco: $\mathrm{PA}=120^{\circ}, i=70^{\circ}, V_{\text {rot }}=400 \mathrm{~km} \mathrm{~s}^{-1}, j=-0.5, a_{\text {cont }}<10 D_{\star} \mathrm{y} a_{\mathrm{Br} \gamma}=14 D_{\star}$. Por otra

Figura 5.58: Espectros de o Aqr en las bandas J (superior) y H (inferior).

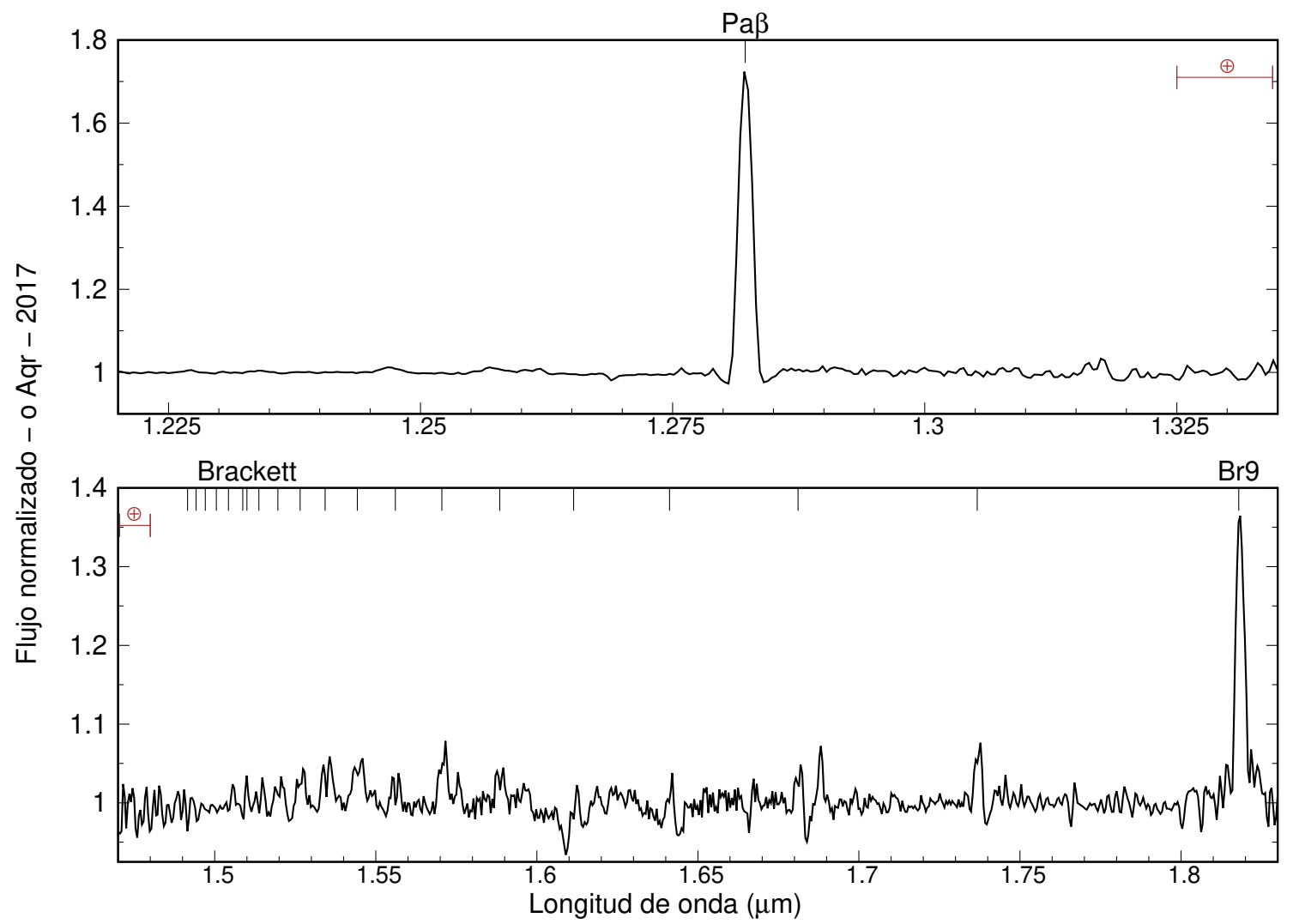


Figura 5.59: Espectro de o Aqr en la banda K.

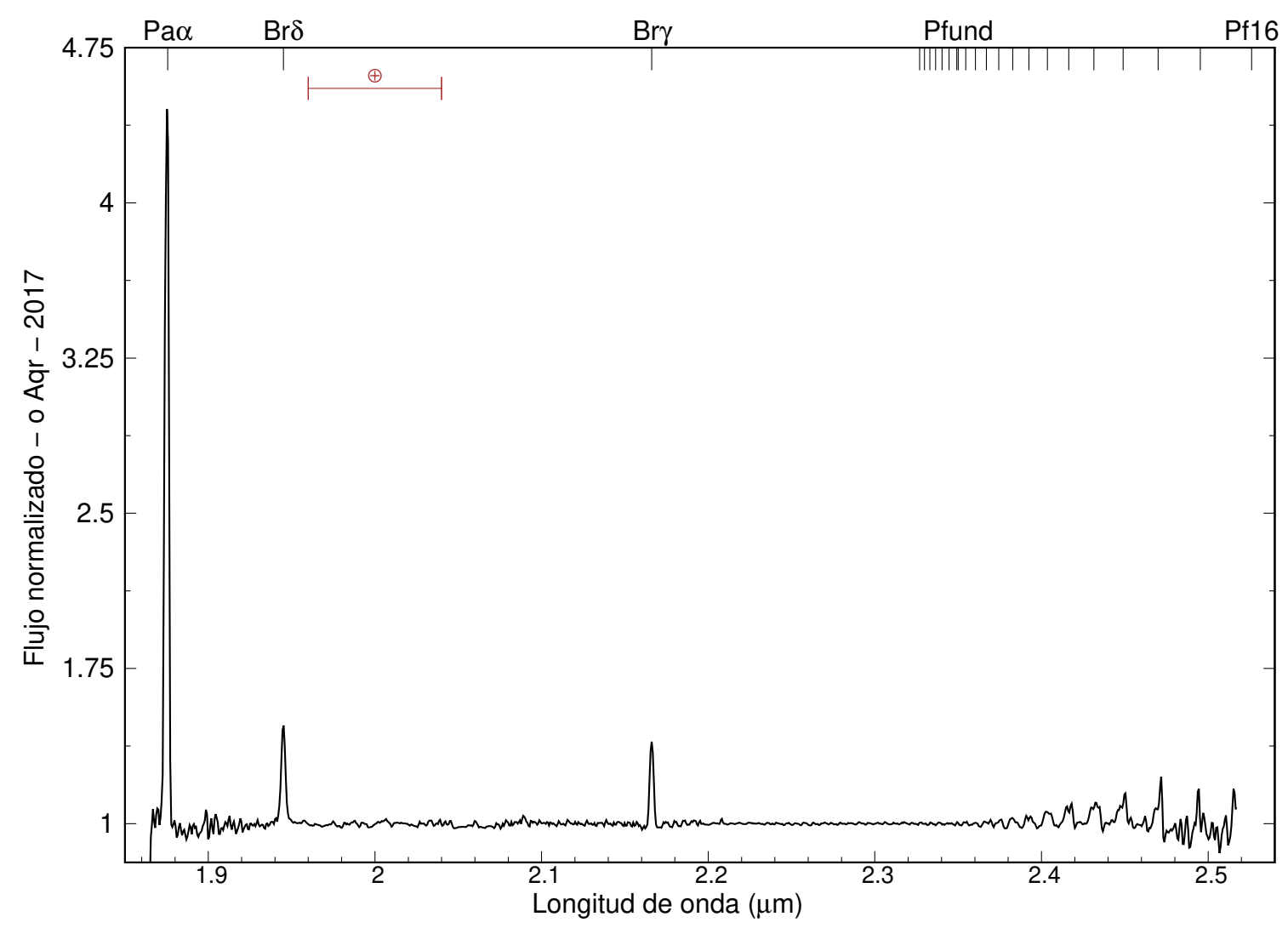

parte, Yudin (2001) encontraron un valor de $\mathrm{PA}=177^{\circ}$ para la polarización observada.

\section{Descripción de los espectros y mediciones realizadas}

Las Figs. 5.58 y 5.59 muestran los espectros obtenidos en el 2017 utilizando el espectrógrafo GNIRS. Las líneas observadas se encuentran en emisión, con perfiles de dos picos en los casos de los miembros más altos de las series de Brackett y Pfund. En algunos miembros de la serie de Brackett se distingue el perfil de absorción fotosférico. La Tabla A.25 muestra los valores de EW, Fl y FWHM obtenidos. Para realizar la corrección por absorción fotosférica se utilizó el espectro sintético correspondiente a una estrella con $T_{\text {eff }}=13000 \mathrm{~K}$ y $\log g=3.5$ (ver Tabla 3.3). Como no obtuvimos observaciones de la banda L, no pudimos ubicar a $o$ Aqr en el diagrama de Lenorzer.

\section{Comportamiento de las series de hidrógeno}

En la Fig. 5.60 vemos los gráficos de EW/ $\lambda$ y FWHM vs. $\lambda$. La serie de Brackett parece alcanzar un valor estable en EW/ $\lambda \sim 10 \cdot 10^{-4}$. Para la serie de Pfund los FWHM obtenidos son similares a $2 \mathrm{~V} \sin (i)$, compatible con regiones de formación cercanas a la fotosfera, mientras que para la serie de Brackett los anchos crecen hacia los miembros más altos de la serie. Algunas líneas 
Figura 5.60: $o$ Aqr - EW/ $\lambda$ y FWHM vs. $\lambda$ de las líneas observadas en las diferentes series. La línea punteada corresponde a un valor de $2 V \sin (i)=564 \mathrm{~km} / \mathrm{s}$, y la región sombreada al error correspondiente (Tabla 3.3).

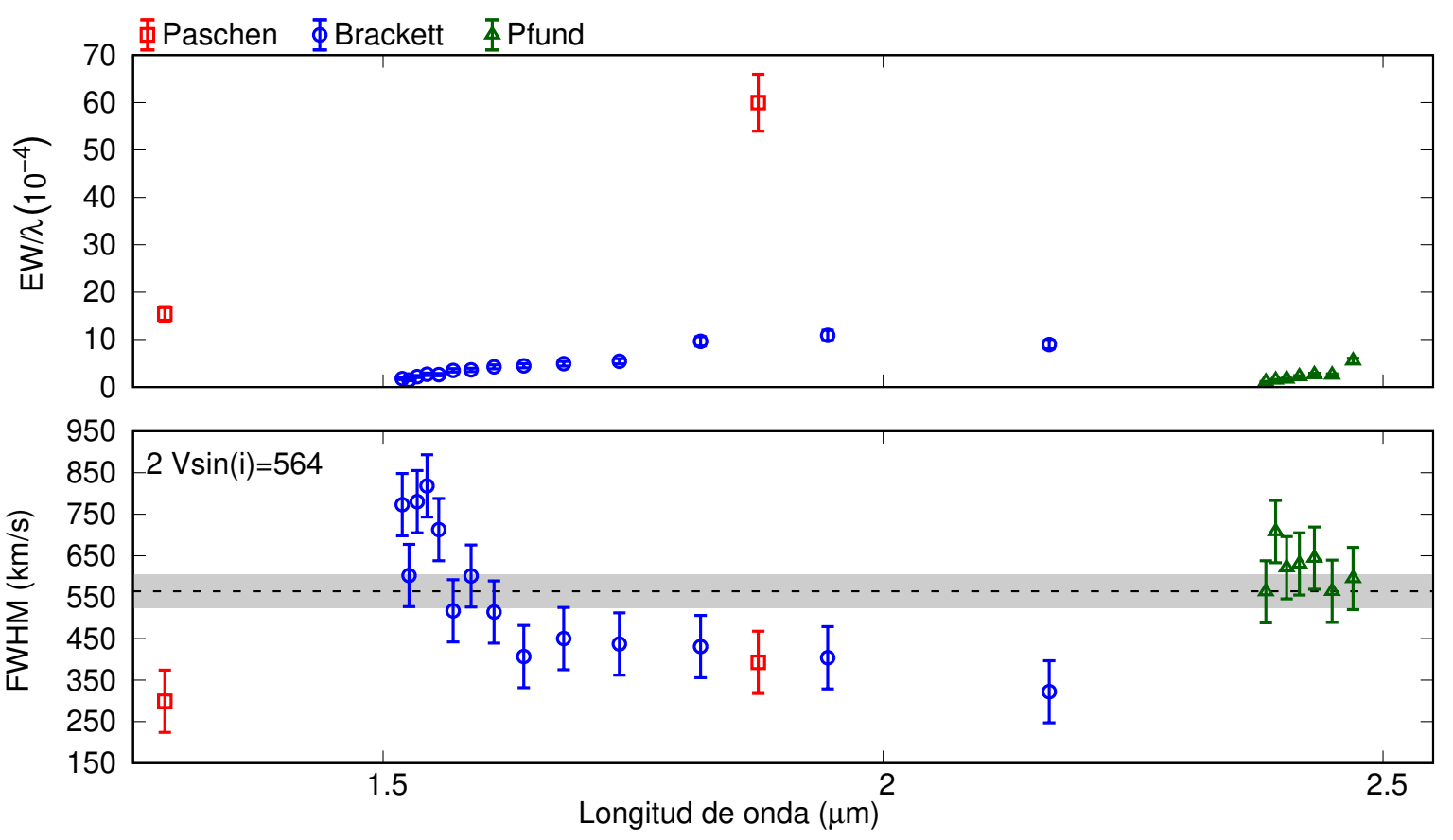

muestran ensanchamientos mayores a $2 \mathrm{~V} \sin (i)$, indicando regiones de formación muy cercanas a la estrella central, o algún mecanismo de ensanchamiento adicional. En la serie de Paschen los FWHM obtenidos son menores, indicando regiones de formación más alejadas de la estrella central.

Los cocientes de los flujos y las comparaciones con los casos ópticamente grueso y delgado se muestran en la Fig. 5.61. Para la serie de Pfund todas las líneas observadas siguen el comportamiento correspondiente a una envoltura ópticamente delgada, mientras que en la serie de Brackett, las líneas hasta $\mathrm{Br}_{14}$ se separan del caso ópticamente delgado. Obtuvimos además que $\mathrm{EW}(\mathrm{Pa} \alpha) / \mathrm{EW}(\mathrm{Pa} \beta) \sim 5.7$.

\section{Determinación de densidades columnares y extensiones de las regiones de formación}

Mediante los métodos $N$ y FWHM obtuvimos las densidades columnares y las extensiones relativas de las regiones de formación para la serie de Brackett, y los radios internos de las regiones de formación relativos a una región de referencia para las líneas de las series de Paschen, Brackett y Pfund. Para estas series obtuvimos también los tamaños en radios estelares con el método $\Delta V$. Los valores obtenidos se muestran en la Tabla A.26. Para las líneas en las cuales no pudimos medir $\Delta V$ obtuvimos valores aproximados para el radio interno de las regiones de formación a partir de los tamaños relativos a $\mathrm{Br}_{10}$ obtenidos mediante el método FWHM. 
Figura 5.61: o Aqr - Cocientes de flujos para las líneas de las series de Pfund y Brackett. En la parte superior se identifican la líneas correspondientes. Los símbolos utilizados son los mismos que en la Fig. 5.60.
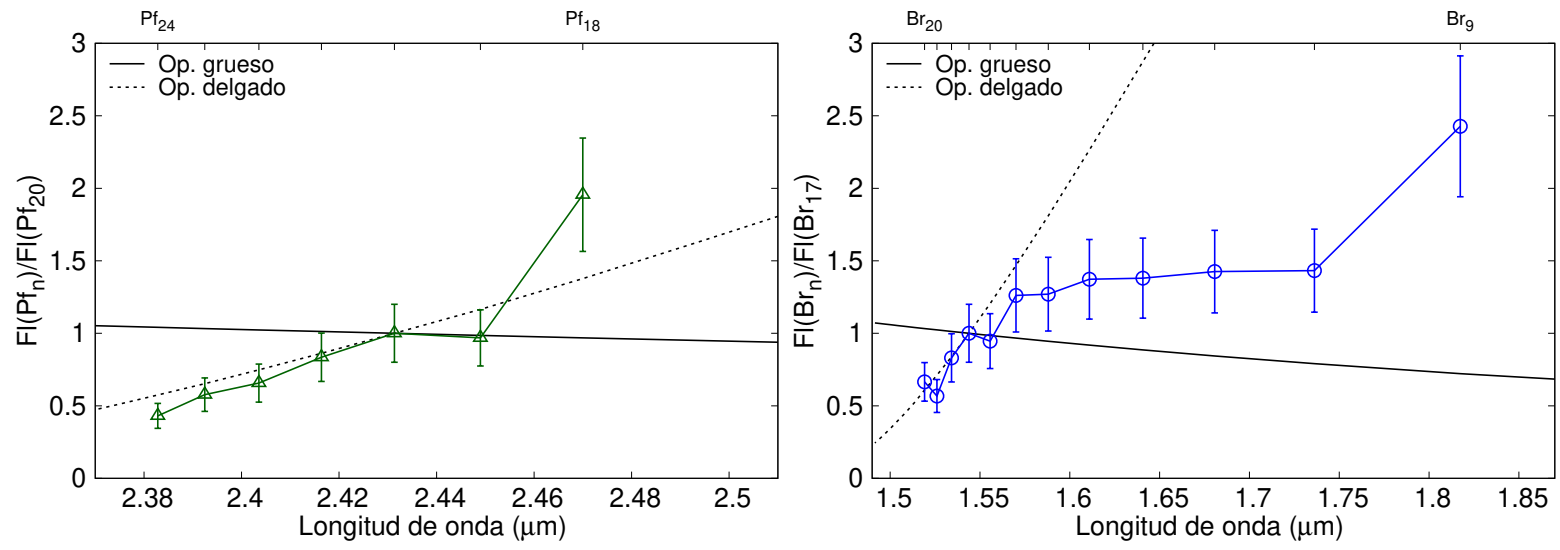

Obtuvimos que en la serie de Brackett, los miembros más altos presentan mayores densidades y extensiones de las regiones de formación, junto con radios menores. Además, los FWHM obtenidos para la serie de Paschen indican regiones de formación más alejadas de la estrella central que las series de Pfund y Brackett.

En radios estelares, obtuvimos valores en el intervalo $1.6-3 R_{\star}$ para la serie de Pfund, y entre 1.6 y $5.2 R_{\star}$ para la serie de Brackett. Las regiones correspondientes a los miembros más altos presentan los tamaños más pequeños. En la Fig. 5.62 se muestran los tamaños de las regiones emisoras respecto a $\operatorname{Br} \delta$ (superior) y en radios estelares (inferior). Las regiones más externas, correspondientes a los primeros miembros de cada serie, alcanzan los $11 R_{\star}$ mientras que los miembros más altos de las series se forman hasta $6 R_{\star}$. 
Figura 5.62: o Aqr - Radios internos de las regiones emisoras de las diferentes líneas relativos a la región emisora de $\operatorname{Br} \delta$ y en radios estelares. Los símbolos utilizados son los mismos que en la Fig. 5.60 .

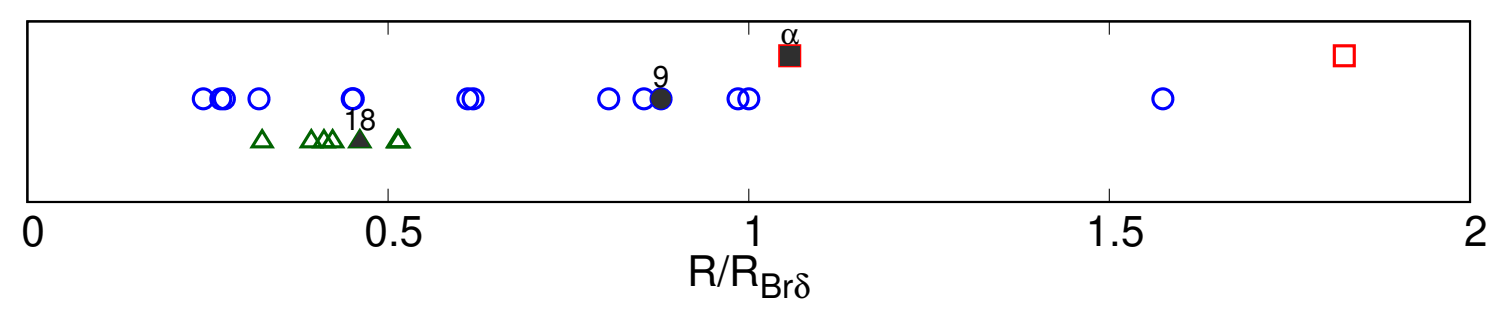

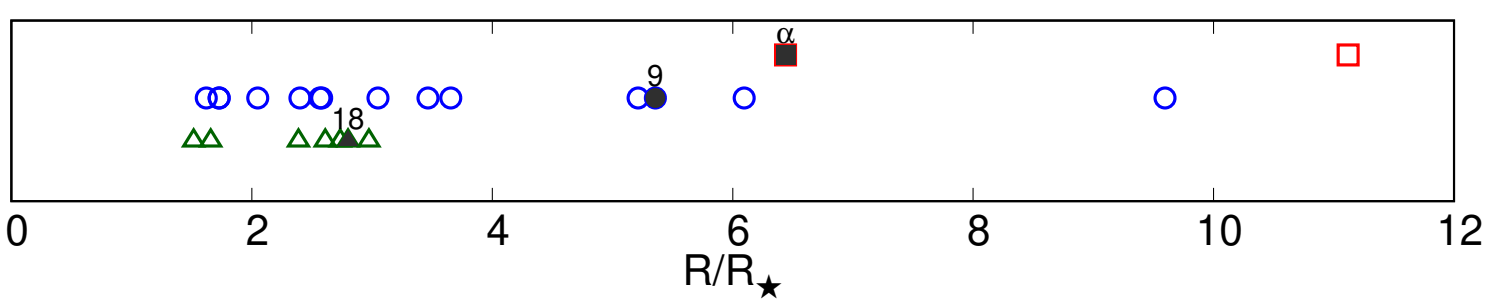




\subsubsection{5 $\pi$ Aqr - HD 212571}

$\pi$ Aqr es una estrella con clasificación espectral B1 III-IV, identificada como Be por Merrill et al. (1925). A partir de un análisis del perfil de $\mathrm{H} \alpha$, Bjorkman et al. (2002) sugirieron que este objeto podría ser un sistema binario con un período orbital de 84.1 días, formado por dos estrellas de masas $M_{1} \sin ^{3} i=12.4 M_{\odot}$ y $M_{2} \sin ^{3} i=2.0 M_{\odot}$. También plantearon que las variaciones fotométricas, espectroscópicas y polarimétricas observadas durante la segunda mitad del siglo XX podrían deberse a la transferencia de masa entre las componentes del sistema binario. Wisniewski et al. (2010) encontraron que el tiempo de escala de la pérdida del disco corresponde a 29 órbitas completas del sistema binario, y determinaron el ángulo de posición de la polarización intrínseca $\left(\mathrm{P}_{\star}=166.7^{\circ}\right)$, indicando que el disco está orientado en el cielo con un ángulo de posición $\mathrm{PA}=76.7^{\circ}$.

Jones et al. (2011) reportaron que el ancho equivalente de la línea $\mathrm{H} \alpha$ decrecía entre 2003 y 2007, mientras que Silaj et al. (2010) ajustaron el perfil de la línea con un doble pico en emisión obteniendo como parámetros del mejor ajuste un tipo espectral B0 para la estrella central y un disco ecuatorial con $\rho_{0}=10^{-11} \mathrm{~g} \mathrm{~cm}^{-3}$ y $n=3.5$, visto con un ángulo de inclinación $i=45^{\circ}$. Arcos et al. (2017) determinaron tres conjuntos de parámetros, utilizando espectros de la línea $\mathrm{H} \alpha$ obtenidos en diferentes épocas: $\rho_{0}=2.5 \cdot 10^{-11} \mathrm{~g} \mathrm{~cm}^{-3}, i=60^{\circ}$ y $n=2.5$ para las observaciones obtenidas en 2012 y 2015 , y $\rho_{0}=7.5 \cdot 10^{-12} \mathrm{~g} \mathrm{~cm}^{-3}, i=60^{\circ}$ y $n=2.5$ para las de 2013. Vieira

Figura 5.63: Espectros de $\pi$ Aqr en las bandas J (superior), H (centro) y K (inferior).

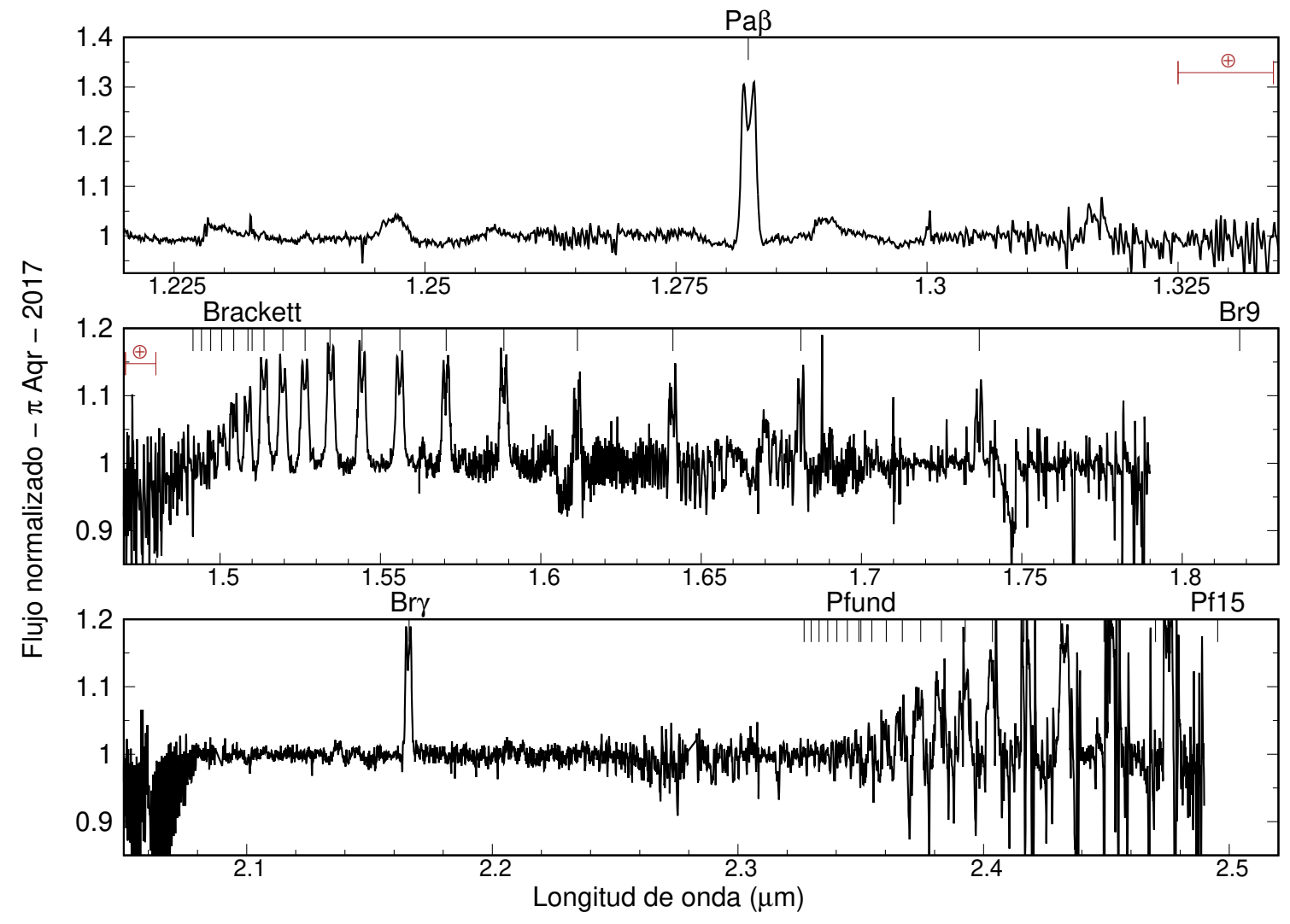


et al. (2017) utilizaron observaciones obtenidas en el año 2010 y mediante un ajuste de la SED obtuvieron $\log \rho_{0}=-12.1 \pm 0.2$ y $n=3.5 \pm 0.4$. Según estos autores, el valor obtenido para $n$ indica la presencia de un disco estable o en formación.

Lenorzer et al. (2002b) presentaron espectros en la banda L obtenidos en 1998, donde no se observaba evidencia de líneas en emisión. Más adelante, Touhami et al. (2010) obtuvieron espectros IR de $\pi$ Aqr, y la ubicaron en la región del diagrama de Lenorzer correspondiente a las estrellas del Grupo II.

\section{Descripción de los espectros y mediciones realizadas}

En la Fig. 5.63 se muestran los espectros obtenidos en el 2017 utilizando el espectrógrafo FIRE. Las líneas de hidrógeno observadas presentan dos picos en emisión. La Tabla A.27 muestra los valores de EW, Fl y FWHM obtenidos, corregidos por absorción fotosférica utilizando el espectro sintético correspondiente a una estrella con $T_{\text {eff }}=26000 \mathrm{~K}$ y $\log g=4.0$ según los parámetros estelares de la Tabla 3.3. Como no obtuvimos observaciones de la banda L, no pudimos ubicar a $\pi$ Aqr en el diagrama de Lenorzer.

\section{Comportamiento de las series de hidrógeno}

En los gráficos de EW/ $\lambda$ y FWHM en función de la longitud de onda vemos que para la serie de Brackett, los valores de EW/ $\lambda$ parecen alcanzan un valor aproximadamente estable para las longitudes de onda mayores en $\sim 4 \cdot 10^{-4}$. La mayor parte de las líneas de la serie de Brackett presentan FWHM $\sim 2 V \sin (i)$ con una tendencia creciente hacia los miembros más altos de la serie. Para $\mathrm{Pa} \beta$, el ensanchamiento obtenido es menor a $2 V \sin (i)$, indicando una región no demasiado alejada de la estrella central.

Los cocientes de flujos se muestran en la Fig. 5.65. Vemos que las líneas observadas de la serie de Pfund se mantienen sobre la recta correspondiente al caso ópticamente delgado, mientras que los miembros de la serie de Brackett hasta $\mathrm{Br}_{17}$ presentan cocientes de flujos cercanos al caso ópticamente grueso.

\section{Determinación de densidades columnares y extensiones de las regiones de formación}

Obtuvimos densidades columnares y extensiones de las regiones de formación utilizando el método $N$, y tamaños relativos entre regiones de formación de distintas líneas y en radios estelares utilizando los métodos FWHM y $\Delta V$.

En la serie de Brackett, los primeros miembros presentan regiones de formación con mayores densidades columnares y extensiones. Los menores tamaños los obtuvimos para los miembros más altos de la serie. Los tamaños relativos al radio interior de la región de formación de $\mathrm{Br} \gamma$ se muestra en la Fig. 5.66 (superior). 
Figura 5.64: $\pi$ Aqr - EW/ $\lambda$ y FWHM vs. $\lambda$ de las líneas observadas en las diferentes series. La línea punteada corresponde a un valor de $2 V \sin (i)=466 \mathrm{~km} / \mathrm{s}$, y la región sombreada al error correspondiente (Tabla 3.3).

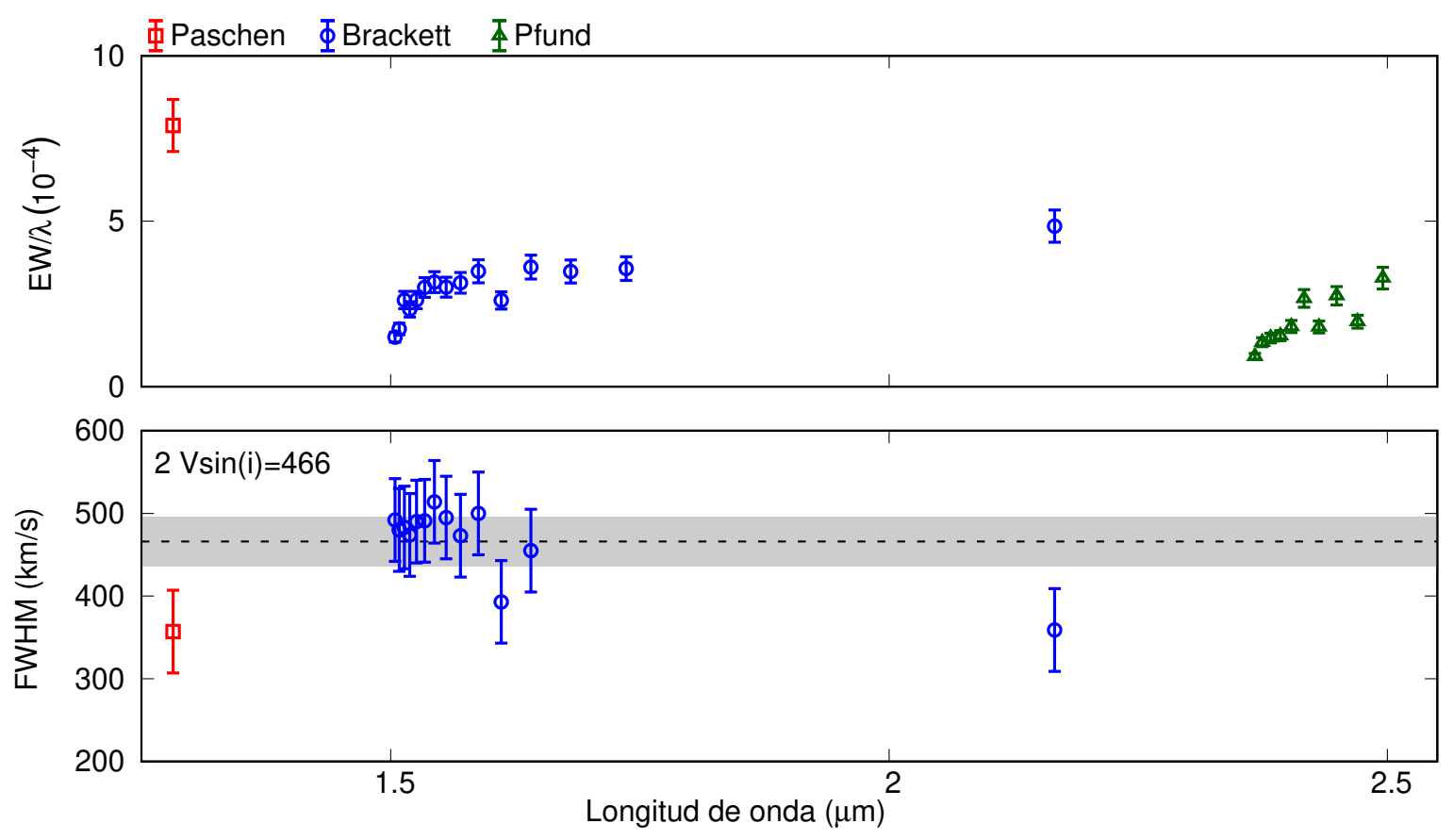

Figura 5.65: $\pi$ Aqr - Cocientes de flujos para las líneas de la serie de Pfund y Brackett. En la parte superior se identifican la líneas correspondientes. Los símbolos utilizados son los mismos que en la Fig. 5.64.
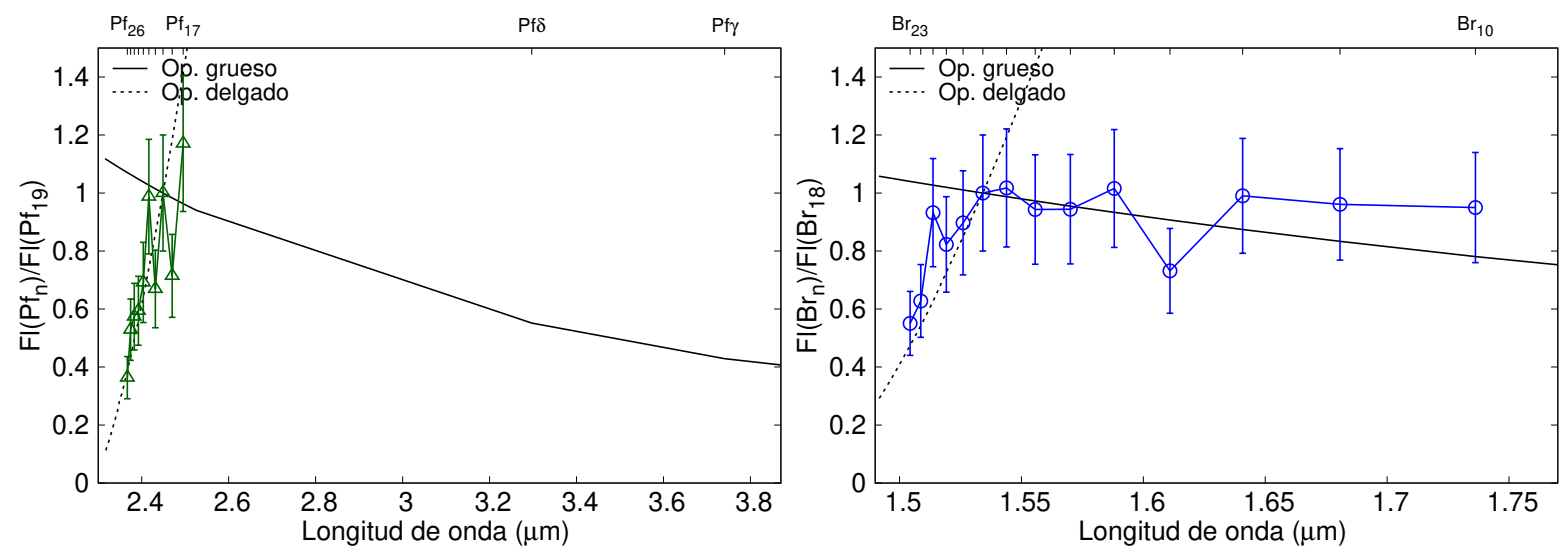

Los miembros de la serie de Pfund se forman en regiones entre $2.3 R_{\star} \mathrm{y} \sim 3.5 R_{\star}$, mientras que para la serie de Brackett alcanzan los $\sim 5 R_{\star}$. Esto puede observarse en la Fig. 5.66 (inferior). Por lo tanto, $\pi$ Aqr presenta un disco denso hasta los $5 R_{\star}$. 
Figura 5.66: $\pi$ Aqr - Radios internos de las regiones emisoras de las diferentes líneas relativos a la región emisora de $\mathrm{Br} \gamma$ y en radios estelares. Los símbolos utilizados son los mismos que en la Fig. 5.64.
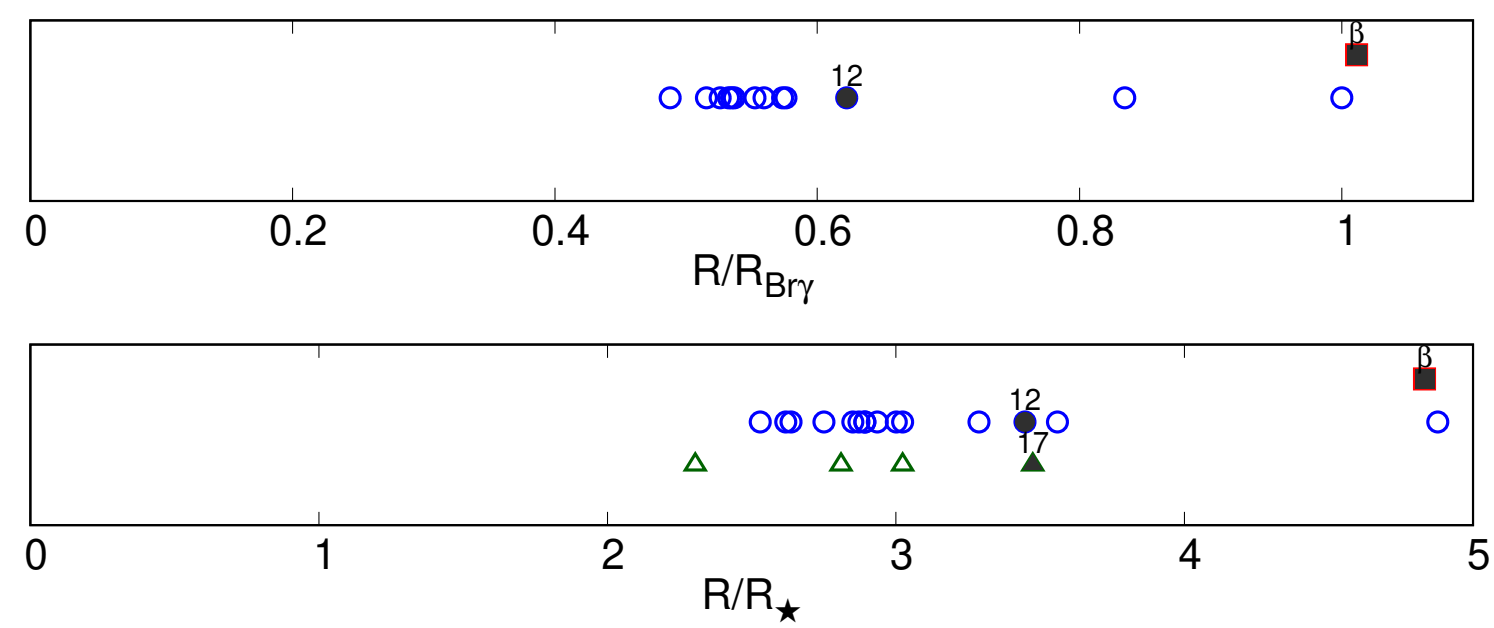


\subsection{Resultados generales}

A partir de los resultados encontrados para los 15 objetos estudiados en este capítulo, analizaremos comportamientos generales que nos permitan separar a las estrellas en grupos con características comunes.

\subsubsection{Ubicación en el diagrama de Lenorzer}

Los 8 objetos presentados en este capítulo que fueron observados en la banda L presentaban emisión en las líneas $\mathrm{Hu}_{14}$, Pf $\gamma$ y $\mathrm{Br} \alpha$. Estos objetos fueron ubicados en el diagrama de Lenorzer (ver Fig. 5.67). Los restantes (MX Pup, 48 Lib, V1075 Sco, HD 171623, V4024 Sgr, o Aqr y $\pi$ Aqr) no pudieron ser ubicados en el diagrama porque no obtuvimos observaciones en la banda L.

Las estrellas clasificadas como pertenecientes al grupo I (V696 Mon y 88 Her) se encuentran en la región $\log \left(\mathrm{Fl}\left(\mathrm{Hu}_{14}\right) / \mathrm{Fl}(\mathrm{Br} \alpha)\right) \geq-0.25$ (límite cercano al valor -0.2 adoptado por Mennickent et al., 2009). Las estrellas que se encuentran por debajo fueron clasificadas como pertenecientes al grupo II según la intensidad de las líneas de la serie de Humphreys. Dentro de este grupo

Figura 5.67: Ubicación en el diagrama de Lenorzer de las estrellas Be estudiadas. Las líneas negra continua y azul punteada indican los límites donde $\log \left(\mathrm{Fl}_{\mathrm{Hu} 14} / \mathrm{Fl}_{\mathrm{Br} \alpha}\right)$ toma los valores -0.25 y -0.50 , respectivamente.

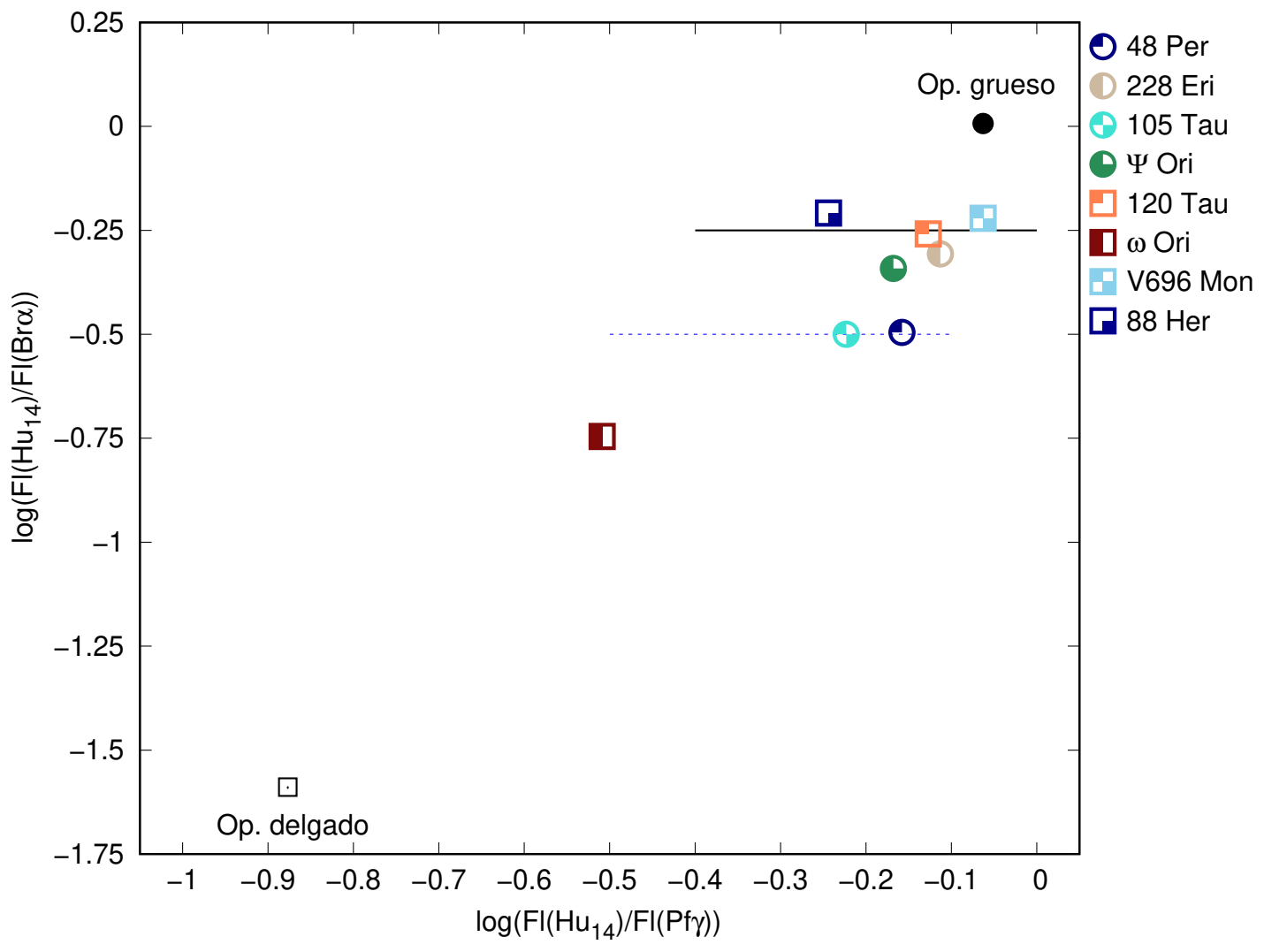


podemos distinguir entre aquellas que presentan cocientes $\mathrm{EW}(\mathrm{Br} \alpha) / \mathrm{EW}(\mathrm{Br} \gamma)$ dentro del intervalo esperado para estrellas del grupo II y aquellas que presentan valores cercanos o dentro de lo esperado para estrellas del grupo I (según los valores reportados por Granada et al., 2010). Las estrellas que presentan valores de $\mathrm{EW}(\mathrm{Br} \alpha) / \mathrm{EW}(\mathrm{Br} \gamma)$ dentro de lo esperado para el grupo II se ubican por debajo de la recta $\log \left(\mathrm{Fl}\left(\mathrm{Hu}_{14}\right) / \mathrm{Fl}(\mathrm{Br} \alpha)\right)=-0.5$ (48 Per, 105 Tau y $\omega$ Ori), quedando en la región intermedia $-0.25>\log \left(\mathrm{Fl}\left(\mathrm{Hu}_{14}\right) / \mathrm{Fl}(\mathrm{Br} \alpha)\right)>-0.5$ aquellas que presentan características asociables al grupo I y II (228 Eri, $\Psi$ Ori y 120 Tau). Esto indicaría que estos objetos se encuentran en transición entre ambos grupos. La línea negra continua y la azul de trazos de la Fig. 5.67 indica donde $\log \left(\mathrm{Fl}\left(\mathrm{Hu}_{14}\right) / \mathrm{Fl}(\mathrm{Br} \alpha)\right)=-0.25$ o -0.5 , respectivamente.

\subsubsection{Comportamiento de las series de hidrógeno}

Observamos que no todas las estrellas muestran un comportamiento igual en el apartamiento del caso ópticamente delgado en los primeros miembros de cada serie. Los diferentes comportamientos se muestran en la Fig. 5.68.

Para la serie de Humphreys, la mayor parte de las estrellas (105 Tau, $\Psi$ Ori, 228 Eri, 120 Tau y V696 Mon) tienen cocientes de flujos decrecientes hacia los primeros miembros, comportamiento que se muestra en la Fig. 5.68 (superior derecha). Para 48 Per, en cambio, las líneas que se apartan del caso ópticamente delgado continúan manteniendo un comportamiento creciente (Fig. 5.68 superior izquierda). Por otro lado, $\omega$ Ori muestra un comportamiento donde los cocientes de los flujos oscilan alrededor de los cocientes esperados para el caso ópticamente delgado, con cocientes similares en los últimos miembros observados.

Para la serie de Pfund observamos un comportamiento similar, donde 48 Per y $\omega$ Ori muestran valores crecientes en los cocientes de los flujos hacia los primeros miembros de la serie con el máximo valor es $\operatorname{Pf} \gamma$ o $\operatorname{Pf} \delta$ (Fig. 5.68 centro izquierda), contrario a lo observado en 105 Tau, $\Psi$ Ori, 228 Eri, 120 Tau y V696 Mon, donde los cocientes de los flujos tienen un máximo en $\mathrm{Pf}_{18}$ (Fig. 5.68 centro derecha). Para $48 \mathrm{Lib}$, o Aqr y $\pi$ Aqr, de las cuales no tenemos observaciones en la banda L y, por lo tanto, no pudimos clasificarlas en los grupos I y II, no observamos los primeros miembros de la serie de Pfund, por lo que no podemos clasificarlas dentro de estos dos comportamientos.

En la serie de Brackett, 48 Per y $\omega$ Ori también muestran un comportamiento creciente en los cocientes de flujos hacia los primeros miembros de la serie, incluso en las líneas que se apartan del caso ópticamente delgado (Fig. 5.68 inferior izquierda). En el caso de $\omega$ Ori, $\mathrm{Br}_{10}$ se aparta del caso ópticamente delgado, pero $\mathrm{Br}_{9}$ vuelve a acercarse, comportamiento similar al observado en la serie de Humphreys. En 105 Tau, $\Psi$ Ori, 228 Eri, 120 Tau y V696 Mon los miembros más altos se mantienen sobre la recta correspondiente al caso ópticamente delgado hasta $\mathrm{Br}_{15}$, luego los cocientes de los flujos disminuyen hasta $\mathrm{Br}_{13}$ para luego volver a crecer (Fig. 5.68 inferior derecha).

En base a esto, y considerando que el apartamiento del caso ópticamente delgado está relacionado con cambios en la profundidad óptica de la envoltura, resulta tentador establecer un 
Figura 5.68: Diferentes comportamiento de los cocientes de flujos de las series de Humphreys (superior), Pfund (centro) y Brackett (inferior).
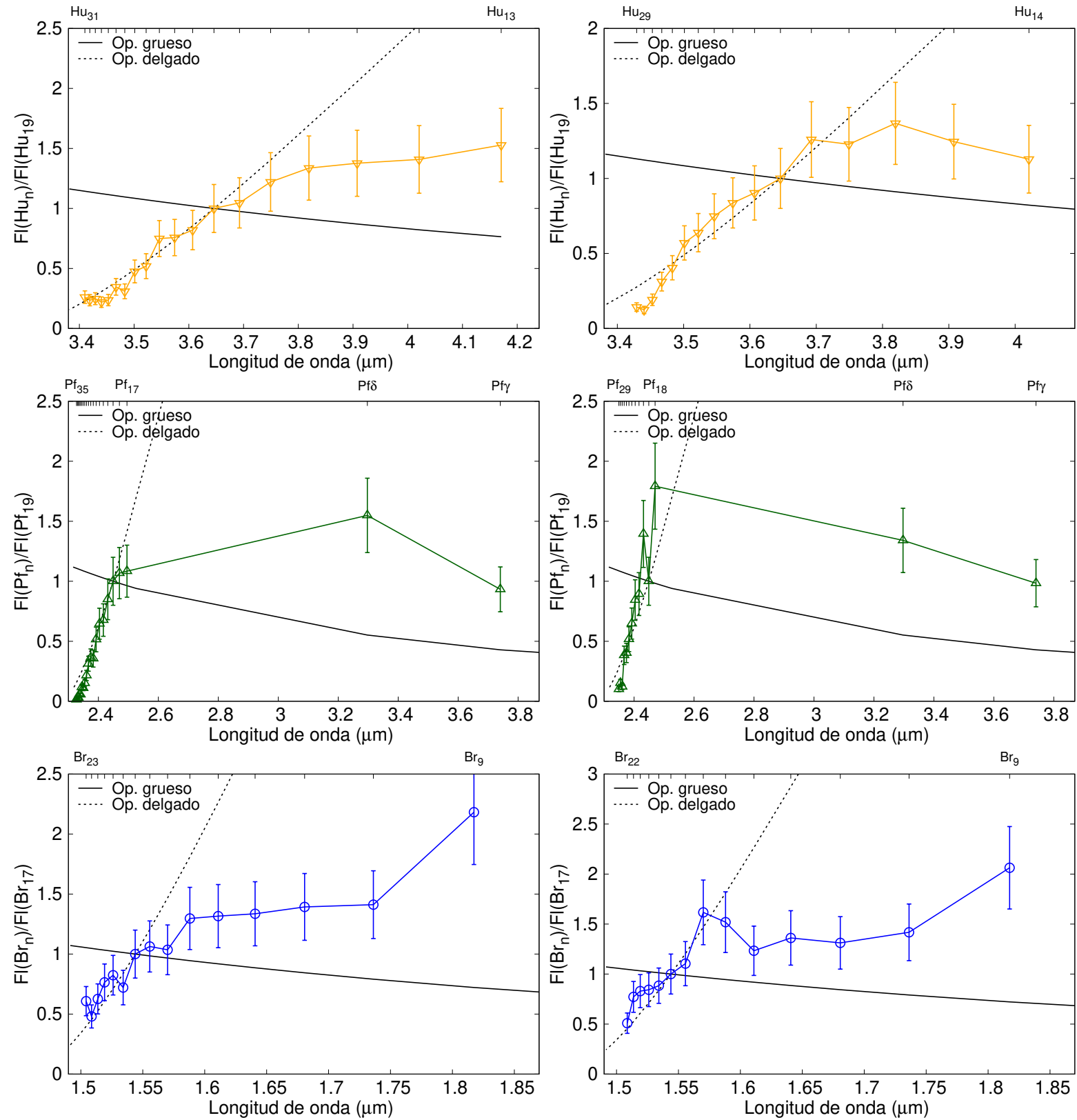

criterio que nos permita separar a las estrellas de los grupos I y II según los comportamientos de los cocientes de los flujos de las líneas de las diferentes series. Sin embargo, veremos en el Capítulo 6 que estos cocientes no están solamente relacionados con las propiedades de la envoltura en ese momento, si no que además brindan información sobre la evolución de la misma.

En la Fig. 5.69 graficamos los cocientes de EW/ $\lambda$ para todos los objetos estudiados en este capítulo. Los colores y líneas indican la pertenencia a los distintos grupos: la línea azul punteada corresponde a las estrellas del grupo II, la línea de trazos verde corresponde a las estrellas del 
Figura 5.69: Comportamiento del cociente EW/ $\lambda$ según los distintos grupos para las líneas de la serie de Humphreys, Pfund y Brackett. La línea azul punteada corresponde a las estrellas del grupo II, la línea de trazos verde corresponde a las estrellas del grupo de transición, y la línea roja punto-raya corresponde a las estrellas del grupo I. Para las estrellas que no fueron clasificadas en los distintos grupos por no haber sido observadas en la banda L utilizamos líneas punto-punto-raya grises.
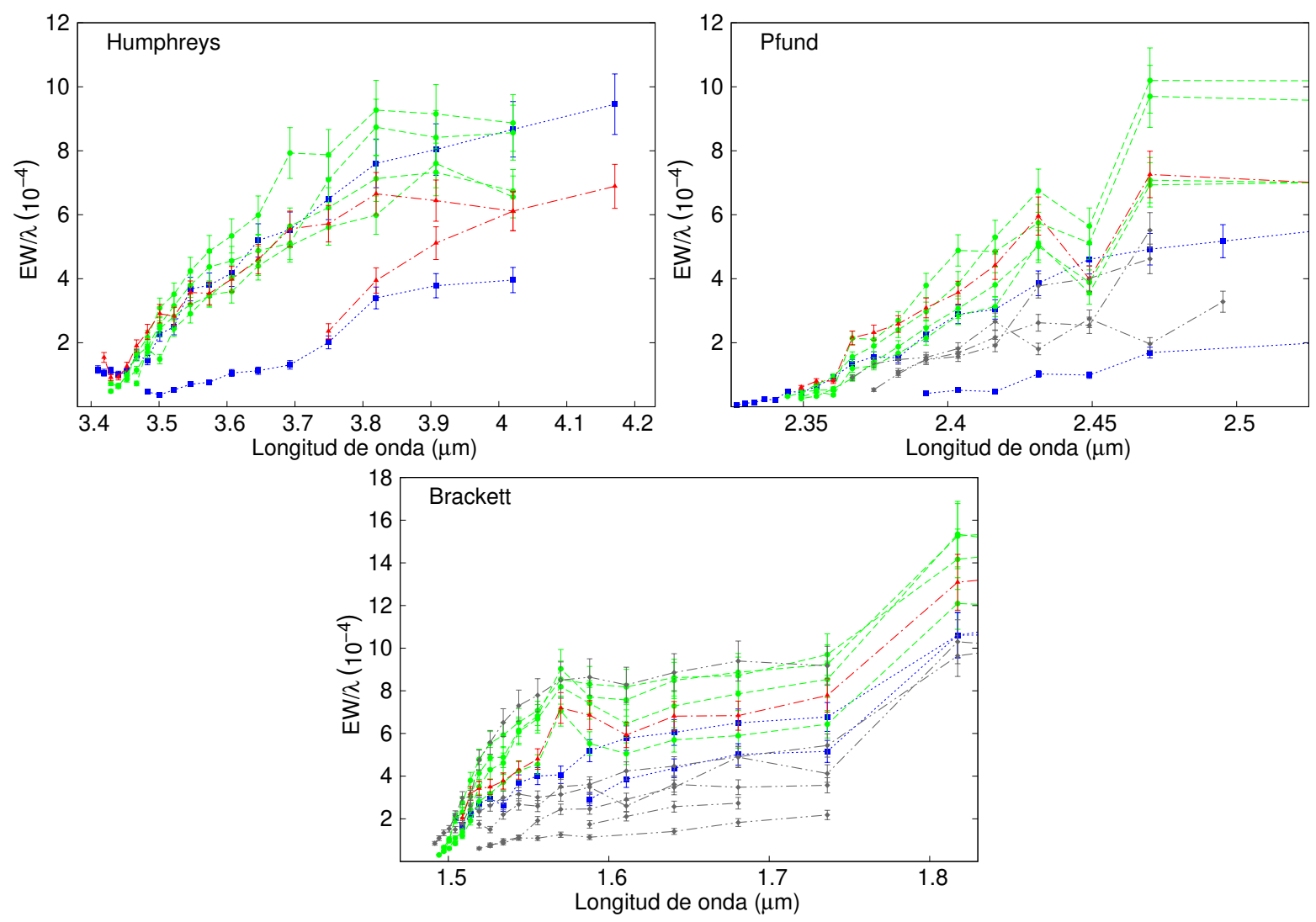

grupo de transición, y la línea roja punto-raya corresponde a las estrellas del grupo I. Para las estrellas que no fueron clasificadas en los distintos grupos por no haber sido observadas en la banda L utilizamos líneas punto-punto-raya grises. Podemos observar que las estrellas del grupo I y del grupo de transición tienen comportamiento similares, con valores mayores a los obtenidos para las estrellas del grupo II. De toda la muestra analizada, solo dos estrellas se apartan de este comportamiento. 48 Per pertenece al grupo II, a pesar de que se ubica justo sobre la recta que separa a las estrellas del grupo II del grupo de transición en el diagrama de Lenorzer, y en el diagrama de cocientes de EW/ $\lambda$ se ubica junto a las estrellas del grupo I. Esto podría indicar que comienza a mostrar características compatibles con el grupo I, debido a la variabilidad en la envoltura. Por otro lado, 88 Her fue clasificada como perteneciente al grupo I, a pesar de que la baja resolución del espectro obtenido no nos permitió observar numerosas líneas. Esta estrella se ubica junto a las estrellas del grupo II, lo que podría deberse a errores en la determinación de los anchos equivalentes debido a la baja intensidad de las líneas. 
En este capítulo presentamos los resultados obtenidos para 15 estrellas de la muestra, estudiando las líneas de recombinación del hidrógeno y definiendo características físicas de sus regiones de formación. A partir del análisis global de los 15 objetos encontramos grupos de objetos con características comunes y logramos refinar la clasificación de los mismos según la profundidad óptica de sus envolturas.

En el siguiente capítulo mostraremos los resultados obtenidos para los 8 objetos restantes, incluyendo el análisis de la variabilidad de los discos. 


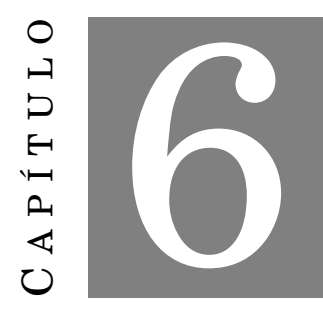

ANÁLisis DE LA VARIABILIDAD

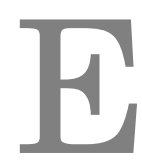

1 estudio de la variabilidad de las estrellas Be es un tema en discusión desde hace más de un siglo. Esta variabilidad se ha asociado a diferentes geometrías para el disco (como por ejemplo un disco en precesión o la presencia de una sobredensidad tipo brazo espiral) o a procesos de crecimiento y disipación del disco (asociados con episodios de eyección esporádica de masa o mecanismos de pérdida de momento angular para objetos que rotan cerca de la velocidad crítica, Krtička et al., 2011). Como vimos en el capítulo anterior, las observaciones espectroscópicas en el IR aportan información sobre las propiedades de los discos, por lo que es interesante realizar un análisis de la evolución temporal de los objetos observados a lo largo de más una década. Este análisis nos permitirá discutir los distintos escenarios propuestos para explicar la variabilidad.

Los resultados individuales para cada objeto se presentarán en la Sección 6.1. En la Sección 6.2 discutiremos algunos resultados que nos permitirán obtener información acerca de la evolución de las envolturas de estos objetos.

\subsection{Mediciones y análisis de cada objeto}

Para cada estrella presentaremos información relevante de la literatura, describiremos los espectros IR y presentaremos los resultados obtenidos. Los parámetros de las líneas observadas en cada banda se muestran en el Apéndice B. A partir de esos valores, analizamos el comportamiento de las series de hidrógeno y calculamos las densidades columnares, extensiones relativas y radios internos de las regiones de formación, mediante la aplicación de la metodología propuesta en el capítulo anterior (métodos $N$, FWHM y $\Delta V$ ). Analizamos además la variabilidad de los parámetros de los discos a lo largo de los años. 


\subsubsection{BK Cam - HD 20336}

BK Cam es una estrella de tipo espectral B2.5 V, identificada como Be por Merrill et al. (1925). Slettebak (1951) reportó la presencia de emisión en las líneas $\mathrm{H} \alpha, \mathrm{H} \beta$ y O I $\lambda 8446 \AA$ A. Esta estrella forma parte de un sistema binario (McLaughlin, 1963) resuelto a partir de observaciones realizadas con interferometría speckle por Mason et al. (2009). La línea $\mathrm{H} \alpha$ ha presentado un incremento en su ancho equivalente entre el 2003 y el 2007 (Granada et al., 2010; Jones et al., 2011). También se reportó variabilidad espectroscópica con un período de 4.5 años (McLaughlin, 1963).

A partir de observaciones IR obtenidas en el año 2008, Granada et al. (2010) ubicaron a BK Cam en la región superior del diagrama de Lenorzer, lo que sugiere la presencia de una región ópticamente gruesa cercana a la estrella central. Esta ubicación, junto con el aumento en la emisión de $\mathrm{H} \alpha$, llevó a los autores a concluir que la misma presentaba una envoltura circunestelar en crecimiento.

Según los datos reportados por Vieira et al. (2017) a partir del modelado del continuo IR, BK Cam presentaba valores de $\log \rho_{0}=-10.8 \pm 0.3$ y $n=3.3 \pm 0.3$ entre mayo del 2006 y agosto del 2007, y $\log \rho_{0}=-11.2 \pm 0.1$ y $n=2.6 \pm 0.1$ durante el 2010. Según los criterios establecidos por estos autores, BK Cam presentaba un disco estable en 2006/2007, que se disipaba hacia el 2010.

Figura 6.1: Espectro de BK Cam en la banda J.

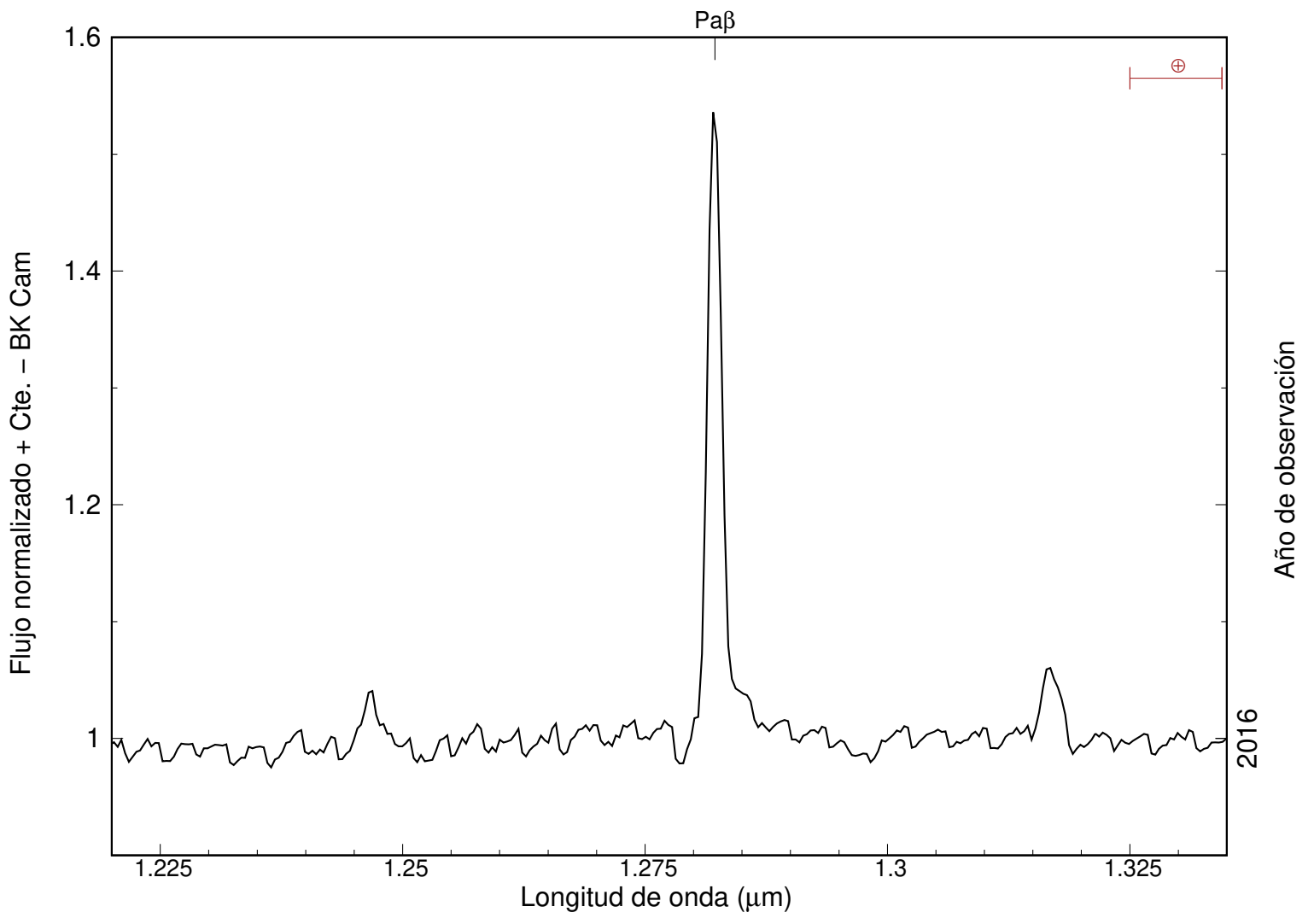


Figura 6.2: Espectros de BK Cam en las bandas H (superior) y K (inferior).
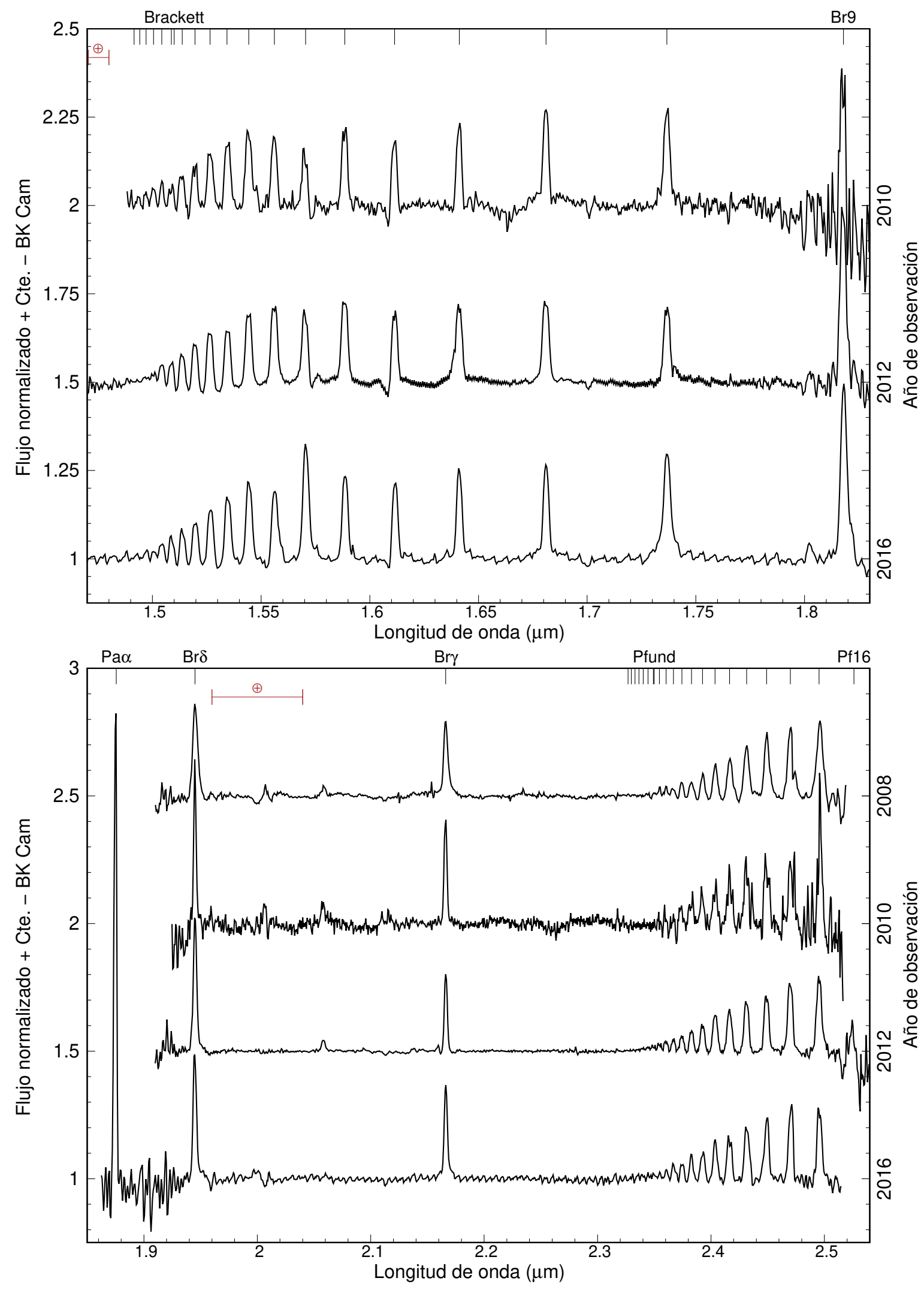
Figura 6.3: Espectros de BK Cam en la banda L (superior) y líneas más intensas de cada banda (inferior). Junto a Pf $\gamma$ se observa $\mathrm{Hu}_{17}$.
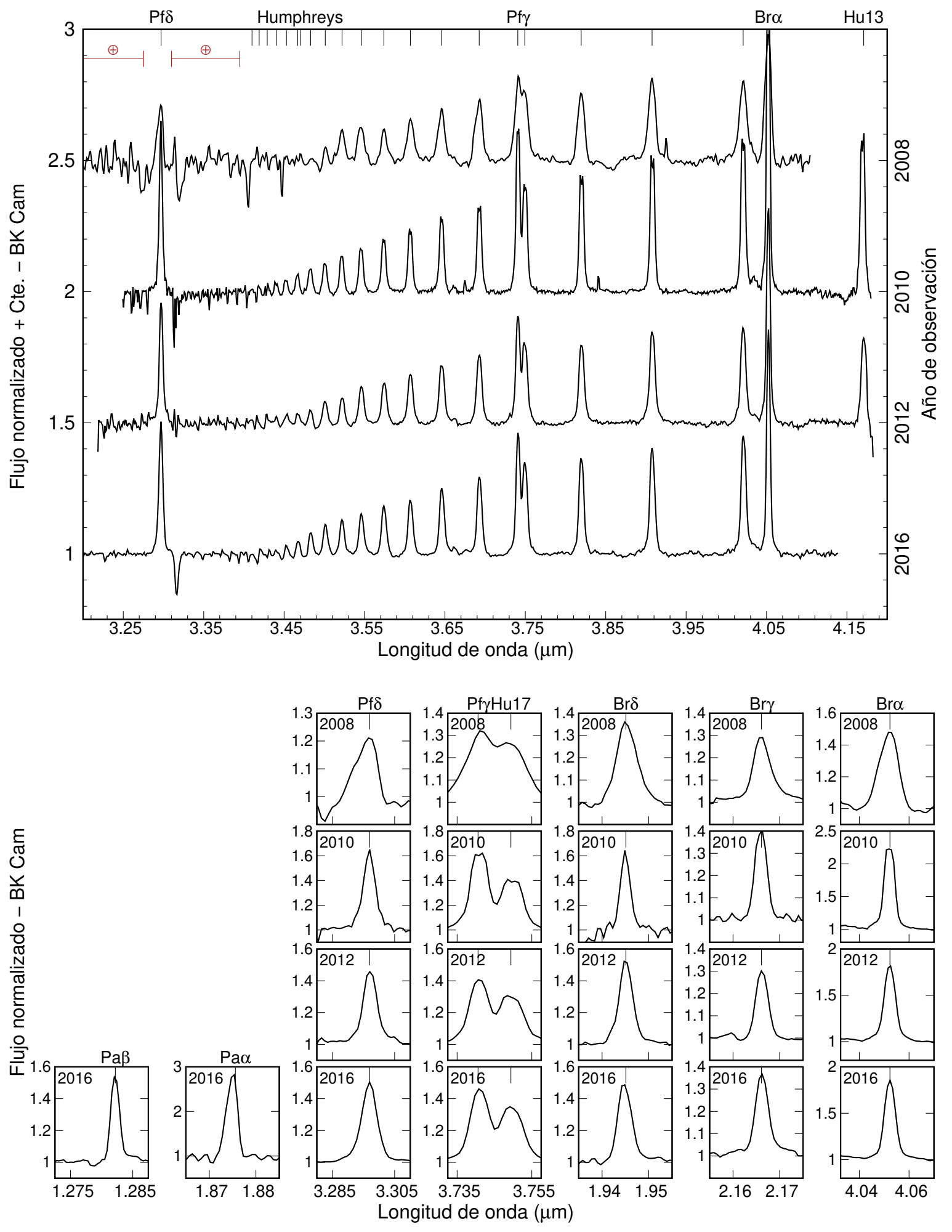


\section{Descripción de los espectros y mediciones realizadas}

En las Figs. 6.1 a 6.3 se presentan los espectros obtenidos en los cinco turnos de observación y las líneas más intensas de cada banda. La observación del 2008 fue realizada con el espectrógrafo NIRI, y a partir del 2010 se utilizó GNIRS. No todas las bandas fueron observadas en todos los turnos (ver Tabla 3.6). Las líneas de hidrógeno observadas se encuentran en emisión en todas las observaciones. Los miembros más altos de las series de Pfund y Brackett presentan perfiles con dos picos en la observación de los años 2010, 2012, al igual que las líneas de la serie de Humphreys en el año 2010. Según la clasificación propuesta por Mennickent et al. (2009), clasificamos a la estrella como perteneciente al grupo I en 2008 y 2016 , y al grupo II en 2010 y 2012. La cantidad de líneas de cada serie que se alcanzan a distinguir y las intensidades de las mismas varían entre cada observación. Los valores de EW, Fl y FWHM obtenidos pueden encontrarse en las Tablas B.1 a B.4, corregidos considerando la absorción fotosférica de una estrella con $T_{\text {eff }}=19000 \mathrm{~K}$ y $\log g=4.0$ según los parámetros estelares de BK Cam (ver Tabla $3.3)$.

\section{Ubicación en el diagrama de Lenorzer}

En la Fig. 6.4 se muestra la posición de BK Cam en el diagrama de Lenorzer para las distintas observaciones (la posición correspondiente a la observación del 2008 fue previamente reportada por Granada et al., 2010). El desplazamiento del objeto en el diagrama es pequeño, alrededor del límite definido por Mennickent et al. (2009) para los grupos I y II.

Figura 6.4: Ubicación de BK Cam en el diagrama de Lenorzer en las diferentes observaciones.

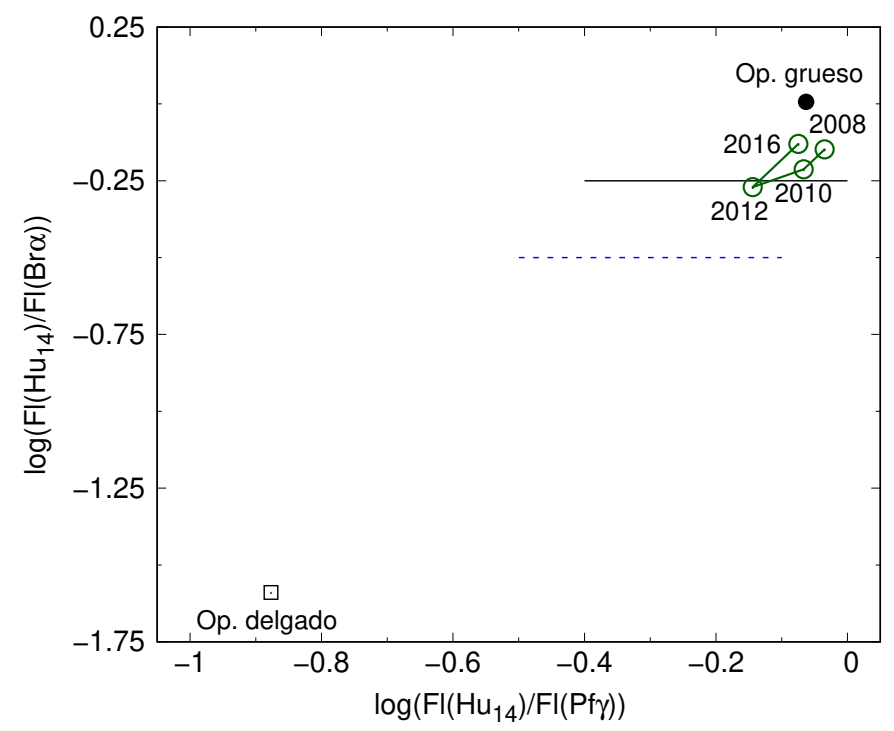




\section{Comportamiento de las series de hidrógeno}

En la Fig. 6.5 se muestran los valores de EW/ $\lambda$ según la longitud de onda. No se observan diferencias significativas entre las distintas observaciones. Los valores obtenidos convergen hacia $9 \cdot 10^{-4}$ en el 2008 y $2016,11 \cdot 10^{-4}$ en el 2010 y $8 \cdot 10^{-4}$ en el 2012.

En los gráficos de FWHM vs longitud de onda de la Fig. 6.6 se ilustran los comportamientos de cada serie a lo largo de los años. En todas las observaciones los valores de FWHM crecen hacia los miembros más altos de cada serie, hasta alcanzar un valor máximo y luego decrecer. Los valores máximos obtenidos se encuentran alrededor de $2 \mathrm{~V} \sin (i)$, salvo para las líneas de la serie de Humphreys en el año 2010 donde son un poco menores que $2 V \sin (i)$. Los valores de FWHM de las líneas $\mathrm{Pa} \alpha$ y $\mathrm{Pa} \beta$ son los más bajos obtenidos, indicando una región de formación más alejada de la estrella central

Los cocientes de los flujos para las líneas de las distintas series y las comparaciones con los casos ópticamente grueso y delgado para las series de Humphreys, Pfund y Brackett son mostrados en la Fig. 6.7. En todas las series observamos que los miembros más altos permanecen sobre la recta correspondiente al caso ópticamente delgado. Las líneas que se apartan del caso ópticamente delgado se listas en la Tabla 6.1.

Figura 6.5: BK Cam - EW/ $\lambda$ vs. $\lambda$ de las líneas observadas en las diferentes series en las distintas observaciones.

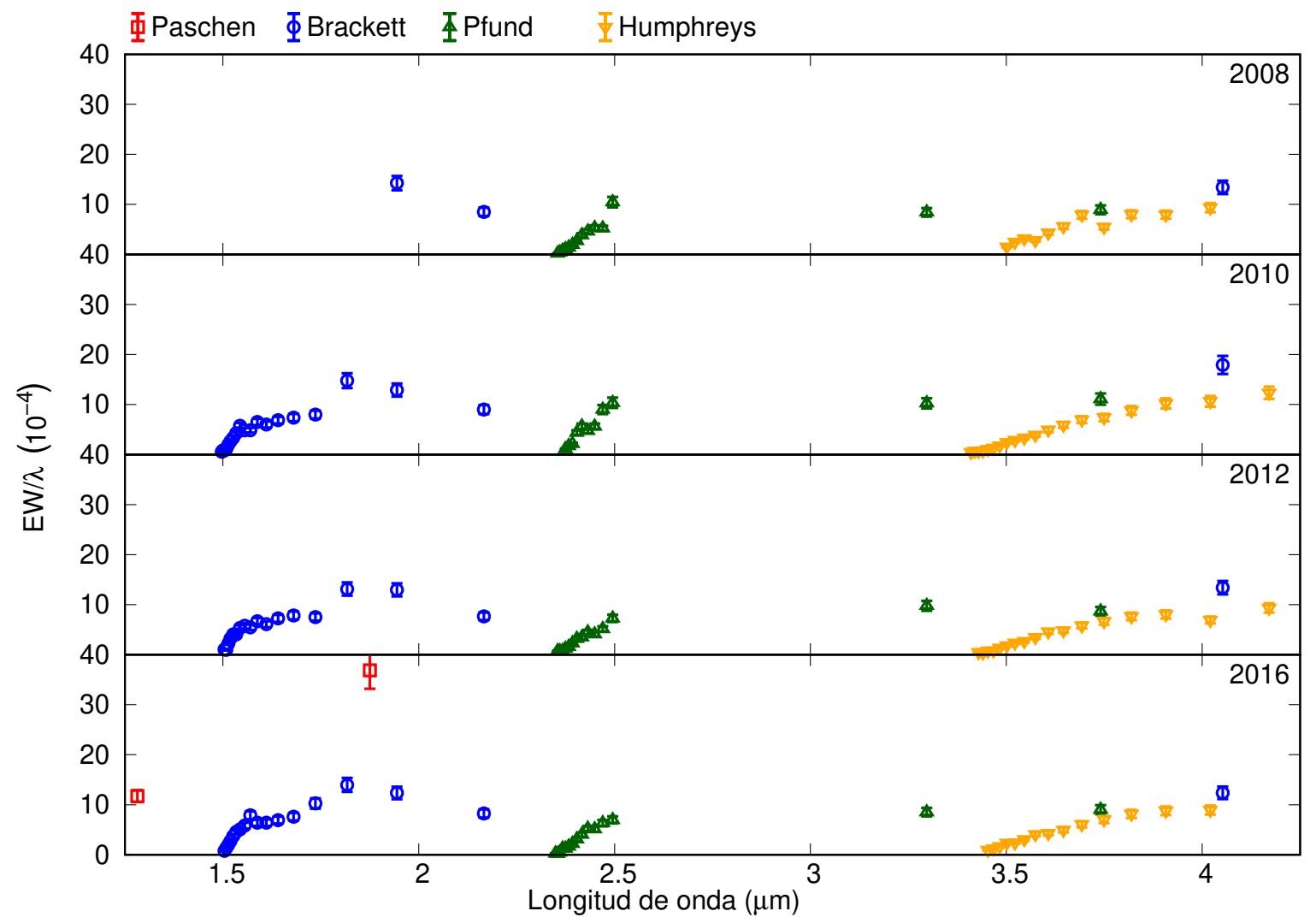


Figura 6.6: BK Cam - FWHM vs. $\lambda$ de las líneas observadas en las diferentes series en las distintas observaciones. La línea punteada corresponde a un valor de $2 V \sin (i)=682 \mathrm{~km} / \mathrm{s}$, y la región sombreada al error correspondiente (Tabla 3.3). Los símbolos utilizados son los mismos que en la Fig. 6.5.

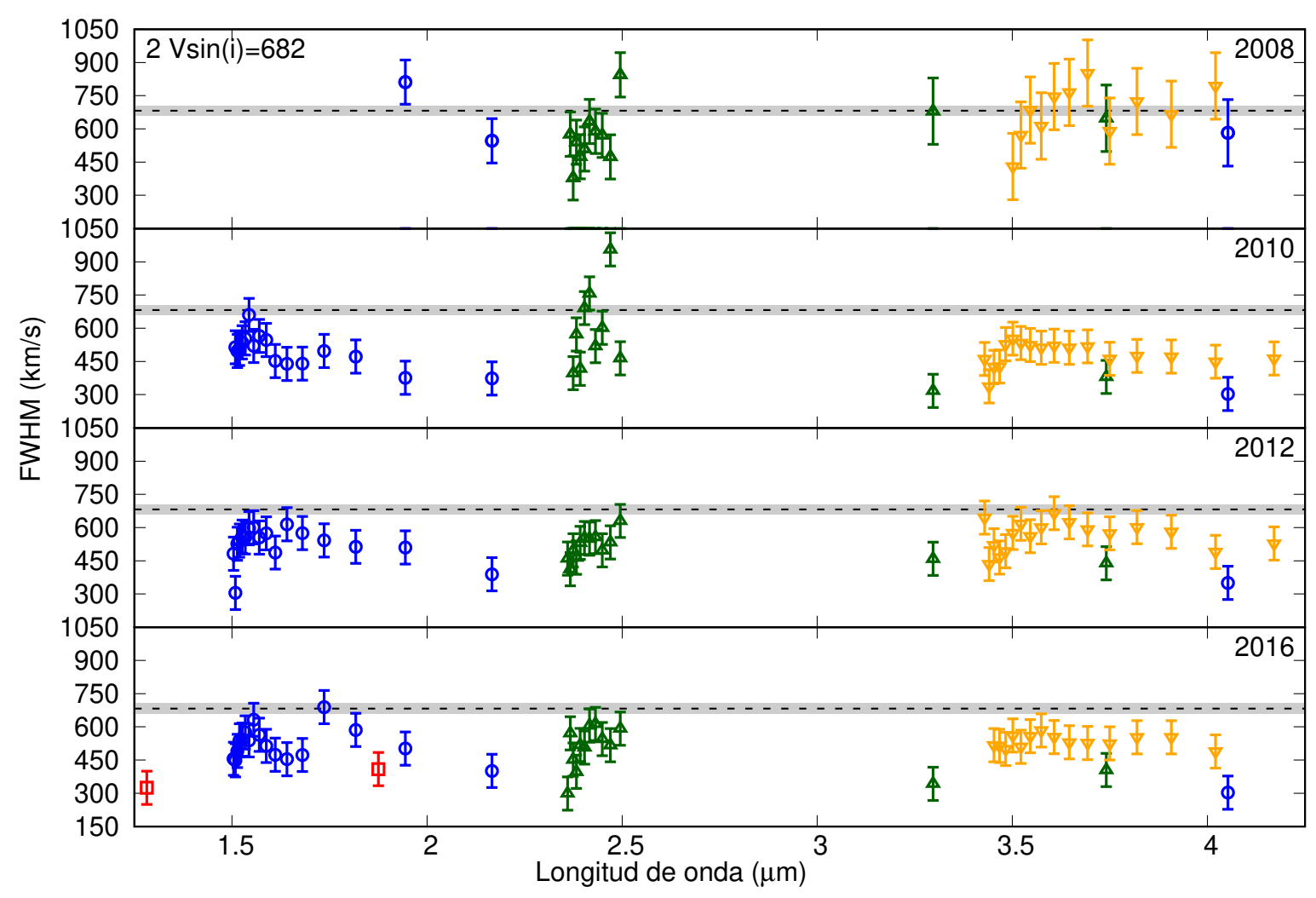

Tabla 6.1: BK Cam - Líneas que se apartan del caso ópticamente delgado. Los miembros más altos de la serie de Brackett no fueron observados en el 2008.

\begin{tabular}{l|cccc}
\hline \hline & 2008 & 2010 & 2012 & 2016 \\
\hline Humphreys & $14-17$ & $13-17$ & $13-16$ & $14-16$ \\
Pfund & $\gamma-\delta$ & $\gamma-\delta$ & $\gamma-\delta$ & $\gamma-\delta$ \\
Brackett & $\times$ & $9-16$ & $9-18$ & $9-14$ \\
\hline
\end{tabular}

Obtuvimos además que los valores para el cociente $\mathrm{EW}(\mathrm{Br} \alpha) / \mathrm{EW}(\mathrm{Br} \gamma)$ se encuentran en el rango 2.8-3.7. Estos valores están en el intervalo correspondiente a objetos del grupo I y en la región entre grupos. El menor valor ( 2.8) corresponde al año 2016, y el mayor ( 3.7) al 2010. Además, en el año 2016 obtuvimos que $\mathrm{EW}(\mathrm{Pa} \alpha) / \mathrm{EW}(\mathrm{Pa} \beta) \sim 4.5$. 


\section{Determinación de densidades columnares y extensiones de las regiones de formación}

En las Tablas B.5 a B.8 se muestran las densidades columnares, extensiones y radios internos de las regiones de formación obtenidos a partir de los métodos $N$, FWHM y $\Delta V$. Presentamos también valores aproximados en radios estelares para las líneas en las cuales no pudimos obtener la separación de los dos picos en emisión.

Las densidades columnares más bajas corresponden a la serie de Pfund, y las más altas a la de Brackett. En todas las series, las mayores densidades se observan en el 2008, seguidas por las obtenidas en el 2012, 2016 y 2010.

La Fig. 6.8 (superior) muestra los radios internos de las regiones de formación relativos a $\mathrm{Br} \delta$. Los radios alcanzan un valor de $\sim 4 R_{\mathrm{Br} \delta}$ en el $2008, \sim 2 R_{\mathrm{Br} \delta}$ en el $2010 \mathrm{y} \sim 3 R_{\mathrm{Br} \delta}$ en el 2012 y 2016. La serie de Humphreys es la que se forma en la región más interna, más extendida en el 2008 y más compacta en el 2016. La serie de Pfund también presenta regiones de formación extendidas en los años 2008 y 2016, y más compacta en 2010 y 2012 . Las regiones de formación

Figura 6.7: BK Cam - Cociente de flujos para las líneas de la serie de Humphreys, Pfund y Brackett. En cada gráfico, la línea continua corresponde al cociente para un caso ópticamente grueso, y la línea punteada al caso ópticamente delgado. En la parte superior se identifican las líneas correspondientes.
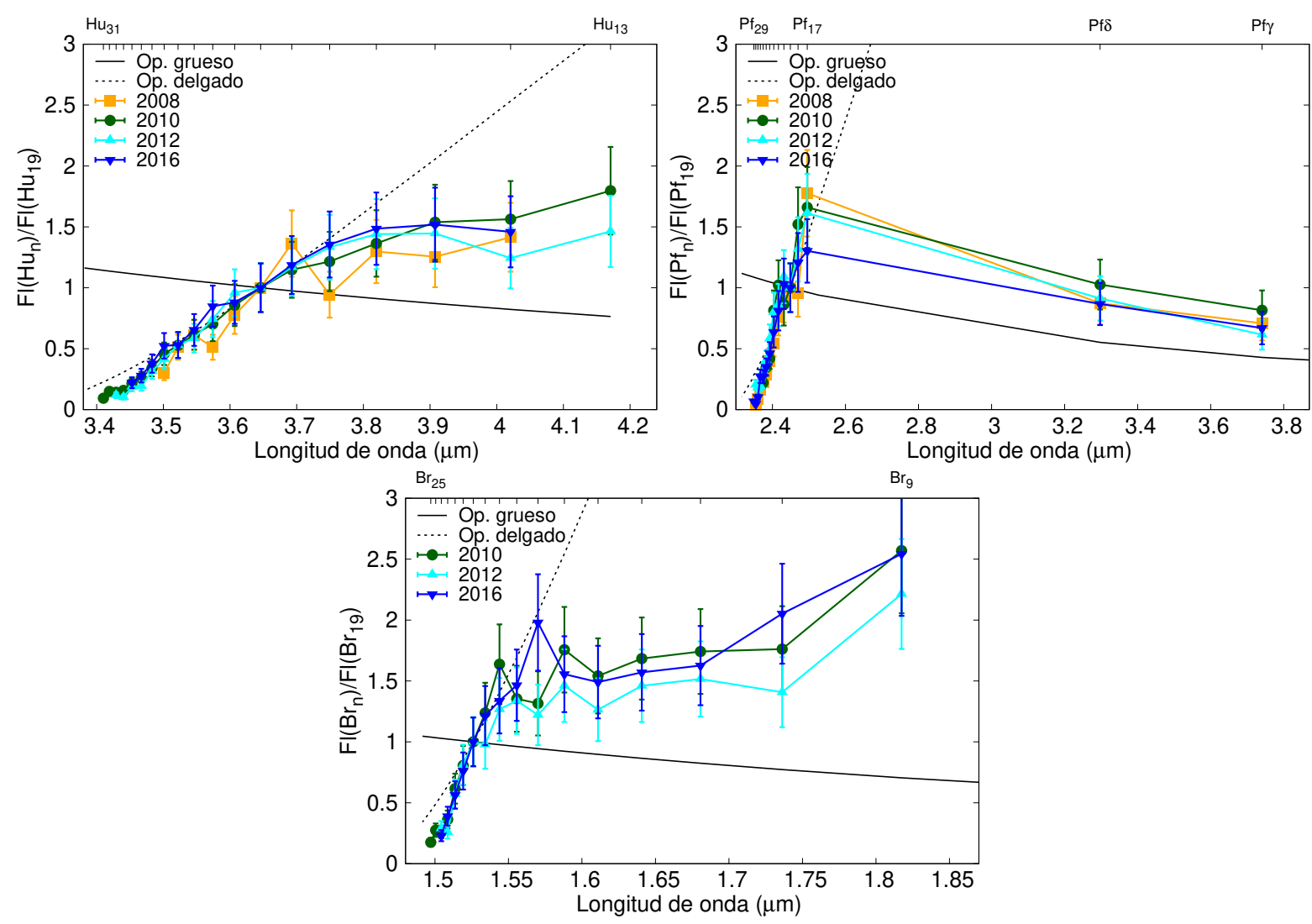
de la serie de Brackett no muestra cambios significativos a lo largo de estos años.

Para las líneas que no pudimos obtener los tamaños en radios estelares, calculamos los valores a partir del tamaño relativos a $\mathrm{Pf}_{18}$ para las observaciones adquiridas en el 2010 y a $\mathrm{Br}_{10}$ en el 2012. Los valores se muestran en la Fig. 6.8 (inferior). La mayoría de las líneas se forman en regiones con radios interiores menores a $\sim 20 R_{\star}$ en la observación del 2010 y menores a $\sim 10 R_{\star}$ en el 2012. Las líneas que se forman en las regiones más alejada de la estrella central son los primeros miembros de la serie de Brackett. 
Figura 6.8: BK Cam - Radios internos de las regiones emisoras de las diferentes líneas relativos a la región emisora de $\operatorname{Br} \delta$ y en radios estelares. Los símbolos utilizados son los mismos que en la Fig. 6.5.
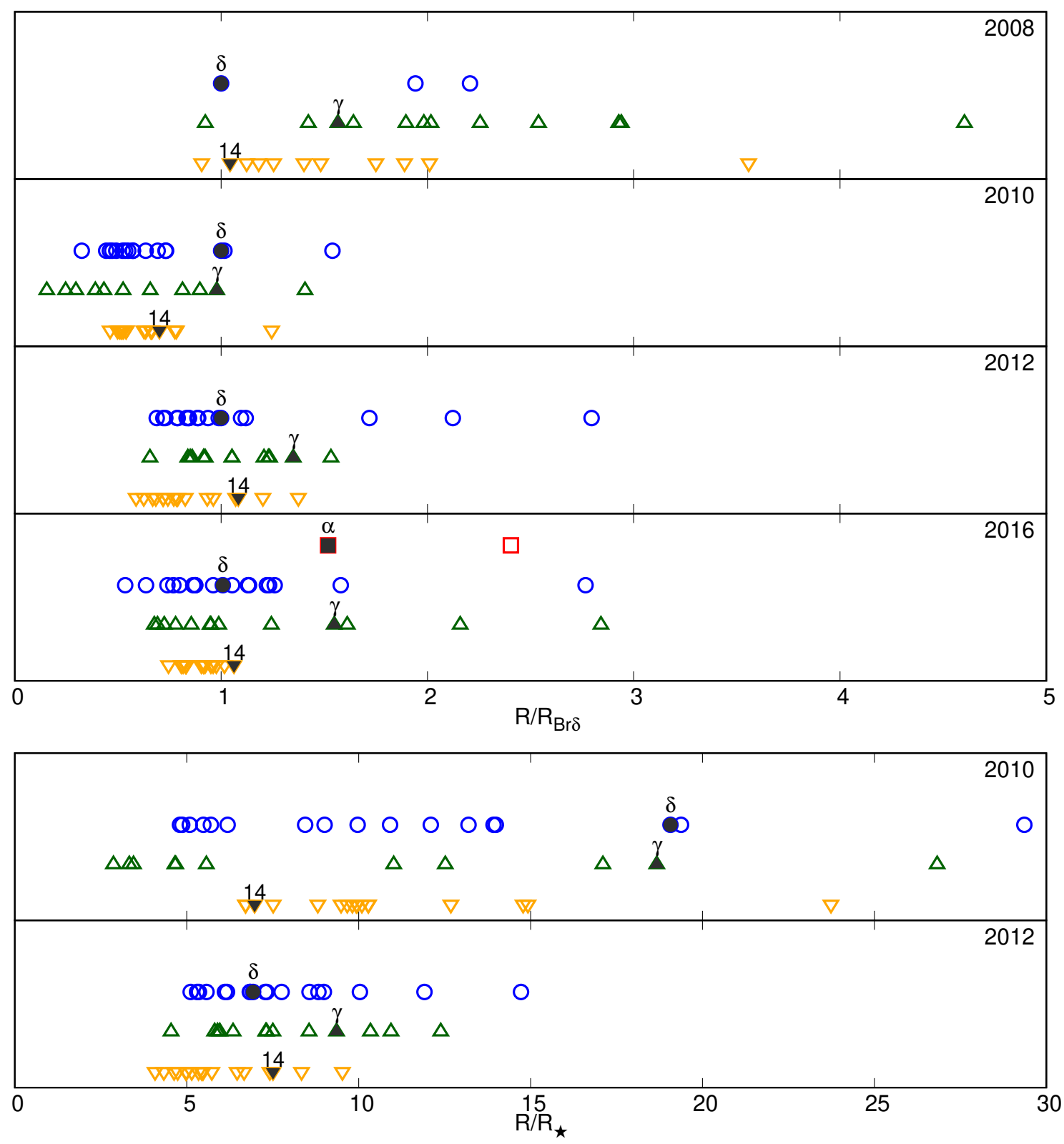


\subsubsection{Tau - HD 23862}

28 Tau es una estrella de tipo espectral B8 V, miembro del cúmulo Pléyades. Slettebak (1951) reportó la presencia de la línea $\mathrm{H} \alpha$ con dos picos en la emisión, junto con una pequeña emisión doble en $\mathrm{H} \beta$. Es una binaria espectroscópica de un solo espectro, con una compañera de baja masa (Nemravová et al., 2010). Tiene un período orbital de 218 días, semiamplitud de $5.9 \mathrm{~km} \mathrm{~s}^{-1}$, y un excentricidad en la órbita de 0.60 (Katahira et al., 1996a,b). La solución orbital propuesta por Nemravová et al. (2010) tiene una órbita con semieje mayor entre 223.5 y $225.5 R \odot$, y una separación en el periastro entre $53 R_{\odot}$ y $53.4 R_{\odot}$, dependiendo de la inclinación del sistema.

Esta estrella ha presentado transiciones entre las fases B, Be y Be-shell, con un período de 34 años (Sadakane et al., 2005). A partir del análisis de la variación de largo período del ángulo de polarización, Hirata (2007) propuso un modelo de un disco en precesión, que explicaría también las variaciones de fase observadas. Pollmann (2018) estudió el perfil de la línea $\mathrm{H} \alpha$ entre octubre de 2004 y marzo de 2018, y encontraron que el máximo en la absorción central del perfil tipo shell coincide con el mínimo valor de V/R y la velocidad radial, como resultado de la precesión del disco.

Taranova et al. (2008) reportaron que la emisión en el IR cercano la estrella se debilitó entre 1999 y 2007. A su vez, el perfil de $\mathrm{H} \alpha$ de doble pico que se mostraba débil en 1990, se intensificó hasta 1996, donde presentó una línea intensa con un solo pico. En 1999 la emisión decayó durante un tiempo, y luego volvió a intensificarse. A partir del 2001 se observaron evidencias de líneas con dos picos en emisión, siendo en el 2002 el pico azul más intenso que el rojo (V>R). Posteriormente, Jones et al. (2011) reportaron que entre el 2003 y 2007 el ancho equivalente de la línea H $\alpha$ estaba decreciendo. Hacia el final del 2007, la línea $\mathrm{H} \alpha$ comenzó a intensificarse, coincidiendo con el inicio de un nuevo período.

El disco de la estrella fue parcialmente resuelto con el arreglo CHARA por Touhami et al. (2013), quienes obtuvieron una relación entre los radios del disco y de la estrella de 1.879, tomando un ángulo de posición $\mathrm{PA}=159^{\circ} \mathrm{y}$ un cociente entre los ejes $r=0.438$. Por otra parte, Silaj et al. (2014) realizan un ajuste de la línea $\mathrm{H} \alpha$, y obtuvieron como parámetros del mejor ajuste $i=76^{\circ}$, $\rho_{0}=6.2 \cdot 10^{-12} \mathrm{~g} \mathrm{~cm}^{-3}$ y $n=2.5$.

Granada et al. (2010) estudiaron espectros obtenidos en el año 2006 y ubicaron a la estrella en la región correspondiente a un disco ópticamente delgado en el diagrama de Lenorzer. Touhami et al. (2010) también estudiaron el espectro IR de este objeto, encontrando características similares a las reportadas por Granada et al. (2010). En el 2011, Sabogal et al. (2017) realizaron observaciones de 28 Tau en la banda L, e indicaron que la misma pertenecía al Grupo II, con líneas de $\mathrm{H}$ muy débiles. Además, contrastaron la apariencia de las líneas observadas en el IR con el comportamiento de $\mathrm{H} \alpha$ a lo largo del tiempo. Dentro de la clasificación definida por estos autores a partir de la ubicación de los objetos y de los cambios en la apariencia de $\mathrm{H} \alpha$, la catalogaron como estrella con disco en disipación, ya que el espectro de $\mathrm{H} \alpha$ mostraba en el 2001 una emisión intensa, y un perfil tipo shell en el 2008. Ese cambio en el perfil de H $\alpha$ debería verse reflejado en un corrimiento de la posición en el diagrama de Lenorzer, hacia la parte inferior del 
mismo donde se ubica en el 2011.

\section{Descripción de los espectros y mediciones realizadas}

Los espectros obtenidos en los diferentes turnos, junto con las líneas más intensas de cada banda, se muestran en las Figs. 6.9 a 6.11. La observación del 2006 fue realizada con el espectrógrafo NIRI, mientras que las siguientes fueron obtenidas con GNIRS. No todas las bandas fueron observadas en todos los turnos (ver Tabla 3.6). En todos los casos las líneas de hidrógeno presentan contribución de la envoltura, ya sea generando perfiles en emisión o estructuras tipo shell superpuestas a la absorción fotosférica (como en los miembros más altos de las series de Brackett y Pfund). En muchos casos los perfiles presentan dos picos en emisión, como los miembros más altos de las series de Pfund y Humphreys en los años 2010, 2012 y 2016, o los primeros miembros de las series de Brackett y Pfund observados en las bandas K y L en los años 2010 y 2012, y los de la banda L en el año 2006. Según el criterio de clasificación de Mennickent et al. (2009), catalogamos a este objeto como perteneciente al Grupo II en todas las observaciones. En las Tablas B.9 a B.12 se listan los valores de EW, Fl y FWHM de las líneas observadas cada año en cada banda, corregidas mediante un espectro sintético correspondiente a una estrella con $T_{\text {eff }}=12000 \mathrm{~K}$ y $\log g=4.0$, según los parámetros de 28 Tau (ver Tabla 3.3).

Figura 6.9: Espectro de 28 Tau en la banda J.

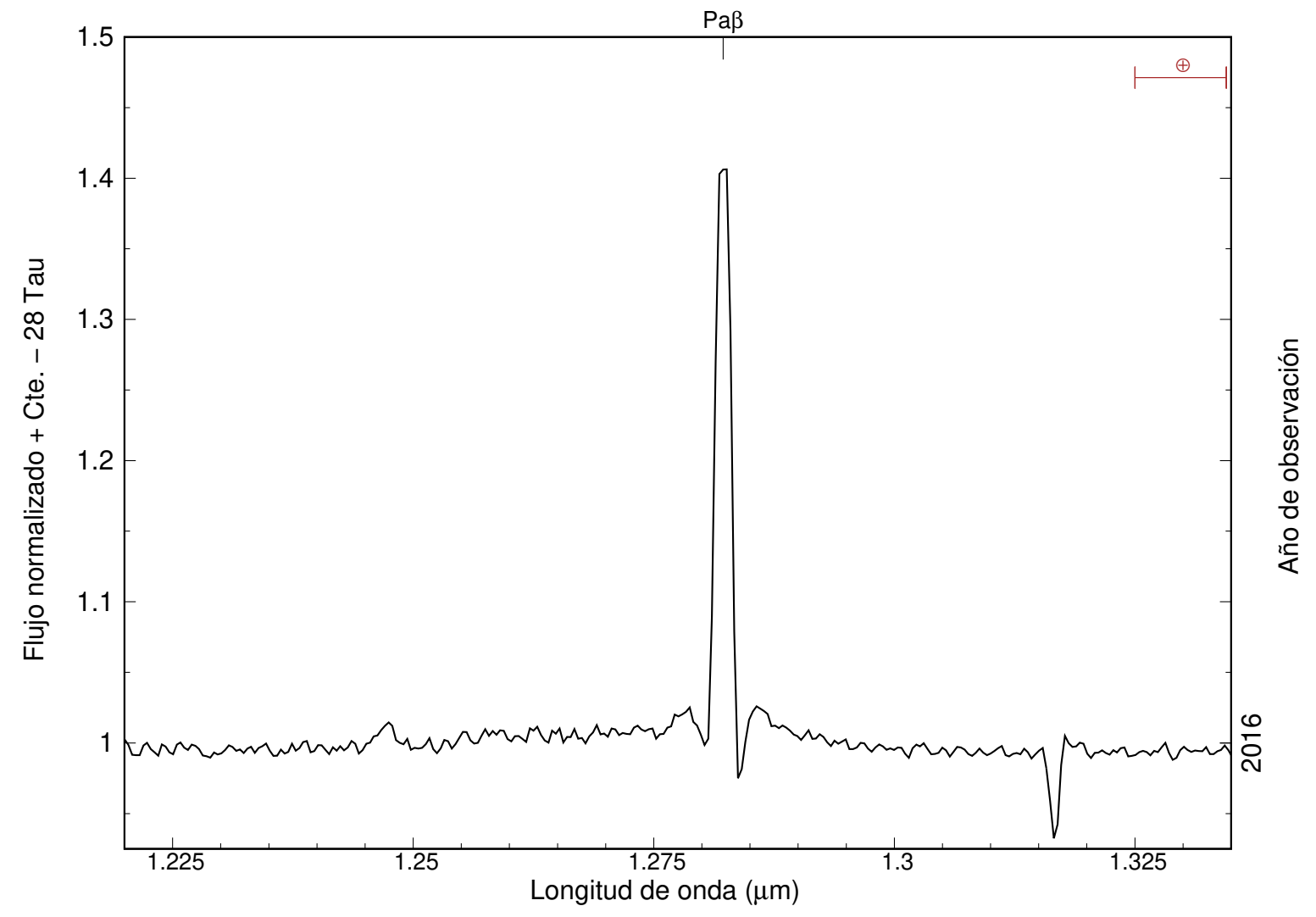


Figura 6.10: Espectros de 28 Tau en las bandas H (superior) y K (inferior).
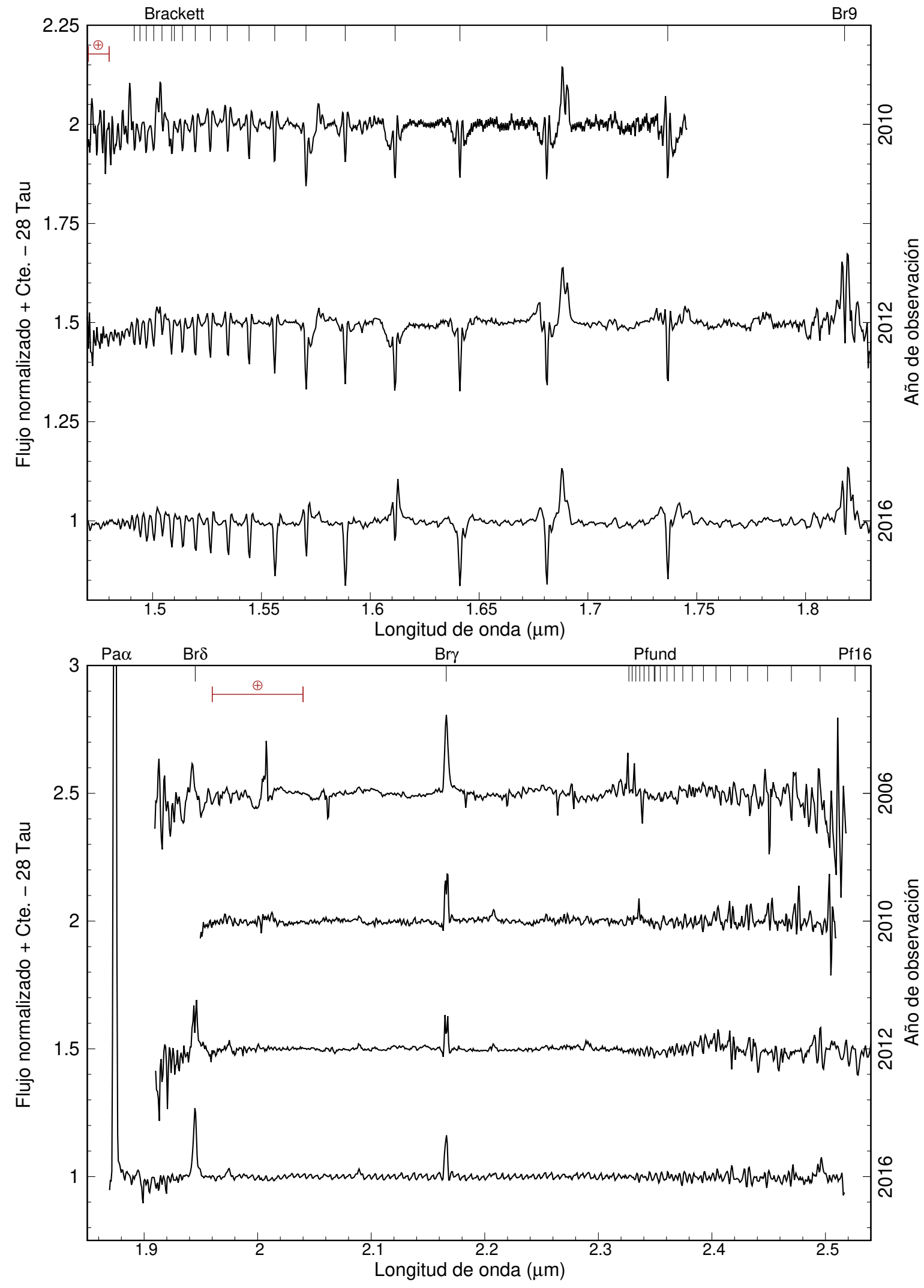
Figura 6.11: Espectros de 28 Tau en la banda L (superior) y líneas más intensas de cada banda (inferior). Junto a Pf $\gamma$ se observa $\mathrm{Hu}_{17}$.
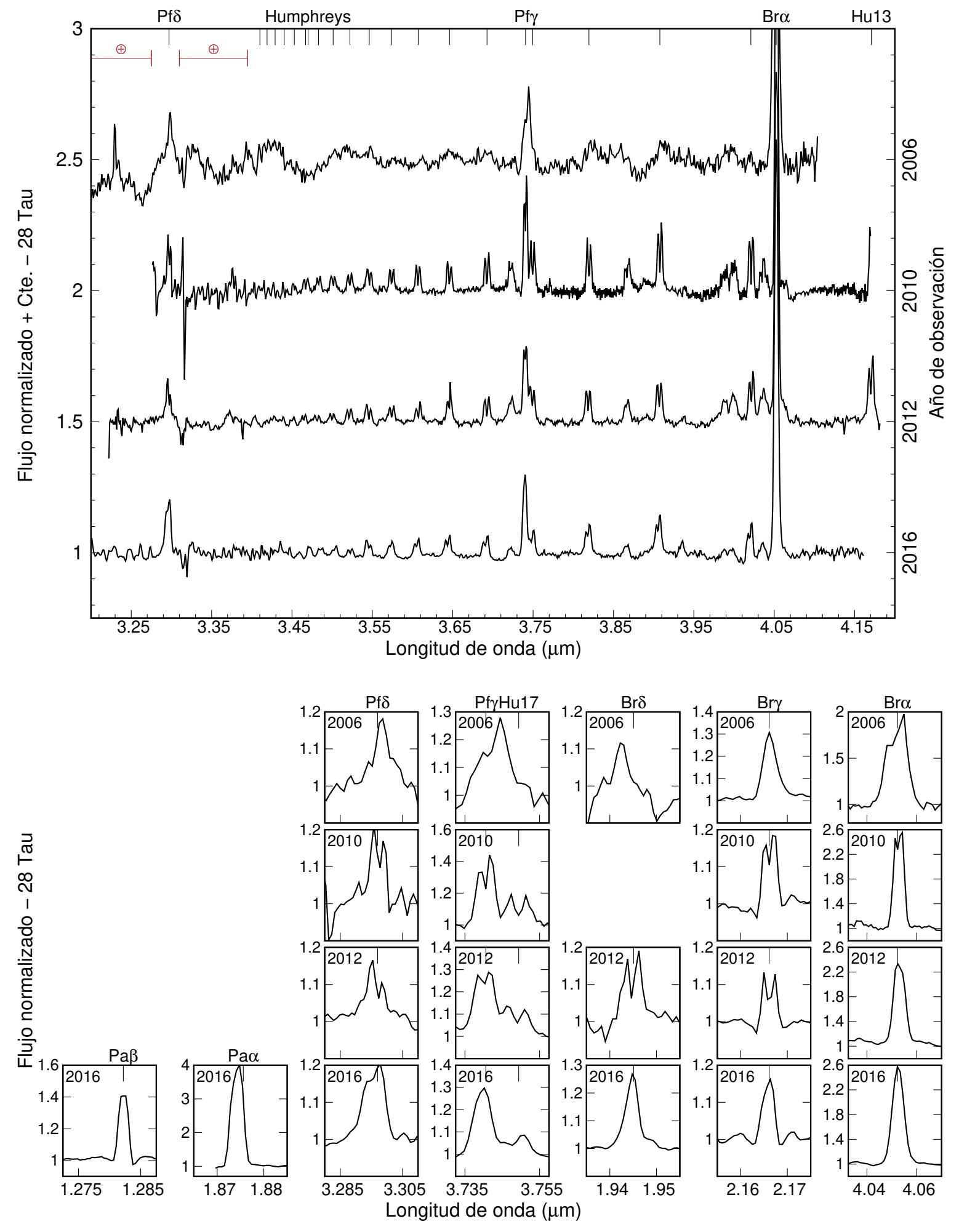
Figura 6.12: Ubicación de 28 Tau en el diagrama de Lenorzer en las diferentes observaciones.

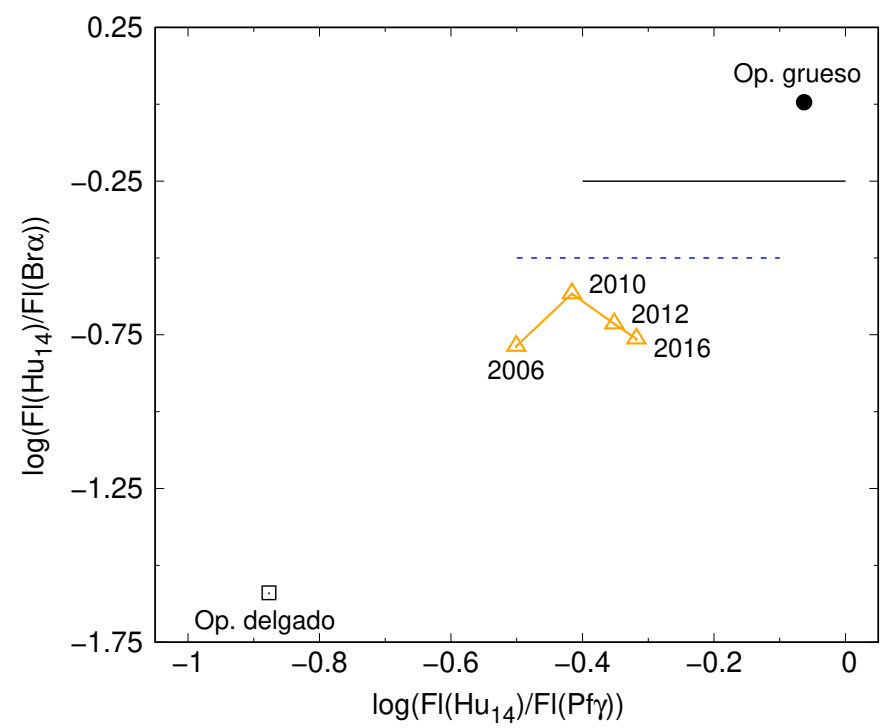

\section{Ubicación en el diagrama de Lenorzer}

Utilizando la observación del año 2006, 28 Tau se ubica en una posición central del diagrama de Lenorzer, en la zona correspondiente a los objetos del Grupo II Granada et al. (previamente reportado por 2010). En las observaciones siguientes se observa que la estrella se mueve sobre la diagonal que une los casos ópticamente grueso y delgado, acercándose a la zona ópticamente gruesa y moviéndose hacia la derecha del diagrama. A pesar de variar su posición en el diagrama, siempre se mantiene en la zona central del mismo, sin llegar a la región correspondiente a las estrellas del Grupo I.

\section{Comportamiento de las series de hidrógeno}

La Fig. 6.13 muestra los valores obtenidos para $\mathrm{EW} / \lambda$ en función de la longitud de onda. Los valores convergen hacia $4 \cdot 10^{-4}$ en la observación del 2012 y hacia $\sim 5$ en 2010 y 2016 . En la observación del año 2006 no tenemos puntos suficientes como para definir una tendencia.

Los valores obtenidos para los FWHM se muestran en la Fig. 6.14. Para la serie de Humphreys los FWHM se encuentran cercanos a $2 \mathrm{~V} \sin (i)$, indicando una región de formación cercana a la fotosfera estelar. En la observación del 2012 los FWHM son mayores a medida que observamos los miembros más altos de la serie, mientras que en el 2010 y 2016 los valores crecen hasta llegar a un máximo en $\mathrm{Hu}_{20}$ y luego decrecen. Los FWHM obtenidos para Pf $\gamma$ son similares en tres de las cuatro observaciones, mientras que los obtenidos para $\mathrm{Pf} \delta$ presentan mayor variabilidad. Los miembros superiores de la serie de Brackett presentan perfiles tipo shell, por lo que solo pudimos medir los FWHM para los primeros miembros de la serie. Para $\operatorname{Br} \delta$ en el 2012 se observa un ensanchamiento mayor a $2 V \sin (i)$. En tres de las cuatro observaciones, los FWHM de Br $\alpha$ son 
Figura 6.13: $28 \mathrm{Tau}-\mathrm{EW} / \lambda$ vs. $\lambda$ de las líneas observadas en las diferentes series en las distintas observaciones.

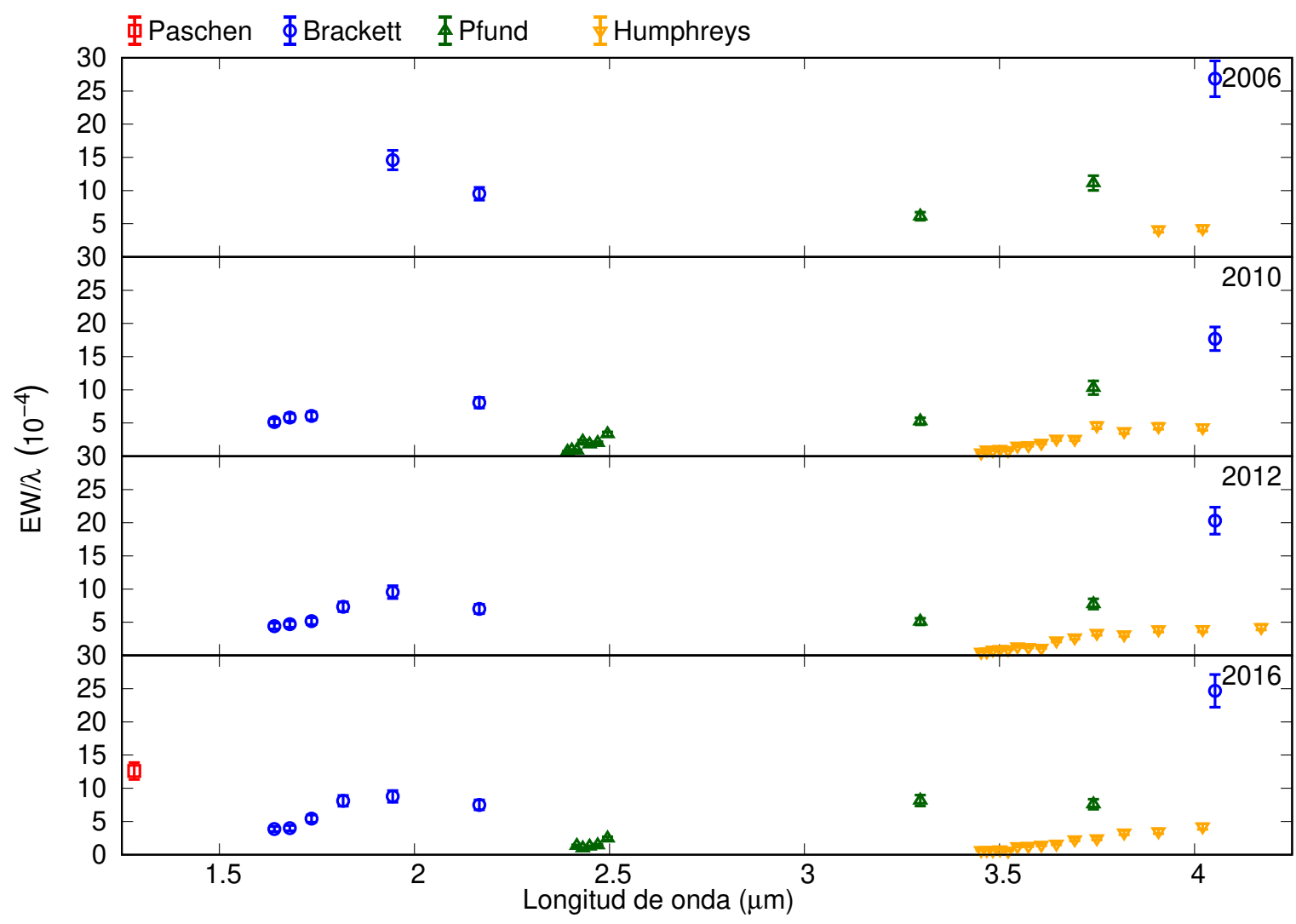

Tabla 6.2: 28 Tau - Líneas que se apartan del caso ópticamente delgado. Las líneas observadas de la serie de Brackett se mantienen sobre el caso ópticamente delgado.

\begin{tabular}{l|ccc}
\hline \hline & 2010 & 2012 & 2016 \\
\hline Humphreys & $14-16$ & $13-16$ & $14-15$ \\
Pfund & $\gamma-\delta$ & - & $\gamma-\delta$ \\
Brackett & - & - & - \\
\hline
\end{tabular}

menores que los de $\mathrm{Br} \gamma$, indicando que $\mathrm{Br} \alpha$ se forma en una región más externa del disco. Para la serie de Paschen, los valores obtenidos en el 2016 también indican regiones de formación alejadas de la fotosfera.

Los cocientes de los flujos y su comparación con los casos ópticamente grueso y delgado se encuentran en la Fig. 6.15. Las líneas que se apartan del caso ópticamente delgado se listas en la Tabla 6.2. Calculamos también los cocientes de flujos para las primeras líneas de la serie de Pfund, y obtuvimos que $\mathrm{Fl}(\mathrm{Pf} \gamma) / \mathrm{Fl}(\mathrm{Pf} \delta)$ toma valores entre 0.75 y 1.5, máximo en 2010 y mínimo en 2016.

Para los cocientes EW(Br $\alpha) / E W(B r \gamma)$ obtuvimos valores $~ 5.4$ en el 2006 y 2012, 4.1 en el 2010 y $\sim 6.1$ en el 2016. Estos valores se encuentran dentro de lo esperado para una estrella del 
Figura 6.14: 28 Tau - FWHM vs. $\lambda$ de las líneas observadas en las diferentes series en las distintas observaciones. La línea punteada corresponde a un valor de $2 V \sin (i)=580 \mathrm{~km} / \mathrm{s}$, y la región sombreada al error correspondiente (Tabla 3.3). Los símbolos utilizados son los mismos que en la Fig. 6.13.

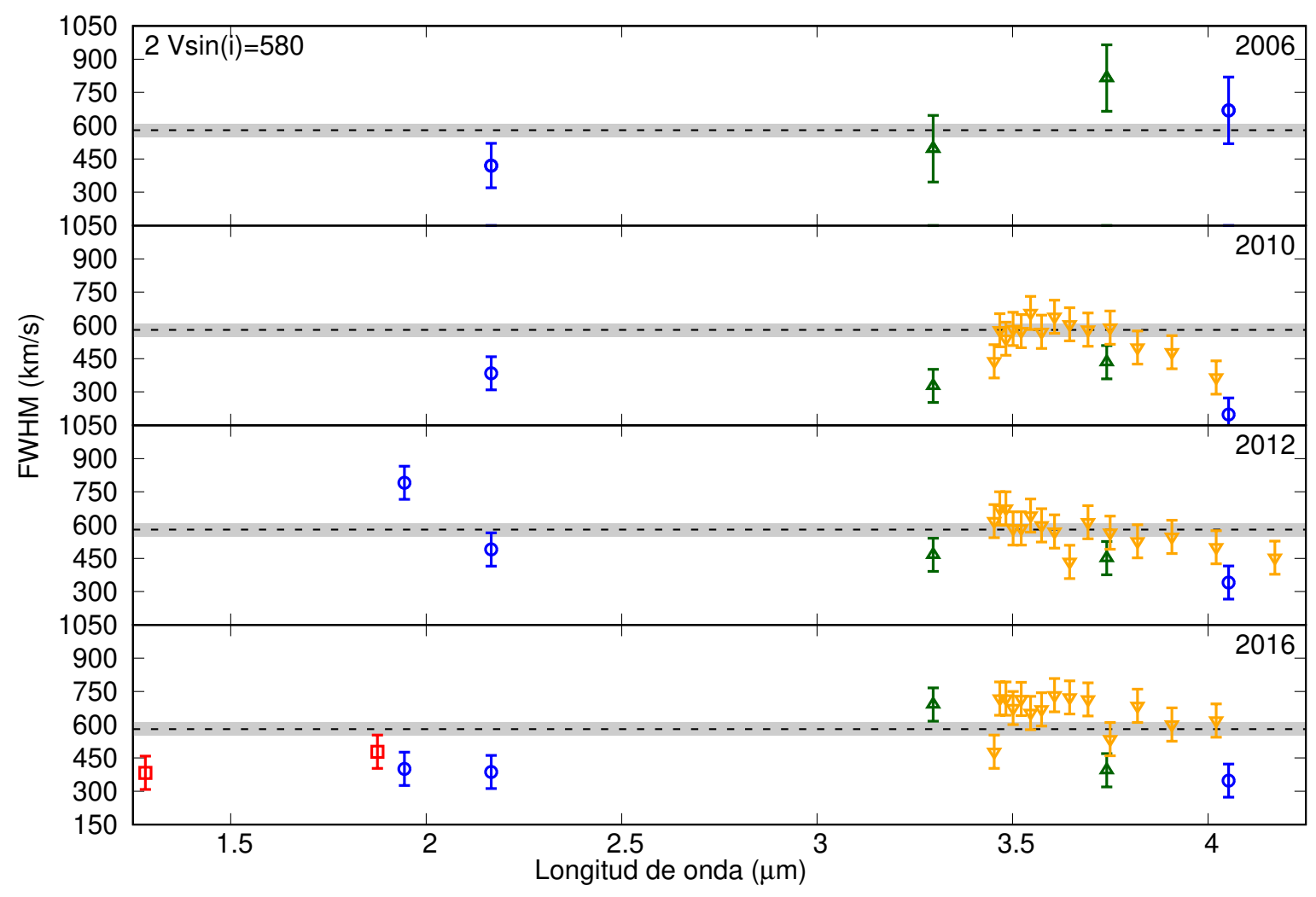

grupo II. Por otro lado, en la observación del $2016 \mathrm{EW}(\mathrm{Pa} \alpha) / \mathrm{EW}(\mathrm{Pa} \beta) \sim 6$.

\section{Determinación de densidades columnares y extensiones de las regiones de formación}

En las Tablas B.13 a B.18 se listan las densidades columnares, extensiones de las regiones de formación y radios internos de las regiones emisoras obtenidos utilizando los métodos $N$, FWHM y $\Delta V$. Para aquellas líneas que no pudimos determinar la separación de los picos, obtuvimos los radios utilizando los tamaños relativos a Pf $\gamma$ para la observación del 2006, $\mathrm{Hu}_{17}$ en el 2010, $\mathrm{Hu}_{15}$ en el 2012 y Pf $\delta$ en el 2016.

Para la serie de Humphreys, las densidades columnares obtenidas en el año 2012 son un poco mayores que las obtenidas en el 2010, mientras que las extensiones relativas de las regiones de formación se mantienen constantes. En la observación del 2016 las densidades columnares obtenidas son menores, al igual que en la serie de Pfund. Para la serie de Brackett no pudimos obtener utilizar el método $N$ ya que no pudimos determinar los FWHM.

En la Fig. 6.16 (superior) se muestran los tamaños relativos a Pf $\gamma$. En las observaciones del 
Figura 6.15: 28 Tau - Cociente de flujos para las líneas de la serie de Humphreys, Pfund y Brackett. En cada gráfico, la línea continua corresponde al cociente para un caso ópticamente grueso, y la línea punteada al caso ópticamente delgado. En la parte superior se identifican las líneas correspondientes.
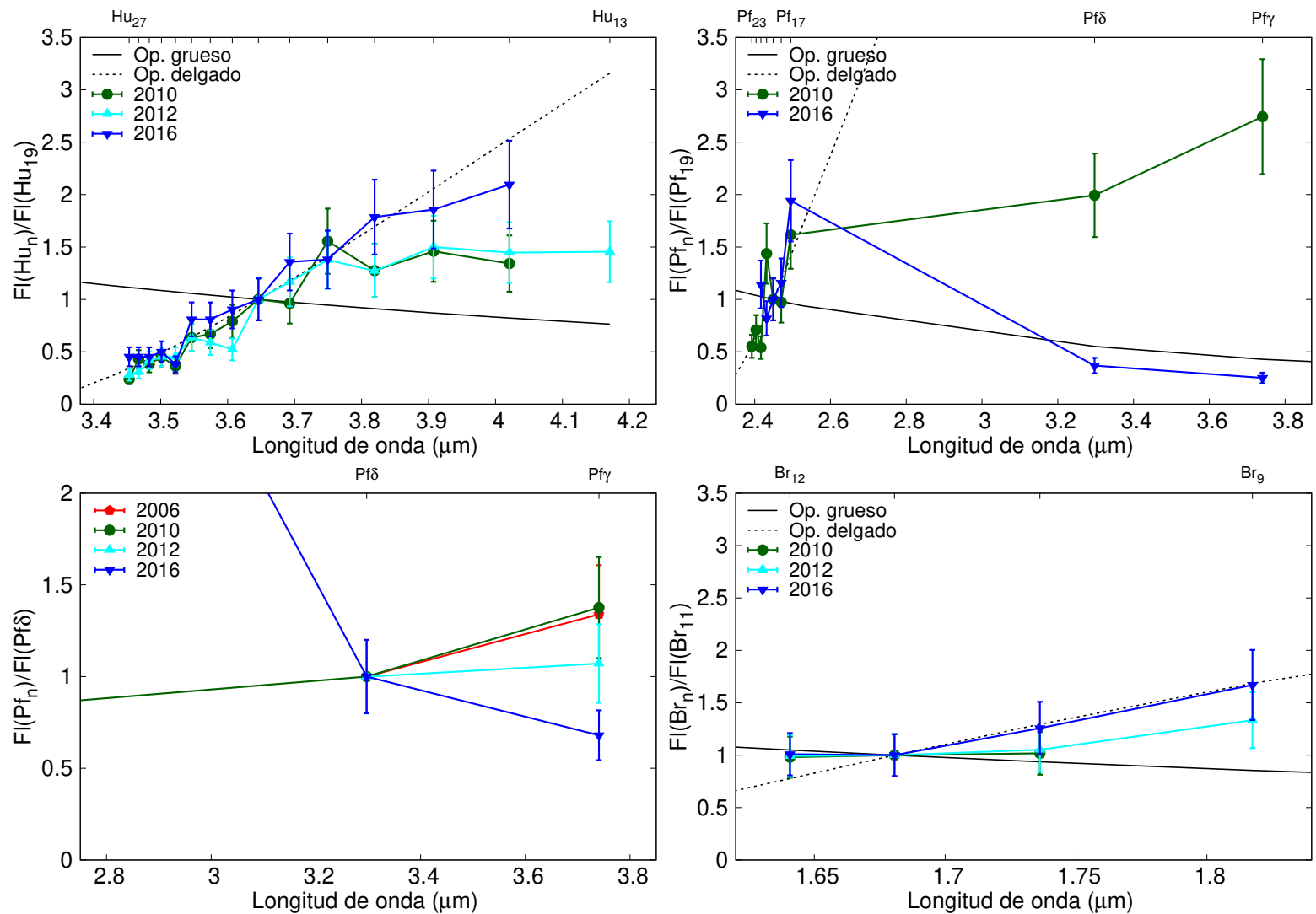

2010, 2012 y 2016, Pf $\gamma$ y $\operatorname{Br} \alpha$ se forma en una región externa de la envoltura, quedando por adentro las regiones de formación de las líneas de la serie de Humphreys y varios miembros de las series de Brackett y Pfund. En la observación del 2006 las regiones de formación están más dispersas y la región de formación de Pf $\gamma$ es la más interna.

La Fig. 6.16 (inferior) muestra los radios de las regiones de formación en radios estelares. Las regiones de formación más externas corresponden a los primeros miembros de cada serie, mientras que los miembros más altos se forman en regiones cercanas a la estrella central (generalmente con valores menores a $4 R_{\star}$ ). Los radios tienden a aumentar levemente entre cada observación. 
Figura 6.16: 28 Tau - Radios internos de las regiones emisoras de las diferentes líneas relativos a la región emisora de Pf $\gamma$ y en radios estelares. Los símbolos utilizados son los mismos que en la Fig. 6.13.
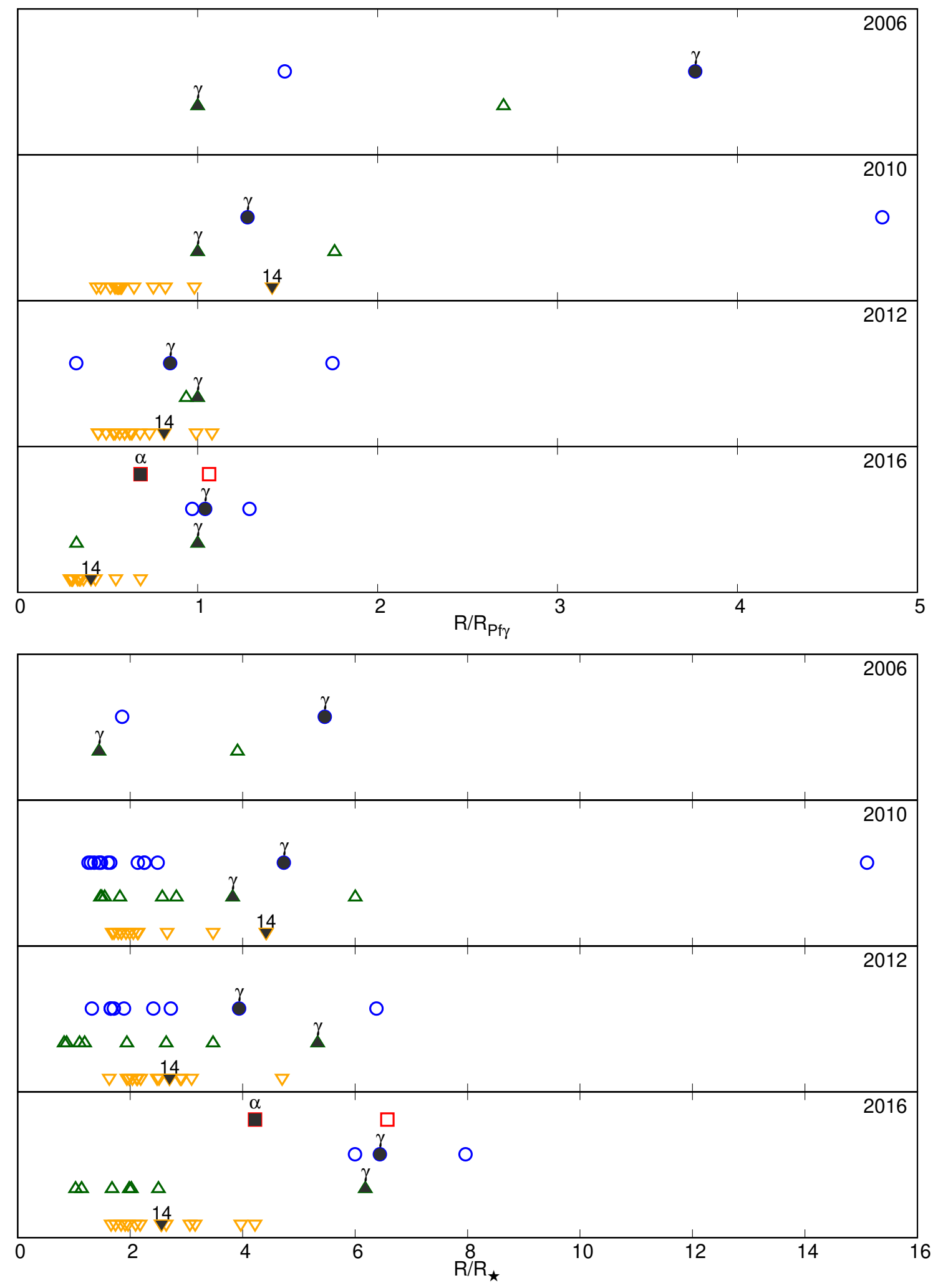


\subsubsection{V1150 Tau - HD 29441}

V1150 Tau es una estrella con clasificación espectral B2.5 V. Mennickent et al. (2009) obtuvieron espectros en el IR en el año 2003 e indicaron que V1150 Tau podría ser un objeto en transición entre los grupos I y II, debido a la intensidad de las líneas de Humphreys y a la ubicación del objeto en el diagrama de Lenorzer, en el límite entre el grupo I y el II.

\section{Descripción de los espectros y mediciones realizadas}

En las Figs. 6.17 a 6.19 se muestran los espectros obtenidos en los dos turnos de observación (2012 y 2016, GNIRS). En todos los casos, las líneas de hidrógeno se observan en emisión. Según el criterio de clasificación de Mennickent et al. (2009), catalogamos a esta estrella como perteneciente al grupo II. En las Tablas B.19 a B.22 se listan los valores de EW, Fl y FWHM obtenidos, considerando la absorción fotosférica correspondiente a una estrella con $T_{\text {eff }}=18000 \mathrm{~K}$ y $\log g=4.0$ (ver Tabla 3.3).

Figura 6.17: Espectro de V1150 Tau en la banda J.

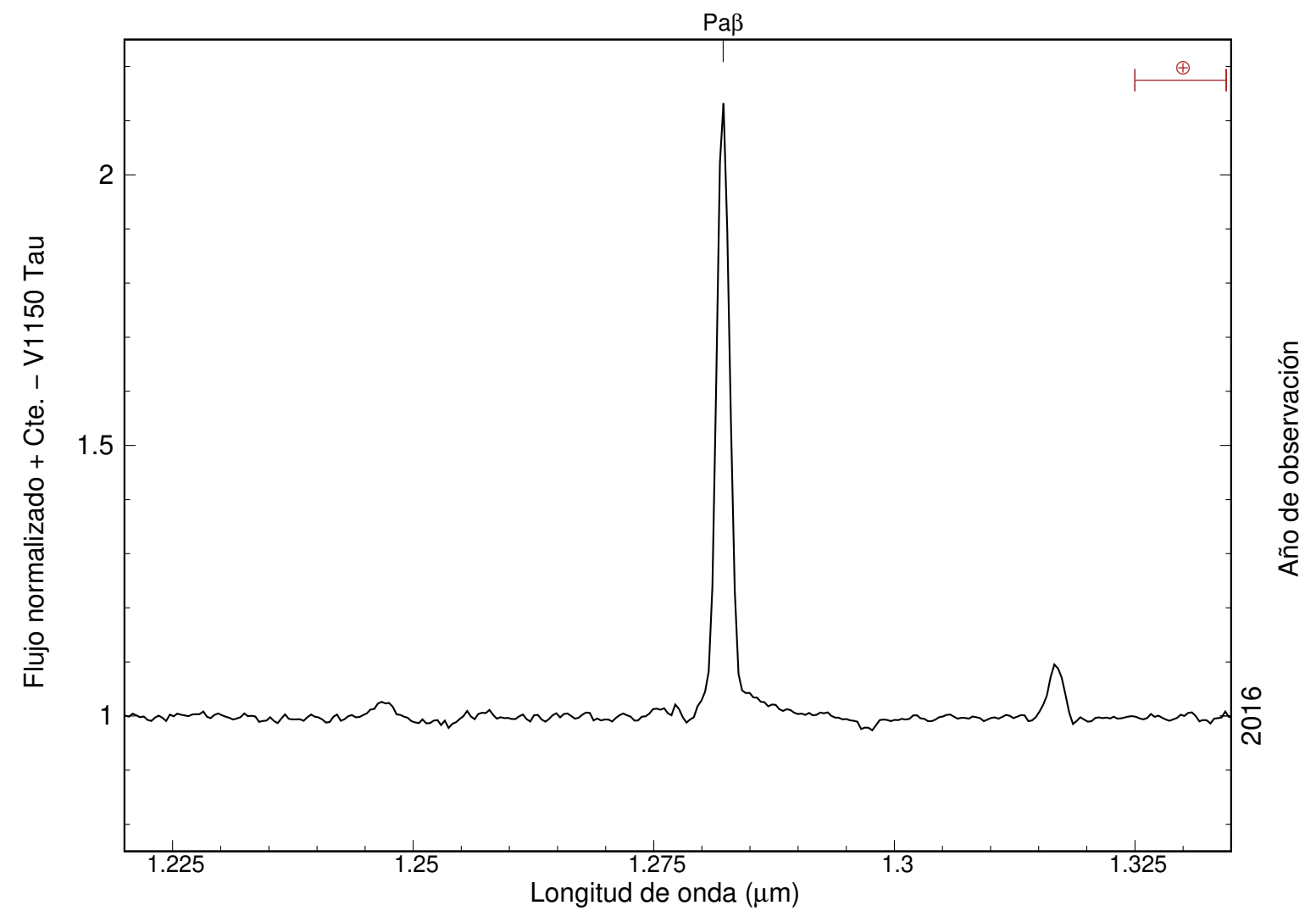


Figura 6.18: Espectros de V1150 Tau en las bandas H (superior) y K (inferior).
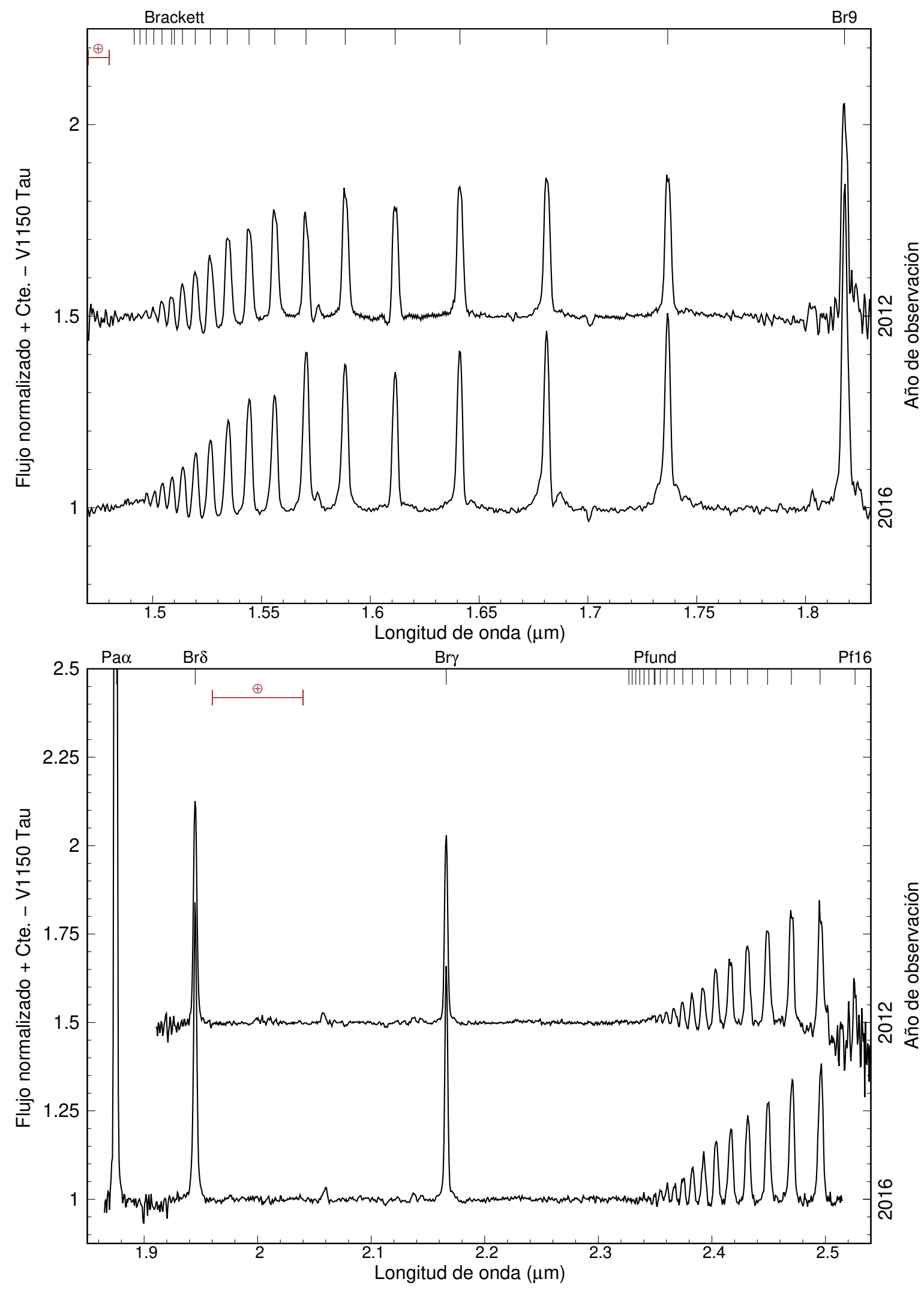
Figura 6.19: Espectro de V1150 Tau en la banda L.

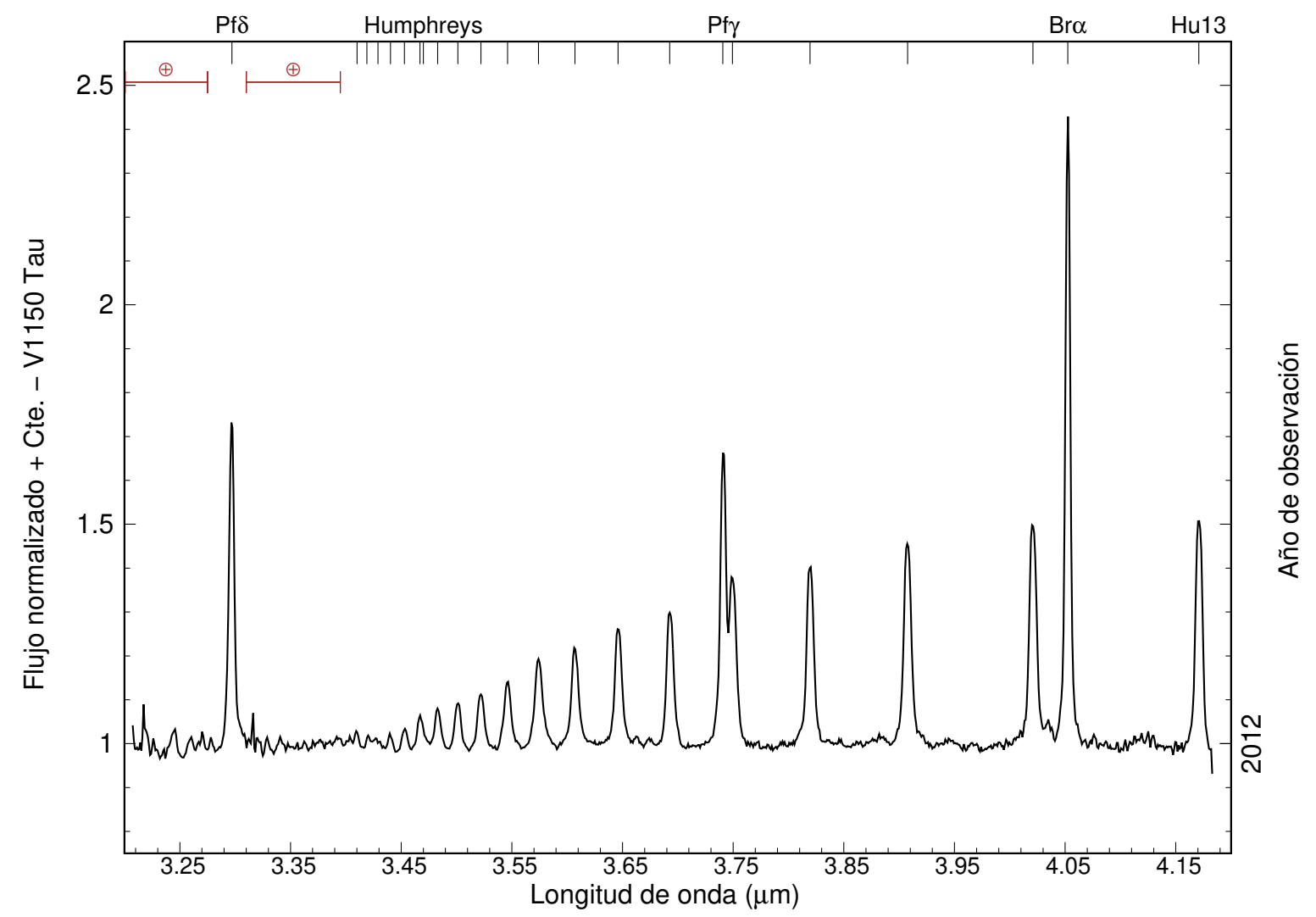

Figura 6.20: Ubicación de V1150 Tau en el diagrama de Lenorzer en la observación del año 2012.

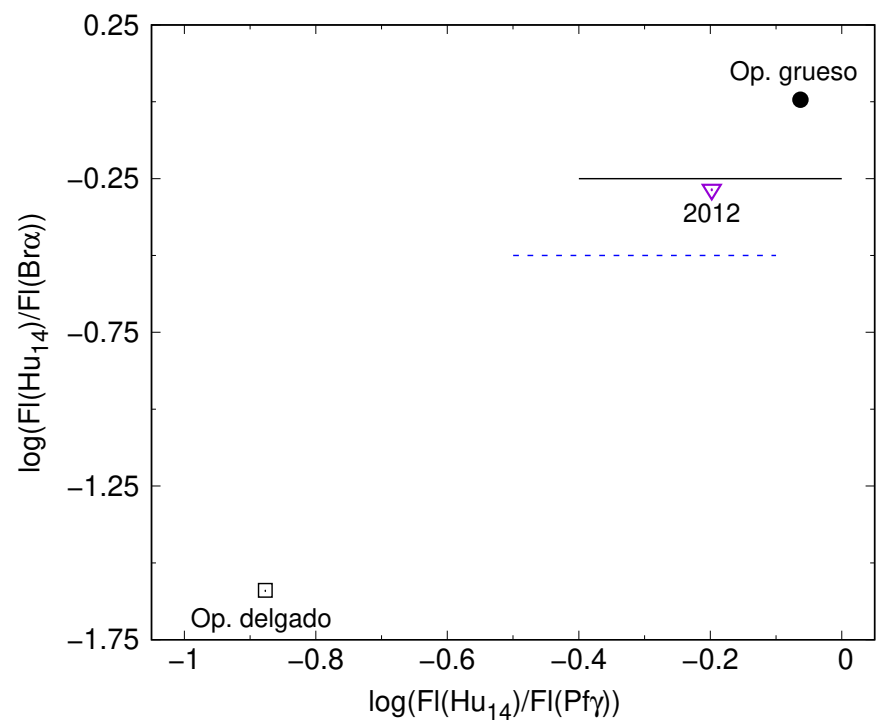


Figura 6.21: V1150 Tau - EW/ $\lambda$ vs. $\lambda$ de las líneas observadas en las diferentes series en las distintas observaciones.

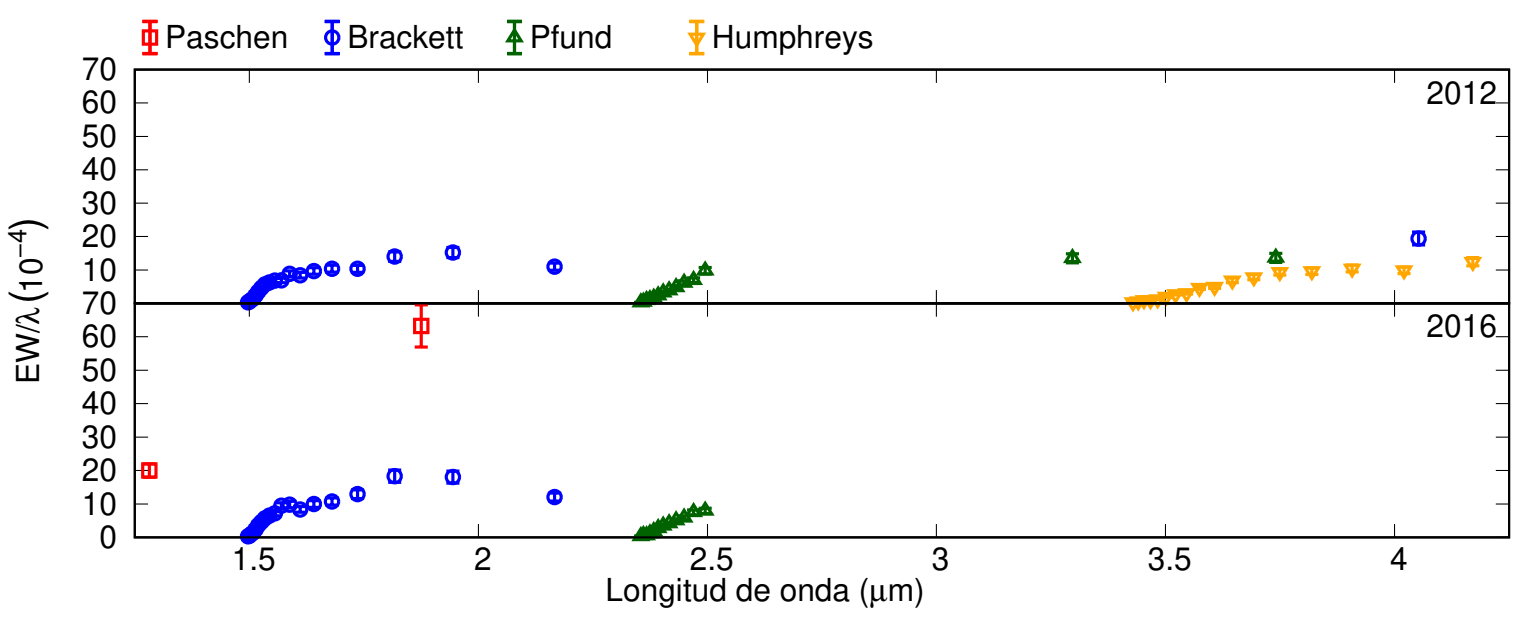

\section{Ubicación en el diagrama de Lenorzer}

La banda L solo fue observada en el 2012, por lo que solo podemos ubicarla en el diagrama de Lenorzer en ese año (ver Fig. 6.20). Vemos que V1150 Tau se encuentra en una región central del diagrama, cerca del límite de las regiones correspondientes a los grupos I y II.

\section{Comportamiento de las series de hidrógeno}

En las Figs. 6.21 a 6.22 se muestran los gráficos de EW/ $\lambda$ y FWHM según la longitud de onda. En el año 2012 los valores de EW/ $\lambda$ para las series de Humphreys y Brackett parecen alcanzar un valor estable $\sim 10 \cdot 10^{-4}$, al igual que las series de Brackett y Pfund en el 2016 en $\sim 12 \cdot 10^{-4}$. Todas las series observadas muestran un comportamiento creciente y luego decreciente a medida que observamos miembros más altos. Ese máximo se alcanza en $\mathrm{Hu}_{19}, \mathrm{Pf}_{17}$ y $\mathrm{Br}_{17}$ en el 2012, y $\mathrm{Pf}_{18}$ y $\mathrm{Br}_{14}$ en el 2016. Los FWHM de las líneas de la serie de Humphreys son menores en la observación del 2016, indicando que las líneas se forman en una región más alejada de la estrella central que en la observación del 2012. $\operatorname{Br} \alpha, \operatorname{Br} \gamma, \operatorname{Pa} \alpha$ y $\operatorname{Pa} \beta$ son las que presentan menor ensanchamiento, indicando regiones de formación más alejadas de la fotosfera. Como esta estrella no tiene $V \sin (i)$ determinado, no podemos comparar los FWHM con los correspondientes si la línea se forma en una región cercana a la fotosfera.

En la Fig. 6.23 se muestran los cocientes de los flujos y las comparaciones con los casos ópticamente grueso y delgado. Las líneas que se apartan del caso ópticamente delgado se listas en la Tabla 6.3. En el año 2012 obtuvimos también que $\mathrm{EW}(\mathrm{Br} \alpha) / \mathrm{EW}(\mathrm{Br} \gamma) \sim 3$ (valor bajo para una estrella del grupo II) mientras que $\mathrm{EW}(\mathrm{Pa} \alpha) / \mathrm{EW}(\mathrm{Pa} \beta) \sim 4.5$ en el 2016. 
Figura 6.22: V1150 Tau - FWHM vs. $\lambda$ de las líneas observadas en las diferentes series en las distintas observaciones. Los símbolos utilizados son los mismos que en la Fig. 6.21.

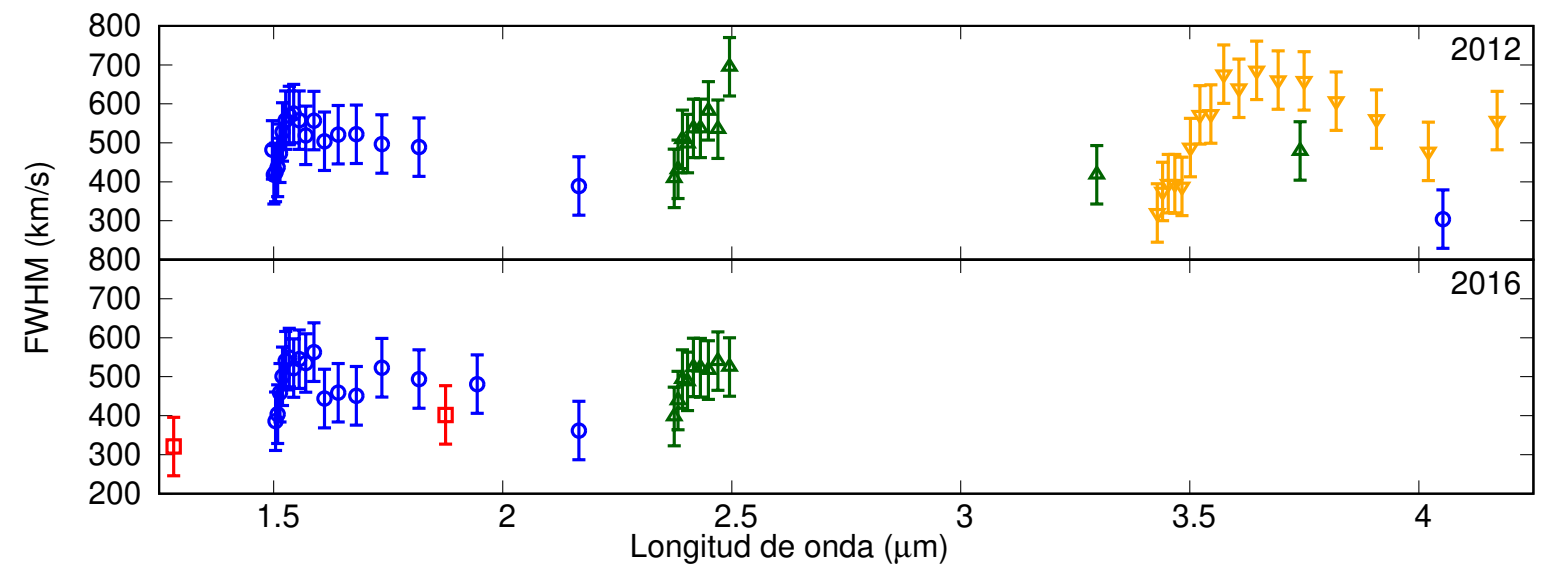

Tabla 6.3: V1150 Tau - Líneas que se apartan del caso ópticamente delgado. La serie de Humphreys y los primeros miembros de la serie de Pfund no fueron observados en el 2016.

\begin{tabular}{l|cc}
\hline \hline & 2012 & 2016 \\
\hline Humphreys & $13-16$ & $\times$ \\
Pfund & $\gamma-\delta$ & $\times$ \\
Brackett & $9-15$ & $9-14$ \\
\hline
\end{tabular}

\section{Determinación de densidades columnares y extensiones de las regiones de formación}

Las Tablas B.23 a B.26 muestran las densidades columnares, extensiones y radios internos de las regiones de formación a partir de los métodos $N$ y FWHM.

Para las densidades columnares y extensiones de las regiones de formación, en la serie de Humphreys los valores obtenidos son similares, y mayores a los de Pf $\delta$ y Pf $\gamma$ para la observación del 2012. En la serie de Brackett, los valores obtenidos en la observación del 2016 son levemente menores a los del 2012.

En la Fig. 6.24 mostramos los tamaños relativos a $\mathrm{Br} \gamma$. La región de formación de esta línea es una de las más alejadas de la estrella central, quedando por dentro la mayor parte de la serie de Humphreys en la observación del 2012 y las líneas de las serie de Brackett y Pfund en ambas observaciones. 
Figura 6.23: V1150 Tau - Cociente de flujos para las líneas de las series de Humphreys, Pfund y Brackett. En cada gráfico, la línea continua corresponde al cociente para un caso ópticamente grueso, y la línea punteada al caso ópticamente delgado. En la parte superior se identifican las líneas correspondientes.

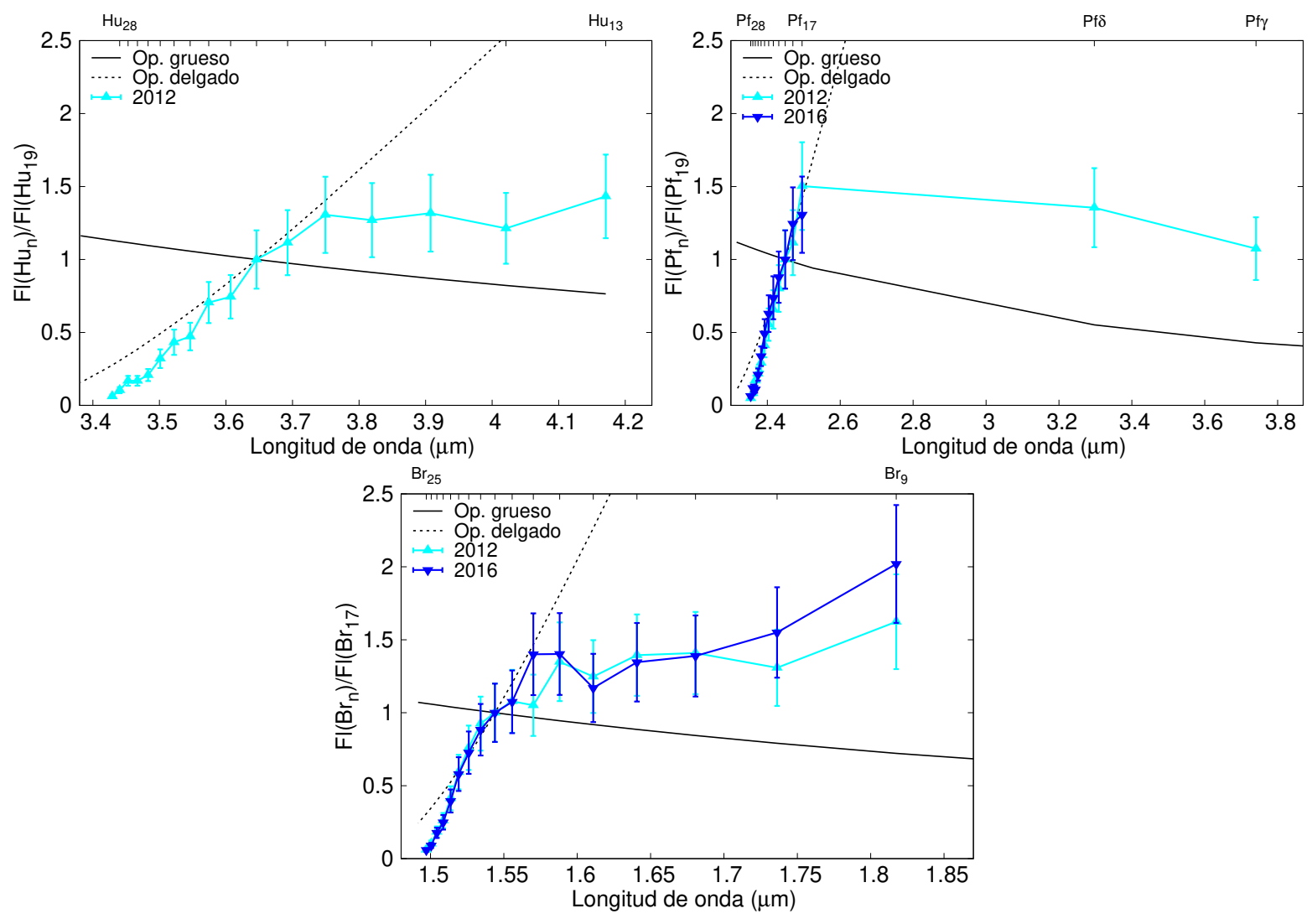

Figura 6.24: V1150 Tau - Radio internos de las regiones emisoras de las diferentes líneas relativos a la región emisora de Bry. Los símbolos utilizados son los mismos que en la Fig. 6.21.

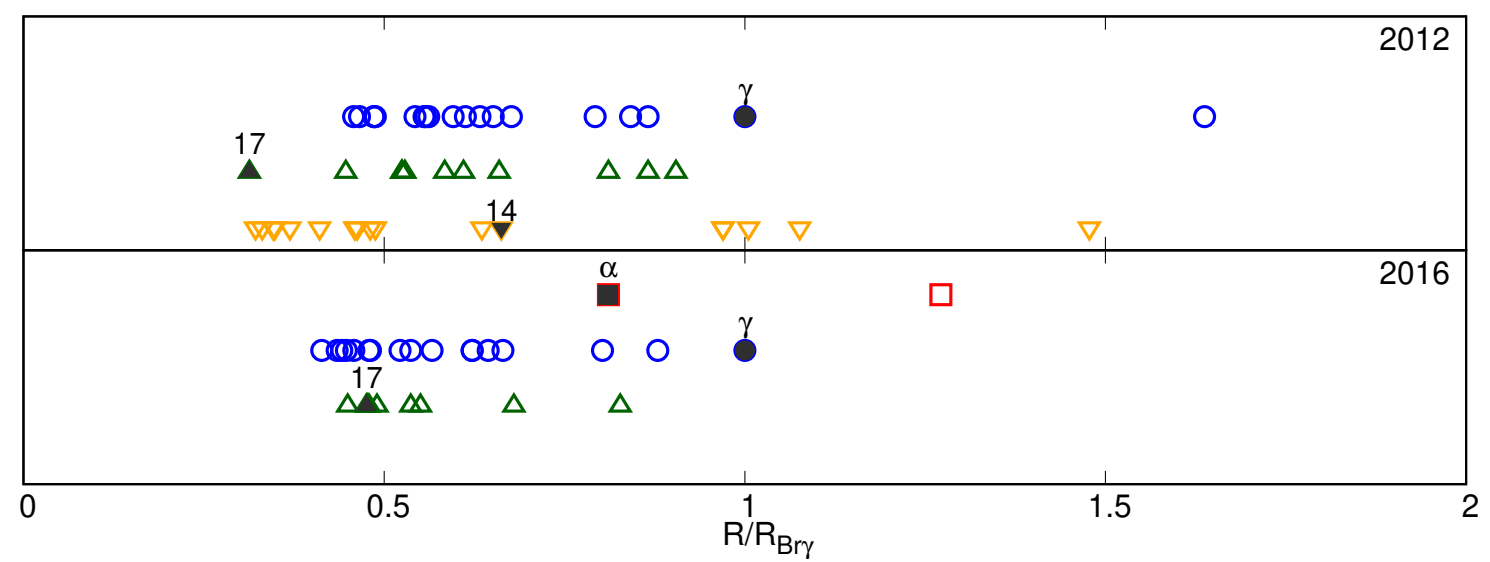




\subsubsection{Oph - HD 164284}

66 Oph es una estrella clasificada como B2 V, que ha presentado importantes variaciones fotométricas y en el perfil de $\mathrm{H} \alpha$ (Floquet et al., 2002). Es una sistema múltiple compuesto por una binaria cercana orbitando la estrella Be primaria (Štefl et al., 2004), cuyo sistema fue resuelto por medio de interferometría speckle por Mason et al. (2009).

A partir de observaciones IR obtenidas en 2008, Granada et al. (2010) la clasificaron como un miembro extremo del grupo II, ya que presentaba emisión en $\operatorname{Br} \alpha$ y $\operatorname{Pf} \gamma$, pero no presentaba líneas de la serie de Humphreys en emisión. Posteriormente, Sabogal et al. (2017) obtuvieron un espectro en la banda L en el 2014, donde no se observaban líneas en emisión, por lo que la clasificaron como perteneciente al grupo III. Esto es consistente con lo observado en la región óptica del espectro, donde $\mathrm{H} \alpha$ presentaba un perfil con dos picos en emisión disminuyendo su intensidad entre el 2001 y 2008, y luego en absorción a partir del 2009 (Granada et al., 2010; Jones et al., 2011; Sabogal et al., 2017). Vieira et al. (2017) modelaron observaciones del continuo IR de 66 Oph adquiridas en 1983 con el siguiente grupo de parámetros, $\log \rho_{0}=-11.3 \pm 0.2$ y $n=2.6 \pm 0.1$, que según el criterio adoptado para los valores de $n$ dan evidencia de la disipación del disco.

\section{Descripción de los espectros y mediciones realizadas}

Fue observada en cuatro oportunidades (2008, 2010, 2016 y 2017) utilizando el espectrógrafo GNIRS. En las Figs. 6.25 y 6.26 se muestran los espectros obtenidos. Los espectros obtenidos en el 2008 fueron presentados previamente por Granada et al. (2010). La mayor parte de las líneas observadas se encuentran en absorción, con excepción de $\operatorname{Br} \gamma$ que presenta una pequeña emisión central sobre la absorción fotosférica, y $\operatorname{Br} \alpha, \operatorname{Br} \delta$, $\operatorname{Pf} \gamma$ y $\operatorname{Pf} \delta$ que presentan emisión por sobre el nivel del continuo en algunas observaciones. Según el criterio de Mennickent et al. (2009), 66 Oph es clasificada como miembro del grupo II en el año 2008 y posteriormente perteneciente al grupo III. En la Tabla B.27 se muestran los valores de EW, Fl y FWHM obtenidos, corregidos utilizó el espectro sintético correspondiente a una estrella con $T_{\text {eff }}=22000 \mathrm{~K}$ y $\log g=4.0$ según los parámetros estelares de la Tabla 3.3.

\section{Comportamiento de las series de hidrógeno}

Debido a que no observamos miembros altos de las series de Pfund y Brackett en emisión, no pudimos comparar los cocientes de flujos con los esperados para los casos ópticamente grueso y delgado para derivar las propiedades del disco. 
Figura 6.25: Espectros de 66 Oph en las bandas J (superior) y H (inferior).
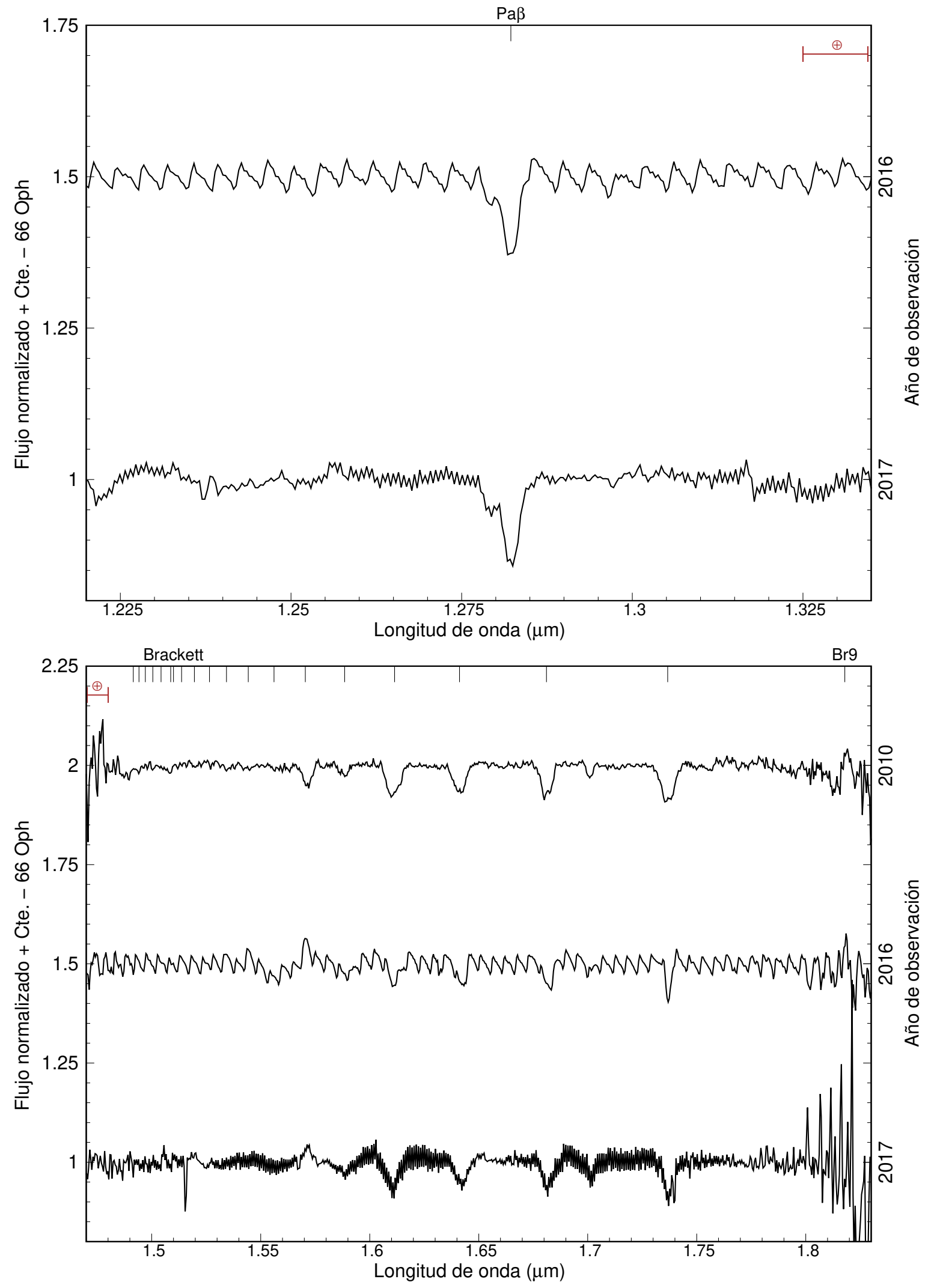
Figura 6.26: Espectros de 66 Oph en las bandas K (superior) y L (inferior).
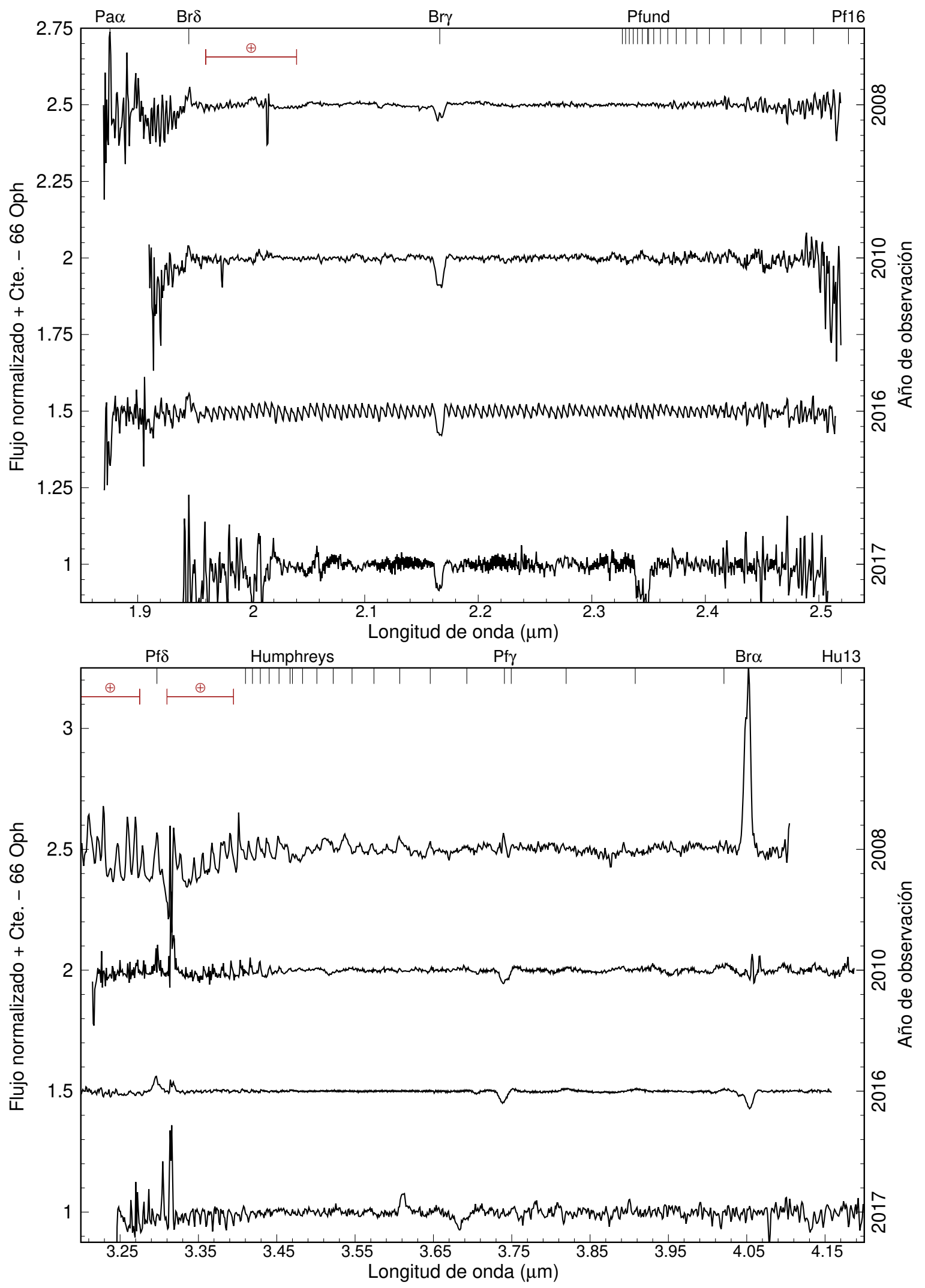
Figura 6.27: 66 Oph - Radios internos de las regiones emisoras de las diferentes líneas relativos a la región emisora de $\operatorname{Br} \alpha$ y en radios estelares.
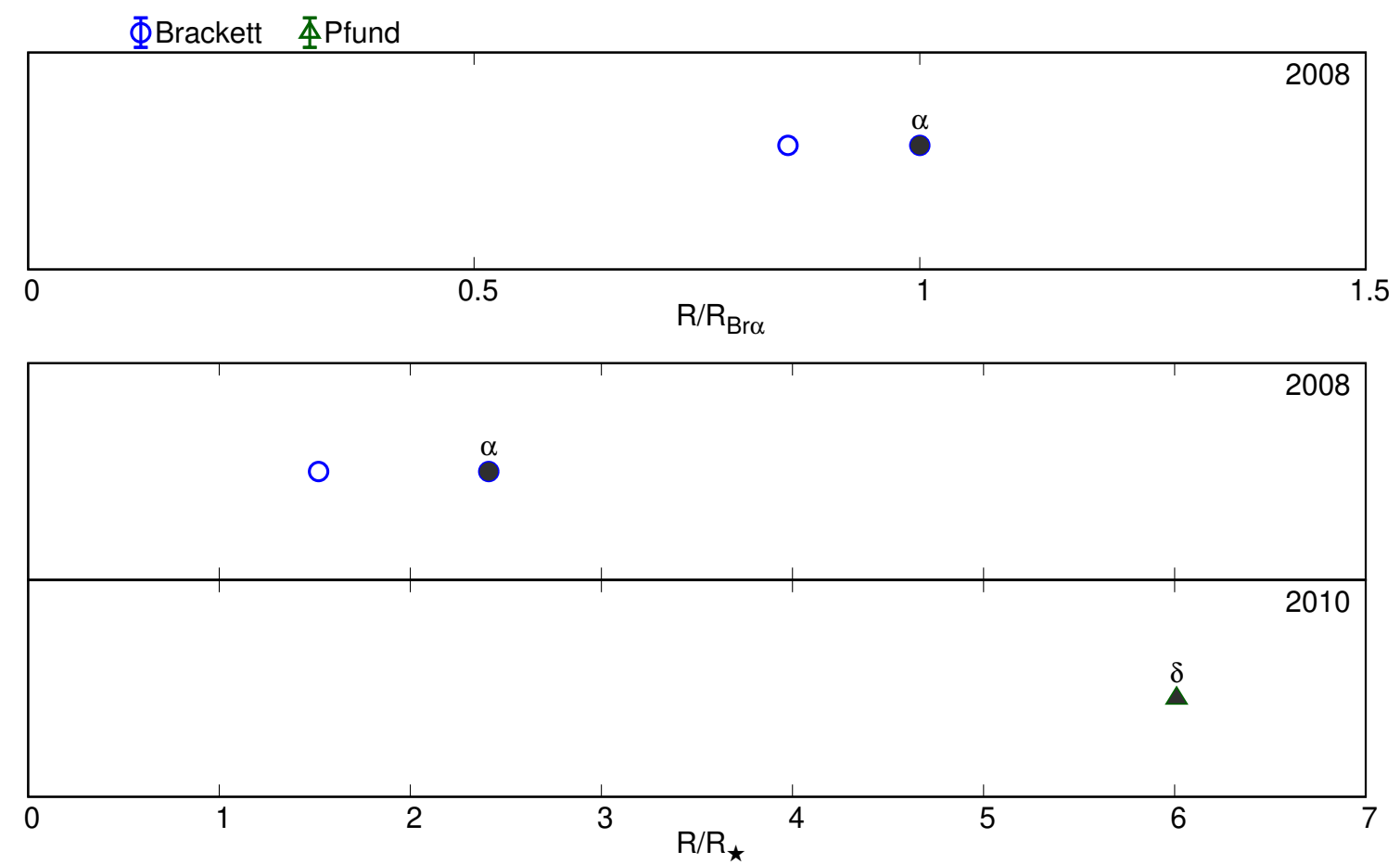

Determinación de densidades columnares y extensiones de las regiones de formación

Para la observación del 2008, pudimos obtener los radios internos de las regiones de formación relativos a $\operatorname{Br} \alpha$ y en radios estelares a partir de los métodos FWHM y $\Delta V$. En la observación del 2010 pudimos obtener el radio interno de la región de formación de $\operatorname{Pf} \delta$ a partir de la separación de los picos en emisión. Los valores obtenidos pueden verse en la Tabla B.28. Los radios internos de las regiones de formación de $\operatorname{Br} \alpha$ y $\operatorname{Br} \gamma$ en el 2008 son $2.4 R_{\star}$ y $1.5 R_{\star}$, respectivamente. Para la región emisora de $\mathrm{Pf} \delta$ obtuvimos en el 2010 un radio interno de $6 R_{\star}$. 


\subsubsection{V923 Aql - HD 183656}

V923 Aql es una estrella con clasificación espectral B7 III, miembro de un sistema binario (Harper, 1937). Ha presentado variaciones espectroscópicas y fotométricas en diferentes escalas de tiempo (Merrill, 1952; Lynds, 1960). Koubský et al. (1989) propusieron que las variaciones observadas en las velocidades radiales eran originadas por la superposición de un ciclo de amplitud variable y uno de período largo ( 1800 - 2400 días), junto con el movimiento orbital de 214.75 días. Estos autores concluyeron que el sistema binario está formado por una estrella primaria de tipo espectral B5-7 y una estrella secundaria de baja masa.

Arias et al. (2004) encontraron variaciones en la intensidad de las líneas de Fe II que correlacionaban con el período orbital (214.75 días) y determinaron un período de 6.5 años para las variaciones en el cociente V/R en la línea $\mathrm{H} \alpha$.

Granada et al. (2010) observaron este objeto en las bandas K y L en el año 2006, la clasificaron en el grupo II y la ubicaron en la región inferior del diagrama de Lenorzer.

Figura 6.28: Espectros de V923 Aql en la banda J.

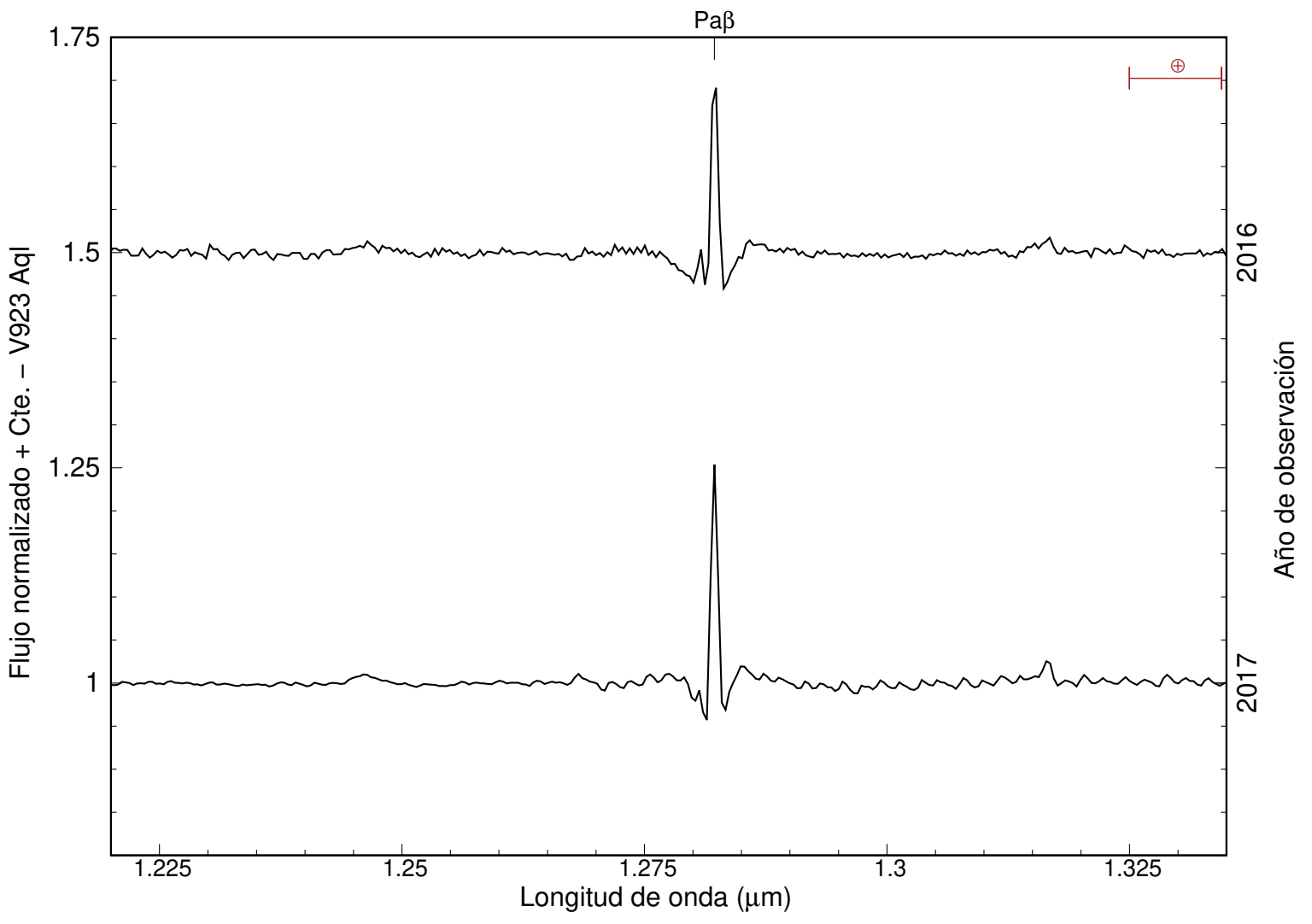


Figura 6.29: Espectros de V923 Aql en las bandas H (superior) y K (inferior).
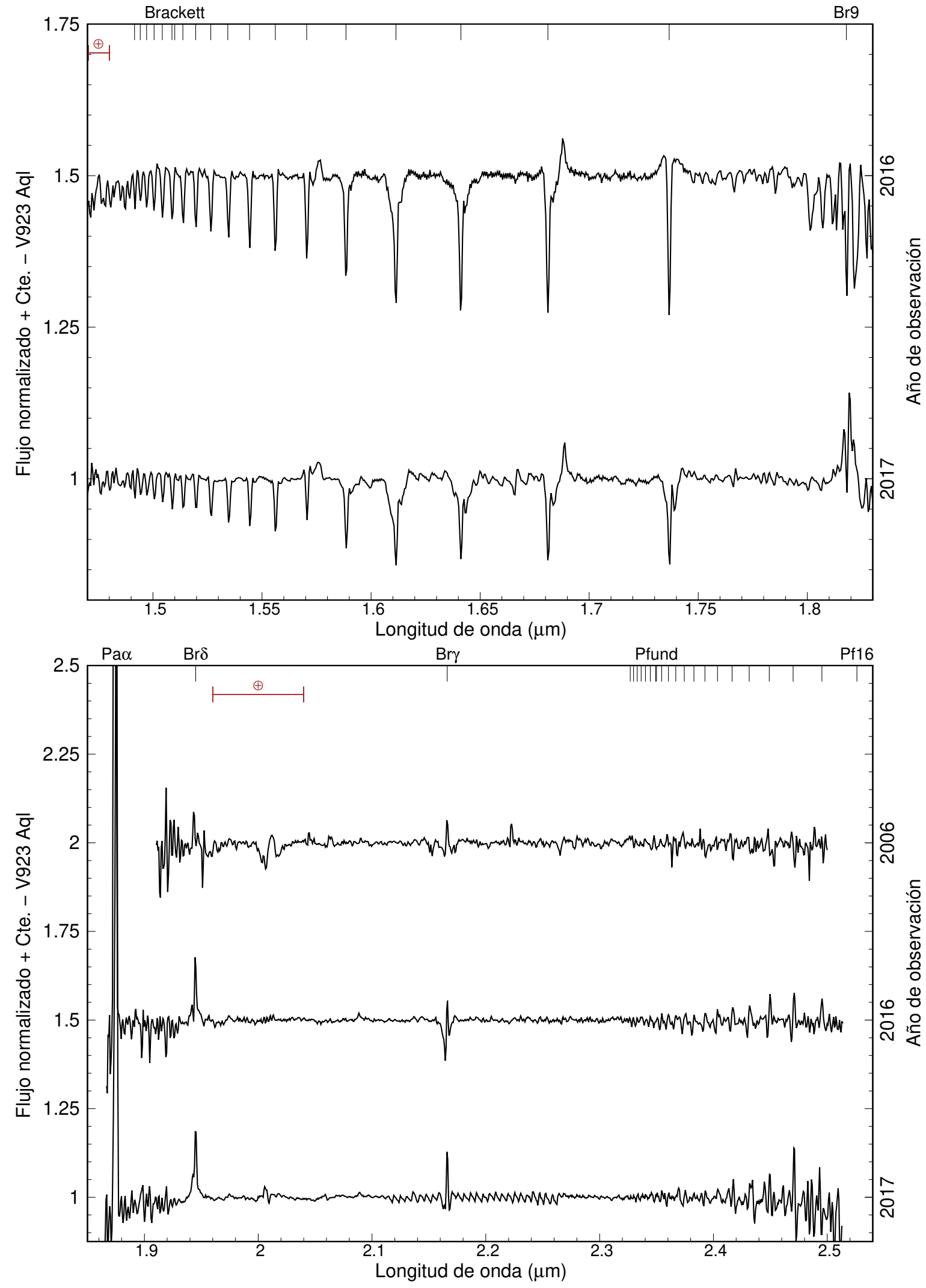
Figura 6.30: Espectros de V923 Aql en la banda L (superior) y líneas más intensas de cada banda (inferior). Junto a $\mathrm{Pf} \gamma$ se observa $\mathrm{Hu}_{17}$.
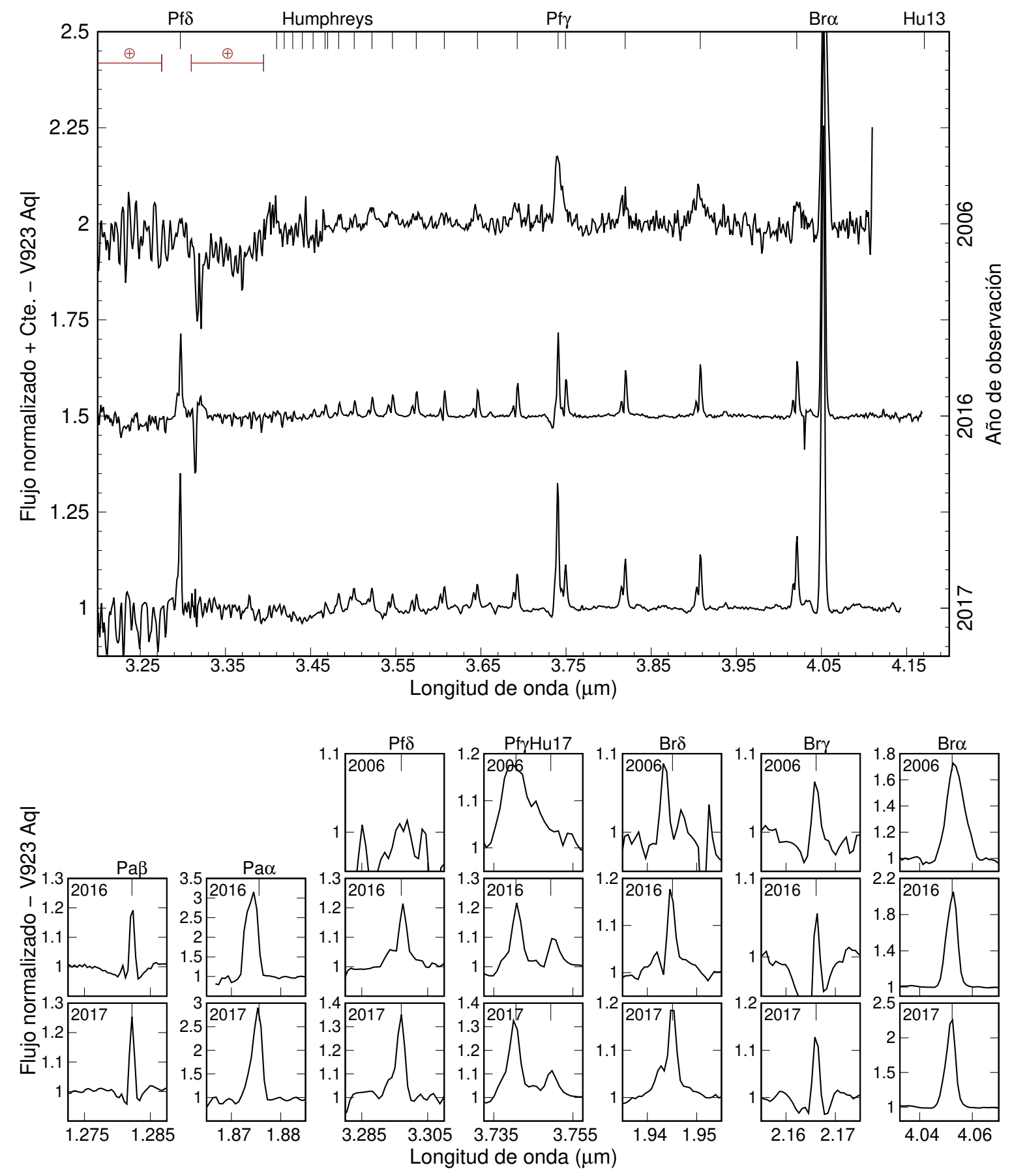


\section{Descripción de los espectros y mediciones realizadas}

En las Figs. 6.28 a 6.30 se muestran los espectros obtenidos en cada banda en los tres turnos de observación (2006, 2016 y 2017), junto con las líneas más intensas a fin de facilitar la comparación. Los espectros obtenidos en el 2006 fueron presentados previamente por Granada et al. (2010). Las líneas de la serie de Humphreys presentan dos picos en emisión, con el pico rojo más intenso que el azul, al igual que $\operatorname{Pa} \beta, \operatorname{Pf} \delta, \operatorname{Pf} \gamma, \operatorname{Br} \delta$ y $\operatorname{Br} \gamma$. Las líneas de la serie de Brackett presentan perfiles tipo shell. Según el criterio de Mennickent et al. (2009), clasificamos a este objeto como perteneciente al Grupo II en todas las observaciones. Los valores para los EW, Fl y FWHM se muestran en las Tablas B.29 a B.32, donde se corrigieron los valores considerando la absorción fotosférica correspondiente a una estrella con $T_{\text {eff }}=13000 \mathrm{~K}$ y $\log g=3.5$ según los valores presentados en la Tabla 3.3.

\section{Ubicación en el diagrama de Lenorzer}

Utilizando la observación del 2006, Granada et al. (2010) ubicaron a V923 Aql en la región central del diagrama de Lenorzer, correspondiente a las estrellas pertenecientes al Grupo II según la clasificación de Mennickent et al. (2009). En las observaciones del 2016 y 2017 se observa que la estrella se ubica aproximadamente en la misma región, desplazada un poco hacia la zona que corresponde a una envoltura ópticamente gruesa.

\section{Comportamiento de las series de hidrógeno}

En la Fig. 6.32 se observa que los valores de EW/ $\lambda$ en función de la longitud de onda parecen estabilizarse en $2 \cdot 10^{-4}$ en el $2006-2016$ y $3 \cdot 10^{-4}$ en el 2017 , consistente con el comportamiento

Figura 6.31: Ubicación de V923 Aql en el diagrama de Lenorzer en las diferentes observaciones.

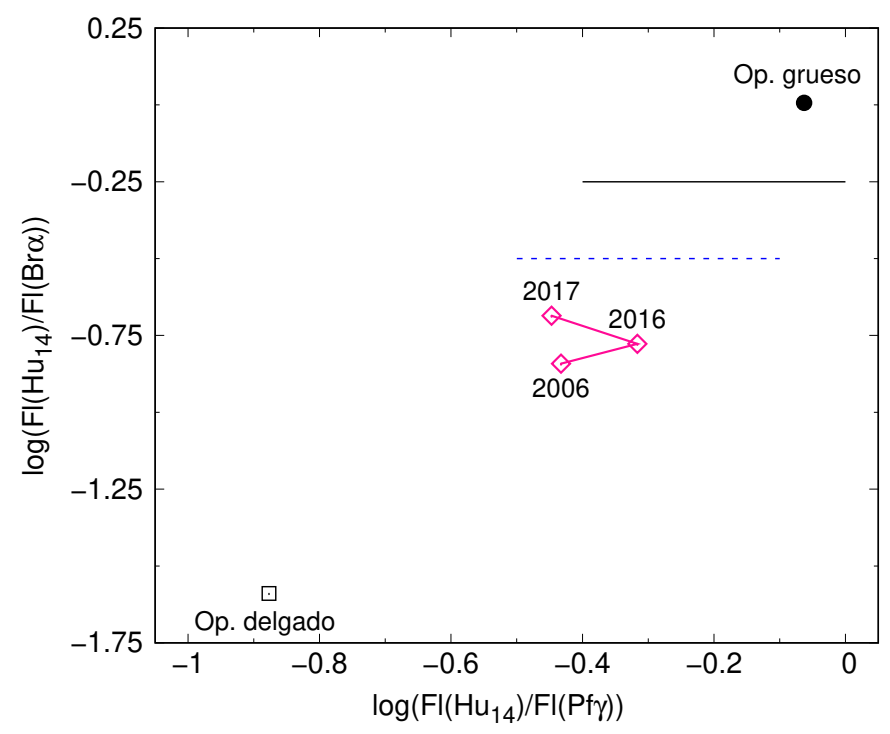


Figura 6.32: V923 Aql - EW/ $\lambda$ vs. $\lambda$ de las líneas observadas en las diferentes series en las distintas observaciones.

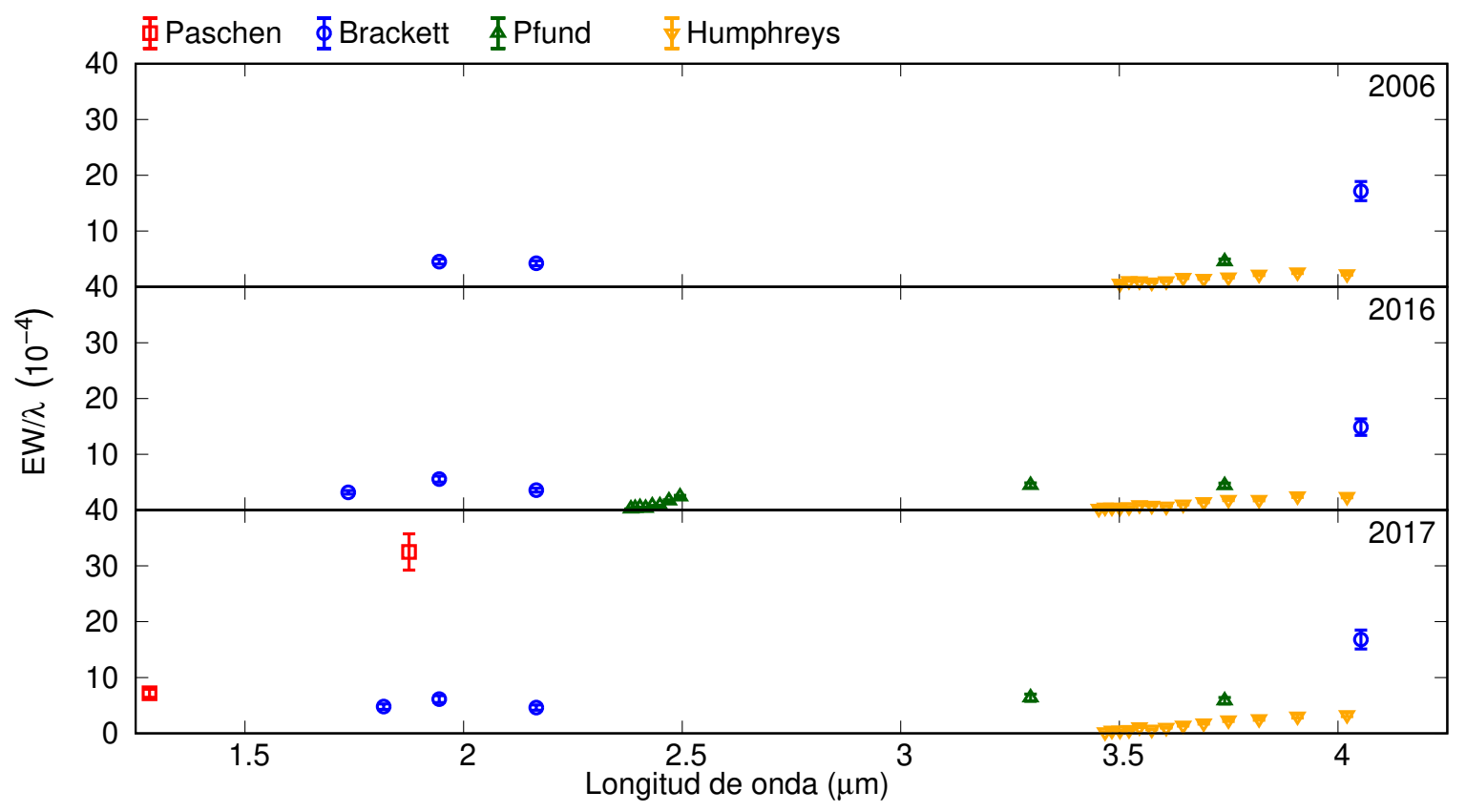

Tabla 6.4: V923 Aql - Líneas que se apartan del caso ópticamente delgado. Para la serie de Pfund, la única observación en la que pudimos obtener los flujos de miembros altos de la serie es la del 2016. La serie de Brackett no fue observada en el 2006, y en los otros años no presentan emisión considerable.

\begin{tabular}{l|ccc}
\hline \hline & 2006 & 2016 & 2017 \\
\hline Humphreys & $14-18$ & 14 & $14-16$ \\
Pfund & - & $\gamma-\delta$ & - \\
Brackett & $\times$ & - & - \\
\hline
\end{tabular}

descripto por Hony et al. (2000). La Fig. 6.33 muestra los FWHM obtenidos en función de la longitud de onda. Los miembros de la serie de Humphreys muestran el comportamiento esperado (creciente para miembros más altos de la serie) en los años 2016 y 2017, y los FWHM indican regiones de formación cercanas a la estrella central. Los FWHM obtenidos para las otras líneas indican regiones de formación más alejadas de la estrella central que los de la serie de Humphreys, con excepción de Bra en el año 2006.

Los cocientes de los flujos obtenidos, y la comparación con los casos ópticamente grueso y delgado se encuentran en la Fig. 6.34. Las líneas que se apartan del caso ópticamente delgado se listas en la Tabla 6.4. Obtuvimos además que la intensidad relativa de Pf $\gamma$ respecto a $\mathrm{Pf} \delta$ disminuyó entre el 2016 y 2017. Por otro lado, $\mathrm{EW}(\mathrm{Br} \alpha) / \mathrm{EW}(\mathrm{Br} \gamma) \sim 7$ en la observación del 2017 y $\sim 8$ en 2006 y 2016, consistente con la pertenencia al Grupo II, mientras que $\mathrm{EW}(\mathrm{Pa} \alpha) / \mathrm{EW}(\mathrm{Pa} \beta) \sim 8$ en el 2016 y $\sim 6.5$ en el 2017. 
Figura 6.33: V923 Aql - FWHM vs. $\lambda$ de las líneas observadas en las diferentes series en las distintas observaciones. La línea punteada corresponde a un valor de $2 V \sin (i)=568 \mathrm{~km} / \mathrm{s}$, y la región sombreada al error correspondiente (Tabla 3.3). Los símbolos utilizados son los mismos que en la Fig. 6.32.

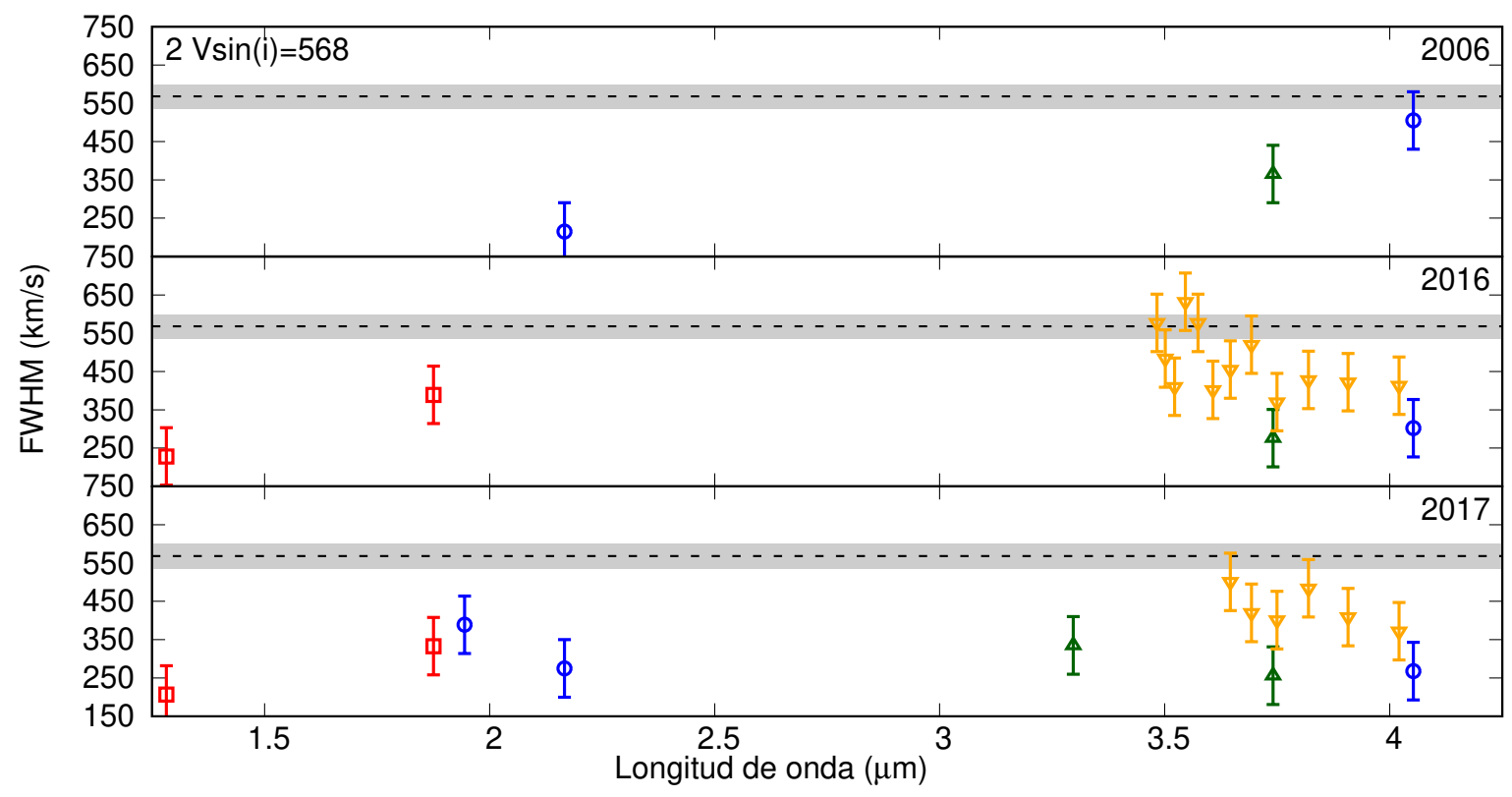

\section{Determinación de densidades columnares y extensiones de las regiones de formación}

Las Tablas B.33 a B.36 muestran las densidades columnares, extensiones de las regiones de formación y radios internos de las mismas respecto a una de referencia y en radios estelares, calculados mediante los métodos $N$, FWHM y $\Delta V$. Presentamos también valores en radios estelares obtenidos a partir de los tamaños relativos a $\mathrm{Hu}_{14}$ en el 2016 y a $\mathrm{Br} \delta$ en el 2017, para aquellas líneas en las cuales no se observaron dos picos en emisión.

En el 2017, donde varias líneas de la serie de Humphreys se apartan del caso ópticamente delgado, las densidades y extensiones relativas son menores para miembros más altos de la serie. La densidad columnar de $\mathrm{Hu}_{14}$ es menor en el 2016 que en 2017. No obtuvimos valores para la observación del 2006 por no tener determinados valores de FWHM.

Para las series de Humphreys y Brackett los radios internos de las regiones de formación son menores para los miembros más altos de las series. Para la serie de Paschen obtuvimos radios mayores para $\mathrm{Pa} \beta$ en las dos observaciones. En la Fig. 6.35 (superior) se muestran los tamaños relativos a Pf $\gamma$, donde vemos que la única línea más externa a Pf $\gamma$ es $\mathrm{Pa} \beta$.

En la Fig. 6.35 (inferior) mostramos los valores obtenidos a partir de $\Delta V$, junto a los obtenidos a partir de los tamaños relativos para las líneas que no presentan dos picos en emisión. Las regiones de formación de las líneas de la serie de Humphreys se mantienen siempre dentro de los $3 R_{\star}$, al igual que los miembros más altos de las series de Pfund y Brackett. No se observan 
Figura 6.34: V923 Aql - Cociente de flujos para las líneas de la serie de Humphreys y Pfund. En cada gráfico, la línea continua corresponde al cociente para un caso ópticamente grueso, y la línea punteada al caso ópticamente delgado. En la parte superior se identifican las líneas correspondientes.
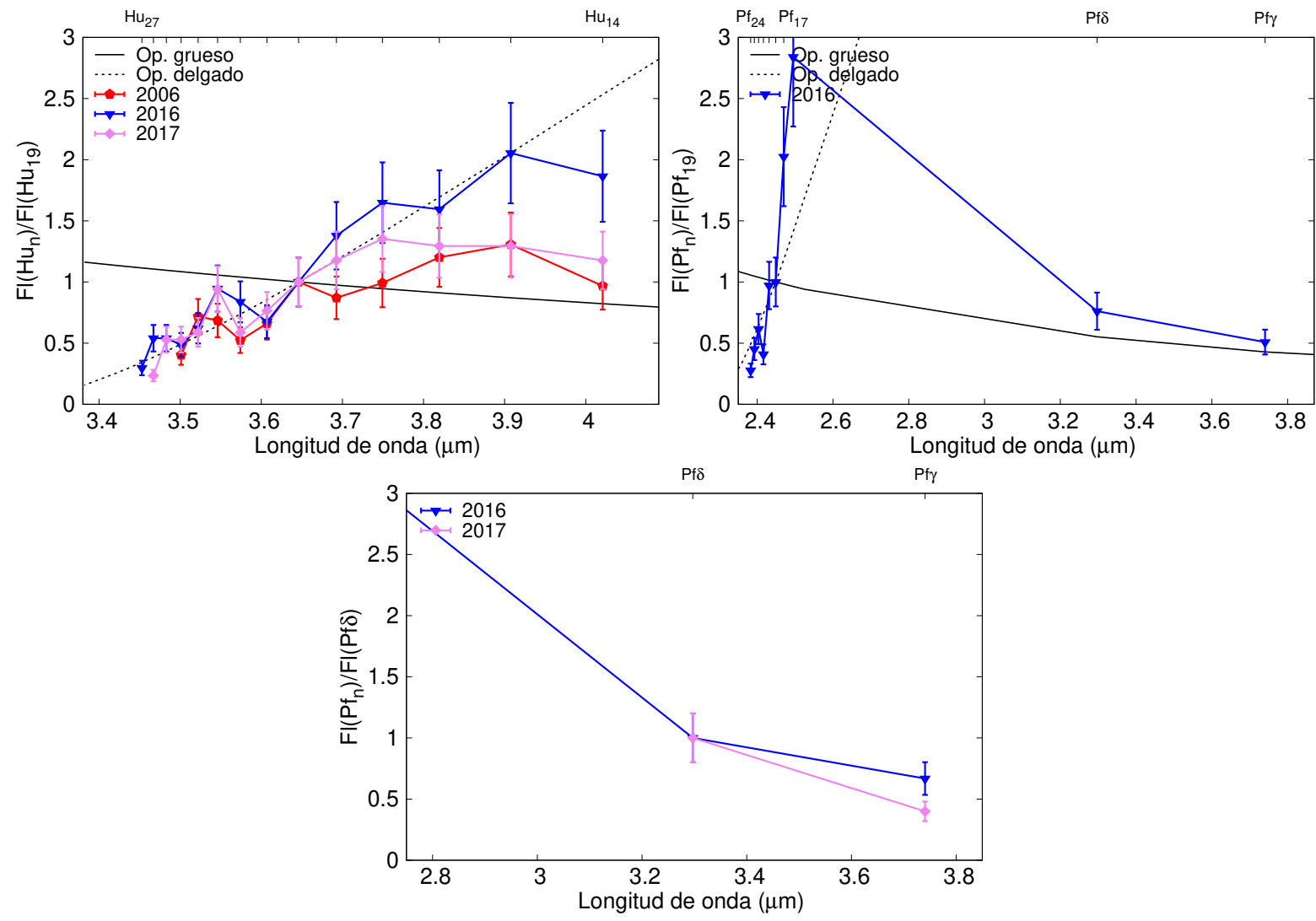

diferencias significativas entre los radios determinados para las dos observaciones. Para $\mathrm{Pa} \beta$ hay una gran diferencia en la ubicación del radio interno de la región de formación cuando la graficamos respecto a $\mathrm{Pf} \gamma$ o en radios estelares, que puede deberse a la dificultad de obtener el FWHM para las líneas que presentan una emisión débil por sobre la absorción fotosférica (medida utilizada en el primer caso) En función del radio estelar, las medidas tienen menor error asociado, por lo que las líneas de la serie de Paschen se formarían las dos entre 2.5 y $3 R_{\star}$. 
Figura 6.35: V923 Aql - Radio internos de las regiones emisoras de las diferentes líneas relativos a la región emisora de $\mathrm{Br} \gamma$ y en radios estelares. Los símbolos utilizados son los mismos que en la Fig. 6.32.
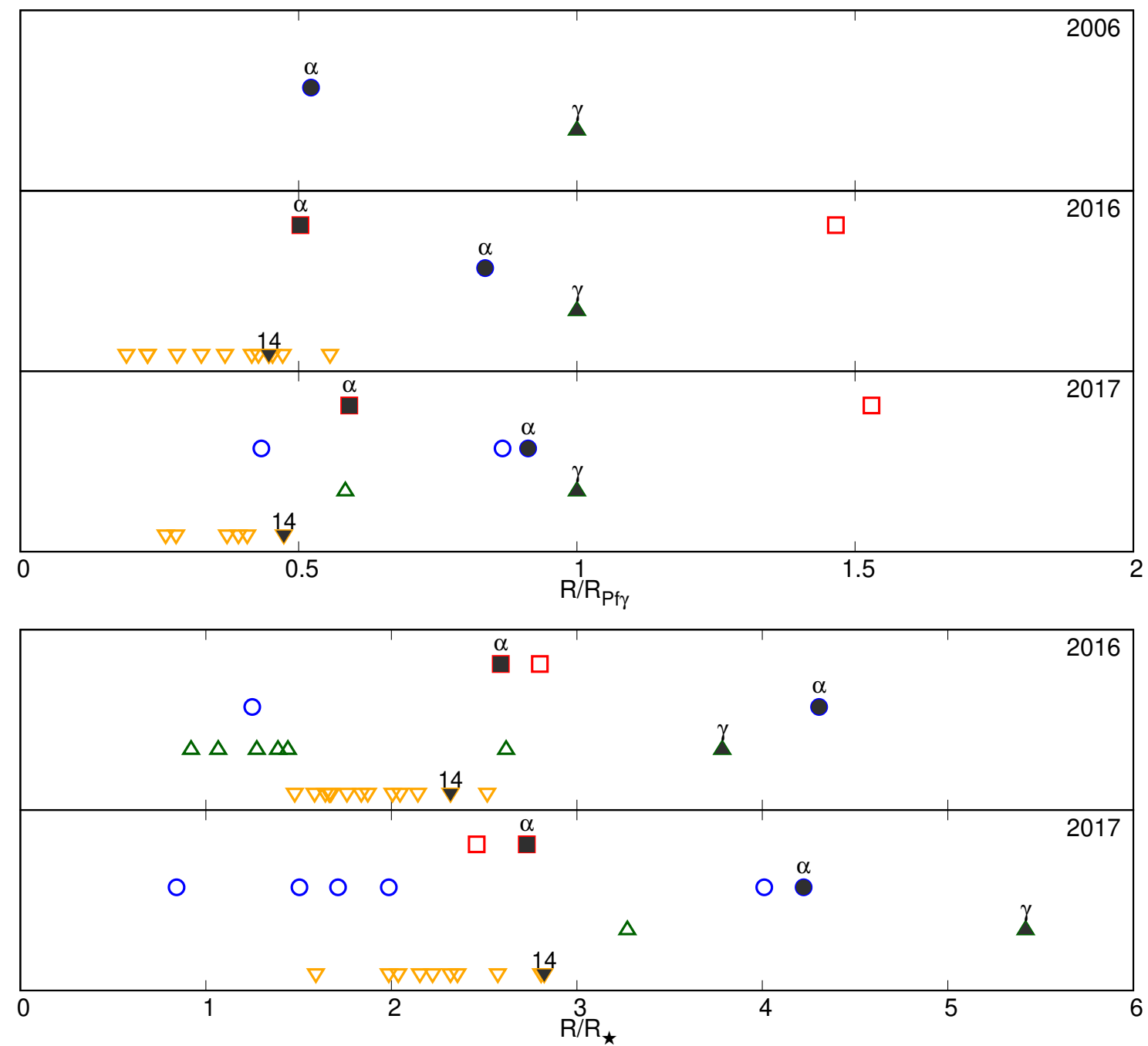


\subsubsection{Vul - HD 187811}

12 Vul es una estrella con clasificación espectral B2.5 V. A lo largo de los años, la línea H $\alpha$ pasó por épocas donde se observaba un perfil en absorción y luego en emisión, para posteriormente decaer nuevamente. En el 2004 presentaba dos picos en emisión que no alcanzaban a rellenar la absorción fotosférica, cuya intensidad aumentó nuevamente hacia el 2005. En el 2009 volvió a decaer su intensidad, llegando a un perfil en absorción en el 2014 (Sabogal et al., 2017).

Lenorzer et al. (2002b) y Mennickent et al. (2009) observaron a 12 Vul en el IR en los años 1998 y 2003, respectivamente. Entre esas dos observaciones, su posición en el diagrama de Lenorzer se modificó levemente, y pasó de pertenecer al grupo I a pertenecer al grupo II. Estas observaciones coinciden con una época en la que $\mathrm{H} \alpha$ comenzaba a decaer. Esto llevó a Sabogal et al. (2017) a pensar que 12 Vul pasó por procesos de disipación y desarrollo del disco varias veces en un intervalo de 15 años, clasificándola como una estrella con disco en desarrollo.

A partir del modelado del continuo IR, Vieira et al. (2017) reportaron valores de $n=5 \pm 1 \mathrm{y}$ $\log \rho_{0}=-12.1 \pm 0.2$ a partir de observaciones en el 2010, lo que según el criterio adoptado por estos autores, indica la presencia de un disco en formación.

Figura 6.36: Espectros de $12 \mathrm{Vul}$ en la banda J.

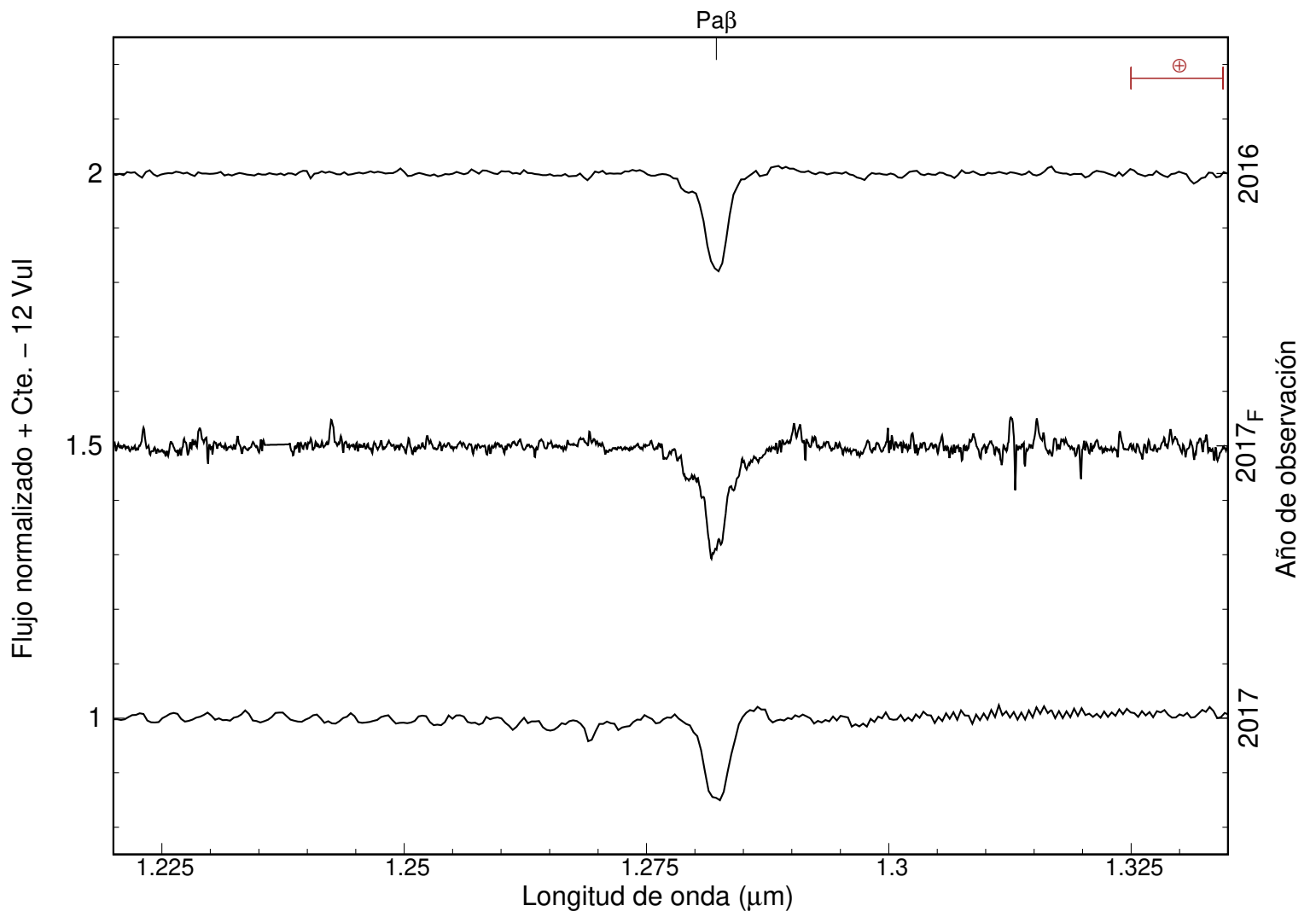


Figura 6.37: Espectros de $12 \mathrm{Vul}$ en las bandas H (superior) y K (inferior).
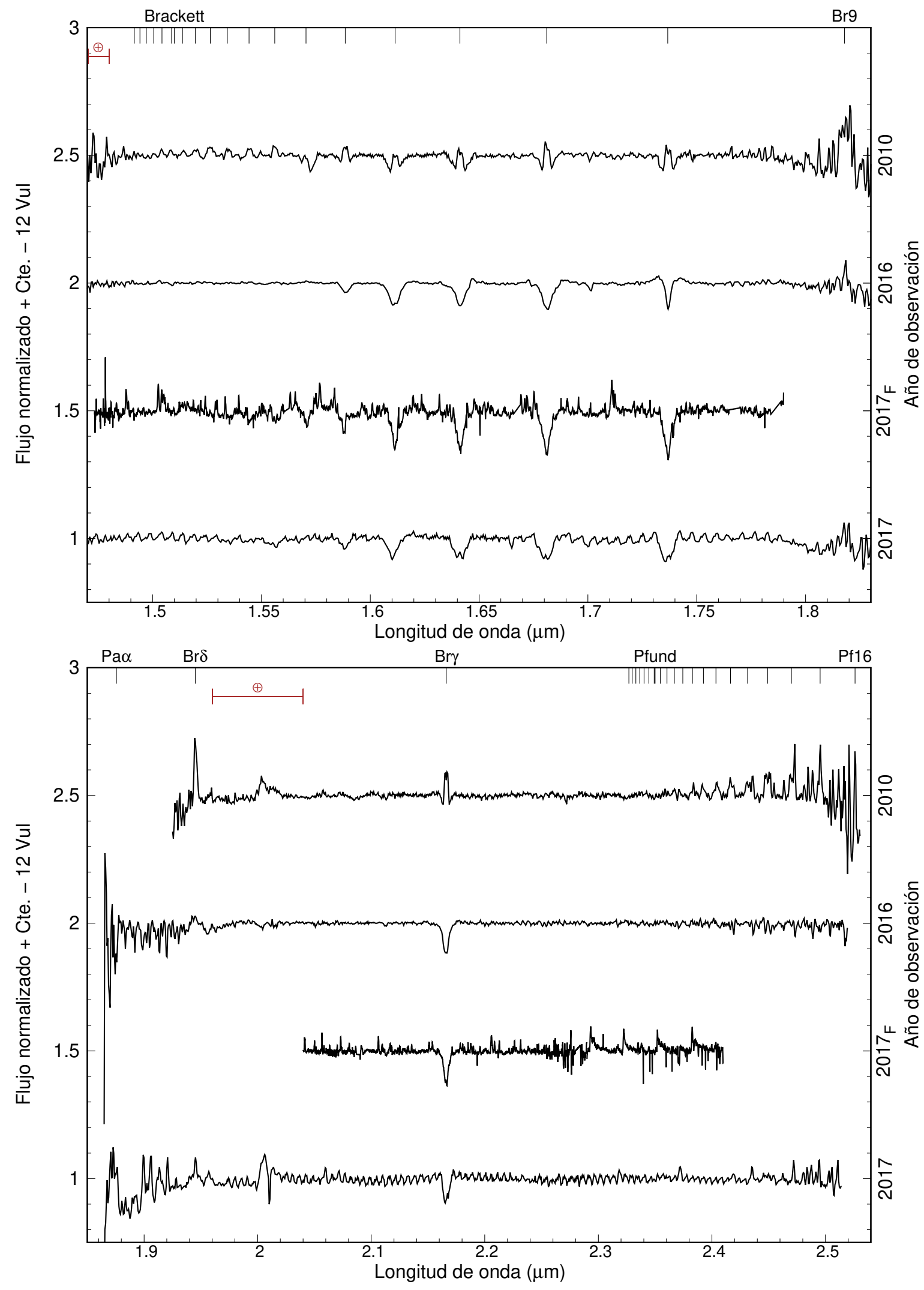
Figura 6.38: Espectros de 12 Vul en la banda L (superior) y líneas más intensas de cada banda (inferior). Junto a Pf $\gamma$ se observa $\mathrm{Hu}_{17}$.
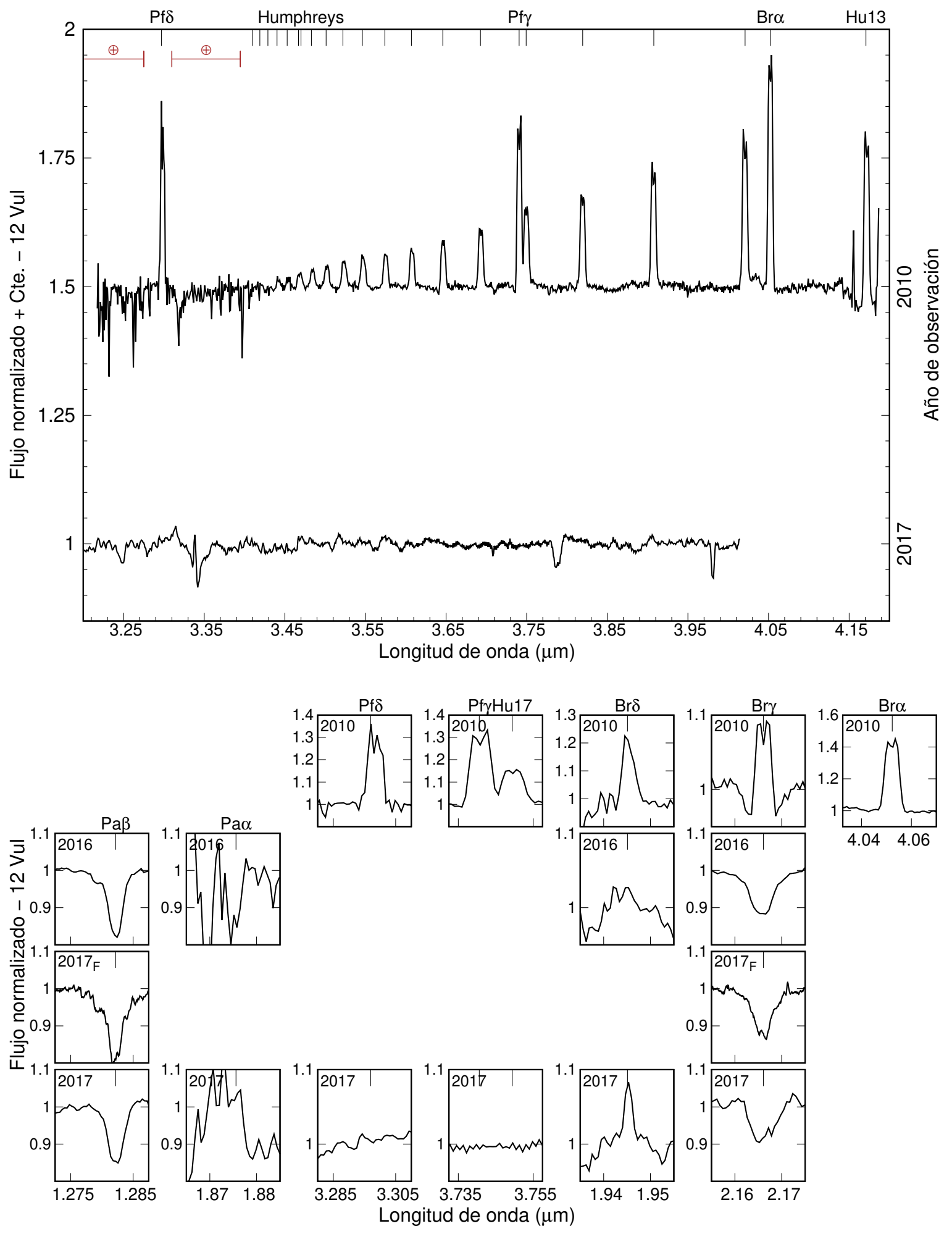


\section{Descripción de los espectros y mediciones realizadas}

En las Figs. 6.36 a 6.38 se muestran los espectros obtenidos en cada banda en las cuatro observaciones realizadas, junto con los primeros miembros de series. Las observaciones se hicieron en el 2010, 2016 y dos en el 2017 (con una separación aproximada de un mes, una de ellas utilizando FIRE). Solamente en el 2010 se observan las líneas de hidrógeno en emisión, superpuestas a la absorción fotosférica en la banda $\mathrm{H}$ y por sobre el nivel del continuo en las bandas K y L. En estos casos, las líneas presentan un perfil con dos picos. Los primeros miembros de las series de Paschen y Brackett presentan perfiles tipo shell o emisiones débiles en las observaciones de los años 2016 y 2017. Según la clasificación de Mennickent et al. (2009) la estrella pertenece al Grupo I en el 2010 y al Grupo III en el 2017. Los valores para los EW, Fl y FWHM de las líneas de hidrógeno en emisión se muestran en las Tablas B.37 a B.40. Para realizar la corrección por absorción fotosférica se utilizó el espectro sintético correspondiente a una estrella con $T_{\text {eff }}=18000 \mathrm{~K}$ y $\log g=4.0$, según los parámetros estelares de la Tabla 3.3.

\section{Ubicación en el diagrama de Lenorzer}

La estrella puede ubicarse en el diagrama de Lenorzer solamente para la observación del 2010, cuando la banda L presentaba líneas en emisión. En el mismo, 12 Vul se ubica en la parte superior, correspondiente a una estrella perteneciente la Grupo I.

\section{Comportamiento de las series de hidrógeno}

En la Fig. 6.40 se muestran los valores obtenidos para los EW/ $\lambda$ y FWHM en función de la longitud de onda en la observación del 2010. Para los EW/ $\lambda$ no se observa un valor de convergencia hacia

Figura 6.39: Ubicación de 12 Vul en el diagrama de Lenorzer en la observación del año 2010.

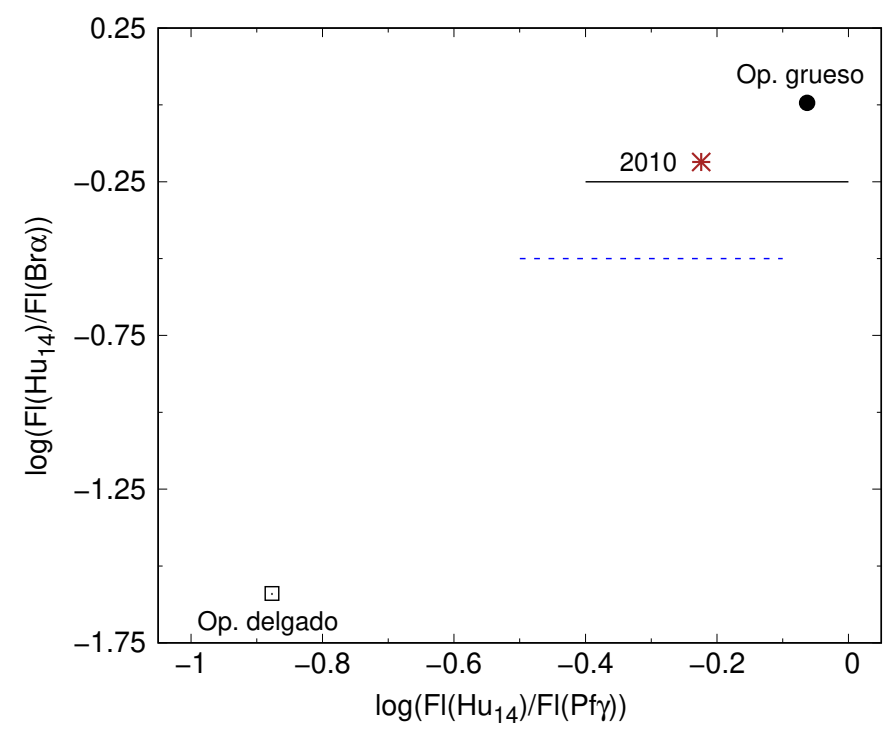


Figura 6.40: 12 Vul - EW/ $\lambda$ y FWHM vs. $\lambda$ de las líneas observadas en la observación del 2010. La línea punteada corresponde a un valor de $2 V \sin (i)=516 \mathrm{~km} / \mathrm{s}$, y la región sombreada al error correspondiente (Tabla 3.3).
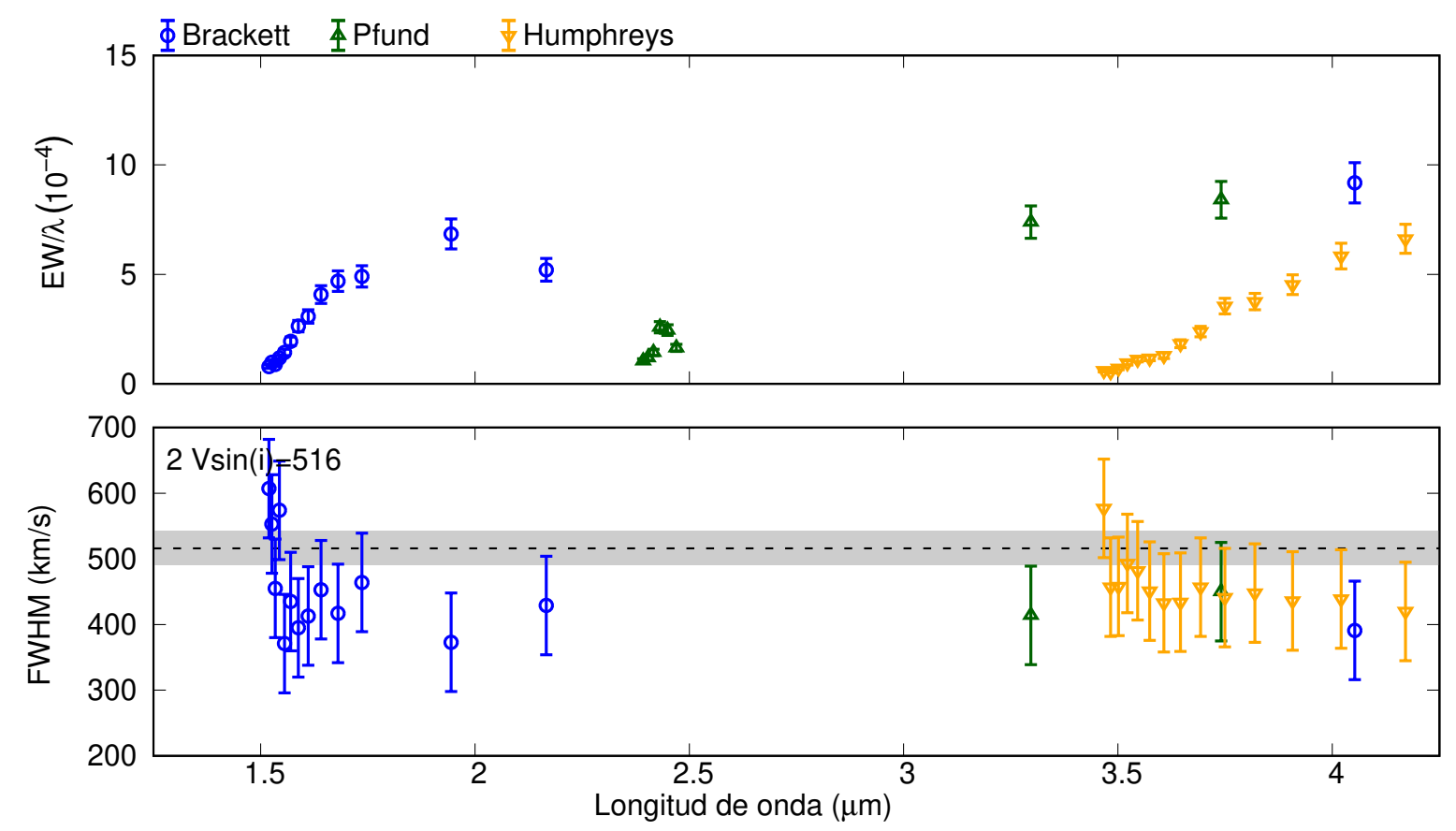

$\lambda$ mayores. Los FWHM son crecientes hacia miembros más altos de las series de Humphreys y Brackett, y la mayor parte de los mismos indican regiones de formación cercanas a la fotosfera.

En la Fig. 6.41 se muestran los cocientes de los flujos y la comparación con los casos ópticamente grueso y delgado. En la serie de Humphreys, todas las líneas se mantienen en la recta correspondiente al caso ópticamente delgado. En las series de Pfund y Brackett los primeros miembros se apartan del caso ópticamente delgado, hasta $\mathrm{Pf} \delta$ y $\mathrm{Br}_{11}$. Obtuvimos además que $\mathrm{EW}(\mathrm{Br} \alpha) / \mathrm{EW}(\mathrm{Br} \gamma) \sim 3.2$, valor en el límite de lo esperado para las estrellas del Grupo I.

\section{Determinación de densidades columnares y extensiones de las regiones de formación}

Las Tablas B.41 a B.43 muestran las densidades columnares, extensiones de las regiones de formación y radios internos de las mismas respecto a una de referencia y en radios estelares, calculados mediante los métodos $N$, FWHM y $\Delta V$. En la observación del 2010 no pudimos medir $\Delta V$ para $\operatorname{Br} \delta$, por lo que obtuvimos un valor aproximado del radio interno de la región de formación en radios estelares mediante el tamaño relativo a $\mathrm{Br} \alpha$ del método FWHM.

Obtuvimos que $\operatorname{Pf} \delta$ se forma en una región más extensa y de mayor densidad que $\operatorname{Pf} \gamma$, mientras que $\mathrm{Br}_{10}$ se forma en una región más extensa que $\mathrm{Br}_{11}$.

En las series de Humphreys y Brackett obtuvimos radios menores para los miembros más altos de la serie. Las dos líneas observadas de la serie de Pfund se forman aproximadamente 
Figura 6.41: 12 Vul - Cociente de flujos para las líneas de la serie de Humphreys, Pfund y Brackett. En cada gráfico, la línea continua corresponde al cociente para un caso ópticamente grueso, y la línea punteada al caso ópticamente delgado. En la parte superior se identifican las líneas correspondientes.
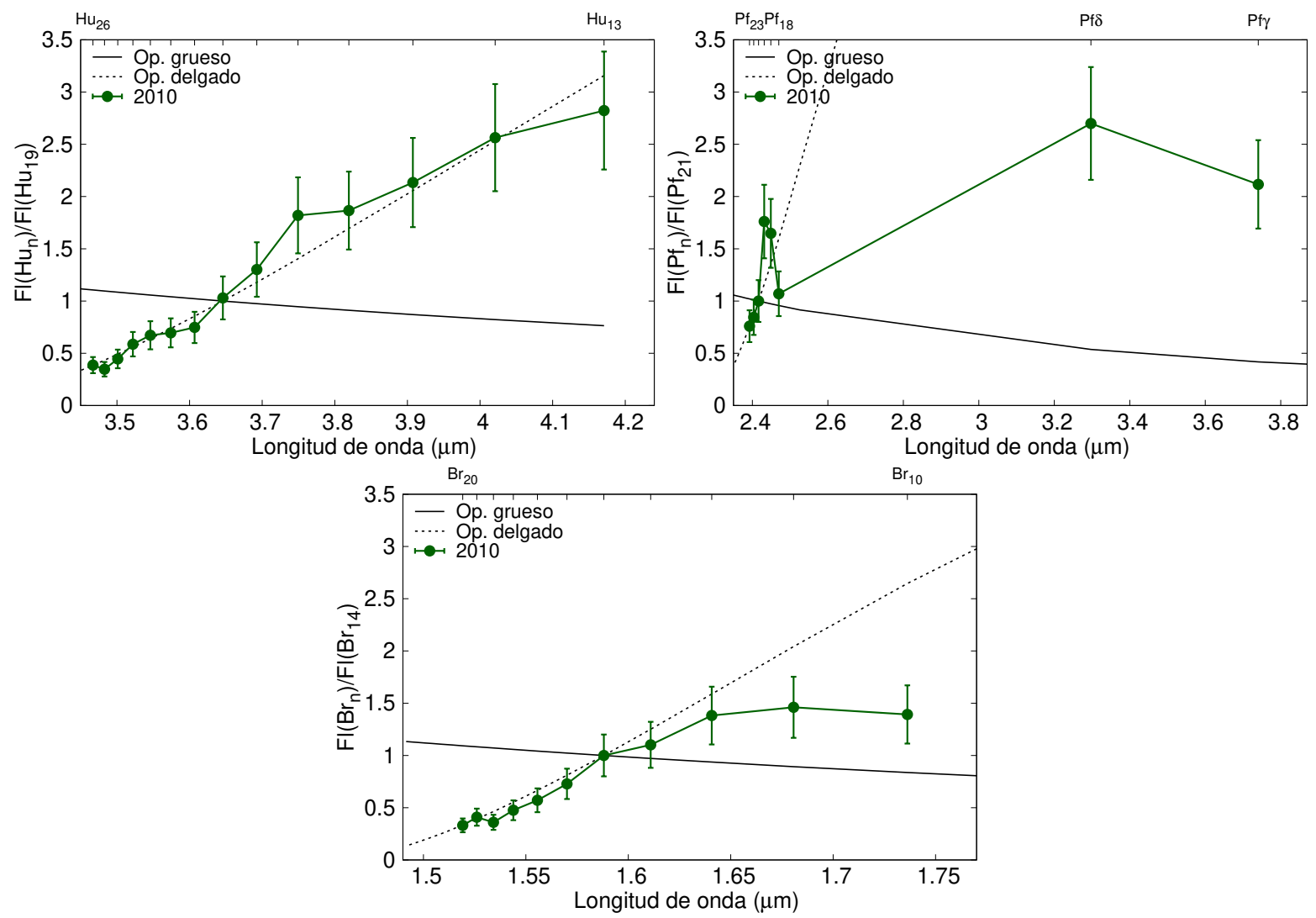

en la misma región. La Fig. 6.42 (superior) muestra los radios internos de todas las regiones de formación respecto al de $\operatorname{Br} \alpha$. La mayor parte de las líneas comienzan a formarse en una región más interna que $\operatorname{Br} \alpha$.

En radios estelares, las líneas de las series de Humphreys y Brackett tienen radios internos en el intervalo $2.75-5 R_{\star}$, mientras que Pf $\gamma$ y Pf $\delta$ se forman a partir de $4 R_{\star}$. En la Fig. Fig. 6.42 (inferior) graficamos los radios internos de las regiones de formación. En la observación del 2010, $12 \mathrm{Vul}$ presenta un disco compacto con radios internos en el rango $2.75-5.5 R_{\star}$. 
Figura 6.42: $12 \mathrm{Vul}$ - Radio internos de las regiones emisoras de las diferentes líneas relativos a la región emisora de $\operatorname{Br} \alpha$ y en radios estelares. Los símbolos utilizados son los mismos que en la Fig. 6.40 .
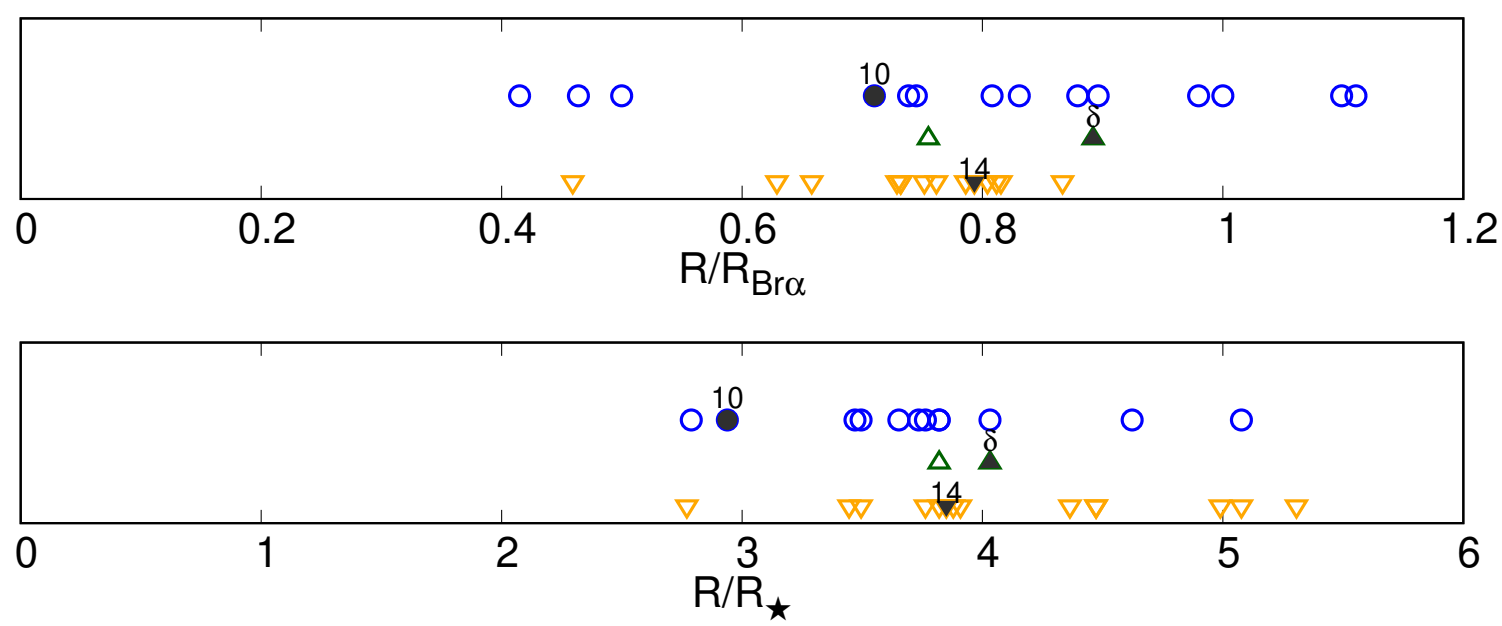


\subsubsection{Cyg - HD 191610}

28 Cyg es una estrella Be con clasificación espectral B2.5 V, que presenta variaciones fotométricas de corto período (Percy et al., 2001). Fue reportada como Be por Merrill et al. (1925), y Peters \& Penrod (1988) confirmaron importantes pérdidas de masa y estructuras en movimiento a partir de perfiles de líneas en el óptico. La línea $\mathrm{H} \alpha$ presenta una gran variabilidad, pasando de una gran emisión con dos picos en el 2001 a una pequeña intensidad el 2009. Entre los años 2011 y 2014, la línea $\mathrm{H} \alpha$ en emisión incrementó un poco su intensidad (Jones et al., 2011; Sabogal et al., 2017).

Lenorzer et al. (2002b) obtuvieron espectros IR en el año 1998, a partir de los cuales ubicaron a la estrella en la región superior del diagrama de Lenorzer. Posteriormente, Touhami et al. (2010) presentaron observaciones en el IR obtenidas en el 2006, y la ubicaron en la parte central del diagrama de Lenorzer, entre los límites ópticamente grueso y delgado. Hasta el año 2008, 28 Cyg continuó cambiando su posición en el diagrama de Lenorzer, ubicándose según lo reportado por Granada et al. (2010) en la región inferior, correspondiente a las estrellas del grupo II. Más adelante, Sabogal et al. (2017) reportan que en el año 2011 la estrella presentaba líneas de H en emisión muy débiles, y la clasifica como perteneciente al grupo II. A partir de los cambios de posición en el diagrama de Lenorzer, y del cambio de apariencia de la línea $\mathrm{H} \alpha$, Sabogal et al. (2017) la clasificaron como una estrella con disco en disipación. Además, remarcaron que es la que presenta el mayor desplazamiento a lo largo del diagrama de Lenorzer.

Vieira et al. (2017) determinaron valores de $\log \rho_{0}=-10 \pm 1$ y $n=4 \pm 1$ en $1983, \log \rho_{0}=-9 \pm 2$ y $n=10 \pm 6$ entre 2006 y 2007 , y $\log \rho_{0}=-11.2 \pm 0.3$ y $n=3.2 \pm 0.2$ en 2010 , a partir del modelado del continuo IR. Según estos valores de $n$ y el criterio definido por estos autores, 28 Cyg presentaba un disco en formación en 1983 y 2006/2007, y estable en el 2010.

\section{Descripción de los espectros y mediciones realizadas}

En las Figs. 6.43 y 6.44 se presentan los espectros obtenidos en los diferentes turnos. Por otro lado, en la Fig. 6.45 se muestran las líneas más intensas de cada banda en diferentes escalas. Los primeros miembros de cada serie se observan en emisión en todos los espectros (o al menos rellenando la absorción fotosférica en el caso de Bry en el año 2010), junto con los miembros más altos de las series de Brackett, Pfund y Humphreys en los años 2016 y 2017. Los miembros de las series de Brackett, Pfund y Humphreys presentan perfiles con dos picos en el 2017, al igual que $\operatorname{Pa} \beta$, los miembros de la serie de Brackett y $\operatorname{Br} \gamma$ en el 2016 y Pf $\gamma$ y $\operatorname{Pf} \delta$ en el 2010. Según el criterio de Mennickent et al. (2009), clasificamos a 28 Cyg como perteneciente al grupo I en el 2017 y al grupo II en las otras observaciones (extremo en el caso de la observación del 2008, donde no presenta emisión en las líneas de Humphreys). En las Tablas B.44 a B.47 se listan las valores de EW, Fl y FWHM de las líneas de hidrógeno observadas en emisión. Según los parámetros estelares presentados en la Tabla 3.3, para realizar la corrección por absorción fotosférica se utilizó el espectro sintético correspondiente a una estrella con $T_{\text {eff }}=18000 \mathrm{~K}$ y $\log g=3.5$. 
Figura 6.43: Espectros de 28 Cyg en las bandas J (superior) y H (inferior).

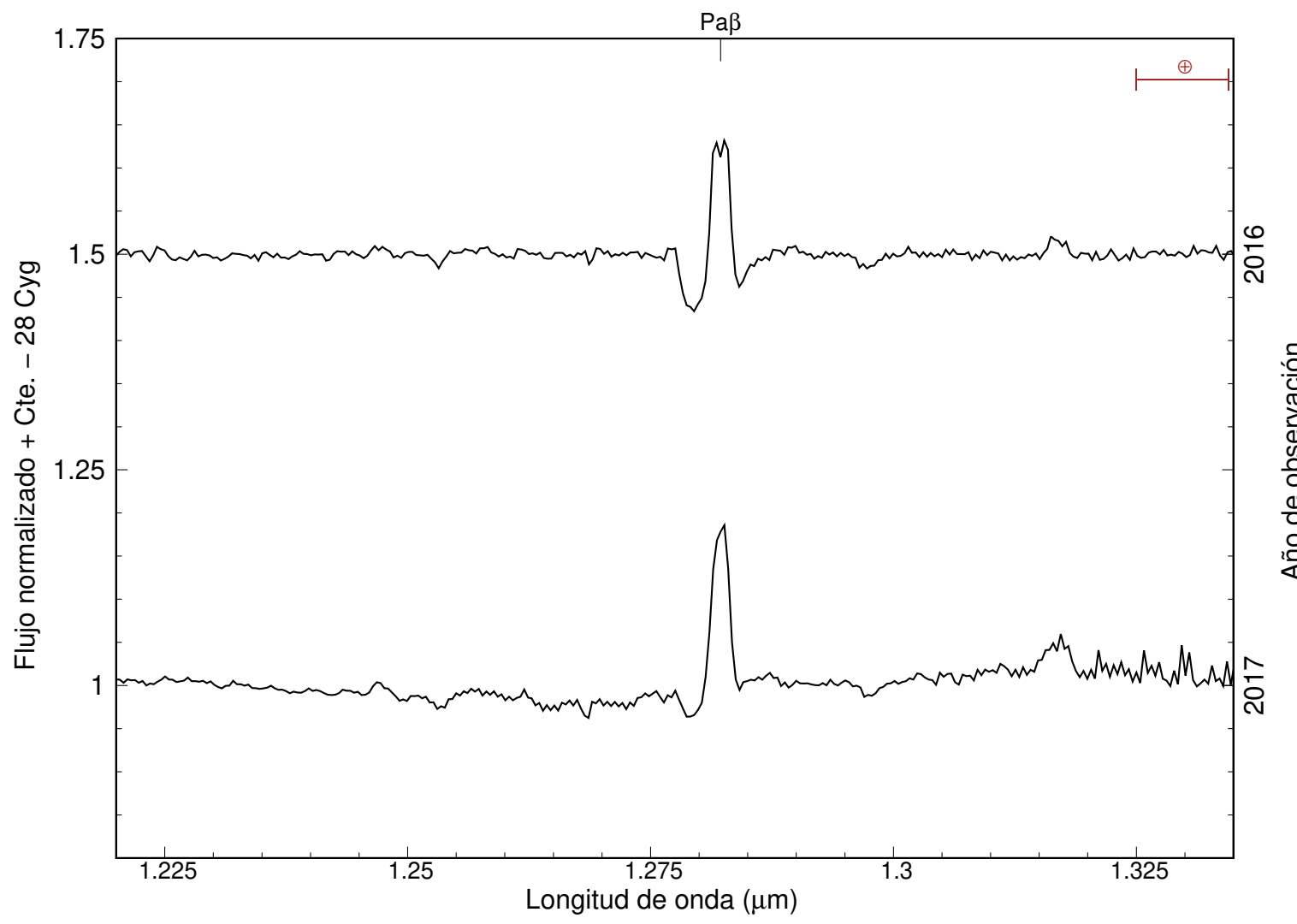

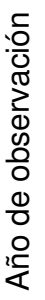

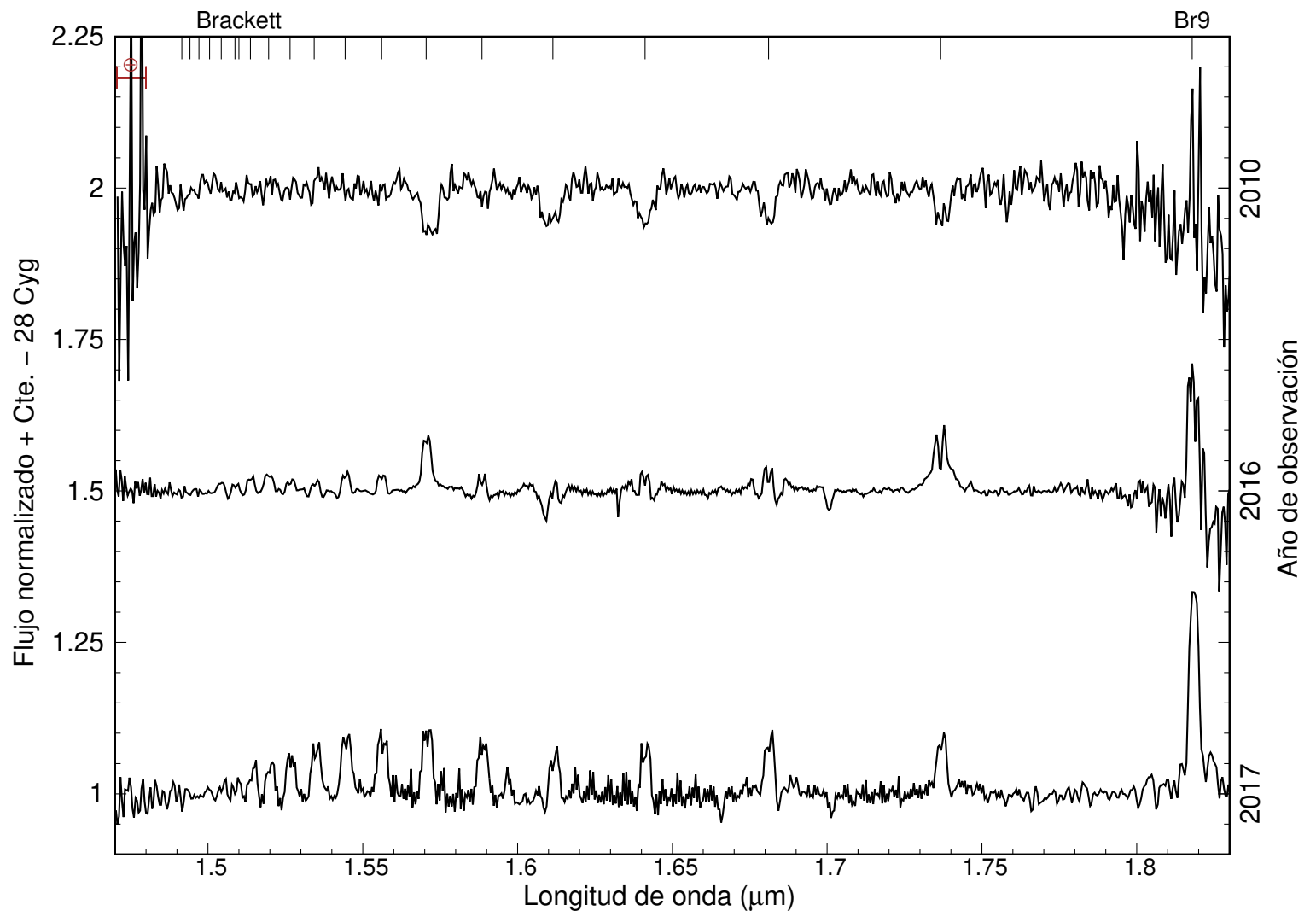


Figura 6.44: Espectros de 28 Cyg en las bandas K (superior) y L (inferior).
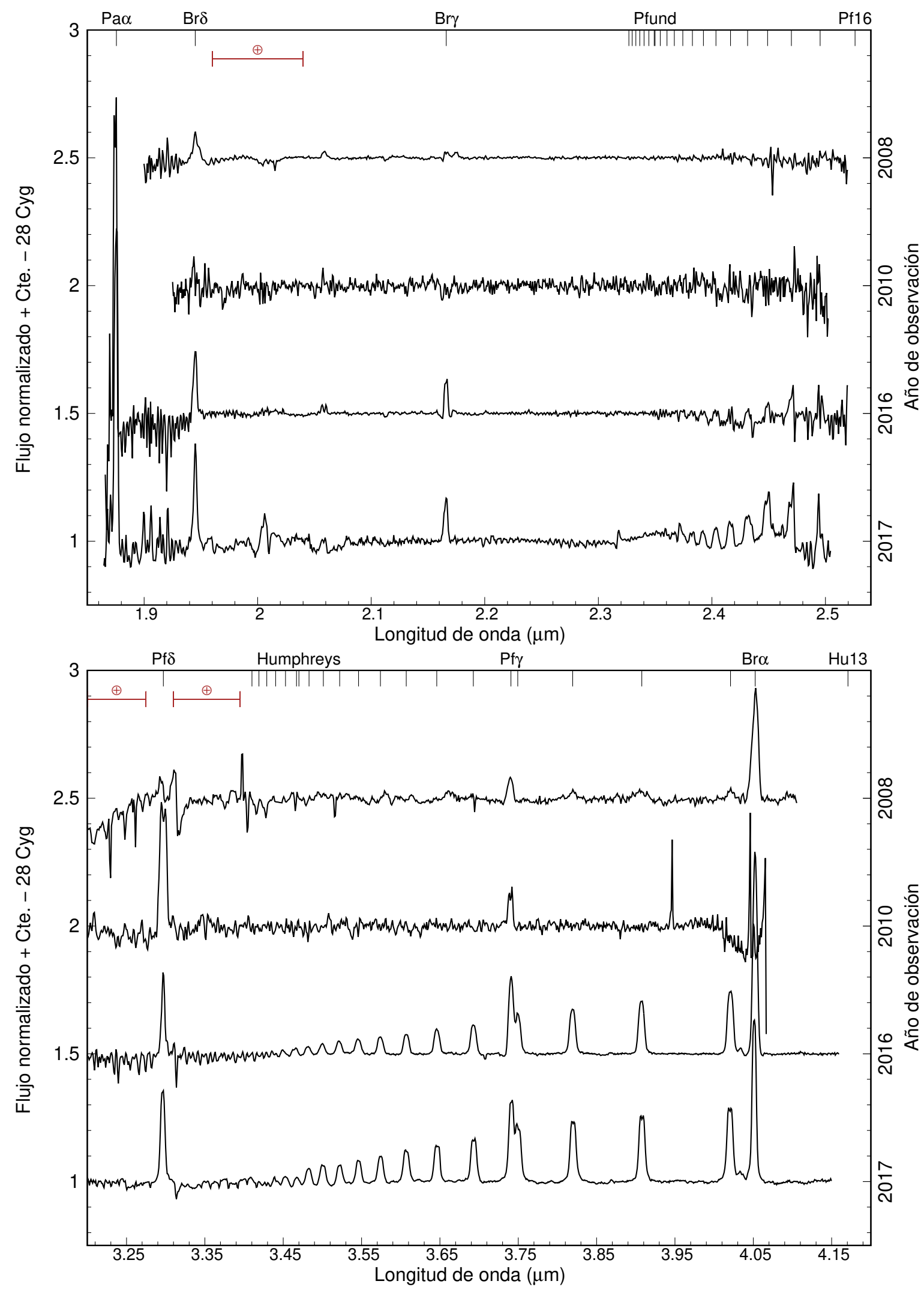
Figura 6.45: 28 Cyg - Líneas más intensas de cada banda. Junto a Pf $\gamma$ se observa $\mathrm{Hu}_{17}$.
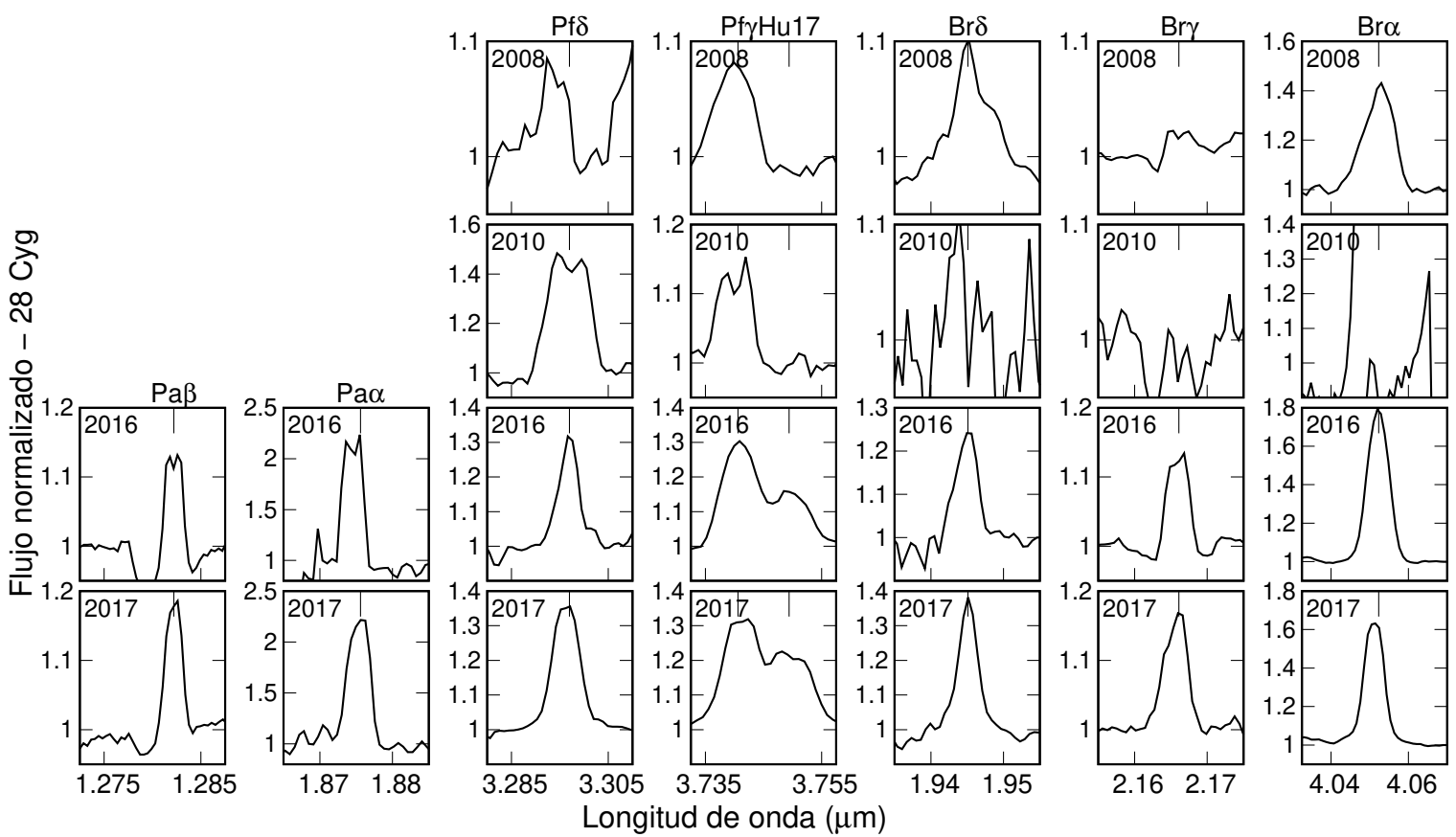

Figura 6.46: Ubicación de 28 Cyg en el diagrama de Lenorzer en las diferentes observaciones.

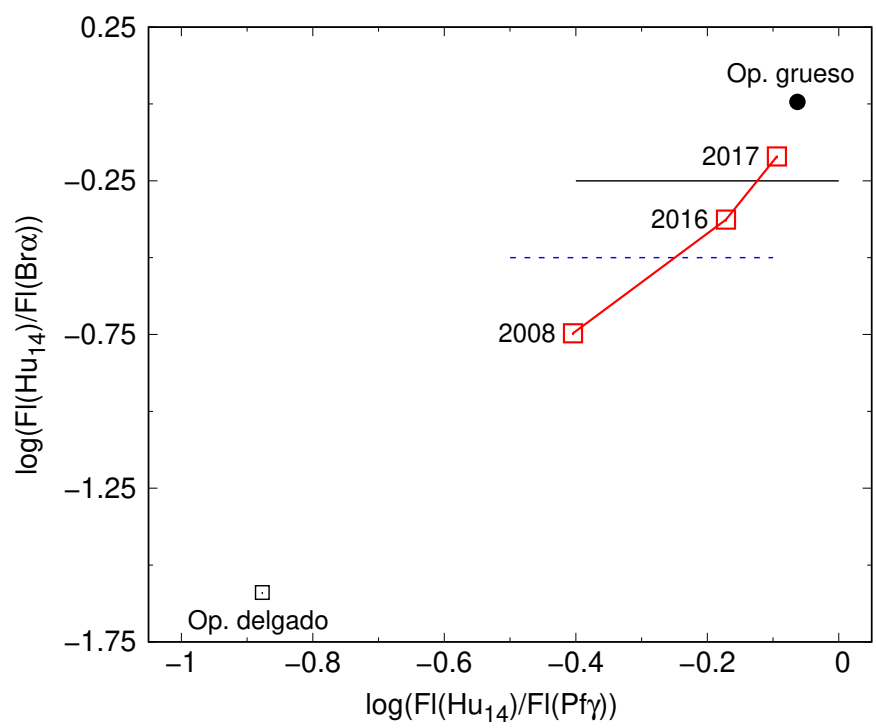

\section{Ubicación en el diagrama de Lenorzer}

En la Fig. 6.46 puede verse como varió la ubicación de la estrella en el diagrama de Lenorzer. Como fue reportado por Granada et al. (2010), en el 2008 la estrella se ubicaba en la región correspondiente al Grupo II. En el año 2010, debido a las pocas líneas observadas, no puede representarse su ubicación en el diagrama. En los años 2016 y 2017 la estrella parece desplazarse 
Tabla 6.5: 28 Cyg - Líneas que se apartan del caso ópticamente delgado.

\begin{tabular}{l|cc}
\hline \hline & 2016 & 2017 \\
\hline Humphreys & 14 & $14-15$ \\
Pfund & $\gamma-\delta$ & $\gamma-\delta$ \\
Brackett & $9-13$ & $9-16$ \\
\hline
\end{tabular}

hacia la región correspondiente a las envolturas ópticamente gruesas, similar a la posición reportada por Lenorzer et al. (2002a) a partir de observaciones realizadas en el año 1998. Esta ubicación en consistente con la pertenencia al grupo I en la observación del 2017.

\section{Comportamiento de las series de hidrógeno}

En las Figs. 6.47 y 6.48 se muestran los diagramas de EW/ $\lambda$ y FWHM según la longitud de onda. En el 2008 y 2010, donde el disco está más disipado, no tenemos suficientes líneas como para discutir un comportamiento en las distintas series. En el 2016 se observa un comportamiento creciente en el valor de EW/ $\lambda$, que parece estabilizarse en la observación del 2017 para valores $\sim 7 \cdot 10^{-4}$. Para los valores obtenidos del FWHM, la serie de Humphreys presenta valores similares a $2 V \sin (i)$ en el 2016 y 2017 , indicando regiones de formación cercanas a la estrella central. Para las series de Pfund y Brackett se observa una tendencia hacia FWHM mayores en los miembros más altos de las series. Los FWHM obtenidos para Pf $\gamma$ y Pf $\delta$ no siempre coinciden, indicando regiones de formación diferentes. En el caso de Pf $\delta$ del 2010 se observa un ensanchamiento mayor al correspondiente según la rotación estelar, lo que podría indicar la existencia de algún mecanismo adicional que agrega momento angular al material del disco. El único caso donde el FWHM correspondiente a $\mathrm{Br} \alpha$ es similar al valor de $2 V \sin (i)$ es el 2008, donde ésta es la única línea en emisión de esta serie, y la estrella está más cercana a la región correspondiente al caso ópticamente delgado del diagrama de Lenorzer. Las líneas de la serie de Paschen observadas en el 2017 se estarían formando en regiones alejadas de la estrella central.

En la Fig. 6.49 se muestran los cocientes de los flujos para las líneas de las distintas series, y la comparación con los casos ópticamente grueso y delgado. En todos los casos los primeros miembros se apartan del caso ópticamente delgado. Las líneas que se apartan del caso ópticamente delgado se listas en la Tabla 6.5. En el año 2016 la serie de Brackett muestra un comportamiento particular, donde se muestra un apartamiento del caso ópticamente delgado en miembros intermedios de la serie, manteniéndose los primeros miembros cercanos a la recta correspondiente al caso ópticamente delgado. En el 2008 y 2010, solo pudimos graficar los cocientes de flujos para los primeros miembros de la serie de Pfund, donde vemos que el flujo de la línea Pf $\gamma$ es menor que el de $\mathrm{Pf} \delta$. Obtuvimos además que $\mathrm{EW}(\mathrm{Br} \alpha) / \mathrm{EW}(\mathrm{Br} \gamma) \sim 7.3$ en el año 2008 (valor esperado para una estrella del grupo II) y que entre el 2016 y 2017, el valor disminuyó desde 5.5 hasta 3.9, consistente con el pasaje del grupo II al I. Para la serie de Paschen calculamos EW(Pa $\alpha) / \mathrm{EW}(\mathrm{Pa} \beta)$ en el 2016 y 2017 , obteniendo valores de $\sim 10$ y $\sim 6$, respectivamente. 
Figura 6.47: 28 Cyg - EW/ $\lambda$ vs. $\lambda$ de las líneas observadas en las diferentes series en las distintas observaciones.

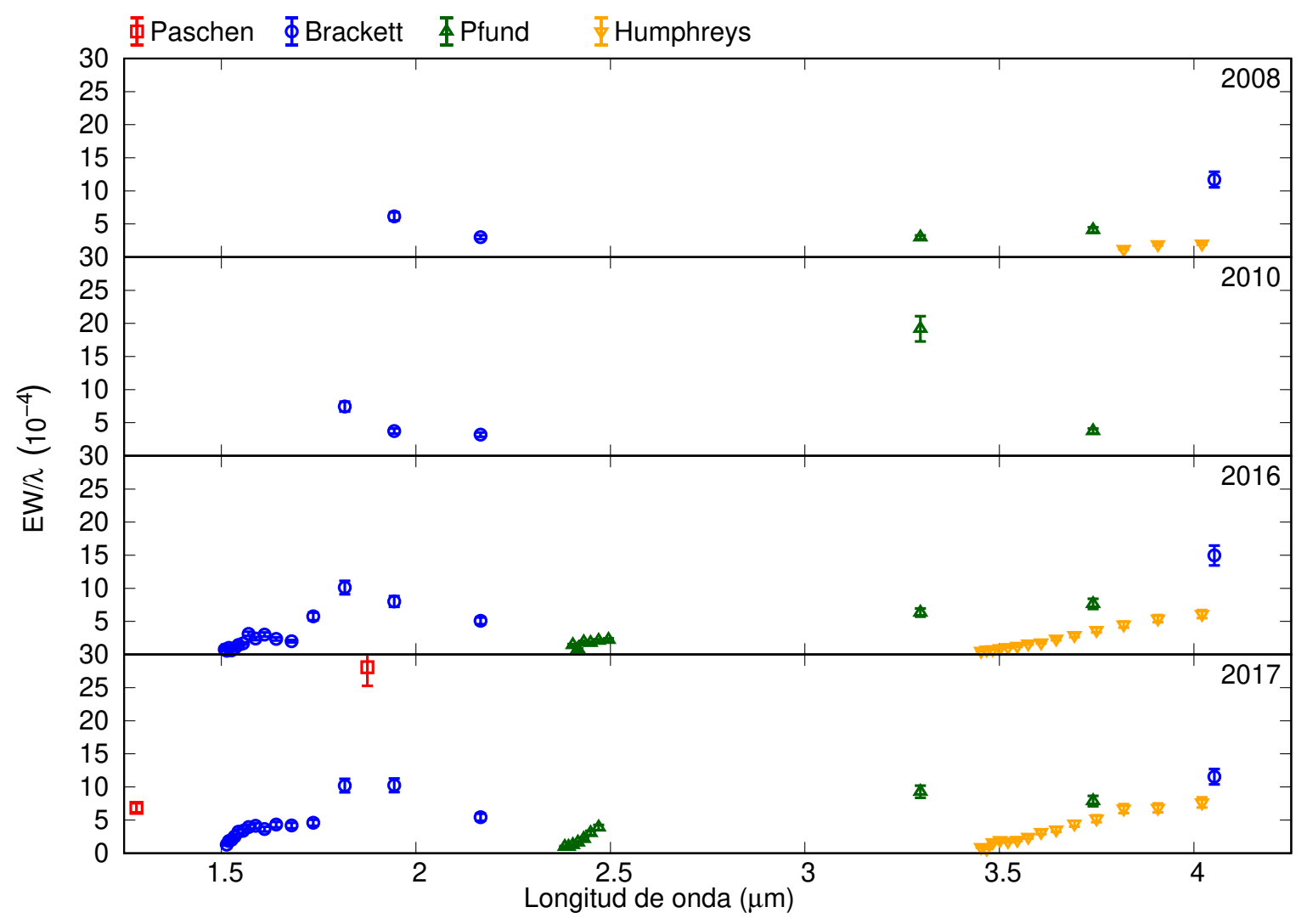

\section{Determinación de densidades columnares y extensiones de las regiones de formación}

Obtuvimos valores de las densidades columnares, extensiones de las regiones de formación de las líneas y radios internos de las mismas con los métodos $N$, FWHM y $\Delta V$. Los mismos se muestran en las Tablas B.48 a B.51. Para las líneas en las cuales no pudimos medir $\Delta V$, presentamos valores aproximados obtenidos a partir de los tamaños relativos a Pf $\gamma$ para la observación del 2010, $\mathrm{Pa} \alpha$ para la observación del 2016 y Hu 14 para la observación del 2017.

Las líneas de la serie de Humphreys presenta menores densidades columnares para sus regiones de formación que las de las series de Pfund y Brackett. Los valores obtenidos a lo largo de la serie de Brackett son similares entre si.

En las líneas de la serie de Humphreys en el 2016 y 2017, y los miembros más altos de la serie de Pfund en el 2016, los radios de las regiones de formación son similares a lo largo de la serie. En la serie de Brackett la línea que se forma desde una región más externa es Bro. Las líneas $\mathrm{Pa} \alpha$ y $\mathrm{Pa} \beta$ tenían radio internos muy similares en 2016, y mayor el de $\mathrm{Pa} \beta$ en 2017. La Fig. 6.50 (superior) muestra los tamaños de las regiones de formación relativos a Pf $\gamma$. Para las observaciones del 2008 y 2010 son pocas las líneas con FWHM medido, por lo que no pudimos 
Figura 6.48: 28 Cyg - FWHM vs. $\lambda$ de las líneas observadas en las diferentes series en las distintas observaciones. La línea punteada corresponde a un valor de $2 V \sin (i)=656 \mathrm{~km} / \mathrm{s}$, y la región sombreada al error correspondiente (Tabla 3.3). Los símbolos utilizados son los mismos que en la Fig. 6.47.

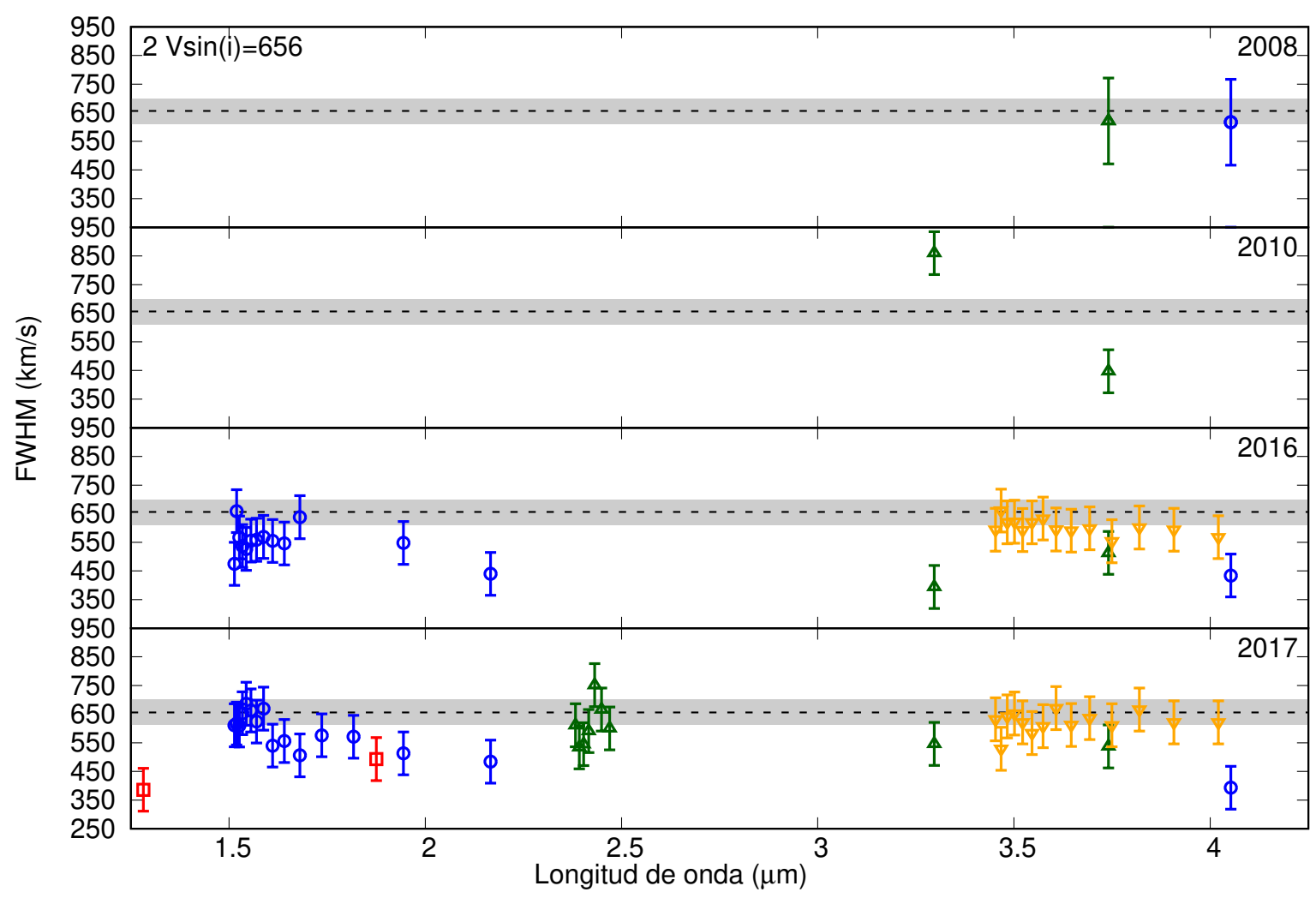

obtener comportamientos generales. Para el 2016 y 2017 se observan numerosas líneas, llegando a alcanzar las regiones de formación extensiones del doble a la correspondiente a Pf $\gamma$. La serie de Humphreys se forma en una región más interna que $\operatorname{Pf} \gamma$ en ambas observaciones, $\operatorname{Pa} \alpha$ y $\operatorname{Pa} \beta$ en una región más externa, y las líneas medidas de las series de Brackett y Pfund se forman tanto antes como después de $\mathrm{Pf} \gamma$.

En la Fig. 6.50 (inferior) se muestran los valores en radios estelares obtenidos a partir de la separación de los dos picos en emisión, junto con valores aproximados obtenidos a partir de los tamaños relativos cuando no se observaron perfiles con dos picos. En el 2010 las pocas líneas en emisión observadas provienen de zonas que llegan a los $5 R_{\star}$. Para el 2016 y 2017 , donde se observan numerosas líneas en emisión, los radios internos de las regiones de formación están entre $2 R_{\star}$ y $8 R_{\star}$ para la observación del 2016 , y entre $2 R_{\star}$ y $10 R_{\star}$ para el 2017 . 
Figura 6.49: 28 Cyg - Cociente de flujos para las líneas de la serie de Humphreys, Pfund y Brackett. En cada gráfico, la línea continua corresponde al cociente para un caso ópticamente grueso, y la línea punteada al caso ópticamente delgado. En la parte superior se identifican las líneas correspondientes.
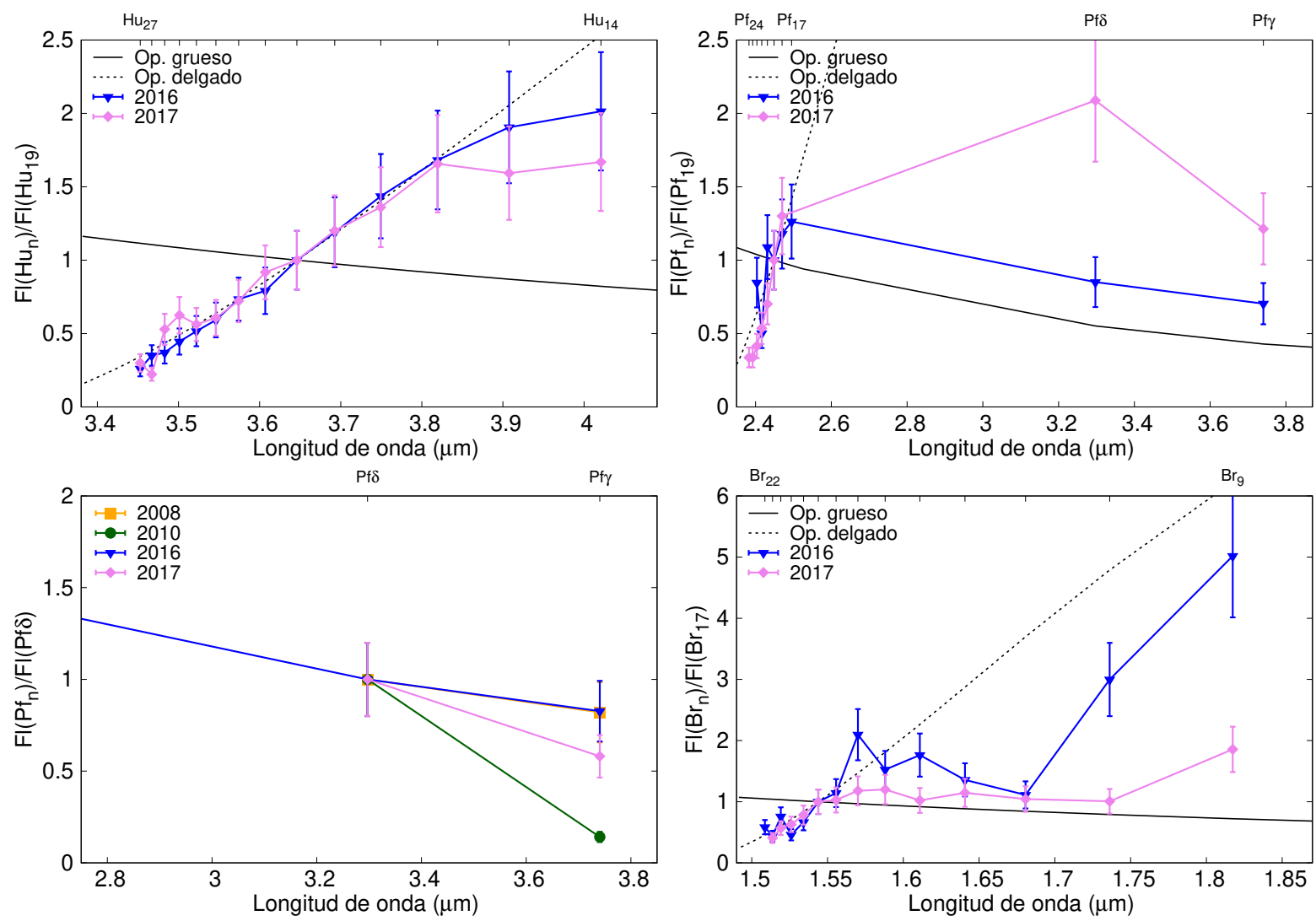
Figura 6.50: 28 Cyg - Radios internos de las regiones emisoras de las diferentes líneas relativos a la región emisora de Pf $\gamma$ y en radios estelares. Los símbolos utilizados son los mismos que en la Fig. 6.47.
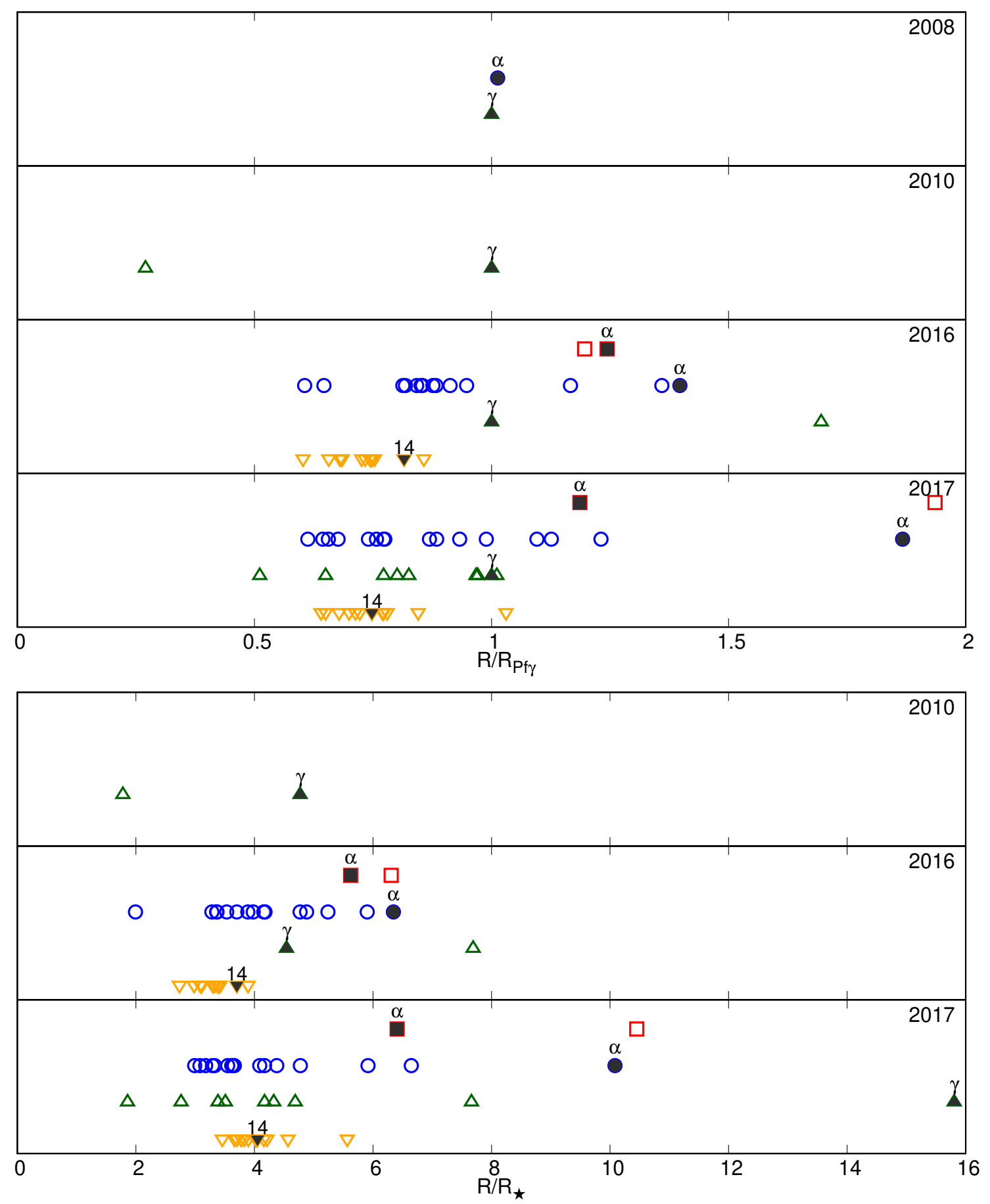


\subsubsection{EW Lac - HD 217050}

EW Lac es una estrella Be shell, con clasificación espectral B4 III (Slettebak, 1982). Slettebak (1951) reportaron la presencia de las líneas $\mathrm{H} \alpha, \mathrm{H} \beta$ y O I $\lambda 8446 \AA$.

Esta estrella ha presentado variaciones espectrales y fotométricas en diferentes escalas de tiempo: Walker (1958) reportó variaciones fotométricas con período 0.7 días, Pavlovski et al. (1993) reportaron multiperiodicidad en este objeto, Jeong et al. (1986) reportaron variaciones fotométricas en 50-60 días y Percy et al. (1996) determinaron otro período de 300 días. También se reportaron variaciones en el cociente V/R (Slettebak \& Reynolds, 1978; Andrillat \& Fehrenbach, 1982). A partir de las velocidades radiales, Antonello et al. (1982) propusieron que EW Lac podría ser un sistema binario con período 40.3 años.

Clark \& Steele (2000) realizaron observaciones en la banda K, y reportaron la presencia de la línea $\mathrm{Br} \gamma$ en emisión con un perfil con dos picos, junto He I $\lambda 2.058 \mu \mathrm{m}$. Estos autores indicaron que esta estrella muestra efectos de diferentes profundidades ópticas en ambas líneas, ya que la línea de He I muestra un perfil tipo shell muy pronunciado, indicando una profundidad óptica grande, mientras que la absorción central de $\mathrm{Br} \gamma$ no sobrepasa el nivel del continuo. Las líneas de la serie de Brackett observadas en la banda $\mathrm{H}$ también presentaban un perfil en emisión con dos picos (Steele \& Clark, 2001).

Granada et al. (2010), utilizando espectros obtenidos en los años 2006 y 2008 indicaron que EW Lac pertenecía al grupo II en el momento de ambas observaciones y que la posición en el diagrama de Lenorzer mostraba que podía ser una estrella en transición entre los grupos I y II.

\section{Descripción de los espectros y mediciones realizadas}

En las Figs. 6.51 y 6.52 se presentan los espectros obtenidos en los cinco turnos de observación. Además, en la Fig. 6.53 se muestran las primeros miembros de cada serie, que no se aprecian bien en la escala de los gráficos anteriores. En las observaciones de los años 2006, 2008 y 2010 las líneas de hidrógeno se muestran en emisión, con dos picos en el caso de la serie de Pfund en el año 2008 y las series de Brackett, Pfund y Humphreys en el 2010. En el año 2016 solo se observan en emisión los miembros de la serie de Humphreys, junto con algunos miembros de la serie de Pfund y los primeros miembros de las otras series. La serie de Brackett presenta perfiles tipo shell en la observación del 2017, mientras que el resto de las líneas se observan en emisión con doble pico. Según la clasificación de Mennickent et al. (2009), en todas las observaciones EW Lac pertenece al grupo II. En las Tablas B.52 a B.55 se listan los EW, Fl y FWHM de las líneas de hidrógeno observadas en emisión. Si bien la estrella está clasificada como B4III (Tabla 3.3, en su espectro presenta líneas de He I, correspondientes a tipos espectrales B3 o más tempranos (Clark $\&$ Steele, 2000). Por lo tanto, la corrección por absorción fotosférica fue realizada considerando $T_{\text {eff }}=18000 \mathrm{~K}$ y $\log g=3.5$, correspondientes a una estrella B3III. 
Figura 6.51: Espectros de EW Lac en las bandas J (superior) y H (inferior).
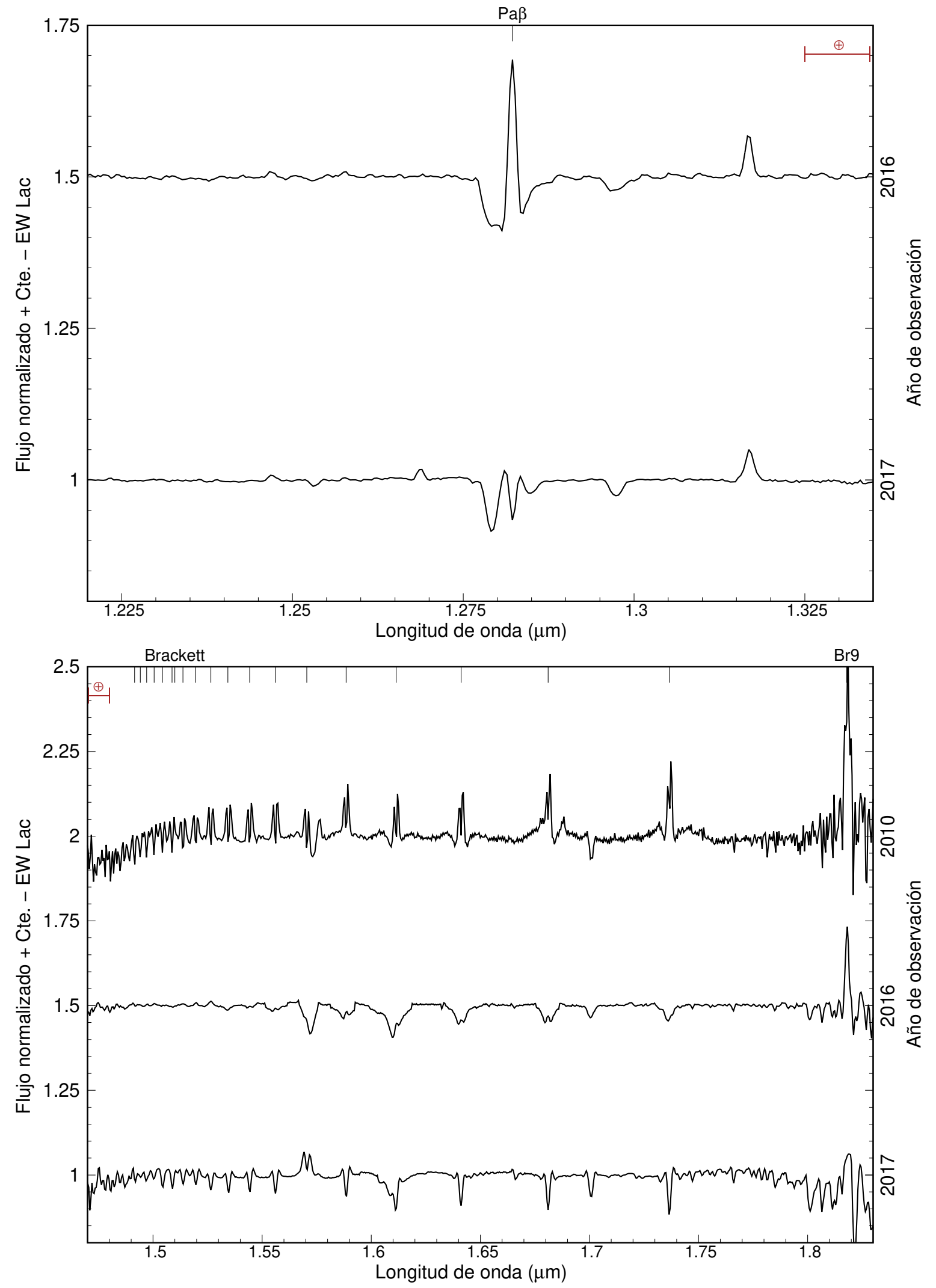
Figura 6.52: Espectros de EW Lac en las bandas K (superior) y L (inferior).
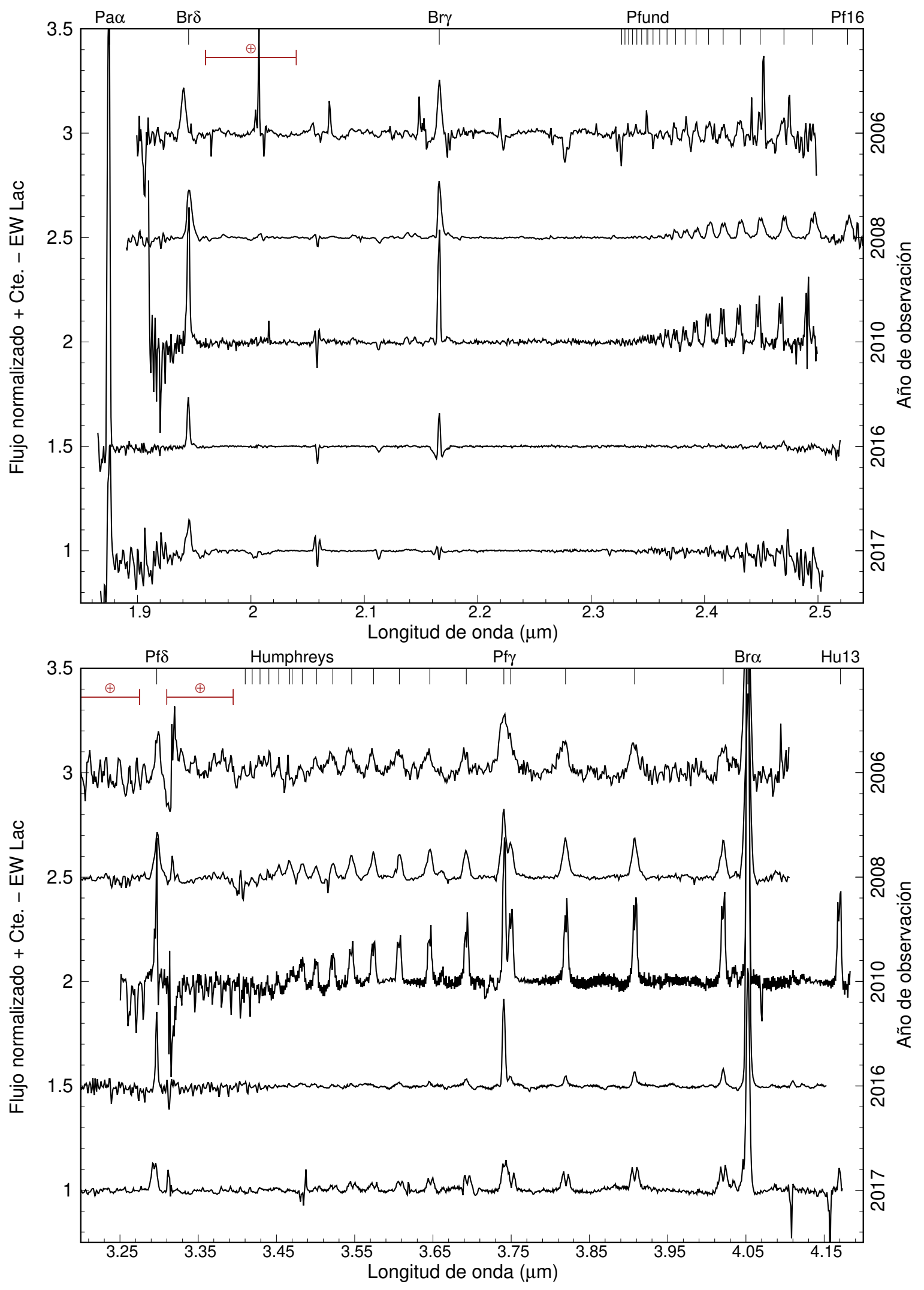
Figura 6.53: EW Lac - Líneas más intensas de cada banda. Junto a Pf $\gamma$ se observa $\mathrm{Hu}_{17}$.
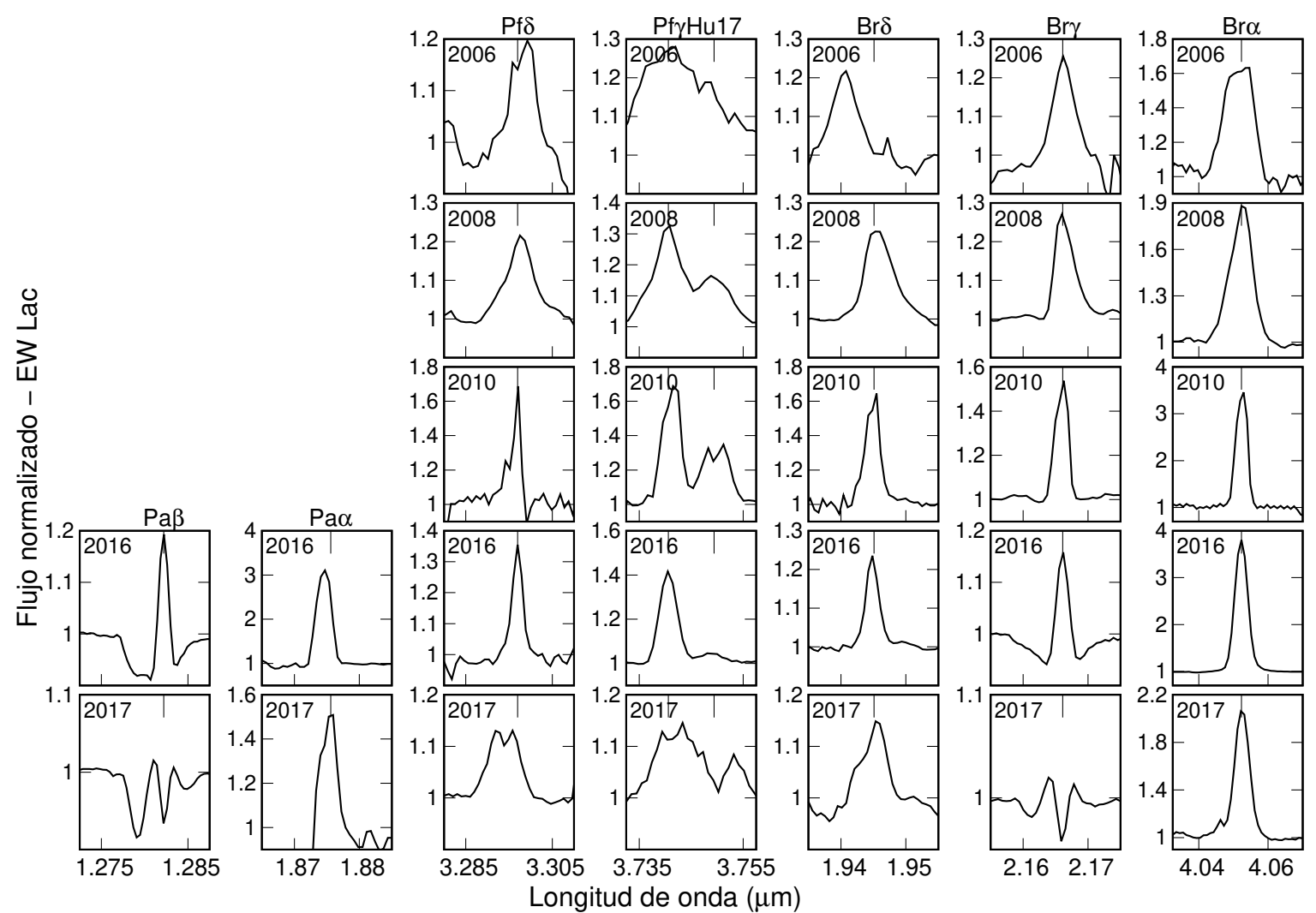

Figura 6.54: Ubicación de EW Lac en el diagrama de Lenorzer en las diferentes observaciones.

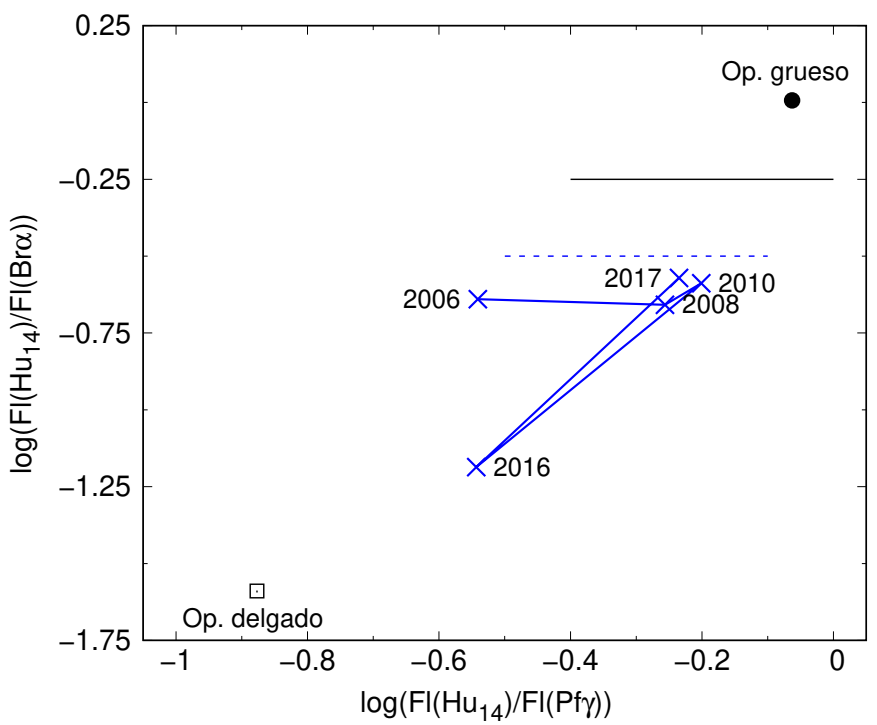




\section{Ubicación en el diagrama de Lenorzer}

En la Fig. 6.54 puede verse la ubicación de EW Lac en el diagrama de Lenorzer en las diferentes observaciones. Granada et al. (2010) habían reportado que EW Lac podría ser un objeto de transición entre los grupos I y II por su ubicación en el diagrama en los años 2006 y 2008. Además, el aumento en la intensidad de $\operatorname{Br} \alpha$ había sido interpretado como un incremento de la superficie emisora de la línea. Luego de eso, en la observación del año 2010, la estrella se encuentra más cerca de la región correspondiente al caso ópticamente grueso. En el 2016 se ubicó en una posición más cercana al caso ópticamente delgado, para volver a una posición similar a las del 2010 en el 2017. Este desplazamiento en el diagrama podría indicar la eyección de la envoltura ópticamente gruesa, que se expande y se transforma en ópticamente delgada hasta el año 2016, para luego volver a desarrollarse.

\section{Comportamiento de las series de hidrógeno}

En las Figs. 6.55 y 6.56 se muestran los gráficos de EW/ $\lambda$ y FWHM según la longitud de onda. Los valores de $\mathrm{EW} / \lambda$ comienza a mostrar un valor estable de $5 \cdot 10^{-4}$ para las longitudes de onda

Figura 6.55: EW Lac - EW/ $\lambda$ vs. $\lambda$ de las líneas observadas en las diferentes series en las distintas observaciones.

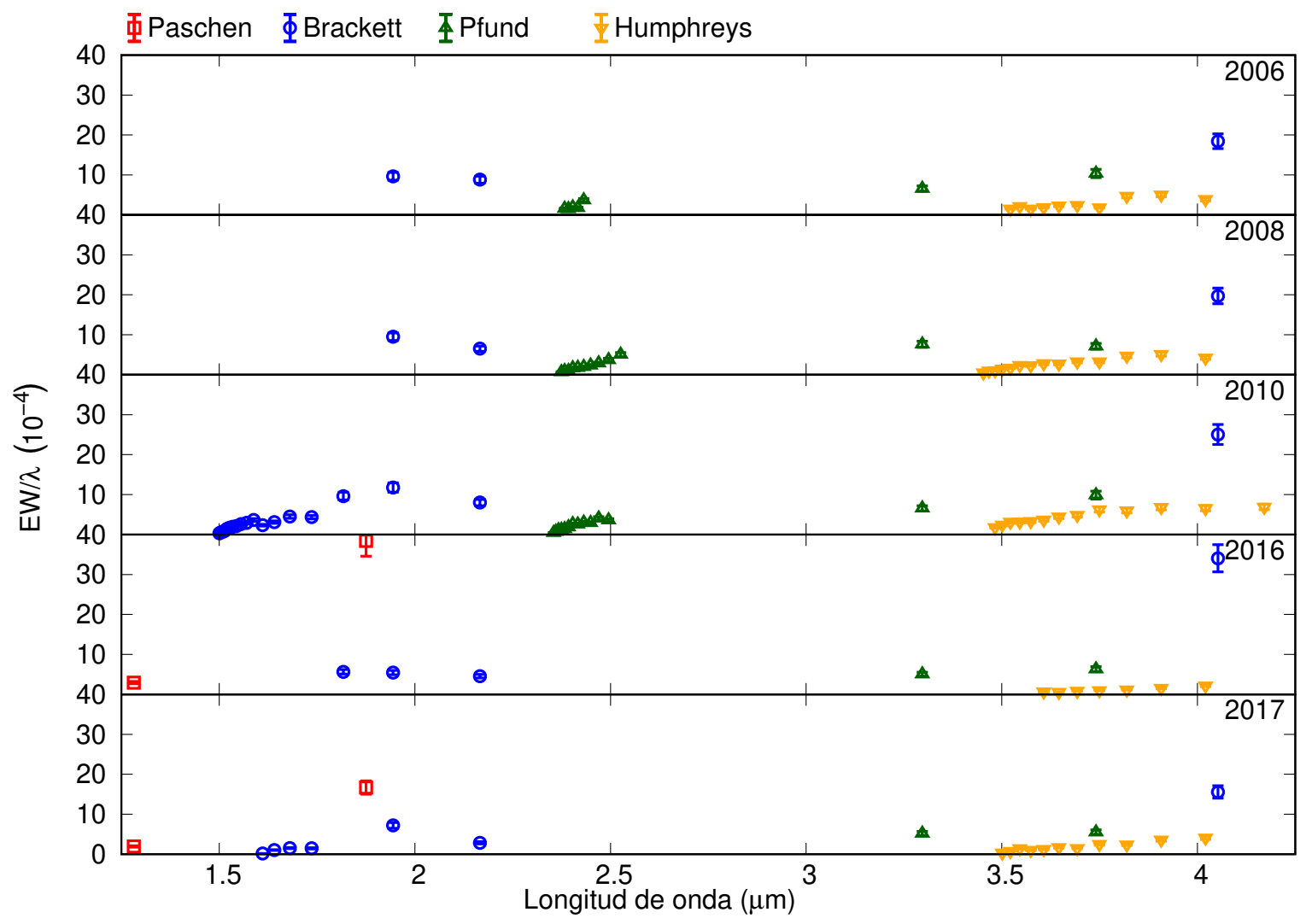


Figura 6.56: EW Lac - FWHM vs. $\lambda$ de las líneas observadas en las diferentes series en las distintas observaciones. La línea punteada corresponde a un valor de $2 V \sin (i)=710 \mathrm{~km} / \mathrm{s}$, y la región sombreada al error correspondiente (Tabla 3.3). Los símbolos utilizados son los mismos que en la Fig. 6.5.

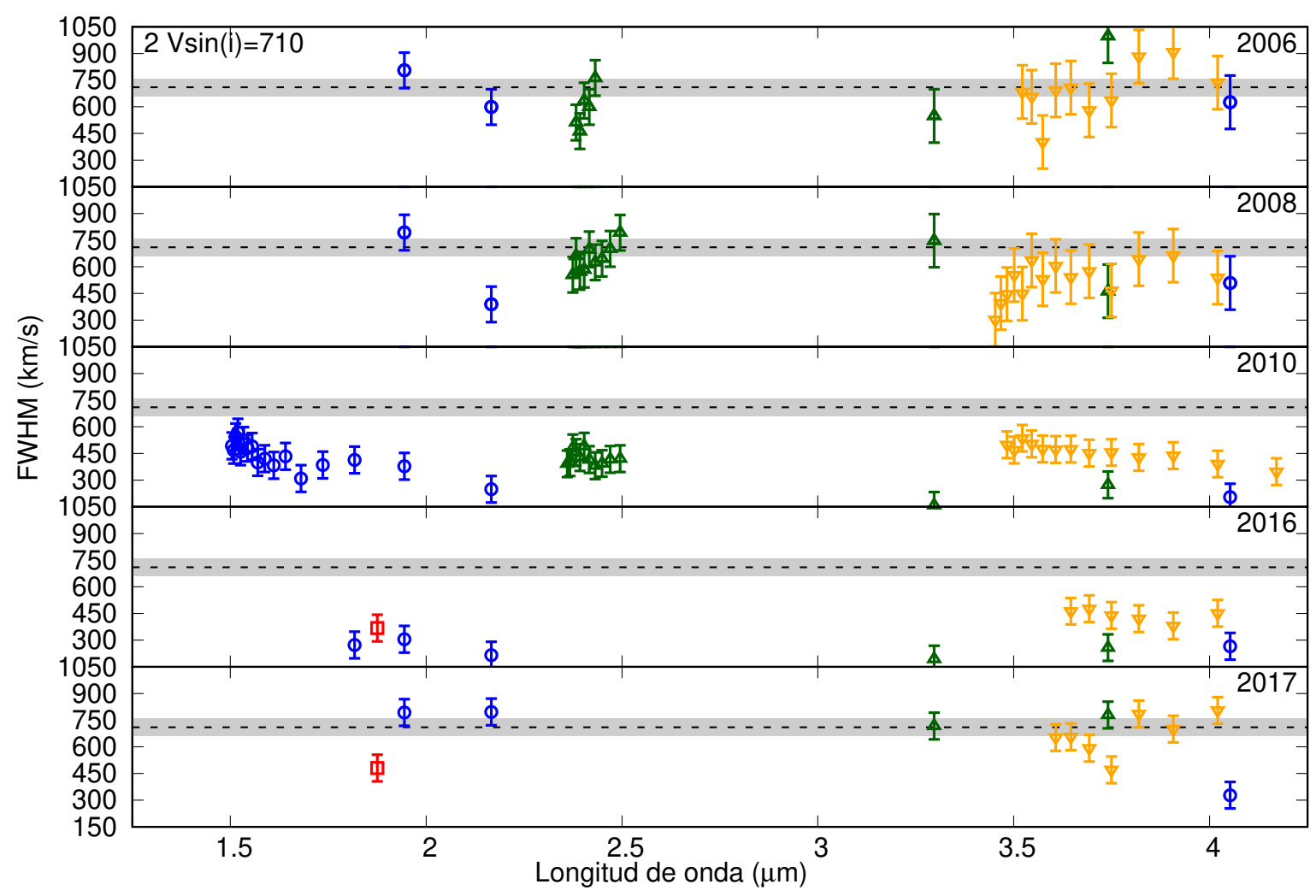

mayores en la observación del 2008, comportamiento más marcado en el año 2010, donde se alcanza un valor $\sim 7 \cdot 10^{-4}$. En el año 2016 se observa un crecimiento de EW/ $\lambda$ con la longitud de onda sin llegar a valores estables, situación que se revierte en la observación del 2017, donde los valores de $\mathrm{EW} / \lambda$ se estabilizan alrededor de $5 \cdot 10^{-4}$. En las observaciones de los años 2008 y 2010 obtuvimos FWHM $\sim 2 V \sin (i)$ en casi todas las líneas, indicando regiones de formación cercanas a la fotosfera estelar. En el año 2010 obtuvimos FWHM crecientes a medida que observamos miembros más altos de las series, sin alcanzar valores similares a $2 V \sin (i)$. El mismo comportamiento se observa para la serie de Humphreys en el 2016. Para las otras series observadas en el 2016 observamos pocos miembros como para poder discutir su comportamiento, los FWHM obtenidos son menores. En el 2017, los FWHM obtenidos son similares a $2 V \sin (i)$, excepto para $\mathrm{Br} \alpha$ y $\mathrm{Pa} \alpha$ que presentan valores menores indicando regiones de formación más alejadas de la estrella central.

Los cocientes de los flujos para las líneas de las distintas series y su comparación con los casos ópticamente grueso y delgado se observan en la Fig. 6.57. En todos los casos los miembros más altos mantienen el comportamiento esperado para un medio ópticamente delgado, 
Figura 6.57: EW Lac - Cociente de flujos para las líneas de la serie de Humphreys, Pfund y Brackett. En cada gráfico, la línea continua corresponde al cociente para un caso ópticamente grueso, y la línea punteada al caso ópticamente delgado. En la parte superior se identifican las líneas correspondientes.
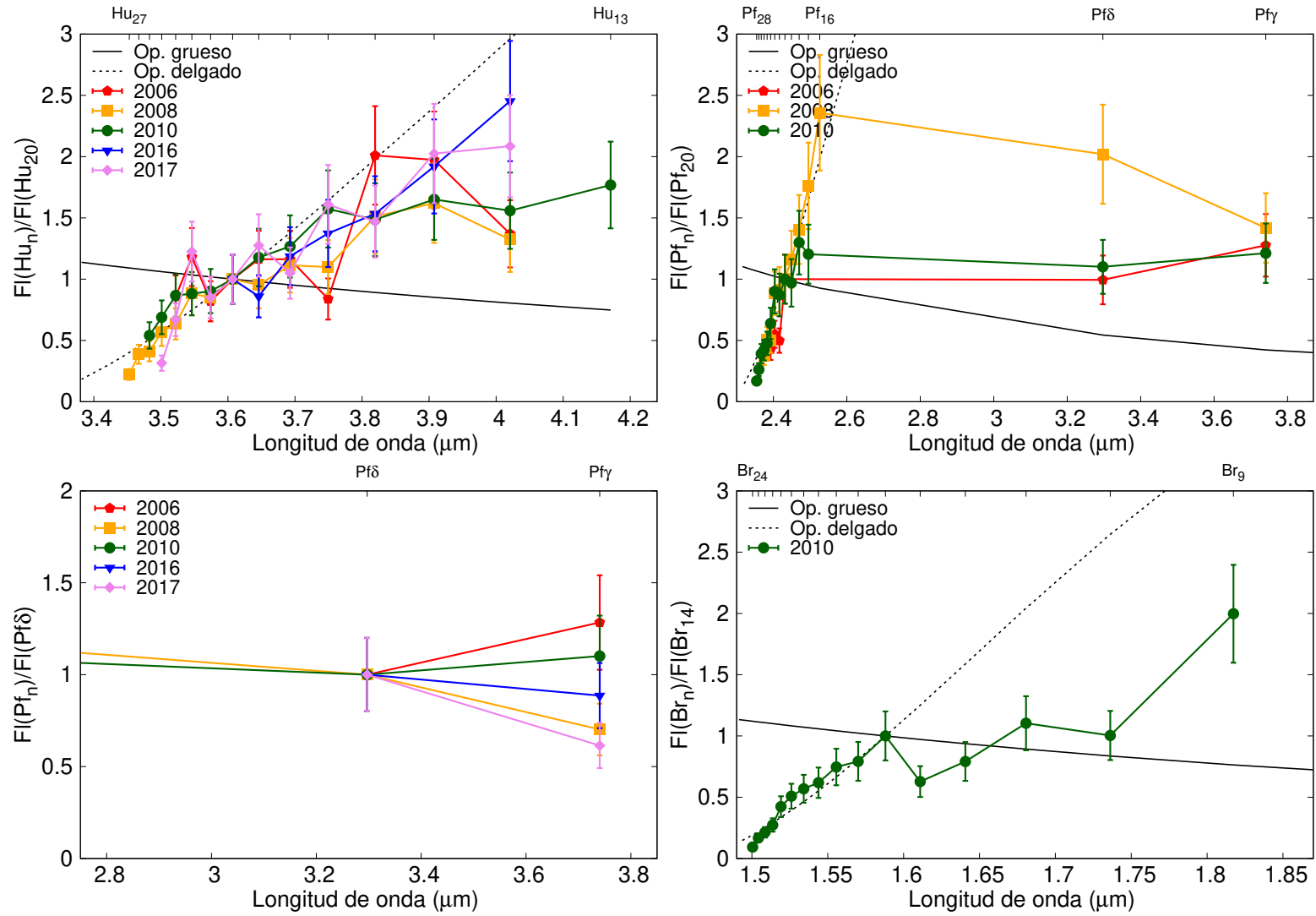

Tabla 6.6: EW Lac - Líneas que se apartan del caso ópticamente delgado.

\begin{tabular}{l|ccccc}
\hline \hline & 2006 & 2008 & 2010 & 2016 & 2017 \\
\hline Humphreys & $14-15$ & $14-19$ & $13-16$ & $14-19$ & $14-16$ \\
Pfund & $\gamma-\delta$ & $\gamma-\delta$ & $\gamma-17$ & - & - \\
Brackett & - & - & $9-13$ & - & - \\
\hline
\end{tabular}

mientras que los primeros miembros se apartan. Las líneas que se apartan del caso ópticamente delgado se listas en la Tabla 6.6. Obtuvimos además que $\mathrm{EW}(\operatorname{Br} \alpha) / \mathrm{EW}(\operatorname{Br} \gamma)=4$ en el 2006, $\mathrm{EW}(\mathrm{Br} \alpha) / \mathrm{EW}(\mathrm{Br} \gamma) \sim 5.75$ en 2008 y 2010, y valores mayores a 10 en 2016 y 2017, todos valores esperables para objetos del Grupo II (Granada et al., 2010). Para EW(Pa $\alpha) / E W(P a \beta)$ obtuvimos valores $\sim 20$ en el 2016 y $\sim 14$ en el 2017. 


\section{Determinación de densidades columnares y extensiones de las regiones de formación}

Las Tablas B.56 a B.61 muestran los valores obtenidos para las densidades columnares, extensiones de las regiones de formación de las líneas y radios internos de las mismas con los métodos $N$, FWHM y $\Delta V$. Para las líneas donde no pudimos medir la separación de los picos, obtuvimos un tamaño aproximado en radios estelares a partir de los tamaños relativos al de $\mathrm{Pf}_{17}$ en el 2008, $\mathrm{Hu}_{13}$ en el 2010 y Bry en el 2017, utilizando los FWHM.

Las mayores densidades columnares se alcanzan en el 2008 para las líneas de la serie de Humphreys y en el 2006 para la serie de Pfund. En la serie de Brackett los miembros más altos de la serie tienen regiones de formación más extensas.

Los radios relativos relativos de las regiones de formación para todas la líneas observadas relativos a $\operatorname{Br} \delta$ se muestra en la Fig. 6.58 (superior). Observamos que en el 2006, 2008 y 2017 una gran cantidad de líneas presentan radios internos de las regiones de formación mayores al de $\operatorname{Br} \delta$, contrario al 2010 y 2016 donde los miembros más altos de las series de Pfund, Brackett y Humphreys comienzan a formarse en una región más interna.

Graficamos los radios internos de las regiones de formación en radios estelares en la Fig. 6.58 (inferior). En el 2008 encontramos que el disco es más extendido, con una gran parte de los radios internos hasta $\sim 25 R_{\star}$. Si bien para esta observación se encuentran miembros de la serie de Humphreys que se formarían hasta una región de $\sim 40 R_{\star}$, estos valores corresponden a los miembros más altos de la serie, donde el FWHM es más complicado de determinar, por lo que esos tamaños tienen mucho error asociado. En el año 2010 los radios son un poco menores, siendo la región donde se forman la mayor parte de las líneas menor a $10 R_{\star}$. El disco es más compacto en la observación del 2017, con numerosas líneas formándose en una región muy cercana a la estrella central. 
Figura 6.58: EW Lac - Radio internos de las regiones emisoras de las diferentes líneas relativos a la región emisora de $\operatorname{Br} \delta$ y en radios estelares. Los símbolos utilizados son los mismos que en la Fig. 6.55.
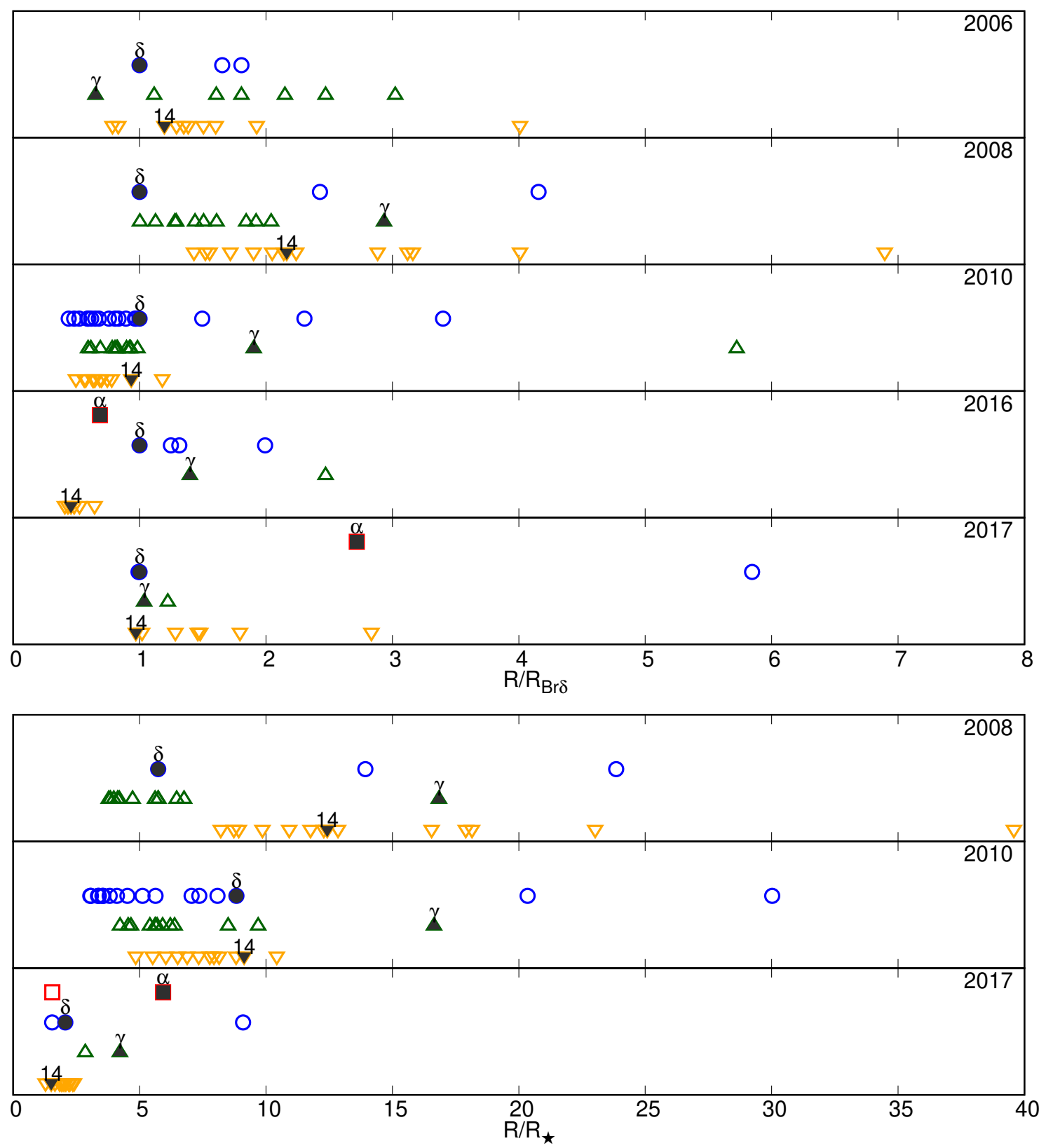


\subsection{Evolución de las envolturas}

\subsubsection{Ley de velocidad en el disco}

Para determinar las extensiones relativas y los radios internos de las regiones de formación a partir de los valores obtenidos para los FWHM y $\Delta V$ utilizamos que el disco sigue un comportamiento Kepleriano, donde la velocidad de rotación decae según $r^{-0.5}$, siendo $r$ la distancia a la estrella. Debido a esto, es esperable que al graficar los FWHM según el radio interno de la región de formación de cada línea, los valores sigan una ley Kepleriana.

En las Figs. 6.59 a 6.61 graficamos los FWHM obtenidos para cada línea en función del radio interno de las regiones de formación. Los puntos en color indican los radios obtenidos a partir del método $\Delta V$, y los grises corresponden a los obtenidos a partir de los valores relativos a otra línea utilizando los FWHM. Los símbolos utilizados son los mismos que en los gráficos de EW/ $\lambda$ vs. $\lambda$. Vemos que en general los valores de FWHM decaen siguiendo una ley Kepleriana. Sin embargo, encontramos regiones donde los FWHM obtenidos se apartan considerablemente de este comportamiento. Este apartamiento lo observamos en BK Cam en las líneas de la serie de Pfund de la observación del 2010, en 28 Tau en líneas de la serie de Humphreys en la observación del 2012 y en EW Lac en las líneas de Pfund en la observación del 2008 y líneas de la serie de Humphreys en la observación del 2017. Debido a los aumentos de densidad en la región interna del disco y los desplazamientos en el diagrama de Lenorzer para estos objetos, es esperable que los mismos hayan tenido eyecciones de material recientes. Esto podría producir una interacción entre la materia eyectada y el material existente en el disco, generando aceleraciones y desaceleraciones en el material , que podrían verse reflejadas como apartamientos de la ley Kepleriana. Una vez que el nuevo material se redistribuye en el disco, las velocidades volverían al comportamiento Kepleriano, como se observa en los años posteriores.

Figura 6.59: BK Cam - FWHM de las líneas observadas en función del radio interno de la región de formación. Se indica el ensanchamiento correspondiente a $2 V \sin (i)$ con el error correspondiente y un ajuste Kepleriano.

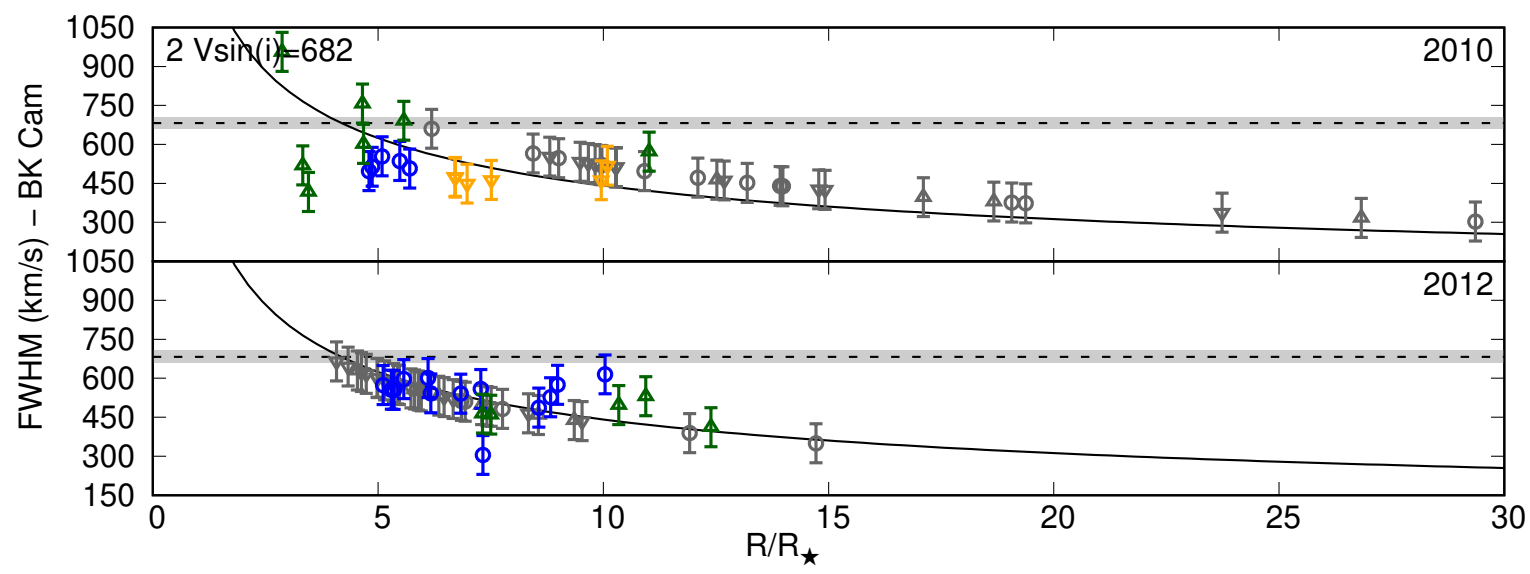


Figura 6.60: 28 Tau, V923 Aql y 12 Vul - FWHM de las líneas observadas en función del radio interno de la región de formación. Se indica el ensanchamiento correspondiente a $2 V \sin (i)$ con el error correspondiente y distintos ajustes Keplerianos.
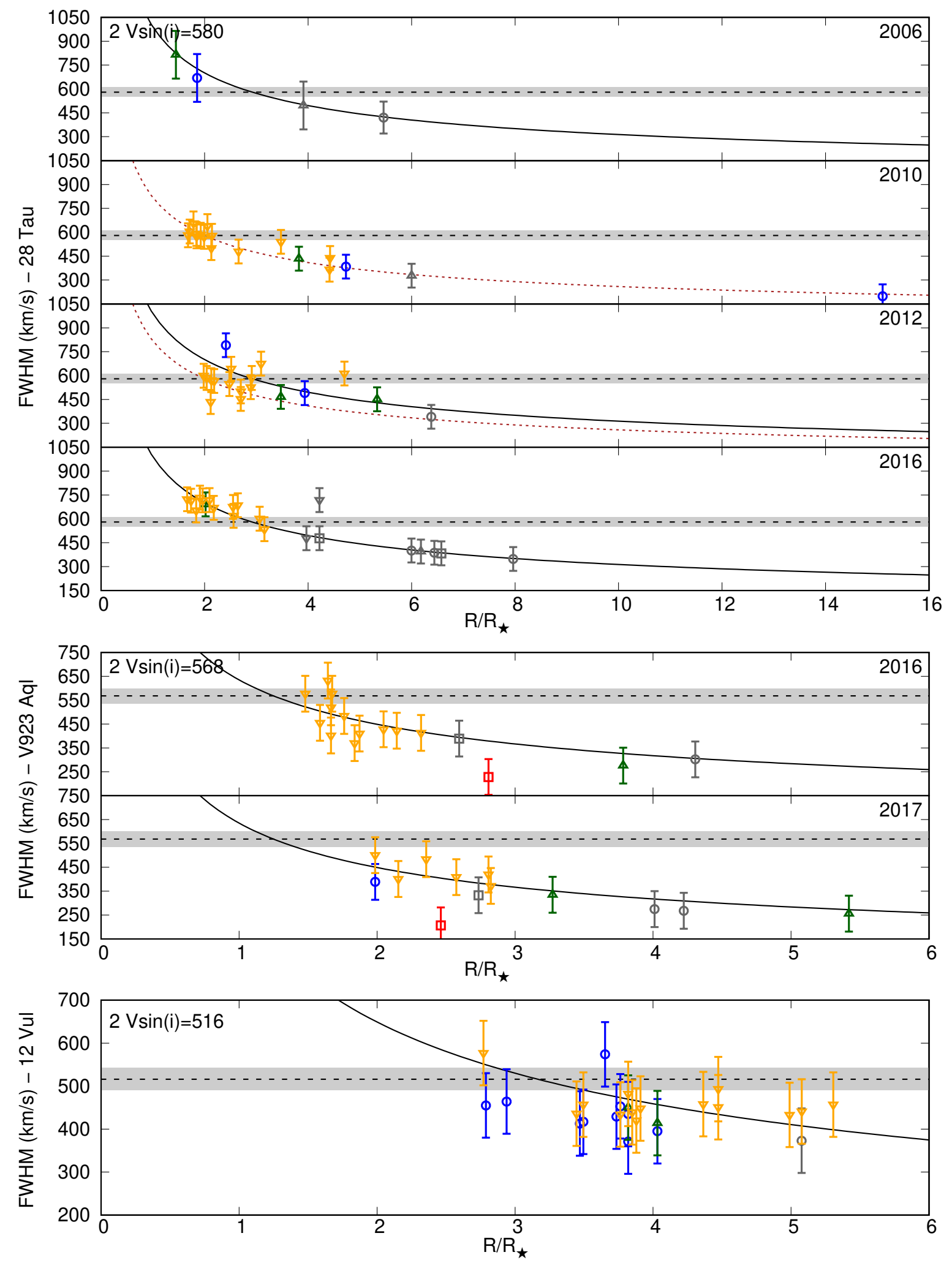
Figura 6.61: 28 Cyg y EW Lac - FWHM de las líneas observadas en función del radio interno de la región de formación. Se indica el ensanchamiento correspondiente a $2 V \sin (i)$ con el error correspondiente y distintos ajustes Keplerianos.
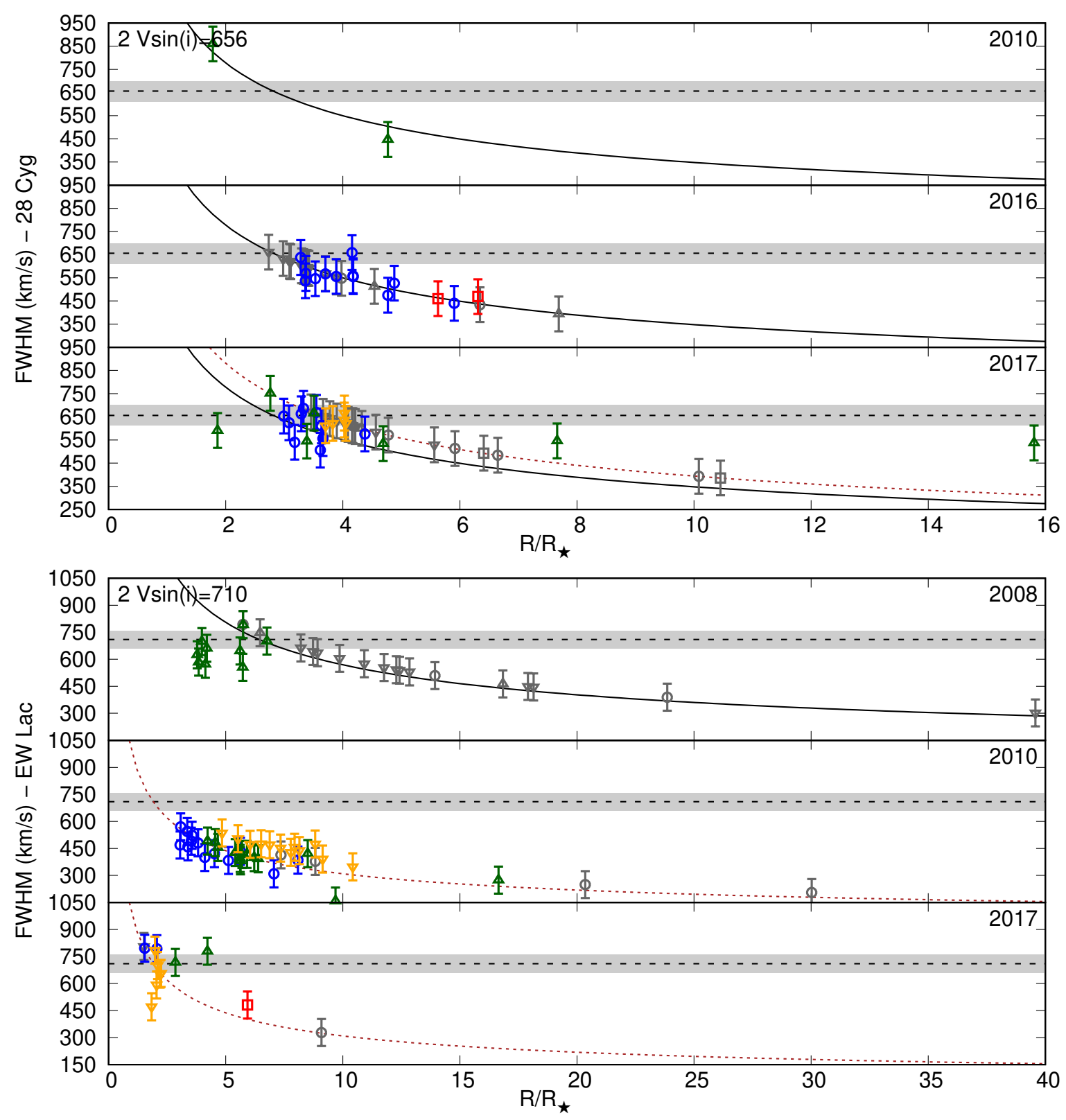


\subsubsection{Evolución de las envolturas}

A lo largo de la Sección 6.1 obtuvimos diferentes parámetros que describen las envolturas de las estrellas Be y su variabilidad a lo largo de las distintas observaciones. Discutimos además cómo es la ley de velocidades presente en el disco y las variaciones observadas en la misma. En base a todo esto, proponemos a continuación cómo podría haber sido la evolución de los discos de esta muestra de estrellas Be a lo largo de estos años.

\section{BK Cam}

El disco de esta estrella presenta pequeñas variaciones en densidad, radio y extensión de las regiones de formación de las líneas. Estas variaciones producen cambios en la estructura del disco, sin llegar a observarse una disipación completa del mismo. El disco se mantiene ópticamente grueso, llegando a radios mínimos en el 2012, donde la mayor parte de las líneas comienzan a formarse entre 5 y $10 R_{\star}$.

Granada et al. (2010) reportaron que BK Cam presentaba un disco en crecimiento en el 2008, mientras que Vieira et al. (2017) indicaba la presencia de un disco en disipación en el 2010. Esto es consistente con la variación de la ubicación de BK Cam en el diagrama de Lenorzer, ya que en el 2008 alcanzó la ubicación más cercana al límite ópticamente grueso y en el 2010 presentaba menores densidades columnares y mayores radios, desplazándose hacia abajo en el diagrama de Lenorzer.

Después del 2013, las altas densidades columnares observadas desde la región más interna del disco indican que continúa eyectándose material desde la estrella central, y la estrella vuelve a desplazarse hacia la región superior del diagrama de Lenorzer.

\section{Tau}

La envoltura de 28 Tau presenta importantes variaciones en las densidades columnares de las regiones de formación, sin variar significativamente los radios de las mismas. La mayoría de las líneas observadas en emisión comienzan a formarse en radios menores a $6 R_{\star}$.

Esta estrella comenzó en el 2006 una nueva fase shell, donde un nuevo disco comenzó a formarse sobre el anterior. En esa época su posición en el diagrama de Lenorzer indicaba un disco ópticamente delgado, con radios internos de las regiones de formación cercanos a la estrella central. En la observación del 2010, la densidad columnar alta observada en la serie de Humphreys indica una región interna densa, manteniéndose la región externa (donde se observan las líneas de Pfund) una densidad columnar baja. Los radios obtenidos aquí son mayores que en el 2006.

Hacia el 2012 se produce una nueva eyección de material mientras la parte externa se disipa. La eyección de material se evidencia por el apartamiento respecto a la ley de rotación Kepleriana de las velocidades en la parte más interna del disco. Esto coincide con un aumento en la emisión 
de $\mathrm{H} \alpha$. Posteriormente, la densidad en la parte interna disminuye y aumenta en la región externa, aumentando los radios internos de las regiones de formación y estabilizándose nuevamente las velocidades de rotación con una ley Kepleriana.

\section{V1150 Tau}

Esta estrella presenta evidencia de un disco denso en la observación del 2012, lo que se ve reflejado en la ubicación en el diagrama de Lenorzer, cerca del límite entre las regiones de los grupos I y II. En la observación del 2016 las densidades columnares de la serie de Brackett son levemente menores, lo que podría indicar el comienzo del proceso de disipación del disco.

\section{Oph}

Para este objeto encontramos evidencia de un disco en proceso de disipación, ya que las líneas observadas en emisión en el 2008 y 2010 se observan en absorción en los años posteriores. Esto es consistente con lo reportado por Granada et al. (2010), Sabogal et al. (2017), Vieira et al. (2017) y el comportamiento observado para $\mathrm{H} \alpha$.

\section{V923 Aql}

La envoltura observada para esta estrella no presenta variaciones significativas a lo largo de los años de observación. En la observación del 2006 presenta una región interna con alta densidad columnar, y se ubica en la posición más baja observada en el diagrama de Lenorzer. Entre el 2016 y 2017 la densidad en la parte interna del disco mostró un aumento, al tiempo que los radios internos de las regiones de formación mostraban tamaños mayores. Esto indicaría que el disco de V923 Aql se encuentra en desarrollo.

\section{Vul}

Esta estrella presentaba evidencia de distintos procesos de formación y disipación del disco. En el 2010, su ubicación en el diagrama de Lenorzer corresponde al límite entre los grupos I y II, a pesar de mostrar una región interna de baja densidad. Ese disco posteriormente se disipó, haciendo que en las observaciones posteriores apenas fueran distinguibles algunas líneas con una pequeña emisión. Este comportamiento es consistente con el decaimiento de la emisión en H $\alpha$ observado luego del 2007.

\section{Cyg}

En el 2002, Lenorzer et al. (2002a) ubicaron a 28 Cyg en una región cercana a la zona ópticamente gruesa de su diagrama. Posteriormente, Granada et al. (2010) reportaron el cambio desde esa posición a la posición que ocupa en el año 2008 , indicando una clara transición entre el Grupo 
I y el Grupo II. Esa transición fue interpretada en términos de la eyección de una envoltura ópticamente gruesa que se expande y se convierte en ópticamente delgada. Esa envoltura continúa expandiéndose hacia el 2010, donde observamos pocas líneas en emisión. Esto es consistente con la clasificación de estrella con disco en disipación reportada por Sabogal et al. (2017).

Nuestras nuevas observaciones en el 2016 y 2017 indican que, luego de ese decaimiento, el disco volvió a generarse y la estrella volvió nuevamente a la región ópticamente gruesa del diagrama de Lenorzer, con una envoltura densa hasta los $6 R_{\star}$. Aún en el 2017 las regiones de alta densidad llegan hasta regiones cercanas a la estrella central, por lo que el disco continuaría en desarrollo, recibiendo material de la estrella central.

\section{EW Lac}

El análisis realizado para EW Lac nos da indicios de la presencia de un disco disipado entre el 2006 y 2008, que mostraba un incremento en la densidad de la región central. Posteriormente, hacia la observación del 2010 la ubicación en el diagrama de Lenorzer se acerca levemente al caso ópticamente grueso. La densidad central obtenida en esa observación es menor que en las anteriores, y posteriormente el disco comienza a disiparse. En el 2016 observamos un disco más cercano al caso ópticamente delgado. Hacia el 2017 la densidad columnar de la región más interna de la envoltura vuelve a aumentar, y la estrella se ubica nuevamente en una región similar a la del 2010 en el diagrama de Lenorzer, a pesar de presentar radios internos de las regiones de formación menores a los obtenidos anteriormente. Esto podría indicar que a esta estrella le falta tiempo para terminar de rellenar el disco, hasta que el mismo sea ópticamente grueso y más extendido, similar al observado en el 2010.

En los 8 objetos analizados encontramos evidencias de distintos tipos de variabilidad: un grupo de objetos presentaría eyecciones de masa recurrentes, mostrando un disco que se mantiene estable a lo largo de varios años (BK Cam y 28 Tau); otro grupo presenta evidencias de haber iniciado un proceso de disipación del disco (V1150 Tau, 66 Oph y $12 \mathrm{Vul}$ ); mientras que las demás muestran un disco en desarrollo (V923 Aql, 28 Cyg y EW Lac). Según esto, los cocientes de los flujos obtenidos en las distintas series nos permiten obtener las densidades del disco en distintas regiones, y a partir de eso inferir como será la evolución del disco.

En el próximo capítulo presentaremos resultados generales a partir del análisis de todos los objetos observados en el IR. 


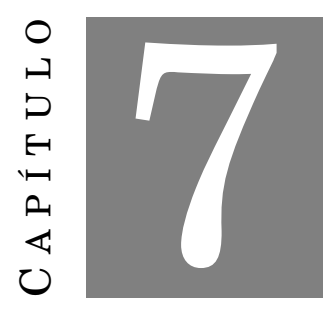

\section{RESULTADOS GENERALES DEL IR}

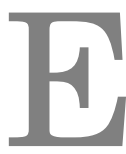

n los Capítulos 5 y 6 describimos las propiedades físicas de las envolturas circunestelares de una muestra significativa de estrellas Be a partir del estudio de sus espectros en el infrarrojo cercano. El análisis de las propiedades físicas de aquellos objetos para los cuales contábamos con un seguimiento temporal nos permitió además estudiar la evolución de sus envolturas.

A partir de la comparación de los resultados encontrados para los objetos analizados en los Capítulos 5 y 6 , sugeriremos estados evolutivos para las envolturas de los objetos que fueron observados en una sola oportunidad (estable, en formación o en disipación). Propondremos además nuevos diagramas de cocientes de flujos que nos permitirán obtener propiedades físicas de las envolturas, y estudiaremos la correlación de los cocientes de anchos equivalentes y flujos de algunas líneas con la pertenencia a los diferentes grupos.

\subsection{Evolución temporal de las envolturas}

A partir del análisis realizado para las estrellas variables, donde observamos cambios en las densidades columnares en las diferentes regiones del disco a medida que las estrellas desarrollaban o disipaban sus envolturas, podemos proponer una evolución temporal para los discos de los objetos presentados en el Capítulo 5. En las estrellas variables, el desarrollo de los discos se manifiesta inicialmente con altas densidades en la región central (llegando a valores $\sim 30 \cdot 10^{14} \mathrm{~cm}^{-2}$ en la serie de Brackett) y apartamientos de la ley de rotación Kepleriana. Así, podemos decir que $228 \mathrm{Eri}, 105 \mathrm{Tau}, \psi$ Ori, $120 \mathrm{Tau}, \mathrm{V} 696 \mathrm{Mon}$ y $\pi$ Aqr presentaban discos en desarrollo al momento de la observación. Por otro lado, 48 Per, $\omega$ Ori, 48 Lib y o Aqr presentan bajas densidades (que no superan los $\sim 15 \cdot 10^{14} \mathrm{~cm}^{-2}$ ) a lo largo de todas las series, lo que nos 
Tabla 7.1: Clasificación en grupos y estados evolutivos propuestos a partir de los espectros IR para los discos de los objetos de la muestra.

\begin{tabular}{|c|c|c|c|c|c|c|c|}
\hline Nombre & Año & Grupo & Estado del disco & Nombre & Año & Grupo & Estado del disco \\
\hline \multirow[t]{4}{*}{ BK Cam } & 2008 & I & en desarrollo & \multirow[t]{4}{*}{66 Oph } & 2008 & II & en disipación \\
\hline & 2010 & I-II & en disipación & & 2010 & III & disipado \\
\hline & 2012 & I-II & en desarrollo & & 2016 & III & disipado \\
\hline & 2016 & I & en desarrollo & & 2017 & III & disipado \\
\hline \multirow[t]{4}{*}{28 Tau } & 2006 & II & \multirow{4}{*}{$\begin{array}{l}\text { en desarrollo } \\
\text { en desarrollo } \\
\text { estable } \\
\text { estable }\end{array}$} & HD 171623 & 2017 & - & disipado \\
\hline & 2010 & II & & V4024 Sgr & 2017 & - & i? \\
\hline & 2012 & II & & \multirow[t]{3}{*}{ V923 Aql } & 2006 & II & en desarrollo \\
\hline & 2016 & II & & & 2016 & II & en desarrollo \\
\hline $48 \mathrm{Per}$ & 2012 & II & en disipación & & 2017 & II & en desarrollo \\
\hline 228 Eri & 2017 & I-II & en desarrollo & \multirow[t]{3}{*}{$12 \mathrm{Vul}$} & 2010 & I & en disipación \\
\hline \multirow[t]{2}{*}{ V1150 Tau } & 2012 & I-II & ¿estable? & & 2016 & - & disipado \\
\hline & 2016 & - & en disipación & & 2017 & III & disipado \\
\hline $105 \mathrm{Tau}$ & 2017 & II & en desarrollo & \multirow[t]{4}{*}{28 Cyg } & 2008 & II & en disipación \\
\hline$\psi_{01}$ Ori & 2017 & I-II & en desarrollo & & 2010 & II & disipado \\
\hline $120 \mathrm{Tau}$ & 2017 & I-II & en desarrollo & & 2016 & I-II & en desarrollo \\
\hline$\omega$ Ori & 2017 & II & en disipación & & 2017 & I & en desarrollo \\
\hline V696 Mon & 2017 & I & en desarrollo & $o$ Aqr & 2017 & - & estable/en disipación \\
\hline MX Pup & 2017 & - & en desarrollo/estable & $\pi \mathrm{Aqr}$ & 2017 & - & en desarrollo \\
\hline $48 \mathrm{Lib}$ & 2017 & - & en disipación & \multirow[t]{4}{*}{ EW Lac } & 2006 & II & en desarrollo \\
\hline V1075 Sco & 2017 & - & en desarrollo/estable & & 2008 & II & en desarrollo \\
\hline \multirow[t]{2}{*}{$88 \mathrm{Her}$} & 2006 & I & ¿? & & 2010 & II & en disipación \\
\hline & & & & & $\begin{array}{l}2016 \\
2017\end{array}$ & II & $\begin{array}{l}\text { disipado } \\
\text { en desarrollo }\end{array}$ \\
\hline
\end{tabular}

da indicios de un proceso de disipación en la envoltura. Para MX Pup y V1075 Sco, la parte externa tiene una densidad considerable y no tenemos información de la parte más interna de la envoltura, por lo que no podemos distinguir entre un disco aún en desarrollo o uno estable. Sobre 88 Her y V4024 Sgr no pudimos asignar un estado evolutivo para el disco debido a las pocas líneas observadas. En la Tabla 7.1 se listan todas las estrellas de la muestra y la evolución temporal propuesta para la envoltura en cada observación.

\subsection{El diagrama de Lenorzer}

En la Fig. 7.1 presentamos la ubicación en el diagrama de Lenorzer de todas las estrellas de nuestra muestra que presentan emisión en las líneas $\mathrm{Br} \alpha, \mathrm{Hu}_{14}$ y $\mathrm{Pf} \delta$. La línea negra continua y la azul de trazos indican donde $\log \left(\mathrm{Fl}\left(\mathrm{Hu}_{14}\right) / \mathrm{Fl}(\mathrm{Br} \alpha)\right)=-0.25$ o -0.5 , respectivamente. Estos valores los definimos en la Sección 5.2 como límites de las regiones donde se ubican las estrellas de los grupos I, II y de transición (que llamaremos grupo I-II). Al igual que las estrellas estudiadas en el Capítulo 5, algunas del Capítulo 6 presentan características comunes a los grupos I y II, y quedan ubicadas en la región de transición del diagrama. La clasificación en los distintos grupos para cada estrella de la muestra se presenta en las columnas 3 y 7 de la Tabla 7.1. 
Figura 7.1: Ubicación en el diagrama de Lenorzer de todas las estrellas de la muestra. La línea negra continua y la azul de trazos marcan los límites de las regiones donde se ubican las estrellas de los grupos I, II y I-II.

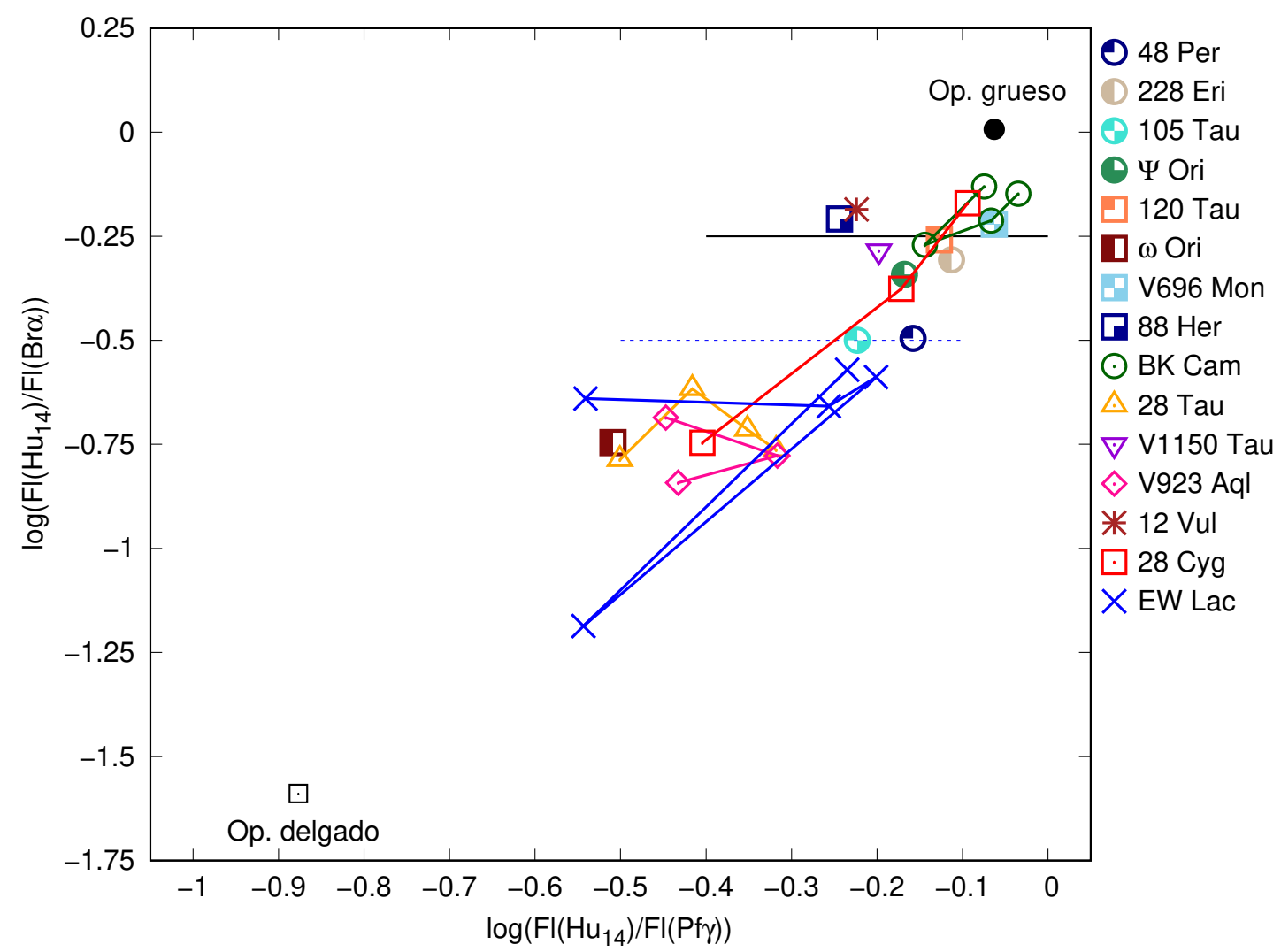

\subsection{Diferentes diagramas de cocientes de flujos}

Hemos visto que los diagramas de cocientes de flujos son una herramienta de diagnóstico de las propiedades de las envolturas de las estrellas Be. En la Fig. 7.2 ubicamos a los objetos de nuestra muestra en los diagramas propuestos por Lenorzer et al. (2002a) y Granada et al. (2010), identificando la pertenencia a los distintos grupos mediante distintos colores. Como estos autores reportaron (y como hemos visto a lo largo de este trabajo para el diagrama de Lenorzer), es posible delimitar regiones separadas para las estrellas de los diferentes grupos. Estos dos diagramas utilizan líneas presentes en la banda $\mathrm{L}\left(\mathrm{Hu}_{14}, \mathrm{Br} \alpha, \mathrm{Pf} \gamma\right)$, por lo que no es posible ubicar en ellos a todas las estrellas de nuestra muestra. Frente a esto, buscamos diagramas equivalentes que utilicen líneas presentes en las bandas J, H y K. En la Fig. 7.3 se muestran dos posibles diagramas, que utilizan las líneas $\mathrm{Pf}_{18}-\mathrm{Br} \gamma-\mathrm{Pa} \beta$ y $\mathrm{Br}_{12}-\mathrm{Pf}_{18}-\mathrm{Br} \gamma$.

El diagrama $\mathrm{Pf}_{18}-\mathrm{Br} \gamma-\mathrm{Pa} \beta$ presenta una distribución similar al de Lenorzer. En la región inferior izquierda se ubica el límite para el caso ópticamente delgado y en la región superior derecha el correspondiente al caso ópticamente grueso. Las estrellas Be observadas se ubican sobre la diagonal, quedando por debajo de la línea $\log \left(\mathrm{Fl}\left(\mathrm{Pf}_{18}\right) / \mathrm{Fl}(\mathrm{Pa} \beta)\right)=-1.25$ las estrellas 
Figura 7.2: Ubicación en los diagramas de Lenorzer (izquierda) y Granada (derecha) de las estrellas de nuestra muestra. Las estrellas quedan separadas según la pertenencia a los diferentes grupos.
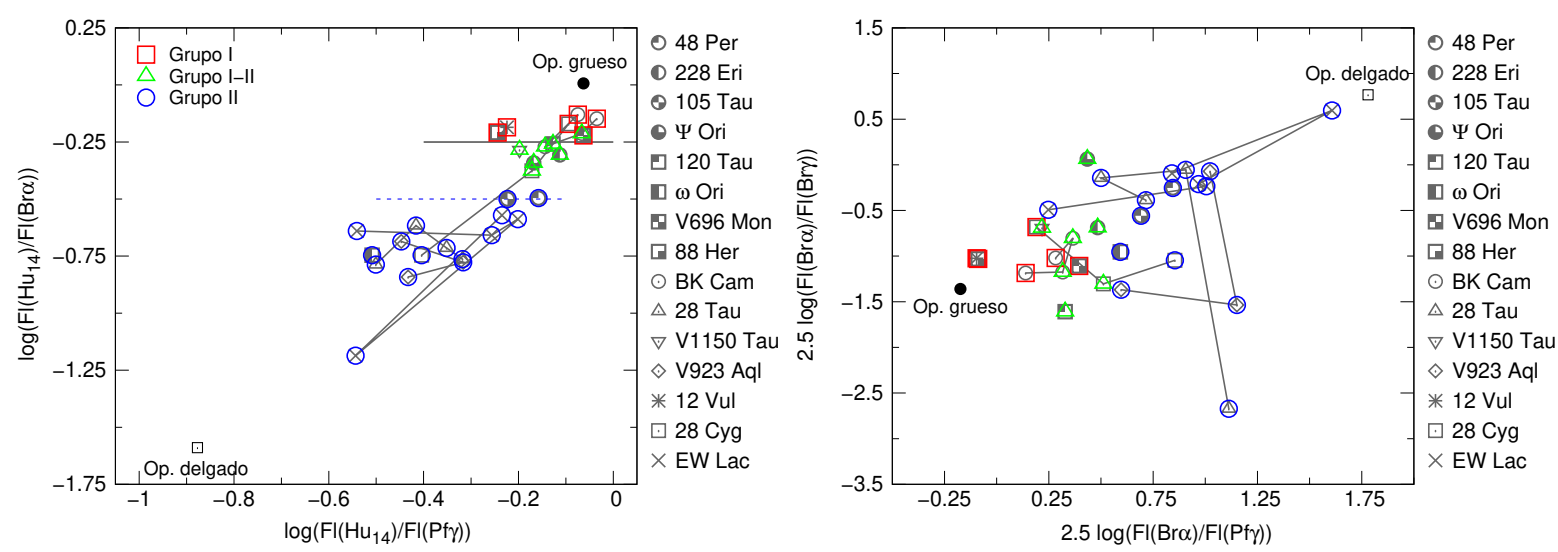

Figura 7.3: Ubicación en los diagramas de cocientes de los flujos de $\mathrm{Pf}_{18}-\mathrm{Br} \gamma-\mathrm{Pa} \beta$ (izquierda) y $\mathrm{Br}_{12}-\mathrm{Pf}_{18}-\mathrm{Br} \gamma$ (derecha). Los símbolos en colores son los mismos que los de la Fig. 7.2, y los símbolos grises representan los objetos sin grupo definido.
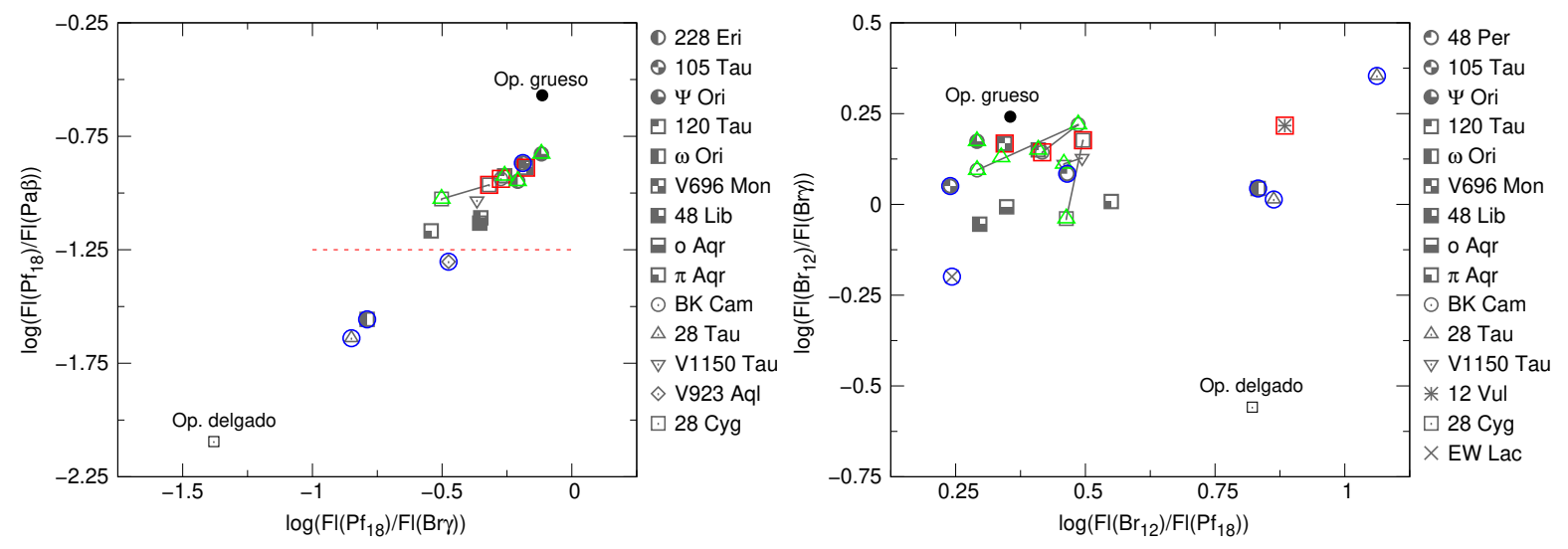

pertenecientes al grupo II. En la región superior se ubican las estrellas correspondientes a los grupos I y I-II, junto con 105 Tau. Si bien 105 Tau fue clasificada como perteneciente al grupo II y en el diagrama de Lenorzer queda sobre el límite con la región de transición, en el nuevo diagrama sería un objeto del grupo I/I-II. Se ubican también en esta región $\pi$ Aqr, que presentaba evidencia de un disco en desarrollo, y 48 Lib y o Aqr, que presentaban evidencia de un disco en proceso de disipación.

En el diagrama $\mathrm{Br}_{12}-\mathrm{Pf}_{18}-\mathrm{Br} \gamma$ las estrellas pertenecientes a los grupos I y I-II se ubican alrededor del límite del caso ópticamente grueso, mientras que las del grupo II se ubican en una región más alejada. El límite entre las diferentes regiones en este caso es más difuso, ubicándose $12 \mathrm{Vul}$ (que presentaba un disco en proceso de disipación) en una región compatible con las estrellas del grupo II. Las estrellas que en el diagrama de Lenorzer quedan en la región límite 
Figura 7.4: Relación entre anchos equivalentes (izquierda) y flujos (derecha) de $\operatorname{Br} \alpha$ y $\operatorname{Br} \gamma$ para los distintos grupos. Los símbolos en colores son los mismos que en la Fig. 7.2. En el panel izquierdo, la línea continua verde corresponde a $\mathrm{EW}(\mathrm{Br} \alpha)=3 \mathrm{EW}(\mathrm{Br} \gamma)$, mientras que la línea punteada negra corresponde a $\mathrm{EW}(\mathrm{Br} \alpha)=5 \mathrm{EW}(\mathrm{Br} \gamma)$. En el panel derecho, la línea continua naranja corresponde a $\mathrm{Fl}(\mathrm{Br} \gamma)=3 \mathrm{Fl}(\mathrm{Br} \alpha)$, mientras que la línea punteada negra corresponde a $\mathrm{Fl}(\mathrm{Br} \gamma)=\mathrm{Fl}(\mathrm{Br} \alpha)$.
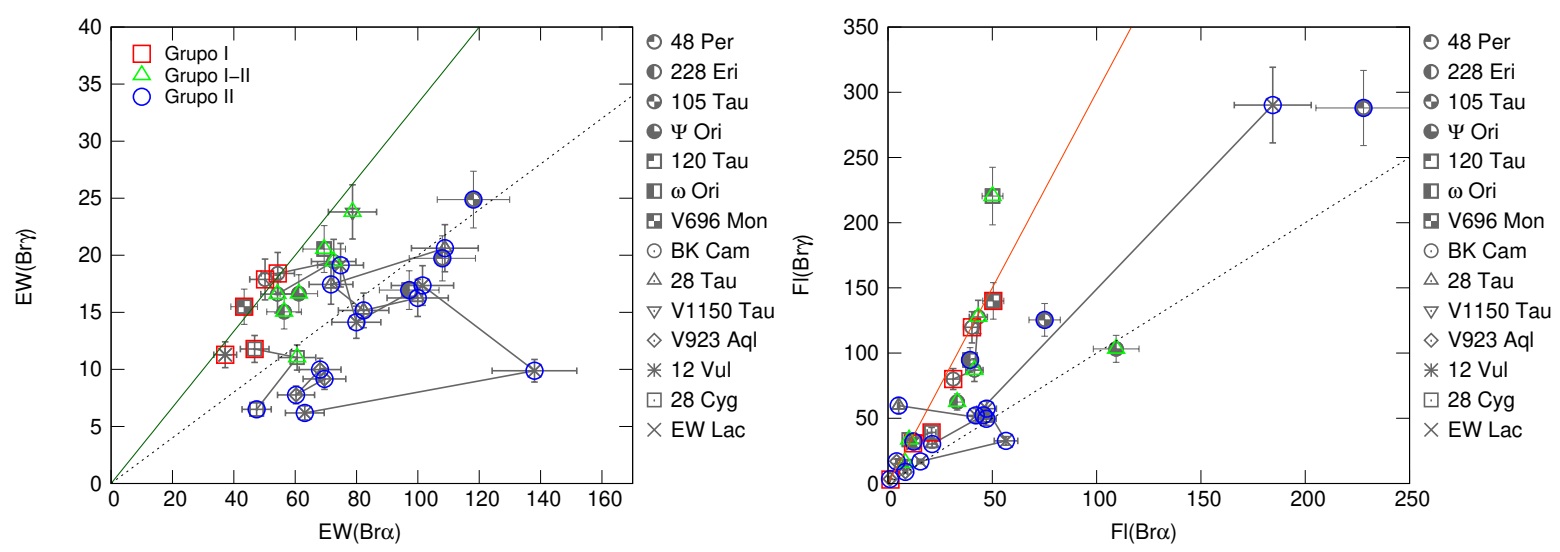

entre los grupos I-II y II (105 Tau y 48 Per) en este diagrama se ubican dentro de la región de las estrellas de los grupos I y I-II, junto con $\pi$ Aqr, o Aqr y 48 Lib.

Los diagramas presentados en la Fig. 7.3 permiten obtener propiedades físicas de las envolturas, de manera similar al diagrama de Lenorzer, y son de utilidad en el caso de no poseer observaciones en la banda L. Según el análisis realizado sobre las líneas en emisión observadas en el IR, las mismas se forman en distintas regiones alrededor de la estrella central, por lo que permiten obtener propiedades físicas de diferentes regiones de las envolturas. Por eso, al comparar diagramas que utilizan cocientes de diferentes líneas, es esperable que las ubicaciones relativas de cada estrella en los mismos presenten pequeñas modificaciones, según la distribución de densidades en la envoltura. Sin embargo, los diagramas propuestos en este trabajo nos permiten diagnosticar de manera general las propiedades de las envolturas de manera similar a los encontrados anteriormente en la literatura.

\subsection{Cocientes de flujos y anchos equivalentes para $\operatorname{Br} \alpha-\operatorname{Br} \gamma$ y $\mathbf{P a} \alpha-\mathbf{P a} \beta$}

Granada et al. (2010) reportaron que para las estrellas pertenecientes al grupo I los flujos obtenidos para la línea $\operatorname{Br} \alpha$ eran menores que los obtenidos para $\mathrm{Br} \gamma$, comportamiento característico para los modelos ópticamente gruesos de Lynch et al. (2000), que predicen una depresión en la emisión de $\operatorname{Br} \alpha$ y $\operatorname{Pa} \alpha$. Además, encontraron que el cociente $\mathrm{EW}(\mathrm{Br} \alpha) / \mathrm{EW}(\mathrm{Br} \gamma)$ tomaba valores entre 2 y 3 en las estrellas pertenecientes al grupo I, mientras que las del grupo II presentaban valores en $\mathrm{EW}(\mathrm{Br} \alpha)>5 \mathrm{EW}(\mathrm{Br} \gamma)$. Ese criterio fue utilizado en este trabajo para separar a las 
Figura 7.5: Relación entre anchos equivalentes (izquierda) y flujos (derecha) de $\mathrm{Pa} \alpha$ y $\mathrm{Pa} \beta$ para los distintos grupos. Los símbolos en colores son los mismos que en la Fig. 7.2. En el panel izquierdo la recta verde representa $\mathrm{EW}(\mathrm{Pa} \alpha)=6 \mathrm{EW}(\mathrm{Pa} \beta)$. En el panel derecho la recta naranja represente $\mathrm{Fl}(\mathrm{Pa} \beta)=0.75 \mathrm{Fl}(\mathrm{Pa} \alpha)$.
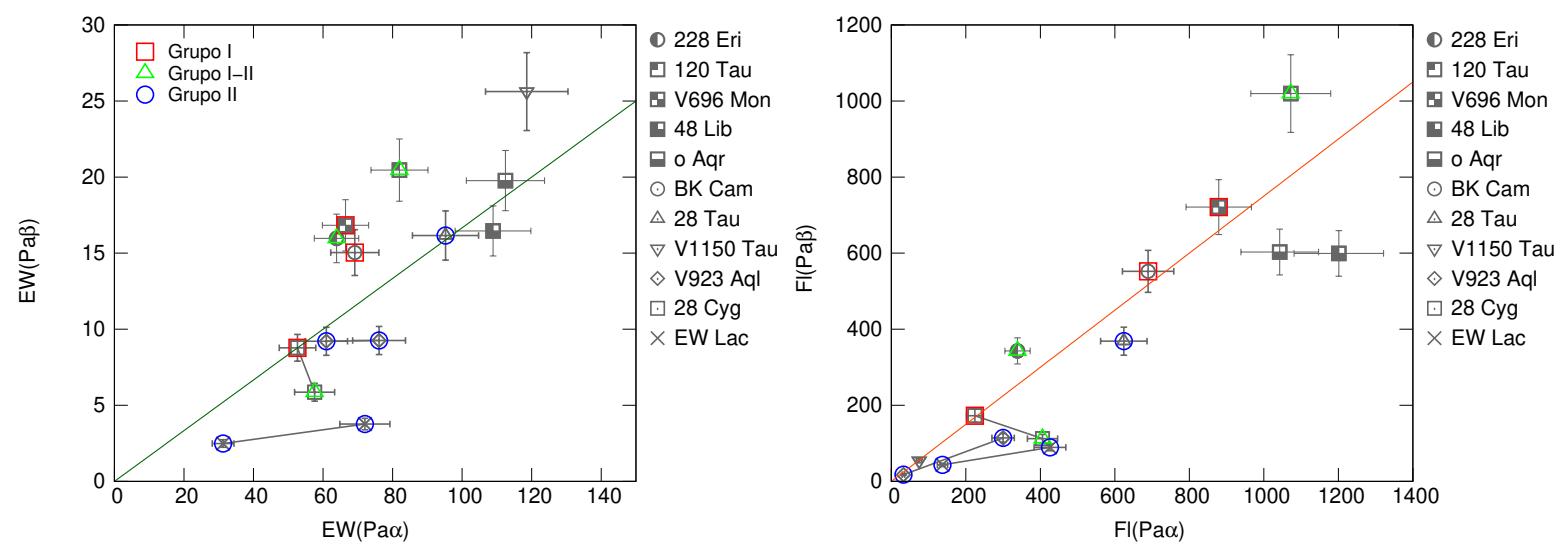

estrellas en grupos I, I-II y II.

En la Fig. 7.4 mostramos los anchos equivalentes y flujos de $\operatorname{Br} \alpha$ y $\operatorname{Br} \gamma$ para todas las estrellas de nuestra muestra. En el gráfico de anchos equivalentes (panel izquierdo) la línea continua verde corresponde a $\mathrm{EW}(\mathrm{Br} \alpha)=3 \mathrm{EW}(\mathrm{Br} \gamma)$, mientras que la línea punteada negra corresponde a $\mathrm{EW}(\mathrm{Br} \alpha)=5 \mathrm{EW}(\mathrm{Br} \gamma)$. En el gráfico de flujos (panel derecho) la línea continua naranja corresponde a $\mathrm{Fl}(\mathrm{Br} \gamma)=3 \mathrm{Fl}(\mathrm{Br} \alpha)$, mientras que la línea punteada negra corresponde a $\mathrm{Fl}(\mathrm{Br} \gamma)=\mathrm{Fl}(\mathrm{Br} \alpha)$. Observamos que en el gráfico de anchos equivalentes, la mayor parte de las estrellas del grupo I se ubican cercanas a la recta verde, $\mathrm{EW}(\mathrm{Br} \alpha)=3 \mathrm{EW}(\mathrm{Br} \gamma)$, mientras que las del grupo II se ubican cerca o por debajo de la recta negra, $\mathrm{EW}(\mathrm{Br} \alpha)=5 \mathrm{EW}(\mathrm{Br} \gamma)$. Las estrellas del grupo I-II se ubican en la región intermedia. Además observamos que los flujos en $\operatorname{Br} \alpha$ son menores a los flujos en $\mathrm{Br} \gamma$ para la mayor parte de los objetos de la muestra. Podemos destacar que para los objetos del grupo I y parte de los objetos del grupo I-II los valores se ubican sobre la recta $\mathrm{Fl}(\mathrm{Br} \gamma)=3 \mathrm{Fl}(\mathrm{Br} \alpha)$.

Un análisis similar puede hacerse para los cocientes de las líneas $\mathrm{Pa} \alpha$ y $\mathrm{Pa} \beta$, como se muestra en la Fig. 7.5. La recta verde representa $\mathrm{EW}(\mathrm{Pa} \alpha)=6 \mathrm{EW}(\mathrm{Pa} \beta)$ en el gráfico de anchos equivalentes (panel izquierdo) y la recta naranja represente $\mathrm{Fl}(\mathrm{Pa} \beta)=0.75 \mathrm{Fl}(\mathrm{Pa} \alpha)$ en el gráfico de flujos (panel derecho). Observamos que la mayor parte de las estrellas de los grupos I y I-II se ubican por encima de la recta verde, mientras que las del grupo II permanecen por debajo. En el gráfico de los flujos, vemos que para valores similares en los flujos de $\mathrm{Pa} \beta$, las estrellas correspondientes a los grupos I y I-II presentan flujos menores en Pa $\alpha$ que las del grupo II. Este comportamiento sigue lo predicho por Lynch et al. (2000), ya que se espera una depresión en la emisión de $\mathrm{Pa} \alpha$ en los modelos ópticamente gruesos. 
En los Capítulos 5, 6 y 7 hemos presentado el análisis realizado a partir de los espectros IR obtenidos para una muestra de 23 estrellas Be. Obtuvimos información sobre las propiedades físicas y estados evolutivos de las envolturas, y planteamos diferentes métodos de diagnóstico de las propiedades de las mismas.

A continuación analizaremos las envolturas de las estrellas Be mediante la implementación de otra técnica útil para analizar la geometría y cinemática de las envolturas de las estrellas Be: la interferometría de larga base con alta resolución espectral. 



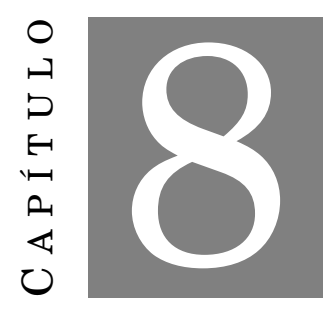

\section{OBSERVACIONES INTERFEROMÉTRICAS ${ }^{1}$}

$\mathrm{P}$ ara lograr describir los discos de las estrellas Be es necesario comprender el mecanismo dominante de eyección de masa desde la estrella central. El estudio de la estructura física, geometría y cinemática de los mismos puede ser usado para poner restricciones a los diferentes procesos involucrados en su formación. Esto no puede realizarse usando únicamente telescopios simples, debido a la necesidad de contar con una alta resolución espacial y espectral. Así, la interferometría de larga base constituye un técnica sumamente valiosa para realizar este estudio. Una introducción a los conceptos de interferometría utilizados en este capítulo pueden encontrarse en el Apéndice C.

Comenzaremos este capítulo con un breve resumen de los resultados previos de la literatura obtenidos a partir de observaciones interferométricas de estrellas Be. A continuación, describiremos las características principales de la instrumentación utilizada para la obtención de nuestros datos, junto con el modelo adoptado para nuestro estudio y los datos obtenidos. Para cada objeto estudiado presentaremos los parámetros del modelo de disco que mejor ajusta a las observaciones interferométricas en $\mathrm{Br} \gamma$ y discutiremos los resultados particulares de nuestra muestra. Analizaremos además una muestra más amplia que incluye mediciones de otros autores.

\subsection{Antecedentes de observaciones interferométricas en estrellas Be}

Thom et al. (1986) fueron los primeros en resolver la envoltura de una estrella Be ( $\gamma$ Cas). Observaron la línea $\mathrm{H} \alpha$ con el interferómetro I2T (Interféromètre à 2 Télescopes, Plateau de Calern, Francia) y encontraron una envoltura con un diámetro angular de 5.2 mas. Posteriormente,

\footnotetext{
${ }^{1}$ Los resultados presentados en este capítulo fueron publicados en Cochetti et al. (2019).
} 
Quirrenbach (1993) resolvió el disco circunestelar de 7 estrellas Be en $\mathrm{H} \alpha$ utilizando el interferómetro Mark III (Interferómetro óptico de larga base, Mount Wilson Observatory, California) y logró determinar inclinaciones y el ángulo de posición del semieje mayor (con orientaciones perpendiculares al ángulo de polarización).

Utilizando datos espectroscópicos e interferométricos, Stee (1995) modeló la envoltura circunestelar de $\gamma$ Cas mediante una región polar dominada por líneas ópticamente gruesas y una región ecuatorial con líneas ópticamente delgadas en rotación Kepleriana.

Cuando la contribución de la envoltura circunestelar es despreciable, la fotosfera puede ser resuelta permitiendo, por ejemplo, la determinación de su achatamiento. Domiciano de Souza et al. (2003) estudiaron Achernar ( $\alpha$ Eri) y encontraron un valor de $2.53 \pm 0.06$ mas para el eje mayor y $1.62 \pm 0.01$ mas para el eje menor $(2 \mathrm{a} / 2 \mathrm{~b}=1.56 \pm 0.05)$. Para este objeto fue reportada también la presencia de un viento polar (Kervella \& Domiciano de Souza, 2006).

El estudio de la correlación entre la intensidad de $\mathrm{H} \alpha$ en emisión y la extensión de la región emisora puede ser utilizada para determinar propiedades de la envoltura circunestelar. A partir de una muestra de 7 estrellas Be, Quirrenbach et al. (1997) encontraron que las estrellas con mayor emisión en $\mathrm{H} \alpha$ tienen una tendencia a tener disco mayores. Además, encontraron que los discos más grandes parecieran darse en los tipos espectrales B intermedios.

Tycner et al. (2005) analizaron mediciones interferométricas en $\mathrm{H} \alpha$ de una muestra de 7 objetos y encontraron una dependencia entre la emisión de $\mathrm{H} \alpha$ y el tamaño de la región emisora, consistente con una línea ópticamente gruesa. Dada la pequeña muestra usada, los mismos autores no encontraron dependencia entre el tamaño de la región emisora y el tipo espectral o la rotación. Sin embargo, sugieren que las estrellas más calientes tienen envolturas más extendidas.

Utilizando VLTI/AMBER, Meilland et al. (2007a) obtuvieron la primera evidencia clara de que el disco de $\alpha$ Ara está en rotación Kepleriana. Luego, Meilland et al. (2007b) reportaron una asimetría en el disco de $\kappa$ CMa y propusieron un disco con un brazo espiral para explicarla. En un trabajo posterior, Meilland et al. (2011) verificaron los parámetros orbitales de la binaria $\delta$ Sco y resolvieron el disco circunestelar en $\mathrm{H} \alpha, \mathrm{Br} \gamma, 2.6 \mu \mathrm{m}$ He I y la banda $\mathrm{K}$.

Meilland et al. (2009) observaron 7 estrellas Be (p Car, $\zeta$ Tau, ${ } \mathrm{CMa}, \alpha \mathrm{Col}, \delta \mathrm{Cen}, \beta \mathrm{CMi}$, $\alpha$ Ara) con VLTI/MIDI y líneas de base mayores a $130 \mathrm{~m}$. Casi todos los objetos no eran resueltos incluso con la línea de base más larga, indicando que la extensión de los discos circunestelares era menor que 10 mas. La única excepción fue $\alpha$ Ara, que fue resuelta y ajustada con una envoltura elíptica con tamaño y achatamiento similar al medido con VLTI/AMBER en la banda K en 2 $\mu \mathrm{m}$. Por otro lado, encontraron que el tamaño de las envolturas no tenía una relación fuerte con la longitud de onda entre los 8 y $12 \mu \mathrm{m}$. Esto es contrario a lo encontrado por Millour et al. (2009), Meilland et al. (2010) y Borges Fernandes et al. (2011) para las estrellas B[e], quienes reportaron que el tamaño aumenta con la longitud de onda. Además, el tamaño y la forma del disco de $\alpha$ Ara era prácticamente idéntico en 2, 8, y $12 \mu \mathrm{m}$, lo que podría explicarse por un efecto de truncamiento producido por la presencia de una compañera. Por otra parte, encontraron que el 
disco de las estrellas de tipo espectral tardío era más chico que en las de tipo espectral temprano.

Con el instrumento CHARA/VEGA (Mount Wilson, California), Delaa et al. (2011) estimaron la extensión del disco en el continuo y en $\mathrm{H} \alpha$ para 48 Per y $\phi$ Per. La interferometría de larga base ha permitido además detectar la compañera en sistemas binarios y determinar los parámetros físicos del sistema (Meilland et al., 2008; Millour et al., 2009; Kanaan et al., 2008).

Los mecanismos de eyección de masa pueden ser testeados ajustando la geometría y cinemática de las envolturas de las estrellas $\mathrm{Be}$, lo que solo puede ser realizado utilizando interferometría de larga base con suficiente resolución espectral. Para estudiar estos mecanismos y verificar si existe una dependencia con los parámetros estelares, Meilland et al. (2012) realizaron observaciones para una muestra de 8 estrellas Be brillantes: $\alpha$ Col, $\kappa$ CMa, $\omega$ Car, p Car, $\delta$ Cen, $\mu$ Cen, $\alpha$ Ara y o Aqr. Estos autores determinaron la extensión del disco en la línea $\mathrm{Br} \gamma$ y el continuo, ajustaron la cinemática del disco mostrando que el mismo presenta una ley de rotación cercana a la Kepleriana y determinaron una tasa de rotación media de $\overline{\Omega / \Omega_{\mathrm{c}}}=0.95 \pm 0.02$. No pudieron detectar ninguna correlación entre los parámetros estelares y la estructura de la envoltura circunestelar, pero plantearon la necesidad de extender la muestra de estrellas Be para tener resultado más concluyentes.

\subsection{VLTI/AMBER}

En la actualidad existen numerosos interferómetros, tanto en el rango óptico como IR, que nos permitirían caracterizar los entornos de las estrellas Be. Aquí solo nos limitaremos a describir las características principales de la instrumentación utilizada para la obtención de los datos utilizados en esta tesis.

El Telescopio de Gran Apertura (Very Large Telescope - VLT), perteneciente al Observatorio Europeo Austral (European Southern Observatory - ESO), está situado en el Cerro Paranal, Atacama-Chile. El mismo consiste en un arreglo de 4 telescopios unitarios (UTs) de $8.2 \mathrm{~m}$ y 4 telescopios auxiliares (ATs) de $1.8 \mathrm{~m}$. Los 4 UTs pueden funcionar individualmente, y pueden combinarse pares de UTs o ATs para formar un interferómetro gigante: el VLT Interferometer (VLTI). Los ATs pueden ubicarse en 30 posibles estaciones, formando líneas de base de hasta 200 m. En la Fig. 8.1 puede verse la distribución espacial de los UTs y las diferentes estaciones para los ATs, y en la Fig. 8.2 un esquema del funcionamiento del VLTI. El rango de longitudes de onda observable por el VLTI abarca el IR cercano y medio.

AMBER (Astronomical Multi-BEam combineR) es un espectrointerferómetro del VLTI que opera en el cercano IR en las bandas H y K (1.6 a $2.4 \mu \mathrm{m})$. Este instrumento permite combinar la información de tres telescopios, obteniendo para cada observación medidas de visibilidad y fase en tres frecuencias espaciales diferentes, y una closure phase. Dependiendo de las líneas de base utilizada, AMBER puede resolver fuentes con tamaños entre los 2 mas y los 50 mas. La resolución espectral puede ser baja $(R \sim 30)$, media $(R \sim 1500)$ o alta $(R \sim 12000)$. Puede ser utilizado 
Figura 8.1: VLTI - Posiciones de los UTs y bases para los ATs. La máxima línea de base (200 m) es la formada al combinar los ATs en las bases B5 y J6 (Fuente: www.eso.org).
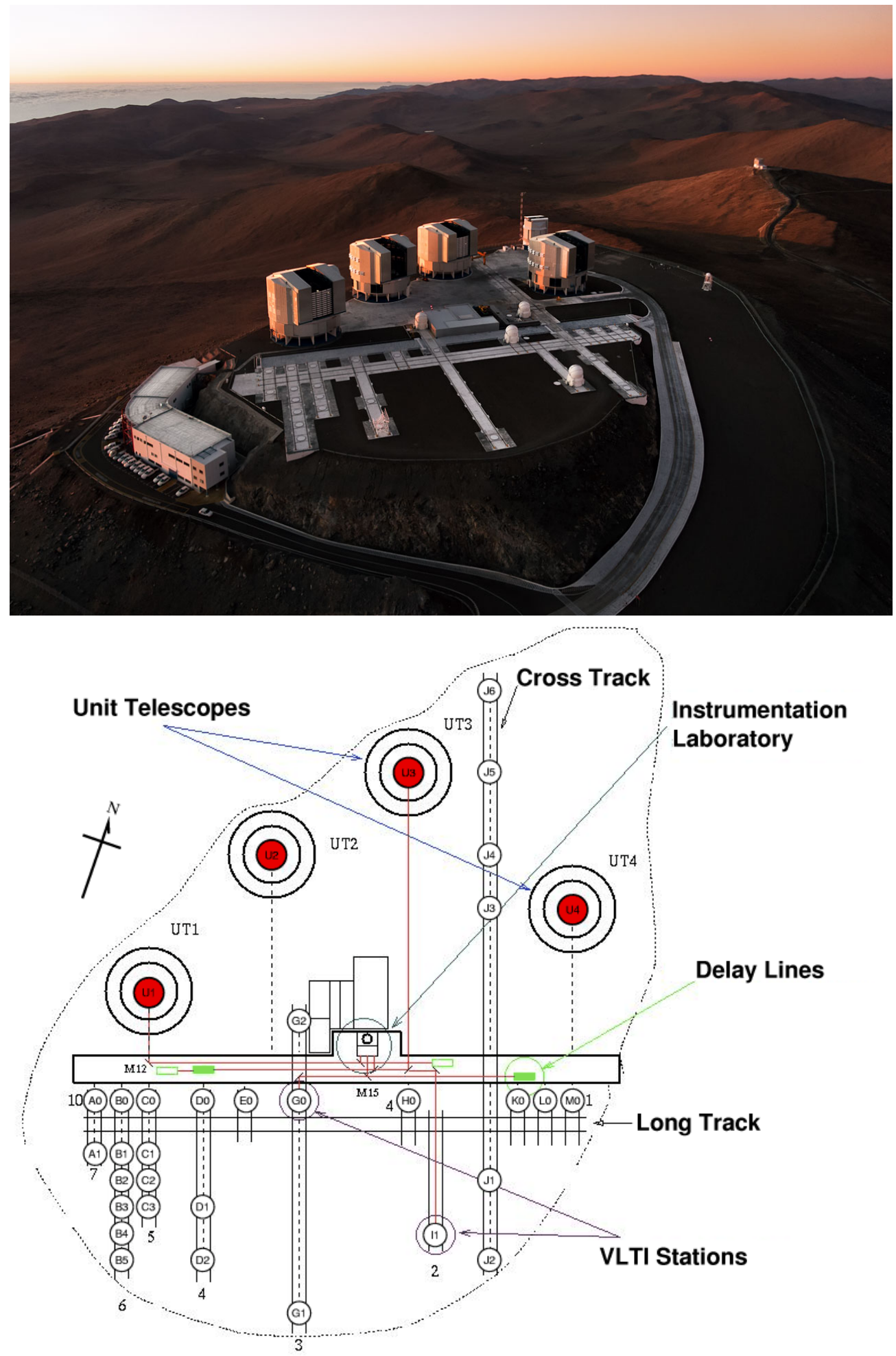
Figura 8.2: Esquema de funcionamiento del VLTI (Fuente: www.eso.org).

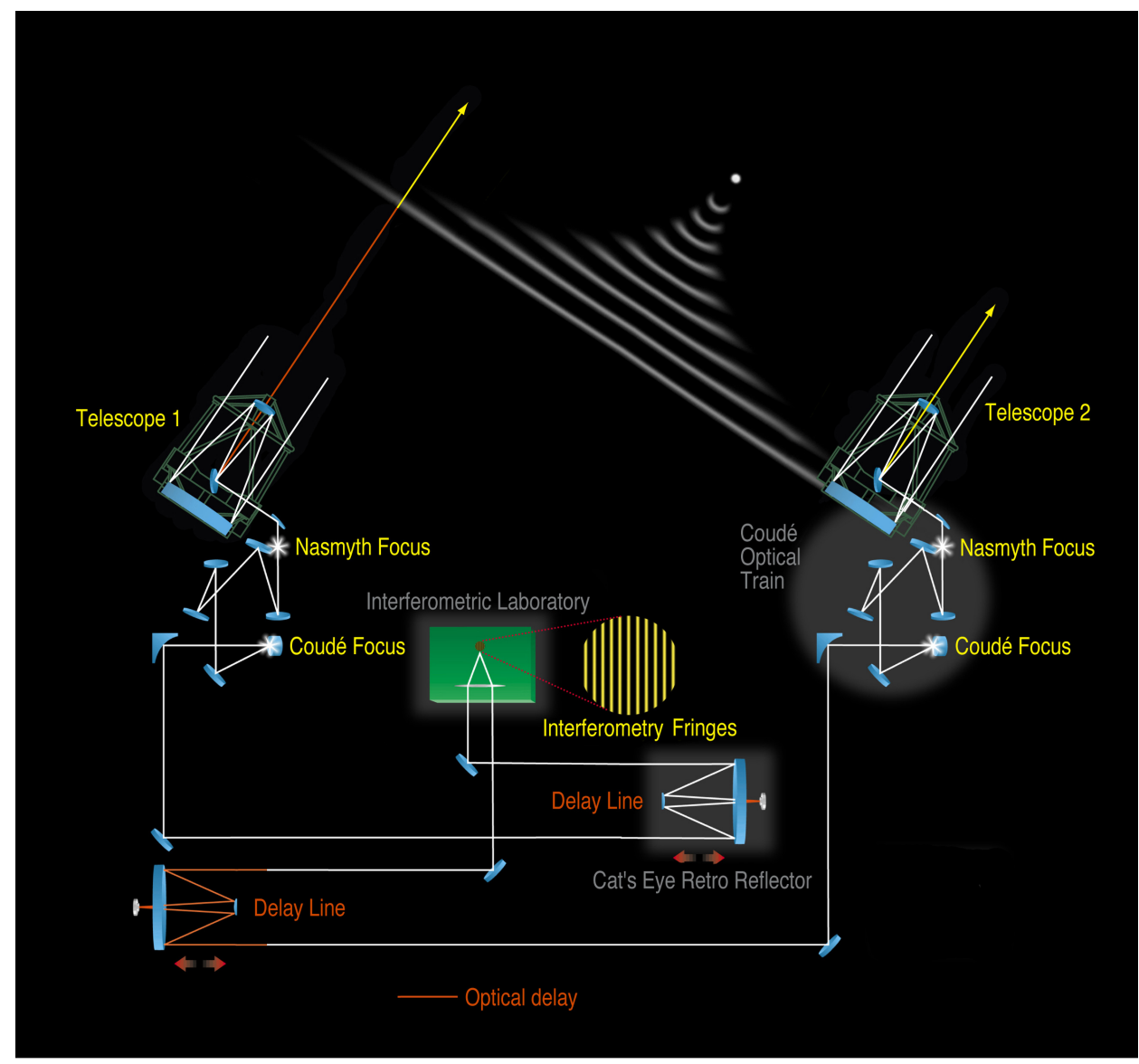

solo, o con FINITO (Fringe-tracking Instrument of NIce and TOrino). La función básica de FINITO es estabilizar la diferencia de camino óptico entre los diferentes telescopios, aumentando la sensibilidad debido a la posibilidad de aumentar el tiempo de exposición. De esta manera, utilizando los UTs con las mejores condiciones y menor resolución espectral, se pueden alcanzar objetos de magnitud $\mathrm{K}=9$, mientras que con los ATs, el límite en magnitud es $\mathrm{K}=6.5$.

Este instrumento fue diseñado con tres objetivos de estudio prioritarios: objetos estelares jóvenes, núcleos de galaxias activos, y planetas extrasolares calientes gigantes (Petrov et al., 2007). Con las especificaciones requeridas para esos programas, AMBER puede también utilizarse para determinar parámetros estelares fundamentales como la masa, radio y edad, y para el estudio de la actividad estelar, como por ejemplo el estudio de manchas y pulsaciones no radiales. De esta manera, se agregan a los temas de estudio las etapas finales de la evolución estelar, las estrellas masivas, y estudio del entorno de las estrellas calientes. Dentro de esto último, podemos mencionar a las estrellas Be. La visibilidad diferencial y la fase permiten poner restricciones al tamaño, la forma y la cinemática de la envoltura. Por otra parte, la closure phase da restricciones independientes a la simetría de la envoltura y podría permitir la reconstrucción de la imagen.

Para cada línea de base $l m$, en cada canal espectral, obtenemos medidas de la intensidad, de 
la amplitud de la visibilidad $V^{l m}(\lambda)$ y de la fase $\phi^{l m}(\lambda)$, utilizadas para obtener los observables de AMBER descriptos a continuación:

- Espectro de la fuente: cada uno de los haces produce un espectro de la fuente en la región espectral correspondiente. El espectro debe ser calibrado en intensidad y longitud de onda. Para observaciones en media y alta resolución es necesario observar calibrador.

- Visibilidad absoluta por canal espectral: esta medida se ve afectada por diferentes efectos, por lo cual es necesario observar un objeto calibrador, cuya visibilidad sea conocida.

- Visibilidad diferencial: es la visibilidad de la fuente en cada canal espectral $\lambda$, calibrada por la visibilidad promedio en un canal de referencia. Es independiente de los efectos que modifican la visibilidad absoluta, por lo que no se necesita de la utilización de un calibrador, y brinda básicamente la misma información física que la visibilidad absoluta espectralmente resuelta.

- Fase diferencial: da información referida a la fase de la visibilidad compleja. Para objetos no resueltos, nos da información sobre la asimetría de la fuente (Petrov, 1989).

- Closure phase CP: para cualquier triplete de líneas de base, la closure phase es cero para un objeto axisimétrico. Para los objetos no axisimétricos, la closure phase decrece con el tamaño angular del objeto sin resolver. Entonces, una closure phase no nula es un fuerte indicador de un objeto no axisimétrico resuelto interferométricamente (Millour et al., 2007; Weigelt et al., 2007).

\subsection{Modelo adoptado}

\subsubsection{Extensión de la región de emisión en $\mathrm{Br} \gamma$}

Como primer paso en la interpretación geométrica de la medida interferométrica, consideramos la región de emisión como un disco Gaussiano elongado. El flujo total emitido en la línea tendrá dos componentes: uno de la absorción fotosférica de la estrella central y otro correspondiente a la emisión de la envoltura. Para este modelo, el módulo de la visibilidad estará dado por:

$$
V_{\mathrm{Br} \gamma}=\frac{V_{\mathrm{Br} \gamma}^{\star} F_{\mathrm{Br} \gamma}^{\star}+V_{\mathrm{Br} \gamma}^{\mathrm{env}} F_{\mathrm{Br} \gamma}^{\mathrm{env}}}{F_{\mathrm{Br} \gamma}^{\mathrm{tot}}}
$$

donde

- $V_{\mathrm{Br} \gamma}^{\star}$ y $F_{\mathrm{Br} \gamma}^{\star}$ son la visibilidad y el flujo de la fotosfera

- $V_{\mathrm{Br} \gamma}^{\mathrm{env}}$ y $F_{\mathrm{Br} \gamma}^{\mathrm{env}}$ son la visibilidad y el flujo de la envoltura

- $F_{\mathrm{Br} \gamma}^{\mathrm{tot}}=F_{\mathrm{Br} \gamma}^{\star}+F_{\mathrm{Br} \gamma}^{\mathrm{env}}$ 
A partir de esta relación podemos despejar

$$
V_{\mathrm{Br} \gamma}^{\mathrm{env}}=\frac{V_{\mathrm{Br} \gamma}-V_{\mathrm{Br} \gamma}^{\star} \frac{F_{\mathrm{B} r \gamma}^{\star}}{F_{\mathrm{Br} \gamma}^{\mathrm{tot}}}}{1-\frac{F_{\mathrm{Br} \gamma}^{\star}}{F_{\mathrm{Br} \gamma}^{\mathrm{tot}}}}
$$

Para estimar $\frac{F_{\mathrm{Br} \gamma}^{\star}}{F_{\mathrm{Br} \gamma}^{\text {tot }}}$ se necesita estimar el perfil de absorción de la línea, para lo que se utiliza una estrella de tipo espectral y $V \sin i$ similar al objeto de estudio. El cociente puede estimarse haciendo

$$
\frac{F_{\mathrm{Br} \gamma}^{\star}}{F_{\mathrm{Br} \gamma}^{\mathrm{tot}}}=\frac{1}{1+\frac{F_{\mathrm{Br \gamma}}^{\text {env }}}{F_{\mathrm{Br} \gamma}^{\text {tot }}}}
$$

El cociente $\frac{F_{\mathrm{Br} \gamma}^{\mathrm{env}}}{F_{\mathrm{Br} \gamma}^{\mathrm{tot}}}$ puede obtenerse a partir del flujo total del perfil de emisión observado y el perfil de absorción de la estrella central, suponiendo despreciable la emisión y absorción en el continuo debido a la envoltura. La contribución de la envoltura en el continuo suele ser de $\sim 10 \%$.

\subsubsection{Extensión del disco en el continuo}

En el continuo, a diferencia de la línea $\mathrm{Br} \gamma$, es difícil separar la componentes estelar y la de la envoltura. Solo podríamos hacerlo si pudiéramos observar la estrella con y sin la envoltura circunestelar. La visibilidad del continuo será

$$
V_{\text {cont }}=\frac{V_{\text {cont }}^{\star} F_{\text {cont }}^{\star}+V_{\text {cont }}^{\text {env }} F_{\text {cont }}^{\text {env }}}{F_{\text {cont }}^{\text {tot }}}
$$

donde

- $V_{\text {cont }}^{\star}$ y $F_{\text {cont }}^{\star}$ son la visibilidad y el flujo de la fotosfera en el continuo

- $V_{\text {cont }}^{\text {env }} F_{\text {cont }}^{\text {env }}$ son la visibilidad y el flujo de la envoltura en el continuo

- $F_{\text {cont }}^{\mathrm{tot}}=F_{\text {cont }}^{\star}+F_{\text {cont }}^{\mathrm{env}}$

En este caso, tanto $\frac{F_{\text {cont }}^{\star}}{F_{\text {cont }}^{\text {tot }}} \operatorname{como} \frac{F_{\text {cont }}^{\text {env }}}{F_{\text {cont }}^{\text {tot }}}$ son parámetros libres.

Si consideramos a $V_{\text {cont }}$ como el resultado de la contribución de una estrella central con un disco uniformemente iluminado rodeada por un disco elíptico que presenta una distribución Gaussiana de brillo, el modelo tiene cinco parámetros libres:

- $\theta_{\star}$ diámetro angular de la estrella

- $\theta_{\text {m.cont }}$ diámetro angular del eje menor del disco Gaussiano en el continuo

- $\frac{F_{\text {cont }}^{\star}}{F_{\text {cont }}^{\text {tot }}}$ contribución en el continuo del flujo de la estrella

- $r_{c}=\frac{R_{\mathrm{cont}}^{\mathrm{M}}}{R_{\mathrm{cont}}^{\mathrm{m}}}$ cociente entre los ejes mayor y menor del disco en el continuo

- PA posición angular del eje mayor del disco respecto a la dirección Norte. 


\subsubsection{Modelo cinemático}

Para ajustar el campo de velocidades del disco de las estrellas Be observadas, se utilizó un modelo simple de dos dimensiones para un disco ecuatorial en rotación y/o expansión. Este modelo fue utilizado para modelar estrellas Be clásicas (Delaa et al., 2011; Meilland et al., 2011, 2012) y una estrella A[e] supergigante (Millour et al., 2011).

En el plano del cielo, de coordenadas cartesianas $x$ e $y$, este modelo incluye una estrella central de brillo constante, $I_{\star}(x, y)$, rodeada por una envoltura circunestelar. La emisión de esta envoltura en la línea $I_{\mathrm{Br} \gamma}(x, y)$ y el continuo $I_{\text {cont }}(x, y)$ son descriptas con dos distribuciones Gaussianas elípticas. Estas distribuciones tienen diferentes FWHM, $a_{\mathrm{Br} \gamma}$ and $a_{\text {cont }}$, pero el mismo achatamiento, producto de la proyección de un disco ecuatorial geométricamente delgado, $f=1 / \cos (i)$.

- Contribución de la envoltura en la línea

Para modelar la intensidad de la emisión de la línea usamos una distribución Gaussiana aplanada $I_{\mathrm{Br} \gamma}(x, y)$. El aplanamiento es debido a la proyección del disco en el plano del cielo debido a la inclinación, según $f=1 / \cos (i)$. El mapa de emisión es combinado con un mapa de dos dimensiones de las velocidades proyectadas, para obtener el mapa de intensidades para cada longitud de onda $\lambda$ y canal espectral $\delta \lambda$. Eso es normalizado por el ancho equivalente (EW) de la línea. Para obtener el mapa de velocidades proyectadas se tiene en cuenta la rotación y la expansión del disco.

Para la velocidad de rotación adoptamos un modelo de ley de potencia (Hutchings, 1970):

$$
V_{\phi}=V_{\text {rot }}\left(\frac{r}{R_{\star}}\right)^{j}
$$

donde $V_{\text {rot }}$ es la velocidad de rotación en la base del disco y $r$ la distancia al centro de la estrella. En este modelo, $j=-0.5$ corresponde a una rotación Kepleriana. $V_{\text {rot }}$ puede ser proporcional o igual a la velocidad crítica de rotación $V_{c}=\sqrt{2 G M_{\star} / 3 R_{\mathrm{p}}}$, donde $M_{\star}$ es la masa de la estrella y $R_{\mathrm{p}}$ el radio polar.

La velocidad de expansión se ajusta con un modelo de viento según la teoría CAK (Castor et al., 1975):

$$
V_{\mathrm{r}}=V_{0}+\left(V_{\infty}-V_{0}\right)\left(1-\frac{R_{\star}}{r}\right)^{\beta}
$$

donde $V_{0}$ es la velocidad de expansión en la fotosfera, $V_{\infty}$ es la velocidad terminal de expansión y $\beta=0.86$ para vientos típicos de estrellas calientes.

A partir de la velocidad de rotación y expansión, teniendo en cuenta la inclinación del disco, obtenemos la velocidad proyectada a lo largo de la línea de la visual:

$$
V_{\text {proy }}(x, y)=\left(V_{\phi} \sin \phi-V_{\mathrm{r}} \cos \phi\right) \cdot \sin i
$$


donde $x$ e $y$ son las coordenadas cartesianas en el plano del cielo.

Para obtener el mapa bidimensional de igual velocidad para la línea en emisión proyectada en el plano del cielo, se obtiene para cada canal espectral $\delta \lambda$ en la línea el mapa de los píxeles que tienen igual velocidad según:

$$
\begin{aligned}
R(x, y, \lambda, \delta \lambda) & =\frac{1}{\sigma \sqrt{2 \pi}} \exp \left[-\left(\frac{V_{\text {proy }}(x, y)-V(\lambda)}{\sqrt{2} \sigma}\right)^{2}\right] \\
\sigma & =\frac{\delta V}{2 \sqrt{2 \ln (2)}}=\frac{\delta \lambda c}{2 \lambda \sqrt{2 \ln (2)}}
\end{aligned}
$$

donde $V(\lambda)$ es el desplazamiento Doppler correspondiente a $\lambda$ en el perfil de la línea.

- Contribución de la envoltura en el continuo

La contribución de la emisión en el continuo por la envoltura $I_{\text {cont }}(x, y)$ es modelada por un disco Gaussiano con el mismo achatamiento que $I_{\mathrm{Br} \gamma}(x, y)$. El flujo de la envoltura en el continuo $F_{\text {cont }}^{\text {env }}$ es constante.

- Contribución de la estrella

Para la estrella central, el modelo considera un disco uniforme con intensidad constante $I_{\star}(x, y)$. El flujo de la estrella central $F_{\star}$ varía con la longitud de onda, considerando las líneas fotosféricas.

$I_{\mathrm{Br \gamma}}(x, y), I_{\text {cont }}(x, y)$ e $I_{\star}(x, y)$ están normalizados por su flujo total.

Considerando entonces la emisión de la estrella, la emisión de la envoltura en el continuo y la línea, junto con los flujos correspondientes, obtenemos la emisión total para cada canal espectral:

$$
I_{\mathrm{tot}}(x, y, \lambda, \delta \lambda)=I_{\mathrm{Br \gamma}}(x, y) \cdot R(x, y, \lambda, \delta \lambda) \cdot \mathrm{EW}+I_{\mathrm{cont}}(x, y) \cdot F_{\mathrm{cont}}^{\mathrm{env}}+I_{\star}(x, y) \cdot F_{\star}(\lambda)
$$

Los parámetros del modelo pueden clasificarse en diferentes categorías:

- parámetros estelares: radio estelar $\left(R_{\star}\right)$, distancia $(d)$, ángulo de inclinación $(i)$, ángulo de posición del eje mayor (PA)

- parámetros cinemáticos: velocidad de rotación en la base del disco $\left(V_{\text {rot }}\right)$, velocidad de expansión $\left(V_{0}\right)$, velocidad terminal $\left(V_{\infty}\right)$ y exponentes de la ley de velocidad de expansión y rotación del disco $(\beta$ y $j)$

- parámetros del disco en el continuo: FWHM del disco en el continuo ( $\left.a_{\text {cont }}\right)$, flujo normalizado del disco en el continuo $\left(F_{\text {cont }}^{\text {env }}\right)$

- parámetros del disco en la línea: FWHM del disco en la línea $\left(a_{\mathrm{Br} \gamma}\right)$, ancho equivalente de la línea (EW). 
Figura 8.3: Ejemplo de mapa de velocidades $\left(i=60^{\circ}, \mathrm{PA}=90^{\circ}, V_{\text {rot }}=400 \mathrm{~km} \mathrm{~s}^{-1}, V_{0}=0, V_{\infty}=0, j=-0.5\right.$ y $\beta=0.86$ ). Un color más oscuro indica velocidades proyectadas acercándose el observador y un color más claro velocidades alejándose del observador.

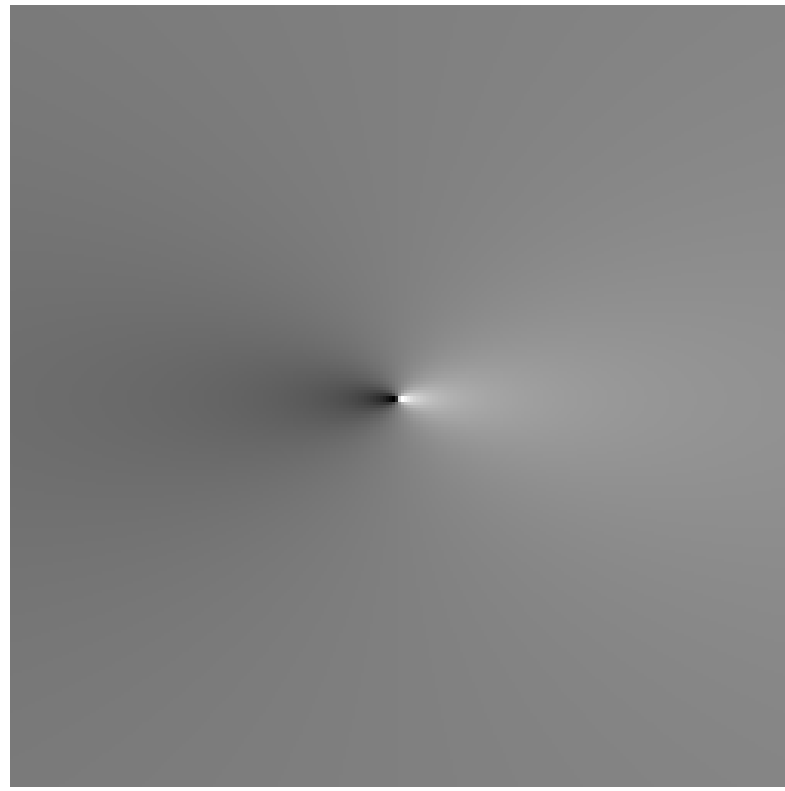

Figura 8.4: Ejemplo de mapa de intensidades para diferentes $\lambda$ (parámetros de la Fig. 8.3 con $R_{\star}=5 R_{\odot}, d=300 \mathrm{pc}, a_{\text {cont }}=5, F_{\text {cont }}^{\mathrm{env}}=0.2, a_{\mathrm{Br} \gamma}=10$ y EW=8).
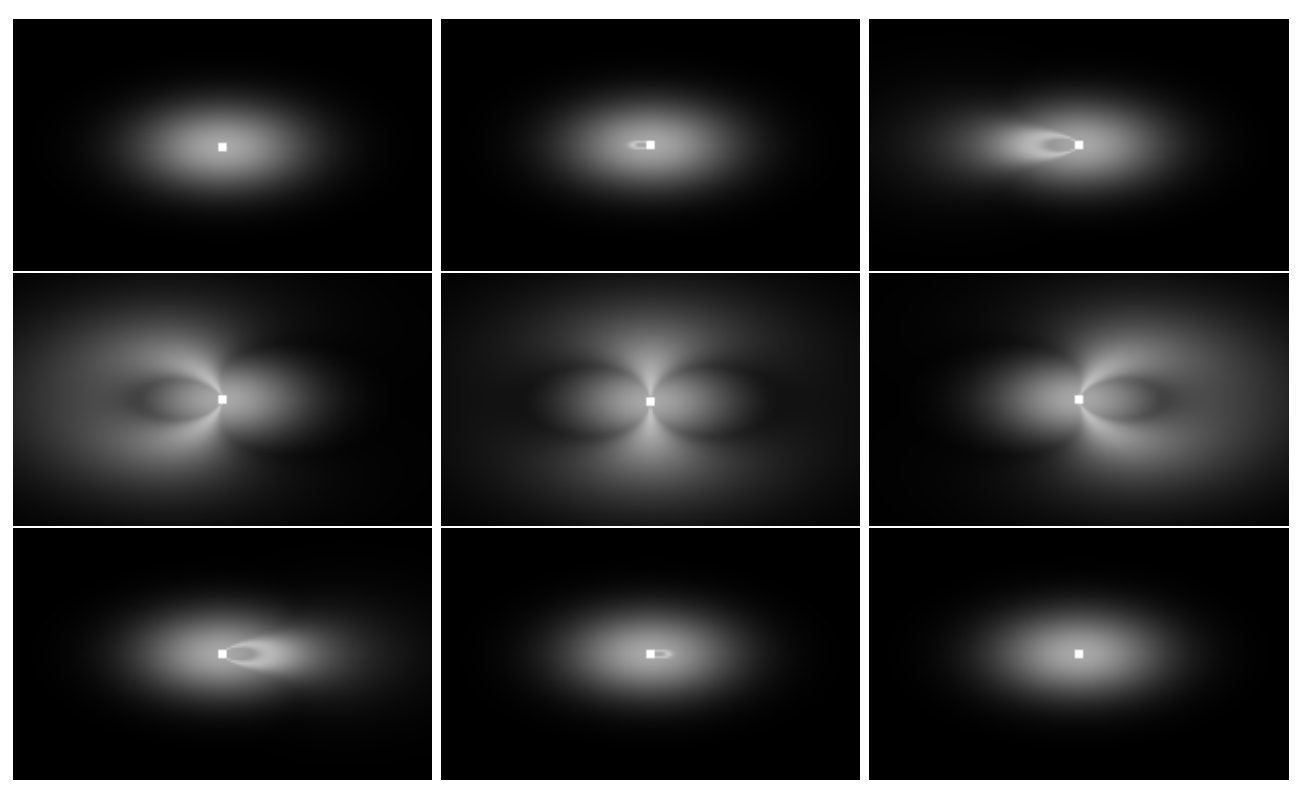

A modo de ejemplo, en la Fig. 8.3 se observa el mapa de velocidades correspondiente a un disco con $i=60^{\circ}, \mathrm{PA}=90^{\circ}, V_{\text {rot }}=400 \mathrm{~km} \mathrm{~s}^{-1}, V_{0}=0, V_{\infty}=0, j=-0.5$ y $\beta=0.86$. Ese mapa de velocidades, combinado con la emisión de un disco correspondiente a $R_{\star}=5 R_{\odot}, d=300 \mathrm{pc}, a_{\text {cont }}=5, F_{\text {cont }}^{\text {env }}=0.2$, $a_{\mathrm{Br} \gamma}=10$ y EW=8, genera los mapas de intensidades de la Fig. 8.4. 


\subsection{Observaciones y reducción de datos}

En este trabajo utilizamos observaciones de 26 estrellas Be, realizadas por Meilland y colaboradores entre el 28 y 31 de octubre de 2014, con el instrumento VLTI/AMBER (Tabla 8.1, ID 094.D-0140). Para las observaciones se utilizaron los telescopios auxiliares (AT, $1.8 \mathrm{~m}$ ) en el modo de alta resolución (HR mode, $R \simeq 12000$ ) centrado en la línea $\operatorname{Br} \gamma 2.16 \mu \mathrm{m}$, lo que permite analizar la cinemática del material circunestelar, a partir del corrimiento Doppler en la emisión de la línea. Para mejorar la estabilidad de los fringes se utilizó también FINITO (Fringe-tracking Instrument of NIce and TOrino). Esto permite obtener mayores tiempos de integración, obteniendo así una mejor relación señal / ruido (S/N). Sin embargo, en las observaciones tomadas con AMBER en el modo HR el uso de FINITO añade grandes incertezas en las visibilidades calibradas, por lo que las observaciones fueron tomadas sin calibradores. Esto no presenta un inconveniente para nuestro estudio, ya que la visibilidad diferencial, la fase diferencial y la closure phase son independientes de los efectos que modifican la visibilidad absoluta.

Los datos fueron reducidos por Meilland utilizando el software de reducción de VLTI/AMBER (amdlib v3.0.9, ver Tatulli et al., 2007; Chelli et al., 2009, para información detallada sobre la reducción de datos).

Tabla 8.1: Observaciones interferométricas en $\mathrm{Br} \gamma$ realizadas con VLTI/AMBER.

\begin{tabular}{lccc}
\hline \hline Estrella & Fecha de observación & Telescopios & DIT \\
\hline HD 23302 & $2014-10-29$ 05:57 & A1-I1-G1 & 5.0 \\
& $2014-10-31$ 06:24 & A1-K0-J3 & 20.0 \\
\hline HD 23338 & $2014-10-29$ 05:39 & A1-G1-I1 & 5.0 \\
\hline HD 23408 & $2014-10-29$ 06:36 & A1-G1-I1 & 5.0 \\
\hline HD 23480 & $2014-10-29$ 06:15 & A1-G1-I1 & 5.0 \\
\hline HD 23630 & $2014-10-29$ 06:55 & A1-G1-I1 & 5.0 \\
& $2014-10-30$ 07:27 & A1-G1-J3 & 5.0 \\
& $2014-10-31$ 06:45 & A1-K0-J3 & 20.0 \\
\hline HD 23862 & $2014-10-30$ 07:47 & A1-G1-J3 & 5.0 \\
& $2014-10-3107: 06$ & A1-K0-J3 & 20.0 \\
\hline HD 28497 & $2014-10-31$ 05:46 & A1-K0-J3 & 10.0 \\
\hline HD 30076 & $2014-10-31$ 06:05 & A1-K0-J3 & 10.0 \\
\hline HD 32991 & $2014-10-31$ 09:20 & A1-K0-J3 & 5.0 \\
\hline HD 33 328 & $2014-10-30$ 07:01 & A1-G1-J3 & 5.0 \\
\hline HD 35439 & $2014-10-31$ 09:02 & A1-K0-J3 & 5.0 \\
\hline HD 36576 & $2014-10-31$ 08:25 & A1-K0-J3 & 5.0 \\
\hline HD 37202 & $2014-10-31$ 08:25 & A1-K0-J3 & 5.0 \\
\hline & Tabla 8.1 continúa en la página siguiente
\end{tabular}


Tabla 8.1 (continuación)

\begin{tabular}{|c|c|c|c|}
\hline HD 37490 & 2014-10-31 08:41 & A1-K0-J3 & 5.0 \\
\hline HD 41335 & $2014-10-3107: 50$ & A1-K0-J3 & 5.0 \\
\hline \multirow[t]{2}{*}{ HD 45725} & 2014-10-29 08:30 & A1-G1-I1 & 4.0 \\
\hline & 2014-10-30 08:08 & A1-G1-J3 & 4.0 \\
\hline HD 60606 & 2014-10-30 08:35 & A1-G1-J3 & 10.0 \\
\hline \multirow[t]{2}{*}{ HD 66194} & 2014-10-31 04:37 & A1-K0-J3 & 10.0 \\
\hline & 2014-10-31 05:21 & A1-K0-J3 & 10. \\
\hline HD 68980 & 2014-10-30 09:16 & A1-G1-J3 & 5.0 \\
\hline HD 75311 & $2014-10-31$ 07:33 & A1-K0-J3 & 5.0 \\
\hline \multirow[t]{7}{*}{ HD 209409} & 2014-10-29 00:48 & A1-G1-I1 & 2.0 \\
\hline & 2014-10-29 01:27 & A1-G1-I1 & 2.0 \\
\hline & 2014-10-30 01:06 & A1-G1-J3 & 2.0 \\
\hline & 2014-10-30 03:31 & A1-G1-J3 & 2.0 \\
\hline & 2014-10-31 00:25 & A1-K0-J3 & 2.0 \\
\hline & 2014-10-31 03:03 & A1-K0-J3 & 2.0 \\
\hline & 2014-10-31 03:39 & A1-K0-J3 & 2.0 \\
\hline \multirow[t]{5}{*}{ HD 212076} & 2014-10-29 02:04 & A1-G1-I1 & 5.0 \\
\hline & 2014-10-29 02:36 & A1-G1-I1 & 5.0 \\
\hline & 2014-10-30 02:02 & A1-G1-J3 & 2.0 \\
\hline & 2014-10-30 02:50 & A1-G1-J3 & 5.0 \\
\hline & 2014-10-31 01:57 & A1-K0-J3 & 5.0 \\
\hline \multirow[t]{2}{*}{ HD 212571} & 2014-10-30 01:42 & A1-G1-J3 & 2.0 \\
\hline & 2014-10-31 01:38 & A1-K0-J3 & 10.0 \\
\hline \multirow[t]{5}{*}{ HD 214748} & 2014-10-29 03:30 & A1-G1-I1 & 5.0 \\
\hline & 2014-10-30 02:28 & A1-G1-J3 & 2.0 \\
\hline & 2014-10-30 04:34 & A1-G1-J3 & 5.0 \\
\hline & $2014-10-3101: 20$ & A1-K0-J3 & 5.0 \\
\hline & 2014-10-31 04:15 & A1-K0-J3 & 5.0 \\
\hline HD 219688 & 2014-10-29 04:15 & A1-G1-I1 & 5.0 \\
\hline HD 224686 & 2014-10-29 03:51 & A1-G1-I1 & 5.0 \\
\hline
\end{tabular}

\subsubsection{Muestra de estrellas Be}

La mayor parte de los objetos observados presentan un perfil de emisión con un doble pico en $\mathrm{Br} \gamma$ (ver Fig. 8.5). 28 Tau (HD 23862) y $\zeta$ Tau (HD 37202) presentan perfiles de tipo shell, la emisión en $\pi$ Aqr (HD 212571) es débil, mientras que ocho estrellas (HD 23302, HD 23338, HD 23408, HD 23480, HD 33 328, HD 75311, HD 219688 y HD 224686) presentan perfiles en 
Figura 8.5: Espectros en la región de $\operatorname{Br} \gamma$ de los objetos observados.
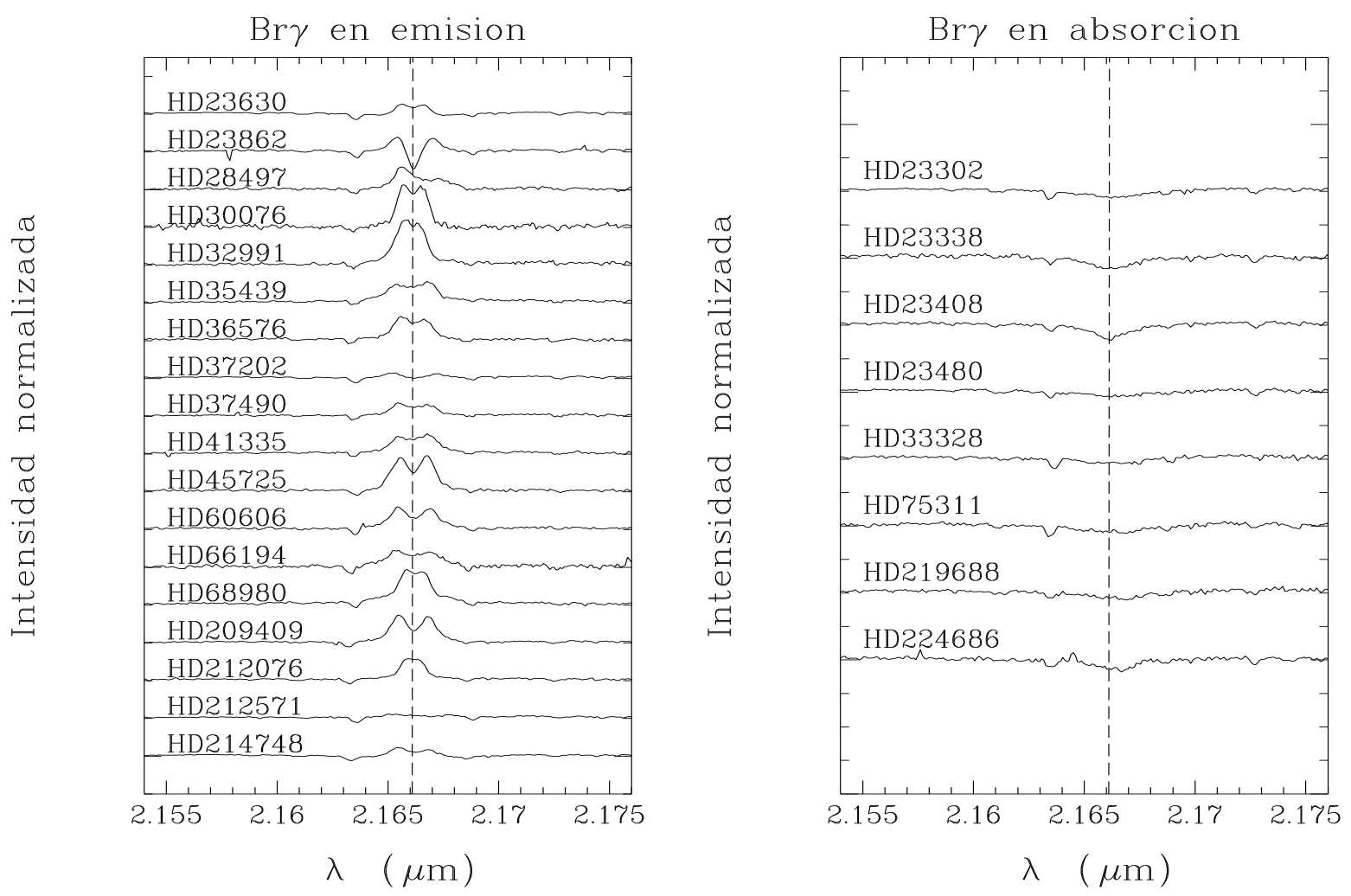

absorción. Estas últimas fueron excluidas de nuestro estudio, debido a que el modelo utilizado solo permite ajustar la emisión del disco. Nuestra muestra final se limita a 18 estrellas Be con $m_{\mathrm{K}} \leq 6$, tipos espectrales entre B1 y B8, y clases de luminosidad entre V y III, distribuidas según el histograma de la Fig. 8.6. Alrededor del 55\% de las estrellas tienen tipos espectrales B1-B2, mientras que el resto está distribuido uniformemente entre B3-B4 y B7-B8. No contamos con observaciones de estrellas con tipos espectrales B5-B6. La clase de luminosidad más frecuente es V, con alrededor del $60 \%$ de la muestra. Estrellas con clases de luminosidad IV son poco más del $20 \%$ y con clase de luminosidad III poco más del 15\%. Las clases de luminosidad III y IV están presentes en los tipos espectrales B1-B2 y B7-B8, mientras que las de clase de luminosidad V están presentes en todos los tipos espectrales de la muestra.

En la Tabla 8.2 presentamos los parámetros fundamentales de cada objeto estudiado. Estos parámetros fueron obtenidos a partir de datos de la literatura o del ajuste de la distribución espectral de energía (SED, ver Subsec. 8.5.1).

- El nombre de la estrella y el número del catálogo HD están en las dos primeras columnas.

- Las columnas 3 y 4 listan la magnitud en la banda $\mathrm{K}\left(m_{\mathrm{K}}\right)$ y tipo espectral. Ambos están tomadas de la base de datos CDS.

- Las columnas 5, 6, 7 y 8 muestran la distancia a la estrella $(d)$, temperatura efectiva ( $\left.T_{\text {eff }}\right)$, 
Figura 8.6: Distribución de TE y CL de las estrellas de la muestra.

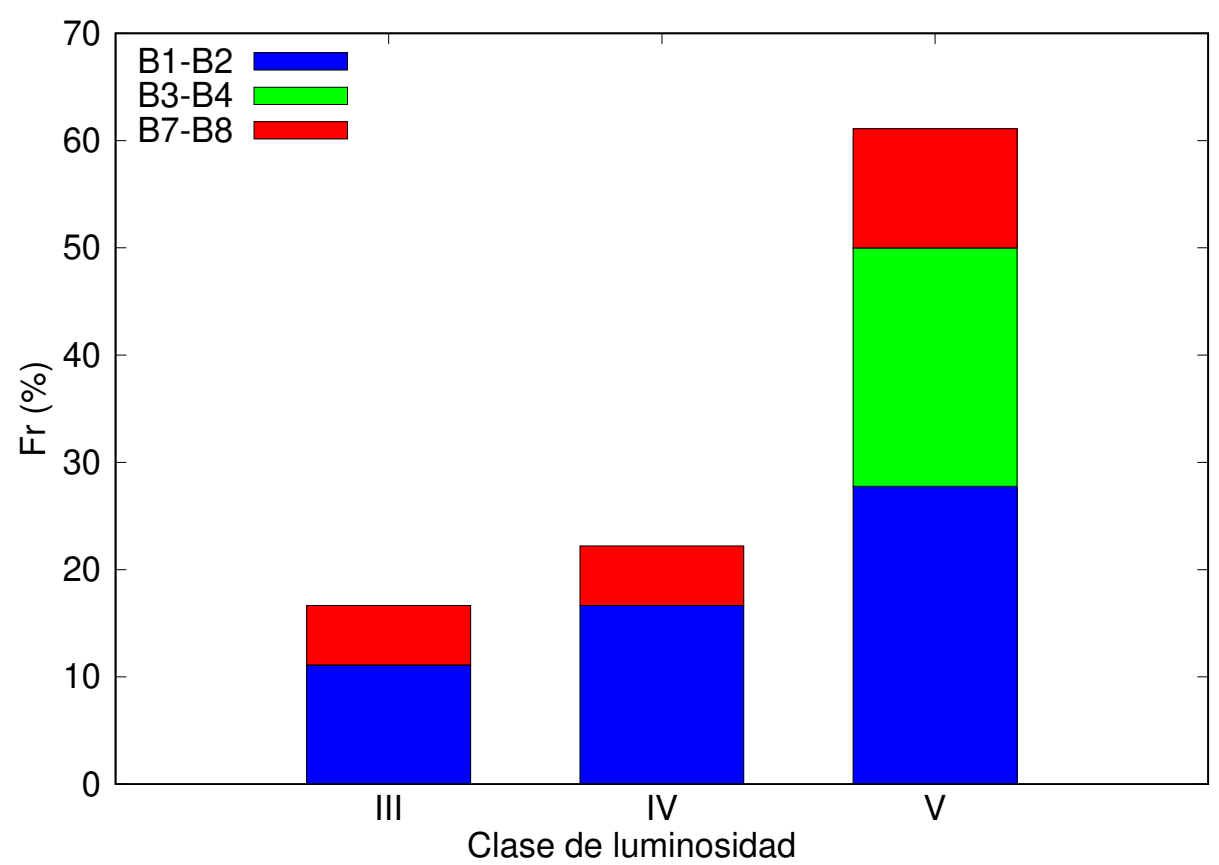

velocidad de rotación proyectada $(V \sin i)$, y velocidad crítica $\left(V_{\text {crit }}\right)$. La distancia $d$ se obtuvo a partir de van Leeuwen (2007), excepto para $\eta$ Car, 56 Eri, 105 Tau y V374 Car, obtenidas a partir de Astraatmadja \& Bailer-Jones (2016). La temperatura efectiva $T_{\text {eff, velocidad de }}$ rotación proyectada $V \sin i$ y velocidad crítica $V_{\text {crit }}$ están sacadas de Frémat et al. (2005), excepto para 105 Tau , $\zeta$ Tau y V374 Car, cuyas temperaturas efectivas están estimadas a partir del ajuste de la SED usando los modelos de Kurucz (1979) para atmósferas estelares y los valores de $V \sin i$ están sacados de Abt et al. (2002) para 105 Tau y Chauville et al. (2001) para $\zeta$ Tau y V374 Car.

- Las columnas 9, 10 y 11 listan el ángulo de polarización $\left(P_{\star}\right)$, el radio estelar $\left(R_{\star}\right)$, y el flujo del disco en el continuo relativo al flujo total en la banda $\mathrm{K}\left(F_{\text {cont }}^{\mathrm{env}}\right)$. El ángulo de polarización fue extraído de Yudin (2001). El radio estelar $R_{\star}$ y el flujo de la envoltura en la banda $\mathrm{K}$ están estimados a partir del ajuste de la SED usando los modelos de Kurucz (1979) para atmósferas estelares, usando los parámetros estelares dados por Frémat et al. (2005). Para evitar la contaminación de la SED por el flujo de la envoltura, el ajuste al continuo estelar se realizó del UV al visible (Meilland et al., 2009). 
Tabla 8.2: Parámetros estelares obtenidos de la literatura o estimados a partir del ajuste de la SED para las 18 estrellas Be de la muestra. Ver la Subsección 8.4.1 para más detalles.

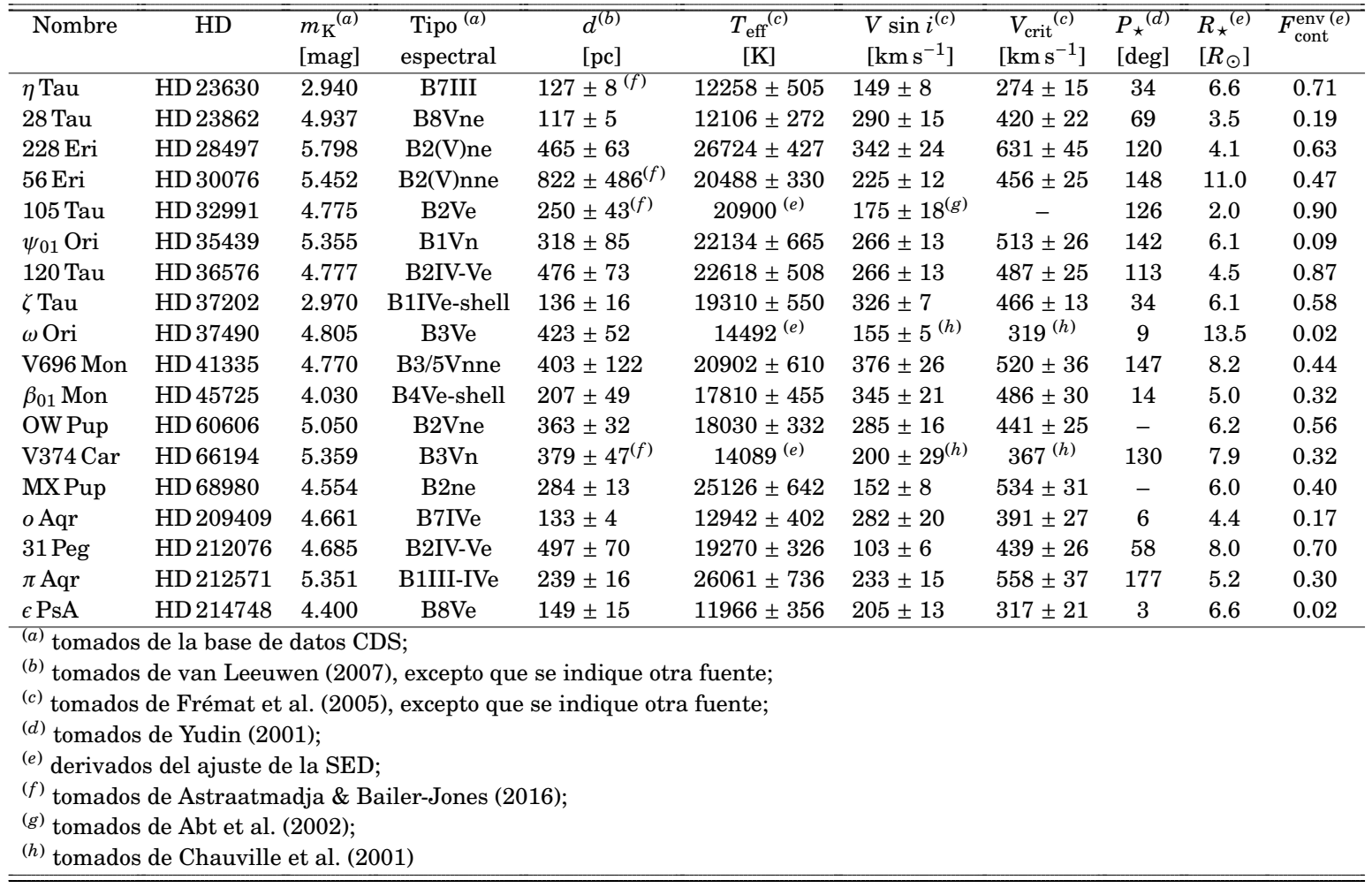

\subsection{Ajustes realizados}

\subsubsection{Parámetros estelares a partir del ajuste de la SED}

Para obtener los valores de $R_{\star}$ y $F_{\text {cont }}^{\text {env }}$ para los objetos de la muestra, realizamos ajustes de la SED. Este método también se utilizó para obtener la $T_{\text {eff }}$ de 105 Tau, $\zeta$ Tau y V374 Car. Para cada objeto, se reconstruyó la SED utilizando información disponible en la base de datos VIZIER. A partir de los datos fotométricos de (Ochsenbein et al., 2000), buscamos el modelo de Kurucz (1979) que mejor ajusta, usando un método de minimización que emplea la rutina de IDL "mpfit" (el algoritmo se describe en forma detallada en Arcos et al., 2018, Sec. 3.1). Los parámetros de entrada de los modelos fueron $T_{\text {eff }} \mathrm{y} \log g$. Los modelos de Kurucz fueron enrojecidos utilizando la ley de extinción de Cardelli et al. (1989) con $R_{v}=3.1$. Para evitar contaminación por el flujo del disco, el ajuste fue realizado en el óptico y UV (Meilland et al., 2009).

Se dejaron fijos los parámetros de la Tabla 8.2 dejando libres $R_{\star}$ (y $T_{\text {eff }}$ en los casos de 105 Tau , $\zeta$ Tau y V374 Car). Del modelo con mejor ajuste, se obtuvo el valor de $R_{\star}$ y $F_{\text {cont }}^{\text {env }}$ (dado automáticamente para todas las bandas).

En la Fig. 8.7 presentamos un ejemplo de ajuste de la SED. Los triángulos rojos representan los valores fotométricos de VIZIER, la línea azul sólida representa el modelo de Kurucz del mejor 
Figura 8.7: Ejemplo de ajuste de la SED para V696 Mon (HD 41335). Los triángulos rojos representan los valores fotométricos de VIZIER, la línea azul sólida representa el modelo de Kurucz del mejor ajuste y la línea rayada muestra el exceso IR.

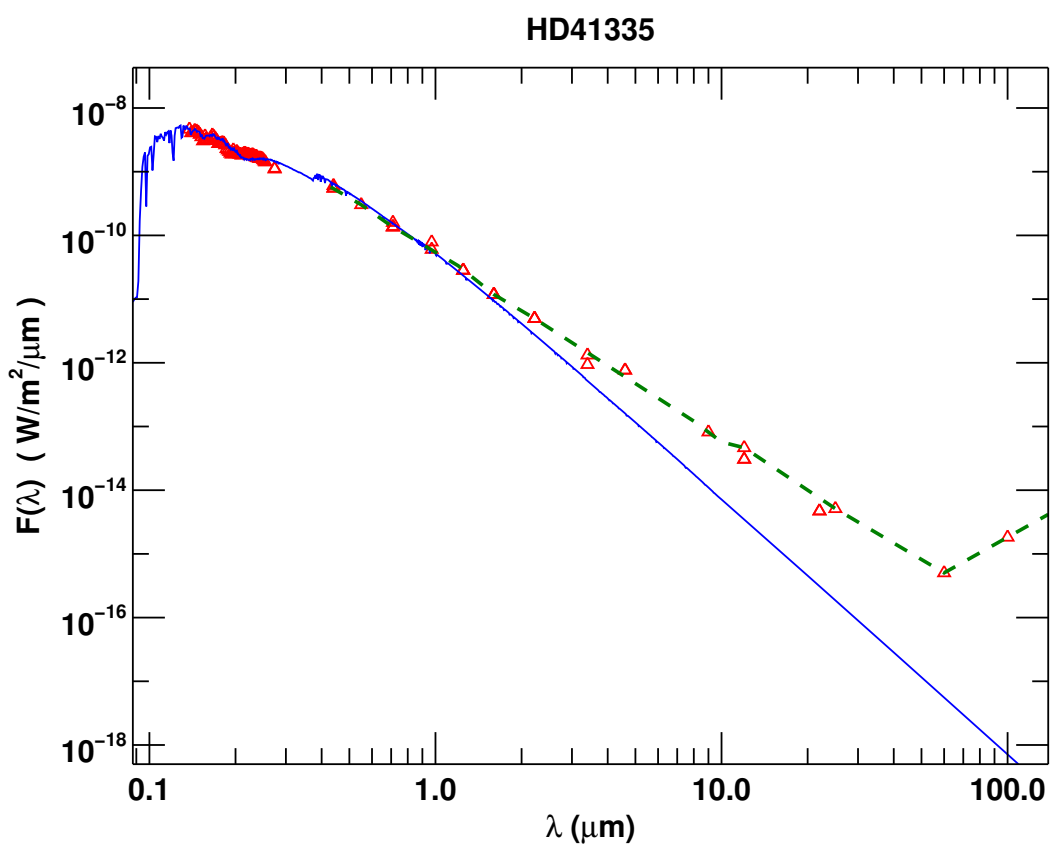

ajuste y la línea rayada muestra el exceso IR.

\subsubsection{Parámetros del disco a partir del ajuste de datos interferométricos}

Para realizar los ajustes de los datos interferométricos se utilizó un código desarrollado por el Dr. Samer Kanaan de la Universidad de Valparaíso, Chile. El código permite ingresar parámetros para el disco y la estrella central, simular un conjunto de observaciones correspondiente a esos parámetros, y compararlo con las observaciones obtenidas para ese objeto.

Debido al gran número de parámetros libres del modelo, un método de ajuste automático requeriría del cálculo de millones de modelos. Dado que conocemos como afecta cada parámetro a la visibilidad y a la fase, resultó más conveniente realizar el ajuste de los parámetros manualmente. La velocidad de expansión fue fijada en 0 , ya que usualmente la misma se considera despreciable sobre la velocidad de rotación (Miroshnichenko et al., 2003; Vinicius et al., 2006; Meilland et al., 2007a,b). Por otro lado, si el disco está directamente conectado a la superficie, $V_{\text {rot }}$ será igual a la velocidad de rotación estelar $V_{\star}$. En algunos casos, si la estrella no rota lo suficientemente rápido y existe un mecanismo adicional que transfiera momento al material circunestelar, $V_{\text {rot }}$ será mayor que $V_{\star}$. En nuestro modelo consideraremos $V_{\text {rot }}$ como un parámetro libre, con valor máximo igual a la velocidad de rotación crítica $V_{\text {crit }}$. 
Para el ajuste de los restantes parámetros, se empezó con un ajuste cualitativo a partir de los datos interferométricos (especialmente PA, $i, a_{\text {cont }}, a_{\mathrm{Br} \gamma} \mathrm{y} \mathrm{EW}$, que afectan fuertemente los observables interferométricos y pueden ser determinados sin ambigüedad) y se exploró el espacio de parámetros a fin de minimizar el valor de $\chi^{2}$. Se exploró también todo el rango de posibles valores con pasos más grandes, a fin de descartar la existencia de otro mínimo.

A continuación describiremos de qué manera afecta cada parámetro a los observables:

- El ángulo de posición del eje mayor (PA) modifica la forma de la visibilidad diferencial y la amplitud de la variación de la fase (Meilland et al., 2011). Cuando la base está alineada con el eje mayor, la amplitud de la caída de la visibilidad diferencial es máxima y toma forma de "W" (por ejemplo la base $B_{1}$ de la Fig. 8.8), pero cuando la base está alineada con el eje menor, la amplitud es mínima y la visibilidad toma forma de "V" (como la base $\mathrm{B}_{5}$ de la Fig. 8.8). Para un disco no resuelto, la amplitud de la forma "S" de la fase diferencial es proporcional a la longitud de la línea de base, y depende de la orientación.

- El ancho equivalente de la línea (EW) es fijado a partir del ajuste del perfil de la línea.

- El FWHM en la línea $\left(a_{\mathrm{Br} \gamma}\right)$ tiene un efecto importante en la amplitud de la caída de la visibilidad y la forma de "S" de la fase diferencial sobre todas las líneas de base. Para bases que resuelven completamente el disco, la fase diferencial pierde la forma de "S" y se hacen visibles efectos secundarios debido a inhomogeneidades en el disco o, como en nuestras observaciones, debido a efectos numéricos por la sobrerresolución del disco ${ }^{2}$ (como se observa en la base $B_{9}$ de la Fig. 8.8). Este parámetro también afecta la separación de los dos picos, siendo menor la separación para discos mayores.

- El FWHM en el continuo ( $\left.a_{\text {cont }}\right)$ afecta el nivel de la visibilidad absoluta en el continuo, siendo la visibilidad menor a medida que aumenta $a_{\text {cont }}$. Además, puede modificar la amplitud de las variaciones de fase, haciendo que mientras más resuelto esté el objeto en el continuo, menor sea la variación de la fase.

- El ángulo de inclinación ( $i$ ) tiene un gran efecto en la amplitud de la caída de la visibilidad para bases cercanas a la orientación polar, y tiene una gran influencia en la separación de los picos ya que afecta a la velocidad de rotación proyectada.

- La velocidad de rotación ( $\left.V_{\text {rot }}\right)$ afecta la separación del doble pico y el ancho de la línea. Mientras más rápido sea la rotación del disco, mayor será la separación de los picos.

- El exponente de la ley de velocidad de rotación $(j)$ también afecta a la separación de los picos. Para valores altos de $j$, la velocidad cae rápidamente con la distancia, y la separación

\footnotetext{
${ }^{2}$ Este efecto numérico se debe a propiedades de la transformada de Fourier. Para un objeto simple, la visibilidad se hace nula cuando el objeto está completamente resuelto, y más allá de eso la fase toma valores negativos. Este efecto es más complejo en el caso de objetos con varias componentes, como un disco rotando alrededor de una estrella $\mathrm{Be}, \mathrm{y}$ genera la pérdida de la forma de "S" en la fase diferencial para un objeto completamente resuelto.
} 
Figura 8.8: $\eta$ Tau (HD 23630): Plano ( $u, v)$, visibilidad diferencial, fase diferencial y closure phase para nuestras observaciones, junto con el modelo correspondiente al mejor ajuste. En el plano $(u, v)$, cada observación (tres líneas de base) está representada con diferente color y símbolo.
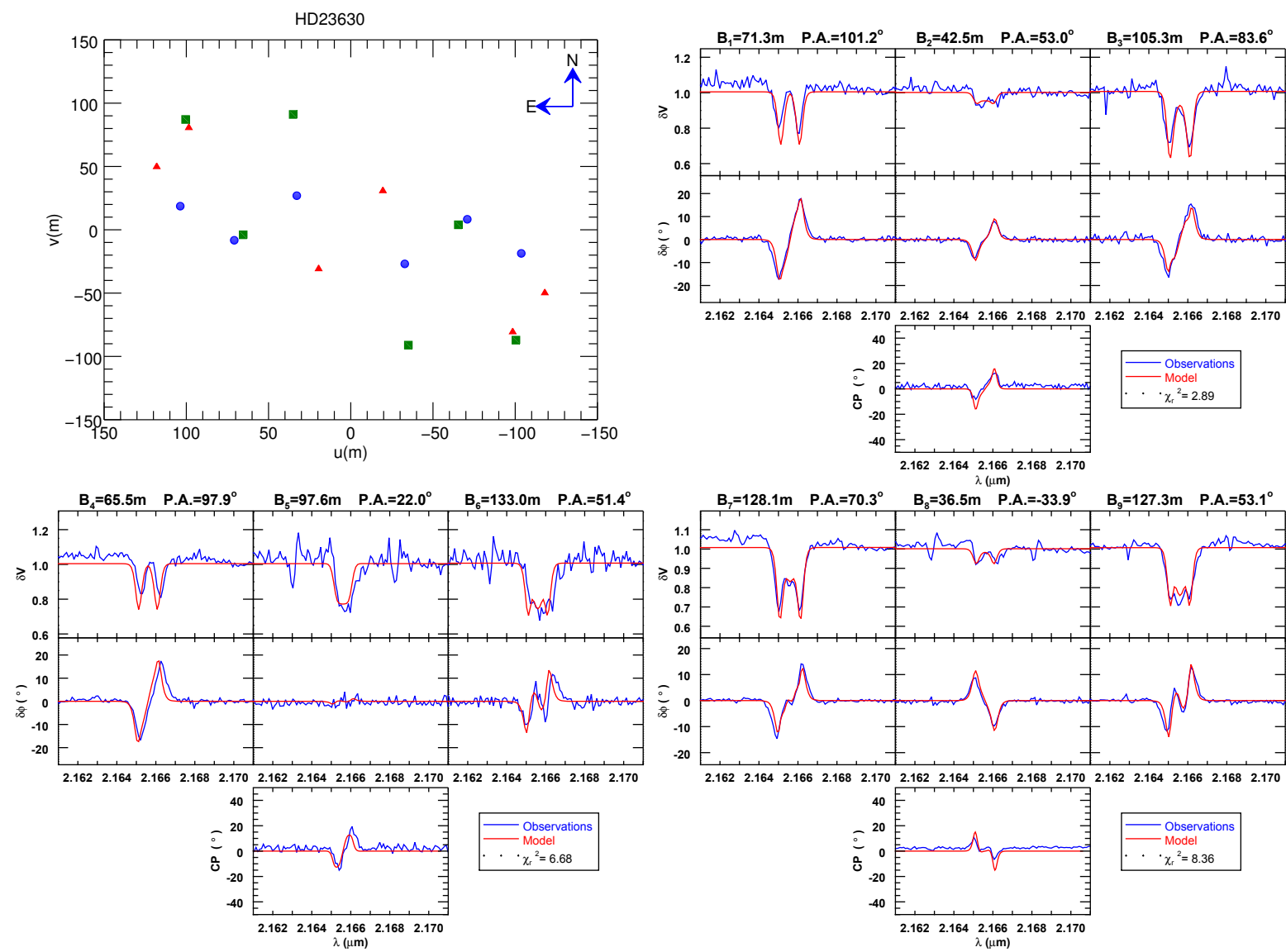

de los picos es menor. Este parámetro también afecta la forma de las alas de la línea, siendo mayores las alas para valores mayores de $j$. Sin embargo, como fue analizado en Delaa et al. (2011) las alas de las líneas en emisión de las estrellas Be pueden ser afectadas por diferentes fenómenos, por lo que es complicado distinguir el efecto de $V_{\text {rot }}$ y $j$ sin ambigüedad.

\subsection{Modelos obtenidos}

En la mayoría de los casos estudiados, observamos una caída en la visibilidad en la línea en emisión, causada por la dependencia del tamaño observado con la longitud de onda y el flujo relativo entre el continuo y la línea. También vemos una forma "W" en la visibilidad diferencial y una forma "S" o más compleja en la variación de la visibilidad diferencial. Estas características evidencian la presencia de un disco ecuatorial en rotación (Meilland et al., 2007b, 2011). 
Figura 8.9: Visibilidad diferencial, fase diferencial y closure phase para las observaciones de HD 23408, que presenta un perfil de Bry en absorción.

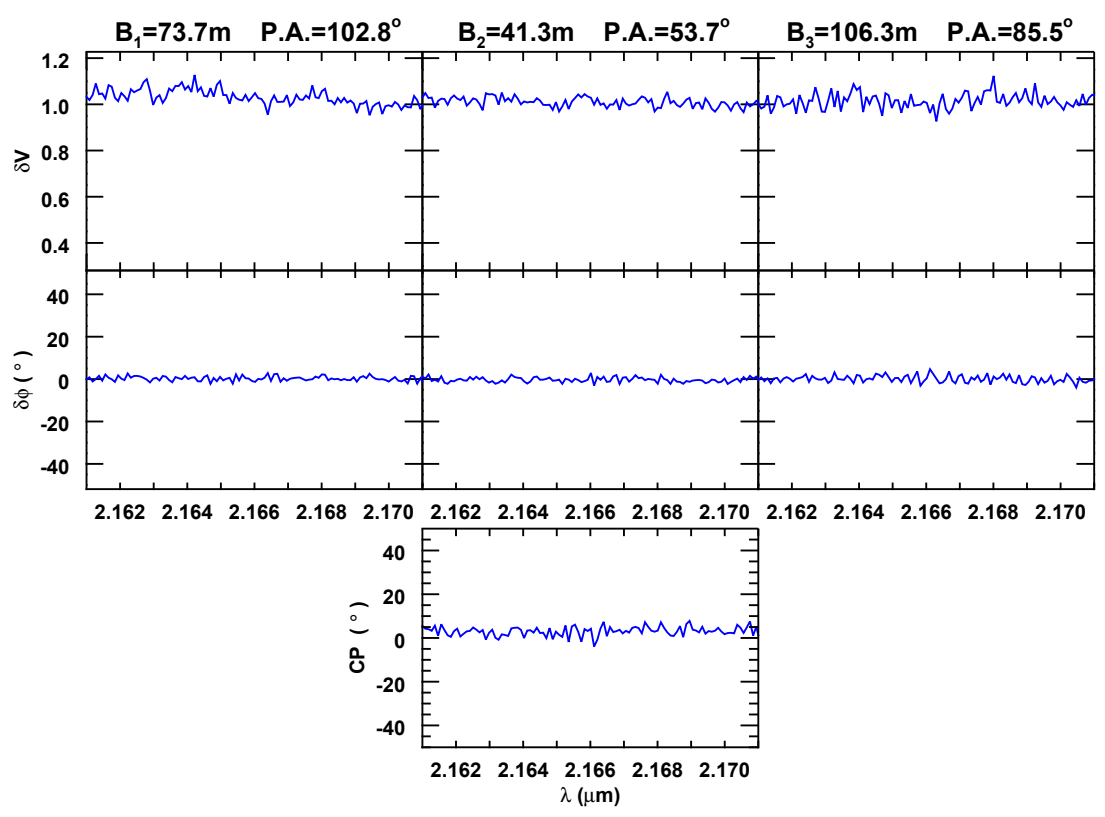

En la Fig. 8.8 se presenta la cobertura del plano $(u, v)$, la visibilidad diferencial, fase diferencial y closure phase, junto con el modelo del mejor ajuste, para $\eta$ Tau (HD 23630). Para los restantes objetos que presentan la línea $\mathrm{Br} \gamma$ en emisión la misma información se muestra en las Figs. 8.108.27. Cada observación en el plano $(u, v)$, i.e. tres líneas de base, está graficada con diferente color y símbolo. Círculos azules corresponden a las líneas de base $\mathrm{B}_{1}, \mathrm{~B}_{2}$ y $\mathrm{B}_{3}$; cuadrados verdes corresponden a las líneas de base $\mathrm{B}_{4}, \mathrm{~B}_{5}$ y $\mathrm{B}_{6}$; triángulos rojos corresponden a las líneas de base $\mathrm{B}_{7}, \mathrm{~B}_{8}$ y $\mathrm{B}_{9}$; diamantes violetas corresponden a las líneas de base $\mathrm{B}_{10}, \mathrm{~B}_{11}$ y $\mathrm{B}_{12}$; hexágonos rosas corresponden a las líneas de base $\mathrm{B}_{13}, \mathrm{~B}_{14}$ y $\mathrm{B}_{15}$; pentágonos cian corresponden a las líneas de base $\mathrm{B}_{16}, \mathrm{~B}_{17}$ y $\mathrm{B}_{18}$; diamantes delgados naranjas corresponden a las líneas de base $\mathrm{B}_{19}, \mathrm{~B}_{20} \mathrm{y}$ $\mathrm{B}_{21}$. La visibilidad diferencial, fase diferencial y closure phase de cada observación está graficada en azul, y el modelo del mejor ajuste en rojo. A modo de ejemplo, en la Fig. 8.9 se muestra la visibilidad diferencial, la fase diferencial y la closure phase para HD 23408, que presenta un perfil de Br $\gamma$ en absorción, donde los observables se mantienen constantes y no se puede realizar un ajuste a partir de un modelo de disco.

En la Tabla 8.3 mostramos los parámetros del mejor ajuste para nuestro modelo bidimensional para cada uno de los objetos de la muestra que presentan evidencia de disco a partir de la presencia de $\mathrm{Br} \gamma$ en emisión. La columna 1 contiene el nombre cada estrella junto a su identificación HD; las columnas 2 y 3 listan los parámetros globales del disco (ángulos de inclinación y posición); las columnas 4 y 5 muestran los parámetros cinemáticos del disco $\left(V_{\text {rot }}\right.$ y $j$ ); las columnas 6 y 7 muestran los valores del eje mayor del disco en el continuo $\left(a_{\text {cont }}\right)$ y en la línea $\left(a_{\mathrm{Br} \gamma}\right)$, respectivamente; la columna 8 muestra en ancho equivalente de $\mathrm{Br} \gamma$ y la columna 9 el 
Tabla 8.3: Parámetros de los mejores ajustes obtenidos para nuestro modelo. En el caso HD 28497, el modelo tuvo que ser modificado para incluir un brazo espiral sobre el disco Gaussiano. El símbolo * indica aquellos casos donde el modelo parece ser independiente del valor de $a_{\text {cont }}$.

\begin{tabular}{|c|c|c|c|c|c|c|c|c|}
\hline \multirow[b]{2}{*}{ Parámetro } & \multicolumn{2}{|c|}{$\begin{array}{l}\text { Parámetros } \\
\text { geométricos }\end{array}$} & \multicolumn{2}{|c|}{$\begin{array}{l}\text { Parámetros } \\
\text { cinemáticos }\end{array}$} & \multirow{2}{*}{$\begin{array}{c}\begin{array}{c}\text { Parámetros en } \\
\text { el continuo }\end{array} \\
a_{\text {cont }} \\
\left(D_{\star}\right)\end{array}$} & \multicolumn{2}{|c|}{$\begin{array}{c}\text { Parámetros en } \\
\text { la línea }\end{array}$} & \multirow{2}{*}{$\begin{array}{l}\chi^{2} \\
- \\
-\end{array}$} \\
\hline & $\begin{array}{c}i \\
\left.{ }^{\circ}\right)\end{array}$ & $\begin{array}{l}P A \\
\left({ }^{\circ}\right)\end{array}$ & $\begin{array}{c}V_{\text {rot }} \\
\left(\mathrm{km} \mathrm{s}^{-1}\right)\end{array}$ & $\begin{array}{l}j \\
-\end{array}$ & & $\begin{array}{l}a_{\mathrm{Br} \gamma} \\
\left(D_{\star}\right)\end{array}$ & $\begin{array}{l}\text { EW } \\
(\AA)\end{array}$ & \\
\hline$\eta$ Tau (HD 23630) & $53 \pm 5$ & $110 \pm 5$ & $270 \pm 25$ & $-0.45 \pm 0.05$ & $1.3 \pm 1.0$ & $7.0 \pm 1.0$ & $5.80 \pm 0.8$ & 5.86 \\
\hline $28 \mathrm{Tau}$ (HD 23862) & $85 \pm 5$ & $333 \pm 10$ & $410 \pm 50$ & $-0.47 \pm 0.05$ & $4.0 *$ & $11.0 \pm 1.0$ & $8.86 \pm 1.0$ & 2.31 \\
\hline 228 Eri (HD 28497) & $44 \pm 10$ & $30 \pm 10$ & $600 \pm 50$ & $-0.35 \pm 0.05$ & $6.0 \pm 2.0$ & $11.0 \pm 2.0$ & $14.00 \pm 3.0$ & 0.96 \\
\hline 56 Eri (HD 30076) & $38 \pm 5$ & $73 \pm 4$ & $400 \pm 20$ & $-0.45 \pm 0.05$ & $6.0 \pm 1.0$ & $10.0 \pm 0.5$ & $20.67 \pm 3.0$ & 0.70 \\
\hline 105 Tau (HD 32991) & $40 \pm 10$ & $157 \pm 5$ & $500 \pm 50$ & $-0.50 \pm 0.05$ & $2.0^{*}$ & $13.0 \pm 2.0$ & $20.00 \pm 2.0$ & 0.62 \\
\hline$\psi_{01}$ Ori (HD 35439) & $55 \pm 5$ & $45 \pm 20$ & $397 \pm 50$ & $-0.50 \pm 0.05$ & $1.7^{*}$ & $4.5 \pm 0.3$ & $20.00 \pm 5.0$ & 1.01 \\
\hline $120 \mathrm{Tau}$ (HD 36576) & $60 \pm 5$ & $198 \pm 10$ & $380 \pm 50$ & $-0.40 \pm 0.02$ & $2.0 \pm 1.0$ & $13.0 \pm 1.0$ & $15.00 \pm 3.0$ & 1.60 \\
\hline$\zeta$ Tau (HD 37202) & $67 \pm 5$ & $127 \pm 5$ & $400 \pm 30$ & $-0.45 \pm 0.03$ & $4.7^{*}$ & $4.8 \pm 0.4$ & $7.43 \pm 2.0$ & 3.19 \\
\hline$\omega$ Ori (HD 37490) & $49 \pm 5$ & $305 \pm 5$ & $308 \pm 30$ & $-0.50 \pm 0.05$ & $1.0^{*}$ & $2.5 \pm 0.5$ & $11.62 \pm 3.0$ & 0.97 \\
\hline V696 Mon (HD 41335) & $68 \pm 5$ & $55 \pm 5$ & $400 \pm 30$ & $-0.60 \pm 0.03$ & $2.0 *$ & $5.0 \pm 0.4$ & $17.00 \pm 4.0$ & 1.98 \\
\hline$\beta_{01}$ Mon (HD 45725) & $72 \pm 5$ & $101 \pm 5$ & $440 \pm 40$ & $-0.50 \pm 0.03$ & $1.2^{*}$ & $12.0 \pm 1.0$ & $22.70 \pm 2.0$ & 2.71 \\
\hline OW Pup (HD 60606) & $70 \pm 10$ & $240 \pm 10$ & $390 \pm 20$ & $-0.50 \pm 0.05$ & $2.0^{*}$ & $7.5 \pm 0.5$ & $17.00 \pm 2.0$ & 1.23 \\
\hline V374 Car (HD 66194) & $55 \pm 5$ & $220 \pm 20$ & $350 \pm 50$ & $-0.40^{*}$ & $3.0^{*}$ & $8.0 \pm 1.0$ & $10.73 \pm 2.0$ & 0.78 \\
\hline MX Pup (HD 68980) & $22 \pm 3$ & $310 \pm 5$ & $410 \pm 40$ & $-0.50 \pm 0.05$ & $1.5^{*}$ & $3.0 \pm 0.5$ & $25.00 \pm 4.0$ & 1.16 \\
\hline$o$ Aqr (HD 209409) & $70 \pm 5$ & $290 \pm 5$ & $355 \pm 50$ & $-0.45 \pm 0.03$ & $3.0^{*}$ & $8.0 \pm 0.5$ & $18.12 \pm 2.0$ & 2.50 \\
\hline 31 Peg (HD 212076) & $22 \pm 5$ & $202 \pm 20$ & $400 \pm 60$ & $-0.50 \pm 0.05$ & $3.0^{*}$ & $4.0 \pm 0.5$ & $12.00 \pm 2.0$ & 1.16 \\
\hline$\pi$ Aqr (HD 212571) & $34 \pm 10$ & $87 \pm 15$ & $440 \pm 40$ & $-0.50 \pm 0.1$ & $1.5^{*}$ & $2.0 \pm 0.3$ & $6.00 \pm 2.0$ & 1.01 \\
\hline$\epsilon$ PsA (HD 214748) & $73 \pm 10$ & $67 \pm 10$ & $244 \pm 20$ & $-0.46 \pm 0.03$ & $2.0^{*}$ & $3.9 \pm 0.4$ & $5.00 \pm 0.5$ & 1.85 \\
\hline
\end{tabular}

valor de $\chi^{2}$ del ajuste.

Debido a que nuestros datos no están calibrados y tenemos la visibilidad diferencial normalizada a la visibilidad del continuo, en algunos casos el ajuste de $a_{\text {cont }}$ es complicado y tiene mucha incerteza. En la Tabla 8.3 se muestran los valores del mejor ajuste según el mínimo de $\chi^{2}$, donde * indica aquellos casos donde el modelo parece ser independiente del valor de $a_{\text {cont }}$.

\subsection{Comentarios y resultados individuales de cada objeto}

A continuación presentamos información de la literatura para los objetos de la muestra, junto con los parámetros del modelo del mejor ajuste:

- $\eta$ Tau (HD 23630) es una estrella Be de tipo espectral B7III, en el cúmulo de las Pléyades, con un perfil de $\mathrm{H} \alpha$ sin cambios significativos en períodos de tiempo largos (Tycner et al., 2005). A partir de observaciones interferométricas y de un ajuste de un disco Gaussiano para la emisión en $\mathrm{H} \alpha$, Tycner et al. (2005) determinaron un valor de $2.08 \pm 0.18$ mas para el diámetro angular del eje mayor (FWHM). A partir de modelos de la emisión en $\mathrm{H} \alpha$, Grundstrom \& Gies (2006) obtuvieron una inclinación $i=44^{\circ}$, un radio para el disco de $\mathrm{H} \alpha$ en emisión $R_{\mathrm{d}} / R_{\star}=5.4 \pm 0.7$ a partir del ancho equivalente de la línea, y un radio interferométrico de $R_{\mathrm{d}} / R_{\star}=2.9 \pm 0.3$. 
Silaj et al. (2010) ajustaron la línea de $\mathrm{H} \alpha$ con un doble pico en emisión usando una estrella central B8 y una distribución de densidad como ley de potencia. Los parámetros del disco que obtienen son los siguientes: inclinación $i=20^{\circ}$, densidad en la base del disco $\rho_{0}=10^{-11} \mathrm{~g} \mathrm{~cm}^{-3}$, y un índice para la ley de potencia de la densidad $n=3.5$.

Touhami et al. (2013) no logró resolver el disco utilizando observaciones interferométricas de CHARA en la Banda K .

Para este objeto se realizaron tres observaciones interferométricas con una buena relación S/N (ver Fig. 8.8). Con el modelo del mejor ajuste se obtuvieron los siguientes parámetros geométricos y cinemáticos para el disco: $\mathrm{PA}=110^{\circ}, i=53^{\circ}, V_{\text {rot }}=270 \mathrm{~km} \mathrm{~s}^{-1}, j=-0.45$, $a_{\text {cont }}=1.3 D_{\star}$ y $a_{\mathrm{Br} \gamma}=7 D_{\star}$. Es importante destacar que a pesar de que las bases no están orientadas según el PA obtenido, la fase diferencial pierde la forma "S" en las bases que resuelven completamente el disco. La inclinación obtenida a partir de los datos interferométricos concuerda con la esperada a partir del perfil de doble pico de la línea $\mathrm{Br} \gamma$ mostrada en la Fig. 8.5, que sugiere una inclinación intermedia.

- 28 Tau (HD 23862) es una estrella Be de tipo espectral B8V, miembro del cúmulo Pléyades. Dentro de los antecedentes presentados para esta estrella en el Capítulo 6, destacamos que el disco de la estrella fue parcialmente resuelto con el arreglo CHARA. Touhami et al. (2013) obtuvieron una relación entre los radios del disco y de la estrella de 1.879, tomando un ángulo de posición $\mathrm{PA}=159^{\circ}$ y un cociente entre los ejes $r=0.438$. Además, Silaj et al. (2014) realizaron un ajuste de la línea $\mathrm{H} \alpha$ y obtuvieron como parámetros del mejor ajuste $i=76^{\circ}, \rho_{0}=6.2 \cdot 10^{-12} \mathrm{~g} \mathrm{~cm}^{-3}$ y $n=2.5$.

Se obtuvieron dos observaciones interferométricas con buena relación S/N (ver Fig. 8.10). El modelo del mejor ajuste proporciona los siguientes parámetros geométricos y cinemáticos para el disco: $\mathrm{PA}=333^{\circ}, i=85^{\circ}, V_{\text {rot }}=410 \mathrm{~km} \mathrm{~s}^{-1}, j=-0.47, a_{\text {cont }}=4 D_{\star} \mathrm{y} a_{\mathrm{Br} \gamma}=11 D_{\star} . \mathrm{El}$ perfil de la línea $\mathrm{Br} \gamma$ exhibe una emisión de doble pico con una absorción tipo shell (Fig. 8.5), típico de estrellas Be vistas con un gran ángulo de inclinación. El ángulo de inclinación obtenido es consistente con la forma de la línea.

- 228 Eri (HD 28497) es una estrella Be de tipo espectral B2V, que muestra emisión variable y episodios tipo shell. La misma fue estudiada en el Capítulo 5. Dentro de los trabajos previos acerca de este objeto, remarcamos que Silaj et al. (2010) ajustaron la línea de $\mathrm{H} \alpha$ con un doble pico en emisión, obteniendo como parámetros del mejor ajuste un tipo espectral B0 para la estrella central, $i=45^{\circ}, \rho_{0}=10^{-10} \mathrm{~g} \mathrm{~cm}^{-3}$ y $n=4$, mientras que Vieira et al. (2017) obtuvieron $\log \rho_{0}=-10.7 \pm 0.3$, y $n=3.4 \pm 0.1$ a partir del continuo IR.

Obtuvimos una observación con buena relación S/N (ver Fig. 8.11). Las formas "W" y "S" de la visibilidad diferencial y la fase diferencial son muy asimétricas, siendo la amplitud menor en la parte roja que en la azul. El perfil de $\mathrm{Br} \gamma$ también es claramente asimétrico, con $V / R \sim 1.29$ (ver Fig. 8.5). Esto no puede ser ajustado con un modelo simple, por 
Figura 8.10: Ídem 8.8, para 28 Tau (HD 23862).
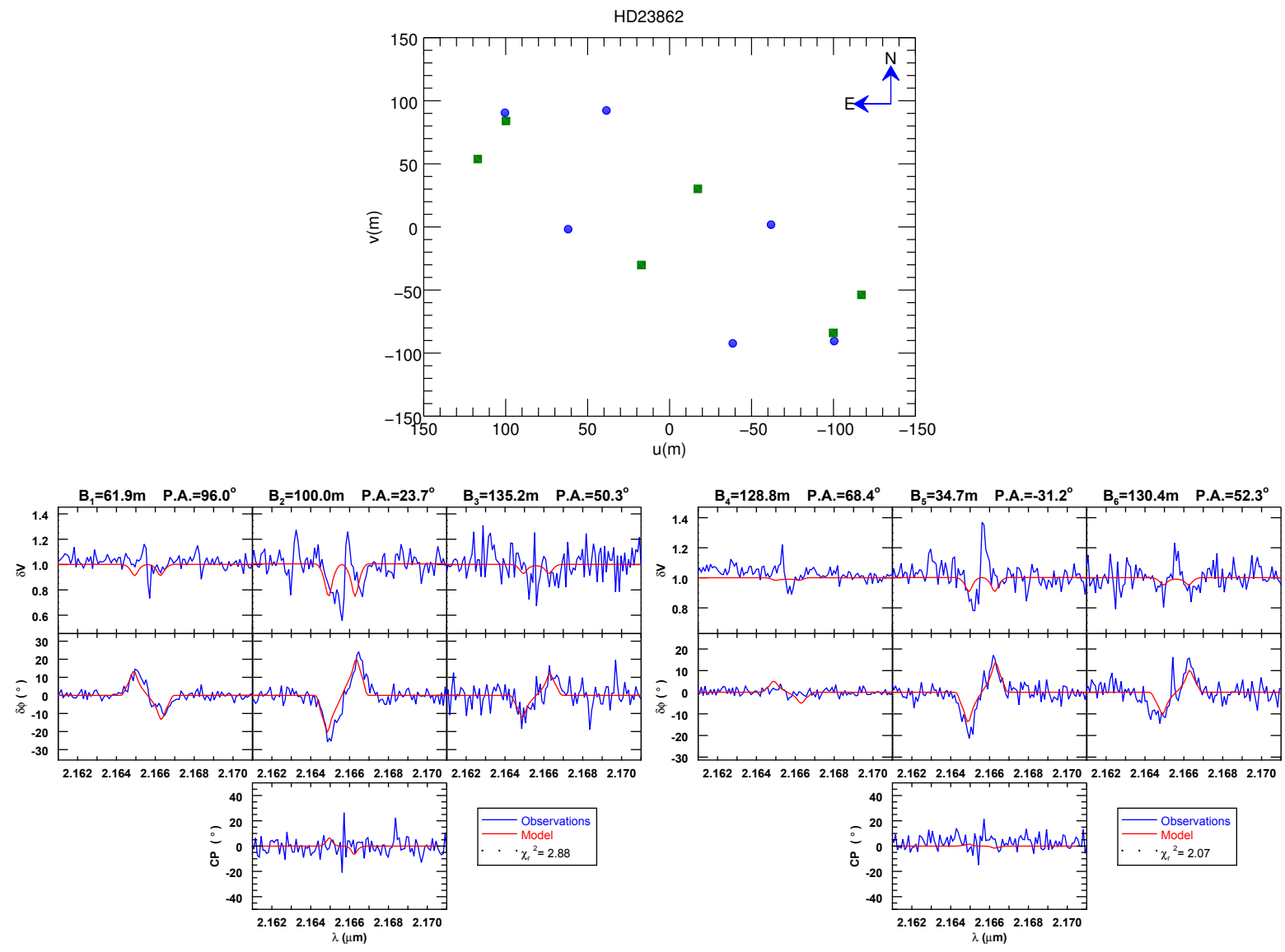

Figura 8.11: Ídem 8.8, para 228 Eri (HD 28497).
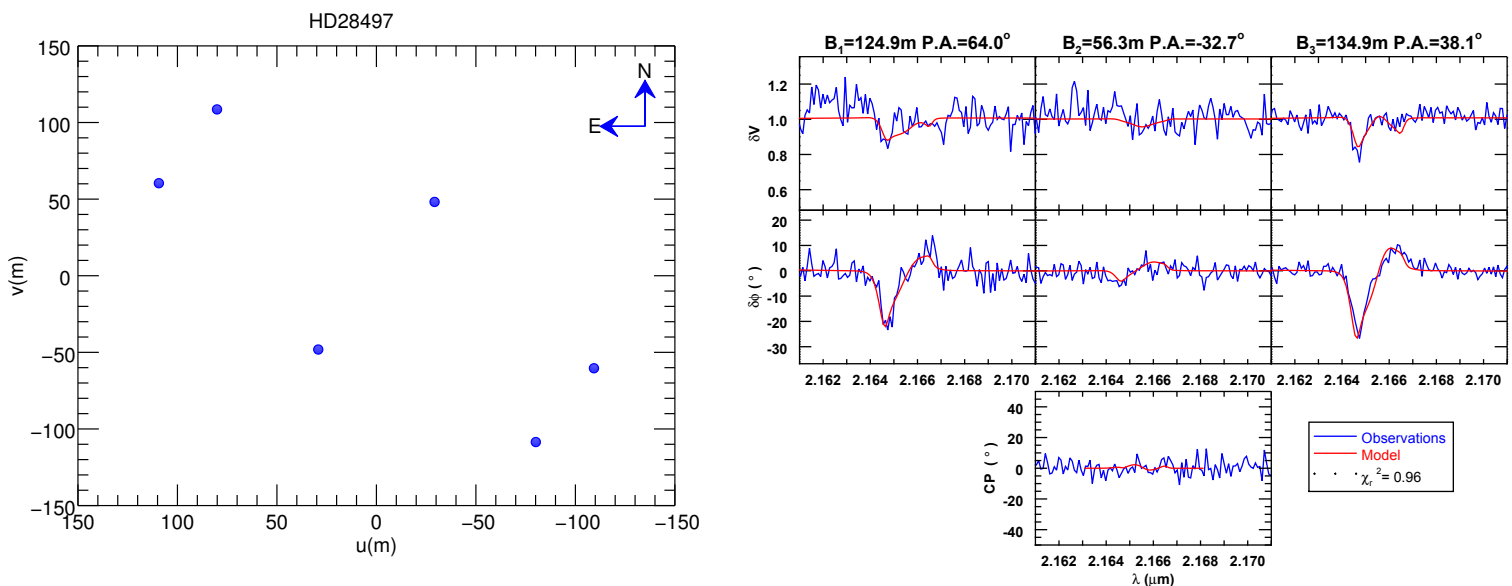
Figura 8.12: Ídem 8.8, para 56 Eri (HD 30076).
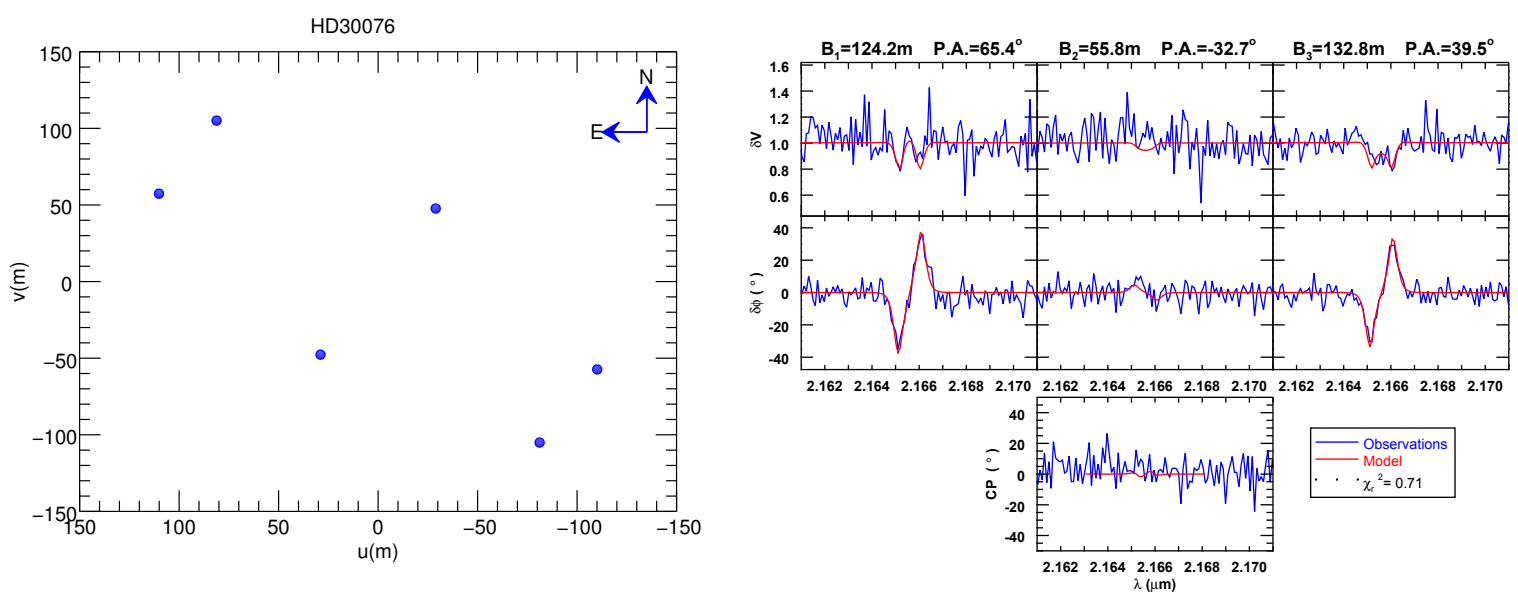

lo que tuvimos que mejorar el ajuste utilizando una sobredensidad del tipo "one armed oscillation". Con el modelo de mejor ajuste obtuvimos los siguientes parámetros geométricos y cinemáticos para el disco: $\mathrm{PA}=30^{\circ}, i=44^{\circ}, V_{\text {rot }}=600 \mathrm{~km} \mathrm{~s}^{-1}, j=-0.35, a_{\text {cont }}=6.0 D_{\star}$ y $a_{\mathrm{Br} \gamma}=11 D_{\star}$. El brazo se orienta con un ángulo de $\phi=50^{\circ}$ desde el eje mayor de la envoltura.

- 56 Eri (HD 30076) es una estrella Be de tipo espectral B2V. Andrillat et al. (1990) encontraron una región emisora en $\mathrm{H} \alpha$ entre $1.37 \mathrm{R}_{\star}$ y $6 \mathrm{R}_{\star}$. Silaj et al. (2010) ajustaron la línea de $\mathrm{H} \alpha$ con un doble pico en emisión, obteniendo como parámetros del mejor ajuste un tipo espectral B2 para la estrella central, una inclinación $i=20^{\circ}, \rho_{0}=5 \cdot 10^{-10} \mathrm{~g} \mathrm{~cm}^{-3}, \mathrm{y} n=3.5$.

A partir de un análisis estadístico, Jones et al. (2011) estudiaron la variabilidad del ancho equivalente de $\mathrm{H} \alpha$, estableciendo que la misma es variable con un $95 \%$ de confianza.

Para este objeto se realizó una observación (ver Fig. 8.12). Los parámetros del disco obtenidos a partir del mejor modelo son: $\mathrm{PA}=73^{\circ}, i=38^{\circ}, V_{\text {rot }}=400 \mathrm{~km} \mathrm{~s}^{-1}, j=-0.45$, $a_{\text {cont }}=6.0 D_{\star}$ y $a_{\mathrm{Br} \gamma}=10 D_{\star}$. La base $\mathrm{B}_{1}$ con ángulo de posición $\mathrm{PA}_{1}=65.4^{\circ}$ está orientada cerca del valor encontrado para el ángulo de posición del disco PA, por lo que las formas "W" y "S" de la visibilidad diferencial y fase diferencial se observan claramente. El doble pico en emisión del perfil de $\mathrm{Br} \gamma$ (Fig. 8.5) es consistente con el ángulo de inclinación obtenido a partir del modelo interferométrico.

- 105 Tau (HD 32991) es una estrella Be-shell de tipo espectral B2V. En el Capítulo 5 presentamos información relevante sobre este objeto. Destacamos que Silaj et al. (2010) realizaron un ajuste de la línea de $\mathrm{H} \alpha$ con un pico en emisión, obteniendo como parámetros del mejor ajuste un tipo espectral B2 para la estrella central, $i=20^{\circ}, \rho_{0}=5 \cdot 10^{-10} \mathrm{~g} \mathrm{~cm}^{-3}$, y $n=3.5$. Este objeto no tiene determinaciones previas del tamaño del disco. 
Figura 8.13: Ídem 8.8, para 105 Tau (HD 32991).
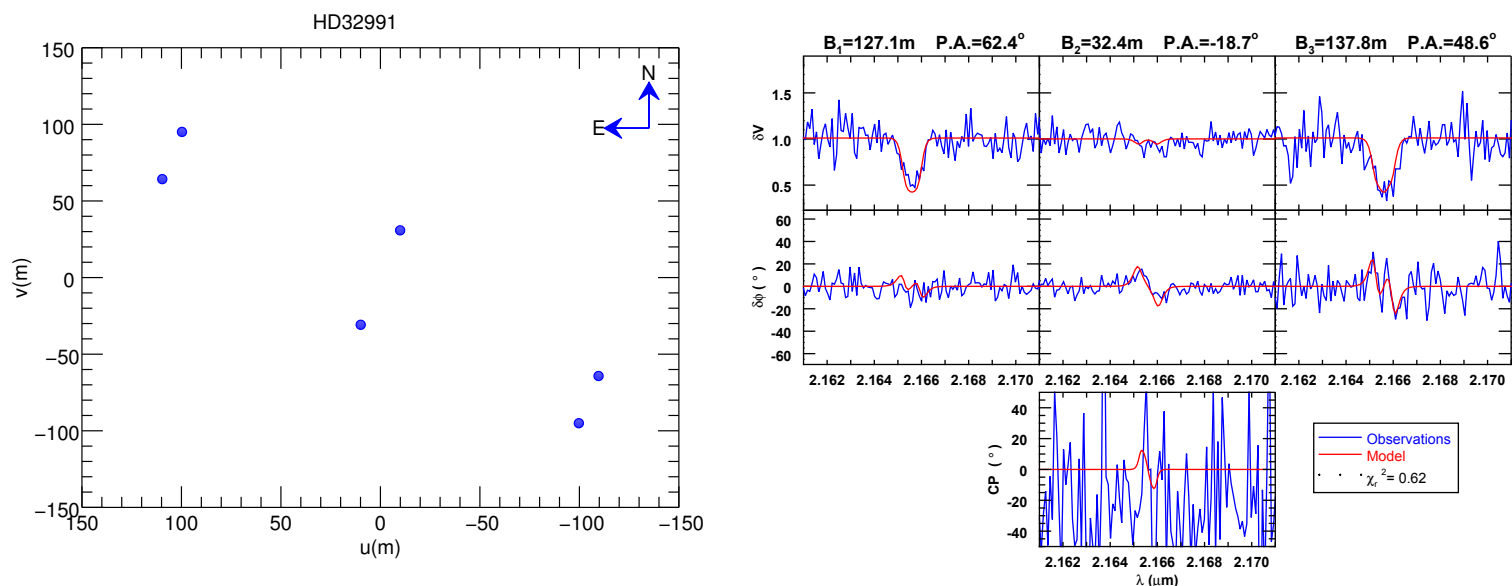

Figura 8.14: Ídem 8.8, para $\psi_{01}$ Ori (HD 35439).
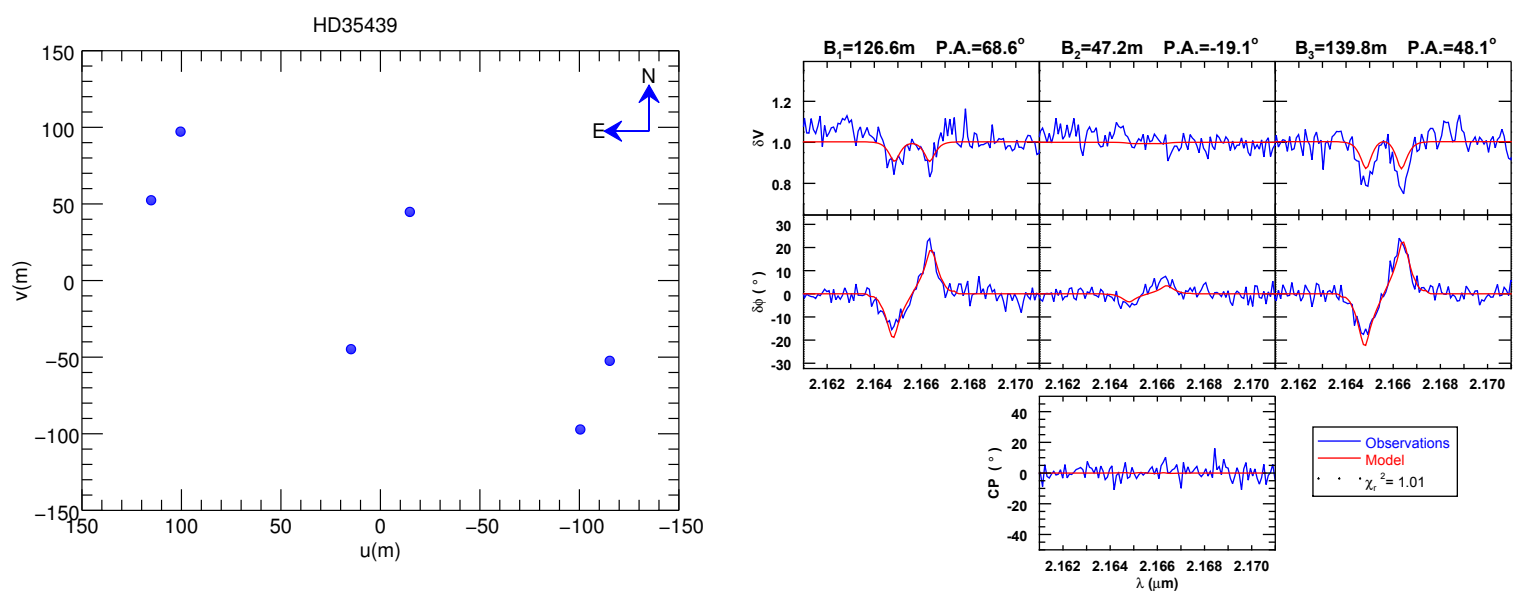

Obtuvimos una observación para este objeto (ver Fig. 8.13). Los parámetros del mejor ajuste dan los siguientes parámetros geométricos y cinemáticos para el disco: $\mathrm{PA}=157^{\circ}, i=40^{\circ}$, $V_{\text {rot }}=500 \mathrm{~km} \mathrm{~s}^{-1}, j=-0.5, a_{\text {cont }}=2.0 D_{\star} \mathrm{y} a_{\mathrm{Br} \gamma}=13 D_{\star}$. Como la base $\mathrm{B}_{2}\left(\mathrm{PA}_{2}=-18.7^{\circ}\right.$, orientada según el eje mayor del disco) es la más corta, las formas "W" y "S" observadas en la visibilidad diferencial y fase diferencial son débiles. El perfil de $\mathrm{Br} \gamma$ de un pico (Fig. 8.5) es consistente con el pequeño ángulo de inclinación obtenido.

- $\psi_{01}$ Ori (HD 35439) es una estrella Be de tipo espectral B1V, que presenta variaciones de fase $\mathrm{Be} \rightarrow \mathrm{B} \rightarrow \mathrm{Be}$. La misma fue estudiada en el Capítulo 5. Dentro de los antecedentes reportados, destacamos que Silaj et al. (2010) ajustaron la línea de $\mathrm{H} \alpha$ con un doble pico en emisión, obteniendo como parámetros del mejor ajuste un tipo espectral B0 para la estrella 
Figura 8.15: Ídem 8.8, para 120 Tau (HD 36576).
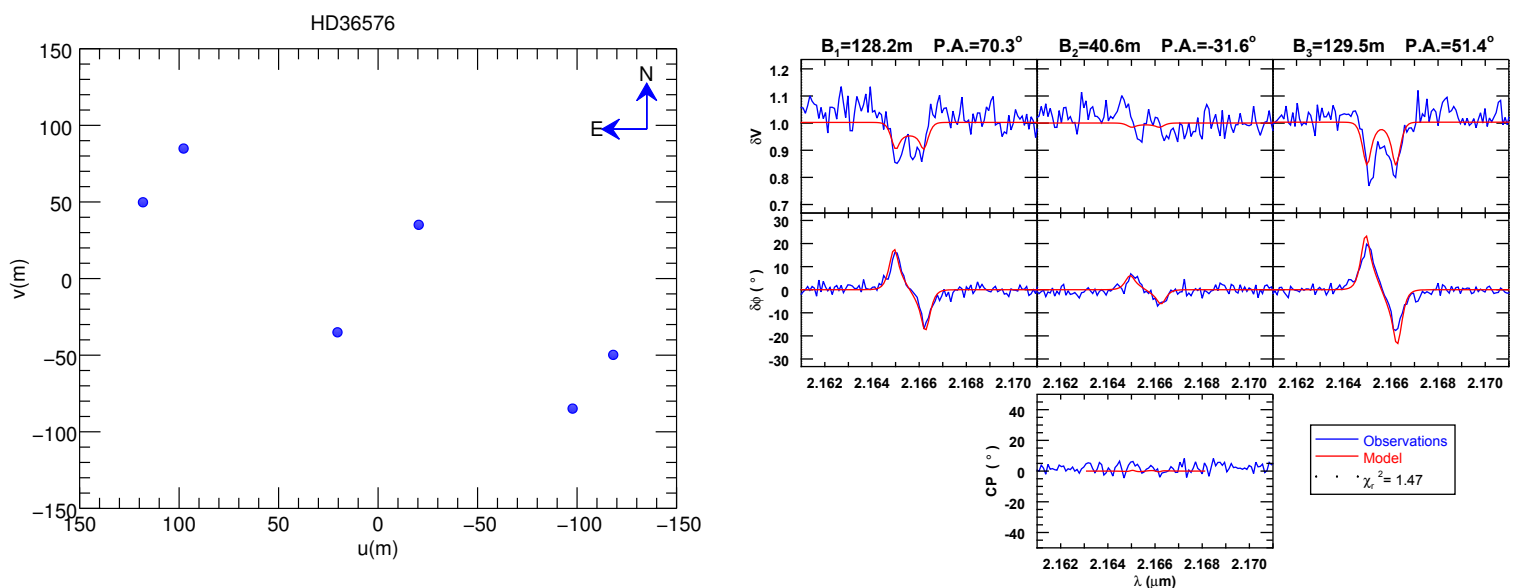

central, $i=45^{\circ}, \rho_{0}=5 \cdot 10^{-11} \mathrm{~g} \mathrm{~cm}^{-3}$ y $n=3.5$. El valor de $\rho_{0}$ es mayor que el encontrado por Vieira et al. (2017) a partir del ajuste de la SED con dados de WISE ( $\log \rho_{0}=-11 \mathrm{y}$ $n=3.4$ ). Por otro lado, Arcos et al. (2017) encontraron dos conjuntos de parámetros: $i=50^{\circ}$, $\rho_{0}=2.5 \cdot 10^{-11} \mathrm{~g} \mathrm{~cm}^{-3}$ y $n=2.5$ modelando observaciones de $\mathrm{H} \alpha$ tomadas en 2012 e $i=50^{\circ}$, $\rho_{0}=5 \cdot 10^{-12} \mathrm{~g} \mathrm{~cm}^{-3}$ y $n=2$ a partir de observaciones tomadas en 2015 .

Se realizó una observación interferométrica (ver Fig. 8.14). Los parámetros del mejor ajuste geométrico y cinemático son los siguientes: $\mathrm{PA}=45^{\circ}, i=55^{\circ}, V_{\text {rot }}=397 \mathrm{~km} \mathrm{~s}^{-1}, j=-0.5$, $a_{\text {cont }}=1.75 D_{\star} \mathrm{y} a_{\mathrm{Br} \gamma}=4.5 D_{\star}$. La base $\mathrm{B}_{3}$ está orientada cerca del PA del eje mayor del disco, por lo que la visibilidad diferencial y la fase diferencial presentan formas "W" $\mathrm{y}$ "S". El perfil de $\mathrm{Br} \gamma$ con doble pico presentado en la Fig. 8.5 es consistente con el ángulo de inclinación obtenido a partir de la interferometría.

- 120 Tau (HD 36576) es una estrella Be de tipo espectral B2IV-V, que fue estudiada en el Capítulo 5. Entre los antecedentes reportados previamente, destacamos que Silaj et al. (2010) modelaron la línea de $\mathrm{H} \alpha$ con un pico en emisión usando una estrella central B2 con un disco con $i=45^{\circ}, \rho_{0}=5 \cdot 10^{-10} \mathrm{~g} \mathrm{~cm}^{-3}$, y $n=3.5$. Por otro lado, a partir de la SED, Vieira et al. (2017) obtuvieron $\log \rho_{0}=-11.4$ y $n=2.36$.

Para este objeto se obtuvo una observación interferométrica (ver Fig. 8.15). El mejor ajuste brinda los siguientes parámetros geométricos y cinemáticos para el disco: $\mathrm{PA}=198^{\circ}, i=60^{\circ}$, $V_{\text {rot }}=380 \mathrm{~km} \mathrm{~s}^{-1}, j=-0.4, a_{\text {cont }}=2.0 D_{\star}$ y $a_{\mathrm{Br} \gamma}=13.0 D_{\star}$. A pesar de que las líneas de base no están orientadas según el PA obtenido para el disco, la forma "W" de la visibilidad diferencial y la forma "S" de la fase diferencial se observan claramente en la base más larga. El perfil de doble pico en $\mathrm{Br} \gamma$ es consistente con el ángulo de inclinación intermedio obtenido del ajuste. 
Figura 8.16: Ídem 8.8, para $\zeta$ Tau (HD 37202).
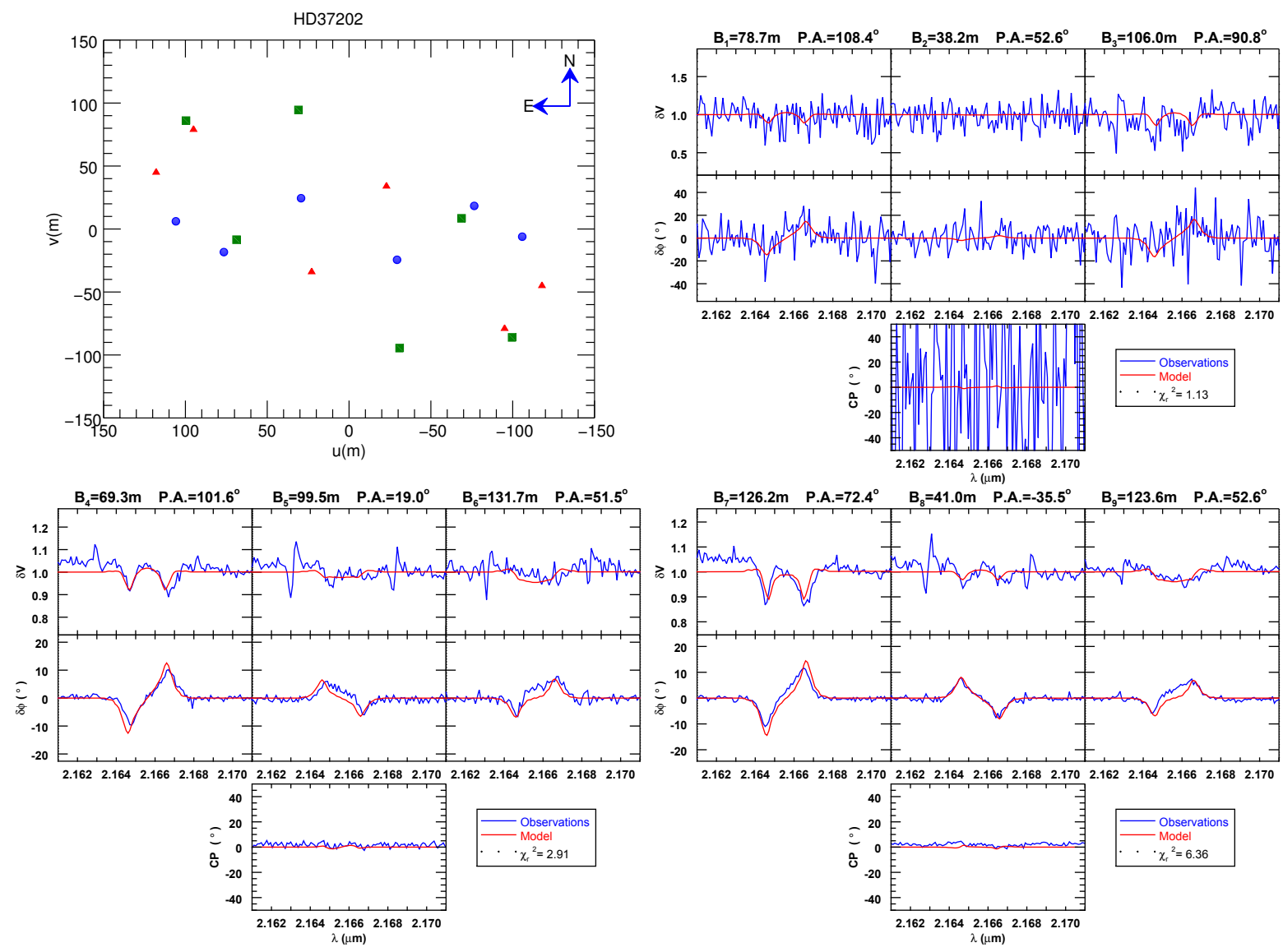

- C Tau (HD 37202) es una de las estrellas Be más observadas, con un tipo espectral B1IV, catalogada como Be-shell. Es conocida como binaria espectroscópica desde hace décadas, y Harmanec (1984) calculó un período de 132.9735 días y una excentricidad de $\sim 0.15$. Ha mostrado complejas variaciones en su perfil de $\mathrm{H} \alpha$ en escalas de meses a años, y variaciones más significativas de largo período (Ballereau et al., 1987; Tycner et al., 2005). A la distancia en la que se encuentra, su disco puede ser resuelto con los actuales instrumentos y la espectrointerferometría permite estudiar la dinámica del disco. El diámetro del disco fotosférico con oscurecimiento al limbo es estimado en 0.4 mas (Tycner et al., 2004; Gies et al., 2007), correspondiente a un radio de entre $5.5 R \odot$ y $6 R_{\odot}$ a la distancia determinada con la paralaje de Hipparcos.

A partir de observaciones interferométricas y de un ajuste de un disco Gaussiano para la emisión en $\mathrm{H} \alpha$, se determinó un valor de 3.14 \pm 0.21 mas para el diámetro angular del eje mayor (FWHM) (Tycner et al., 2005). Basados en estos datos, Grundstrom \& Gies (2006) encontraron una inclinación $i=75^{\circ}$, un radio interferométrico $R_{\mathrm{d}} / R_{\star}=7.3 \pm 0.5$, 
mientras que a partir del ancho equivalente de $\mathrm{H} \alpha$ calcularon una extensión del disco de $R_{\mathrm{d}} / R_{\star}=8.7 \pm 1.5$.

Touhami et al. (2011), utilizando observaciones de la Banda K con el arreglo CHARA, presentaron un análisis de la emisión en el continuo proveniente del gas circunestelar usando un código de transferencia radiativa para un viscous decretion disk model. Los parámetros para el disco son: $\rho_{0}=1.4 \cdot 10^{-10} \mathrm{~g} \mathrm{~cm}^{-3}, n=2.9, i=87^{\circ}$ y $T_{d}=2 / 3 T_{\text {eff. }}$.

Gies et al. (2007), usando CHARA en la Banda K, obtuvieron una medida del semieje mayor del disco de 1.79 mas o $4.5 R_{\star}$. Este valor es menor que el estimado para el lóbulo de Roche para la estrella primaria del sistema, que Tycner et al. (2004) estimó en $144 \pm 12 R_{\odot}$ (5.3 mas), con una separación entre las binarias de $254 \pm 20 R_{\odot}$ (9.2 mas). Esta separación de las binarias es lo suficientemente grande como para que la estrella secundaria quede fuera del disco circunestelar, en acuerdo con muchos modelos (Castle, 1977).

Štefl et al. (2009) estudió tres ciclos V/R entre 1997 y 2008. Encontraron que luego de cada mínimo de V/R, la absorción shell se debilita y se separa en dos componentes, resultando en tres picos en emisión. Además, la fase de la línea $\mathrm{Br} \gamma$ en emisión muestra que el fotocentro de la región que emite la línea se encuentra dentro del plano del disco pero desplazado respecto a la fuente del continuo. El plano del disco permanece igual a lo largo de los ciclos V/R observados.

Schaefer et al. (2010) resolvieron el disco entre 2007-2009, con el MIRC beam combiner en CHARA. Ellos ajustaron un disco visto casi de ecuador, con un FWHM del eje mayor de $\sim 1.8$ mas en la Banda H. Además, encontraron una correlación entre el ángulo de posición del disco y el cociente espectroscópico V/R, lo que sugiere que el disco está precesando.

Se obtuvieron tres observaciones interferométricas para este objeto, dos con una buen cociente $\mathrm{S} / \mathrm{N}$ y una con menor $\mathrm{S} / \mathrm{N}$ (ver Fig. 8.16). Los parámetros geométricos y cinemáticos del mejor ajuste son: $\mathrm{PA}=127^{\circ}, i=67^{\circ}, V_{\text {rot }}=400 \mathrm{~km} \mathrm{~s}^{-1}, j=-0.45, a_{\text {cont }}=4.7 D_{\star}$ y $a_{\mathrm{Br} \gamma}=$ $4.8 D_{\star}$. El perfil de $\mathrm{Br} \gamma$ es típico de una estrella Be vista con un ángulo de inclinación grande (doble pico con una absorción central tipo shell), en acuerdo con su clasificación de Be shell. En las bases $\mathrm{B}_{4}$ y $\mathrm{B}_{7}$ las formas "W" y "S" de la visibilidad diferencial y fase diferencial se observan claramente.

- $\omega$ Ori (HD 37490) es una estrella Be de tipo espectral B3V, que ha sido estudiada por muchos años. En el Capítulo 5 presentamos antecedentes de la misma, y realizamos un análisis de la envoltura de este objeto a partir de espectros en el IR.

Silaj et al. (2010) ajustaron la línea de $\mathrm{H} \alpha$ con un doble pico en emisión, obteniendo como parámetros del mejor ajuste un tipo espectral B2 para la estrella central, $i=20^{\circ}$, $\rho_{0}=10^{-11} \mathrm{~g} \mathrm{~cm}^{-3}$ y $n=3.5$. van Belle (2012) sugirió que este objeto es un posible target para observaciones interferométricas, con un tamaño angular estimado de 0.5 mas y achatamiento $R_{\mathrm{b}} / R_{\mathrm{a}}-1=0.13$. 
Figura 8.17: Ídem 8.8, para $\omega$ Ori (HD 37490).
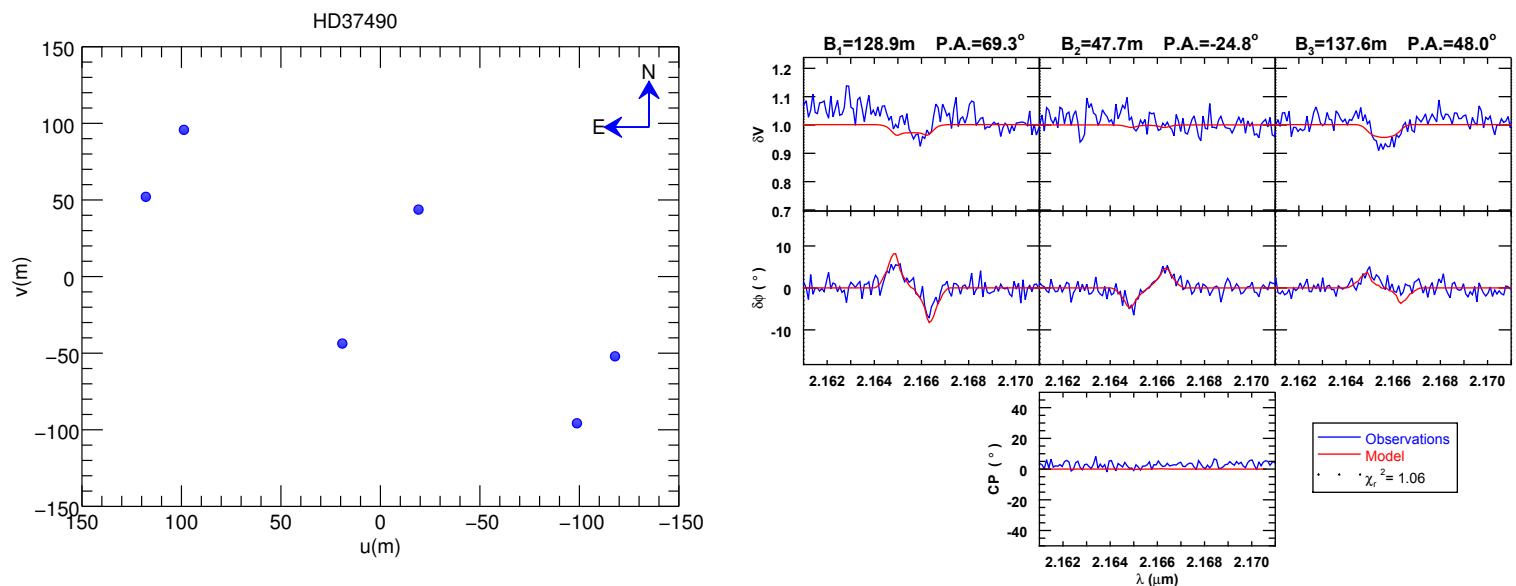

Figura 8.18: Ídem 8.8, para V696 Mon (HD 41335).
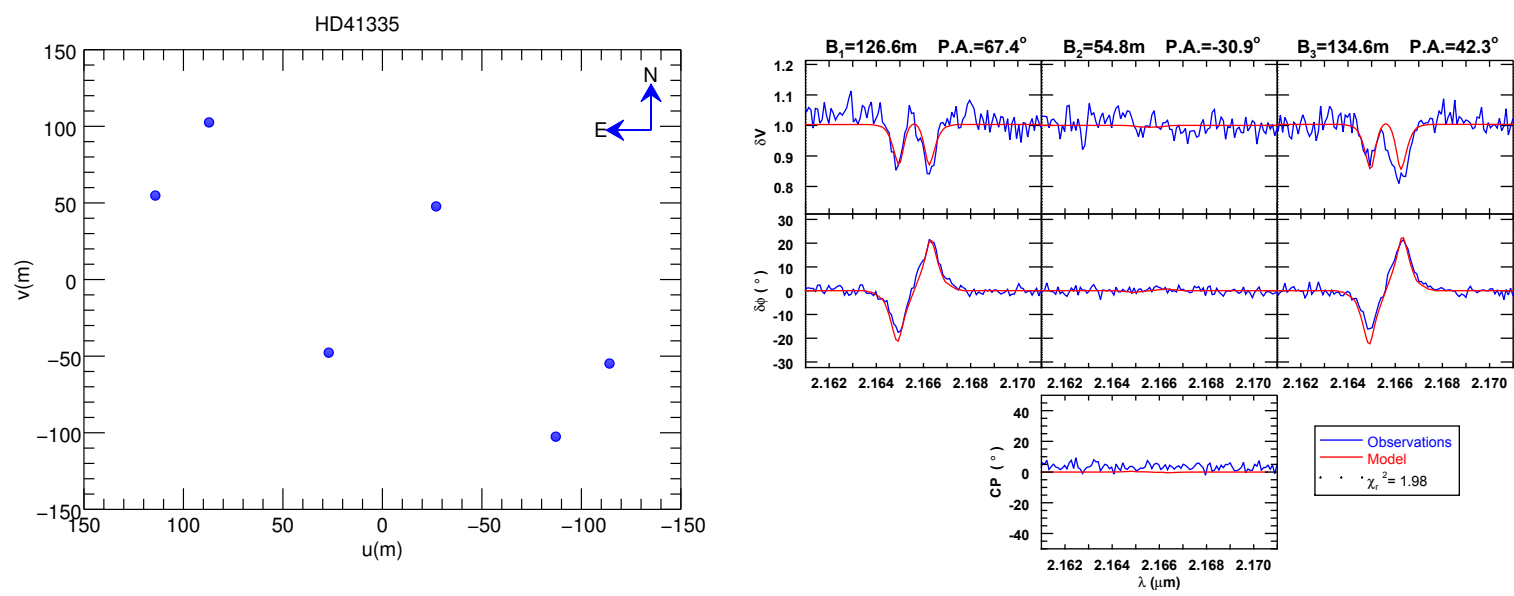

Para esta estrella se obtuvo una observación interferométrica (ver Fig. 8.17) y se ajustaron los siguientes parámetros para el disco: $\mathrm{PA}=305^{\circ}, i=49^{\circ}, V_{\text {rot }}=308 \mathrm{~km} \mathrm{~s}^{-1}, j=-0.5$, $a_{\text {cont }}=1.0 D_{\star}$ y $a_{\mathrm{Br} \gamma}=2.5 D_{\star}$. Los dos picos observados en el perfil de $\mathrm{Br} \gamma$ indican que el objeto es visto con un ángulo de inclinación intermedio, consistente con el valor obtenido con nuestro ajuste y el valor de $i=42 \pm 7^{\circ}$ reportado por Neiner et al. (2003).

- V696 Mon (HD 41335) es un sistema binario interactuante (Peters, 1972). Algunos trabajos relevantes sobre este objeto fueron presentados en el Capítulo 5. Dentro de éstos, destacamos que a partir de la SED, Vieira et al. (2017) obtuvieron $\log \rho_{0}=-10.3 \pm 0.3 \mathrm{y}$ $n=3.0 \pm 0.1$, mientras que Arcos et al. (2017) obtuvieron, a partir de modelos de $\mathrm{H} \alpha$ un $\rho_{0}=5 \cdot 10^{-12}, n=2.0$ e $i=80^{\circ}$. 
Figura 8.19: Ídem 8.8, para $\beta_{01}$ Mon (HD 45725).
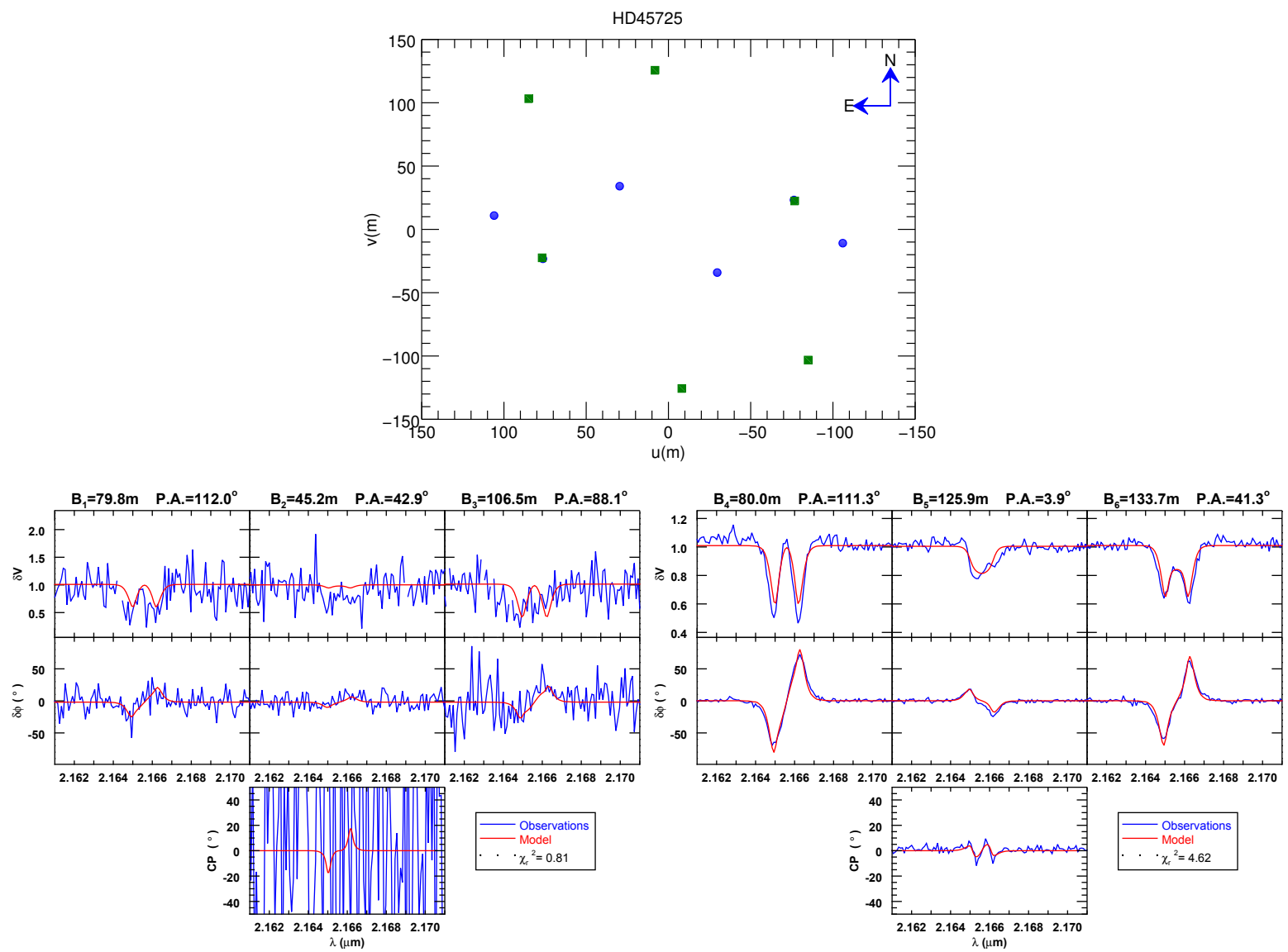

Se obtuvo una observación para este objeto (Fig. 8.18). El modelo del mejor ajuste brinda los siguientes parámetros geométricos y cinemáticos para el disco: $\mathrm{PA}=55^{\circ}, i=68.5^{\circ}$, $V_{\text {rot }}=400 \mathrm{~km} \mathrm{~s}^{-1}, j=-0.6, a_{\text {cont }}=2.0 D_{\star}$ y $a_{\mathrm{Br} \gamma}=5.0 D_{\star}$. El perfil de doble pico, la caída en la visibilidad diferencial y las variaciones de fase presentan pequeñas asimetrías. Las líneas de base más largas $\left(\mathrm{B}_{1}\right.$ y $\left.\mathrm{B}_{3}\right)$ están orientadas con ángulos de posición cercanos al valor obtenido para PA, por lo que las formas "W" y "S" de la visibilidad y fase diferencial son claras. El perfil de doble pico es consistente con la inclinación intermedia encontrada.

A pesar de que Peters et al. (2016) propusieron la presencia de una sobredensidad en forma de brazo espiral, no necesitamos incluir una sobredensidad para ajustar nuestros datos.

- $\beta_{01}$ Mon (HD 45725) es una estrella Be-shell de tipo espectral B4V, catalogada como estrella triple por Struve (1925).

Abt \& Cardona (1984) midieron la separación entre las componentes, obteniendo separaciones de 7.1", 2.8" y 25.9" y, considerando que las separaciones son los semiejes mayores de las órbitas, obtuvieron períodos de 10000, 2600 y 100000 años. Oudmaijer \& Parr (2010), 
Figura 8.20: Ídem 8.8, para OW Pup (HD 60606).
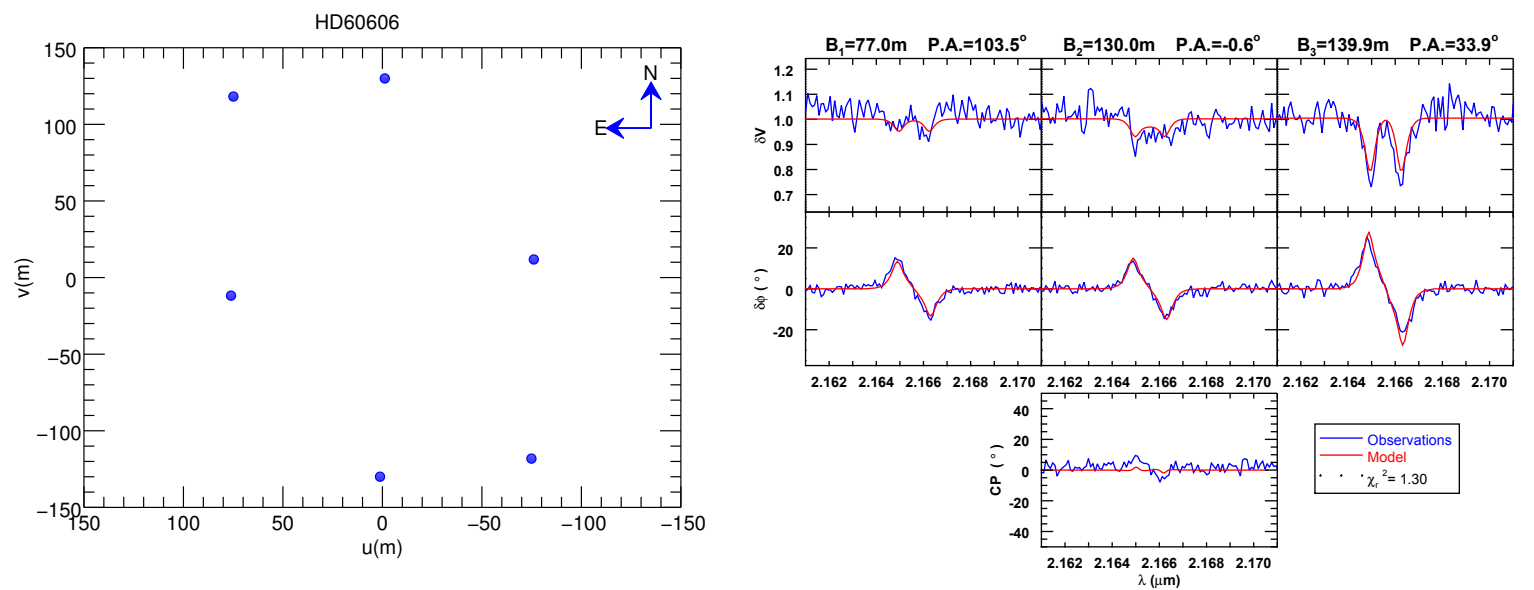

ajustando Gaussianas bidimensionales a los perfiles espaciales, encontraron una separación entre las componentes de 7.14" y un ángulo de posición de $133^{\circ}$.

Andrillat et al. (1990) encontraron que la región emisora de $\mathrm{H} \alpha$ se localiza entre $1.86 \mathrm{R}_{\star} \mathrm{y}$ $3.64 \mathrm{R}_{\star}$.

van Belle (2012) indicó que HD 45725 es una buena candidata para realizar observaciones interferométricas, dado que es un alto rotador $\left(V \sin i=325 \mathrm{~km} \mathrm{~s}^{-1}\right)$ y presenta un disco con tamaño angular estimado de 0.70 mas y un achatamiento $R_{\mathrm{b}} / R_{\mathrm{a}}-1=0.17$.

A partir de ajustes de la línea $\mathrm{H} \alpha$, Arcos et al. (2017) obtuvieron $\rho_{0}=5 \cdot 10^{-12}$, $\mathrm{n}=2$ e $i=70^{\circ}$. Se realizaron dos observaciones interferométricas, una con una buena relación $\mathrm{S} / \mathrm{N}$ y una con una $\mathrm{S} / \mathrm{N}$ baja (ver Fig. 8.19). El modelo del mejor ajuste provee los siguientes parámetros geométricos y cinemáticos para el disco: $\mathrm{PA}=101^{\circ}, i=72^{\circ}, V_{\text {rot }}=440 \mathrm{~km} \mathrm{~s}^{-1}$, $j=-0.5, a_{\text {cont }}=1.20 D_{\star} \mathrm{y} a_{\mathrm{Br} \gamma}=12.0 D_{\star}$. A pesar del alto ángulo de inclinación encontrado, el perfil de $\mathrm{Br} \gamma$ no presenta una absorción central tipo shell. Las bases $\mathrm{B}_{1}$ y $_{4}$ son muy similares y están orientadas cercanas al PA obtenido. Las bases $\mathrm{B}_{2}$ y $\mathrm{B}_{6}$ tiene casi la misma orientación, pero diferente tamaño, por lo que las formas de la visibilidad diferencial y fase diferencial son más marcadas en la base $\mathrm{B}_{6}$.

- OW Pup (HD 60606) es una estrella Be de tipo espectral B2V. Fue catalogada como variable de tipo $\gamma$ Cas por Lefèvre et al. (2009). La SED fue modelada por Vieira et al. (2017), quienes derivaron valores de $\log \rho_{0}=-11.7 \pm 0.1$ y $n=2.5 \pm 0.1$, mientras que a partir de la línea $\mathrm{H} \alpha$, Arcos et al. (2017) obtuvieron los siguientes parámetros del disco: $\rho_{0}=1 \cdot 10^{-10}, \mathrm{n}=3 \mathrm{e}$ $i=70^{\circ}$. Este objeto no tiene determinaciones previas del tamaño del disco.

Basado en una observación interferométrica (Fig. 8.20), el modelo del mejor ajuste provee los siguientes parámetros geométricos y cinemáticos para el disco: $\mathrm{PA}=240^{\circ}, i=70^{\circ}$, 
$V_{\text {rot }}=390 \mathrm{~km} \mathrm{~s}^{-1}, j=-0.5, a_{\text {cont }}=2.0 D_{\star} \mathrm{y} a_{\mathrm{Br} \gamma}=7.5 D_{\star}$. La línea de base más larga está orientada cercana al PA del disco, por lo que las formas "W" de la visibilidad diferencial y "S" de la fase diferencial están bien definidas.

- V374 Car (HD 66194) es una estrella Be de tipo espectral B3V, miembro del cúmulo abierto NGC 2516. Esta estrella fue identificada como un blue straggler por Ahumada \& Lapasset (2007). González \& Lapasset (2000) reportaron variabilidad en la medición de las velocidades y sugirieron que ese objeto podría ser una binaria espectroscópica con transferencia de masa. Esta estrella presenta variaciones fotométricas del tipo $\gamma$ Cas (Lefèvre et al., 2009). No hay determinaciones previas del tamaño del disco para este objeto. Obtuvimos dos observaciones interferométricas con una relación $\mathrm{S} / \mathrm{N}$ baja (Fig. 8.21). El modelo del mejor ajuste provee los siguientes parámetros geométricos y cinemáticos para el disco: $\mathrm{PA}=220^{\circ}, i=55^{\circ}, V_{\text {rot }}=350 \mathrm{~km} \mathrm{~s}^{-1}, j=-0.4, a_{\text {cont }}=3.0 D_{\star} \mathrm{y} a_{\mathrm{Br} \gamma}=9.0 D_{\star}$. Las dos observaciones fueron realizadas con líneas de base similares, y ninguna muestra claramente la caída de la visibilidad o la forma "S" en la fase diferencial. El perfil de la línea Bry es consistente con el ángulo de inclinación intermedio encontrado (ver Fig. 8.5).

- MX Pup (HD 68980) es una binaria espectroscópica de tipo espectral B1.5 IVe (Slettebak, 1982), con un período de 5.1526 días y una órbita muy excéntrica (Carrier et al., 2002). La misma fue presentada en el Capítulo 5 , y entre los antecedentes reportados se destaca que Silaj et al. (2010) ajustaron la línea de $\mathrm{H} \alpha$ mediante un modelo simple de ley de potencia, obteniendo como parámetros del mejor ajuste un tipo espectral B2 para la estrella central, $i=20^{\circ}, \rho_{0}=2 \cdot 10^{-10} \mathrm{~g} \mathrm{~cm}^{-3}$ y $n=3.5$. Arcos et al. (2017) obtuvieron dos conjuntos de parámetros del disco, $\rho_{0}=5 \cdot 10^{-12}, n=2$ e $i=50^{\circ}$ usando observaciones tomadas en 2013 , y $\rho_{0}=2.5 \cdot 10^{-11}, n=2.5$ e $i=50^{\circ}$ con datos adquiridos en 2015. Por otro lado Vieira et al. (2017), a partir de la SED, determinaron $\log \rho_{0}=-11.5$ y $n=2.8$. Este objeto no tiene determinaciones previas del tamaño del disco.

Se obtuvo una observación interferométrica (Fig. 8.22). El modelo del mejor ajuste provee los siguientes parámetros geométricos y cinemáticos para el disco: $\mathrm{PA}=310^{\circ}, i=22^{\circ}$, $V_{\text {rot }}=410 \mathrm{~km} \mathrm{~s}^{-1}, j=-0.5, a_{\text {cont }}=1.5 D_{\star} \mathrm{y} a_{\mathrm{Br} \gamma}=3.0 D_{\star}$. La línea de base más larga, $\mathrm{B}_{3}$, está orientada casi perpendicular al PA obtenido para el disco, por lo que la visibilidad diferencial tiene una forma " $\mathrm{V}$ " $\mathrm{y}$ la fase diferencial tiene forma de "S" con pequeña amplitud. El perfil de la línea $\mathrm{Br} \gamma$ en emisión presenta una pequeña asimetría (ver Fig. 8.5) y es consistente con el pequeño ángulo de inclinación encontrado.

- o Aqr (HD 209409) es una estrella Be de tipo espectral B7IV. La misma fue estudiada en el Capítulo 5, donde presentamos antecedentes relevantes de la literatura.

Como se comentó en el capitulo 5, la estrella fue resuelta a partir de observaciones interferométricas. Touhami et al. (2013) encontraron una relación disco-estrella $R_{\mathrm{d}} / R_{\star}=5.776$ 
Figura 8.21: Ídem 8.8, para V374 Car (HD 66194).
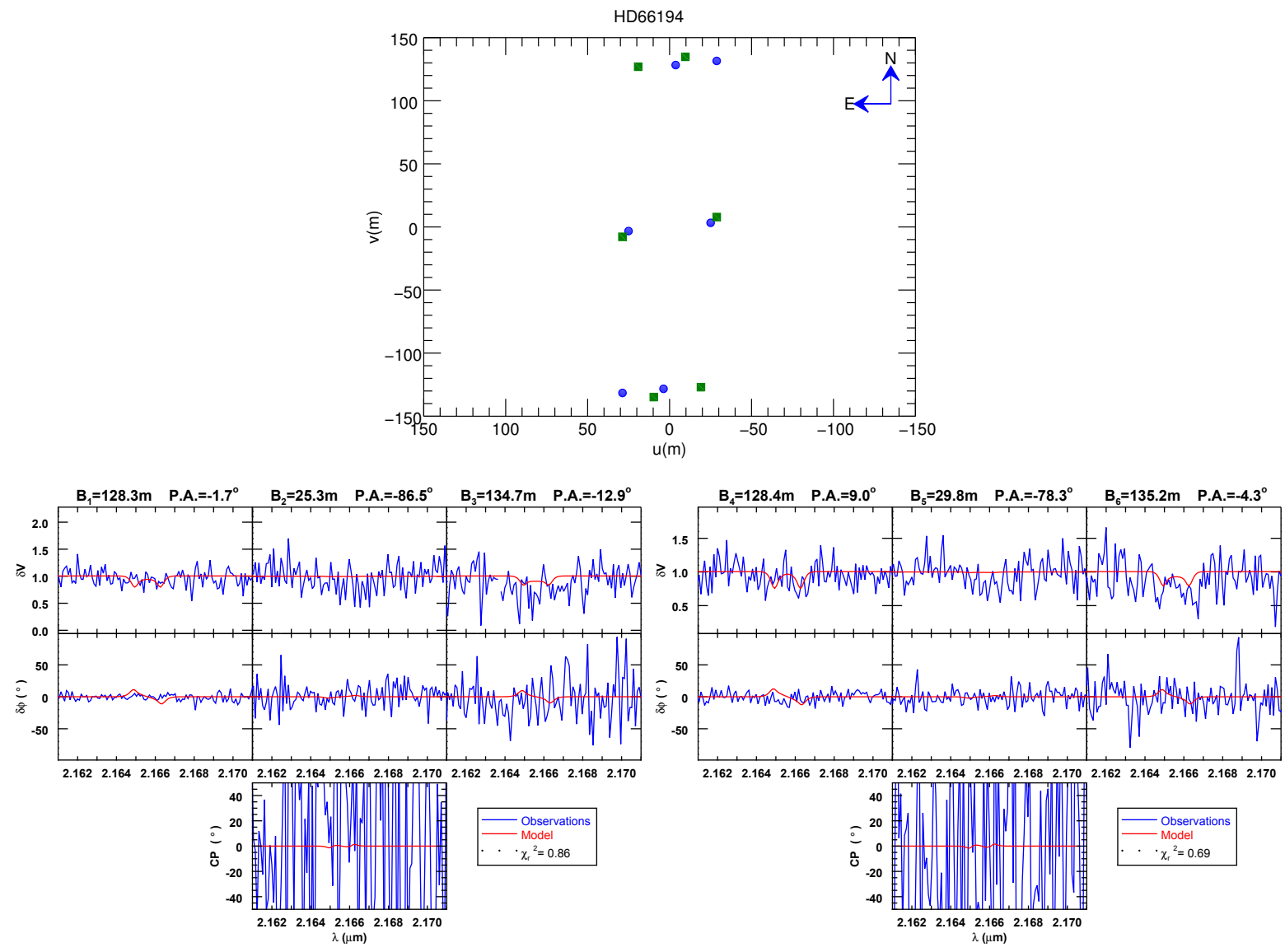

Figura 8.22: Ídem 8.8, para MX Pup (HD 68980).
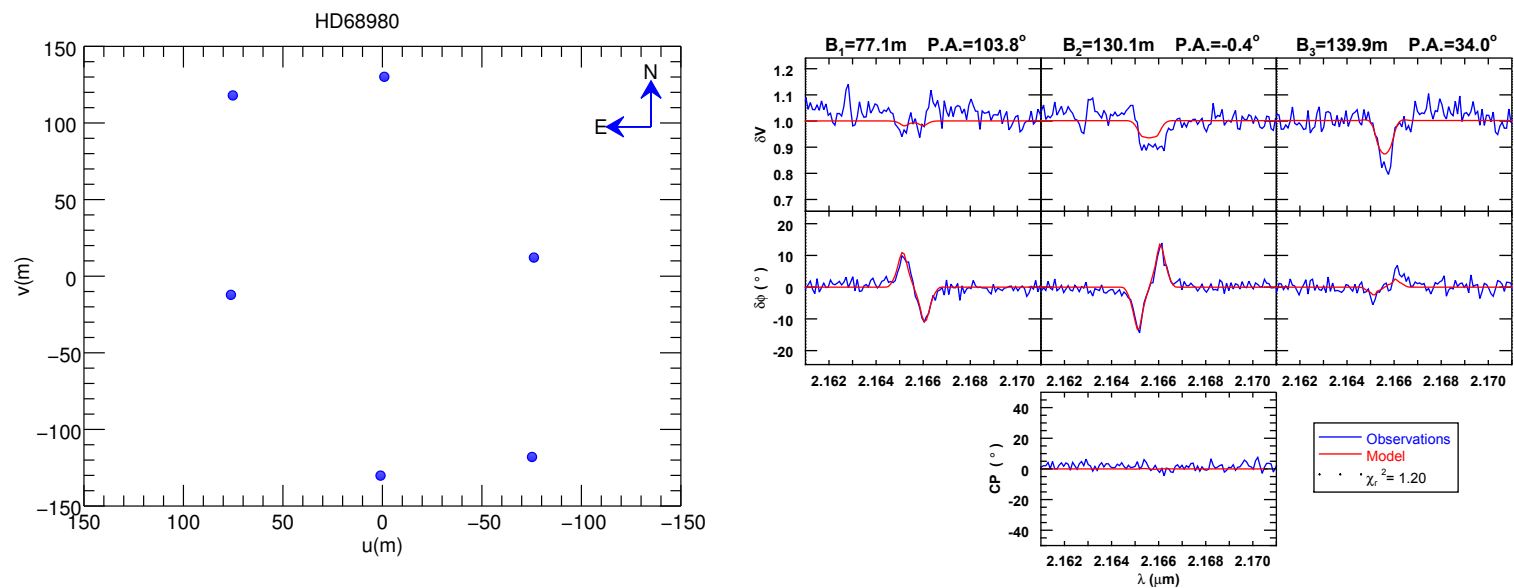

y $\mathrm{PA}=107.5^{\circ}$, mientras que Cyr et al. (2015) determinaron un cociente entre los ejes de 
Figura 8.23: Ídem 8.8, para o Aqr (HD 209409).
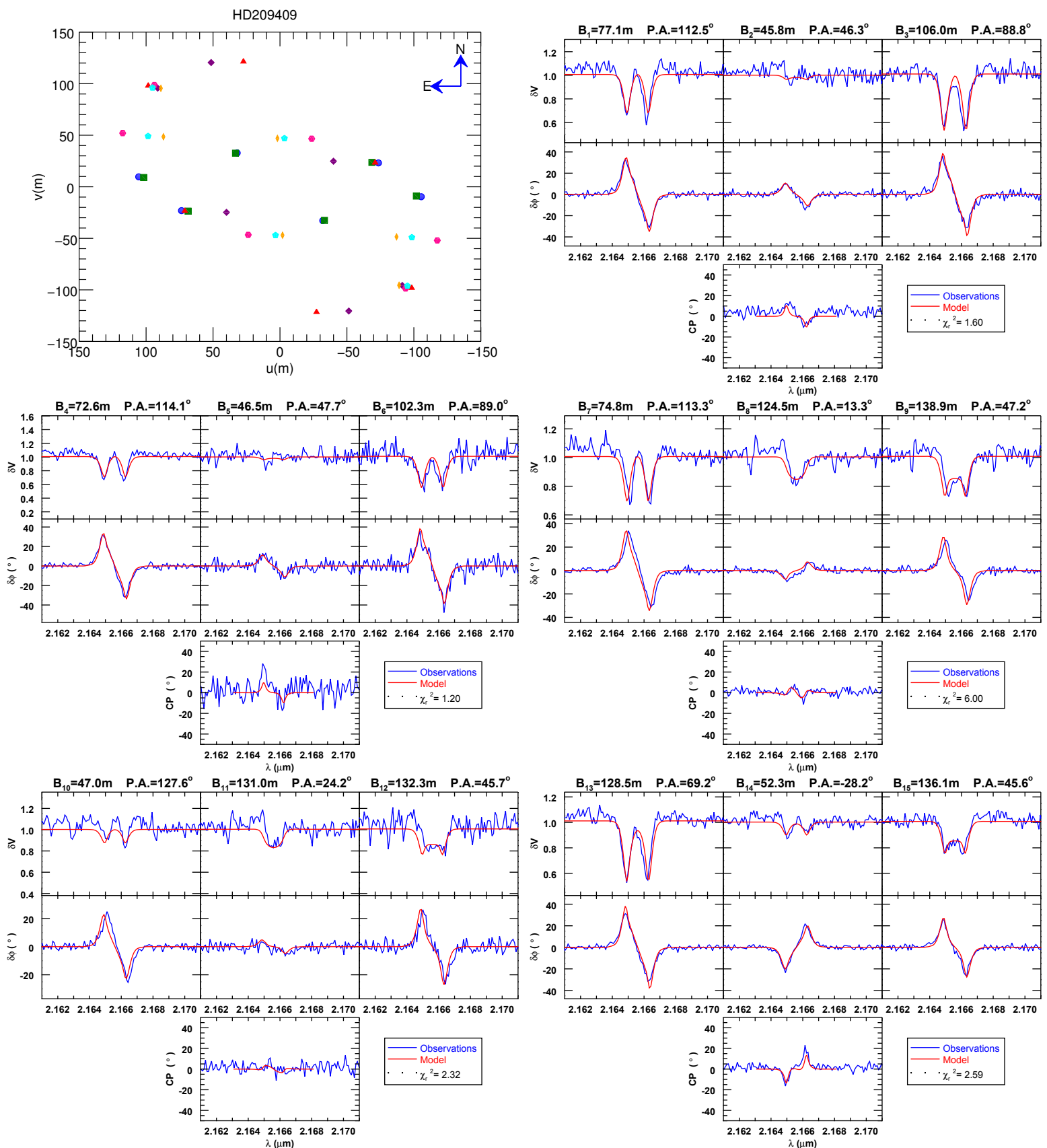

0.25 en la Banda K. Sigut et al. (2015), combinando observaciones interferométricas y en $\mathrm{H} \alpha$ durante un período de 7 años, concluyeron que las observaciones son consistentes con un disco circunestelar visto con una inclinación $i=75^{\circ}$ y $\mathrm{PA}=110^{\circ}$ con $\rho_{0}=6.6 \cdot 10^{-11}$ $\mathrm{g} \mathrm{cm}^{-3}$ y $n=2.7$. Meilland et al. (2012) obtuvieron los siguientes parámetros para el disco: $\mathrm{PA}=120^{\circ}, i=70^{\circ}, V_{\text {rot }}=400 \mathrm{~km} \mathrm{~s}^{-1}, j=-0.5, a_{\text {cont }}<10 D_{\star} \mathrm{y} a_{\mathrm{Br} \gamma}=14 D_{\star}$. Por otra parte, 
Figura 8.24: Ídem 8.8, para o Aqr (HD 209409) (continuación).
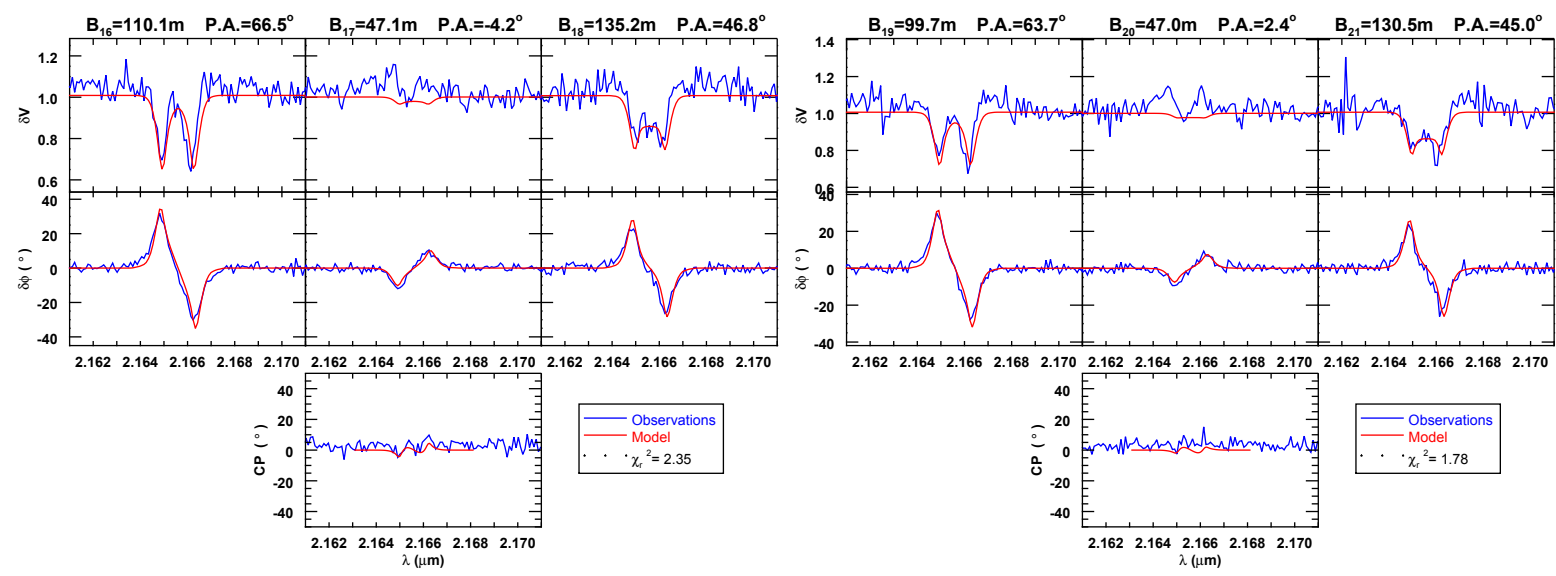

Yudin (2001) determinó un valor $\mathrm{PA}=177^{\circ}$ para la polarización observada.

Silaj et al. (2010) ajustaron la línea de $\mathrm{H} \alpha$ con un doble pico, obteniendo como parámetros del mejor ajuste un tipo espectral B8 para la estrella central, $i=45^{\circ}, \rho_{0}=5 \cdot 10^{-11}$ $\mathrm{g} \mathrm{cm}^{-3}$ y $n=3.5$, mientras que Arcos et al. (2017) obtuvieron densidades e índice menores ( $\rho_{0}=5 \cdot 10^{-10} \mathrm{~g} \mathrm{~cm}^{-3}$ y $n=2.0$ ), y dos diferentes inclinaciones: $i=25^{\circ}$ (en 2013) e $i=50^{\circ}$ (en 2015).

Para este objeto obtuvimos siete observaciones interferométricas con alta relación $\mathrm{S} / \mathrm{N}$ (Fig. 8.23 y 8.24). A partir del modelo del mejor ajuste obtuvimos los siguientes parámetros geométricos y cinemáticos para el disco: $\mathrm{PA}=290^{\circ}, i=70^{\circ}, V_{\text {rot }}=355 \mathrm{~km} \mathrm{~s}^{-1}, j=-0.45$, $a_{\text {cont }}=3.0 D_{\star}$ y $a_{\mathrm{Br} \gamma}=8.0 D_{\star}$. Las líneas de base $\mathrm{B}_{1}, \mathrm{~B}_{4}$ y $\mathrm{B}_{7}$ están orientadas cercanas al PA obtenido para el disco, por lo que la forma "W" de la visibilidad diferencial y la forma "S" de la fase diferencial están bien definidas. La base $\mathrm{B}_{11}$ es una de las más largas y está orientada perpendicular al PA obtenido para el disco, por lo que la visibilidad diferencial tiene forma "V" y la fase diferencial presenta una forma "S" con pequeña amplitud. El perfil de doble pico de $\mathrm{Br} \gamma$, la forma "W" en la caída de la visibilidad diferencial y la forma "S" de la fase diferencial son cuasi-simétrico. Esto indica que el objeto está visto desde un alto ángulo de inclinación y que no presenta grandes inhomogeneidades en el disco.

- 31 Peg (HD 212076) es una estrella Be de tipo espectral B2IV-V. La estrella fue parcialmente resuelta con el arreglo CHARA. Adoptando valores $\mathrm{PA}=148^{\circ}$ y un cociente de radios $r=0.955$, Touhami et al. (2013) encontraron un cociente entre el tamaño del disco y la estrella de 1.852. Silaj et al. (2010) ajustaron la línea de $\mathrm{H} \alpha$ con un pico en emisión, obteniendo como parámetros del mejor ajuste un tipo espectral B2 para la estrella central, con $i=20^{\circ}, \rho_{0}=10^{-11} \mathrm{~g} \mathrm{~cm}^{-3}$ y $n=2.5$. Estos parámetros difieren de los encontrados por Arcos et al. (2017, $n=2, i=30^{\circ}, \rho_{0}=2.5 \cdot 10^{-11} \mathrm{~g} \mathrm{~cm}^{-3}$ en $2012 \mathrm{y} \rho_{0}=2.5 \cdot 10^{-12} \mathrm{~g} \mathrm{~cm}^{-3}$ en 2015 ) 
Figura 8.25: Ídem 8.8, para 31 Peg (HD 212076).
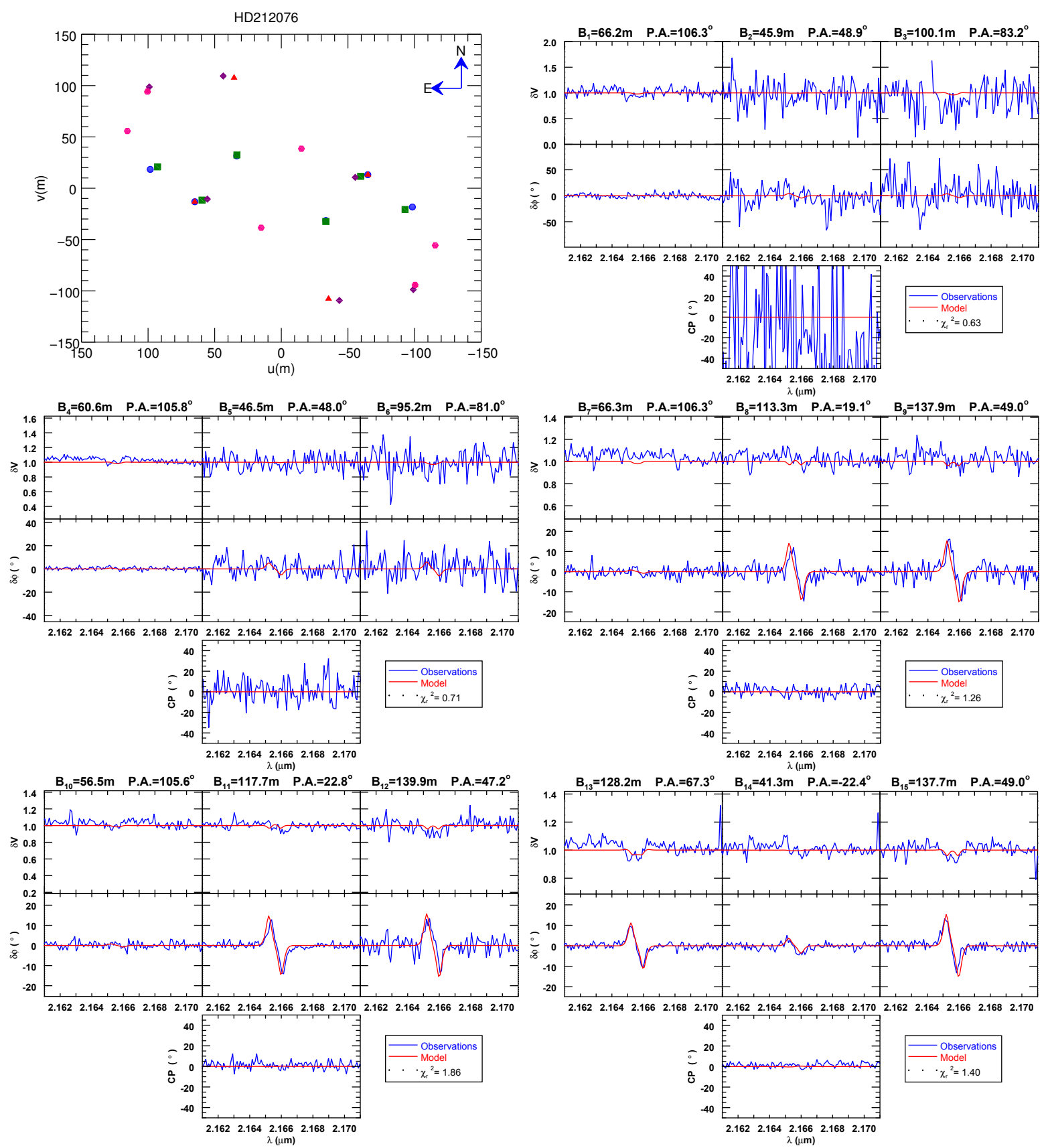
Figura 8.26: Ídem 8.8, para $\pi$ Aqr (HD 212571).
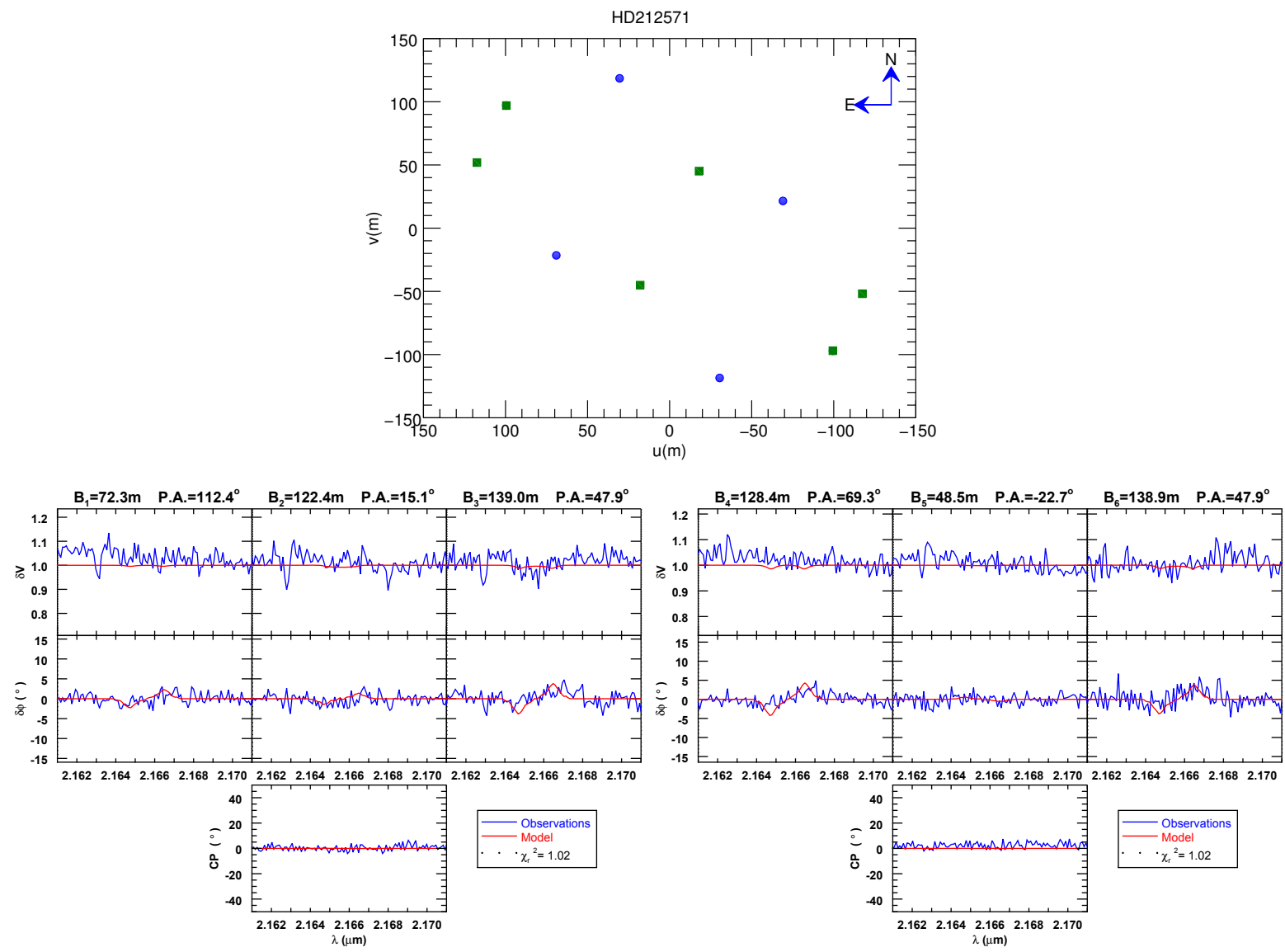

y por Vieira et al. $\left(2017, \log \rho_{0}=-11.7\right.$ y $\left.n=2.13.\right)$.

Obtuvimos cinco observaciones interferométricas, tres con alta relación $\mathrm{S} / \mathrm{N}$ y dos con baja relación $\mathrm{S} / \mathrm{N}$. El modelo del mejor ajuste brinda los siguientes parámetros geométricos y cinemáticos para el disco: $\mathrm{PA}=202^{\circ}, i=22^{\circ}, V_{\text {rot }}=400 \mathrm{~km} \mathrm{~s}^{-1}, j=-0.5, a_{\text {cont }}=3.0 D_{\star}$ y $a_{\mathrm{Br} \gamma}=4.0 D_{\star}$. Formas "W" en la visibilidad diferencial y "S" en la fase diferencial son observadas en algunas líneas de base (generalmente las más largas), tal como se muestra en la Fig. 8.25.

- $\pi$ Aqr (HD 212571) es una estrella Be de tipo espectral B1III-IV. Dentro de los antecedentes reportados en el Capítulo 5, remarcamos que Silaj et al. (2010) ajustaron la línea de $\mathrm{H} \alpha$ con un doble pico en emisión obteniendo como parámetros del mejor ajuste un tipo espectral B0 para la estrella central y un disco ecuatorial con $\rho_{0}=10^{-11} \mathrm{~g} \mathrm{~cm}^{-3}$ y $n=3.5$, visto con un ángulo de inclinación $i=45^{\circ}$. Arcos et al. (2017) encontraron tres conjuntos de parámetros, usando espectros obtenidos en diferentes épocas: $\rho_{0}=2.5 \cdot 10^{-11} \mathrm{~g} \mathrm{~cm}^{-3}, i=60^{\circ}$ y $n=2.5$ para las observaciones obtenidas en 2012 y 2015 , y $\rho_{0}=7.5 \cdot 10^{-12} \mathrm{~g} \mathrm{~cm}^{-3}, i=60^{\circ}$ 
y $n=2.5$ para las de 2013, mientras que Vieira et al. (2017) obtuvieron a partir de la SED, $\log \rho_{0}=-12.1$ y $n=3.5$.

Este objeto no tiene determinaciones previas del tamaño del disco.

Se realizaron dos observaciones interferométricas con una buena relación S/N (Fig. 8.26). El modelo del mejor ajuste provee los siguientes parámetros geométricos y cinemáticos para el disco: $\mathrm{PA}=87^{\circ}, i=34^{\circ}, V_{\text {rot }}=440 \mathrm{~km} \mathrm{~s}^{-1}, j=-0.5, a_{\text {cont }}=1.5 D_{\star} \mathrm{y} a_{\mathrm{Br} \gamma}=2.0 D_{\star}$. La forma "W" de la visibilidad diferencial es apenas perceptible en las bases $\mathrm{B}_{3}$ y $\mathrm{B}_{4}$. La forma "S" de la fase diferencial está presente solo en algunas bases. El perfil de doble pico de la línea Bry es muy débil, como se ve en la Fig. 8.5.

- $\epsilon$ PsA (HD 214748) es una estrella Be de tipo B8V. Arcos et al. (2017) modelaron la línea $\mathrm{H} \alpha$ en emisión usando una distribución de densidades de ley de potencia, y encontraron que $\rho_{0}=2.5 \cdot 10^{-10} \mathrm{gr} \mathrm{cm}^{-3}, n=3.5$ e $i=50^{\circ}$. La densidad en la base del disco es más alta que la calculada por Vieira et al. $\left(2017, \log \rho_{0}=-11.9\right.$ y $\left.n=2.7\right)$, basados en un modelo de pseudo-fotosfera a partir de la emisión del disco en el continuo.

Se obtuvieron cinco observaciones interferométricas con alta relación $\mathrm{S} / \mathrm{N}$ (ver Fig. 8.27). A partir del modelo del mejor ajuste se obtuvieron los siguientes parámetros geométricos y cinemáticos para el disco: $\mathrm{PA}=67^{\circ}, i=73^{\circ}, V_{\text {rot }}=244 \mathrm{~km} \mathrm{~s}^{-1}, j=-0.46, a_{\text {cont }}=2.0 D_{\star} \mathrm{y}$ $a_{\mathrm{Br} \gamma}=3.9 D_{\star}$. La línea de base $\mathrm{B}_{10}$ está orientada cercana al PA observado. La línea $\mathrm{Br} \gamma$ en emisión presenta una fuerte asimetría (ver Fig. 8.5).

\subsection{Comentarios y resultados generales}

Para casi todos los objetos, el modelo cinemático simple reproduce las observaciones. En el caso de 228 Eri, tuvimos que agregar una sobredensidad en forma de brazo espiral para mejorar el ajuste de nuestros datos debido a la gran asimetría de los mismos. La cinemática del disco está dominada por rotación, con una ley cercana a la rotación Kepleriana para casi todos los objetos. Las mayores discrepancias se encuentran en 120 Tau $(j=-0.4)$, V696 Mon $(j=-0.4)$, V374 Car $(j=-0.6)$ y 228 Eri $(j=-0.35)$. Este último corresponde al objeto con una sobredensidad en el disco.

\subsection{1 Ángulo de polarización}

Generalmente, el ángulo de polarización en un disco ópticamente delgado es perpendicular al plano del disco, debido a que la polarización es generada por dispersión de electrones (Brown \& McLean, 1977). Sin embargo, Delaa et al. (2011) reportaron que para 48 Per el eje mayor está orientado casi paralelo al ángulo de polarización, y propusieron que esto puede deberse a la alta profundidad óptica del disco. 
Figura 8.27: Ídem 8.8, para $\epsilon$ PsA (HD 214748).
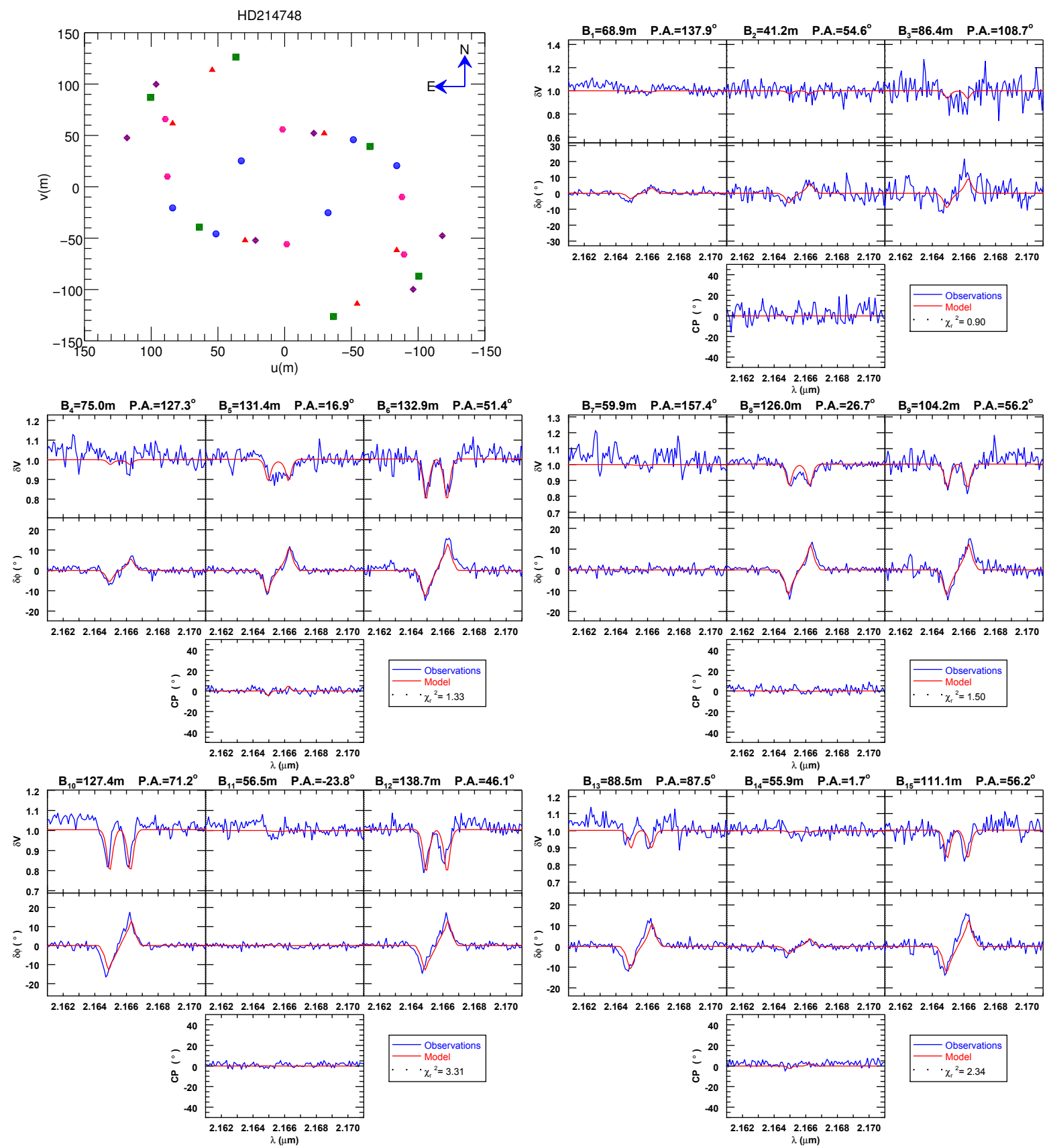

Para la mayor parte de los objetos de nuestra muestra, el eje mayor del disco es aproximadamente perpendicular al ángulo de polarización, como se muestra en la Fig. 8.28. Las excepciones son 105 Tau y 31 Peg. En ambos casos, los objetos tienen pequeños ángulos de inclinación, lo que hace que la determinación del ángulo de posición sea más imprecisa, especialmente cuando la observación tiene una mala relación $\mathrm{S} / \mathrm{N}$ como en 105 Tau o cuando se observa un disco pequeño 
Figura 8.28: Ángulo de posición del disco PA vs. ángulo de polarización $P_{\star}$. La línea continua negra corresponde a la situación donde $\mathrm{PA}$ es perpendicular a $P_{\star}\left(\mathrm{PA}=P_{\star} \pm 90\right)$. La línea continua roja corresponde a la situación donde PA es paralelo a $P_{\star}\left(\mathrm{PA}=P_{\star} \pm 180\right)$. Las líneas punteadas corresponden a una incerteza de $15^{\circ}$.

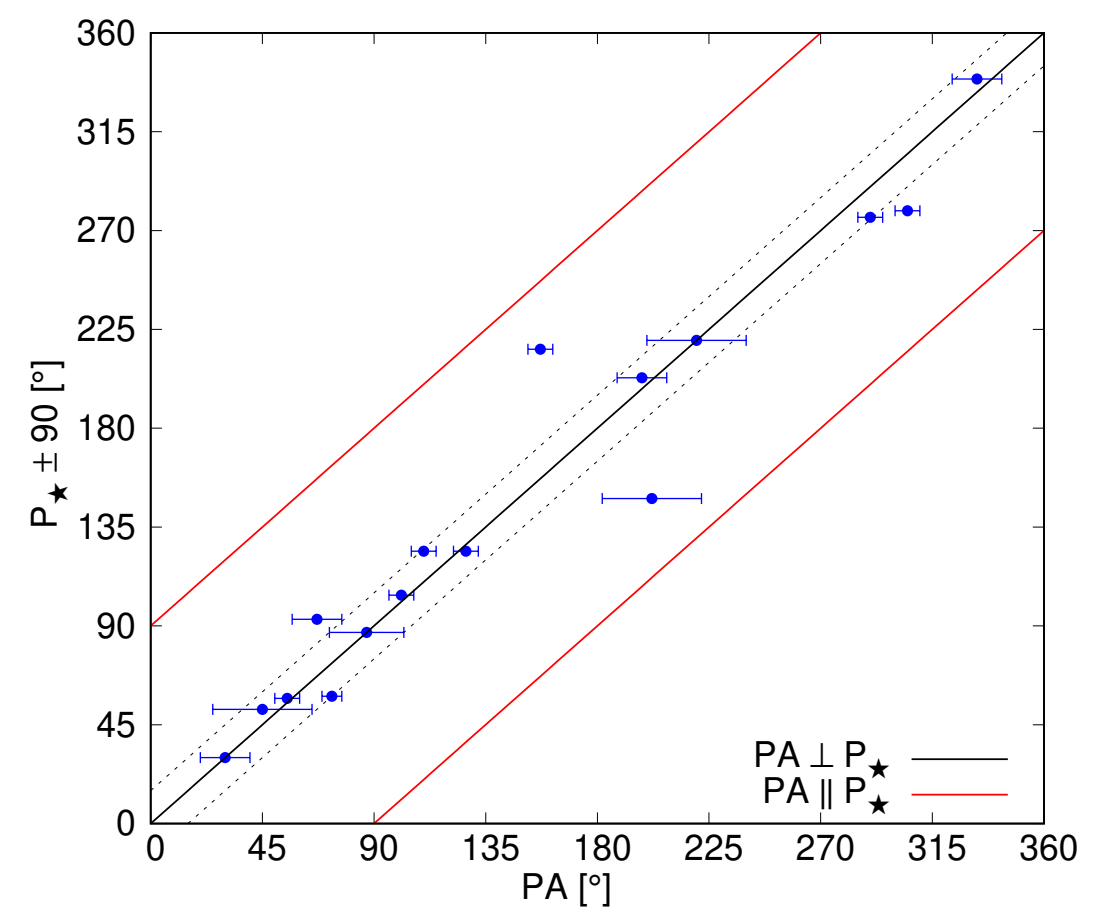

como en $31 \mathrm{Peg}$.

En el caso de $28 \mathrm{Tau}, \mathrm{PA}$ es casi perpendicular al ángulo de polarización medido por Yudin (2001), pero diferente a valores previos reportados. Esto puede deberse a la precesión del disco causada por una compañera (Hirata, 2007).

\subsubsection{Distribución de la extensión del disco en $\operatorname{Br} \gamma\left(a_{\mathrm{Br} \gamma}\right)$ y comparación con $\mathbf{H} \alpha$}

La distribución de la extensión del disco en $\mathrm{Br} \gamma$ es presentada en la Fig. 8.29 ${ }^{3}$. Alrededor de un tercio de la muestra presenta discos chicos, con $a_{\mathrm{Br} \gamma}$ entre $2 D_{\star}$ y $5.7 D_{\star}$. La distribución es casi constante en el intervalo $7.5-13 D_{\star}$ y la mayor medida corresponde a $13 D_{\star}$. Las estrellas de clase de luminosidad $\mathrm{V}$ presentan discos con diferentes tamaños, mientras que las de clase de luminosidad III solo presentan discos pequeños. Los tamaños que determinamos para $a_{\mathrm{Br} \gamma}$ están dentro del mismo rango que los valores obtenidos por Meilland et al. (2012) para una muestra de 8 estrellas Be a partir del modelado de datos interferométricos, y son sistemáticamente más grandes que los calculados por Gies et al. (2007) y Touhami et al. (2013) utilizando el interferómetro

\footnotetext{
${ }^{3}$ En este y todos los histogramas presentados, se utilizó un método de optimización del bin para encontrar el número y tamaño adecuados (Shimazaki \& Shinomoto, 2007).
} 
Figura 8.29: Distribución de $a_{\mathrm{Br} \gamma}$ según tipo espectral y clase de luminosidad.
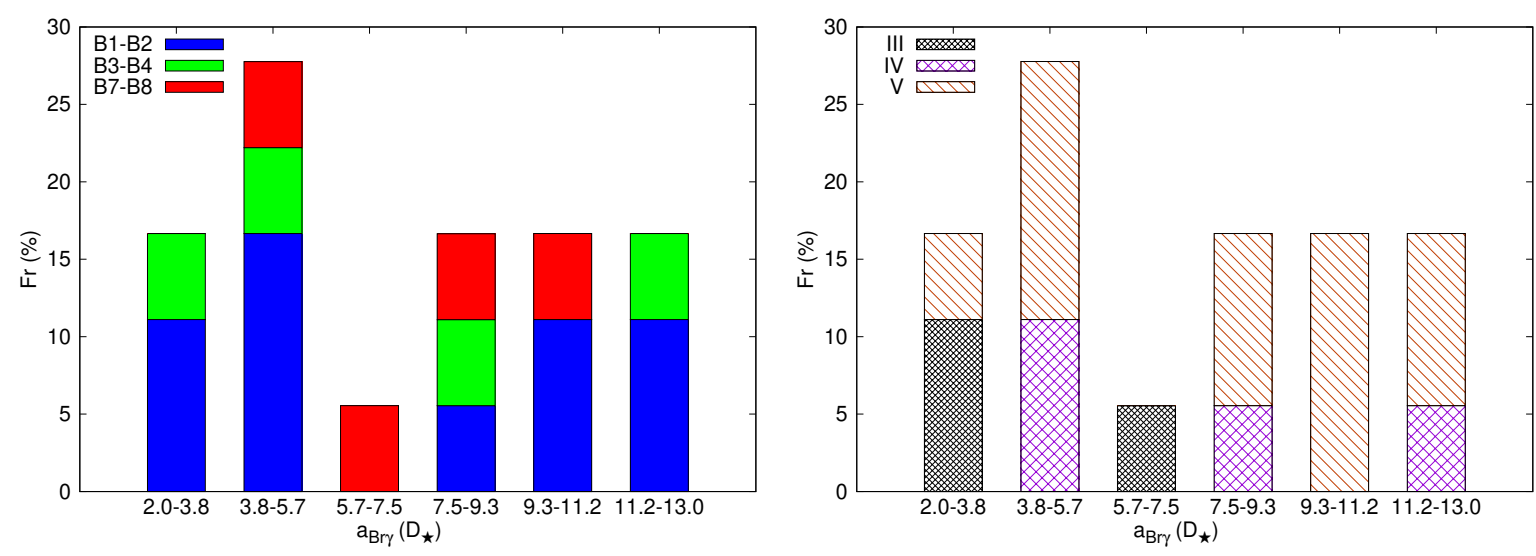

CHARA en la Banda K.

Utilizando 48 estrellas Be del norte, Catanzaro (2013) encontró que los tamaños de los discos en $\mathrm{H} \alpha$ tienen valores entre $2 R_{\star}$ y $14 R_{\star}$, con una concentración máxima en el intervalo $6-8 R_{\star}$, similar a lo que encontramos para la línea $\mathrm{Br} \gamma$. Sin embargo, no encontramos una correlación clara entre los tamaños medidos en las líneas $\mathrm{H} \alpha$ y $\mathrm{Br} \gamma$ a partir de las 10 estrellas de nuestra muestra que tienen reportadas medidas del disco en la línea $\mathrm{H} \alpha$ (Hanuschik et al., 1988; Andrillat et al., 1990; Grundstrom \& Gies, 2006). El tamaño en $\operatorname{Br} \gamma$ para $\eta$ Tau es mayor que el encontrado para $\mathrm{H} \alpha$ por Grundstrom \& Gies (2006), pero menor que el encontrado por Hanuschik et al. (1988), incluso a pesar de que esta estrella tiene un perfil de $\mathrm{H} \alpha$ estable. Para $\omega$ Ori, el tamaño de la envoltura en $\mathrm{Br} \gamma$ y $\mathrm{H} \alpha$ (Andrillat et al., 1990; Hanuschik et al., 1988) es similar. Para $\zeta$ Tau y $\epsilon$ PsA, el tamaño en la línea $\mathrm{Br} \gamma$ es menor que los obtenidos para $\mathrm{H} \alpha$, como se reportó para $\delta$ Sco por Meilland et al. (2011). Al contrario, para 228 Eri, 56 Eri, $\psi_{01}$ Ori, 120 Tau, V696 Mon y $\beta_{01}$ Mon se encontraron tamaños en $\mathrm{Br} \gamma$ mayores que los obtenidos para $\mathrm{H} \alpha$. En particular, todos estos objetos tienen perfiles de $\mathrm{H} \alpha$ variables, por lo que esto podría indicar que el disco aumentó su tamaño entre las diferentes observaciones.

Considerando la variabilidad de las estrellas $\mathrm{Be}$, y los diferentes métodos utilizados para obtener los tamaños de los discos, sería interesante realizar observaciones en varias longitudes de onda de manera simultánea.

\subsubsection{Distribución del ángulo de inclinación $(i)$}

En la Fig. 8.30 presentamos la distribución de inclinaciones por tipo espectral y clase de luminosidad. Las inclinaciones están distribuidas casi de manera uniforme en el intervalo $22-64^{\circ}$, presentando un pico en el intervalo 64-74.5 ${ }^{\circ}$. Algunas estrellas B tardías fueron observadas desde el plano ecuatorial (5\%). Las estrellas con ángulos de inclinación pequeños (menores que $43^{\circ}$ ) son todas de tipos espectrales B1-B2, con cualquier clase de luminosidad. Para inclinaciones interme- 
Figura 8.30: Distribución de inclinaciones según tipo espectral y clase de luminosidad.
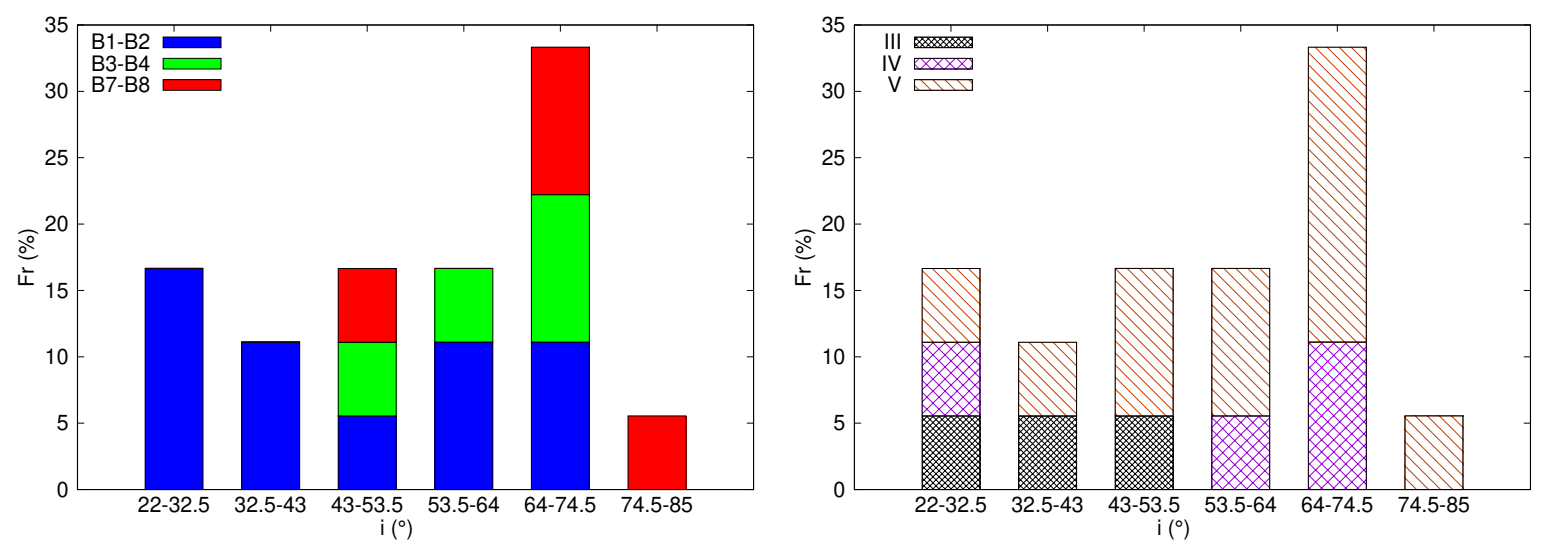

Figura 8.31: Distribución de $V_{\text {rot }}$ según tipo espectral y clase de luminosidad.
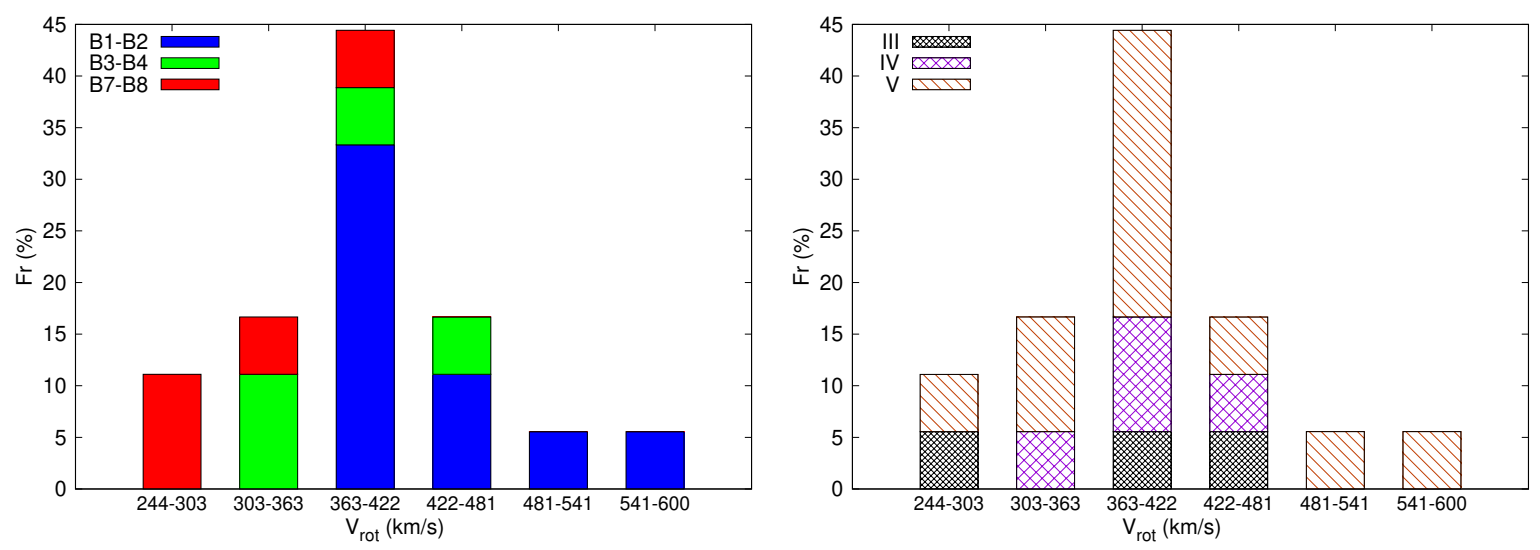

dias y altas la distribución es casi uniforme entre los tipos espectrales. En nuestra muestra no tenemos estrellas de clase de luminosidad III vistas con ángulos de inclinación grandes.

Un análisis más profundo de la distribución de $i$ puede encontrarse en la Subsec. 8.9.2.

\subsubsection{Distribución de la velocidad de rotación en la base del disco $\left(V_{\text {rot }}\right)$}

En la Fig. 8.31 presentamos la distribución de la velocidad de rotación en la base del disco ( $\left.V_{\text {rot }}\right)$. La distribución tiene un máximo en $363-422 \mathrm{~km} / \mathrm{s}$. El máximo contiene estrellas de todos los tipos espectrales y clases de luminosidad. Las estrellas con tipos espectrales más tempranos tienen discos con mayores velocidades de rotación, mientras que las más tardías muestran discos con menores velocidades de rotación. La distribución no parece depender de la clase de luminosidad. Solo un objeto (228 Eri) presenta un valor más alto, de $600 \mathrm{~km} \mathrm{~s}^{-1}$.

En la Fig. 8.32 presentamos el semieje mayor del disco en Br $\gamma$ en función de $V_{\text {rot }}$. A pesar de que no hay una correlación clara entre la extensión del disco en la línea y $V_{\text {rot }}$, observamos que 
Figura 8.32: Relación entre $V_{\text {rot }}$ y la extensión del disco en $\mathrm{Br} \gamma$.

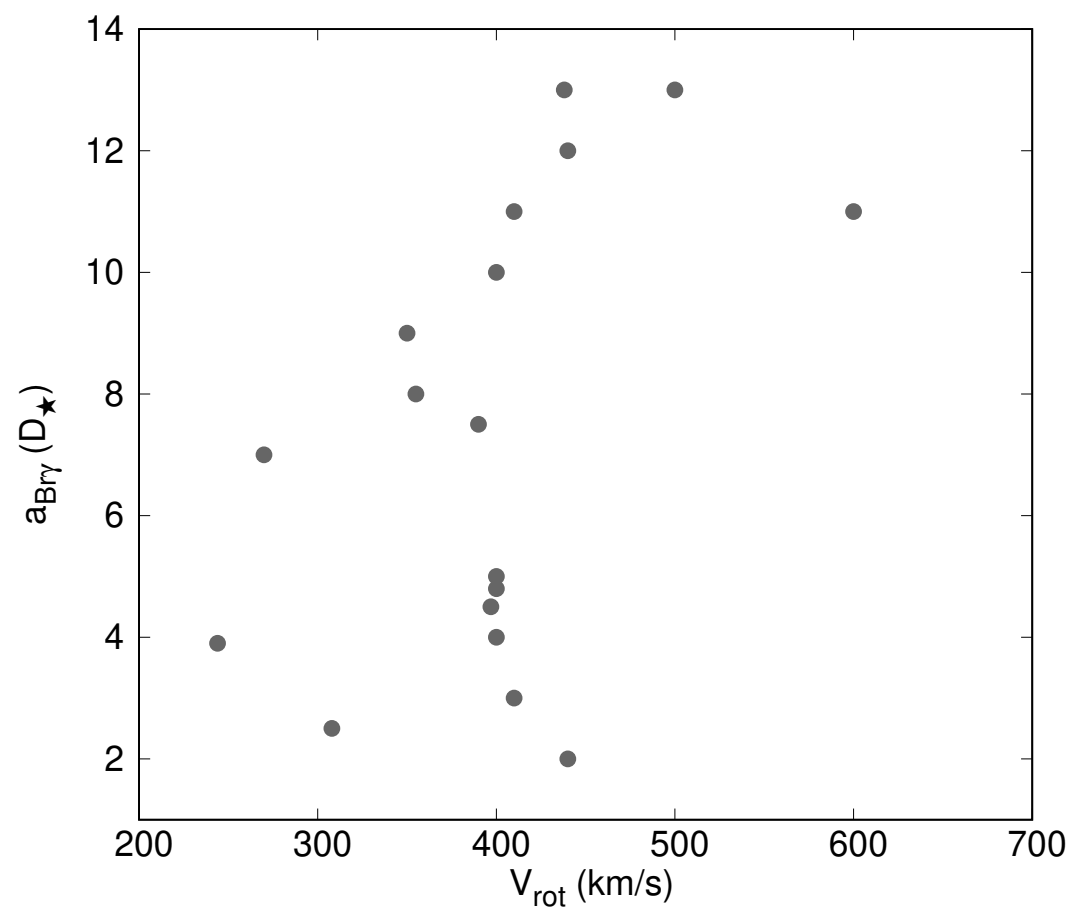

los discos con extensiones mayores a $10 D_{\star}$ solo están presentes cuando $V_{\text {rot }}>400 \mathrm{~km} \mathrm{~s}^{-1}$. Esto será discutido en el Capítulo 9.

\subsubsection{Distribución de velocidad de rotación lineal $(V)$ y angular $(\Omega)$}

Con los valores de $V \sin i$ y $V_{\text {crit }}$ obtenidos de la literatura, junto a nuestros valores de $i$, obtuvimos los cocientes $V / V_{\text {crit }}$ presentados en la Tabla $8.4 . V / V_{\text {crit }}$ tiene valores entre 0.62 y 0.79 , con la distribución presentada en la Fig. 8.33. La distribución no tiene un pico máximo y no encontramos correlación con el tipo espectral o la clase de luminosidad. Es valor medio es de $\overline{V / V_{\text {crit }}}=0.71 \pm 0.06$.

La tasa de rotación es definida en Frémat et al. (2005) como el cociente entre la velocidad angular de rotación y la velocidad angular de rotación crítica, $\frac{\Omega}{\Omega_{\text {crit }}}=\frac{V}{V_{\text {crit }}} \frac{R_{\text {eq crit }}}{R_{\text {eq }}}$, donde $R_{\text {eq crit }} \mathrm{y}$ $R_{\text {eq }}$ son los radios ecuatoriales (en radios polares) para estrellas rotando a la velocidad $V_{\text {crit }} \mathrm{y} V$, respectivamente. El valor medio para nuestra muestra es $\overline{\Omega / \Omega_{\text {crit }}}=0.86 \pm 0.04$, y la distribución es la que se muestra en la Fig. 8.34.

\subsection{Discusión de los resultados del capítulo}

Con el objetivo de estudiar una muestra mayor y más representativa de estrellas Be para entender mejor los mecanismos físicos involucrados en el origen de las discos circunestelares, analizamos nuestros resultados junto con los obtenidos por Meilland et al. (2012). La Tabla 8.5 presenta los parámetros determinados por estos autores para los discos estudiados. Excluimos a la estrella 
Figura 8.33: Distribución de $V / V_{\text {crit }}$ según tipo espectral y clase de luminosidad.
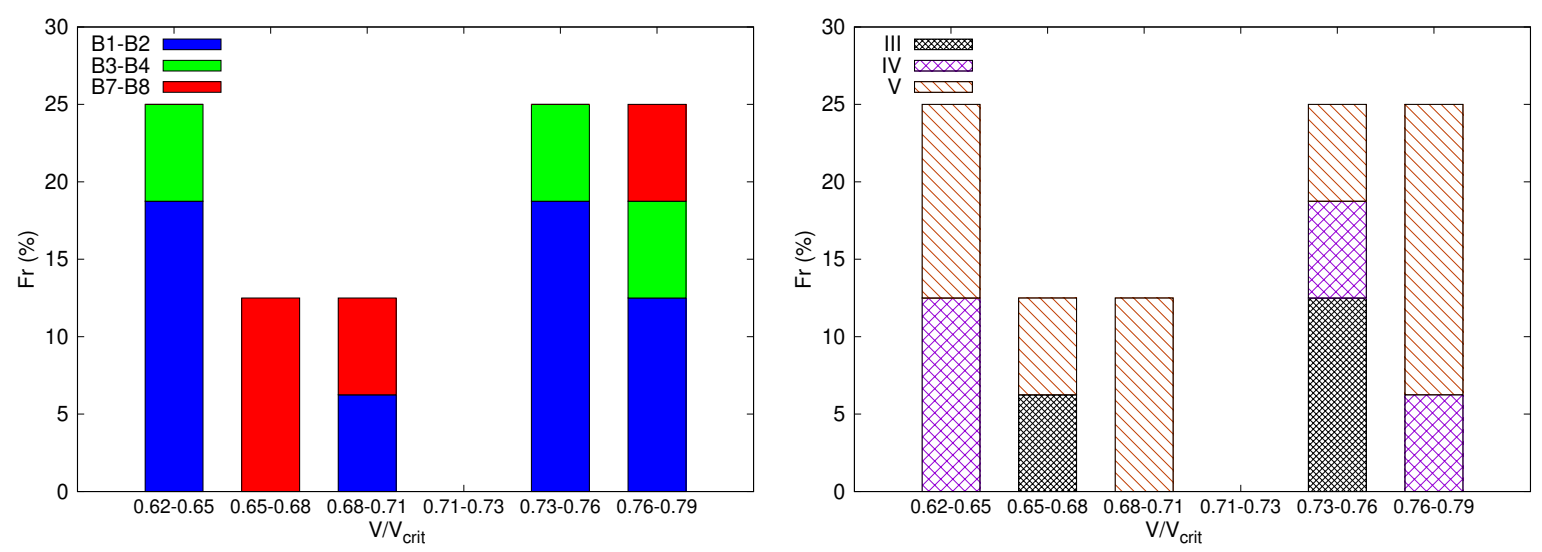

Figura 8.34: Distribución de $\Omega / \Omega_{\text {crit }}$ según tipo espectral y clase de luminosidad.
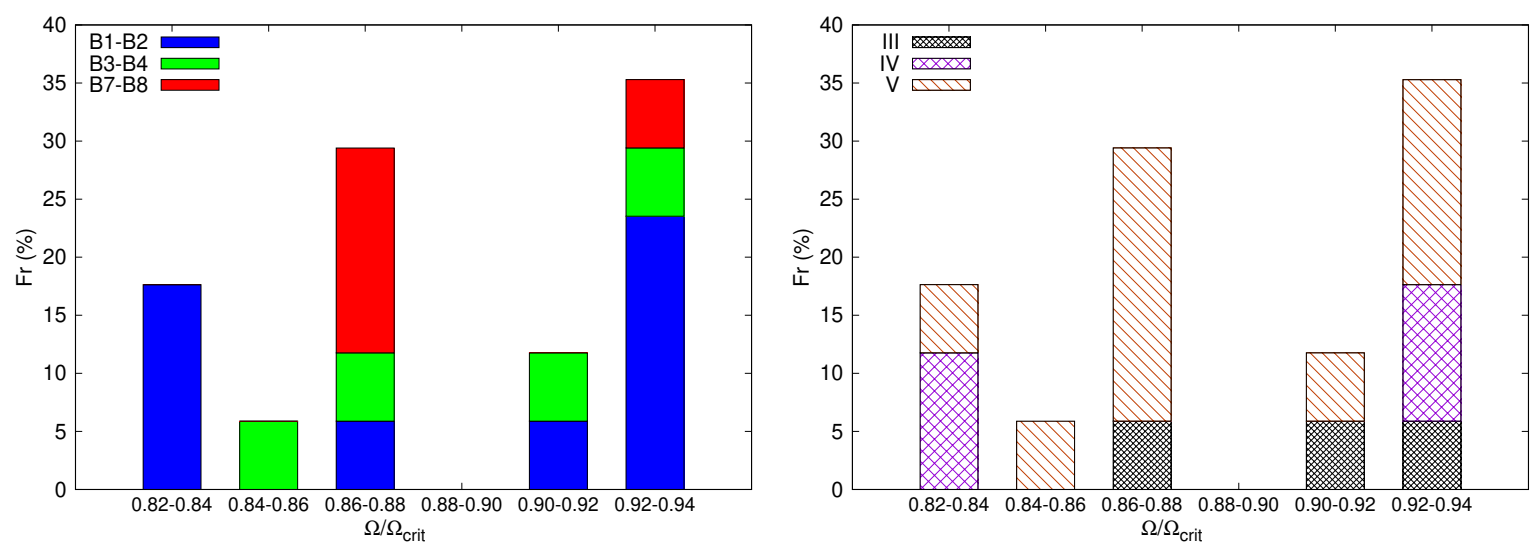

$o$ Aqr de la muestra de Meilland et al. ya que nuestras observaciones tienen una mejor relación señal/ruido. De esta manera, la muestra combinada contiene 25 estrellas $\mathrm{Be}$, con tipos espectrales en el rango B1-B8 y clases de luminosidades entre V y III, distribuidas según los histogramas de la Fig. 8.35.

En la Fig. 8.36 presentamos las distribuciones de $a_{\mathrm{Br} \gamma}, i, V_{\text {rot }}, V / V_{\text {crit }} \mathrm{y} \Omega / \Omega_{\text {crit }}$ para la muestra combinada. Según los tipos espectrales y clases de luminosidad, las distribuciones de $a_{\mathrm{Br} \gamma}, i$ y $V_{\text {rot }}$ para esta muestra tienen el mismo comportamiento que el descripto para los objetos de nuestra muestra. Para $V / V_{\text {crit }}$ encontramos que la distribución es casi constante entre 0.62 y 0.81 , y contiene aproximadamente el $80 \%$ de las estrellas de la muestra. El $20 \%$ restante se distribuye entre 0.81 y 0.99 . No observamos correlaciones con el TE o CL. La distribución de $\Omega / \Omega_{\text {crit }}$ es similar para estrellas con diferentes TE y CL. 
Tabla 8.4: $V / V_{\text {crit }}$ y $\Omega / \Omega_{\text {crit }}$ para nuestra muestra de estrellas Be.

\begin{tabular}{l|c|c}
\hline \hline & $V / V_{\text {crit }}$ & $\Omega / \Omega_{\text {crit }}$ \\
\hline$\eta$ Tau (HD 23630) & $0.68 \pm 0.12$ & $0.87 \pm 0.09$ \\
28 Tau (HD 23862) & $0.69 \pm 0.08$ & $0.87 \pm 0.06$ \\
228 Eri (HD 28497) & $0.78 \pm 0.25$ & $0.93 \pm 0.13$ \\
56 Eri (HD 30076) & $0.79 \pm 0.18$ & $0.94 \pm 0.09$ \\
105 Tau (HD 32991) & - & - \\
$\psi_{01}$ Ori (HD 35439) & $0.63 \pm 0.10$ & $0.83 \pm 0.09$ \\
120 Tau (HD 36576) & $0.63 \pm 0.09$ & $0.83 \pm 0.08$ \\
$\zeta$ Tau (HD 37202) & $0.76 \pm 0.07$ & $0.92 \pm 0.04$ \\
$\omega$ Ori (HD 37490) & $0.64 \pm 0.07$ & $0.84 \pm 0.06$ \\
V696 Mon (HD 41335) & $0.78 \pm 0.14$ & $0.93 \pm 0.07$ \\
$\beta_{01}$ Mon (HD 45725) & $0.75 \pm 0.11$ & $0.91 \pm 0.07$ \\
OW Pup (HD 60606) & $0.69 \pm 0.12$ & $0.87 \pm 0.09$ \\
V374 Car (HD 66194) & $0.67 \pm 0.14$ & $0.86 \pm 0.11$ \\
MX Pup (HD 68980) & $0.76 \pm 0.18$ & $0.92 \pm 0.10$ \\
$o$ Aqr (HD 209409) & $0.77 \pm 0.13$ & $0.93 \pm 0.07$ \\
31 Peg (HD 212076) & $0.62 \pm 0.21$ & $0.82 \pm 0.18$ \\
$\pi$ Aqr (HD 212571) & $0.75 \pm 0.29$ & $0.91 \pm 0.17$ \\
$\epsilon$ PsA (HD 214748) & $0.68 \pm 0.12$ & $0.87 \pm 0.09$ \\
\hline \hline
\end{tabular}

Tabla 8.5: Parámetros de los mejores ajustes obtenidos por Meilland et al. (2012).

\begin{tabular}{|c|c|c|c|c|c|c|c|c|}
\hline \multirow[b]{2}{*}{ Parámetro } & \multicolumn{2}{|c|}{$\begin{array}{l}\text { Parámetros } \\
\text { geométricos }\end{array}$} & \multicolumn{2}{|c|}{$\begin{array}{l}\text { Parámetros } \\
\text { cinemáticos }\end{array}$} & \multirow{2}{*}{$\begin{array}{c}\begin{array}{c}\text { Parámetros en } \\
\text { el continuo }\end{array} \\
a_{\text {cont }} \\
\left(D_{\star}\right)\end{array}$} & \multicolumn{2}{|c|}{$\begin{array}{c}\text { Parámetros en } \\
\text { la línea }\end{array}$} & \multirow{2}{*}{$\begin{array}{l}\chi^{2} \\
- \\
-\end{array}$} \\
\hline & $\begin{array}{l}i \\
\left(^{\circ}\right)\end{array}$ & $\begin{array}{l}P A \\
\left({ }^{\circ}\right)\end{array}$ & $\begin{array}{c}V_{\text {rot }} \\
\left(\mathrm{km} \mathrm{s}^{-1}\right)\end{array}$ & $\begin{array}{l}j \\
-\end{array}$ & & $\begin{array}{l}a_{\mathrm{Br} \gamma} \\
\left(D_{\star}\right)\end{array}$ & $\begin{array}{l}\text { EW } \\
(\AA)\end{array}$ & \\
\hline$\alpha \operatorname{Col}(\mathrm{HD} 37$ 795) & $35 \pm 5$ & 10 & $350 \pm 10$ & $-0.5 \pm 0.1$ & $2.0 \pm 0.5$ & $5.5 \pm 0.3$ & $7.0 \pm 0.5$ & 4.0 \\
\hline$\kappa \mathrm{CMa}(\mathrm{HD} 50013)$ & $35 \pm 10$ & $25 \pm 10$ & $480 \pm 40$ & $-0.5 \pm 0.2$ & $3.5 \pm 0.5$ & $6.2 \pm 2.0$ & $13.0 \pm 2.0$ & 6.8 \\
\hline$\omega \mathrm{Car}(\mathrm{HD} 89080)$ & $65 \pm 10$ & $5 \pm 5$ & $300 \pm 20$ & $-0.45 \pm 0.1$ & $3.0 \pm 1.0$ & $6.5 \pm 1.0$ & $5.8 \pm 0.5$ & 1.1 \\
\hline P Car (HD 91465$)$ & $70 \pm 10$ & $-25 \pm 10$ & $400 \pm 30$ & $-0.45 \pm 0.1$ & $2.0 \pm 0.5$ & $11.0 \pm 2.0$ & $10.0 \pm 1.0$ & 2.5 \\
\hline$\delta$ Cen (HD 105435) & $35 \pm 15$ & $40 \pm 10$ & $500 \pm 50$ & $-0.5 \pm 0.3$ & $2.0 \pm 1.0$ & $9.0 \pm 2.0$ & $19.2 \pm 2.0$ & 2.3 \\
\hline$\mu$ Cen (HD 120324$)$ & $25 \pm 5$ & $80 \pm 15$ & $510 \pm 20$ & $-0.5 \pm 0.1$ & $<3.0$ & $4.0 \pm 1.0$ & $5.6 \pm 0.3$ & 1.3 \\
\hline$\alpha$ Ara (HD 158 427) & $45 \pm 5$ & $88 \pm 2$ & $480 \pm 20$ & $-0.5 \pm 0.1$ & $<2.0$ & $5.8 \pm 0.5$ & $14.5 \pm 1.0$ & 1.7 \\
\hline
\end{tabular}


Figura 8.35: Distribución de estrellas Be por tipo espectral y CL para la muestra combinada.

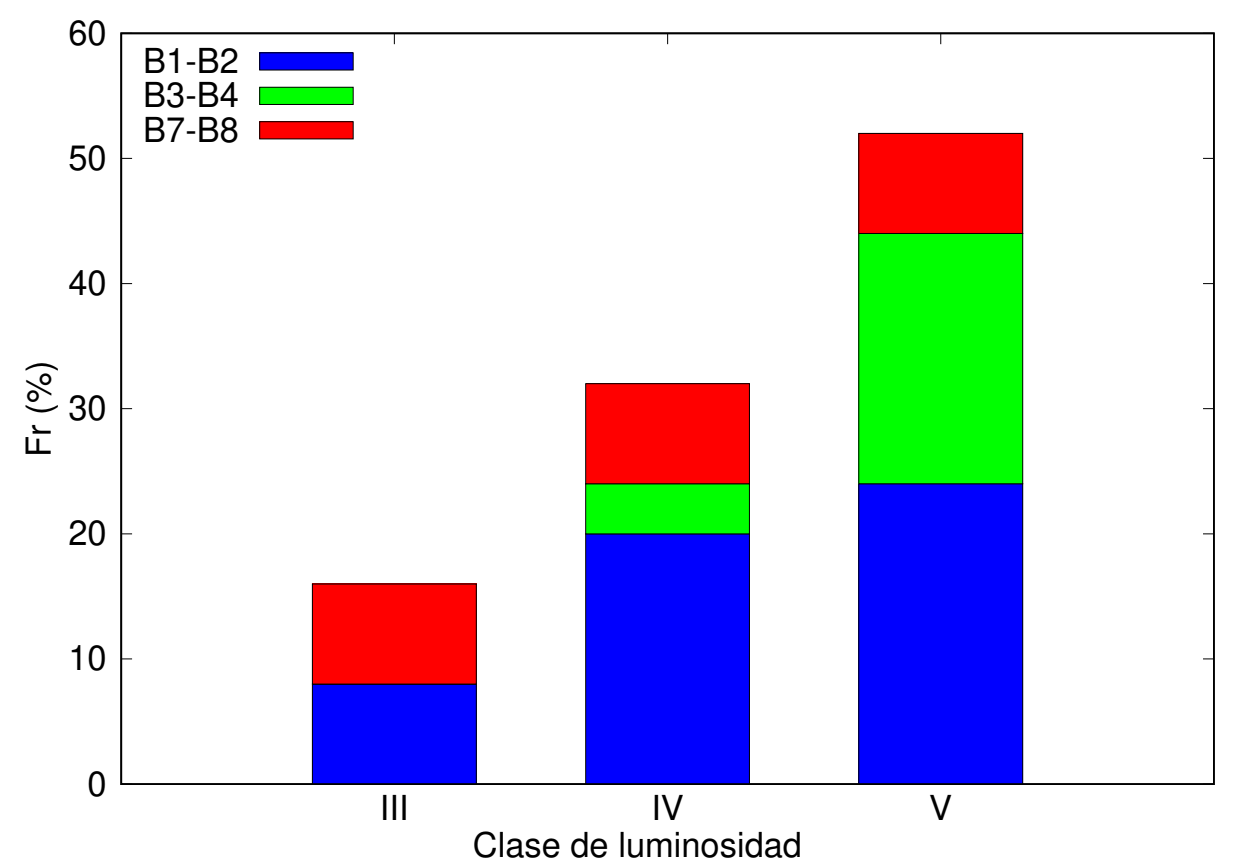

\subsubsection{Tasas de rotación}

Considerando la muestra combinada, encontramos los siguientes valores medios para las tasas de rotación: $\overline{V / V_{\text {crit }}}=0.75 \pm 0.08 \mathrm{y} \overline{\Omega / \Omega_{\text {crit }}}=0.90 \pm 0.05$. Estos valores están de acuerdo con los determinados por Frémat et al. (2005) a partir de una muestra de 130 estrellas Be, i.e. $\overline{V / V_{\text {crit }}} \simeq 0.75 \mathrm{y} \overline{\Omega / \Omega_{\text {crit }}} \simeq 0.88$, y por Zorec et al. $\left(2016, \overline{V / V_{\text {crit }}}=0.77\right)$ sin tener en cuenta correcciones por oscurecimiento gravitatorio, macroturbulencia y binaridad. Sin embargo, como nuestra muestra tiene 7 estrellas binarias, nuestros valores deberían ser considerados límites superiores. En base a este resultado concluimos que, en promedio, nuestra muestra de estrellas Be no rota a la velocidad crítica.

Cranmer (2005) propuso que las tasas de rotación tienen diferentes límites dependiendo de la temperatura efectiva. Para probar esta hipótesis, graficamos los valores de $V / V_{\text {crit }}$ y $\Omega / \Omega_{\text {crit }}$ según la temperatura efectiva. No encontramos ninguna correlación entre los valores de $T_{\text {eff }} \mathrm{y}$ las tasas de rotación, tal como se observa en la Fig. 8.37.

\subsubsection{Orientación de los ejes de rotación}

Con el objetivo de discutir si la distribución de los ejes de rotación es al azar, se comparó la distribución de los ángulos de inclinación de nuestra muestra con una distribución teórica (ver por ejemplo Curé et al., 2014).

Definimos un vector unitario arbitrario $\mathbf{v}$ en el espacio, donde $i$ el ángulo formado entre $\mathbf{v}$ y la línea de la visual. La proyección de $\mathbf{v}$ en el plano normal a la línea de la visual será 
Figura 8.36: Distribución de $i, a_{\mathrm{Br} \gamma}, V_{\text {rot }}, V / V_{\text {crit }} \mathrm{y} \Omega / \Omega_{\text {crit }}$ para la muestra combinada.
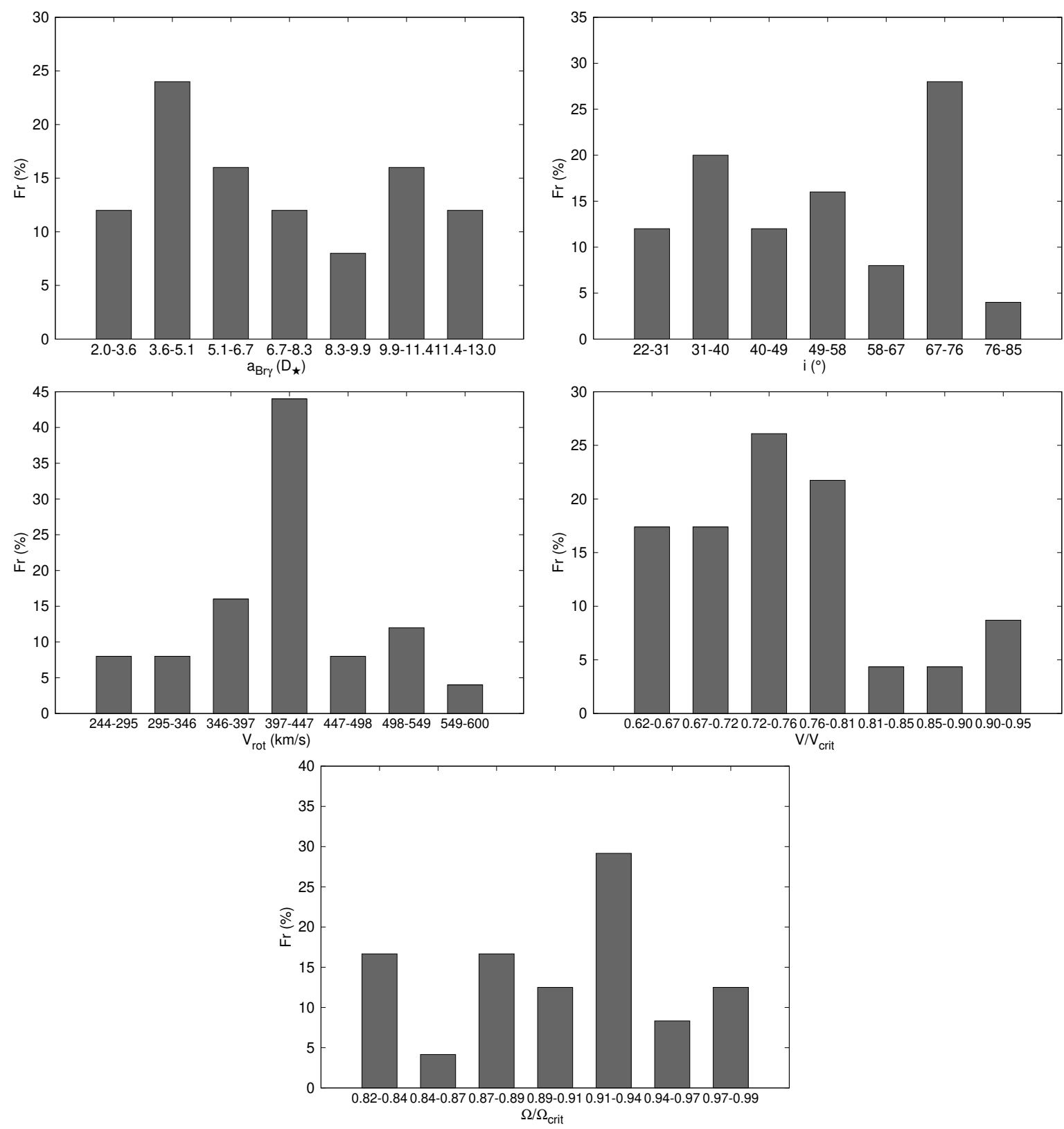

$s=\sin i$. Si consideramos que $\mathbf{v}$ está uniformemente distribuido en la esfera de radio 1 , entonces la función de distribución de probabilidades para $s$ (probability distribution function - PDF) verifica $f(s)=s / \sqrt{1-s^{2}}$ (Curé et al., 2014).

En la Fig. 8.38 presentamos, en línea negra continua, una estimación de la función de distribución de probabilidad de los valores de $\sin i$ observados, obtenida mediante el método de Kernel (kernel density estimation - KDE) junto con la PDF teórica $f(s)$. Para obtener una mejor aproximación a la función de distribución de probabilidad observada, utilizamos el método 
Figura 8.37: Distribuciones de $V / V_{\text {crit }}$ y $\Omega / \Omega_{\text {crit }}$ con la temperatura efectiva. No se observa la correlación propuesta por Cranmer (2005).
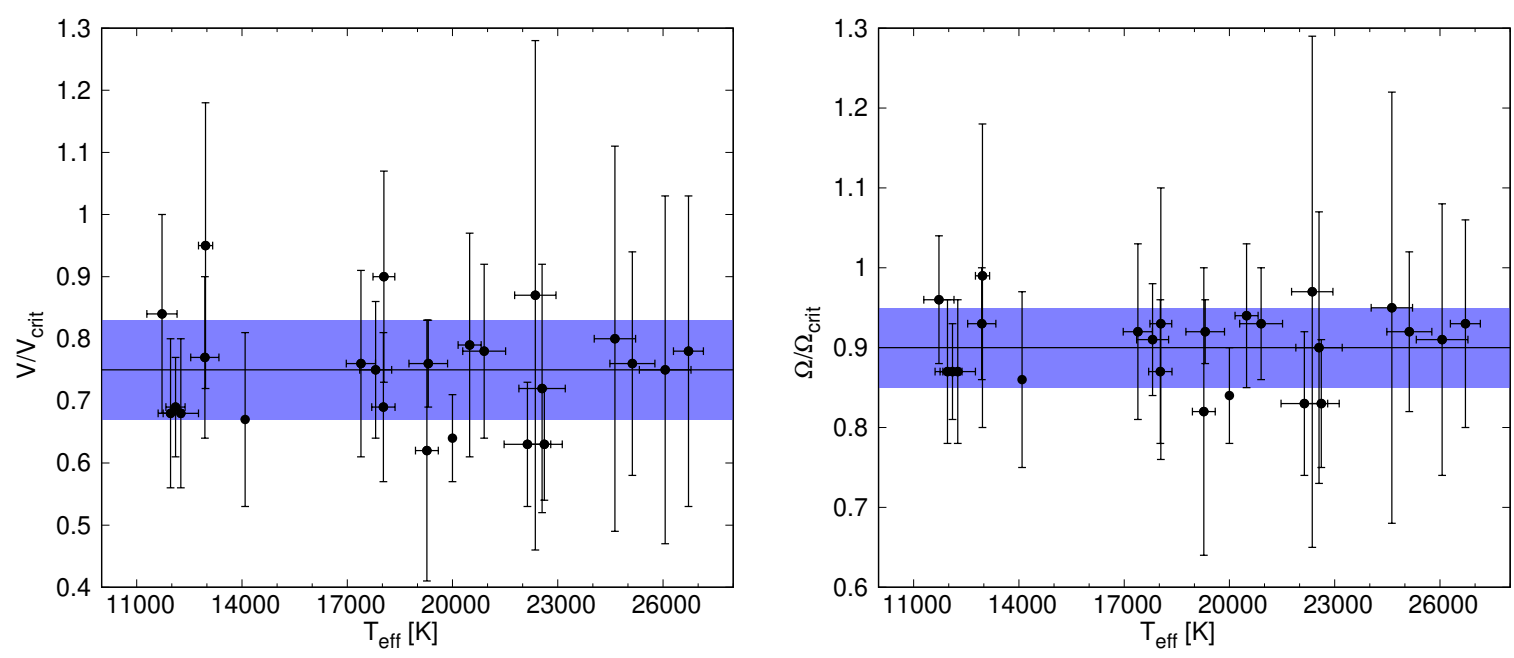

Figura 8.38: La PDF (línea negra continua) fue generada a partir de nuestra muestra con $N=25$. Fue calculada utilizando un algoritmo KDE con un kernel Gaussiano y un ancho de banda Silverman de 0.1029. El intervalo de $95 \%$ de confianza se muestra como la región gris y fue calculado utilizando los cuantiles para 2000 muestra de bootstrap con $N=25$. La PDF teórica $f(s)$ se muestra como una línea punteada.

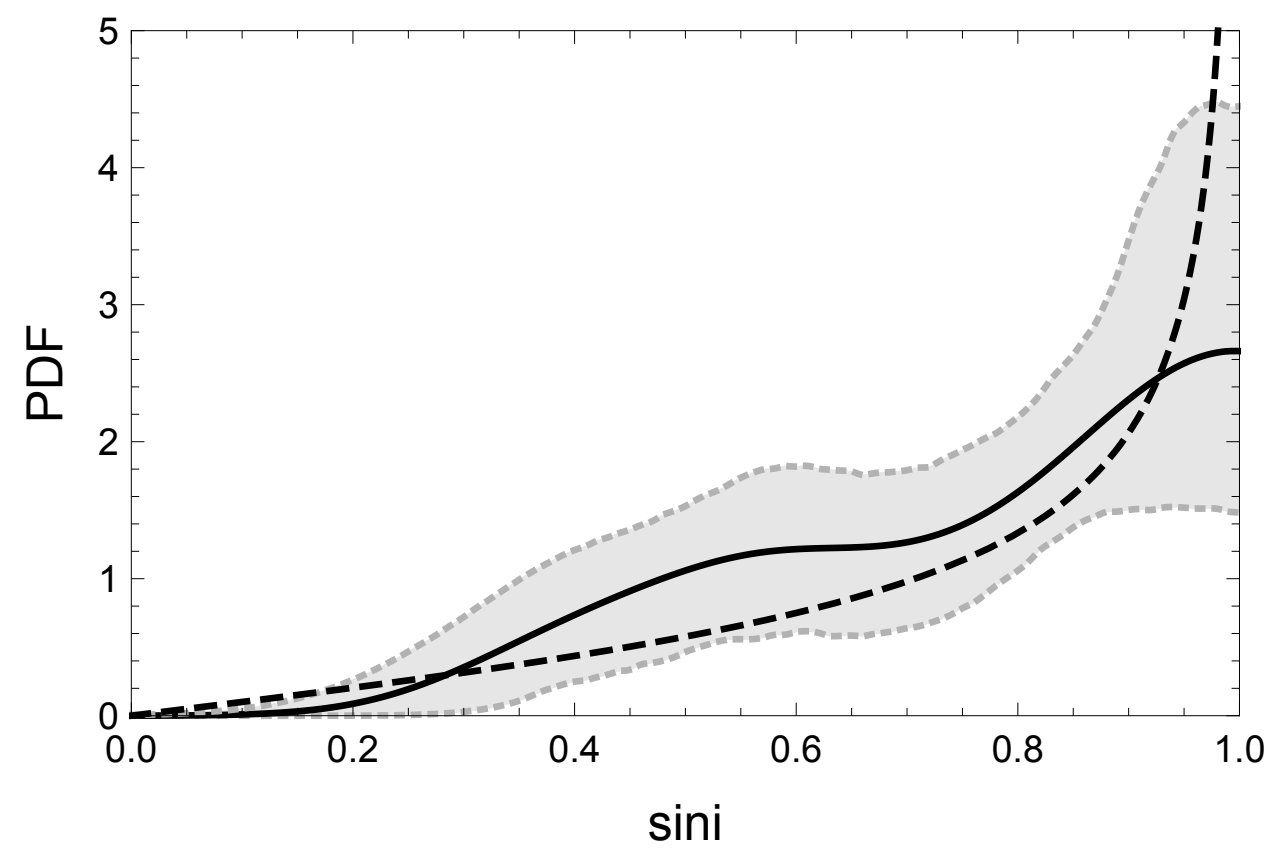

Bootstrap, para lo que generamos 2000 muestras al azar a partir de nuestro valores de $\sin i$, cada una con 25 datos. El intervalo de confidencia resultante, usando los cuantiles 0.025 y 0.975 también se grafican en la figura (región sombreada gris), mostrando que la PDF teórica se 
encuentra casi siempre dentro del intervalo de confianza.

En vista de esto, para verificar sin los datos observados están distribuidos según la PDF $f(s)$ esperada, realizamos tests de Kolmorogov-Smirnov y Anderson-Darling. Obtuvimos un valor $p$ de 0.0686 y 0.0997 , respectivamente. Ambos tests no rechazan la hipótesis, por lo que la muestra puede provenir de una orientación al azar de los ejes de rotación. Sin embargo, los resultados no son del todo concluyentes debido al pequeño valor de $p$, por lo que sugerimos realizar un estudio más detallado con una muestra más grande de datos para dilucidar la distribución subyacente.

A lo largo de este capítulo describimos los parámetros del disco obtenidos mediante el ajuste de modelos a las observaciones interferométricas en $\mathrm{Br} \gamma$. Analizamos además las distribuciones de estos parámetros según el TE y CL de la estrella.

En el próximo capítulo presentaremos un análisis conjunto de los resultados obtenidos mediante las dos técnicas utilizadas. 


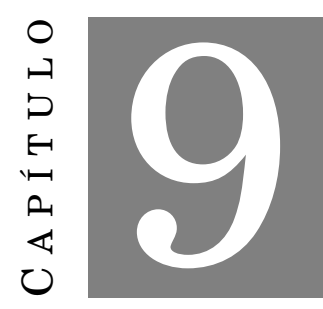

\section{ANÁLISIS CONJUNTO DE LOS DATOS IR E INTERFEROMÉTRICOS}

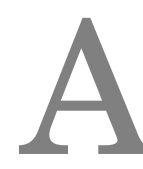

lo largo de este trabajo presentamos, por un lado, el estudio realizado sobre un conjunto de observaciones IR que abarcan la región espectral de la línea $\mathrm{Br} \gamma$ para una muestra de 23 estrellas Be, y por otro el modelado de los datos interferométricos obtenidos para 18 objetos. Estas dos muestras tienen 10 objetos en común, lo que constituye la muestra más extensa observada con técnicas de espectroscopía IR e interferometría. Tanto el análisis de los espectros IR como el ajuste de modelos interferométricos en la línea $\mathrm{Br} \gamma$ nos permitieron obtener propiedades físicas y dinámicas de las envolturas circunestelares. A continuación presentaremos un análisis conjunto de los resultados obtenidos a partir de las dos técnicas.

\subsection{Correlación entre el tamaño del disco y la velocidad de rotación}

Para discutir el origen de las envolturas circunestelares y el papel de la rotación estelar, analizamos la existencia de una relación entre la extensión del disco en la línea Br $\gamma$ y la velocidad de rotación en la base del mismo $V_{\text {rot }}$, obtenidas a partir del modelado de datos interferométricos. Utilizando la muestra combinada de estrellas, que incluye las estrellas para las cuales los parámetros de las envolturas fueron determinados en este trabajo junto con las estudiadas anteriormente por Meilland et al. (2012), notamos que no hay estrellas con discos extensos en la línea $\mathrm{Br} \gamma$ que presenten velocidades de rotación bajas. Además, pudimos definir un límite superior para el tamaño del disco en función de las velocidades de rotación. En la Fig. 9.1 graficamos los tamaños obtenidos para $a_{\mathrm{Br} \gamma}$ en función de $V_{\text {rot }}$, junto con la recta definida como límite.

Considerando la variabilidad observada en muchas estrellas Be, buscamos un criterio para determinar cuales estrellas poseen discos estables y cuales no. Con este propósito, para cada 
Figura 9.1: Relación entre $V_{\text {rot }}$ y la extensión del disco en la línea Br $\gamma$ para la muestra combinada. La línea azul ( $\left.a_{\mathrm{Br} \gamma}=0.043271 \cdot V_{\text {rot }}-5.98045\right)$ marca un límite superior para el tamaño del disco en función de $V_{\text {rot }}$.

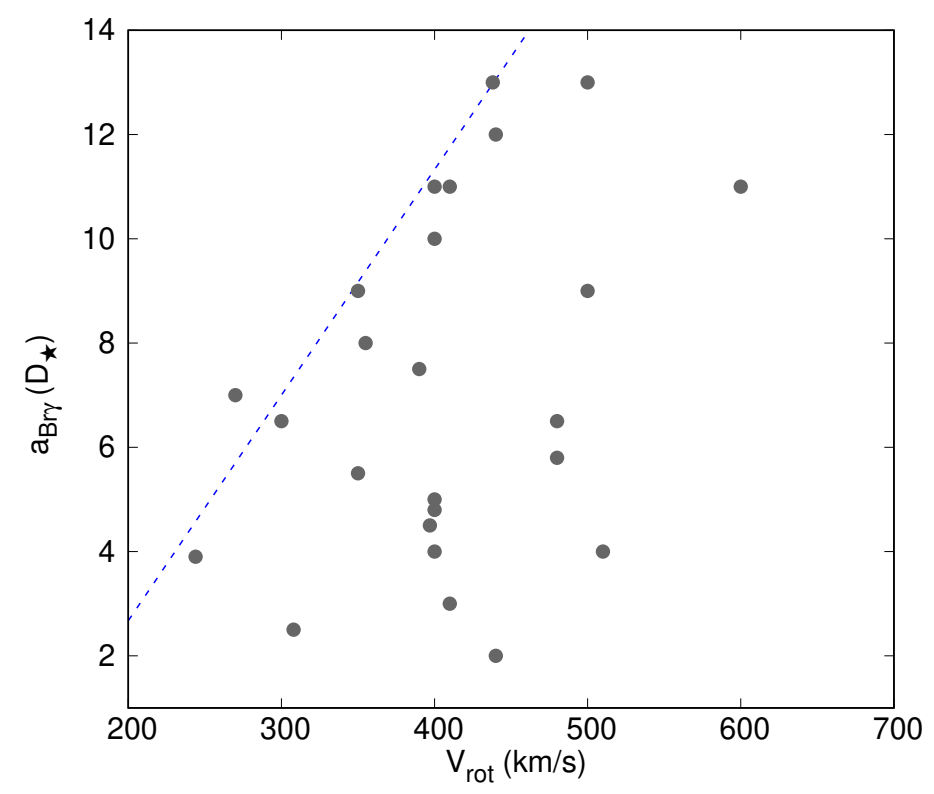

Figura 9.2: Relación entre $V_{\text {rot }}$ y la extensión del disco en la línea $\mathrm{Br} \gamma$ para la muestra combinada. Izquierda: La línea rayada azul y la punteada naranja podrían estar relacionadas con estrellas con discos estables, identificados según los diferentes criterios utilizados. Derecha:

La escala de colores indica la masa de la estrella central. Para una dada $V_{\text {rot }}$, la línea azul rayada marca la ubicación de las estrellas de baja masa con discos estables y la línea naranja punteada la de las estrellas Be de alta masa con discos estables. Se identifican también las estrellas pertenecientes a sistemas binarios.
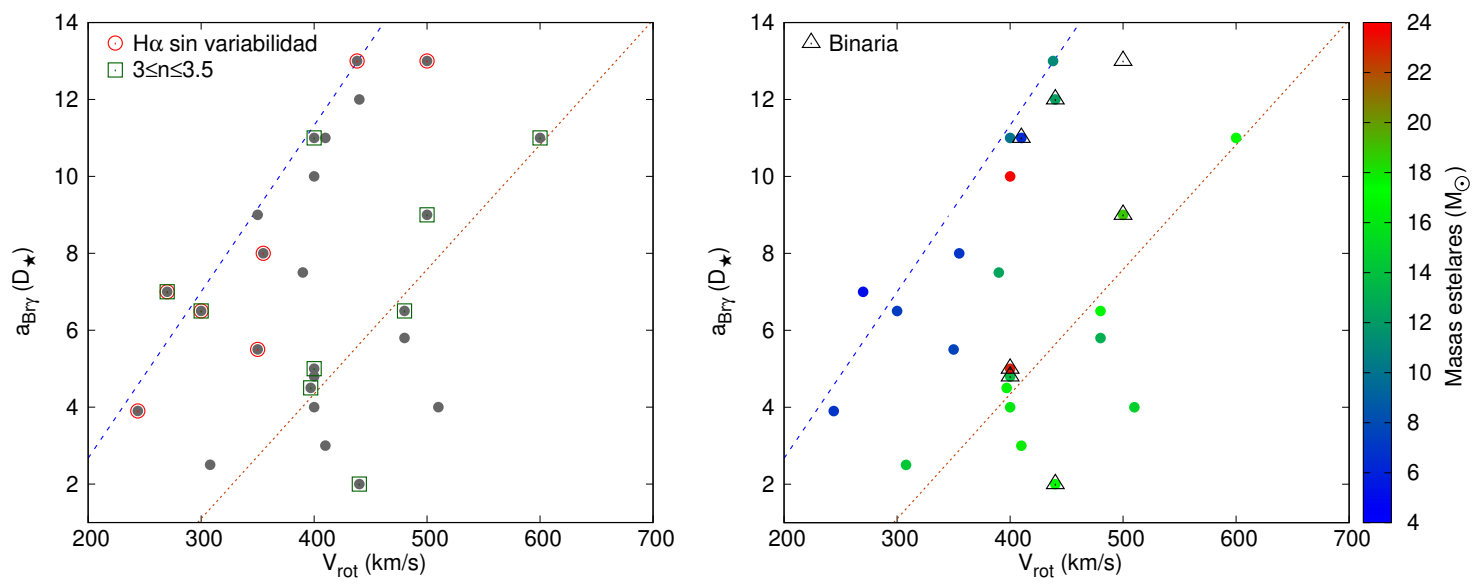

estrella comparamos la intensidad y apariencia de la línea $\mathrm{H} \alpha$ usando observaciones publicadas por Doazan et al. (1991), Silaj et al. (2010), Silaj et al. (2014), Arcos et al. (2017) y Arcos (2017). A partir de esta búsqueda, identificamos las estrellas en las cuales la línea $\mathrm{H} \alpha$ se mantuvo estable en un intervalo de varios años alrededor de la fecha de obtención de los datos interferométricos. 
Figura 9.3: Relación entre $V_{\text {rot }} / V_{\text {crit }}$ y el tamaño del disco en la línea $\mathrm{Br} \gamma$ para la muestra combinada. Las estrellas con mayores tamaño del disco tienen $V_{\text {rot }}$ cercanos a la velocidad de rotación critica.

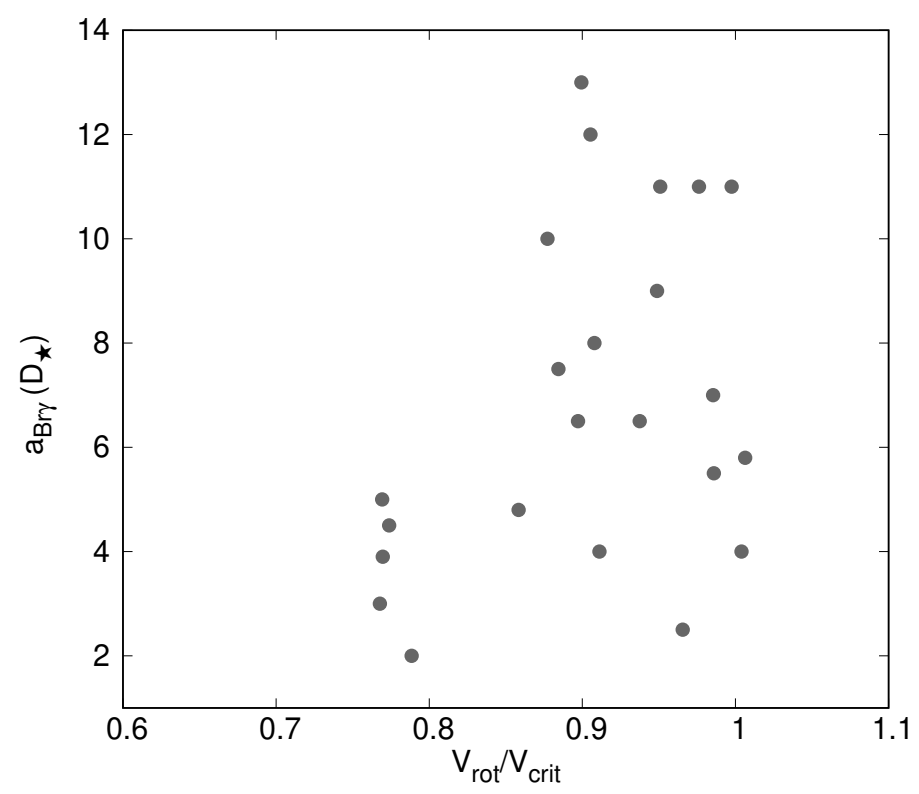

Basados en este criterio, $\eta$ Tau, 105 Tau, 120 Tau, $\alpha$ Col, $\omega$ Car, $o$ Aqr y $\epsilon$ PsA deberían presentar discos estables. Las mismas se encuentran indicadas como "H $\alpha$ sin variabilidad" en la Fig. 9.2 (izquierda).

Otro criterio de estabilidad se basa en los resultados encontrados por Vieira et al. (2017), donde los distintos valores de $n$ están asociados a distintos estados evolutivos para el disco (ver Subsección 2.5.2). Mediante este criterio, Vieira et al. (2017) encontraron que, dentro de las estrellas de nuestra muestra, $\eta$ Tau, 228 Eri, $\Psi_{01}$ Ori, V696 Mon, $\kappa$ CMa, $\omega$ Car, p Car, $\delta$ Cen y $\pi$ Aqr presentaban discos estables en observaciones tomadas en el 2010. En la Fig. 9.2 (izquierda) estos objetos están identificados como " $3 \leq n \leq 3.5$ ". Algunos se ubican en la relación lineal encontrada anteriormente, pero otras parecen formar una segunda regresión lineal graficada con una línea punteada naranja. Esto podría indicar otra condición de estabilidad para la extensión del disco.

En la Fig. 9.3 graficamos el tamaño del disco contra $V_{\text {rot }} / V_{\text {crit }}$. Observamos que las estrellas con discos mayores presentan valores de $V_{\text {rot }} / V_{\text {crit }} \gtrsim 0.9$, aunque no se observa correlación con el tipo espectral (ver Fig. 8.37).

Dado que se ha reportado que las estrellas Be de tipo espectral tardío son menos variables que las tempranas (Hubert \& Floquet, 1998; Jones et al., 2011; Barnsley \& Steele, 2013; LabadieBartz et al., 2017), y que no hemos observado una clara dependencia con el tipo espectral y la clase de luminosidad de la estrella, es posible que las relaciones encontradas anteriormente para los discos estables estén relacionadas con la masa de la estrella. Utilizando los valores obtenidos para $R_{\star}$ y $V_{\text {crit }}$, obtuvimos una estimación para las masas estelares a partir de la 
expresión $V_{\text {crit }}=\sqrt{G M_{\star} / R_{\star}}$, donde $G$ es la constante de gravitación universal. En la Fig. 9.2 (derecha) observamos que para una dada $V_{\text {rot }}$ las estrellas menos masivas están más cerca del límite superior, mientras que las más masivas están cerca del límite inferior. Este resultado señala que las estrellas Be con mayor masa tienen discos más densos y compactos que las de baja masa. A medida que la estrella central es más luminosa, el disco denso y compacto será mas brillante en la línea, resultado que apoya las conclusiones obtenidas por Arcos et al. (2017) a partir del modelado de la línea $\mathrm{H} \alpha$.

Es interesante mencionar que $\pi$ Aqr es una estrella binaria que se ubica por debajo de la línea naranja y presentaba un disco estable según Vieira et al. (2017). Sin embargo, es posible que la separación temporal entre las observaciones utilizadas por Vieira et al. (2017, realizadas entre enero y noviembre del 2010) y nuestras observaciones interferométricas (tomadas en octubre del 2014) no sea despreciable frente a la escala de tiempo de evolución del disco, ya que Arcos et al. (2017) observaron este objeto en 2012, 2013 y 2015 y en todos los casos determinaron valores de $n$ que la ubicaba en la región de disipación del disco. Por lo tanto, es posible que el tamaño del disco encontrado en este trabajo a partir del modelado de datos interferométricos no corresponda al tamaño correspondiente a la fase estable.

En base a este resultado, es interesante analizar si existe alguna correlación entre la velocidad de rotación de la estrella y los radios internos de las regiones de formación encontrados a partir del análisis de los espectros IR. A partir de los valores de $V \sin i$ e $i$ obtuvimos $V=V \sin i / \sin i$, utilizando los valores determinados en el Capítulo 8 o los determinados por Frémat et al. (2005). En la Fig. 9.4 se muestran los radios internos de las regiones de formación de $\mathrm{Br} \gamma$ obtenidos a partir de la espectroscopía IR en función de $V \sin i / \sin i$. Al observar esta figura deben tenerse en cuenta no solo los diferentes valores de los radios internos de las regiones de formación obtenidos para la línea $\mathrm{Br} \gamma$, sino también el estado evolutivo del disco (en formación - en disipación estable). Por ejemplo, los radios obtenido para las distintas observaciones de EW Lac y BK Cam (que presentan velocidades de rotación en el rango $350-400 \mathrm{~km} / \mathrm{s}$ ) son mayores que los obtenidos para $28 \mathrm{Tau}$ (con $V \sim 300 \mathrm{~km} / \mathrm{s}$ ), presentando las tres estrellas en alguna observación un disco estable. Por otro lado, $48 \mathrm{Lib}$ presenta un radio pequeño en $\mathrm{Br} \gamma$ a pesar de presentar el mayor valor para la velocidad de rotación, y según en análisis presentado en el Capítulo 7 presentaba un disco en disipación. Por lo tanto, el tamaño medido podría no ser el máximo alcanzado.

Si bien las dos técnicas utilizadas nos permiten obtener diferentes parámetros de las envolturas ${ }^{1}$, a partir de ambas encontramos que existiría una diferencia en los tamaños alcanzados por los discos circunestelares en función de la velocidad de rotación. Según este análisis, sería posible que las estrellas con velocidades de rotación menores no lleguen a desarrollar envolturas tan grandes como aquellas con mayor velocidad de rotación.

\footnotetext{
${ }^{1}$ A partir del análisis de los espectros IR logramos obtener los radios internos de las regiones de formación de las líneas, mientras que los modelos interferométricos nos permiten determinar velocidades de rotación en la base del disco junto con el FWHM de la Gaussiana utilizada para modelar la emisión del disco en $\mathrm{Br} \gamma$.
} 
Figura 9.4: Radios internos de las regiones de formación de $\operatorname{Br} \gamma \operatorname{según} V=V \sin i / \sin i$.

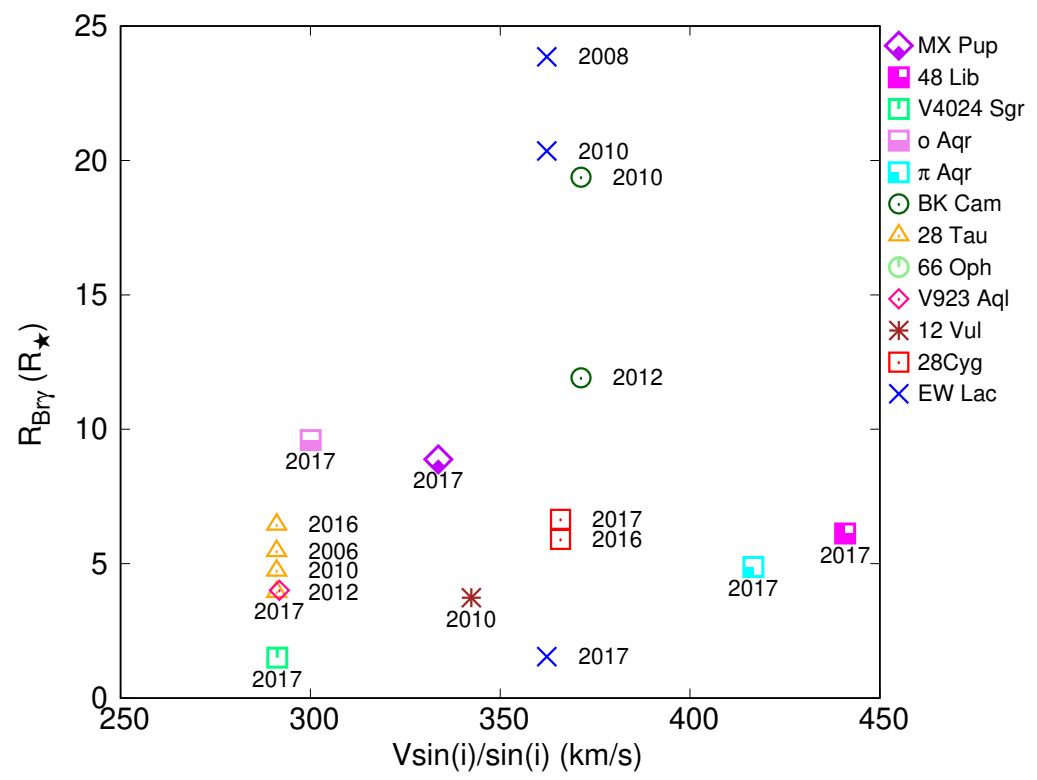

\subsection{Comentarios sobre objetos particulares estudiados con ambas técnicas}

A partir de los espectros obtenidos en el IR y del modelado de datos interferométricos obtuvimos parámetros para las envolturas de 31 estrellas Be. Dentro de esa muestra de objetos, 10 fueron estudiados utilizando ambas técnicas. A continuación presentamos algunos comentarios sobre estos objetos.

- $28 \mathrm{Tau}$ : Las observaciones en el IR realizadas a partir del 2006 muestran un perfil con $\mathrm{Br} \gamma$ en emisión. A partir de ellas obtuvimos evidencias del desarrollo y la estabilización de un nuevo disco. Esto es consistente con los parámetros obtenidos a partir del modelado de las observaciones interferométricas realizadas en 2014, ya que los mismos la ubican en una región cercana a la recta correspondiente al límite superior para los tamaños del disco según $V_{\text {rot }}$.

- 228 Eri: En el ajuste de los datos interferométricos de este objeto fue necesario incluir una sobredensidad en forma de brazo espiral debido a la gran asimetría observada. La estrella presentaba un disco estable (Vieira et al., 2017) y se ubicaba sobre la recta límite correspondiente a las estrellas de alta masa. Esta asimetría no se observó en los espectros IR obtenidos en el 2017. A partir de los espectros IR, propusimos que la estrella presentaba un disco en desarrollo, lo cual es consistente con pequeñas variaciones observadas en la emisión de la línea $\mathrm{H} \alpha^{2}$.

\footnotetext{
${ }^{2} \mathrm{Los}$ espectros en la línea $\mathrm{H} \alpha$ fueron analizados a partir de de los datos disponibles en la base de datos de estrellas Be (Be Star Spectra database - BeSS, http://basebe.obspm.fr/basebe/)
} 
- 105 Tau: A partir de los parámetros del disco obtenidos mediante el modelado de los datos interferométricos, determinamos que el tamaño de la envoltura era inferior al valor máximo esperado según $V_{\text {rot }}$. Este objeto presentaba en los años cercanos a 2014 un perfil estable en la línea $\mathrm{H} \alpha$ en emisión. La intensidad de $\mathrm{H} \alpha$ decayó débilmente alrededor del 2016, y luego volvió a intensificarse, lo cual es consistente con la clasificación de un disco en desarrollo obtenida a partir de los espectros IR adquiridos en el 2017. Es probable que el tamaño alcanzado en el 2017 sea aún mayor que en el 2014.

- $\Psi_{01}$ Ori: En 2014, los parámetros obtenidos a partir del ajuste de modelos interferométricos la ubican sobre la recta correspondiente a las estrellas de alta masa, con un disco estable según Vieira et al. (2017). Esto coincide con un máximo en la intensidad de la línea H $\alpha$. La intensidad de esta línea luego decae y vuelve a aumentar en el 2015, consistente con la hipótesis de un disco en desarrollo obtenida a partir de los espectros IR del 2017.

- 120 Tau: A partir de los datos interferométricos obtenidos en 2014, ubicamos a este objeto sobre la recta límite para el tamaño de la envoltura en función de $V_{\text {rot }}$. Sin embargo, a partir del análisis de los espectros IR, obtuvimos que el disco se encontraba en desarrollo. Esto es consistente con pequeñas variaciones observadas en la intensidad de $\mathrm{H} \alpha$, que indicarían diferentes episodios de crecimiento y decrecimiento del disco.

- $\omega$ Ori: Se encuentra sobre la recta correspondiente a las estrellas de alta masa a partir de los parámetros determinados mediante el ajuste de modelos interferométricos. Alrededor de la fecha de obtención de estos datos $\mathrm{H} \alpha$ mantuvo una intensidad estable, que comenzó a decaer a partir de 2016. Esto concuerda con el proceso de disipación del disco propuesto a partir de los espectros IR.

- V696 Mon: Los parámetros obtenidos a partir del ajuste de modelos interferométricos la ubican sobre la recta correspondiente a las estrellas de alta masa. A partir de las observaciones espectroscópicas en el IR obtuvimos que el disco estaba en desarrollo en el año 2017, lo que se corresponde con un aumento en la intensidad de $\mathrm{H} \alpha$.

- MX Pup: Según Vieira et al. (2017), la envoltura de este objeto se encontraba en disipación en el 2010. A partir de los datos interferométricos, en 2014 obtuvimos un tamaño pequeño para la envoltura. Luego, a partir de lo obtenido en el IR en 2017, propusimos que el disco estaba en desarrollo o estable. En esta observación el radio interno para la región de formación de $\mathrm{Br} \gamma$ era mayor al FWHM de la Gaussiana usada para modelar el disco en emisión en 2014. Esto indicaría que a lo largo de estos años la estrella ha pasado por uno o varios eventos de disipación/formación del disco.

- o Aqr: El tamaño obtenido para el disco a partir del modelado de datos interferométricos la ubicaba cerca de la recta límite según su velocidad de rotación. A partir de los espectros 
obtenidos en la región IR en 2017 propusimos que el disco habría comenzado un proceso de disipación, y dado que el radio obtenido es aún grande creemos que el mismo era reciente.

- $\pi$ Aqr: Según los parámetros obtenidos a partir de los datos interferométricos, la envoltura presentaba en 2014 un tamaño inferior al valor definido como límite superior según $V_{\text {rot }}$, y habría comenzado el proceso de disipación. A partir de los espectros obtenidos en el IR en 2017, determinamos que esta estrella presentaba un disco en desarrollo. El radio interno de la región de formación de $\mathrm{Br} \gamma$ obtenido en 2017 es mayor que el FWHM de la Gaussiana usada para modelar el disco en 2014. Esto apoya la hipótesis de que el tamaño obtenido a partir de la interferometría no corresponde al máximo posible para esta envoltura.

Notamos que muchas estrellas presentan variaciones relacionadas con procesos de formación y disipación del disco en escalas de tiempo relativamente cortas (de algunos meses a unos pocos años). Esto nos motiva a hacer un seguimiento temporal en un amplio rango espectral de objetos individuales, a fin de describir más precisamente la evolución de las envolturas, su escala temporal y su relación con los procesos que intervienen en la formación/disipación de los discos. En base a nuestros resultados proponemos que la velocidad de rotación establece un rango para la variabilidad y una cota máxima para el tamaño de los discos. Sin embargo, la rotación no parece ser el mecanismo disparador de la variabilidad. 



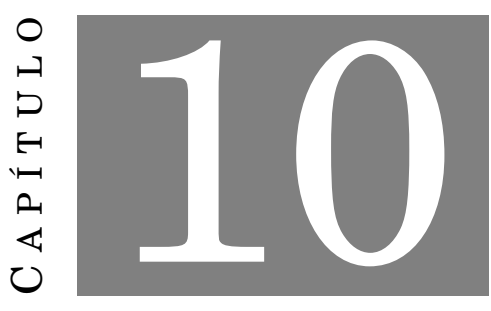

Conclusiones

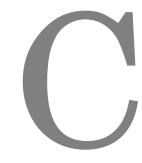

on el fin de contribuir a mejorar el conocimiento acerca de las características físicas y cinemáticas de los discos de las estrellas Be, hemos analizado espectros IR de una muestra de 23 estrellas Be obtenidos en un amplio rango espectral en forma simultánea. Esto es un avance respecto a los trabajos previos encontrados en la literatura, centrados en el análisis de unos pocos objetos o en un rango espectral acotado. Además, para 8 de los objetos de la muestra estos son los primeros espectros IR reportados. El análisis de estos espectros nos permitió determinar propiedades físicas de las envolturas y analizar su evolución temporal.

De manera complementaria, analizamos datos interferométricos de una muestra de 26 estrellas Be, a partir de los cuales obtuvimos parámetros geométricos y cinemáticos de las envolturas de 18 objetos que presentaban la línea $\mathrm{Br} \gamma$ en emisión. Para 5 de estos objetos se reportan por primera vez los parámetros del disco.

A continuación presentamos los principales resultados de esta tesis:

- Realizamos un Atlas de espectros IR que contiene una gran muestra de estrellas Be observadas en un amplio rango espectral.

- Clasificamos a las estrellas según la intensidad de las líneas en emisión observadas en la banda L en diferentes grupos (según la clasificación propuesta por Mennickent et al., 2009), y las ubicamos en diagramas de cocientes de flujos utilizados previamente en la literatura (Lenorzer et al., 2002b; Granada et al., 2010). Al analizar los cocientes entre los anchos equivalentes (o flujos) de las líneas $\mathrm{Br} \alpha$ y $\mathrm{Br} \gamma$, encontramos que hay estrellas que presentan propiedades intermedias entre los grupos I y II. Esto nos permitió delimitar una región de transición entre ambos grupos en el diagrama de Lenorzer. Los cocientes entre los anchos equivalentes de $\mathrm{Pa} \alpha$ y $\mathrm{Pa} \beta$ también permiten obtener información sobre la pertenencia a los distintos grupos. 
- Propusimos diagramas de cocientes de flujos que utilizan líneas presentes en las bandas J, H y K. Los mismos permiten obtener propiedades físicas de las envolturas, de manera similar al diagrama de Lenorzer, y son de utilidad en el caso de no poseer observaciones en la banda L.

- Determinamos densidades columnares, extensiones relativas y radios internos de las regiones de formación de las distintas líneas observadas.

- Analizamos la variabilidad de estos parámetros en las estrellas para las cuales realizamos un seguimiento temporal, lo que nos permitió estudiar la evolución de las envolturas. Además, pudimos proponer estados evolutivos para las envolturas de las estrellas que fueron observadas en una sola oportunidad, comparando sus propiedades con las obtenidas para los objetos observados en varias oportunidades.

- A partir del modelado de datos interferométricos obtuvimos parámetros geométricos y cinemáticos del disco para las 18 estrellas Be que presentaban la línea $\mathrm{Br} \gamma$ en emisión al momento de la observación.

- Los tamaños de los discos en $\mathrm{Br} \gamma$ son similares a los obtenidos a partir de la línea $\mathrm{H} \alpha$, entre $2 D \star$ y $13 D \star$. No observamos correlación entre el tamaño del disco y la $T_{\text {eff }}$ o TE de la estrella central. Encontramos que un tercio de la muestra tiene discos menores a $6 D \star y$ las estrellas con CL III tienden a presentar los discos más pequeños.

- En general, los discos rotan siguiendo una rotación cercana a la Kepleriana, con la excepción de HD 28 497. El disco de esta estrella debió ser modelad considerando una sobredensidad de tipo brazo espiral.

- La función de distribución de las velocidades de rotación es simétrica, con un máximo en $V_{\text {rot }} \sim 365 \mathrm{~km} \mathrm{~s}^{-1}$. Las estrellas con tipos espectrales tempranos tienden a tener mayor velocidad de rotación, mientras que las tardías presentan valores más bajos. Determinamos valores medios de $\overline{V / V_{\text {crit }}}=0.75 \pm 0.08$ y $\overline{\Omega / \Omega_{\text {crit }}}=0.90 \pm 0.05$, por lo que confirmamos que, en promedio, las estrellas Be no rotan a la velocidad crítica. Este resultado sugiere la necesidad de considerar un mecanismo adicional a la rotación que contribuya a la formación de la envoltura circunestelar. No encontramos correlación entre $V / V_{\text {crit }} \mathrm{y}$ el tipo espectral, contrario a lo sugerido por Cranmer (2005).

- La distribución de inclinaciones de los ejes de rotación con respecto al plano del cielo es uniforme. Sin embargo, este resultado no es del todo concluyente por lo que es necesario realizar más observaciones.

- Utilizando una muestra extendida de estrellas Be (nuestras 18 estrellas más 7 estudiadas por Meilland et al., 2012) encontramos un límite superior para la extensión del disco en 
$\mathrm{Br} \gamma$ que correlaciona con la velocidad de rotación en la parte interna del disco y con la masa estelar. Las estrellas más masivas tienen discos más compactos y densos que sus contrapartes de menor masa. Este resultado es reforzado con lo obtenido a partir de los espectros IR, donde los radios internos de las regiones de formación de la línea Bry de las estrellas con mayores velocidades de rotación abarcan un rango de valores más amplio que aquellas con menor velocidad de rotación.

- Encontramos evidencia de variaciones relacionadas con procesos de formación/disipación del disco en escalas de tiempo relativamente cortas (de algunos meses a unos pocos años).

A partir de los resultados encontrados, resaltamos la importancia de realizar un seguimiento temporal de una muestra de estrellas Be para estudiar la variabilidad de las envolturas. Hemos podido detectar la presencia de discos estables o en fases de formación y disipación, donde el rango de variabilidad de los parámetros del disco se relaciona con la velocidad de rotación. Esto nos motiva a realizar un muestreo temporal sistemático en intervalos más cortos de tiempo y en un amplio rango espectral de objetos de interés particular, a fin de seguir el desarrollo de los discos y comprender mejor los procesos de formación y disipación de los mismos. Además, sería interesante complementar los resultados con el modelado de la línea Br $\gamma$ utilizando modelos de discos, para discutir si el tamaño máximo encontrado (alrededor de $13 D_{\star}$ ) tiene un significado físico en términos de la estructura de densidad y ionización de un disco completamente desarrollado. 



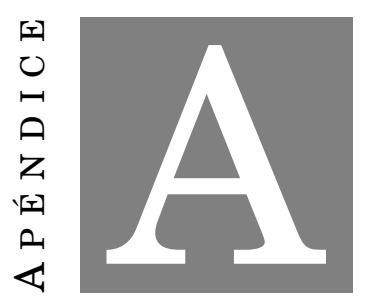

\section{Mediciones CORRESPONDientes AL CAPÍtulo 5}

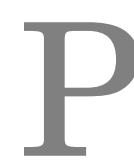
ara cada objeto presentado en el Capítulo 5 mostramos dos tablas. La primera lista los valores obtenidos para los anchos equivalentes (EW) en $\AA$, los flujos (Fl) en unidades de $10^{-13} \frac{\mathrm{erg}}{\mathrm{cm}^{2} \mathrm{~s} \AA}$ y los anchos a mitad de altura (FWHM) en $\mathrm{km} / \mathrm{s}$ de las líneas de hidrógeno presentes en cada banda. Aquellas líneas distinguibles por sobre el nivel del continuo, pero cuyos anchos equivalentes y flujos son difíciles de medir están indicadas con "em", en el caso de líneas en emisión y "shell", en el caso de líneas de envoltura. Para los anchos equivalentes consideramos valores positivos para representar las líneas en emisión (contrario a lo estándar). La segunda tabla de cada objeto muestra los valores de las densidades columnares $(N)$ en unidades de $\frac{10^{14}}{\mathrm{~cm}^{2}} \mathrm{y}$ las extensiones relativas de las regiones de formación $\left(\frac{\Delta R}{\Delta R(\text { ref })}\right)$ obtenidos con el método $N$; los radios internos de las regiones de formación relativos a una de referencia $\left(\frac{R}{R(\text { ref })}\right)$ obtenidos con el método FWHM; y los valores de la separación de los picos para las líneas con dos picos en emisión $(\Delta V)$ en $\mathrm{km} / \mathrm{s}$, los radios internos de las regiones de formación $(R)$ en $R_{\star} \mathrm{y}$ los radios internos de las regiones de formación respecto a una línea de referencia $\left(\frac{R}{R(\mathrm{ref})}\right)$, calculados utilizando el método $\Delta V$. 
Tabla A.1: 48 Per - Anchos equivalentes, flujos y FWHM de las líneas de hidrógeno.

\begin{tabular}{|c|c|c|c|c|c|c|c|c|c|c|c|}
\hline & \multicolumn{3}{|c|}{ Banda H } & & \multicolumn{3}{|c|}{ Banda K } & & \multicolumn{3}{|c|}{ Banda L } \\
\hline & EW & $\mathrm{Fl}$ & FWHM & & $\mathrm{EW}$ & $\mathrm{Fl}$ & FWHM & & EW & $\mathrm{Fl}$ & FWHM \\
\hline $\mathrm{Br}_{9}$ & 19.28 & 571.26 & 242 & $\mathrm{Pf}_{16}$ & \multicolumn{3}{|c|}{ em } & $\mathrm{Hu}_{13}$ & 39.43 & 78.99 & 286 \\
\hline $\mathrm{Br}_{10}$ & 11.76 & 369.51 & 188 & $\mathrm{Pf}_{17}$ & 12.91 & 121.67 & 265 & $\operatorname{Br} \alpha$ & 107.97 & 227.92 & 170 \\
\hline $\mathrm{Br}_{11}$ & 10.92 & 364.62 & 206 & $\mathrm{Pf}_{18}$ & 12.16 & 119.86 & 288 & $\mathrm{Hu}_{14}$ & 34.86 & 72.82 & 292 \\
\hline $\mathrm{Br}_{12}$ & 9.92 & 349.66 & 216 & $\mathrm{Pf}_{19}$ & 11.29 & 112.26 & 310 & $\mathrm{Hu}_{15}$ & 31.41 & 71.15 & 304 \\
\hline $\mathrm{Br}_{13}$ & 9.31 & 344.56 & 250 & $\mathrm{Pf}_{20}$ & 9.38 & 95.37 & 328 & $\mathrm{Hu}_{16}$ & 29.05 & 69.09 & 337 \\
\hline $\mathrm{Br}_{14}$ & 8.25 & 339.40 & 246 & $\mathrm{Pf}_{21}$ & 7.35 & 75.98 & 317 & $\mathrm{Hu}_{17}$ & 24.36 & 63.10 & 337 \\
\hline $\mathrm{Br}_{15}$ & 6.38 & 271.02 & 224 & $\mathrm{Pf}_{22}$ & 6.94 & 72.56 & 359 & Pf $\gamma$ & 39.96 & 104.71 & 228 \\
\hline $\mathrm{Br}_{16}$ & 6.23 & 278.35 & 248 & $\mathrm{Pf}_{23}$ & 5.43 & 57.81 & 311 & $\mathrm{Hu}_{18}$ & 20.43 & 54.11 & 332 \\
\hline $\mathrm{Br}_{17}$ & 5.70 & 261.76 & 279 & $\mathrm{Pf}_{24}$ & 3.64 & 39.94 & 285 & $\mathrm{Hu}_{19}$ & 18.95 & 51.69 & 358 \\
\hline $\mathrm{Br}_{18}$ & 4.01 & 188.70 & 220 & $\mathrm{Pf}_{25}$ & 3.71 & 40.80 & 312 & $\mathrm{Hu}_{20}$ & 15.06 & 42.40 & 351 \\
\hline $\mathrm{Br}_{19}$ & 4.51 & 215.70 & 306 & $\mathrm{Pf}_{26}$ & 3.19 & 35.11 & 341 & $\mathrm{Hu}_{21}$ & 13.58 & 39.16 & 377 \\
\hline $\mathrm{Br}_{20}$ & 4.13 & 200.08 & 310 & $\mathrm{Pf}_{27}$ & 2.14 & 24.12 & 254 & $\mathrm{Hu}_{22}$ & 13.04 & 38.72 & 439 \\
\hline $\mathrm{Br}_{21}$ & 3.32 & 163.87 & 300 & $\mathrm{Pf}_{28}$ & 1.48 & 16.95 & 216 & $\mathrm{Hu}_{23}$ & 8.79 & 26.83 & 347 \\
\hline $\mathrm{Br}_{22}$ & 2.50 & 125.70 & 288 & $\mathrm{Pf}_{29}$ & 1.06 & 12.34 & 209 & $\mathrm{Hu}_{24}$ & 7.96 & 24.57 & 378 \\
\hline $\mathrm{Br}_{23}$ & 3.06 & 159.10 & 331 & $\mathrm{Pf}_{30}$ & 1.12 & 13.14 & 306 & $\mathrm{Hu}_{25}$ & 5.07 & 15.99 & 334 \\
\hline $\mathrm{Br}_{24}$ & & em & & $\mathrm{Pf}_{31}$ & 0.52 & 6.24 & - & $\mathrm{Hu}_{26}$ & 5.60 & 17.91 & 317 \\
\hline $\mathrm{Br}_{25}$ & & em & & $\mathrm{Pf}_{32}$ & 0.53 & 6.38 & - & $\mathrm{Hu}_{27}$ & 3.78 & 12.22 & 326 \\
\hline $\mathrm{Br}_{26}$ & & em & & $\mathrm{Pf}_{33}$ & 0.28 & 3.35 & - & $\mathrm{Hu}_{28}$ & 3.47 & 11.34 & 347 \\
\hline \multirow[t]{4}{*}{$\mathrm{Br}_{27}$} & \multirow{4}{*}{\multicolumn{3}{|c|}{ em }} & $\mathrm{Pf}_{34}$ & 0.27 & 3.21 & - & $\mathrm{Hu}_{29}$ & 3.86 & 12.82 & 311 \\
\hline & & & & $\mathrm{Pf}_{35}$ & 0.13 & 1.52 & - & $\mathrm{Hu}_{30}$ & 3.64 & 12.17 & 337 \\
\hline & & & & $\mathrm{Br} \gamma$ & 19.73 & 287.95 & 197 & $\mathrm{Hu}_{31}$ & 3.97 & 13.49 & 449 \\
\hline & & & & $\operatorname{Br} \delta$ & 23.39 & 504.61 & 285 & $\operatorname{Pf} \delta$ & 43.62 & 173.86 & 255 \\
\hline
\end{tabular}

Tabla A.2: 48 Per - Densidades columnares y tamaños de las regiones emisoras de las líneas de las series de Humphreys, Pfund y Brackett a partir de los métodos $N$ y FWHM.

\begin{tabular}{|c|c|c|c|c|c|c|c|c|c|c|c|}
\hline & \multicolumn{2}{|c|}{ Método $N$} & \multirow{2}{*}{$\begin{array}{c}\text { Método FWHM } \\
\frac{R\left(\mathrm{Hu}_{\mathrm{n}}\right)}{R\left(\mathrm{Hu}_{14}\right)}\end{array}$} & & \multicolumn{2}{|c|}{ Método $N$} & \multirow{2}{*}{$\begin{array}{c}\text { Método FWHM } \\
\frac{R\left(\mathrm{Pf}_{\mathrm{n}}\right)}{R(\mathrm{Pf} \gamma)}\end{array}$} & & \multicolumn{2}{|c|}{ Método $N$} & \multirow{2}{*}{$\begin{array}{c}\text { Método FWHM } \\
\frac{R\left(\mathrm{Br}_{\mathrm{n}}\right)}{R\left(\mathrm{Br}_{9}\right)}\end{array}$} \\
\hline & $N$ & $\frac{\Delta R\left(\mathrm{Hu}_{\mathrm{n}}\right)}{\Delta R\left(\mathrm{Hu}_{14}\right)}$ & & & $N$ & $\frac{\Delta R\left(\mathrm{Pf}_{\mathrm{n}}\right)}{\Delta R(\mathrm{Pf} \gamma)}$ & & & $N$ & $\frac{\Delta R\left(\mathrm{Br}_{\mathrm{n}}\right)}{\Delta R\left(\mathrm{Br}_{9}\right)}$ & \\
\hline $\mathrm{Hu}_{13}$ & 3.21 & 0.84 & 1.04 & $\operatorname{Pf} \gamma$ & 1.30 & 1.00 & 1.00 & $\operatorname{Br} \alpha$ & - & - & 2.03 \\
\hline $\mathrm{Hu}_{14}$ & 3.80 & 1.00 & 1.00 & $\operatorname{Pf} \delta$ & 2.33 & 1.79 & 0.8 & $\mathrm{Br} \gamma$ & - & - & 1.51 \\
\hline $\mathrm{Hu}_{15}$ & 3.78 & 0.99 & 0.92 & $\mathrm{Pf}_{17}$ & 8.40 & 6.45 & 0.74 & $\operatorname{Br} \delta$ & - & - & 0.72 \\
\hline $\mathrm{Hu}_{16}$ & 3.31 & 0.87 & 0.75 & $\mathrm{Pf}_{18}$ & 4.47 & 3.43 & 0.63 & $\mathrm{Br}_{9}$ & 3.82 & 1.00 & 1.00 \\
\hline $\mathrm{Hu}_{17}$ & 2.56 & 0.67 & 0.75 & $\mathrm{Pf}_{19}$ & - & - & 0.54 & $\mathrm{Br}_{10}$ & 5.78 & 1.52 & 1.66 \\
\hline $\mathrm{Hu}_{18}$ & 2.75 & 0.72 & 0.77 & $\mathrm{Pf}_{20}$ & - & - & 0.48 & $\mathrm{Br}_{11}$ & 7.59 & 1.99 & 1.38 \\
\hline $\mathrm{Hu}_{19}$ & - & - & 0.67 & $\mathrm{Pf}_{21}$ & - & - & 0.52 & $\mathrm{Br}_{12}$ & 8.99 & 2.35 & 1.26 \\
\hline $\mathrm{Hu}_{20}$ & - & - & 0.69 & $\mathrm{Pf}_{22}$ & - & - & 0.4 & $\mathrm{Br}_{13}$ & 10.52 & 2.75 & 0.94 \\
\hline $\mathrm{Hu}_{21}$ & - & - & 0.60 & $\mathrm{Pf}_{23}$ & - & - & 0.54 & $\mathrm{Br}_{14}$ & 9.26 & 2.43 & 0.97 \\
\hline $\mathrm{Hu}_{22}$ & - & - & 0.44 & $\mathrm{Pf}_{24}$ & - & - & 0.64 & $\mathrm{Br}_{15}$ & 14.01 & 3.67 & 1.17 \\
\hline $\mathrm{Hu}_{23}$ & - & - & 0.71 & $\mathrm{Pf}_{25}$ & - & - & 0.53 & $\mathrm{Br}_{16}$ & 7.66 & 2.01 & 0.95 \\
\hline $\mathrm{Hu}_{24}$ & - & - & 0.60 & $\mathrm{Pf}_{26}$ & - & - & 0.45 & $\mathrm{Br}_{17}$ & - & - & 0.75 \\
\hline $\mathrm{Hu}_{25}$ & - & - & 0.76 & $\mathrm{Pf}_{27}$ & - & - & 0.81 & $\mathrm{Br}_{18}$ & - & - & 1.21 \\
\hline $\mathrm{Hu}_{26}$ & - & - & 0.85 & $\mathrm{Pf}_{28}$ & - & - & 1.11 & $\mathrm{Br}_{19}$ & - & - & 0.63 \\
\hline $\mathrm{Hu}_{27}$ & - & - & 0.80 & $\mathrm{Pf}_{29}$ & - & - & 1.19 & $\mathrm{Br}_{20}$ & - & - & 0.61 \\
\hline $\mathrm{Hu}_{28}$ & - & - & 0.71 & $\mathrm{Pf}_{30}$ & - & - & 0.56 & $\mathrm{Br}_{21}$ & - & - & 0.43 \\
\hline $\mathrm{Hu}_{29}$ & - & - & 0.88 & & & & & $\mathrm{Br}_{22}$ & - & - & 0.47 \\
\hline $\mathrm{Hu}_{30}$ & - & - & 0.75 & & & & & $\mathrm{Br}_{23}$ & - & - & 0.35 \\
\hline $\mathrm{Hu}_{31}$ & - & - & 0.42 & & & & & & & & \\
\hline
\end{tabular}


Tabla A.3: 228 Eri - Anchos equivalentes, flujos y FWHM de las líneas de hidrógeno.

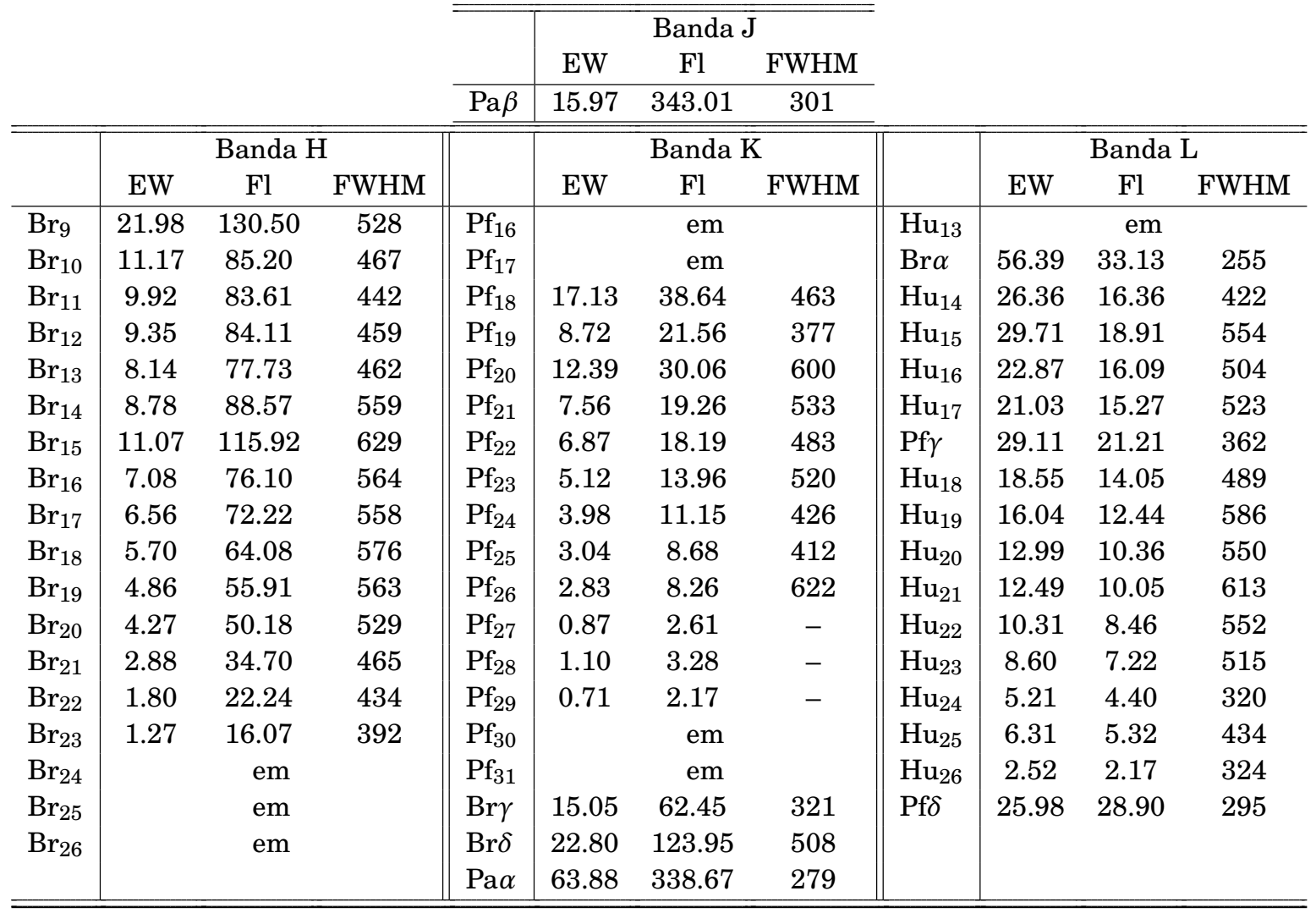

Tabla A.4: 228 Eri - Densidades columnares y tamaños de las regiones emisoras de las líneas de las series de Humphreys, Pfund, Brackett y Paschen a partir de los métodos $N$ y FWHM.

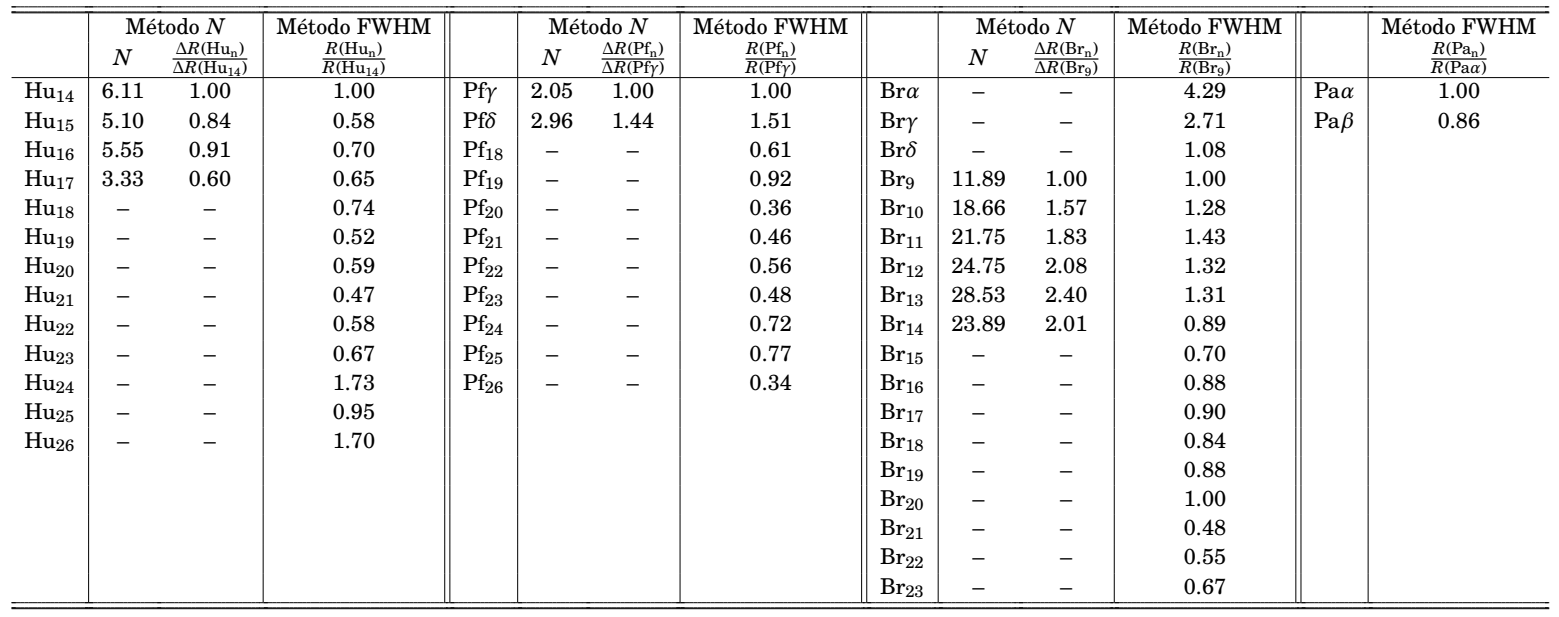


Tabla A.5: 105 Tau - Anchos equivalentes, flujos y FWHM de las líneas de hidrógeno.

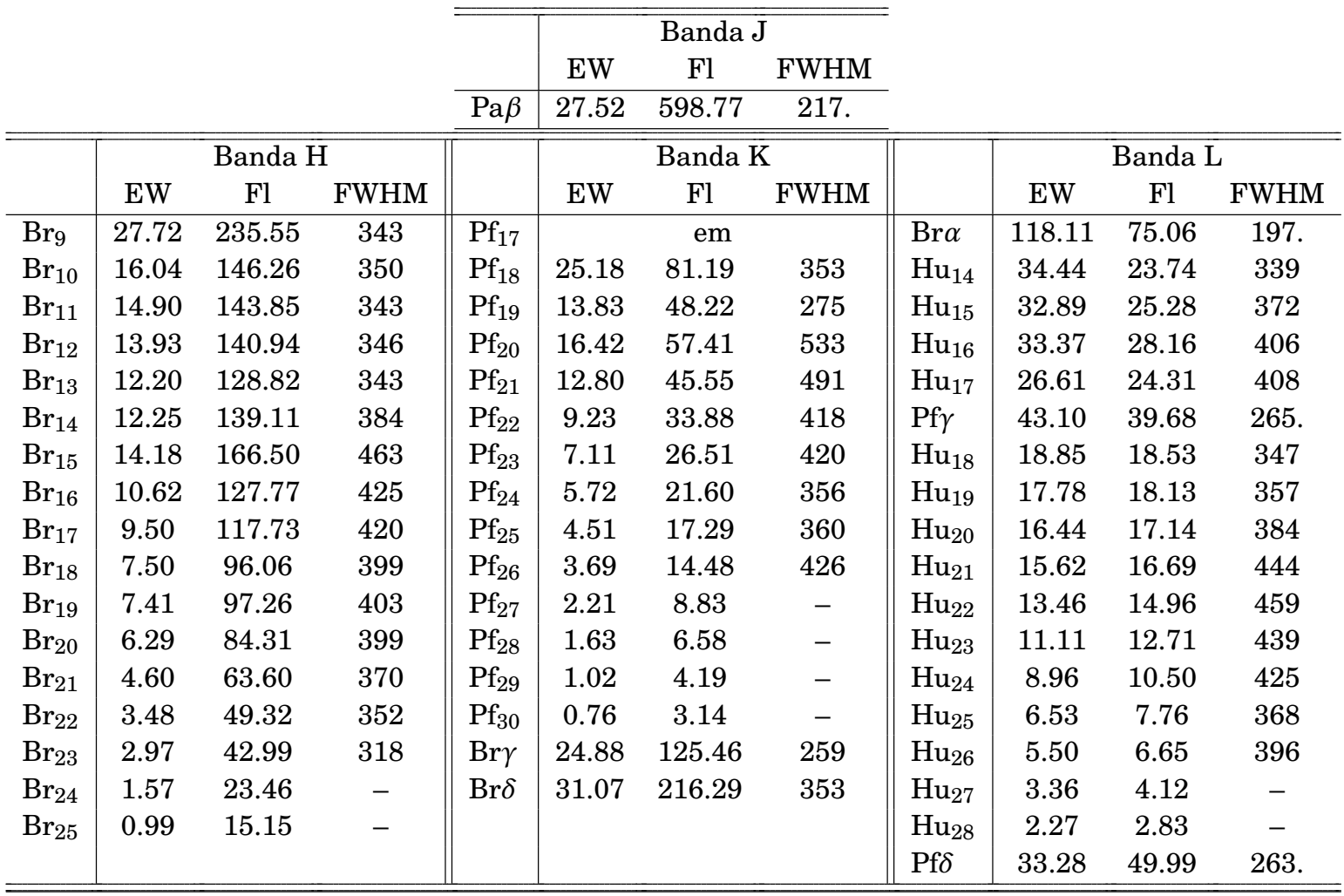

Tabla A.6: 105 Tau - Densidades columnares y tamaños de las regiones emisoras de las líneas de las series de Humphreys, Pfund y Brackett a partir de los métodos $N$ y FWHM.

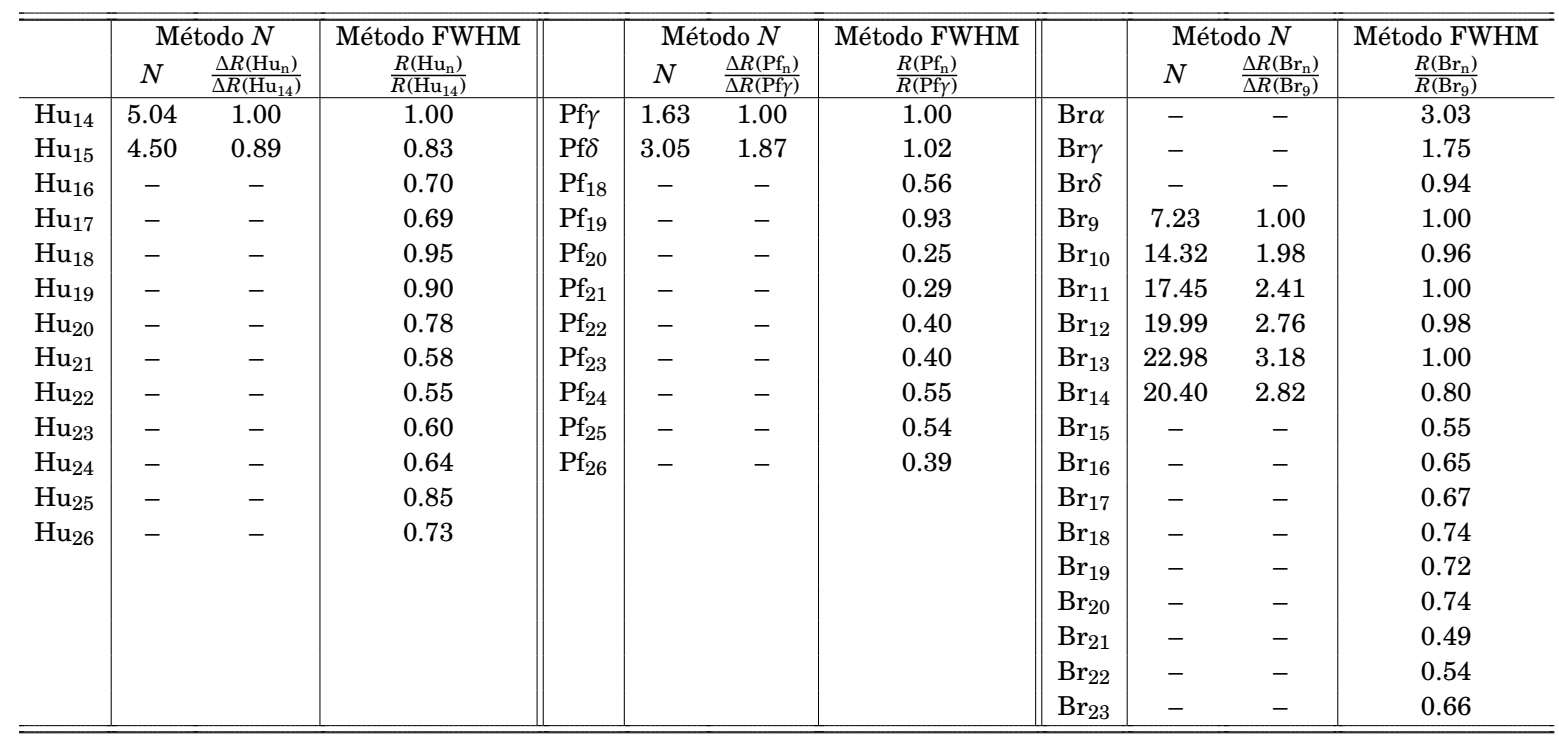


Tabla A.7: $\Psi$ Ori - Anchos equivalentes, flujos y FWHM de las líneas de hidrógeno.

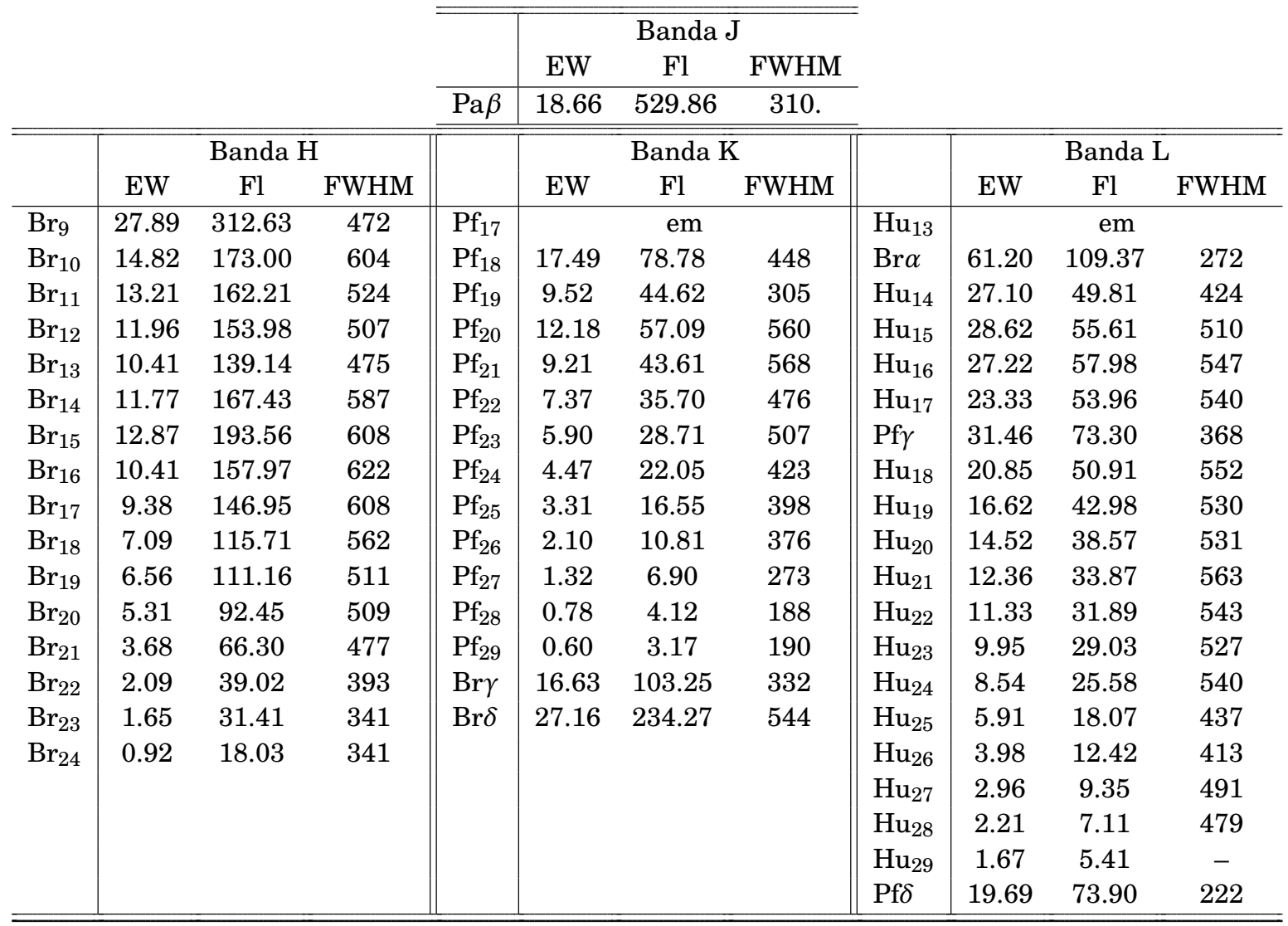

Tabla A.8: $\Psi$ Ori - Densidades columnares y tamaños de las regiones emisoras de las líneas de las series de Humphreys, Pfund y Brackett a partir de los métodos $N$ y FWHM.

\begin{tabular}{|c|c|c|c|c|c|c|c|c|c|c|c|}
\hline & \multicolumn{2}{|c|}{ Método $N$} & \multirow{2}{*}{$\begin{array}{c}\text { Método FWHM } \\
\frac{R\left(\mathrm{Hu}_{\mathrm{n}}\right)}{R\left(\mathrm{Hu}_{14}\right)}\end{array}$} & & \multicolumn{2}{|c|}{ Método $N$} & \multirow{2}{*}{$\begin{array}{c}\text { Método FWHM } \\
\frac{R\left(\mathrm{Pf}_{\mathrm{n}}\right)}{R(\mathrm{Pf} \gamma)} \\
\end{array}$} & & \multicolumn{2}{|c|}{ Método $N$} & \multirow{2}{*}{$\begin{array}{c}\text { Método FWHM } \\
\frac{R\left(\mathrm{Br}_{\mathrm{n}}\right)}{R\left(\mathrm{Br}_{9}\right)}\end{array}$} \\
\hline & $N$ & $\frac{\Delta R\left(\mathrm{Hu}_{\mathrm{n}}\right)}{\Delta R\left(\mathrm{Hu}_{14}\right)}$ & & & $N$ & $\frac{\Delta R\left(\mathrm{Pf}_{\mathrm{n}}\right)}{\Delta R(\mathrm{Pf} \gamma)}$ & & & $N$ & $\frac{\Delta R\left(\mathrm{Br}_{\mathrm{n}}\right)}{\Delta R\left(\mathrm{Br}_{9}\right)}$ & \\
\hline $\mathrm{Hu}_{14}$ & 7.54 & 1.00 & 1.00 & Pf $\gamma$ & 1.64 & 1.00 & 1.00 & $\operatorname{Br} \alpha$ & - & - & 3.01 \\
\hline $\mathrm{Hu}_{16}$ & 5.22 & 0.69 & 0.60 & $\mathrm{Pf}_{18}$ & - & - & 0.67 & $\operatorname{Br} \delta$ & - & - & 0.75 \\
\hline $\mathrm{Hu}_{17}$ & 3.29 & 0.44 & 0.62 & $\mathrm{Pf}_{19}$ & - & - & 1.46 & $\mathrm{Br}_{9}$ & 9.53 & 1.00 & 1.00 \\
\hline $\mathrm{Hu}_{18}$ & - & - & 0.59 & $\mathrm{Pf}_{20}$ & - & - & 0.43 & $\mathrm{Br}_{10}$ & 26.47 & 2.78 & 0.61 \\
\hline $\mathrm{Hu}_{20}$ & - & - & 0.64 & $\mathrm{Pf}_{22}$ & - & - & 0.60 & $\mathrm{Br}_{12}$ & 35.51 & 3.73 & 0.87 \\
\hline $\mathrm{Hu}_{21}$ & - & - & 0.57 & $\mathrm{Pf}_{23}$ & - & - & 0.53 & $\mathrm{Br}_{13}$ & 40.18 & 4.22 & 0.99 \\
\hline $\mathrm{Hu}_{22}$ & - & - & 0.61 & $\mathrm{Pf}_{24}$ & - & - & 0.76 & $\mathrm{Br}_{14}$ & 35.47 & 3.72 & 0.65 \\
\hline $\mathrm{Hu}_{23}$ & - & - & 0.65 & $\mathrm{Pf}_{25}$ & - & - & 0.85 & $\mathrm{Br}_{15}$ & - & - & 0.60 \\
\hline $\mathrm{Hu}_{24}$ & - & - & 0.62 & $\mathrm{Pf}_{26}$ & - & - & 0.96 & $\mathrm{Br}_{16}$ & - & - & 0.58 \\
\hline $\mathrm{Hu}_{25}$ & - & - & 0.94 & $\mathrm{Pf}_{27}$ & - & - & 1.82 & $\mathrm{Br}_{17}$ & - & - & 0.60 \\
\hline & & & & & & & & $\mathrm{Br}_{21}$ & - & - & 0.48 \\
\hline & & & & & & & & $\mathrm{Br}_{22}$ & - & - & 0.71 \\
\hline & & & & & & & & $\mathrm{Br}_{23}$ & - & - & 0.95 \\
\hline & & & & & & & & $\mathrm{Br}_{24}$ & - & - & 0.95 \\
\hline
\end{tabular}


Tabla A.9: 120 Tau - Anchos equivalentes, flujos y FWHM de las líneas de hidrógeno.

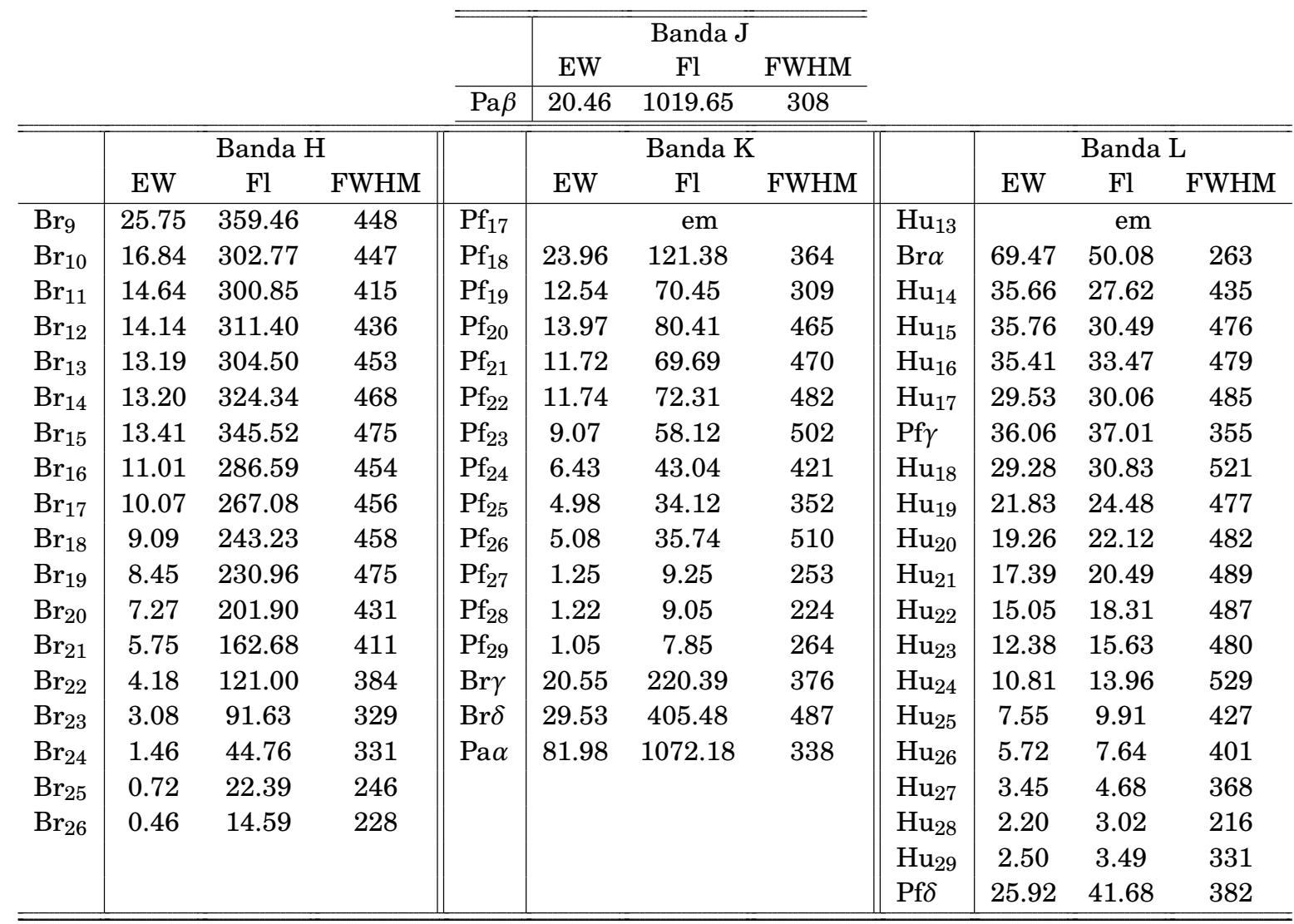

Tabla A.10: 120 Tau - Densidades columnares y tamaños de las regiones emisoras de las líneas de las series de Humphreys, Pfund, Brackett y Paschen a partir de los métodos $N$ y FWHM.

\begin{tabular}{|c|c|c|c|c|c|c|c|c|c|c|c|c|c|}
\hline & \multicolumn{2}{|c|}{ Método $N$} & \multirow{2}{*}{$\begin{array}{c}\text { Método FWHM } \\
\frac{R\left(\mathrm{Hu}_{\mathrm{n}}\right)}{R\left(\mathrm{Hu}_{14}\right)}\end{array}$} & & \multicolumn{2}{|c|}{ Método $N$} & \multirow{2}{*}{$\begin{array}{c}\text { Método FWHM } \\
\frac{R\left(\mathrm{Pf}_{\mathrm{n}}\right)}{R(\mathrm{Pf} \gamma)}\end{array}$} & & \multicolumn{2}{|c|}{ Método $N$} & \multirow{2}{*}{$\begin{array}{c}\text { Método FWHM } \\
\frac{R\left(\mathrm{Br}_{\mathrm{n}}\right)}{R\left(\mathrm{Br}_{9}\right)}\end{array}$} & & \multirow{2}{*}{$\begin{array}{c}\text { Método FWHM } \\
\frac{R\left(\mathrm{~Pa}_{\mathrm{n}}\right)}{R(\mathrm{~Pa} \alpha)}\end{array}$} \\
\hline & $N$ & $\frac{\Delta R\left(\mathrm{Hu}_{\mathrm{n}}\right)}{\Delta R\left(\mathrm{Hu}_{14}\right)}$ & & & $N$ & $\frac{\Delta R\left(\mathrm{Pf}_{\mathrm{n}}\right)}{\Delta R(\mathrm{Pf} \gamma)}$ & & & $N$ & $\frac{\Delta R\left(\mathrm{Br}_{\mathrm{n}}\right)}{\Delta R\left(\mathrm{Br}_{9}\right)}$ & & & \\
\hline $\mathrm{Hu}_{14}$ & 8.01 & 1 & 1 & Pf $\gamma$ & 2.61 & 1 & 1.00 & $\operatorname{Br} \alpha$ & - & - & 2.9 & $\mathrm{~Pa} \alpha$ & 1 \\
\hline $\mathrm{Hu}_{15}$ & 7.47 & 0.93 & 0.84 & $\operatorname{Pf} \delta$ & 5.72 & 2.19 & 0.86 & $\mathrm{Br} \gamma$ & - & - & 1.42 & $\mathrm{~Pa} \beta$ & 1.2 \\
\hline $\mathrm{Hu}_{17}$ & 3.48 & 0.43 & 0.8 & $\mathrm{Pf}_{19}$ & - & - & 1.32 & $\mathrm{Br}_{9}$ & 13.47 & 1 & 1.00 & & \\
\hline $\mathrm{Hu}_{18}$ & - & - & 0.7 & $\mathrm{Pf}_{20}$ & - & - & 0.58 & $\mathrm{Br}_{10}$ & 20.56 & 1.53 & 1.00 & & \\
\hline $\mathrm{Hu}_{19}$ & - & - & 0.83 & $\mathrm{Pf}_{21}$ & - & - & 0.57 & $\mathrm{Br}_{11}$ & 23.65 & 1.76 & 1.17 & & \\
\hline $\mathrm{Hu}_{22}$ & - & - & 0.8 & $\mathrm{Pf}_{24}$ & - & - & 0.71 & $\mathrm{Br}_{14}$ & 22.62 & 1.68 & 0.92 & & \\
\hline $\mathrm{Hu}_{23}$ & - & - & 0.82 & $\mathrm{Pf}_{25}$ & - & - & 1.02 & $\mathrm{Br}_{15}$ & - & - & 0.89 & & \\
\hline $\mathrm{Hu}_{24}$ & - & - & 0.68 & $\mathrm{Pf}_{26}$ & - & - & 0.48 & $\mathrm{Br}_{16}$ & - & - & 0.97 & & \\
\hline $\mathrm{Hu}_{25}$ & - & - & 1.04 & $\mathrm{Pf}_{27}$ & - & - & 1.97 & $\mathrm{Br}_{17}$ & - & - & 0.97 & & \\
\hline $\mathrm{Hu}_{26}$ & - & - & 1.18 & $\mathrm{Pf}_{28}$ & - & - & 2.51 & $\mathrm{Br}_{18}$ & - & - & 0.96 & & \\
\hline $\mathrm{Hu}_{27}$ & - & - & 1.4 & $\mathrm{Pf}_{29}$ & - & - & 1.81 & $\mathrm{Br}_{19}$ & - & - & 0.89 & & \\
\hline & & & & & & & & $\mathrm{Br}_{25}$ & - & - & 2.34 & & \\
\hline & & & & & & & & $\mathrm{Br}_{26}$ & - & - & 2.72 & & \\
\hline
\end{tabular}


Tabla A.11: $\omega$ Ori - Anchos equivalentes, flujos y FWHM de las líneas de hidrógeno.

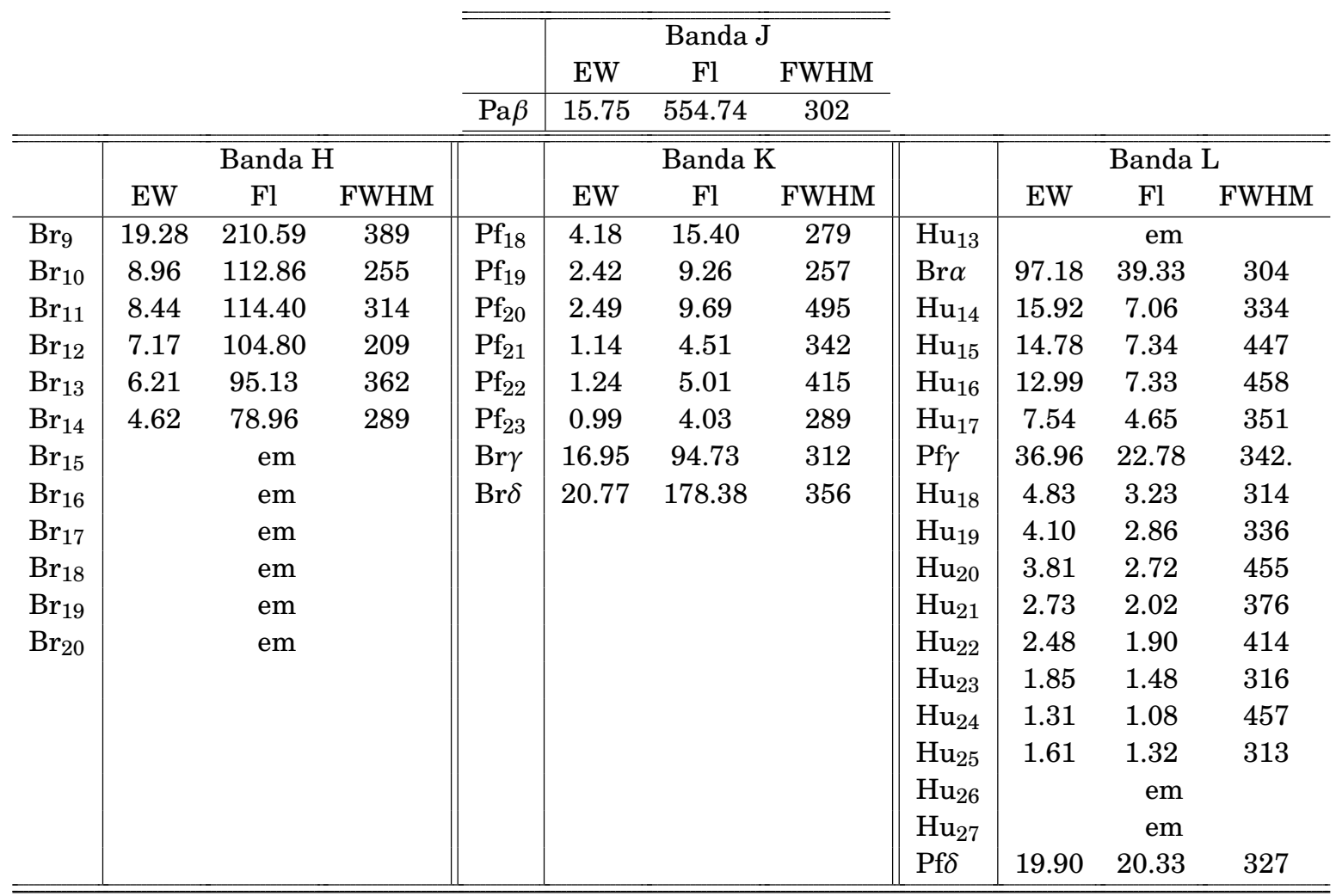

Tabla A.12: $\omega$ Ori - Densidades columnares y tamaños de las regiones emisoras de las líneas de las series de Humphreys, Pfund Brackett a partir de los métodos $N$ y FWHM.

\begin{tabular}{l|c||c|cc|c||c|c}
\hline \hline & $\begin{array}{c}\text { Método FWHM } \\
\frac{R\left(\mathrm{Hu}_{\mathrm{n}}\right)}{R\left(\mathrm{Hu}_{14}\right)}\end{array}$ & & \multicolumn{2}{|c|}{ Método $N$} & Método FWHM & & Método FWHM \\
& 1.00 & $\mathrm{Pf} \gamma$ & 1.11 & $\frac{\Delta R\left(\mathrm{Pf}_{\mathrm{n}}\right)}{\Delta R\left(\mathrm{Pf}^{\prime}\right)}$ & $\frac{R\left(\mathrm{Pf}_{\mathrm{n}}\right)}{R(\mathrm{Pf} \gamma)}$ & & $\frac{R\left(\mathrm{Br}_{\mathrm{n}}\right)}{R\left(\mathrm{Br}_{9}\right)}$ \\
\hline $\mathrm{Hu}_{14}$ & 0.56 & $\mathrm{Pf} \delta$ & 2.26 & 2.05 & 1.00 & $\mathrm{Br} \alpha$ & 1.64 \\
$\mathrm{Hu}_{15}$ & 0.53 & $\mathrm{Pf}_{18}$ & - & - & 1.09 & $\mathrm{Br} \gamma$ & 1.55 \\
$\mathrm{Hu}_{16}$ & 0.91 & $\mathrm{Pf}_{19}$ & - & - & 1.50 & $\mathrm{Br} \delta$ & 1.19 \\
$\mathrm{Hu}_{17}$ & 1.13 & $\mathrm{Pf}_{20}$ & - & - & 0.48 & $\mathrm{Br}_{9}$ & 1.00 \\
$\mathrm{Hu}_{18}$ & 0.99 & $\mathrm{Pf}_{21}$ & - & - & 1.00 & $\mathrm{Br}_{10}$ & 2.33 \\
$\mathrm{Hu}_{19}$ & 0.54 & $\mathrm{Pf}_{22}$ & - & - & 0.68 & $\mathrm{Br}_{12}$ & 1.53 \\
$\mathrm{Hu}_{20}$ & 0.79 & $\mathrm{Pf}_{23}$ & - & - & 1.40 & $\mathrm{Br}_{13}$ & 3.46 \\
$\mathrm{Hu}_{21}$ & 0.65 & & & & & $\mathrm{Br}_{14}$ & 1.15 \\
$\mathrm{Hu}_{22}$ & 1.12 & & & & & & 1.81 \\
$\mathrm{Hu}_{23}$ & 0.53 & & & & & & \\
$\mathrm{Hu}_{24}$ & 1.14 & & & & & & \\
$\mathrm{Hu}_{25}$ & 1.50
\end{tabular}


Tabla A.13: V696 Mon - Anchos equivalentes, flujos y FWHM de las líneas de hidrógeno.

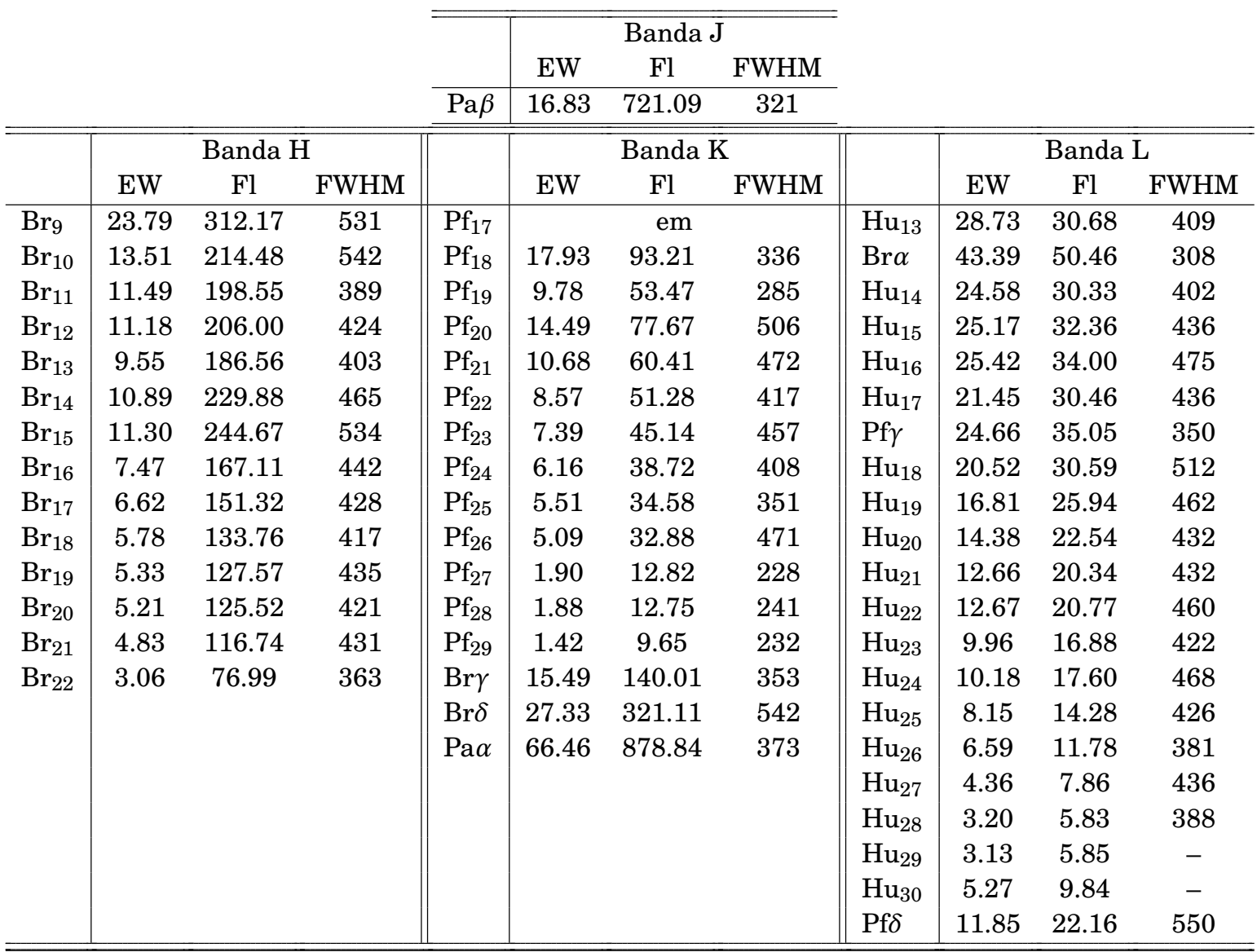

Tabla A.14: V696 Mon - Densidades columnares y tamaños de las regiones emisoras de las líneas de las series de Humphreys, Pfund, Brackett y Paschen a partir de los métodos $N$ y FWHM.

\begin{tabular}{|c|c|c|c|c|c|c|c|c|c|c|c|c|c|}
\hline & \multicolumn{2}{|c|}{ Método $N$} & \multirow{2}{*}{$\begin{array}{c}\text { Método FWHM } \\
\frac{R\left(\mathrm{Hu}_{\mathrm{n}}\right)}{R\left(\mathrm{Hu}_{14}\right)} \\
\end{array}$} & & \multicolumn{2}{|c|}{ Método $N$} & \multirow{2}{*}{$\begin{array}{c}\text { Método FWHM } \\
\frac{R\left(\mathrm{Pf}_{\mathrm{n}}\right)}{R\left(\mathrm{Pf}^{\prime}\right)} \\
\end{array}$} & & \multicolumn{2}{|c|}{ Método $N$} & \multirow{2}{*}{$\begin{array}{c}\text { Método FWHM } \\
\frac{R\left(\mathrm{Br}_{\mathrm{n}}\right)}{R\left(\mathrm{Br}_{9}\right)} \\
\end{array}$} & & \multirow{2}{*}{$\begin{array}{c}\text { Método FWHM } \\
\frac{\left.R(\operatorname{Pa})^{\prime}\right)}{R(\operatorname{Pa} \alpha)} \\
\end{array}$} \\
\hline & $N$ & $\frac{\Delta R\left(\mathrm{Hu}_{\mathrm{n}}\right)}{\Delta R\left(\mathrm{Hu}_{14}\right)}$ & & & $N$ & $\frac{\Delta R\left(\mathrm{Pf}_{\mathrm{n}}\right)}{\Delta R(\mathrm{Pf} \gamma)}$ & & & $N$ & $\frac{\Delta R\left(\mathrm{Br}_{\mathrm{n}}\right)}{\Delta R\left(\mathrm{Br}_{9}\right)}$ & & & \\
\hline $\mathrm{Hu}_{13}$ & 6.38 & 0.9 & 0.97 & $\mathrm{Pf} \gamma$ & 2.64 & 1 & 1 & $\operatorname{Br} \alpha$ & - & - & 2.97 & $\mathrm{~Pa} \alpha$ & 1.00 \\
\hline $\mathrm{Hu}_{14}$ & 7.11 & 1 & 1.00 & $\mathrm{Pf} \delta$ & 10.1 & 3.82 & 0.4 & $\mathrm{Br} \gamma$ & - & - & 2.26 & $\mathrm{~Pa} \beta$ & 1.35 \\
\hline $\mathrm{Hu}_{16}$ & 5.43 & 0.76 & 0.72 & $\mathrm{Pf}_{19}$ & - & - & 1.51 & $\mathrm{Br}_{9}$ & 11.68 & 1 & 1.00 & & \\
\hline $\mathrm{Hu}_{17}$ & 4.64 & 0.65 & 0.85 & $\mathrm{Pf}_{20}$ & - & - & 0.48 & $\mathrm{Br}_{10}$ & 20.79 & 1.78 & 0.96 & & \\
\hline $\mathrm{Hu}_{18}$ & - & - & 0.62 & $\mathrm{Pf}_{21}$ & - & - & 0.55 & $\mathrm{Br}_{11}$ & 19.32 & 1.65 & 1.86 & & \\
\hline $\mathrm{Hu}_{21}$ & - & - & 0.87 & $\mathrm{Pf}_{24}$ & - & - & 0.74 & $\mathrm{Br}_{14}$ & 14.86 & 1.27 & 1.30 & & \\
\hline $\mathrm{Hu}_{22}$ & - & - & 0.76 & $\mathrm{Pf}_{25}$ & - & - & 0.99 & $\mathrm{Br}_{15}$ & - & - & 0.99 & & \\
\hline $\mathrm{Hu}_{23}$ & - & - & 0.91 & $\mathrm{Pf}_{26}$ & - & - & 0.55 & $\mathrm{Br}_{16}$ & - & - & 1.44 & & \\
\hline $\mathrm{Hu}_{24}$ & - & - & 0.74 & $\mathrm{Pf}_{27}$ & - & - & 2.36 & $\mathrm{Br}_{17}$ & - & - & 1.54 & & \\
\hline $\mathrm{Hu}_{25}$ & - & - & 0.89 & $\mathrm{Pf}_{28}$ & - & - & 2.11 & $\mathrm{Br}_{18}$ & - & - & 1.62 & & \\
\hline $\mathrm{Hu}_{26}$ & - & - & 1.11 & $\mathrm{Pf}_{29}$ & - & - & 2.28 & $\mathrm{Br}_{19}$ & - & - & 1.49 & & \\
\hline
\end{tabular}


Tabla A.15: MX Pup - Anchos equivalentes, flujos y FWHM de las líneas de hidrógeno.

\begin{tabular}{|c|c|c|c|c|c|c|c|c|c|c|c|}
\hline & \multicolumn{3}{|c|}{$\overline{\text { Banda J }}$} & & \multicolumn{3}{|c|}{ Banda $\mathrm{H}$} & & \multicolumn{3}{|c|}{ Banda K } \\
\hline & EW & Fl & FWHM & & EW & $\mathrm{Fl}$ & FWHM & & EW & $\mathrm{Fl}$ & FWHM \\
\hline \multirow[t]{20}{*}{$\mathrm{Pa} \beta$} & 18.52 & 39.71 & 191 & $\mathrm{Br}_{10}$ & 15.93 & 12.55 & 290 & \multirow[t]{20}{*}{$\mathrm{Br} \gamma$} & \multirow[t]{20}{*}{20.90} & \multirow[t]{20}{*}{8.65} & \multirow[t]{20}{*}{229} \\
\hline & & & & $\mathrm{Br}_{11}$ & 15.79 & 13.06 & 310 & & & & \\
\hline & & & & $\mathrm{Br}_{12}$ & 14.53 & 13.13 & 310 & & & & \\
\hline & & & & $\mathrm{Br}_{13}$ & 13.34 & 12.73 & 316 & & & & \\
\hline & & & & $\mathrm{Br}_{14}$ & 13.72 & 13.52 & 339 & & & & \\
\hline & & & & $\mathrm{Br}_{15}$ & 13.35 & 13.49 & 338 & & & & \\
\hline & & & & $\mathrm{Br}_{16}$ & 12.12 & 12.86 & 335 & & & & \\
\hline & & & & $\mathrm{Br}_{17}$ & 11.28 & 12.32 & 345 & & & & \\
\hline & & & & $\mathrm{Br}_{18}$ & 9.99 & 11.19 & 340 & & & & \\
\hline & & & & $\mathrm{Br}_{19}$ & 8.52 & 9.82 & 340 & & & & \\
\hline & & & & $\mathrm{Br}_{20}$ & 7.21 & 8.51 & 336 & & & & \\
\hline & & & & $\mathrm{Br}_{21}$ & 4.60 & 5.71 & 286 & & & & \\
\hline & & & & $\mathrm{Br}_{22}$ & 4.49 & 5.55 & 308 & & & & \\
\hline & & & & $\mathrm{Br}_{23}$ & 3.25 & 4.14 & 295 & & & & \\
\hline & & & & $\mathrm{Br}_{24}$ & 2.30 & 2.99 & 266 & & & & \\
\hline & & & & $\mathrm{Br}_{25}$ & 2.01 & 2.64 & 285 & & & & \\
\hline & & & & $\mathrm{Br}_{26}$ & 1.63 & 2.15 & 286 & & & & \\
\hline & & & & $\mathrm{Br}_{27}$ & 1.27 & 1.71 & 297 & & & & \\
\hline & & & & $\mathrm{Br}_{28}$ & & em & & & & & \\
\hline & & & & $\mathrm{Br}_{29}$ & & em & & & & & \\
\hline
\end{tabular}


Tabla A.16: MX Pup - Densidades columnares y tamaños de las regiones emisoras de las líneas de las series de Brackett y Paschen a partir de los métodos $N$, FWHM y $\Delta V$.

\begin{tabular}{|c|c|c|c|c|c|c|c|c|c|c|}
\hline & \multicolumn{2}{|c|}{ Método $N$} & \multirow{2}{*}{$\begin{array}{c}\text { Método FWHM } \\
\frac{R\left(\mathrm{Br}_{\mathrm{n}}\right)}{R\left(\mathrm{Br}_{10}\right)}\end{array}$} & \multicolumn{3}{|c|}{ Método $\Delta V$} & & \multicolumn{3}{|c|}{ Método $\Delta V$} \\
\hline & $N$ & $\frac{\Delta R\left(\mathrm{Br}_{\mathrm{n}}\right)}{\Delta R\left(\mathrm{Br}_{10}\right)}$ & & $\Delta V_{\mathrm{n}}$ & $R_{\mathrm{n}}$ & $\frac{R\left(\mathrm{Br}_{\mathrm{n}}\right)}{R\left(\mathrm{Br}_{10}\right)}$ & & $\Delta V_{\mathrm{n}}$ & $R_{\mathrm{n}}$ & $\frac{R\left(\mathrm{~Pa}_{\mathrm{n}}\right)}{R(\mathrm{~Pa} \beta)}$ \\
\hline $\mathrm{Br} \gamma$ & - & - & 1.60 & 102 & 8.88 & 1.29 & $\mathrm{~Pa} \beta$ & 98 & 9.62 & 1.00 \\
\hline $\mathrm{Br}_{10}$ & 14.19 & 1.00 & 1.00 & 116 & 6.87 & 1.00 & & & & \\
\hline $\mathrm{Br}_{11}$ & 18.38 & 1.29 & 0.88 & 117 & 6.75 & 0.98 & & & & \\
\hline $\mathrm{Br}_{12}$ & 20.59 & 1.45 & 0.88 & 112 & 7.37 & 1.07 & & & & \\
\hline $\mathrm{Br}_{13}$ & 22.55 & 1.59 & 0.84 & 144 & 4.46 & 0.65 & & & & \\
\hline $\mathrm{Br}_{14}$ & 20.24 & 1.43 & 0.73 & 137 & 4.92 & 0.72 & & & & \\
\hline $\mathrm{Br}_{15}$ & 15.19 & 1.07 & 0.74 & 127 & 5.73 & 0.83 & & & & \\
\hline $\mathrm{Br}_{16}$ & 9.22 & 0.65 & 0.75 & 106 & 8.22 & 1.20 & & & & \\
\hline $\mathrm{Br}_{17}$ & - & - & 0.71 & 115 & 6.99 & 1.02 & & & & \\
\hline $\mathrm{Br}_{18}$ & - & - & 0.73 & 101 & 9.06 & 1.32 & & & & \\
\hline $\mathrm{Br}_{19}$ & - & - & 0.73 & 113 & 7.24 & 1.05 & & & & \\
\hline $\mathrm{Br}_{20}$ & - & - & 0.74 & 114 & 7.11 & 1.04 & & & & \\
\hline $\mathrm{Br}_{21}$ & - & - & 0.64 & 124 & 6.01 & 0.88 & & & & \\
\hline $\mathrm{Br}_{22}$ & - & - & 0.55 & 111 & 7.50 & 1.09 & & & & \\
\hline $\mathrm{Br}_{23}$ & - & - & 0.60 & 161 & 3.57 & 0.52 & & & & \\
\hline $\mathrm{Br}_{24}$ & - & - & 0.74 & 133 & 5.22 & 0.76 & & & & \\
\hline $\mathrm{Br}_{25}$ & - & - & 0.65 & 140 & 4.72 & 0.68 & & & & \\
\hline $\mathrm{Br}_{26}$ & - & - & 0.64 & 134 & 5.15 & 0.75 & & & & \\
\hline $\mathrm{Br}_{27}$ & - & - & 0.59 & - & - & - & & & & \\
\hline
\end{tabular}

Tabla A.17: 48 Lib - Anchos equivalentes, flujos y FWHM de las líneas de hidrógeno.

\begin{tabular}{l|ccc||c|ccc||cc|cc}
\hline \hline & \multicolumn{3}{|c|}{ Banda J } & & \multicolumn{3}{c|}{ Banda $\mathrm{H}$} & & \multicolumn{3}{c}{ Banda K } \\
& EW & Fl & FWHM & & EW & Fl & FWHM & & EW & Fl & FWHM \\
\hline $\mathrm{Pa} \beta$ & 16.46 & 599.27 & 373 & $\mathrm{Br}_{9}$ & 18.72 & 229.36 & - & $\mathrm{Pf}_{18}$ & 11.41 & 46.61 & - \\
& & & & $\mathrm{Br}_{10}$ & 7.16 & 102.19 & - & $\mathrm{Pf}_{19}$ & 9.82 & 42.60 & - \\
& & & & $\mathrm{Br}_{11}$ & 8.22 & 126.03 & - & $\mathrm{Pf}_{20}$ & 9.18 & 40.54 & - \\
& & & & $\mathrm{Br}_{12}$ & 5.70 & 92.23 & - & $\mathrm{Pf}_{21}$ & 4.62 & 21.80 & - \\
& & & & $\mathrm{Br}_{13}$ & 4.69 & 80.35 & - & $\mathrm{Pf}_{22}$ & 3.78 & 18.40 & - \\
& & & $\mathrm{Br}_{14}$ & 3.91 & 73.99 & - & $\mathrm{Pf}_{23}$ & 3.52 & 17.18 & - \\
& & & $\mathrm{Br}_{15}$ & 3.84 & 77.32 & - & $\mathrm{Pf}_{24}$ & 2.45 & 12.30 & - \\
& & & $\mathrm{Br}_{16}$ & 2.98 & 61.05 & - & $\mathrm{Pf}_{25}$ & 1.26 & 6.44 & - \\
& & & $\mathrm{Br}_{17}$ & 1.74 & 37.03 & - & $\mathrm{Br} \gamma$ & 15.39 & 104.56 & 377 \\
& & & $\mathrm{Br}_{18}$ & 1.49 & 32.35 & - & $\mathrm{Br} \delta$ & 18.63 & 194.60 & 520 \\
& & & $\mathrm{Br}_{19}$ & 1.12 & 25.01 & - & $\mathrm{Pa} \alpha$ & 108.90 & 1201.24 & 374 \\
& & & $\mathrm{Br}_{20}$ & 0.93 & 21.18 & - & & & & \\
\hline \hline
\end{tabular}


Tabla A.18: 48 Lib - Tamaños de las regiones emisoras de las líneas de las series de Pfund, Brackett y Paschen a partir de los métodos FWHM y $\Delta V$. El valor indicado entre paréntesis fue obtenido a partir del tamaño relativo a $\mathrm{Pa} \beta$.

\begin{tabular}{|c|c|c|c|c|c|c|c|c|c|c|c|c|}
\hline & \multicolumn{3}{|c|}{ Método $\Delta V$} & & \multirow{2}{*}{$\begin{array}{c}\text { Método FWHM } \\
\frac{R\left(\mathrm{Br}_{\mathrm{n}}\right)}{R(\mathrm{~B} \delta)}\end{array}$} & \multicolumn{3}{|c|}{ Método $\Delta V$} & & \multirow{2}{*}{$\begin{array}{c}\text { Método FWHM } \\
\frac{R\left(\mathrm{~Pa}_{\mathrm{n}}\right)}{R(\mathrm{~Pa} \beta)}\end{array}$} & \multicolumn{2}{|c|}{$\overline{\overline{\Delta \Delta V}}$} \\
\hline & $\Delta V_{\mathrm{n}}$ & $R_{\mathrm{n}}$ & $\frac{R\left(\mathrm{Pf}_{\mathrm{n}}\right)}{R\left(\mathrm{Pf}_{18}\right)}$ & & & $\Delta V_{\mathrm{n}}$ & $R_{\mathrm{n}}$ & $\frac{R\left(\mathrm{Br} \mathrm{r}_{\mathrm{n}}\right)}{R(\mathrm{Br} \delta)}$ & & & $\Delta V_{\mathrm{n}}$ & $R_{\mathrm{n}}$ \\
\hline $\mathrm{Pf}_{18}$ & 320 & 6.47 & 1.00 & $\mathrm{Br} \gamma$ & 1.90 & 234 & 12.10 & 1.98 & $\mathrm{~Pa} \alpha$ & 0.99 & - & (12.07) \\
\hline $\mathrm{Pf}_{19}$ & 262 & 9.65 & 1.49 & $\operatorname{Br} \delta$ & 1.00 & 329 & 6.12 & 1.00 & $\mathrm{~Pa} \beta$ & 1.00 & 233 & 12.20 \\
\hline $\mathrm{Pf}_{20}$ & 491 & 2.75 & 0.42 & $\mathrm{Br}_{9}$ & - & 378 & 4.64 & 0.76 & & & & \\
\hline $\mathrm{Pf}_{21}$ & 426 & 3.65 & 0.56 & $\mathrm{Br}_{10}$ & - & 400 & 4.14 & 0.68 & & & & \\
\hline $\mathrm{Pf}_{22}$ & 407 & 4.00 & 0.62 & $\mathrm{Br}_{11}$ & - & 356 & 5.23 & 0.85 & & & & \\
\hline $\mathrm{Pf}_{23}$ & 484 & 2.83 & 0.44 & $\mathrm{Br}_{12}$ & - & 434 & 3.52 & 0.57 & & & & \\
\hline $\mathrm{Pf}_{24}$ & 441 & 3.41 & 0.53 & $\mathrm{Br}_{13}$ & - & 423 & 3.70 & 0.60 & & & & \\
\hline & & & & $\mathrm{Br}_{14}$ & - & 488 & 2.78 & 0.45 & & & & \\
\hline & & & & $\mathrm{Br}_{15}$ & - & 478 & 2.90 & 0.47 & & & & \\
\hline & & & & $\mathrm{Br}_{16}$ & - & 519 & 2.46 & 0.40 & & & & \\
\hline & & & & $\mathrm{Br}_{17}$ & - & 498 & 2.67 & 0.44 & & & & \\
\hline & & & & $\mathrm{Br}_{18}$ & - & 519 & 2.46 & 0.40 & & & & \\
\hline & & & & $\mathrm{Br}_{19}$ & - & 508 & 2.57 & 0.42 & & & & \\
\hline & & & & $\mathrm{Br}_{20}$ & - & 540 & 2.27 & 0.37 & & & & \\
\hline & & & & $\mathrm{Br}_{21}$ & - & 533 & 2.33 & 0.38 & & & & \\
\hline
\end{tabular}

Tabla A.19: V1075 Sco - Anchos equivalentes, flujos y FWHM de las líneas de hidrógeno.

\begin{tabular}{l|ccc||c|ccc||cc|cc}
\hline \hline & \multicolumn{3}{|c|}{ Banda J } & & \multicolumn{3}{c|}{ Banda H } & & \multicolumn{3}{c}{ Banda K } \\
& EW & Fl & FWHM & & EW & Fl & FWHM & & EW & Fl & FWHM \\
\hline $\mathrm{Pa} \beta$ & 4.44 & 535.85 & 197 & $\mathrm{Br}_{10}$ & 3.79 & 157.54 & 313 & $\mathrm{Br} \gamma$ & 6.20 & 121.38 & 244 \\
& & & & $\mathrm{Br}_{11}$ & 3.07 & 144.84 & 438 & & & & \\
& & & & $\mathrm{Br}_{12}$ & 2.31 & 115.05 & 529 & & & & \\
& & & & $\mathrm{Br}_{13}$ & & $\mathrm{em}$ & & & & & \\
& & & $\mathrm{Br}_{14}$ & 1.81 & 104.26 & 502 & & & & \\
& & & $\mathrm{Br}_{15}$ & 1.97 & 116.70 & 478 & & & & \\
& & & $\mathrm{Br}_{16}$ & 1.70 & 103.57 & 509 & & & & \\
& & & $\mathrm{Br}_{17}$ & 1.70 & 104.69 & 540 & & & & \\
& & & $\mathrm{Br}_{18}$ & 1.33 & 83.74 & 521 & & & & \\
& & & $\mathrm{Br}_{19}$ & 1.20 & 77.31 & 587 & & & & \\
\hline \hline
\end{tabular}


Tabla A.20: V1075 Sco - Tamaños de las regiones emisoras de las líneas de la serie de Brackett a partir de los métodos $N$ y FWHM.

\begin{tabular}{l||cc||c}
\hline \hline & \multicolumn{2}{|c|}{ Método $N$} & Método FWHM \\
& $N$ & $\frac{\Delta R\left(\mathrm{Br}_{\mathrm{n}}\right)}{\Delta R\left(\mathrm{Br}_{10}\right)}$ & $\frac{R\left(\mathrm{Br}_{\mathrm{n}}\right)}{R\left(\mathrm{Br}_{10}\right)}$ \\
\hline $\mathrm{Br} \gamma$ & - & - & 1.65 \\
$\mathrm{Br}_{10}$ & 11.41 & 1.00 & 1.00 \\
$\mathrm{Br}_{11}$ & 21.23 & 1.86 & 0.51 \\
$\mathrm{Br}_{12}$ & 37.41 & 3.28 & 0.35 \\
$\mathrm{Br}_{13}$ & - & - & - \\
$\mathrm{Br}_{14}$ & 44.13 & 3.87 & 0.39 \\
$\mathrm{Br}_{15}$ & 25.91 & 2.27 & 0.43 \\
$\mathrm{Br}_{16}$ & 40.87 & 3.58 & 0.38 \\
$\mathrm{Br}_{17}$ & - & - & 0.34 \\
$\mathrm{Br}_{18}$ & - & - & 0.36 \\
$\mathrm{Br}_{19}$ & - & - & 0.28 \\
\hline \hline
\end{tabular}

Tabla A.21: 88 Her - Anchos equivalentes, flujos y FWHM de las líneas de hidrógeno.

\begin{tabular}{l|ccc||l|ccc}
\hline \hline & \multicolumn{3}{|c||}{ Banda K } & & \multicolumn{3}{c}{ Banda L } \\
& EW & Fl & FWHM & & EW & Fl & FWHM \\
\hline $\operatorname{Br} \delta$ & 18.29 & 10.90 & - & $\mathrm{Br} \alpha$ & 54.99 & 1.18 & 481 \\
& & & & $\mathrm{Hu}_{14}$ & 24.57 & 0.73 & 641 \\
& & & & $\mathrm{Hu}_{15}$ & 19.98 & 0.72 & 674 \\
& & & & $\mathrm{Hu}_{16}$ & 15.07 & 0.65 & - \\
& & & & $\mathrm{Hu}_{17}$ & 8.84 & 0.41 & 506 \\
& & & & $\mathrm{Pf} \gamma$ & 27.45 & 1.28 & 827 \\
\hline \hline
\end{tabular}

Tabla A.22: 88 Her - Tamaños de las regiones emisoras de las líneas de las series de Brackett, Pfund y Humphreys a partir del método FWHM.

\begin{tabular}{l||c}
\hline \hline & $\begin{array}{c}\text { Método FWHM } \\
\end{array}$ \\
\hline $\mathrm{Br} \alpha$ & 2.95 \\
$\mathrm{Hu}_{14}$ & 1.66 \\
$\mathrm{Hu}_{15}$ & 1.50 \\
$\mathrm{Hu}_{17}$ & 2.67 \\
$\mathrm{Pf} \gamma$ & 1.00 \\
\hline \hline
\end{tabular}


Tabla A.23: V4024 Sgr - Anchos equivalentes, flujos y FWHM de las líneas de hidrógeno.

\begin{tabular}{|c|c|c|c|c|c|c|c|c|c|c|c|}
\hline & \multicolumn{3}{|c|}{ Banda J } & & \multicolumn{3}{|c|}{ Banda $\mathrm{H}$} & & \multicolumn{3}{|c|}{ Banda K } \\
\hline & EW & $\mathrm{Fl}$ & FWHM & & EW & $\mathrm{Fl}$ & FWHM & & EW & $\mathrm{Fl}$ & FWHM \\
\hline \multirow[t]{5}{*}{$\mathrm{Pa} \beta$} & 6.19 & 1282.20 & - & $\mathrm{Br}_{10}$ & \multicolumn{3}{|c|}{ em } & $\mathrm{Br} \gamma$ & 8.18 & 285.61 & 295 \\
\hline & & & & $\mathrm{Br}_{11}$ & 4.59 & 367.65 & - & & & & \\
\hline & & & & $\mathrm{Br}_{12}$ & 4.22 & 352.06 & - & & & & \\
\hline & & & & $\mathrm{Br}_{13}$ & 3.40 & 320.73 & - & & & & \\
\hline & & & & $\mathrm{Br}_{14}$ & 2.76 & 276.09 & - & & & & \\
\hline
\end{tabular}

Tabla A.24: V4024 Sgr - Tamaños de las regiones emisoras de las líneas $\mathrm{Br} \gamma$ y $\mathrm{Pa} \beta$ a partir del método $\Delta V$.

\begin{tabular}{c|ccc||c|ccc}
\hline \hline & \multicolumn{3}{|c|}{ Método $\Delta V$} & & \multicolumn{3}{c}{ Método $\Delta V$} \\
& $\Delta V_{\mathrm{n}}$ & $R_{\mathrm{n}}$ & $\frac{R\left(\mathrm{Br}_{\mathrm{n}}\right)}{R(\mathrm{Br} \gamma)}$ & & $\Delta V_{\mathrm{n}}$ & $R_{\mathrm{n}}$ & $\frac{R\left(\mathrm{~Pa}_{\mathrm{n}}\right)}{R(\mathrm{~Pa} \beta)}$ \\
\hline $\mathrm{Br} \gamma$ & 181 & 1.50 & 1.00 & $\mathrm{~Pa} \beta$ & 187 & 1.41 & 1.00 \\
\hline \hline
\end{tabular}

Tabla A.25: o Aqr - Anchos equivalentes, flujos y FWHM de las líneas de hidrógeno.

\begin{tabular}{c|ccc||c|ccc||c|ccc}
\hline \hline & \multicolumn{3}{|c|}{ Banda J } & & \multicolumn{3}{c|}{ Banda $\mathrm{H}$} & & \multicolumn{3}{c}{ Banda K } \\
& EW & Fl & FWHM & & EW & Fl & FWHM & & EW & Fl & FWHM \\
\hline $\mathrm{Pa} \beta$ & 19.77 & 603.00 & 299 & $\mathrm{Br}_{9}$ & 17.52 & 174.20 & 431 & $\mathrm{Pf}_{17}$ & & $\mathrm{em}$ & \\
& & & & $\mathrm{Br}_{10}$ & 9.44 & 102.77 & 437 & $\mathrm{Pf}_{18}$ & 13.62 & 44.43 & 595 \\
& & & $\mathrm{Br}_{11}$ & 8.26 & 102.29 & 450 & $\mathrm{Pf}_{19}$ & 6.23 & 22.00 & 564 \\
& & & $\mathrm{Br}_{12}$ & 7.33 & 99.06 & 407 & $\mathrm{Pf}_{20}$ & 6.38 & 22.72 & 644 \\
& & & $\mathrm{Br}_{13}$ & 6.84 & 98.50 & 514 & $\mathrm{Pf}_{21}$ & 5.24 & 18.97 & 630 \\
& & & $\mathrm{Br}_{14}$ & 5.73 & 91.13 & 601 & $\mathrm{Pf}_{22}$ & 4.04 & 14.92 & 621 \\
& & $\mathrm{Br}_{15}$ & 5.50 & 90.52 & 517 & $\mathrm{Pf}_{23}$ & 3.52 & 13.11 & 708 \\
& & & $\mathrm{Br}_{16}$ & 4.04 & 67.89 & 713 & $\mathrm{Pf}_{24}$ & 2.59 & 9.78 & 563 \\
& & $\mathrm{Br}_{17}$ & 4.13 & 71.76 & 818 & $\mathrm{Br} \gamma$ & 19.43 & 100.61 & 322 \\
& & $\mathrm{Br}_{18}$ & 3.38 & 59.62 & 780 & $\mathrm{Br} \delta$ & 21.22 & 166.60 & 404 \\
& & $\mathrm{Br}_{19}$ & 2.28 & 40.72 & 602 & $\mathrm{~Pa} \alpha$ & 112.46 & 1042.52 & 393 \\
& & & $\mathrm{Br}_{20}$ & 2.66 & 47.72 & 773 & & & & \\
\hline \hline
\end{tabular}

Tabla A.26: o Aqr - Densidades columnares y tamaños de las regiones emisoras de las líneas de las series de Humphreys, Pfund, Brackett y Paschen a partir de los métodos $N$ y FWHM. Los valores indicados entre paréntesis fueron obtenidos a partir del tamaño relativo a $\mathrm{Br}_{10}$.

\begin{tabular}{|c|c|c|c|c|c|c|c|c|c|c|c|c|c|c|}
\hline & \multirow{2}{*}{$\begin{array}{c}\text { Método FWHM } \\
\frac{R(\operatorname{Pa})}{R(\operatorname{Pa} \alpha)} \\
\end{array}$} & \multirow{2}{*}{$\begin{array}{c}\text { Método } \Delta V \\
R_{\mathrm{n}}\end{array}$} & & \multirow{2}{*}{$\begin{array}{c}\text { Método FWHM } \\
\frac{R\left(\mathrm{Pf}_{\mathrm{n}}\right)}{R\left(\mathrm{Pf}_{18}\right)}\end{array}$} & \multicolumn{3}{|c|}{ Método $\Delta V$} & & \multicolumn{2}{|c|}{ Método $N$} & \multirow{2}{*}{$\begin{array}{c}\text { Método FWHM } \\
\frac{R\left(\mathrm{Br}_{\mathrm{n}}\right)}{R\left(\mathrm{Br}_{\mathrm{g}}\right)} \\
\end{array}$} & \multicolumn{3}{|c|}{ Método $\Delta V$} \\
\hline & & & & & $\Delta V_{\mathrm{n}}$ & $R_{\mathrm{n}}$ & $\frac{R\left(\mathrm{Pf}_{\mathrm{n}}\right)}{R\left(\mathrm{Pf}_{18}\right)}$ & & $N$ & $\frac{\Delta R\left(\mathrm{Br}_{\mathrm{n}}\right)}{\Delta R\left(\mathrm{Br}_{9}\right)}$ & & $\Delta V_{\mathrm{n}}$ & $R_{\mathrm{n}}$ & $\frac{R\left(\mathrm{Br}_{\mathrm{n}}\right)}{R\left(\mathrm{Br}_{10}\right)}$ \\
\hline $\mathrm{Pa} \alpha$ & 1.00 & $(6.44)$ & $\mathrm{Pf}_{18}$ & 1.00 & 337 & 2.80 & 1.00 & $\mathrm{Br} \gamma$ & - & - & 1.79 & - & (9.59) & - \\
\hline $\mathrm{Pa} \beta$ & 1.73 & (11.12) & $\mathrm{Pf}_{19}$ & 1.11 & 349 & 2.61 & 0.93 & $\operatorname{Br} \delta$ & - & - & 1.14 & - & (6.09) & - \\
\hline & & & $\mathrm{Pf}_{21}$ & 0.89 & 365 & 2.39 & 0.85 & $\mathrm{Br}_{10}$ & 12.78 & 2.11 & 0.97 & 247 & 5.21 & 1.00 \\
\hline & & & $\mathrm{Pf}_{22}$ & 0.92 & 341 & 2.74 & 0.98 & $\mathrm{Br}_{11}$ & 14.58 & 2.41 & 0.92 & 295 & 3.66 & 0.70 \\
\hline & & & $\mathrm{Pf}_{23}$ & 0.71 & 327 & 2.97 & 1.06 & $\mathrm{Br}_{12}$ & 13.19 & 2.18 & 1.12 & 303 & 3.46 & 0.66 \\
\hline & & & & & & & & $\mathrm{Br}_{15}$ & - & - & 0.69 & 352 & 2.57 & 0.49 \\
\hline & & & & & & & & $\mathrm{Br}_{16}$ & - & - & 0.37 & 394 & 2.05 & 0.39 \\
\hline & & & & & & & & $\mathrm{Br}_{17}$ & - & - & 0.28 & 429 & 1.73 & 0.33 \\
\hline & & & & & & & & $\mathrm{Br}_{18}$ & - & - & 0.31 & 429 & 1.73 & 0.33 \\
\hline & & & & & & & & $\mathrm{Br}_{19}$ & - & - & 0.51 & 364 & 2.40 & 0.46 \\
\hline & & & & & & & & $\mathrm{Br}_{20}$ & - & - & 0.31 & 443 & 1.62 & 0.31 \\
\hline
\end{tabular}


Tabla A.27: $\pi$ Aqr - Anchos equivalentes, flujos y FWHM de las líneas de hidrógeno.

\begin{tabular}{|c|c|c|c|c|c|c|c|c|c|c|c|}
\hline & \multicolumn{3}{|c|}{ Banda J } & & \multicolumn{3}{|c|}{ Banda H } & & \multicolumn{3}{|c|}{ Banda K } \\
\hline & EW & $\mathrm{Fl}$ & FWHM & & EW & $\mathrm{Fl}$ & FWHM & & EW & $\mathrm{Fl}$ & FWHM \\
\hline \multirow[t]{14}{*}{$\mathrm{Pa} \beta$} & 10.12 & 41.82 & 357 & $\mathrm{Br}_{10}$ & 6.20 & 9.70 & - & $\mathrm{Pf}_{17}$ & 8.19 & 4.67 & - \\
\hline & & & & $\mathrm{Br}_{11}$ & 5.85 & 9.81 & - & $\mathrm{Pf}_{18}$ & 4.85 & 2.85 & - \\
\hline & & & & $\mathrm{Br}_{12}$ & 5.93 & 10.11 & 455 & $\mathrm{Pf}_{19}$ & 6.73 & 3.99 & - \\
\hline & & & & $\mathrm{Br}_{13}$ & 4.20 & 7.47 & 393 & $\mathrm{Pf}_{20}$ & 4.39 & 2.67 & - \\
\hline & & & & $\mathrm{Br}_{14}$ & 5.54 & 10.37 & 500 & $\mathrm{Pf}_{21}$ & 6.44 & 3.94 & - \\
\hline & & & & $\mathrm{Br}_{15}$ & 4.93 & 9.64 & 473 & $\mathrm{Pf}_{22}$ & 4.37 & 2.76 & - \\
\hline & & & & $\mathrm{Br}_{16}$ & 4.68 & 9.63 & 495 & $\mathrm{Pf}_{23}$ & 3.69 & 2.37 & - \\
\hline & & & & $\mathrm{Br}_{17}$ & 4.88 & 10.39 & 514 & $\mathrm{Pf}_{24}$ & 3.51 & 2.29 & - \\
\hline & & & & $\mathrm{Br}_{18}$ & 4.60 & 10.21 & 491 & $\mathrm{Pf}_{25}$ & 3.19 & 2.11 & - \\
\hline & & & & $\mathrm{Br}_{19}$ & 4.00 & 9.16 & 490 & $\mathrm{Pf}_{26}$ & 2.16 & 1.45 & - \\
\hline & & & & $\mathrm{Br}_{20}$ & 3.55 & 8.40 & 474 & $\mathrm{Pf}_{27}$ & & em & \\
\hline & & & & $\mathrm{Br}_{21}$ & 3.96 & 9.52 & 483 & $\mathrm{Pf}_{28}$ & & em & \\
\hline & & & & $\mathrm{Br}_{22}$ & 2.63 & 6.41 & 480 & $\mathrm{Br} \gamma$ & 10.51 & 9.93 & 359 \\
\hline & & & & $\mathrm{Br}_{23}$ & 2.25 & 5.62 & 492 & & & & \\
\hline
\end{tabular}

Tabla A.28: $\pi$ Aqr - Densidades columnares y tamaños de las regiones emisoras de las líneas de las series de Pfund, Brackett y Paschen a partir del método $\Delta V$.

\begin{tabular}{|c|c|c|c|c|c|c|c|c|c|c|c|c|c|c|}
\hline & \multicolumn{3}{|c|}{ Método $\Delta V$} & & \multicolumn{2}{|c|}{ Método $N$} & \multirow{2}{*}{$\begin{array}{c}\text { Método FWHM } \\
\frac{R\left(\mathrm{Br}_{\mathrm{n}}\right)}{R\left(\mathrm{Br}_{12}\right)}\end{array}$} & \multicolumn{3}{|c|}{ Método $\Delta V$} & & \multicolumn{3}{|c|}{ Método $\Delta V$} \\
\hline & $\Delta V_{\mathrm{n}}$ & $R_{\mathrm{n}}$ & $\frac{R\left(\mathrm{Pf}_{\mathrm{n}}\right)}{R\left(\mathrm{Pf}_{17}\right)}$ & & $N$ & $\frac{\Delta R\left(\mathrm{Br}_{\mathrm{n}}\right)}{\Delta R\left(\mathrm{Br}_{12}\right)}$ & & $\Delta V_{\mathrm{n}}$ & $R_{\mathrm{n}}$ & $\frac{R\left(\mathrm{Br}_{\mathrm{n}}\right)}{R\left(\mathrm{Br}_{12}\right)}$ & & $\Delta V_{\mathrm{n}}$ & $R$ & $\frac{R\left(\mathrm{~Pa}_{\mathrm{n}}\right)}{R(\mathrm{~Pa} \beta)}$ \\
\hline $\mathrm{Pf}_{17}$ & 250 & 3.47 & 1.00 & $\mathrm{Br} \gamma$ & - & - & 1.61 & 211 & 4.88 & 1.41 & $\mathrm{~Pa} \beta$ & 212 & 4.8 & 1.00 \\
\hline $\mathrm{Pf}_{18}$ & 268 & 3.02 & 0.87 & $\mathrm{Br}_{10}$ & - & - & - & 257 & 3.29 & 0.95 & & & & \\
\hline $\mathrm{Pf}_{19}$ & 307 & 2.30 & 0.66 & $\mathrm{Br}_{11}$ & - & - & - & 247 & 3.56 & 1.03 & & & & \\
\hline $\mathrm{Pf}_{20}$ & 278 & 2.81 & 0.81 & $\mathrm{Br}_{12}$ & 39.11 & 1.00 & 1.00 & 251 & 3.45 & 1.00 & & & & \\
\hline & & & & $\mathrm{Br}_{13}$ & 48.49 & 1.24 & 1.34 & 272 & 2.94 & 0.85 & & & & \\
\hline & & & & $\mathrm{Br}_{14}$ & 47.03 & 1.20 & 0.83 & 288 & 2.62 & 0.76 & & & & \\
\hline & & & & $\mathrm{Br}_{15}$ & 46.87 & 1.20 & 0.93 & 268 & 3.02 & 0.88 & & & & \\
\hline & & & & $\mathrm{Br}_{16}$ & 42.01 & 1.07 & 0.84 & 293 & 2.53 & 0.73 & & & & \\
\hline & & & & $\mathrm{Br}_{17}$ & 20.38 & 0.52 & 0.78 & 287 & 2.64 & 0.76 & & & & \\
\hline & & & & $\mathrm{Br}_{18}$ & - & - & 0.86 & 274 & 2.89 & 0.84 & & & & \\
\hline & & & & $\mathrm{Br}_{19}$ & - & - & 0.86 & 281 & 2.75 & 0.80 & & & & \\
\hline & & & & $\mathrm{Br}_{20}$ & - & - & 0.92 & 274 & 2.89 & 0.84 & & & & \\
\hline & & & & $\mathrm{Br}_{21}$ & - & - & 0.55 & 275 & 2.87 & 0.83 & & & & \\
\hline & & & & $\mathrm{Br}_{22}$ & - & - & 0.56 & 276 & 2.85 & 0.83 & & & & \\
\hline & & & & $\mathrm{Br}_{23}$ & - & - & 0.53 & 269 & 3.00 & 0.87 & & & & \\
\hline
\end{tabular}




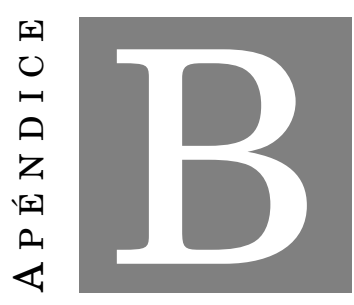

\section{Mediciones CORREspondientes AL CAPÍtulo 6}

\section{$\mathrm{P}$} ara cada objeto presentado en el Capítulo 6, las primeras tablas listan los valores obtenidos para los anchos equivalentes (EW) en $\AA$, los flujos (Fl) en unidades de $10^{-13} \frac{\mathrm{erg}}{\mathrm{cm}^{2} \mathrm{~s} \AA}$ y los anchos a mitad de altura (FWHM) en $\mathrm{km} / \mathrm{s}$ de las líneas de hidrógeno, separados por bandas y por año de observación. Aquellas líneas distinguibles por sobre el continuo, pero cuyos anchos equivalentes y flujos son difíciles de medir están indicadas con "em", en el caso de líneas en emisión y "shell", en el caso de líneas de envoltura. Para los anchos equivalentes consideramos valores positivos para representar las líneas en emisión (contrario a lo estándar). Las siguientes tablas muestran los valores de las densidades columnares $(N)$ en unidades de $\frac{10^{14}}{\mathrm{~cm}^{2}}$ y las extensiones relativas de las regiones de formación $\left(\frac{\Delta R}{\Delta R(\mathrm{ref})}\right)$ obtenidos con el método $N$; los radios internos de las regiones de formación relativos a una de referencia $\left(\frac{R}{R(\mathrm{ref})}\right)$ obtenidos con el método FWHM; y los valores de la separación de los picos para las líneas con dos picos en emisión $(\Delta V)$ en $\mathrm{km} / \mathrm{s}$, los radios internos de las regiones de formación $(R)$ en $R_{\star} \mathrm{y}$ los radios internos de las regiones de formación respecto a una línea de referencia $\left(\frac{R}{R(\text { ref })}\right)$, calculados utilizando el método $\Delta V$. Las tablas que incluyen los parámetros de los discos se encuentra separadas por serie espectral.

Tabla B.1: BK Cam - Anchos equivalentes, flujos y FWHM de las líneas de hidrógeno presentes en la banda J.

\begin{tabular}{c|ccc}
\hline \hline & & 3 & 2016 \\
& EW & Fl & FWHM \\
\hline $\mathrm{Pa} \beta$ & 15.04 & 552.22 & 325. \\
\hline \hline
\end{tabular}


Tabla B.2: BK Cam - Anchos equivalentes, flujos y FWHM de las líneas de hidrógeno presentes en la banda $\mathrm{H}$.

\begin{tabular}{l|ccc|ccc|ccc}
\hline \hline & \multicolumn{3}{|c|}{2010} & \multicolumn{3}{c|}{2012} & \multicolumn{3}{c}{2016} \\
& EW & Fl & FWHM & EW & Fl & FWHM & EW & Fl & FWHM \\
\hline $\mathrm{Br}_{9}$ & 26.83 & 164.83 & 472 & 23.84 & 321.00 & 513 & 25.40 & 269.77 & 586 \\
$\mathrm{Br}_{10}$ & 13.85 & 112.98 & 497 & 13.06 & 204.11 & 542 & 17.82 & 217.73 & 689 \\
$\mathrm{Br}_{11}$ & 12.39 & 111.73 & 440 & 13.16 & 219.82 & 575 & 12.78 & 172.52 & 473 \\
$\mathrm{Br}_{12}$ & 11.24 & 108.01 & 439 & 11.92 & 211.78 & 615 & 11.38 & 166.64 & 454 \\
$\mathrm{Br}_{13}$ & 9.65 & 98.86 & 452 & 9.80 & 183.32 & 487 & 10.36 & 158.14 & 474 \\
$\mathrm{Br}_{14}$ & 10.34 & 112.65 & 547 & 10.59 & 211.90 & 574 & 10.20 & 165.02 & 514 \\
$\mathrm{Br}_{15}$ & 7.61 & 84.40 & 565 & 8.56 & 177.05 & 555 & 12.41 & 210.03 & 564 \\
$\mathrm{Br}_{16}$ & 7.50 & 86.85 & 520 & 8.96 & 194.09 & 601 & 9.07 & 155.40 & 632 \\
$\mathrm{Br}_{17}$ & 8.88 & 105.01 & 660 & 8.25 & 183.69 & 597 & 7.86 & 141.84 & 539 \\
$\mathrm{Br}_{18}$ & 6.51 & 79.40 & 554 & 6.19 & 141.86 & 556 & 7.02 & 128.89 & 575 \\
$\mathrm{Br}_{19}$ & 5.07 & 64.14 & 536 & 6.17 & 145.00 & 559 & 5.65 & 106.08 & 542 \\
$\mathrm{Br}_{20}$ & 4.00 & 51.71 & 507 & 4.87 & 117.59 & 541 & 4.17 & 80.68 & 539 \\
$\mathrm{Br}_{21}$ & 2.95 & 39.51 & 497 & 3.32 & 82.94 & 527 & 3.03 & 59.79 & 491 \\
$\mathrm{Br}_{22}$ & 1.70 & 23.30 & 514 & 1.47 & 37.41 & 305 & 2.05 & 41.35 & 449 \\
$\mathrm{Br}_{23}$ & 1.18 & 16.68 & - & 1.60 & 42.09 & 482 & 1.18 & 24.46 & 456 \\
$\mathrm{Br}_{24}$ & 1.24 & 17.63 & - & - & - & - & & em & \\
$\mathrm{Br}_{25}$ & 0.79 & 11.25 & - & - & - & - & & em & \\
\hline \hline
\end{tabular}

Tabla B.3: BK Cam - Anchos equivalentes, flujos y FWHM de las líneas de hidrógeno presentes en la banda $\mathrm{K}$.

\begin{tabular}{c|ccc|ccc|ccc|ccc}
\hline \hline & \multicolumn{3}{|c|}{2008} & & \multicolumn{3}{c|}{2010} & \multicolumn{3}{c|}{2012} & \multicolumn{3}{c}{2016} \\
& $\mathrm{EW}$ & $\mathrm{Fl}$ & $\mathrm{FWHM}$ & $\mathrm{EW}$ & $\mathrm{Fl}$ & $\mathrm{FWHM}$ & $\mathrm{EW}$ & $\mathrm{Fl}$ & FWHM & EW & Fl & $\mathrm{FWHM}$ \\
\hline $\mathrm{Pf}_{17}$ & 26.07 & 60.39 & 844 & 25.79 & 60.27 & 464 & 18.08 & 84.77 & 630 & 17.39 & 68.83 & 592 \\
$\mathrm{Pf}_{18}$ & 12.81 & 32.43 & 473 & 22.22 & 55.22 & 956 & 12.70 & 69.15 & 533 & 15.65 & 63.82 & 517 \\
$\mathrm{Pf}_{19}$ & 12.92 & 34.00 & 571 & 13.74 & 36.31 & 602 & 10.03 & 52.54 & 497 & 12.54 & 52.83 & 545 \\
$\mathrm{Pf}_{20}$ & 11.12 & 30.34 & 589 & 11.51 & 31.32 & 519 & 10.91 & 57.33 & 557 & 12.77 & 54.57 & 613 \\
$\mathrm{Pf}_{21}$ & 9.27 & 25.87 & 633 & 13.60 & 37.10 & 757 & 8.35 & 45.17 & 550 & 9.76 & 42.90 & 606 \\
$\mathrm{Pf}_{22}$ & 6.42 & 18.52 & 509 & 10.54 & 29.60 & 691 & 7.61 & 42.06 & 553 & 7.46 & 33.66 & 507 \\
$\mathrm{Pf}_{23}$ & 4.61 & 13.55 & 474 & 5.12 & 15.19 & 417 & 5.44 & 30.59 & 531 & 5.28 & 24.41 & 518 \\
$\mathrm{Pf}_{24}$ & 3.24 & 9.77 & 540 & 4.07 & 12.53 & 572 & 3.69 & 21.25 & 464 & 3.93 & 18.52 & 397 \\
$\mathrm{Pf}_{25}$ & 2.46 & 7.56 & 378 & 2.55 & 8.01 & 397 & 2.81 & 16.45 & 497 & 2.99 & 14.32 & 452 \\
$\mathrm{Pf}_{26}$ & 1.74 & 5.46 & 576 & - & - & - & 1.68 & 10.06 & 412 & 2.97 & 14.48 & 571 \\
$\mathrm{Pf}_{27}$ & 0.92 & 2.94 & - & - & - & - & 1.59 & 9.61 & 460 & 1.10 & 5.44 & 299 \\
$\mathrm{Pf}_{28}$ & 0.39 & 1.27 & - & - & - & - & 1.74 & 10.70 & - & 0.49 & 2.48 & - \\
$\mathrm{Pf}_{29}$ & - & - & - & - & - & - & & em & & 0.71 & 3.59 & - \\
$\mathrm{Pf}_{30}$ & - & - & - & - & - & - & & $\mathrm{em}$ & & - & - & - \\
$\mathrm{Pf}_{31}$ & - & - & - & - & - & - & & $\mathrm{em}$ & & - & - & - \\
$\mathrm{Pf}_{32}$ & - & - & - & - & - & - & & $\mathrm{em}$ & & - & - & - \\
$\mathrm{Pf}_{33}$ & - & - & - & - & - & - & & em & & - & - & - \\
$\mathrm{Br} \gamma$ & 18.41 & 80.06 & 546 & 19.45 & 86.92 & 373. & 16.60 & 127.68 & 389 & 17.88 & 119.76 & 401 \\
$\mathrm{Br} \delta$ & 27.71 & 164.15 & 811 & 25.07 & 152.28 & 376. & 25.18 & 262.51 & 510 & 24.03 & 221.82 & 502 \\
$\mathrm{~Pa} \alpha$ & - & - & - & - & - & - & - & - & - & 69.13 & 689.22 & 409 \\
\hline \hline
\end{tabular}


Tabla B.4: BK Cam - Anchos equivalentes, flujos y FWHM de las líneas de hidrógeno presentes en la banda L.

\begin{tabular}{l|ccc|ccc|ccc|ccc}
\hline \hline & \multicolumn{3}{|c|}{2008} & \multicolumn{3}{c|}{2010} & \multicolumn{3}{c|}{2012} & \multicolumn{3}{c}{2016} \\
& EW & Fl & FWHM & EW & Fl & FWHM & EW & Fl & FWHM & EW & Fl & FWHM \\
\hline $\mathrm{Hu}_{13}$ & - & - & - & 51.52 & 29.18 & 463 & 38.95 & 27.29 & 528 & - & - & - \\
$\mathrm{Br} \alpha$ & 54.32 & 31.28 & 582 & 72.56 & 41.42 & 303 & 54.23 & 43.27 & 350 & 50.20 & 40.22 & 303 \\
$\mathrm{Hu}_{14}$ & 37.53 & 22.25 & 794 & 42.78 & 25.39 & 449 & 27.86 & 23.20 & 490 & 35.93 & 29.80 & 489 \\
$\mathrm{Hu}_{15}$ & 30.98 & 19.75 & 666 & 39.91 & 24.98 & 472 & 31.42 & 26.97 & 581 & 34.39 & 31.00 & 553 \\
$\mathrm{Hu}_{16}$ & 30.60 & 20.42 & 724 & 33.63 & 22.15 & 475 & 29.25 & 26.87 & 602 & 31.22 & 30.33 & 553 \\
$\mathrm{Hu}_{17}$ & 20.59 & 14.84 & 590 & 27.71 & 19.74 & 462 & 25.04 & 24.86 & 574 & 26.45 & 27.67 & 525 \\
$\mathrm{Pf} \gamma$ & 33.32 & 24.10 & 648 & 41.46 & 29.59 & 380 & 32.37 & 32.32 & 439 & 33.67 & 35.39 & 405 \\
$\mathrm{Hu}_{18}$ & 29.14 & 21.44 & 852 & 25.75 & 18.64 & 518 & 21.52 & 21.79 & 592 & 22.48 & 24.24 & 527 \\
$\mathrm{Hu}_{19}$ & 20.41 & 15.73 & 765 & 21.58 & 16.24 & 512 & 17.67 & 18.65 & 624 & 18.17 & 20.42 & 530 \\
$\mathrm{Hu}_{20}$ & 15.61 & 12.22 & 746 & 17.86 & 13.92 & 521 & 16.70 & 17.90 & 665 & 15.62 & 17.98 & 554 \\
$\mathrm{Hu}_{21}$ & 10.07 & 8.05 & 613 & 14.02 & 11.41 & 512 & 12.48 & 13.86 & 601 & 14.71 & 17.32 & 584 \\
$\mathrm{Hu}_{22}$ & 11.23 & 9.62 & 685 & 11.91 & 9.99 & 524 & 9.57 & 10.93 & 561 & 10.96 & 13.35 & 558 \\
$\mathrm{Hu}_{23}$ & 8.83 & 8.05 & 572 & 10.11 & 8.57 & 533 & 8.37 & 9.87 & 617 & 8.66 & 10.84 & 510 \\
$\mathrm{Hu}_{24}$ & 5.29 & 4.76 & 430 & 8.58 & 7.45 & 553 & 6.44 & 7.75 & 576 & 8.39 & 10.69 & 561 \\
$\mathrm{Hu}_{25}$ & - & - & - & 6.20 & 5.53 & 528 & 4.79 & 5.86 & 493 & 5.95 & 7.72 & 500 \\
$\mathrm{Hu}_{26}$ & - & - & - & 4.66 & 4.21 & 427 & 2.91 & 3.61 & 465 & 4.33 & 5.69 & 514 \\
$\mathrm{Hu}_{27}$ & - & - & - & 3.77 & 3.48 & 425 & 2.77 & 3.49 & 520 & 3.38 & 4.51 & 517 \\
$\mathrm{Hu}_{28}$ & - & - & - & 2.72 & 2.55 & 337 & 1.49 & 1.91 & 435 & - & - & - \\
$\mathrm{Hu}_{29}$ & - & - & - & 2.44 & 2.31 & 461 & 1.69 & 2.19 & 645 & - & - & - \\
$\mathrm{Hu}_{30}$ & - & - & - & 2.52 & 2.42 & - & - & - & - & - & - & - \\
$\mathrm{Hu}_{31}$ & - & - & - & 1.56 & 1.52 & - & - & - & - & - & - & - \\
$\mathrm{Pf} \delta^{27.68}$ & 29.68 & 680 & 33.76 & 37.28 & 317 & 32.20 & 47.97 & 459 & 28.09 & 45.81 & 343 \\
\hline \hline
\end{tabular}

Tabla B.5: BK Cam - Densidades columnares y tamaños de las regiones emisoras de las líneas de la serie de Humphreys a partir de los métodos $N$, FWHM y $\Delta V$. Los valores indicados entre paréntesis fueron obtenidos a partir del tamaño relativo a $\mathrm{Pf}_{18}$.

\begin{tabular}{|c|c|c|c|c|c|c|c|c|c|c|c|c|c|c|c|}
\hline & \multicolumn{8}{|c|}{ Método $N$} & \multicolumn{4}{|c|}{ Método FWHM } & \multicolumn{3}{|c|}{ Método $\Delta V$} \\
\hline & \multicolumn{2}{|c|}{2008} & \multicolumn{2}{|c|}{2010} & \multicolumn{2}{|c|}{2012} & \multicolumn{2}{|c|}{2016} & \multirow{2}{*}{$\begin{array}{c}2008 \\
\frac{R\left(\mathrm{Hu}_{\mathrm{n}}\right)}{R\left(\mathrm{Hu}_{14}\right)}\end{array}$} & \multirow{2}{*}{$\begin{array}{c}2010 \\
\frac{R\left(\mathrm{Hu}_{\mathrm{n}}\right)}{R\left(\mathrm{Hu}_{14}\right)}\end{array}$} & \multirow{2}{*}{$\begin{array}{c}2012 \\
\frac{R\left(\mathrm{Hu}_{\mathrm{n}}\right)}{R\left(\mathrm{Hu}_{14}\right)}\end{array}$} & \multirow{2}{*}{$\begin{array}{c}2016 \\
\frac{R\left(\mathrm{Hu}_{\mathrm{n}}\right)}{R\left(\mathrm{Hu}_{14}\right)}\end{array}$} & \multicolumn{3}{|c|}{2010} \\
\hline & $N$ & $\frac{\Delta R\left(\mathrm{Hu}_{\mathrm{n}}\right)}{\Delta R\left(\mathrm{Hu}_{14}\right)}$ & $N$ & $\frac{\Delta R\left(\mathrm{Hu}_{\mathrm{n}}\right)}{\Delta R\left(\mathrm{Hu}_{14}\right)}$ & $N$ & $\frac{\Delta R\left(\mathrm{Hu}_{\mathrm{n}}\right)}{\Delta R\left(\mathrm{Hu}_{14}\right)}$ & $N$ & $\frac{\Delta R\left(\mathrm{Hu}_{\mathrm{n}}\right)}{\Delta R\left(\mathrm{Hu}_{14}\right)}$ & & & & & $\Delta V_{\mathrm{n}}$ & $R_{\mathrm{n}}$ & $\frac{R\left(\mathrm{Hu}_{\mathrm{n}}\right)}{R\left(\mathrm{Hu}_{14}\right)}$ \\
\hline $\mathrm{Hu}_{13}$ & - & - & 4.08 & 0.84 & 6.36 & 0.81 & - & - & - & 0.94 & 0.86 & - & 249 & 7.52 & 1.08 \\
\hline $\mathrm{Hu}_{15}$ & 10.29 & 0.99 & 4.27 & 0.88 & 6.38 & 0.81 & 5.23 & 0.86 & 1.42 & 0.90 & 0.71 & 0.78 & 263 & 6.71 & 0.96 \\
\hline $\mathrm{Hu}_{16}$ & 8.03 & 0.77 & 4.28 & 0.88 & 4.05 & 0.51 & 3.02 & 0.50 & 1.20 & 0.89 & 0.66 & 0.78 & 263 & 6.71 & 0.96 \\
\hline $\mathrm{Hu}_{17}$ & 12.71 & 1.22 & 3.60 & 0.74 & - & - & - & - & 1.81 & 0.94 & 0.73 & 0.87 & 216 & 9.95 & 1.43 \\
\hline $\mathrm{Hu}_{20}$ & - & - & - & - & - & - & - & - & 1.13 & 0.74 & 0.54 & 0.78 & - & $(9.93)$ & - \\
\hline $\mathrm{Hu}_{21}$ & - & - & - & - & - & - & - & - & 1.68 & 0.77 & 0.66 & 0.70 & - & (10.28) & - \\
\hline $\mathrm{Hu}_{22}$ & - & - & - & - & - & - & - & - & 1.34 & 0.73 & 0.76 & 0.77 & - & $(9.82)$ & - \\
\hline $\mathrm{Hu}_{23}$ & - & - & - & - & - & - & - & - & 1.93 & 0.71 & 0.63 & 0.92 & - & (9.49) & - \\
\hline $\mathrm{Hu}_{24}$ & - & - & - & - & - & - & - & - & 3.41 & 0.66 & 0.72 & 0.76 & - & $(8.81)$ & - \\
\hline $\mathrm{Hu}_{29}$ & - & - & - & - & - & - & - & - & - & 0.95 & 0.58 & - & - & (12.68) & - \\
\hline
\end{tabular}


Tabla B.6: BK Cam - Densidades columnares y tamaños de las regiones emisoras de las líneas de la serie de Pfund a partir de los métodos $N$, FWHM y $\Delta V$. Los valores indicados entre paréntesis fueron obtenidos a partir del tamaño relativo a $\mathrm{Pf}_{18}$ en el 2010 y a $\mathrm{Br}_{10}$ en el 2012.

\begin{tabular}{|c|c|c|c|c|c|c|c|c|c|c|c|c|c|c|c|c|c|c|}
\hline & \multicolumn{8}{|c|}{ Método $N$} & \multicolumn{4}{|c|}{ Método FWHM } & \multicolumn{6}{|c|}{ Método $\Delta V$} \\
\hline & \multicolumn{2}{|c|}{2008} & \multicolumn{2}{|c|}{2010} & \multicolumn{2}{|c|}{2012} & \multicolumn{2}{|c|}{2016} & $\begin{array}{l}2008 \\
\frac{R\left(\mathrm{Pf}_{\mathrm{f}}\right)}{R(\mathrm{P} \gamma \mathrm{\gamma})}\end{array}$ & $\frac{2010}{R\left(\mathrm{Pf}_{\mathrm{n}}\right)}$ & $\begin{array}{l}2012 \\
\frac{R\left(\mathrm{Pf}_{\mathrm{f}}\right)}{R(\mathrm{Pf})}\end{array}$ & $\begin{array}{l}2016 \\
\frac{R\left(\mathrm{Pf}_{\mathrm{f}}\right)}{R(\mathrm{Pf} \gamma)}\end{array}$ & \multicolumn{2}{|r|}{2010} & $\frac{R\left(\mathrm{Pf}_{\mathrm{n}}\right)}{R\left(\mathrm{Pf}_{18}\right)}$ & $\Delta V_{\mathrm{n}}$ & $\begin{array}{l}2012 \\
R_{\mathrm{n}}\end{array}$ & $\frac{R\left(\mathrm{Pf}_{\mathrm{n}}\right)}{R\left(\mathrm{Pf}_{\mathrm{f}_{3}}\right)}$ \\
\hline $\mathrm{Pf} \gamma$ & 4.20 & 1.00 & 2.34 & 1.00 & 2.99 & 1.00 & 2.68 & 1.00 & 1.00 & 1.00 & 1.00 & 1.00 & - & (18.67) & - & - & (9.36) & - \\
\hline $\mathrm{Pf}_{17}$ & - & - & - & - & - & - & - & - & 0.59 & 0.67 & 0.49 & 0.47 & - & (12.52) & - & - & $(4.54)$ & - \\
\hline $\mathrm{Pf}_{18}$ & - & - & - & - & - & - & - & - & 1.88 & 0.16 & 0.68 & 0.61 & 403 & 2.87 & 1.00 & - & (6.35) & - \\
\hline $\mathrm{Pf}_{19}$ & - & - & - & - & - & - & - & - & 1.29 & 0.40 & 0.78 & 0.55 & 315 & 4.68 & 1.63 & - & $(7.30)$ & - \\
\hline $\mathrm{Pf}_{22}$ & - & - & - & - & - & - & - & - & 1.62 & 0.30 & 0.63 & 0.64 & 289 & 5.57 & 1.94 & - & (5.90) & - \\
\hline $\mathrm{Pf}_{23}$ & - & - & - & - & - & - & - & - & 1.87 & 0.83 & 0.68 & 0.61 & 367 & 3.45 & 1.20 & 206 & 10.94 & 1.00 \\
\hline $\mathrm{Pf}_{24}$ & - & - & - & - & - & - & - & - & 1.44 & 0.44 & 0.90 & 1.04 & 205 & 11.02 & 3.84 & 252 & 7.32 & 0.67 \\
\hline $\mathrm{Pf}_{25}$ & - & - & - & - & - & - & - & - & 2.94 & 0.92 & 0.78 & 0.80 & - & $(17.10)$ & - & 212 & 10.34 & 0.94 \\
\hline $\mathrm{Pf}_{26}$ & - & - & - & - & - & - & - & - & 1.27 & - & 1.14 & 0.50 & - & - & - & 194 & 12.39 & 1.13 \\
\hline $\mathrm{Pf}_{27}$ & - & - & - & - & - & - & - & - & - & - & 0.91 & 1.83 & - & - & - & 249 & 7.50 & 0.66 \\
\hline
\end{tabular}

Tabla B.7: BK Cam - Densidades columnares y tamaños de las regiones emisoras de las líneas de la serie de Brackett a partir de los métodos $N$, FWHM y $\Delta V$. Los valores indicados entre paréntesis fueron obtenidos a partir del tamaño relativo a $\mathrm{Pf}_{18}$ en el 2010 y a $\mathrm{Br}_{10}$ en el 2012.

\begin{tabular}{|c|c|c|c|c|c|c|c|c|c|c|c|c|c|c|c|c|}
\hline & \multicolumn{6}{|c|}{ Método $N$} & \multicolumn{4}{|c|}{ Método FWHM } & \multicolumn{6}{|c|}{ Método $\Delta V$} \\
\hline & \multicolumn{2}{|c|}{2010} & \multicolumn{2}{|c|}{2012} & \multicolumn{2}{|c|}{2016} & \multirow{2}{*}{$\begin{array}{c}2008 \\
\frac{R\left(\mathrm{Br}{ }_{\mathrm{n}}\right)}{R(\mathrm{Br} \delta)}\end{array}$} & \multirow{2}{*}{$\begin{array}{c}2010 \\
\frac{R\left(\mathrm{Br}_{\mathrm{n}}\right)}{R\left(\mathrm{Br}_{11}\right)}\end{array}$} & \multirow{2}{*}{$\begin{array}{c}2012 \\
\frac{R\left(\mathrm{Br}_{\mathrm{n}}\right)}{R\left(\mathrm{Br}_{11}\right)}\end{array}$} & \multirow{2}{*}{$\begin{array}{c}2016 \\
\frac{R\left(\mathrm{Br}_{\mathrm{n}}\right)}{R\left(\mathrm{Br}_{11}\right)}\end{array}$} & \multicolumn{2}{|r|}{2010} & \multirow[b]{2}{*}{$\frac{R\left(\mathrm{Br}_{\mathrm{n}}\right)}{R\left(\mathrm{Br}_{18}\right)}$} & \multirow[b]{2}{*}{$\Delta V_{\mathrm{n}}$} & \multirow{2}{*}{$\begin{array}{l}2012 \\
R_{\mathrm{n}}\end{array}$} & \multirow[b]{2}{*}{$\frac{R\left(\mathrm{Br}_{\mathrm{n}}\right)}{R\left(\mathrm{Br}_{18}\right)}$} \\
\hline & $N$ & $\frac{\Delta R\left(\mathrm{Br}_{\mathrm{n}}\right)}{\Delta R\left(\mathrm{Br}_{11}\right)}$ & $N$ & $\frac{\Delta R\left(\mathrm{Br}_{\mathrm{n}}\right)}{\Delta R\left(\mathrm{Br}_{11}\right)}$ & $N$ & $\frac{\Delta R\left(\mathrm{Br}_{\mathrm{n}}\right)}{\Delta R\left(\mathrm{Br}_{11}\right)}$ & & & & & $\Delta V_{\mathrm{n}}$ & $R_{\mathrm{n}}$ & & & & \\
\hline $\mathrm{Br} \alpha$ & - & - & - & - & - & - & 1.94 & 2.11 & 2.70 & 2.44 & - & (29.36) & - & - & (14.72) & - \\
\hline $\mathrm{Br} \gamma$ & - & - & - & - & - & - & 2.20 & 1.39 & 2.18 & 1.39 & - & (19.37) & - & - & (11.92) & - \\
\hline $\operatorname{Br} \delta$ & - & - & - & - & - & - & 1.00 & 1.37 & 1.27 & 0.89 & - & (19.07) & - & - & (6.93) & - \\
\hline $\mathrm{Br}_{9}$ & 10.83 & 0.49 & 13.28 & 0.40 & 13.58 & 0.53 & - & 0.87 & 1.26 & 0.65 & - & (12.10) & - & - & $(6.85)$ & - \\
\hline $\mathrm{Br}_{10}$ & 20.25 & 0.91 & 26.02 & 0.78 & 24.99 & 0.98 & - & 0.78 & 1.13 & 0.47 & - & (10.91) & - & 274 & 6.17 & 1.17 \\
\hline $\mathrm{Br}_{11}$ & 22.27 & 1.00 & 33.15 & 1.00 & 25.60 & 1.00 & - & 1.00 & 1.00 & 1.00 & - & (13.92) & - & 227 & 8.99 & 1.70 \\
\hline $\mathrm{Br}_{12}$ & 25.82 & 1.16 & 42.43 & 1.28 & 28.97 & 1.13 & - & 1.00 & 0.87 & 1.09 & - & (13.99) & - & 215 & 10.03 & 1.89 \\
\hline $\mathrm{Br}_{13}$ & 31.24 & 1.40 & 42.95 & 1.30 & 34.27 & 1.34 & - & 0.95 & 1.39 & 1.00 & - & (13.19) & - & 233 & 8.57 & 1.62 \\
\hline $\mathrm{Br}_{14}$ & 26.89 & 1.21 & 41.60 & 1.25 & 33.25 & 1.30 & - & 0.65 & 1.00 & 0.85 & - & $(9.01)$ & - & 302 & 5.11 & 0.97 \\
\hline $\mathrm{Br}_{15}$ & 44.76 & 2.01 & 50.32 & 1.52 & - & - & - & 0.61 & 1.07 & 0.70 & - & $(8.44)$ & - & 295 & 5.36 & 1.01 \\
\hline $\mathrm{Br}_{16}$ & 26.80 & 1.20 & 31.77 & 0.96 & - & - & - & 0.72 & 0.92 & 0.56 & - & $(9.97)$ & - & 276 & 6.11 & 1.15 \\
\hline $\mathrm{Br}_{17}$ & - & - & 18.08 & 0.55 & - & - & - & 0.44 & 0.93 & 0.77 & - & (6.189) & - & 289 & 5.57 & 1.05 \\
\hline $\mathrm{Br}_{18}$ & - & - & 33.27 & 1.00 & - & - & - & 0.63 & 1.07 & 0.68 & 302 & 5.09 & 1.00 & 296 & 5.30 & 1.00 \\
\hline $\mathrm{Br}_{19}$ & - & - & - & - & - & - & - & 0.67 & 1.06 & 0.76 & 291 & 5.48 & 1.08 & 253 & 7.28 & 1.37 \\
\hline $\mathrm{Br}_{20}$ & - & - & - & - & - & - & - & 0.75 & 1.13 & 0.77 & 285 & 5.70 & 1.12 & 261 & 6.83 & 1.29 \\
\hline $\mathrm{Br}_{21}$ & - & - & - & - & - & - & - & 0.56 & 0.54 & 0.67 & 311 & 4.80 & 0.94 & 229 & 8.83 & 1.67 \\
\hline $\mathrm{Br}_{22}$ & - & - & - & - & - & - & - & 0.53 & 1.63 & 0.80 & 309 & 4.87 & 0.96 & 252 & 7.33 & 1.38 \\
\hline $\mathrm{Br}_{23}$ & - & - & - & - & - & - & - & - & 0.65 & 0.77 & - & - & - & - & $(7.76)$ & - \\
\hline
\end{tabular}

Tabla B.8: BK Cam - Tamaños de las regiones emisoras de las líneas de la serie de Paschen a partir del método FWHM.

\begin{tabular}{c||c}
\hline \hline & Método FWHM \\
& 2016 \\
& $\frac{R(\mathrm{~Pa})}{R(\mathrm{~Pa} \alpha)}$ \\
\hline $\mathrm{Pa} \alpha$ & 1.00 \\
$\mathrm{~Pa} \beta$ & 1.58 \\
\hline \hline
\end{tabular}


Tabla B.9: 28 Tau - Anchos equivalentes, flujos y FWHM de las líneas de hidrógeno presentes en la banda J.

\begin{tabular}{c|ccc}
\hline \hline & & 3 & 2016 \\
& EW & Fl & FWHM \\
\hline $\mathrm{Pa} \beta$ & 16.16 & 368.68 & 383 \\
\hline \hline
\end{tabular}

Tabla B.10: 28 Tau - Anchos equivalentes, flujos y FWHM de las líneas de hidrógeno presentes en la banda $\mathrm{H}$.

\begin{tabular}{|c|c|c|c|c|c|c|c|c|c|}
\hline & \multicolumn{3}{|c|}{2010} & \multicolumn{3}{|c|}{2012} & \multicolumn{3}{|c|}{2016} \\
\hline & EW & $\mathrm{Fl}$ & FWHM & EW & $\mathrm{Fl}$ & FWHM & EW & $\mathrm{Fl}$ & FWHM \\
\hline $\mathrm{Br}_{9}$ & - & - & - & 13.33 & 93.64 & - & 14.73 & 102.04 & - \\
\hline $\mathrm{Br}_{10}$ & 10.54 & 122.46 & - & 8.97 & 73.83 & - & 9.43 & 76.86 & - \\
\hline $\mathrm{Br}_{11}$ & 9.79 & 120.35 & - & 7.94 & 70.19 & - & 6.73 & 61.09 & - \\
\hline $\mathrm{Br}_{12}$ & 8.43 & 118.08 & - & 7.25 & 69.61 & - & 6.34 & 61.66 & - \\
\hline $\mathrm{Br}_{13}$ & & shell & & & shell & & & shell & \\
\hline$\ldots$ & & shell & & & shell & & & shell & \\
\hline $\mathrm{Br}_{21}$ & & shell & & & shell & & & shell & \\
\hline $\mathrm{Br}_{22}$ & - & - & - & & shell & & & shell & \\
\hline $\mathrm{Br}_{23}$ & - & - & - & & shell & & & shell & \\
\hline
\end{tabular}

Tabla B.11: 28 Tau - Anchos equivalentes, flujos y FWHM de las líneas de hidrógeno presentes en la banda K.

\begin{tabular}{l|ccc|ccc|ccc|ccc}
\hline \hline & \multicolumn{3}{|c|}{2006} & \multicolumn{3}{c|}{2010} & \multicolumn{3}{c|}{2012} & \multicolumn{3}{c}{2016} \\
& EW & Fl & FWHM & EW & Fl & FWHM & EW & Fl & FWHM & EW & Fl & FWHM \\
\hline $\mathrm{Pf}_{17}$ & - & - & - & 8.28 & 16.99 & - & & shell & & 6.13 & 14.15 & - \\
$\mathrm{Pf}_{18}$ & - & - & - & 4.94 & 10.24 & - & & shell & & 3.57 & 8.45 & - \\
$\mathrm{Pf}_{19}$ & - & - & - & 4.38 & 10.52 & - & & shell & & 3.02 & 7.29 & - \\
$\mathrm{Pf}_{20}$ & - & - & - & 5.37 & 15.12 & - & & shell & & 2.40 & 5.97 & - \\
$\mathrm{Pf}_{21}$ & - & - & - & 2.08 & 5.68 & - & & shell & & 3.29 & 8.33 & - \\
$\mathrm{Pf}_{22}$ & - & - & - & 2.15 & 7.46 & - & & shell & & - & - & - \\
$\mathrm{Pf}_{23}$ & - & - & - & 1.65 & 5.81 & - & & shell & & - & - & - \\
$\mathrm{Pf}_{24}$ & - & - & - & & shell & & & shell & & - & - & - \\
$\mathrm{Br} \gamma$ & 20.62 & 30.21 & 420 & 17.45 & 52.27 & 384 & 15.17 & 49.58 & 490 & 16.26 & 59.77 & 387 \\
$\mathrm{Br} \delta$ & 28.40 & 61.46 & - & - & - & - & 18.57 & 89.27 & 791 & 17.08 & 96.17 & 401 \\
$\mathrm{~Pa} \alpha$ & - & - & - & - & - & - & - & - & - & 95.23 & 623.99 & 478 \\
\hline \hline
\end{tabular}


Tabla B.12: 28 Tau - Anchos equivalentes, flujos y FWHM de las líneas de hidrógeno presentes en la banda L.

\begin{tabular}{l|ccc|ccc|ccc|ccc}
\hline & \multicolumn{3}{|c|}{2006} & \multicolumn{3}{c|}{2010} & \multicolumn{3}{c}{2012} & \multicolumn{3}{c}{2016} \\
& EW & Fl & FWHM & EW & Fl & FWHM & EW & Fl & FWHM & EW & Fl & FWHM \\
\hline $\mathrm{Hu}_{13}$ & - & - & - & - & - & - & 17.77 & 9.15 & 453 & - & - & - \\
$\mathrm{Br} \alpha$ & 108.79 & 21.13 & 669 & 71.68 & 45.77 & 198 & 82.30 & 47.13 & 341 & 99.94 & 5.10 & 348 \\
$\mathrm{Hu}_{14}$ & 17.30 & 3.45 & - & 17.45 & 11.07 & 365 & 15.89 & 9.10 & 500 & 17.03 & 0.88 & 619 \\
$\mathrm{Hu}_{15}$ & 16.14 & 3.42 & - & 17.58 & 12.04 & 479 & 15.29 & 9.43 & 547 & 13.79 & 0.78 & 601 \\
$\mathrm{Hu}_{16}$ & - & - & - & 14.33 & 10.52 & 500 & 12.14 & 8.00 & 527 & 12.70 & 0.75 & 685 \\
$\mathrm{Hu}_{17}$ & - & - & - & 17.31 & 12.83 & 590 & 12.64 & 8.66 & 566 & 9.20 & 0.58 & 535 \\
$\mathrm{Pf}$ & 41.60 & 10.93 & 815 & 38.57 & 28.86 & 434 & 29.01 & 20.45 & 451 & 28.41 & 1.83 & 395 \\
$\mathrm{Hu}$ & - & - & - & 9.56 & 7.94 & 581 & 10.04 & 7.34 & 613 & 8.49 & 0.57 & 714 \\
$\mathrm{Hu}_{19}$ & - & - & - & 9.55 & 8.25 & 605 & 8.19 & 6.29 & 434 & 5.98 & 0.42 & 723 \\
$\mathrm{Hu}_{20}$ & - & - & - & 7.24 & 6.52 & 639 & 4.11 & 3.29 & 571 & 5.33 & 0.38 & 733 \\
$\mathrm{Hu}_{21}$ & - & - & - & 5.95 & 5.52 & 571 & 4.49 & 3.70 & 599 & 4.69 & 0.34 & 669 \\
$\mathrm{Hu}_{22}$ & - & - & - & 5.53 & 5.24 & 656 & 4.76 & 4.00 & 643 & 4.54 & 0.34 & 653 \\
$\mathrm{Hu}_{23}$ & - & - & - & 3.10 & 3.00 & 574 & 3.29 & 2.84 & 585 & 2.13 & 0.16 & 716 \\
$\mathrm{Hu}_{24}$ & - & - & - & 3.79 & 3.72 & 584 & 3.00 & 2.64 & 675 & 2.70 & 0.21 & 675 \\
$\mathrm{Hu}_{25}$ & - & - & - & 3.14 & 3.14 & 540 & 2.30 & 2.08 & 760 & 2.40 & 0.19 & 718 \\
$\mathrm{Hu}_{26}$ & - & - & - & 3.49 & 3.55 & 578 & 2.10 & 1.92 & 675 & 2.38 & 0.19 & 478 \\
$\mathrm{Hu}_{27}$ & - & - & - & 1.90 & 1.97 & 438 & 1.90 & 1.76 & 618 & 1.27 & 0.10 & 493 \\
$\mathrm{Hu}_{28}$ & - & - & - & & em & & - & - & - & - & - & - \\
$\mathrm{Pf}$ & 20.21 & 8.16 & 496 & 17.37 & 20.97 & 327 & 16.83 & 19.07 & 466 & 26.90 & 2.69 & 691 \\
\hline \hline
\end{tabular}

Tabla B.13: 28 Tau - Densidades columnares y tamaños de las regiones emisoras de las líneas de la serie de Humphreys a partir de los métodos $N$ y FWHM.

\begin{tabular}{|c|c|c|c|c|c|c|c|c|c|}
\hline & \multicolumn{6}{|c|}{ Método $N$} & \multicolumn{3}{|c|}{ Método FWHM } \\
\hline & \multicolumn{2}{|c|}{2010} & \multicolumn{2}{|c|}{2012} & \multicolumn{2}{|c|}{2016} & \multirow{2}{*}{$\begin{array}{c}2010 \\
\frac{R\left(\mathrm{Hu}_{\mathrm{n}}\right)}{R\left(\mathrm{Hu}_{14}\right)}\end{array}$} & \multirow{2}{*}{$\begin{array}{c}2012 \\
\frac{R\left(\mathrm{Hu}_{\mathrm{n}}\right)}{R\left(\mathrm{Hu}_{14}\right)}\end{array}$} & \multirow{2}{*}{$\begin{array}{c}2016 \\
\frac{R\left(\mathrm{Hu}_{\mathrm{n}}\right)}{R\left(\mathrm{Hu}_{14}\right)}\end{array}$} \\
\hline & $N$ & $\frac{\Delta R\left(\mathrm{Hu}_{\mathrm{n}}\right)}{\Delta R\left(\mathrm{Hu}_{14}\right)}$ & $N$ & $\frac{\Delta R\left(\mathrm{Hu}_{\mathrm{n}}\right)}{\Delta R\left(\mathrm{Hu}_{14}\right)}$ & $N$ & $\frac{\Delta R\left(\mathrm{Hu}_{\mathrm{n}}\right)}{\Delta R\left(\mathrm{Hu}_{14}\right)}$ & & & \\
\hline $\mathrm{Hu}_{13}$ & - & - & 5.37 & 1.03 & - & - & - & 1.22 & - \\
\hline $\mathrm{Hu}_{14}$ & 5.14 & 1.00 & 6.16 & 1.00 & 2.36 & 1.00 & 1.00 & 1.00 & 1.00 \\
\hline $\mathrm{Hu}_{15}$ & 5.31 & 1.03 & 5.65 & 1.03 & 2.41 & 1.02 & 0.58 & 0.84 & 1.06 \\
\hline $\mathrm{Hu}_{16}$ & 6.43 & 1.25 & 6.97 & 1.25 & - & - & 0.53 & 0.90 & 0.82 \\
\hline $\mathrm{Hu}_{17}$ & - & - & - & - & - & - & 0.38 & 0.78 & 1.34 \\
\hline $\mathrm{Hu}_{18}$ & - & - & - & - & - & - & 0.39 & 0.67 & 0.75 \\
\hline $\mathrm{Hu}_{19}$ & - & - & - & - & - & - & 0.36 & 1.33 & 0.73 \\
\hline $\mathrm{Hu}_{20}$ & - & - & - & - & - & - & 0.33 & 0.77 & 0.71 \\
\hline $\mathrm{Hu}_{21}$ & - & - & - & - & - & - & 0.41 & 0.70 & 0.86 \\
\hline $\mathrm{Hu}_{22}$ & - & - & - & - & - & - & 0.31 & 0.60 & 0.90 \\
\hline $\mathrm{Hu}_{23}$ & - & - & - & - & - & - & 0.40 & 0.73 & 0.75 \\
\hline $\mathrm{Hu}_{24}$ & - & - & - & - & - & - & 0.39 & 0.55 & 0.84 \\
\hline $\mathrm{Hu}_{25}$ & - & - & - & - & - & - & 0.46 & 0.43 & 0.74 \\
\hline $\mathrm{Hu}_{26}$ & - & - & - & - & - & - & 0.40 & 0.55 & 1.68 \\
\hline $\mathrm{Hu}_{27}$ & - & - & - & - & - & - & 0.69 & 0.65 & 1.58 \\
\hline
\end{tabular}


Tabla B.14: 28 Tau - Tamaños de las regiones emisoras de las líneas de la serie de Humphreys a partir del método $\Delta V$. Los valores indicados entre paréntesis fueron obtenidos a partir del tamaño relativo a $\mathrm{Hu}_{15}$ en 2012 y $\mathrm{Pf} \delta$ en 2016.

\begin{tabular}{|c|c|c|c|c|c|c|c|c|c|}
\hline & \multicolumn{9}{|c|}{ Método $\Delta V$} \\
\hline & \multicolumn{3}{|c|}{2010} & \multicolumn{3}{|c|}{2012} & \multicolumn{3}{|c|}{2016} \\
\hline & $\Delta V_{\mathrm{n}}$ & $R_{\mathrm{n}}$ & $\frac{R\left(\mathrm{Hu}_{\mathrm{n}}\right)}{R\left(\mathrm{Hu}_{14}\right)}$ & $\Delta V_{\mathrm{n}}$ & $R_{\mathrm{n}}$ & $\frac{R\left(\mathrm{Hu}_{\mathrm{n}}\right)}{R\left(\mathrm{Hu}_{14}\right)}$ & $\Delta V_{\mathrm{n}}$ & $R_{\mathrm{n}}$ & $\frac{R\left(\mathrm{Hu}_{\mathrm{n}}\right)}{R\left(\mathrm{Hu}_{14}\right)}$ \\
\hline $\mathrm{Hu}_{13}$ & - & - & - & 353 & 2.70 & 0.99 & - & - & - \\
\hline $\mathrm{Hu}_{14}$ & 276 & 4.41 & 1.00 & 353 & 2.70 & 1.00 & 363 & 2.56 & 1.00 \\
\hline $\mathrm{Hu}_{15}$ & 356 & 2.66 & 0.60 & 369 & 2.48 & 0.92 & 331 & 3.06 & 1.20 \\
\hline $\mathrm{Hu}_{16}$ & 397 & 2.13 & 0.48 & 341 & 2.89 & 1.07 & 357 & 2.64 & 1.03 \\
\hline $\mathrm{Hu}_{17}$ & 427 & 1.84 & 0.42 & 392 & 2.19 & 0.81 & 327 & 3.15 & 1.23 \\
\hline $\mathrm{Hu}_{18}$ & 447 & 1.68 & 0.38 & 267 & 4.70 & 1.74 & 440 & 1.74 & 0.68 \\
\hline $\mathrm{Hu}_{19}$ & 443 & 1.71 & 0.39 & 399 & 2.12 & 0.78 & 450 & 1.66 & 0.65 \\
\hline $\mathrm{Hu}_{20}$ & 404 & 2.06 & 0.46 & 397 & 2.13 & 0.79 & 420 & 1.91 & 0.75 \\
\hline $\mathrm{Hu}_{21}$ & 411 & 1.99 & 0.45 & 412 & 1.98 & 0.73 & 393 & 2.18 & 0.85 \\
\hline $\mathrm{Hu}_{22}$ & 434 & 1.79 & 0.40 & 366 & 2.51 & 0.93 & 428 & 1.84 & 0.72 \\
\hline $\mathrm{Hu}_{23}$ & 427 & 1.84 & 0.42 & 340 & 2.91 & 1.08 & 414 & 1.96 & 0.77 \\
\hline $\mathrm{Hu}_{24}$ & 418 & 1.92 & 0.44 & 406 & 2.04 & 0.75 & 363 & 2.55 & 1.00 \\
\hline $\mathrm{Hu}_{25}$ & 311 & 3.47 & 0.79 & 330 & 3.09 & 1.14 & 401 & 2.09 & 0.82 \\
\hline $\mathrm{Hu}_{26}$ & 396 & 2.14 & 0.48 & - & (1.63) & - & - & $(4.22)$ & - \\
\hline $\mathrm{Hu}_{27}$ & 276 & 4.42 & 1.00 & - & (1.94) & - & - & (3.97) & - \\
\hline
\end{tabular}

Tabla B.15: 28 Tau - Densidades columnares y tamaños de las regiones emisoras de las líneas de la serie de Pfund a partir de los métodos $N$ y FWHM.

\begin{tabular}{l||cc|cc||c|c|c|c}
\hline \hline \multicolumn{1}{l||}{} & \multicolumn{4}{c||}{ Método $N$} & \multicolumn{4}{c}{ Método FWHM } \\
& \multicolumn{2}{c|}{2010} & \multicolumn{2}{c|}{2016} & 2006 & 2010 & 2012 & 2016 \\
& $N$ & $\frac{\Delta R\left(\mathrm{Pf}_{\mathrm{n}}\right)}{\Delta R(\mathrm{Pf} \gamma)}$ & $N$ & $\frac{\Delta R\left(\mathrm{Pf}_{\mathrm{n}}\right)}{\Delta R(\mathrm{Pf} \gamma)}$ & $\frac{R\left(\mathrm{Pf}_{\mathrm{n}}\right)}{R(\mathrm{Pf} \gamma)}$ & $\frac{R\left(\mathrm{Pf}_{\mathrm{n}}\right)}{R(\mathrm{Pf} \gamma)}$ & $\frac{R\left(\mathrm{Pf}_{\mathrm{n}}\right)}{R\left(\mathrm{Pf}_{\gamma}\right)}$ & $\frac{R\left(\mathrm{Pf}_{\mathrm{n}}\right)}{R(\mathrm{Pf} \gamma)}$ \\
\hline Pf $\gamma$ & 1.76 & 1.00 & 3.20 & 1.00 & 1.00 & 1.00 & 1.00 & 1.00 \\
$\mathrm{Pf} \delta$ & 3.07 & 1.75 & 10.52 & 3.29 & 2.70 & 1.76 & 0.94 & 0.33 \\
\hline \hline
\end{tabular}


Tabla B.16: 28 Tau - Tamaños de las regiones emisoras de las líneas de la serie de Pfund a partir del método $\Delta V$. Los valores indicados entre paréntesis fueron obtenidos a partir del tamaño relativo a $\mathrm{Hu}_{17}$ en 2010 y $\mathrm{Pf} \delta$ en 2016.

\begin{tabular}{l||cc|ccc|ccc|ccc}
\hline \hline \multicolumn{1}{l||}{} & \multicolumn{10}{c}{ Método $\Delta V$} \\
& \multicolumn{2}{c|}{2006} & \multicolumn{10}{c}{2010} & \multicolumn{3}{c}{2012} & \multicolumn{3}{c}{2016} \\
& $\Delta V_{\mathrm{n}}$ & $R_{\mathrm{n}}$ & $\Delta V_{\mathrm{n}}$ & $R_{\mathrm{n}}$ & $\frac{R\left(\mathrm{Pf}_{\mathrm{n}}\right)}{R\left(\mathrm{P}_{23}\right)}$ & $\Delta V_{\mathrm{n}}$ & $R_{\mathrm{n}}$ & $\frac{R\left(\mathrm{Pf}_{\mathrm{n}}\right)}{R\left(\mathrm{P}_{23}\right)}$ & $\Delta V_{\mathrm{n}}$ & $R_{\mathrm{n}}$ & $\frac{R\left(\mathrm{Pf}_{\mathrm{n}}\right)}{R\left(\mathrm{Pf}_{23}\right)}$ \\
\hline $\mathrm{Pf} \gamma$ & 482 & 1.45 & 297 & 3.82 & 1.00 & 251 & 5.33 & 1.00 & - & $(6.18)$ & - \\
$\mathrm{Pf} \delta$ & - & - & - & $(6.00)$ & - & 311 & 3.47 & 0.65 & 408 & 2.02 & 1.00 \\
$\mathrm{Pf}_{17}$ & - & - & 345 & 2.82 & 0.74 & 622 & 0.87 & 0.16 & 366 & 2.50 & 1.24 \\
$\mathrm{Pf}_{18}$ & - & - & 474 & 1.50 & 0.39 & 638 & 0.83 & 0.15 & 448 & 1.68 & 0.83 \\
$\mathrm{Pf}_{19}$ & - & - & 466 & 1.55 & 0.45 & 553 & 1.10 & 0.21 & 412 & 1.98 & 0.98 \\
$\mathrm{Pf}_{20}$ & - & - & 478 & 1.47 & 0.38 & 357 & 2.64 & 0.49 & 544 & 1.14 & 0.56 \\
$\mathrm{Pf}_{21}$ & - & - & 362 & 2.57 & 0.67 & 416 & 1.94 & 0.36 & 571 & 1.03 & 0.51 \\
$\mathrm{Pf}_{22}$ & - & - & 430 & 1.82 & 0.48 & 532 & 1.19 & 0.22 & - & - & - \\
\hline \hline
\end{tabular}

Tabla B.17: 28 Tau - Tamaños de las regiones emisoras de las líneas de la serie de Brackett a partir de los métodos FWHM y $\Delta V$. Los valores indicados entre paréntesis fueron obtenidos a partir del tamaño relativo a Pf $\gamma$ en 2006, $\mathrm{Hu}_{15}$ en 2012 y $\mathrm{Pf} \delta$ en 2016.

\begin{tabular}{|c|c|c|c|c|c|c|c|c|c|c|c|c|c|}
\hline & \multicolumn{4}{|c|}{ Método FWHM } & \multicolumn{9}{|c|}{ Método $\Delta V$} \\
\hline & \multirow{2}{*}{$\begin{array}{l}2006 \\
\frac{R\left(\mathrm{Br}_{\mathrm{n}}\right)}{R(\mathrm{Br} \alpha)}\end{array}$} & \multirow{2}{*}{$\begin{array}{l}2010 \\
\left.\frac{R(\mathrm{Br})}{R(\operatorname{rr} \alpha}\right)\end{array}$} & \multirow{2}{*}{$\begin{array}{l}2012 \\
\frac{R\left(\mathrm{Br}_{\mathrm{n}}\right)}{R(\mathrm{r} \alpha}\end{array}$} & \multirow{2}{*}{$\begin{array}{l}2016 \\
\frac{R\left(\operatorname{Br} r_{n}\right)}{R(\operatorname{Br} \alpha}\end{array}$} & \multicolumn{2}{|c|}{2006} & \multicolumn{3}{|c|}{2010} & \multicolumn{3}{|c|}{2012} & \multirow{2}{*}{$\begin{array}{c}2016 \\
R_{\mathrm{n}}\end{array}$} \\
\hline & & & & & $\Delta V_{\mathrm{n}}$ & $R_{\mathrm{n}}$ & $\Delta V_{\mathrm{n}}$ & $R_{\mathrm{n}}$ & $\frac{\left.R(\operatorname{Br})_{n}\right)}{R(\operatorname{Br} \alpha)}$ & $\Delta V_{\mathrm{n}}$ & $R_{\mathrm{n}}$ & $\frac{R\left(\mathrm{Br}_{\mathrm{n}}\right)}{R(\mathrm{rr} \gamma)}$ & \\
\hline $\operatorname{Br} \alpha$ & 1.00 & 1.00 & 1.00 & 1.00 & 426 & 1.86 & 149 & 15.10 & 1.00 & - & $(6.38)$ & - & (7.96) \\
\hline $\mathrm{Br} \gamma$ & 2.53 & 0.26 & 0.48 & 0.81 & - & $(5.46)$ & 267 & 4.73 & 0.31 & 292 & 3.94 & 1.00 & (6.44) \\
\hline $\operatorname{Br} \delta$ & - & - & 0.19 & 0.75 & - & - & - & - & - & 375 & 2.41 & 0.61 & (6.00) \\
\hline $\mathrm{Br}_{9}$ & - & - & - & - & - & - & - & - & - & 422 & 1.89 & 0.48 & - \\
\hline $\mathrm{Br}_{10}$ & - & - & - & - & - & - & 368 & 2.49 & 0.16 & 444 & 1.71 & 0.43 & - \\
\hline $\mathrm{Br}_{11}$ & - & - & - & - & - & - & 386 & 2.26 & 0.15 & 451 & 1.65 & 0.42 & - \\
\hline $\mathrm{Br}_{12}$ & - & - & - & - & - & - & 397 & 2.13 & 0.14 & 351 & 2.72 & 0.69 & - \\
\hline $\mathrm{Br}_{13}$ & - & - & - & - & - & - & 387 & 2.25 & 0.15 & 451 & 1.65 & 0.42 & - \\
\hline $\mathrm{Br}_{14}$ & - & - & - & - & - & - & 484 & 1.44 & 0.09 & 505 & 1.32 & 0.33 & - \\
\hline $\mathrm{Br}_{15}$ & - & - & - & - & - & - & 477 & 1.48 & 0.10 & - & - & - & - \\
\hline $\mathrm{Br}_{16}$ & - & - & - & - & - & - & 452 & 1.65 & 0.11 & - & - & - & - \\
\hline $\mathrm{Br}_{17}$ & - & - & - & - & - & - & 480 & 1.46 & 0.10 & - & - & - & - \\
\hline $\mathrm{Br}_{18}$ & - & - & - & - & - & - & 517 & 1.26 & 0.08 & - & - & - & - \\
\hline $\mathrm{Br}_{19}$ & - & - & - & - & - & - & 509 & 1.30 & 0.09 & - & - & - & - \\
\hline $\mathrm{Br}_{20}$ & - & - & - & - & - & - & 497 & 1.36 & 0.09 & - & - & - & - \\
\hline $\mathrm{Br}_{21}$ & - & - & - & - & - & - & 458 & 1.61 & 0.11 & - & - & - & - \\
\hline
\end{tabular}

Tabla B.18: 28 Tau - Tamaños de las regiones emisoras de las líneas de la serie de Paschen a partir de los métodos FWHM.

\begin{tabular}{c||c}
\hline \hline & Método FWHM \\
& 2016 \\
& $\frac{R(\mathrm{~Pa})}{R(\mathrm{~Pa} \alpha)}$ \\
\hline $\mathrm{Pa} \alpha$ & 1.00 \\
$\mathrm{~Pa} \beta$ & 1.56 \\
\hline \hline
\end{tabular}


Tabla B.19: V1150 Tau - Anchos equivalentes, flujos y FWHM de las líneas de hidrógeno presentes en la banda J.

\begin{tabular}{c|ccc}
\hline \hline & \multicolumn{3}{|c}{2016} \\
& EW & Fl & FWHM \\
\hline $\mathrm{Pa} \beta$ & 25.62 & 54.24 & 321 \\
\hline \hline
\end{tabular}

Tabla B.20: V1150 Tau - Anchos equivalentes, flujos y FWHM de las líneas de hidrógeno presentes en la banda $\mathrm{H}$.

\begin{tabular}{l|ccc|ccc}
\hline \hline & \multicolumn{3}{|c|}{2012} & & 2016 \\
& EW & Fl & FWHM & EW & Fl & FWHM \\
\hline $\mathrm{Br}_{9}$ & 25.45 & 23.66 & 489 & 33.26 & 23.53 & 494 \\
$\mathrm{Br}_{10}$ & 17.94 & 19.06 & 497 & 22.46 & 18.06 & 523 \\
$\mathrm{Br}_{11}$ & 17.45 & 20.53 & 522 & 18.07 & 16.18 & 451 \\
$\mathrm{Br}_{12}$ & 15.88 & 20.32 & 521 & 16.37 & 15.68 & 459 \\
$\mathrm{Br}_{13}$ & 13.56 & 18.18 & 504 & 13.47 & 13.63 & 444 \\
$\mathrm{Br}_{14}$ & 14.08 & 19.66 & 557 & 15.54 & 16.34 & 563 \\
$\mathrm{Br}_{15}$ & 10.78 & 15.31 & 519 & 14.82 & 16.32 & 535 \\
$\mathrm{Br}_{16}$ & 10.61 & 15.71 & 558 & 11.16 & 12.52 & 545 \\
$\mathrm{Br}_{17}$ & 9.70 & 14.57 & 575 & 10.03 & 11.65 & 522 \\
$\mathrm{Br}_{18}$ & 8.69 & 13.48 & 570 & 8.72 & 10.29 & 549 \\
$\mathrm{Br}_{19}$ & 7.01 & 11.07 & 558 & 7.01 & 8.46 & 541 \\
$\mathrm{Br}_{20}$ & 5.32 & 8.64 & 528 & 5.46 & 6.75 & 501 \\
$\mathrm{Br}_{21}$ & 3.61 & 6.02 & 473 & 3.61 & 4.60 & 459 \\
$\mathrm{Br}_{22}$ & 2.22 & 3.80 & 437 & 2.22 & 2.90 & 404 \\
$\mathrm{Br}_{23}$ & 1.53 & 2.68 & 424 & 1.54 & 2.06 & 386 \\
$\mathrm{Br}_{24}$ & 0.84 & 1.50 & 418 & 0.78 & 1.05 & - \\
$\mathrm{Br}_{25}$ & 0.49 & 0.89 & 482 & 0.50 & 0.69 & - \\
\hline \hline
\end{tabular}


Tabla B.21: V1150 Tau - Anchos equivalentes, flujos y FWHM de las líneas de hidrógeno presentes en la banda $\mathrm{K}$.

\begin{tabular}{l|ccc|ccc}
\hline \hline & \multicolumn{3}{|c|}{2012} & & 2016 \\
& EW & Fl & FWHM & EW & Fl & FWHM \\
\hline $\mathrm{Pf}_{17}$ & 24.38 & 9.53 & 695 & 19.81 & 5.28 & 525 \\
$\mathrm{Pf}_{18}$ & 17.23 & 7.07 & 535 & 18.49 & 5.03 & 540 \\
$\mathrm{Pf}_{19}$ & 15.05 & 6.34 & 582 & 14.35 & 4.04 & 517 \\
$\mathrm{Pf}_{20}$ & 11.70 & 5.08 & 537 & 12.33 & 3.55 & 523 \\
$\mathrm{Pf}_{21}$ & 9.32 & 4.17 & 537 & 10.03 & 2.98 & 524 \\
$\mathrm{Pf}_{22}$ & 7.67 & 3.51 & 498 & 8.41 & 2.54 & 488 \\
$\mathrm{Pf}_{23}$ & 5.61 & 2.61 & 509 & 6.45 & 1.99 & 494 \\
$\mathrm{Pf}_{24}$ & 3.90 & 1.85 & 432 & 4.32 & 1.36 & 439 \\
$\mathrm{Pf}_{25}$ & 2.85 & 1.38 & 409 & 2.64 & 0.85 & 398 \\
$\mathrm{Pf}_{26}$ & 2.19 & 1.08 & 502 & 1.33 & 0.44 & 309 \\
$\mathrm{Pf}_{27}$ & 1.06 & 0.53 & 345 & 1.44 & 0.48 & 287 \\
$\mathrm{Pf}_{28}$ & 0.62 & 0.31 & 301 & 0.78 & 0.26 & 261 \\
$\mathrm{Br} \gamma$ & 23.80 & 15.72 & 389 & 26.15 & 11.68 & 362 \\
$\mathrm{Br} \delta$ & 29.56 & 25.97 & 498 & 35.06 & 20.36 & 481 \\
$\mathrm{~Pa} \alpha$ & - & - & - & 118.6 & 74.74 & 402 \\
\hline \hline
\end{tabular}

Tabla B.22: V1150 Tau - Anchos equivalentes, flujos y FWHM de las líneas de hidrógeno presentes en la banda $\mathrm{L}$.

\begin{tabular}{l|ccc}
\hline & & 2012 \\
& $\mathrm{EW}$ & $\mathrm{Fl}$ & $\mathrm{FWHM}$ \\
\hline $\mathrm{Hu} 13$ & 51.98 & 5.10 & 557 \\
$\mathrm{Br} \alpha$ & 78.68 & 8.32 & 304 \\
$\mathrm{Hu}$ & 39.76 & 4.32 & 478 \\
$\mathrm{Hu}_{15}$ & 40.75 & 4.69 & 561 \\
$\mathrm{Hu}_{16}$ & 36.86 & 4.52 & 607 \\
$\mathrm{Hu}_{17}$ & 35.19 & 4.65 & 659 \\
$\mathrm{Pf} \gamma$ & 51.00 & 6.81 & 479 \\
$\mathrm{Hu}$ & 28.97 & 3.97 & 661 \\
$\mathrm{Hu}$ & 25.11 & 3.56 & 686 \\
$\mathrm{Hu}$ & 18.01 & 2.65 & 640 \\
$\mathrm{Hu}_{21}$ & 16.67 & 2.51 & 676 \\
$\mathrm{Hu}_{22}$ & 10.92 & 1.68 & 574 \\
$\mathrm{Hu}_{23}$ & 9.77 & 1.54 & 572 \\
$\mathrm{Hu}_{24}$ & 7.06 & 1.14 & 488 \\
$\mathrm{Hu}_{25}$ & 4.46 & 0.74 & 388 \\
$\mathrm{Hu}_{26}$ & 3.54 & 0.60 & 395 \\
$\mathrm{Hu}_{27}$ & 2.21 & 0.37 & 375 \\
$\mathrm{Hu}_{28}$ & 1.26 & 0.22 & 320 \\
$\mathrm{Pf} \delta$ & 44.55 & 8.59 & 418 \\
\hline \hline
\end{tabular}


Tabla B.23: V1150 Tau - Tamaños de las regiones emisoras de las líneas de la serie de Humphreys a partir de los métodos $N$ y FWHM.

\begin{tabular}{l||cc||c}
\hline \hline & \multicolumn{2}{|c||}{ Método $N$} & Método FWHM \\
& \multicolumn{2}{c}{2012} & 2012 \\
& $N$ & $\frac{\Delta R\left(\mathrm{Hu}_{\mathrm{n}}\right)}{\Delta R\left(\mathrm{Hu}_{14}\right)}$ & $\frac{R\left(\mathrm{Hu}_{\mathrm{n}}\right)}{R\left(\mathrm{Hu}_{14}\right)}$ \\
\hline $\mathrm{Hu}_{13}$ & 6.93 & 0.87 & 0.74 \\
$\mathrm{Hu}_{14}$ & 8.01 & 1.00 & 1.00 \\
$\mathrm{Hu}_{15}$ & 7.87 & 0.98 & 0.73 \\
$\mathrm{Hu}_{16}$ & 7.29 & 0.91 & 0.62 \\
$\mathrm{Hu}_{17}$ & - & - & 0.53 \\
$\mathrm{Hu}_{18}$ & - & - & 0.52 \\
$\mathrm{Hu}_{19}$ & - & - & 0.49 \\
$\mathrm{Hu}_{20}$ & - & - & 0.56 \\
$\mathrm{Hu}_{21}$ & - & - & 0.50 \\
$\mathrm{Hu}_{22}$ & - & - & 0.69 \\
$\mathrm{Hu}_{23}$ & - & - & 0.70 \\
$\mathrm{Hu}_{24}$ & - & - & 0.96 \\
$\mathrm{Hu}_{25}$ & - & - & 1.52 \\
$\mathrm{Hu}_{26}$ & - & - & 1.46 \\
$\mathrm{Hu}_{27}$ & - & - & 1.62 \\
$\mathrm{Hu}_{28}$ & - & - & 2.23 \\
\hline \hline
\end{tabular}

Tabla B.24: V1150 Tau - Tamaños de las regiones emisoras de las líneas de la serie de Pfund a partir de los métodos $N$ y FWHM.

\begin{tabular}{l||cc||c|c}
\hline \hline \multicolumn{1}{|c||}{} & \multicolumn{2}{c||}{ Método $N$} & \multicolumn{2}{c}{ Método FWHM } \\
& \multicolumn{2}{c|}{2012} & 2012 & 2016 \\
& $N$ & $\frac{\Delta R\left(\mathrm{Pf}_{\mathrm{n}}\right)}{\Delta R(\mathrm{Pf} \gamma)}$ & $\frac{R\left(\mathrm{Pf}_{\mathrm{n}}\right)}{R\left(\mathrm{Pf}_{\gamma}\right)}$ & $\frac{R\left(\mathrm{Pf}_{\mathrm{n}}\right)}{R\left(\mathrm{Pf}_{\gamma}\right)}$ \\
\hline $\mathrm{Pf} \gamma$ & 2.65 & 0.62 & 2.11 & - \\
$\mathrm{Pf} \delta$ & 4.25 & 1.00 & 2.76 & - \\
$\mathrm{Pf}_{17}$ & - & - & 1.00 & 1.00 \\
$\mathrm{Pf}_{18}$ & - & - & 1.69 & 0.95 \\
$\mathrm{Pf}_{19}$ & - & - & 1.43 & 1.03 \\
$\mathrm{Pf}_{20}$ & - & - & 1.68 & 1.01 \\
$\mathrm{Pf}_{21}$ & - & - & 1.68 & 1.00 \\
$\mathrm{Pf}_{22}$ & - & - & 1.95 & 1.16 \\
$\mathrm{Pf}_{23}$ & - & - & 1.86 & 1.13 \\
$\mathrm{Pf}_{24}$ & - & - & 2.59 & 1.43 \\
$\mathrm{Pf}_{25}$ & - & - & 2.89 & 1.74 \\
\hline \hline
\end{tabular}


Tabla B.25: V1150 Tau - Tamaños de las regiones emisoras de las líneas de la serie de Brackett a partir de los métodos $N$ y FWHM.

\begin{tabular}{l||cc|cc||c|c}
\hline \hline \multicolumn{1}{l||}{} & \multicolumn{5}{c||}{ Método $N$} & \multicolumn{2}{c}{ Método FWHM } \\
& \multicolumn{2}{c|}{2012} & \multicolumn{2}{c}{2016} & 2012 & 2016 \\
& $N$ & $\frac{\Delta R\left(\mathrm{Br}_{\mathrm{n}}\right)}{\Delta R\left(\mathrm{Br}_{11}\right)}$ & $N$ & $\frac{\Delta R\left(\mathrm{Br}_{\mathrm{n}}\right)}{\Delta R\left(\mathrm{Br}_{11}\right)}$ & $\frac{R\left(\mathrm{Br} \mathrm{B}_{\mathrm{n}}\right)}{R(\mathrm{Br} \delta)}$ & $\frac{R\left(\mathrm{Br} \mathrm{Br}_{\mathrm{n}}\right)}{R(\mathrm{Br} \delta)}$ \\
\hline $\mathrm{Br} \alpha$ & - & - & - & - & 2.95 & - \\
$\mathrm{Br} \gamma$ & - & - & - & - & 1.80 & 1.55 \\
$\mathrm{Br} \delta$ & - & - & - & - & 1.10 & 0.88 \\
$\mathrm{Br} 9$ & 12.30 & 0.53 & 10.38 & 0.50 & 1.14 & 0.83 \\
$\mathrm{Br}_{10}$ & 19.78 & 0.84 & 18.04 & 0.88 & 1.10 & 0.74 \\
$\mathrm{Br}_{11}$ & 23.42 & 1.00 & 20.58 & 1.00 & 1.00 & 1.00 \\
$\mathrm{Br}_{12}$ & 25.30 & 1.08 & 23.46 & 1.14 & 1.00 & 0.97 \\
$\mathrm{Br}_{13}$ & 28.44 & 1.21 & 27.80 & 1.35 & 1.07 & 1.03 \\
$\mathrm{Br}_{14}$ & 21.17 & 0.90 & 18.40 & 0.89 & 0.88 & 0.64 \\
$\mathrm{Br}_{15}$ & 29.78 & 1.27 & - & - & 1.01 & 0.71 \\
$\mathrm{Br}_{16}$ & - & - & - & - & 0.88 & 0.68 \\
$\mathrm{Br}_{17}$ & - & - & - & - & 0.82 & 0.75 \\
$\mathrm{Br}_{18}$ & - & - & - & - & 0.84 & 0.67 \\
$\mathrm{Br}_{19}$ & - & - & - & - & 0.88 & 0.69 \\
$\mathrm{Br}_{20}$ & - & - & - & - & 0.98 & 0.81 \\
$\mathrm{Br}_{21}$ & - & - & - & - & 0.68 & 0.62 \\
$\mathrm{Br}_{22}$ & - & - & - & - & 0.79 & 0.80 \\
$\mathrm{Br}_{23}$ & - & - & - & - & 0.84 & 0.88 \\
$\mathrm{Br}_{24}$ & - & - & - & - & 0.87 & - \\
$\mathrm{Br}_{25}$ & - & - & - & - & 0.65 & - \\
\hline \hline
\end{tabular}

Tabla B.26: V1150 Tau - Tamaños de las regiones emisoras de las líneas de la serie de Paschen a partir de lo método FWHM.

\begin{tabular}{c||c}
\hline \hline & Método FWHM \\
& 2016 \\
\hline $\mathrm{Pa} \alpha$ & 1.00 \\
$\mathrm{~Pa} \beta$ & 1.57 \\
\hline \hline
\end{tabular}

Tabla B.27: 66 Oph - Anchos equivalentes, flujos y FWHM de las líneas presentes en las bandas K y L.

\begin{tabular}{c|ccc|ccc|ccc}
\hline \hline & \multicolumn{3}{|c|}{2008} & \multicolumn{3}{|c|}{2010} & \multicolumn{3}{c}{2016} \\
& EW & Fl & FWHM & EW & Fl & FWHM & EW & Fl & FWHM \\
\hline Br $\delta$ & 7.94 & 55.44 & 637 & 8.01 & 27.86 & 514 & 11.26 & 48.90 & - \\
Br $\alpha$ & 73.70 & 38.12 & 588 & - & - & - & - & - & - \\
Pf $\gamma$ & 9.57 & 6.69 & - & - & - & - & - & - & - \\
Pf $\delta$ & - & - & - & 9.72 & 5.07 & - & 10.28 & 1.81 & 663 \\
\hline \hline
\end{tabular}


Tabla B.28: 66 Oph - Tamaños de las regiones emisoras de las líneas de las series de Brackett y Pfund a partir de los métodos FWHM y $\Delta V$.

\begin{tabular}{l||c||ccc|ccc}
\hline \hline \multicolumn{1}{l|}{} & \multicolumn{1}{c||}{ Método FWHM } & \multicolumn{6}{c}{ Método $\Delta V$} \\
& 2008 & & 2008 & & 2010 \\
& $\frac{R\left(\mathrm{Br}_{\mathrm{n}}\right)}{R(\mathrm{r} \delta)}$ & $\Delta V_{\mathrm{n}}$ & $R_{\mathrm{n}}$ & $\frac{R\left(\mathrm{Br}_{\mathrm{n}}\right)}{R\left(\mathrm{Br}_{11}\right)}$ & $\Delta V_{\mathrm{n}}$ & $R_{\mathrm{n}}$ & $\frac{R(\mathrm{Br})}{R(\mathrm{P} \mathrm{f} \delta)}$ \\
\hline $\operatorname{Br} \alpha$ & 1.00 & 370 & 2.41 & 1.00 & - & - & - \\
$\operatorname{Br} \delta$ & 0.85 & 466 & 1.52 & 0.63 & - & - & - \\
\hline $\operatorname{Pf} \delta$ & - & - & - & - & 234 & 6.02 & 1.00 \\
\hline \hline
\end{tabular}

Tabla B.29: V923 Aql - Anchos equivalentes, flujos y FWHM de las líneas de hidrógeno presentes en la banda J.

\begin{tabular}{c|ccc|ccc}
\hline \hline & \multicolumn{3}{|c|}{2016} & \multicolumn{3}{c}{2017} \\
& EW & Fl & FWHM & EW & Fl & FWHM \\
\hline $\mathrm{Pa} \beta$ & 9.26 & 114.24 & 228 & 9.21 & 17.63 & 207 \\
\hline \hline
\end{tabular}

Tabla B.30: V923 Aql - Anchos equivalentes, flujos y FWHM de las líneas de hidrógeno presentes en la banda $\mathrm{H}$.

\begin{tabular}{l|ccc|ccc}
\hline \hline & \multicolumn{3}{|c|}{2016} & \multicolumn{3}{c}{2017} \\
& EW & Fl & FWHM & EW & Fl & FWHM \\
\hline $\mathrm{Br}_{9}$ & - & - & - & 8.70 & 5.75 & - \\
$\mathrm{Br}_{10}$ & 5.51 & 26.32 & - & \multicolumn{3}{|c}{ em } \\
$\mathrm{Br}_{11-14}$ & \multicolumn{3}{|c|}{ shell } & & \multicolumn{3}{c}{ em } \\
\hline \hline
\end{tabular}

Tabla B.31: V923 Aql - Anchos equivalentes, flujos y FWHM de las líneas de hidrógeno presentes en la banda $\mathrm{K}$.

\begin{tabular}{c|ccc|ccc|ccc}
\hline \hline & \multicolumn{7}{|c|}{2006} & \multicolumn{7}{c|}{2016} & \multicolumn{3}{c}{2017} \\
& EW & Fl & FWHM & EW & Fl & FWHM & EW & Fl & FWHM \\
\hline $\mathrm{Pf}_{17}$ & - & - & - & 5.95 & 7.98 & - & - & - & - \\
$\mathrm{Pf}_{18}$ & - & - & - & 4.11 & 5.69 & - & - & - & - \\
$\mathrm{Pf}_{19}$ & - & - & - & 1.95 & 2.81 & - & - & - & - \\
$\mathrm{Pf}_{20}$ & - & - & - & 1.86 & 2.73 & - & - & - & - \\
$\mathrm{Pf}_{21}$ & - & - & - & 0.76 & 1.15 & - & - & - & - \\
$\mathrm{Pf}_{22}$ & - & - & - & 1.12 & 1.73 & - & - & - & - \\
$\mathrm{Pf}_{23}$ & - & - & - & 0.81 & 1.27 & - & - & - & - \\
$\mathrm{Pf}_{24}$ & - & - & - & 0.49 & 0.78 & - & - & - & - \\
$\mathrm{Br} \gamma$ & 9.16 & 8.91 & 215 & 7.76 & 16.99 & - & 9.98 & 3.42 & 275 \\
$\mathrm{Br} \delta$ & 8.78 & 16.41 & - & 10.81 & 36.83 & - & 11.93 & 6.07 & 389 \\
$\mathrm{~Pa} \alpha$ & - & - & - & 76.12 & 299.93 & 389 & 60.95 & 32.55 & 333 \\
\hline \hline
\end{tabular}


Tabla B.32: V923 Aql - Anchos equivalentes, flujos y FWHM de las líneas de hidrógeno presentes en la banda $\mathrm{L}$.

\begin{tabular}{|c|c|c|c|c|c|c|c|c|c|}
\hline & \multicolumn{3}{|c|}{2006} & \multicolumn{3}{|c|}{2016} & \multicolumn{3}{|c|}{2017} \\
\hline & EW & $\mathrm{Fl}$ & FWHM & EW & $\mathrm{Fl}$ & FWHM & EW & $\mathrm{Fl}$ & FWHM \\
\hline $\operatorname{Br} \alpha$ & 69.55 & 8.34 & 505 & 60.29 & 4.13 & 302 & 68.12 & 0.97 & 268 \\
\hline $\mathrm{Hu}_{14}$ & 9.35 & 1.20 & - & 9.76 & 0.69 & 413 & 13.41 & 0.20 & 372 \\
\hline $\mathrm{Hu}_{15}$ & 10.28 & 1.62 & - & 9.91 & 0.76 & 422 & 11.95 & 0.22 & 409 \\
\hline $\mathrm{Hu}_{16}$ & 8.61 & 1.49 & - & 7.12 & 0.59 & 428 & 9.96 & 0.22 & 484 \\
\hline $\mathrm{Hu}_{17}$ & 6.46 & 1.23 & - & 7.02 & 0.61 & 370 & 8.88 & 0.23 & 401 \\
\hline $\operatorname{Pf} \gamma$ & 16.94 & 3.25 & 365 & 16.46 & 1.43 & 276 & 21.87 & 0.56 & 256 \\
\hline $\mathrm{Hu}_{18}$ & 5.41 & 1.08 & - & 5.44 & 0.51 & 520 & 6.85 & 0.20 & 420 \\
\hline $\mathrm{Hu}_{19}$ & 6.02 & 1.24 & - & 3.81 & 0.37 & 455 & 5.30 & 0.17 & 501 \\
\hline $\mathrm{Hu}_{20}$ & 3.82 & 0.82 & - & 2.45 & 0.25 & 402 & 3.83 & 0.13 & - \\
\hline $\mathrm{Hu}_{21}$ & 2.94 & 0.65 & - & 2.94 & 0.31 & 577 & 2.59 & 0.10 & - \\
\hline $\mathrm{Hu}_{22}$ & 3.75 & 0.85 & - & 3.28 & 0.35 & 632 & 4.08 & 0.16 & - \\
\hline $\mathrm{Hu}_{23}$ & 3.82 & 0.89 & - & 2.04 & 0.23 & 410 & 2.25 & 0.10 & - \\
\hline $\mathrm{Hu}_{24}$ & 2.12 & 0.50 & - & 1.58 & 0.18 & 484 & 1.84 & 0.09 & - \\
\hline $\mathrm{Hu}_{25}$ & - & - & - & 1.73 & 0.20 & 577 & 1.84 & 0.09 & - \\
\hline $\mathrm{Hu}_{26}$ & - & - & - & 1.72 & 0.20 & - & 0.85 & 0.04 & - \\
\hline $\mathrm{Hu}_{27}$ & - & - & - & 0.95 & 0.11 & - & - & - & - \\
\hline $\operatorname{Pf} \delta$ & - & - & - & 14.65 & 2.14 & - & 21.07 & 1.40 & 335 \\
\hline
\end{tabular}

Tabla B.33: V923 Aql - Densidades columnares y tamaños de las regiones emisoras de las líneas de la serie de Humphreys a partir de los métodos $N$, FWHM y $\Delta V$.

\begin{tabular}{|c|c|c|c|c|c|c|c|c|c|c|c|c|}
\hline & \multicolumn{4}{|c|}{ Método $N$} & \multicolumn{2}{|c|}{ Método FWHM } & \multicolumn{6}{|c|}{ Método $\Delta V$} \\
\hline & \multicolumn{2}{|c|}{2016} & \multicolumn{2}{|c|}{2017} & \multirow{2}{*}{$\begin{array}{c}2016 \\
\frac{R\left(\mathrm{Hu}_{\mathrm{n}}\right)}{R\left(\mathrm{Hu}_{4}\right)}\end{array}$} & \multirow{2}{*}{$\begin{array}{c}2017 \\
\frac{R\left(\mathrm{Hu}_{\mathrm{n}}\right)}{R\left(\mathrm{Hu}_{14}\right)}\end{array}$} & \multicolumn{3}{|c|}{2016} & \multicolumn{3}{|c|}{2017} \\
\hline & $N$ & $\frac{\Delta R\left(\mathrm{Hu}_{\mathrm{n}}\right)}{\Delta R\left(\mathrm{Hu}_{14}\right)}$ & $N$ & $\frac{\Delta R\left(\mathrm{Hu}_{\mathrm{n}}\right)}{\Delta R\left(\mathrm{Hu}_{14}\right)}$ & & & $\Delta V_{\mathrm{n}}$ & $R_{\mathrm{n}}$ & $\frac{R\left(\mathrm{Br}_{\mathrm{n}}\right)}{R\left(\mathrm{Br}_{11}\right)}$ & $\Delta V_{\mathrm{n}}$ & $R_{\mathrm{n}}$ & $\frac{R\left(\mathrm{Br}_{\mathrm{n}}\right)}{R\left(\mathrm{Br}_{11}\right)}$ \\
\hline $\mathrm{Hu}_{14}$ & 2.88 & 1.00 & 6.51 & 1.00 & 1.00 & 1.00 & 373 & 2.32 & 1.00 & 338 & 2.82 & 1.00 \\
\hline $\mathrm{Hu}_{15}$ & - & - & 5.94 & 0.91 & 0.96 & 0.83 & 388 & 2.14 & 0.92 & 354 & 2.57 & 0.91 \\
\hline $\mathrm{Hu}_{16}$ & - & - & 5.42 & 0.83 & 0.93 & 0.59 & 397 & 2.05 & 0.89 & 370 & 2.36 & 0.83 \\
\hline $\mathrm{Hu}_{17}$ & - & - & - & - & 1.25 & 0.86 & 419 & 1.84 & 0.79 & 387 & 2.15 & 0.76 \\
\hline $\mathrm{Hu}_{18}$ & - & - & - & - & 0.63 & 0.78 & 440 & 1.67 & 0.72 & 339 & 2.81 & 0.99 \\
\hline $\mathrm{Hu}_{19}$ & - & - & - & - & 0.82 & 0.55 & 451 & 1.59 & 0.68 & 403 & 1.99 & 0.70 \\
\hline $\mathrm{Hu}_{20}$ & - & - & - & - & 1.06 & - & 440 & 1.67 & 0.72 & 398 & 2.04 & 0.72 \\
\hline $\mathrm{Hu}_{21}$ & - & - & - & - & 0.51 & - & 439 & 1.67 & 0.72 & 450 & 1.59 & 0.56 \\
\hline $\mathrm{Hu}_{22}$ & - & - & - & - & 0.43 & - & 443 & 1.64 & 0.71 & 381 & 2.22 & 0.79 \\
\hline $\mathrm{Hu}_{23}$ & - & - & - & - & 1.01 & - & 415 & 1.87 & 0.81 & 373 & 2.32 & 0.82 \\
\hline $\mathrm{Hu}_{24}$ & - & - & - & - & 0.73 & - & 428 & 1.76 & 0.76 & - & - & - \\
\hline $\mathrm{Hu}_{25}$ & - & - & - & - & 0.51 & - & 467 & 1.48 & 0.64 & - & - & - \\
\hline $\mathrm{Hu}_{26}$ & - & - & - & - & - & - & 358 & 2.52 & 1.08 & - & - & - \\
\hline $\mathrm{Hu}_{27}$ & - & - & - & - & - & - & 401 & 2.01 & 0.86 & - & - & - \\
\hline
\end{tabular}


Tabla B.34: V923 Aql - Tamaños de las regiones emisoras de las líneas de la serie de Pfund a partir del método $\Delta V$.

\begin{tabular}{|c|c|c|c|c|c|c|}
\hline & \multicolumn{5}{|c|}{ Método $\Delta V$} & \\
\hline & \multicolumn{3}{|c|}{2016} & \multicolumn{3}{|c|}{2017} \\
\hline & $\Delta V_{\mathrm{n}}$ & $R_{\mathrm{n}}$ & $\frac{R\left(\mathrm{Pf}_{\mathrm{n}}\right)}{R(\mathrm{Pf} \delta)}$ & $\Delta V_{\mathrm{n}}$ & $R_{\mathrm{n}}$ & $\frac{R\left(\mathrm{Pf}_{\mathrm{n}}\right)}{R(\mathrm{Pf} \delta)}$ \\
\hline Pf $\gamma$ & 292 & 3.78 & 1.00 & 244 & 5.42 & 1.00 \\
\hline $\operatorname{Pf} \delta$ & 351 & 2.62 & 0.69 & 314 & 3.27 & 0.60 \\
\hline $\mathrm{Pf}_{17}$ & 473 & 1.44 & 0.38 & - & - & - \\
\hline $\mathrm{Pf}_{18}$ & 550 & 1.07 & 0.28 & - & - & - \\
\hline $\mathrm{Pf}_{19}$ & 482 & 1.39 & 0.37 & - & - & - \\
\hline $\mathrm{Pf}_{20}$ & 592 & 0.92 & 0.24 & - & - & - \\
\hline $\mathrm{Pf}_{21}$ & 503 & 1.28 & 0.45 & - & - & - \\
\hline
\end{tabular}

Tabla B.35: V923 Aql - Tamaños de las regiones emisoras de las líneas de la serie de Brackett a partir de los métodos FWHM y $\Delta V$. Los valores indicados entre paréntesis fueron obtenidos a partir del tamaño relativo a $\mathrm{Hu}_{14}$ en 2016 y $\mathrm{Br} \delta$ en 2017.

\begin{tabular}{l||c||cc|ccc}
\hline \hline \multicolumn{1}{|c||}{} & \multicolumn{1}{c||}{ Método FWHM } & \multicolumn{5}{c}{ Método $\Delta V$} \\
& 2017 & \multicolumn{2}{c}{2016} & \multicolumn{3}{c}{2017} \\
& $\frac{R(\mathrm{Br})}{R(\mathrm{Br} \alpha)}$ & $\Delta V_{\mathrm{n}}$ & $R_{\mathrm{n}}$ & $\Delta V_{\mathrm{n}}$ & $R_{\mathrm{n}}$ & $\frac{R\left(\mathrm{Br}_{\mathrm{n}}\right)}{R(\mathrm{Br} \delta)}$ \\
\hline $\mathrm{Br} \alpha$ & 1.00 & - & $(4.31)$ & - & $(4.22)$ & - \\
$\mathrm{Br} \gamma$ & 0.95 & - & - & - & $(4.01)$ & - \\
$\mathrm{Br} \delta$ & 0.47 & 508 & 1.25 & 403 & 1.99 & 1.00 \\
$\mathrm{Br} 9$ & - & - & - & 434 & 1.71 & 0.86 \\
$\mathrm{Br}_{10}$ & - & - & - & 463 & 1.50 & 0.76 \\
$\mathrm{Br}_{11}$ & - & - & - & 619 & 0.84 & 0.42 \\
\hline \hline
\end{tabular}

Tabla B.36: V923 Aql - Tamaños de las regiones emisoras de las líneas de la serie de Paschen a partir de los métodos FWHM y $\Delta V$. Los valores indicados entre paréntesis fueron obtenidos a partir del tamaño relativo a $\mathrm{Hu}_{14}$ en 2016 y $\mathrm{Br} \delta$ en 2017.

\begin{tabular}{c||c|c||cc|cc}
\hline \hline \multicolumn{1}{l||}{} & \multicolumn{3}{c||}{ Método FWHM } & \multicolumn{4}{c}{ Método $\Delta V$} \\
& 2016 & 2017 & \multicolumn{2}{c}{2016} & \multicolumn{2}{c}{2017} \\
& $\frac{R(\mathrm{~Pa})}{R(\mathrm{~Pa} \alpha)}$ & $\frac{R(\mathrm{~Pa})}{R(\mathrm{~Pa} \alpha)}$ & $\Delta V_{\mathrm{n}}$ & $R_{\mathrm{n}}$ & $\Delta V_{\mathrm{n}}$ & $R_{\mathrm{n}}$ \\
\hline $\mathrm{Pa} \alpha$ & 1.00 & 1.00 & - & $(2.59)$ & - & $(2.74)$ \\
$\mathrm{Pa} \beta$ & 2.91 & 2.59 & 339 & 2.81 & 362 & 2.46 \\
\hline \hline
\end{tabular}

Tabla B.37: 12 Vul - Anchos equivalentes, flujos y FWHM de las líneas de hidrógeno presentes en la banda J.

\begin{tabular}{l|cc}
\hline \hline & \multicolumn{2}{|c}{2017} \\
& EW & Fl FWHM \\
\hline $\mathrm{Pa} \beta$ & \multicolumn{2}{|c}{ shell } \\
\hline \hline
\end{tabular}


Tabla B.38: 12 Vul - Anchos equivalentes, flujos y FWHM de las líneas de hidrógeno presentes en la banda $\mathrm{H}$.

\begin{tabular}{l|ccc|ccc|ccc}
\hline \hline & \multicolumn{3}{|c|}{2010} & \multicolumn{3}{c|}{2016} & \multicolumn{3}{c}{2017} \\
& EW & Fl & FWHM & EW & Fl & FWHM & EW & Fl & FWHM \\
\hline $\mathrm{Br}_{9}$ & \multicolumn{3}{|c|}{ em } & & \multicolumn{3}{|c|}{ em } & & \multicolumn{3}{c}{ em } \\
$\mathrm{Br}_{10}$ & 8.52 & 51.11 & 464 & - & - & - & - & - & - \\
$\mathrm{Br}_{11}$ & 7.88 & 53.63 & 417 & - & - & - & - & - & - \\
$\mathrm{Br}_{12}$ & 6.69 & 50.72 & 453 & - & - & - & - & - & - \\
$\mathrm{Br}_{13}$ & 4.96 & 40.44 & 413 & - & - & - & - & - & - \\
$\mathrm{Br}_{14}$ & 4.18 & 36.70 & 395 & - & - & - & - & - & - \\
$\mathrm{Br}_{15}$ & 3.05 & 26.73 & 435 & - & - & - & - & - & - \\
$\mathrm{Br}_{16}$ & 2.23 & 20.95 & 371 & - & - & - & - & - & - \\
$\mathrm{Br}_{17}$ & 1.82 & 17.45 & 574 & - & - & - & - & - & - \\
$\mathrm{Br}_{18}$ & 1.35 & 13.22 & 455 & - & - & - & - & - & - \\
$\mathrm{Br}_{19}$ & 1.50 & 15.02 & 553 & - & - & - & - & - & - \\
$\mathrm{Br}_{20}$ & 1.19 & 12.14 & 607 & - & - & - & - & - & - \\
\hline \hline
\end{tabular}

Tabla B.39: 12 Vul - Anchos equivalentes, flujos y FWHM de las líneas de hidrógeno presentes en la banda K.

\begin{tabular}{c|ccc|ccc|ccc}
\hline \hline & \multicolumn{3}{|c|}{2010} & \multicolumn{3}{c|}{2016} & \multicolumn{3}{c}{2017} \\
& EW & Fl & FWHM & EW & Fl & FWHM & EW & Fl & FWHM \\
\hline $\mathrm{Pf}_{18}$ & 4.05 & 6.62 & - & - & - & - & - & - & - \\
$\mathrm{Pf}_{19}$ & 6.01 & 10.20 & - & - & - & - & - & - & - \\
$\mathrm{Pf}_{20}$ & 6.29 & 10.90 & - & - & - & - & - & - & - \\
$\mathrm{Pf}_{21}$ & 3.46 & 6.19 & - & - & - & - & - & - & - \\
$\mathrm{Pf}_{22}$ & 2.86 & 5.23 & - & - & - & - & - & - & - \\
$\mathrm{Pf}_{23}$ & 2.50 & 4.70 & - & - & - & - & - & - & - \\
$\mathrm{Br} \gamma$ & 11.28 & 30.78 & 429 & - & - & - & - & - & - \\
$\mathrm{Br} \delta$ & 13.31 & 54.40 & 373 & & em & & \multicolumn{3}{|c|}{ em } \\
$\mathrm{Pa} \alpha$ & - & - & - & - & - & - & \multicolumn{3}{c|}{ em } \\
\hline \hline
\end{tabular}


Tabla B.40: 12 Vul - Anchos equivalentes, flujos y FWHM de las líneas de hidrógeno presentes en la banda L.

\begin{tabular}{l|ccc}
\hline \hline & & 2010 & \\
& $\mathrm{EW}$ & Fl & FWHM \\
\hline $\mathrm{Hu} 13$ & 27.62 & 8.61 & 420 \\
$\mathrm{Br} \alpha$ & 37.20 & 11.99 & 391 \\
$\mathrm{Hu}$ & 23.46 & 7.82 & 439 \\
$\mathrm{Hu}_{15}$ & 17.69 & 6.51 & 436 \\
$\mathrm{Hu}_{16}$ & 14.34 & 5.69 & 448 \\
$\mathrm{Hu}_{17}$ & 13.30 & 5.55 & 441 \\
$\mathrm{Pf} \gamma$ & 31.45 & 13.10 & 450 \\
$\mathrm{Hu}_{18}$ & 8.80 & 3.97 & 457 \\
$\mathrm{Hu}_{19}$ & 6.67 & 3.14 & 434 \\
$\mathrm{Hu}_{20}$ & 4.64 & 2.28 & 433 \\
$\mathrm{Hu}_{21}$ & 4.19 & 2.12 & 451 \\
$\mathrm{Hu}_{22}$ & 3.96 & 2.05 & 482 \\
$\mathrm{Hu}_{23}$ & 3.37 & 1.79 & 493 \\
$\mathrm{Hu}_{24}$ & 2.50 & 1.36 & 458 \\
$\mathrm{Hu}_{25}$ & 1.91 & 1.06 & 457 \\
$\mathrm{Hu}_{26}$ & 2.10 & 1.18 & 577 \\
$\mathrm{Pf} \delta^{24.36}$ & 16.71 & 414 \\
\hline \hline
\end{tabular}

Tabla B.41: 12 Vul - Tamaños de las regiones emisoras de las líneas de la serie de Humphreys a partir de los métodos FWHM y $\Delta V$.

\begin{tabular}{l||c||ccc}
\hline \hline \multicolumn{1}{l||}{} & \multicolumn{1}{c||}{ Método FWHM } & \multicolumn{3}{c}{ Método $\Delta V$} \\
& 2010 & \multicolumn{3}{c}{2010} \\
& $\frac{R\left(\mathrm{Hu}_{\mathrm{n}}\right)}{R\left(\mathrm{Hu}_{14}\right)}$ & $\Delta V_{\mathrm{n}}$ & $R_{\mathrm{n}}$ & $\frac{R\left(\mathrm{Hu}_{\mathrm{n}}\right)}{R\left(\mathrm{Hu}_{14}\right)}$ \\
\hline $\mathrm{Hu}_{13}$ & 1.09 & 262 & 3.88 & 1.01 \\
$\mathrm{Hu}_{14}$ & 1.00 & 263 & 3.85 & 1.00 \\
$\mathrm{Hu}_{15}$ & 1.01 & 278 & 3.44 & 0.89 \\
$\mathrm{Hu}_{16}$ & 0.96 & 261 & 3.99 & 1.01 \\
$\mathrm{Hu}_{17}$ & 0.99 & 229 & 5.08 & 1.32 \\
$\mathrm{Hu}_{18}$ & 0.92 & 276 & 3.49 & 0.91 \\
$\mathrm{Hu}_{19}$ & 1.02 & 266 & 3.76 & 0.98 \\
$\mathrm{Hu}_{20}$ & 1.03 & 231 & 4.99 & 1.30 \\
$\mathrm{Hu}_{21}$ & 0.95 & 244 & 4.47 & 1.16 \\
$\mathrm{Hu}_{22}$ & 0.83 & 264 & 3.82 & 0.99 \\
$\mathrm{Hu}_{23}$ & 0.79 & 244 & 4.47 & 1.16 \\
$\mathrm{Hu}_{24}$ & 0.92 & 247 & 4.36 & 1.13 \\
$\mathrm{Hu}_{25}$ & 0.92 & 224 & 5.31 & 1.38 \\
$\mathrm{Hu}_{26}$ & 0.58 & 310 & 2.77 & 0.72 \\
\hline \hline
\end{tabular}


Tabla B.42: 12 Vul - Tamaños de las regiones emisoras de las líneas de la serie de Pfund a partir de los métodos $N$, FWHM y $\Delta V$.

\begin{tabular}{c||cc|c||ccc}
\hline \hline & \multicolumn{1}{|c||}{ Método $N$} & Método FWHM & \multicolumn{3}{c}{ Método $\Delta V$} \\
& 2010 & 2010 & \multicolumn{3}{c}{2010} \\
& $N$ & $\frac{\Delta R\left(\mathrm{Pf}_{\mathrm{n}}\right)}{\Delta R(\mathrm{Pf} \gamma)}$ & $\frac{R\left(\mathrm{Pf}_{\mathrm{n}}\right)}{R(\mathrm{Pf} \gamma)}$ & $\Delta V_{\mathrm{n}}$ & $R_{\mathrm{n}}$ & $\frac{R\left(\mathrm{Pf}_{\mathrm{n}}\right)}{R(\mathrm{Pf} \gamma)}$ \\
\hline Pf $\gamma$ & 2.08 & 0.63 & 0.85 & 264 & 3.82 & 0.95 \\
$\operatorname{Pf} \delta$ & 3.31 & 1.00 & 1.00 & 257 & 4.03 & 1.00 \\
\hline \hline
\end{tabular}

Tabla B.43: 12 Vul - Tamaños de las regiones emisoras de las líneas de la serie de Brackett a partir de los métodos $N$, FWHM y $\Delta V$. El valor indicado entre paréntesis fue obtenido a partir del tamaño relativo a $\operatorname{Br} \alpha$.

\begin{tabular}{|c|c|c|c|c|c|c|}
\hline & \multicolumn{2}{|c|}{$\begin{array}{l}\text { Método } N \\
2010\end{array}$} & \multirow{2}{*}{$\begin{array}{c}\text { Método FWHM } \\
2010 \\
\frac{R\left(\mathrm{Br}_{\mathrm{n}}\right)}{R(\mathrm{Br} \delta)} \\
\end{array}$} & \multicolumn{3}{|c|}{$\begin{array}{c}\text { Método } \Delta V \\
2010\end{array}$} \\
\hline & $N$ & $\frac{\Delta R\left(\mathrm{Br}_{\mathrm{n}}\right)}{\Delta R\left(\mathrm{Br}_{11}\right)}$ & & $\Delta V_{\mathrm{n}}$ & $R_{\mathrm{n}}$ & $\frac{R\left(\mathrm{Br}_{\mathrm{n}}\right)}{R(\mathrm{Br} \gamma)}$ \\
\hline $\operatorname{Br} \alpha$ & - & - & 1.41 & 240 & 4.62 & 1.00 \\
\hline $\operatorname{Br} \gamma$ & - & - & 1.17 & 267 & 3.73 & 0.81 \\
\hline $\operatorname{Br} \delta$ & - & - & 1.55 & - & (5.08) & - \\
\hline $\mathrm{Br}_{10}$ & 6.18 & 1.00 & 1.00 & 301 & 2.94 & 0.64 \\
\hline $\mathrm{Br}_{11}$ & 3.02 & 0.49 & 1.24 & 276 & 3.50 & 0.76 \\
\hline $\mathrm{Br}_{12}$ & - & - & 1.05 & 266 & 3.76 & 0.81 \\
\hline $\mathrm{Br}_{13}$ & - & - & 1.26 & 277 & 3.47 & 0.75 \\
\hline $\mathrm{Br}_{14}$ & - & - & 1.38 & 257 & 4.03 & 0.87 \\
\hline $\mathrm{Br}_{15}$ & - & - & 1.14 & 264 & 3.82 & 0.83 \\
\hline $\mathrm{Br}_{16}$ & - & - & 1.56 & 264 & 3.82 & 0.83 \\
\hline $\mathrm{Br}_{17}$ & - & - & 0.65 & 270 & 3.65 & 0.79 \\
\hline $\mathrm{Br}_{18}$ & - & - & 1.04 & 309 & 2.79 & 0.60 \\
\hline $\mathrm{Br}_{19}$ & - & - & 0.70 & - & - & - \\
\hline $\mathrm{Br}_{20}$ & - & - & 0.58 & - & - & - \\
\hline
\end{tabular}

Tabla B.44: 28 Cyg - Anchos equivalentes, flujos y FWHM de las líneas de hidrógeno presentes en la banda J.

\begin{tabular}{c|ccc|ccc}
\hline \hline & \multicolumn{3}{|c|}{2016} & \multicolumn{3}{c}{2017} \\
& EW & Fl & FWHM & EW & Fl & FWHM \\
\hline $\mathrm{Pa} \beta$ & 5.86 & 112.26 & 469 & 8.78 & 172.70 & 386 \\
\hline \hline
\end{tabular}


Tabla B.45: 28 Cyg - Anchos equivalentes, flujos y FWHM de las líneas de hidrógeno presentes en la banda $\mathrm{H}$.

\begin{tabular}{l|ccc|ccc|ccc}
\hline \hline & \multicolumn{3}{|c|}{2010} & \multicolumn{3}{c|}{2016} & \multicolumn{3}{c}{2017} \\
& EW & Fl & FWHM & EW & Fl & FWHM & EW & Fl & FWHM \\
\hline $\mathrm{Br}_{9}$ & 13.53 & 60.11 & - & 18.38 & 113.56 & - & 18.55 & 95.20 & 571 \\
$\mathrm{Br}_{10}$ & - & - & - & 10.00 & 67.87 & - & 7.99 & 51.67 & 576 \\
$\mathrm{Br}_{11}$ & - & - & - & 3.37 & 25.15 & 638 & 7.04 & 53.51 & 506 \\
$\mathrm{Br}_{12}$ & - & - & - & 3.86 & 30.71 & 546 & 7.08 & 58.72 & 556 \\
$\mathrm{Br}_{13}$ & - & - & - & 4.86 & 39.89 & 555 & 5.87 & 52.36 & 540 \\
$\mathrm{Br}_{14}$ & - & - & - & 3.83 & 34.50 & 569 & 6.56 & 61.52 & 669 \\
$\mathrm{Br}_{15}$ & - & - & - & 4.91 & 47.43 & 559 & 6.18 & 60.45 & 624 \\
$\mathrm{Br}_{16}$ & - & - & - & 2.60 & 25.83 & 556 & 5.25 & 52.74 & 663 \\
$\mathrm{Br}_{17}$ & - & - & - & 2.21 & 22.63 & 527 & 5.02 & 51.29 & 686 \\
$\mathrm{Br}_{18}$ & - & - & - & 1.44 & 15.07 & 537 & 3.87 & 40.04 & 653 \\
$\mathrm{Br}_{19}$ & - & - & - & 0.96 & 10.41 & 567 & 3.10 & 32.27 & 610 \\
$\mathrm{Br}_{20}$ & - & - & - & 1.54 & 17.14 & 659 & 2.79 & 29.18 & 617 \\
$\mathrm{Br}_{21}$ & - & - & - & 0.87 & 9.90 & 475 & 1.96 & 20.86 & 611 \\
$\mathrm{Br}_{22}$ & - & - & - & 1.15 & 13.22 & - & & $\mathrm{em}$ & \\
$\mathrm{Br}_{23}$ & - & - & - & & em & & & $\mathrm{em}$ & \\
\hline \hline
\end{tabular}

Tabla B.46: 28 Cyg - Anchos equivalentes, flujos y FWHM de las líneas de hidrógeno presentes en la banda K.

\begin{tabular}{l|ccc|ccc|ccc|ccc}
\hline \hline & \multicolumn{3}{|c|}{2008} & \multicolumn{3}{|c|}{2010} & \multicolumn{3}{c|}{2016} & \multicolumn{3}{c}{2017} \\
& EW & Fl & FWHM & EW & Fl & FWHM & EW & Fl & FWHM & EW & Fl & FWHM \\
\hline $\mathrm{Pf}_{17}$ & - & - & - & - & - & - & 5.55 & 11.33 & - & & em & \\
$\mathrm{Pf}_{18}$ & - & - & - & - & - & - & 5.16 & 10.57 & - & 11.92 & 21.86 & 600 \\
$\mathrm{Pf}_{19}$ & - & - & - & - & - & - & 4.33 & 8.97 & - & 9.60 & 18.77 & 666 \\
$\mathrm{Pf}_{20}$ & - & - & - & - & - & - & 4.57 & 9.77 & - & 7.50 & 14.43 & 751 \\
$\mathrm{Pf}_{21}$ & - & - & - & - & - & - & 2.04 & 4.50 & - & 5.25 & 10.13 & 591 \\
$\mathrm{Pf}_{22}$ & - & - & - & - & - & - & 3.42 & 7.60 & - & 3.94 & 7.74 & 545 \\
$\mathrm{Pf}_{23}$ & - & - & - & - & - & - & & em & & 2.97 & 5.99 & 534 \\
$\mathrm{Pf}_{24}$ & - & - & - & - & - & - & & em & & 2.32 & 4.86 & 611 \\
$\mathrm{Br} \gamma$ & 6.50 & 32.14 & - & 6.94 & 19.66 & - & 11.04 & 33.61 & 440 & 11.79 & 39.11 & 484 \\
$\mathrm{Br} \delta$ & 11.95 & 87.34 & - & 7.28 & 32.40 & - & 15.58 & 78.65 & 548 & 19.94 & 87.21 & 513 \\
$\mathrm{~Pa} \alpha$ & - & - & - & - & - & - & 57.57 & 405.76 & 460 & 52.66 & 224.28 & 493 \\
\hline \hline
\end{tabular}


Tabla B.47: 28 Cyg - Anchos equivalentes, flujos y FWHM de las líneas de hidrógeno presentes en la banda L.

\begin{tabular}{l|ccc|ccc|ccc|ccc}
\hline & \multicolumn{3}{|c|}{2008} & \multicolumn{3}{c|}{2010} & \multicolumn{3}{c|}{2016} & \multicolumn{3}{c}{2017} \\
& EW & Fl & FWHM & EW & Fl & FWHM & EW & Fl & FWHM & EW & Fl & FWHM \\
\hline $\mathrm{Br} \alpha$ & 47.44 & 12.26 & 617 & - & - & - & 60.64 & 10.11 & 434 & 46.79 & 20.88 & 393 \\
$\mathrm{Hu}$ & 8.21 & 2.20 & - & - & - & - & 24.56 & 4.25 & 568 & 30.82 & 14.10 & 621 \\
$\mathrm{Hu}_{15}$ & 7.50 & 2.28 & - & - & - & - & 21.18 & 4.02 & 594 & 26.74 & 13.46 & 621 \\
$\mathrm{Hu}_{16}$ & 4.70 & 1.59 & - & - & - & - & 17.29 & 3.55 & 602 & 25.76 & 14.00 & 666 \\
$\mathrm{Hu}$ & - & - & - & - & - & - & 13.83 & 3.03 & 554 & 19.55 & 11.50 & 611 \\
$\mathrm{Pf} \gamma$ & 15.29 & 5.58 & 621 & 14.01 & 1.42 & 447 & 28.60 & 6.31 & 513 & 29.47 & 17.51 & 537 \\
$\mathrm{Hu}$ & - & - & - & - & - & - & 10.80 & 2.51 & 599 & 16.45 & 10.15 & 636 \\
$\mathrm{Hu}_{19}$ & - & - & - & - & - & - & 8.64 & 2.11 & 591 & 13.06 & 8.45 & 612 \\
$\mathrm{Hu}_{20}$ & - & - & - & - & - & - & 6.57 & 1.67 & 595 & 11.53 & 7.75 & 671 \\
$\mathrm{Hu}_{21}$ & - & - & - & - & - & - & 5.87 & 1.55 & 633 & 8.76 & 6.10 & 608 \\
$\mathrm{Hu}_{22}$ & - & - & - & - & - & - & 4.59 & 1.25 & 620 & 7.13 & 5.13 & 584 \\
$\mathrm{Hu}_{23}$ & - & - & - & - & - & - & 3.91 & 1.09 & 593 & 6.42 & 4.75 & 621 \\
$\mathrm{Hu}_{24}$ & - & - & - & - & - & - & 3.28 & 0.94 & 622 & 6.95 & 5.28 & 652 \\
$\mathrm{Hu}_{25}$ & - & - & - & - & - & - & 2.69 & 0.78 & 620 & 5.75 & 4.47 & 642 \\
$\mathrm{Hu}_{26}$ & - & - & - & - & - & - & 2.49 & 0.74 & 661 & 2.37 & 1.88 & 529 \\
$\mathrm{Hu}_{27}$ & - & - & - & - & - & - & 1.84 & 0.55 & 594 & 3.13 & 2.53 & 632 \\
$\mathrm{Hu}_{28}$ & - & - & - & - & - & - & - & - & - & & em \\
$\mathrm{Hu}_{29}$ & - & - & - & - & - & - & - & - & - & & em & \\
$\mathrm{Pf}$ & 9.84 & 6.80 & - & 63.25 & 10.05 & 860 & 20.89 & 7.63 & 394 & 30.60 & 30.14 & 546 \\
\hline \hline
\end{tabular}

Tabla B.48: 28 Cyg - Densidades columnares y tamaños de las regiones emisoras de las líneas de la serie de Humphreys a partir de los métodos $N$, FWHM y $\Delta V$. Los valores indicados entre paréntesis fueron obtenidos a partir del tamaño relativo a $\mathrm{Pa} \alpha$ en 2016 y $\mathrm{Hu}_{14}$ en 2017.

\begin{tabular}{l||cc|cc||c|c||c|ccc}
\hline \hline \multicolumn{1}{l||}{} & \multicolumn{4}{c||}{ Método $N$} & \multicolumn{3}{c||}{ Método FWHM } & \multicolumn{4}{c}{ Método $\Delta V$} \\
& \multicolumn{2}{c|}{2016} & \multicolumn{2}{c}{2017} & 2016 & 2017 & 2016 & \multicolumn{2}{c}{2017} \\
& $N$ & $\frac{\Delta R\left(\mathrm{Hu}_{\mathrm{n}}\right)}{\Delta R\left(\mathrm{Hu}_{14}\right)}$ & $N$ & $\frac{\Delta R\left(\mathrm{Hu}_{\mathrm{n}}\right)}{\Delta R\left(\mathrm{Hu}_{14}\right)}$ & $\frac{R\left(\mathrm{Hu}_{\mathrm{n}}\right)}{R\left(\mathrm{Hu}_{14}\right)}$ & $\frac{R\left(\mathrm{Hu}_{\mathrm{n}}\right)}{R\left(\mathrm{Hu}_{14}\right)}$ & $R_{\mathrm{n}}$ & $\Delta V_{\mathrm{n}}$ & $R_{\mathrm{n}}$ & $\frac{R\left(\mathrm{Hu}_{\mathrm{n}}\right)}{R\left(\mathrm{Hu}_{14}\right)}$ \\
\hline $\mathrm{Hu}_{14}$ & 2.46 & 1.00 & 5.54 & 1.00 & 1.00 & 1.00 & $(3.70)$ & 326 & 4.05 & 1.00 \\
$\mathrm{Hu}_{15}$ & - & - & 4.71 & 0.85 & 0.91 & 1.00 & $(3.39)$ & 336 & 3.83 & 0.94 \\
$\mathrm{Hu}_{16}$ & - & - & - & - & 0.89 & 0.87 & $(3.30)$ & 327 & 4.03 & 0.99 \\
$\mathrm{Hu}_{17}$ & - & - & - & - & 1.05 & 1.03 & $(3.89)$ & 341 & 3.71 & 0.92 \\
$\mathrm{Hu}_{18}$ & - & - & - & - & 0.90 & 0.95 & $(3.33)$ & 327 & 4.02 & 0.99 \\
$\mathrm{Hu}_{19}$ & - & - & - & - & 0.92 & 1.03 & $(3.42)$ & - & $(4.16)$ & - \\
$\mathrm{Hu}_{20}$ & - & - & - & - & 0.91 & 0.86 & $(3.37)$ & - & $(3.46)$ & - \\
$\mathrm{Hu}_{21}$ & - & - & - & - & 0.82 & 1.04 & $(2.98)$ & - & $(4.21)$ & - \\
$\mathrm{Hu}_{22}$ & - & - & - & - & 0.84 & 1.13 & $(3.11)$ & - & $(4.57)$ & - \\
$\mathrm{Hu}_{23}$ & - & - & - & - & 0.92 & 1.00 & $(3.40)$ & - & $(4.04)$ & - \\
$\mathrm{Hu}_{24}$ & - & - & - & - & 0.83 & 0.91 & $(3.09)$ & - & $(3.66)$ & - \\
$\mathrm{Hu}_{25}$ & - & - & - & - & 0.84 & 0.94 & $(3.11)$ & - & $(3.78)$ & - \\
$\mathrm{Hu}_{26}$ & - & - & - & - & 0.74 & 1.38 & $(2.73)$ & - & $(5.56)$ & - \\
$\mathrm{Hu}_{27}$ & - & - & - & - & 0.91 & 0.97 & $(3.39)$ & - & $(3.90)$ & - \\
\hline \hline
\end{tabular}


Tabla B.49: 28 Cyg - Densidades columnares y tamaños de las regiones emisoras de las líneas de la serie de Pfund a partir de los métodos $N$, FWHM y $\Delta V$. Los valores indicados entre paréntesis fueron obtenidos a partir del tamaño relativo a $\mathrm{Pa} \alpha$ en 2016 y Hu 14 en 2017.

\begin{tabular}{|c|c|c|c|c|c|c|c|c|c|c|c|c|c|c|}
\hline & \multicolumn{4}{|c|}{ Método $N$} & \multicolumn{3}{|c|}{ Método FWHM } & \multicolumn{7}{|c|}{ Método $\Delta V$} \\
\hline & \multicolumn{2}{|c|}{2016} & \multicolumn{2}{|c|}{2017} & \multirow{2}{*}{$\begin{array}{l}2010 \\
\frac{R\left(\mathrm{Pf}_{\mathrm{n}}\right)}{R\left(\mathrm{Pf}_{\gamma}\right)}\end{array}$} & \multirow{2}{*}{$\begin{array}{l}2016 \\
\frac{R\left(\mathrm{Pf}_{\mathrm{n}}\right)}{R(\mathrm{Pf} \gamma)}\end{array}$} & \multirow{2}{*}{$\begin{array}{l}2017 \\
\frac{R\left(\mathrm{Pf}_{\mathrm{n}}\right)}{R(\mathrm{Pf} \gamma)}\end{array}$} & \multirow[b]{2}{*}{$\Delta V_{\mathrm{n}}$} & \multicolumn{2}{|c|}{2010} & \multirow{2}{*}{$\begin{array}{c}2016 \\
R_{\mathrm{n}}\end{array}$} & \multirow[b]{2}{*}{$\Delta V_{\mathrm{n}}$} & \multicolumn{2}{|l|}{2017} \\
\hline & $N$ & $\frac{\Delta R\left(\mathrm{Pf}_{\mathrm{n}}\right)}{\Delta R(\mathrm{Pf} \gamma)}$ & $N$ & $\frac{\Delta R\left(\mathrm{Pf}_{\mathrm{n}}\right)}{\Delta R(\mathrm{Pf} \gamma)}$ & & & & & $R_{\mathrm{n}}$ & $\frac{R\left(\mathrm{Pf}_{\mathrm{n}}\right)}{R(\mathrm{Pf} \gamma)}$ & & & $R_{\mathrm{n}}$ & $\frac{R\left(\mathrm{Pf}_{\mathrm{n}}\right)}{R(\mathrm{Pf} \gamma)}$ \\
\hline $\mathrm{Pf} \gamma$ & 3.15 & 1.00 & 3.00 & 1.00 & 1.00 & 1.00 & 1.00 & 300 & 4.77 & 1.00 & $(4.54)$ & 165 & 15.8 & 1.00 \\
\hline $\operatorname{Pf} \delta$ & 4.64 & 1.47 & 4.76 & 1.59 & 0.27 & 1.70 & 0.97 & 492 & 1.78 & 0.37 & (7.69) & 237 & 7.66 & 0.49 \\
\hline $\mathrm{Pf}_{18}$ & - & - & - & - & - & - & 0.80 & - & - & - & - & - & $(4.32)$ & - \\
\hline $\mathrm{Pf}_{19}$ & - & - & - & - & - & - & 0.65 & - & - & - & - & 350 & 3.51 & 0.22 \\
\hline $\mathrm{Pf}_{20}$ & - & - & - & - & - & - & 0.51 & - & - & - & - & 395 & 2.76 & 0.17 \\
\hline $\mathrm{Pf}_{21}$ & - & - & - & - & - & - & 0.83 & - & - & - & - & 481 & 1.86 & 0.11 \\
\hline $\mathrm{Pf}_{22}$ & - & - & - & - & - & - & 0.97 & - & - & - & - & 357 & 3.39 & 0.21 \\
\hline $\mathrm{Pf}_{23}$ & - & - & - & - & - & - & 1.01 & - & - & - & - & 303 & 4.69 & 0.29 \\
\hline $\mathrm{Pf}_{24}$ & - & - & - & - & - & - & 0.77 & - & - & - & - & - & $(4.17)$ & - \\
\hline
\end{tabular}

Tabla B.50: 28 Cyg - Densidades columnares y tamaños de las regiones emisoras de las líneas de la serie de Brackett a partir de los métodos $N$, FWHM y $\Delta V$. Los valores indicados entre paréntesis fueron obtenidos a partir del tamaño relativo a $\mathrm{Pa} \alpha$ en 2016 y $\mathrm{Hu}_{14}$ en 2017.

\begin{tabular}{l||cc||c|c||ccc|ccc}
\hline \hline \multicolumn{1}{l||}{} & \multicolumn{2}{c||}{ Método $N$} & \multicolumn{7}{c|}{ Método FWHM } & \multicolumn{6}{c}{ Método $\Delta V$} \\
& \multicolumn{2}{c||}{2017} & 2016 & 2017 & & 2016 & & & 2017 & \\
& $N$ & $\frac{\Delta R\left(\mathrm{Br}_{\mathrm{n}}\right)}{\Delta R\left(\mathrm{Br}_{11}\right)}$ & $\frac{R\left(\mathrm{Br}_{\mathrm{n}}\right)}{R\left(\mathrm{Br}_{11}\right)}$ & $\frac{R\left(\mathrm{Br}_{\mathrm{n}}\right)}{R\left(\mathrm{Br}_{11}\right)}$ & $\Delta V_{\mathrm{n}}$ & $R_{\mathrm{n}}$ & $\frac{R\left(\mathrm{Br}_{\mathrm{n}}\right)}{R\left(\mathrm{Br}_{11}\right)}$ & $\Delta V_{\mathrm{n}}$ & $R_{\mathrm{n}}$ & $\frac{R\left(\mathrm{Br}_{\mathrm{n}}\right)}{R\left(\mathrm{Br}_{11}\right)}$ \\
\hline $\mathrm{Br} \alpha$ & - & - & 2.16 & 1.65 & - & $(6.34)$ & - & - & $(10.08)$ & - \\
$\mathrm{Br} \gamma$ & - & - & 2.10 & 1.09 & 270 & 5.90 & 1.80 & - & $(6.65)$ & - \\
$\mathrm{Br} \delta$ & - & - & 1.36 & 0.97 & - & $(3.98)$ & - & - & $(5.92)$ & - \\
$\mathrm{Br}$ & 10.90 & 0.44 & - & 0.79 & - & - & - & - & $(4.78)$ & - \\
$\mathrm{Br}_{10}$ & 23.05 & 0.93 & - & 0.77 & 465 & 1.99 & 0.61 & 314 & 4.38 & 1.21 \\
$\mathrm{Br}_{11}$ & 24.74 & 1.00 & 1.00 & 1.00 & 362 & 3.28 & 1.00 & 345 & 3.62 & 1.00 \\
$\mathrm{Br}_{12}$ & 28.34 & 1.15 & 1.37 & 0.83 & 349 & 3.53 & 1.08 & 343 & 3.66 & 1.01 \\
$\mathrm{Br}_{13}$ & 35.80 & 1.45 & 1.32 & 0.88 & 321 & 4.18 & 1.27 & 368 & 3.18 & 0.88 \\
$\mathrm{Br}_{14}$ & 31.62 & 1.28 & 1.26 & 0.57 & 357 & 3.37 & 1.03 & 348 & 3.55 & 0.98 \\
$\mathrm{Br}_{15}$ & 22.80 & 0.92 & 1.30 & 0.66 & 321 & 4.17 & 1.27 & 374 & 3.08 & 0.85 \\
$\mathrm{Br}_{16}$ & 24.93 & 1.01 & 1.32 & 0.58 & 333 & 3.89 & 1.18 & 362 & 3.29 & 0.91 \\
$\mathrm{Br}_{17}$ & - & - & 1.47 & 0.54 & 297 & 4.88 & 1.49 & 359 & 3.33 & 0.92 \\
$\mathrm{Br}_{18}$ & - & - & 1.41 & 0.60 & 358 & 3.36 & 1.03 & 379 & 2.99 & 0.83 \\
$\mathrm{Br}_{19}$ & - & - & 1.27 & 0.69 & 341 & 3.70 & 1.13 & 344 & 3.64 & 1.01 \\
$\mathrm{Br}_{20}$ & - & - & 0.94 & 0.67 & 322 & 4.16 & 1.27 & - & $(4.09)$ & - \\
$\mathrm{Br}_{21}$ & - & - & 0.86 & 0.63 & 300 & 4.77 & 1.45 & - & $(4.17)$ & - \\
\hline \hline
\end{tabular}


Tabla B.51: 28 Cyg - Tamaños de las regiones emisoras de las líneas de la serie de Paschen a partir de los métodos FWHM y $\Delta V$. Los valores indicados entre paréntesis fueron obtenidos a partir del tamaño relativo a $\mathrm{Hu}_{14}$.

\begin{tabular}{|c|c|c|c|c|c|c|}
\hline & \multicolumn{2}{|c|}{ Método FWHM } & \multicolumn{4}{|c|}{ Método $\Delta V$} \\
\hline & 2016 & 2017 & & 2016 & & 2017 \\
\hline & $\frac{R\left(\mathrm{~Pa}_{\mathrm{n}}\right)}{R(\mathrm{~Pa} \alpha)}$ & $\frac{R\left(\mathrm{~Pa}_{\mathrm{n}}\right)}{R(\mathrm{~Pa} \alpha)}$ & $\Delta V_{\mathrm{n}}$ & $R_{\mathrm{n}}$ & $\frac{R\left(\mathrm{~Pa}_{\mathrm{n}}\right)}{R(\mathrm{~Pa} \alpha)}$ & $R_{\mathrm{n}}$ \\
\hline $\mathrm{Pa} \alpha$ & 1.00 & 1.00 & 277 & 5.63 & 1.00 & $(6.41)$ \\
\hline $\mathrm{Pa} \beta$ & 0.96 & 1.63 & 260 & 6.31 & 1.12 & (10.45) \\
\hline
\end{tabular}

Tabla B.52: EW Lac - Anchos equivalentes, flujos y FWHM de las líneas de hidrógeno presentes en la banda J.

\begin{tabular}{c|ccc|ccc}
\hline \hline & \multicolumn{3}{|c|}{2016} & \multicolumn{3}{c}{2017} \\
& EW & Fl & FWHM & EW & Fl & FWHM \\
\hline $\mathrm{Pa} \beta$ & 3.77 & 89.44 & - & 2.49 & 43.31 & - \\
\hline \hline
\end{tabular}

Tabla B.53: EW Lac - Anchos equivalentes, flujos y FWHM de las líneas de hidrógeno presentes en la banda $\mathrm{H}$.

\begin{tabular}{l|ccc|ccc|ccc}
\hline \hline & \multicolumn{3}{|c|}{2010} & & 2016 & & \multicolumn{3}{c}{2017} \\
& EW & Fl & FWHM & EW & Fl & FWHM & EW & Fl & FWHM \\
\hline $\mathrm{Br}_{9}$ & 17.48 & 91.56 & 414 & 10.25 & 70.21 & 273 & - & - & - \\
$\mathrm{Br}_{10}$ & 7.57 & 46.01 & 385 & - & - & - & 2.56 & 16.08 & - \\
$\mathrm{Br}_{11}$ & 7.55 & 50.60 & 309 & - & - & - & 2.58 & 17.63 & - \\
$\mathrm{Br}_{12}$ & 5.09 & 36.30 & 434 & - & - & - & 1.71 & 12.65 & - \\
$\mathrm{Br}_{13}$ & 3.79 & 28.77 & 383 & - & - & - & 0.28 & 2.15 & - \\
$\mathrm{Br}_{14}$ & 5.77 & 45.84 & 421 & - & - & - & & shell & \\
$\mathrm{Br}_{15}$ & 4.57 & 36.33 & 400 & - & - & - & & shell & \\
$\mathrm{Br}_{16}$ & 4.08 & 34.22 & 490 & - & - & - & & shell & \\
$\mathrm{Br}_{17}$ & 3.30 & 28.36 & 481 & - & - & - & & shell & \\
$\mathrm{Br}_{18}$ & 2.97 & 26.09 & 523 & - & - & - & & shell & \\
$\mathrm{Br}_{19}$ & 2.60 & 23.30 & 459 & - & - & - & & shell & \\
$\mathrm{Br}_{20}$ & 2.14 & 19.39 & 570 & - & - & - & - & - & - \\
$\mathrm{Br}_{21}$ & 1.36 & 12.53 & 544 & - & - & - & - & - & - \\
$\mathrm{Br}_{22}$ & 1.05 & 9.80 & 469 & - & - & - & - & - & - \\
$\mathrm{Br}_{23}$ & 0.81 & 7.68 & 493 & - & - & - & - & - & - \\
$\mathrm{Br}_{24}$ & 0.45 & 4.27 & - & - & - & - & - & - & - \\
\hline \hline
\end{tabular}


Tabla B.54: EW Lac - Anchos equivalentes, flujos y FWHM de las líneas de hidrógeno presentes en la banda $\mathrm{K}$.

\begin{tabular}{|c|c|c|c|c|c|c|c|c|c|c|c|c|c|c|c|}
\hline & \multicolumn{3}{|c|}{2006} & \multicolumn{3}{|c|}{2008} & \multicolumn{3}{|c|}{2010} & \multicolumn{3}{|c|}{2016} & \multicolumn{3}{|c|}{2017} \\
\hline & EW & $\mathrm{Fl}$ & FWHM & EW & $\mathrm{Fl}$ & FWHM & EW & $\mathrm{Fl}$ & FWHM & EW & Fl & FWHM & EW & $\mathrm{Fl}$ & FWHM \\
\hline $\mathrm{Pf}_{16}$ & - & - & - & 12.72 & 27.73 & - & - & - & - & - & - & - & - & - & - \\
\hline $\mathrm{Pf}_{17}$ & - & - & - & 9.26 & 20.72 & 792 & 8.98 & 19.24 & 421 & & em & & & shell & \\
\hline $\mathrm{Pf}_{19}$ & - & - & - & 5.71 & 13.67 & 646 & 7.19 & 15.50 & 394 & & em & & & shell & \\
\hline $\mathrm{Pf}_{20}$ & 9.03 & 115.01 & 762 & 4.81 & 11.76 & 625 & 7.31 & 15.98 & 381 & & em & & & shell & \\
\hline $\mathrm{Pf}_{21}$ & 4.26 & 57.34 & 599 & 4.29 & 10.77 & 698 & 6.24 & 13.92 & 416 & - & - & - & & shell & \\
\hline $\mathrm{Pf}_{24}$ & 3.72 & 51.81 & 512 & 2.25 & 5.96 & 661 & 3.28 & 7.61 & 455 & - & - & - & - & - & - \\
\hline $\mathrm{Pf}_{25}$ & & $\mathrm{em}$ & & 1.62 & 4.39 & 555 & 2.85 & 6.74 & 482 & - & - & - & - & - & - \\
\hline $\mathrm{Pf}_{26}$ & - & - & - & & em & & 2.62 & 6.26 & 399 & - & - & - & - & - & - \\
\hline $\mathrm{Pf}_{27}$ & - & - & - & & em & & 1.73 & 4.19 & 392 & - & - & - & - & - & - \\
\hline $\mathrm{Pf}_{28}$ & - & - & - & - & - & - & 1.09 & 2.70 & - & - & - & - & - & - & - \\
\hline
\end{tabular}

Tabla B.55: EW Lac - Anchos equivalentes, flujos y FWHM de las líneas de hidrógeno presentes en la banda $\mathrm{L}$.

\begin{tabular}{|c|c|c|c|c|c|c|c|c|c|c|c|c|c|c|c|}
\hline & \multicolumn{3}{|c|}{2006} & \multicolumn{3}{|c|}{2008} & \multicolumn{3}{|c|}{2010} & \multicolumn{3}{|c|}{2016} & \multicolumn{3}{|c|}{2017} \\
\hline & EW & Fl & FWHM & EW & $\mathrm{Fl}$ & FWHM & EW & $\mathrm{Fl}$ & FWHM & EW & $\mathrm{Fl}$ & FWHM & EW & $\mathrm{Fl}$ & FWHM \\
\hline $\mathrm{Hu}_{13}$ & - & - & - & - & - & - & 28.86 & 13.83 & 348. & - & - & - & - & - & - \\
\hline $\operatorname{Br} \alpha$ & 74.79 & 184.41 & 626 & 79.93 & 42.08 & 509 & 101.50 & 47.23 & 205 & 138.00 & 56.55 & 266 & 63.13 & 15.52 & 328 \\
\hline $\mathrm{Hu}_{15}$ & 19.75 & 60.86 & 908 & 20.13 & 11.31 & 663 & 26.79 & 12.91 & 438 & 6.13 & 2.88 & 380 & 14.08 & 4.05 & 700 \\
\hline $\mathrm{Hu}_{16}$ & 18.14 & 61.99 & 883 & 18.07 & 10.55 & 643 & 22.95 & 11.62 & 428 & 4.55 & 2.30 & 421 & 9.19 & 2.94 & 785 \\
\hline $\mathrm{Hu}_{17}$ & 6.87 & 25.85 & 636 & 12.33 & 7.67 & 467 & 23.48 & 12.30 & 456 & 3.77 & 2.06 & 439 & 9.47 & 3.22 & 471 \\
\hline $\mathrm{Hu}_{19}$ & 8.41 & 35.85 & 708 & 9.99 & 6.65 & 542 & 16.27 & 9.20 & 475 & 2.15 & 1.29 & 463 & 6.25 & 2.55 & 656 \\
\hline $\mathrm{Hu}_{20}$ & 6.86 & 30.84 & 693 & 10.17 & 6.98 & 605 & 13.39 & 7.82 & 473 & 2.41 & 1.50 & - & 4.57 & 2.00 & 652 \\
\hline $\mathrm{Hu}_{21}$ & 5.48 & 25.28 & 402 & 8.34 & 5.93 & 530 & 11.85 & 7.06 & 476 & & em & & 3.64 & 1.70 & - \\
\hline $\mathrm{Hu}_{22}$ & 7.61 & 36.41 & 656 & 8.54 & 6.16 & 636 & 11.33 & 6.89 & 504 & & em & & 5.06 & 2.45 & - \\
\hline $\mathrm{Hu}_{23}$ & 5.43 & 26.48 & 684 & 6.00 & 4.43 & 449 & 11.07 & 6.78 & 536 & & em & & 2.66 & 1.34 & - \\
\hline $\mathrm{Hu}_{28}$ & - & - & - & & em & & & em & & - & - & - & - & - & - \\
\hline $\mathrm{Hu}_{29}$ & - & - & - & & em & & - & - & - & - & - & - & - & - & - \\
\hline $\operatorname{Pf} \delta$ & 21.72 & 114.32 & 549 & 25.17 & 23.75 & 747 & 21.68 & 17.59 & 158 & 16.66 & 14.51 & 194 & 17.14 & 11.65 & 717 \\
\hline
\end{tabular}


Tabla B.56: EW Lac - Densidades columnares y tamaños de las regiones emisoras de las líneas de la serie de Humphreys a partir de los métodos $N$ y FWHM.

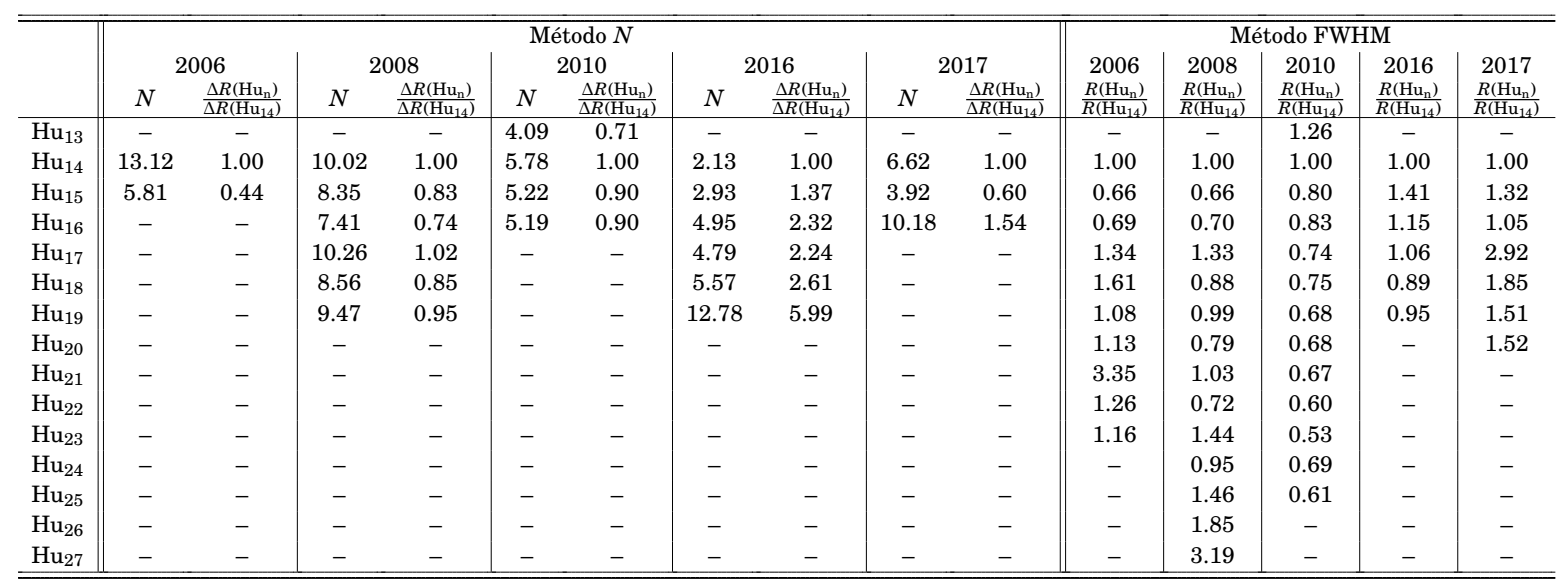

Tabla B.57: EW Lac - Tamaños de las regiones emisoras de las líneas de la serie de Humphreys a partir del método $\Delta V$. Los valores indicados entre paréntesis fueron obtenidos a partir del tamaño relativo a $\mathrm{Pf}_{17}$ en 2008 y $\mathrm{Br} \gamma$ en 2017.

\begin{tabular}{l||c|ccc|ccc}
\hline \hline \multicolumn{1}{l||}{} & \multicolumn{7}{c}{ Método $\Delta V$} \\
& 2008 & \multicolumn{7}{c}{2010} & & & \\
& $R_{\mathrm{n}}$ & $\Delta V_{\mathrm{n}}$ & $R_{\mathrm{n}}$ & $\frac{R\left(\mathrm{Hu}_{\mathrm{n}}\right)}{R\left(\mathrm{u}_{14}\right)}$ & $\Delta V_{\mathrm{n}}$ & $R_{\mathrm{n}}$ & $\frac{R\left(\mathrm{Hu}_{\mathrm{n}}\right)}{R\left(\mathrm{Hu}_{14}\right)}$ \\
\hline $\mathrm{Hu}_{13}$ & - & 220 & 10.43 & 1.14 & - & $(1.51)$ & - \\
$\mathrm{Hu}_{14}$ & $(12.42)$ & 235 & 9.14 & 1.00 & 491 & 2.09 & 1.00 \\
$\mathrm{Hu}_{15}$ & $(8.21)$ & 249 & 8.15 & 0.89 & 505 & 1.98 & 0.95 \\
$\mathrm{Hu}_{16}$ & $(8.73)$ & 255 & 7.78 & 0.85 & 524 & 1.84 & 0.88 \\
$\mathrm{Hu}_{17}$ & $(16.55)$ & 252 & 7.94 & 0.87 & 496 & 2.05 & 0.98 \\
$\mathrm{Hu}_{18}$ & $(10.92)$ & 262 & 7.34 & 0.80 & 474 & 2.24 & 1.07 \\
$\mathrm{Hu}_{19}$ & $(12.29)$ & 239 & 8.82 & 0.97 & 479 & 2.20 & 1.05 \\
$\mathrm{Hu}_{20}$ & $(9.86)$ & 289 & 6.04 & 0.66 & 458 & 2.41 & 1.15 \\
$\mathrm{Hu}_{21}$ & $(12.85)$ & 278 & 6.51 & 0.71 & 552 & 1.65 & 0.79 \\
$\mathrm{Hu}_{22}$ & $(8.92)$ & 302 & 5.52 & 0.60 & 628 & 1.28 & 0.61 \\
$\mathrm{Hu}_{23}$ & $(17.90)$ & 322 & 4.85 & 0.53 & 514 & 1.91 & 0.91 \\
$\mathrm{Hu}_{24}$ & $(11.76)$ & 271 & 6.88 & 0.75 & 462 & 2.36 & 1.13 \\
$\mathrm{Hu}_{25}$ & $(18.15)$ & - & - & - & - & - & - \\
$\mathrm{Hu}_{26}$ & $(23.02)$ & - & - & - & - & - & - \\
$\mathrm{Hu}_{27}$ & $(39.58)$ & - & - & - & - & - & - \\
\hline \hline
\end{tabular}


Tabla B.58: EW Lac - Densidades columnares y tamaños de las regiones emisoras de las líneas de la serie de Pfund a partir de los métodos $N$ y FWHM.

\begin{tabular}{|c|c|c|c|c|c|c|c|c|c|c|c|}
\hline & \multicolumn{6}{|c|}{ Método $N$} & \multicolumn{5}{|c|}{ Método FWHM } \\
\hline & \multicolumn{2}{|c|}{2006} & \multicolumn{2}{|c|}{2008} & \multicolumn{2}{|c|}{2010} & \multirow{2}{*}{$\begin{array}{l}2006 \\
R\left(\mathrm{Pf}_{\mathrm{n}}\right) \\
R(\mathrm{Pf} \gamma)\end{array}$} & \multirow{2}{*}{$\begin{array}{l}2008 \\
R\left(\mathrm{Pf}_{\mathrm{n}}\right) \\
R(\mathrm{Pf} \gamma)\end{array}$} & \multirow{2}{*}{$\begin{array}{l}2010 \\
\frac{R\left(\mathrm{Pf}_{\mathrm{n}}\right)}{R(\mathrm{Pf} \gamma)}\end{array}$} & \multirow{2}{*}{$\begin{array}{l}2016 \\
\frac{R\left(\mathrm{Pf}_{\mathrm{n}}\right)}{R(\mathrm{Pf} \gamma)}\end{array}$} & \multirow{2}{*}{$\begin{array}{l}2017 \\
\frac{R\left(\mathrm{Pf}_{\mathrm{n}}\right)}{R(\mathrm{Pf} \gamma)}\end{array}$} \\
\hline & $N$ & $\frac{\Delta R\left(\mathrm{Pf}_{\mathrm{n}}\right)}{\Delta R(\mathrm{Pf} \gamma)}$ & $N$ & $\frac{\Delta R\left(\mathrm{Pf}_{\mathrm{n}}\right)}{\Delta R(\mathrm{Pf} \gamma)}$ & $N$ & $\frac{\Delta R\left(\mathrm{Pf}_{\mathrm{n}}\right)}{\Delta R(\mathrm{Pf} \gamma)}$ & & & & & \\
\hline $\mathrm{Pf} \gamma$ & 5.55 & 1.00 & 2.46 & 1.00 & 1.56 & 1.00 & 1.00 & 1.00 & 1.00 & 1.00 & 1.00 \\
\hline $\operatorname{Pf} \delta$ & 7.11 & 1.28 & 6.72 & 2.73 & 1.96 & 1.25 & 3.30 & 0.38 & 3.01 & 1.77 & 1.18 \\
\hline $\mathrm{Pf}_{17}$ & - & - & - & - & 14.89 & 9.54 & - & 0.34 & 0.42 & - & - \\
\hline $\mathrm{Pf}_{18}$ & - & - & - & - & - & - & - & 0.44 & 0.43 & - & - \\
\hline $\mathrm{Pf}_{19}$ & - & - & - & - & - & - & - & 0.51 & 0.48 & - & - \\
\hline $\mathrm{Pf}_{20}$ & - & - & - & - & - & - & 1.71 & 0.55 & 0.52 & - & - \\
\hline $\mathrm{Pf}_{21}$ & - & - & - & - & - & - & 2.77 & 0.44 & 0.43 & - & - \\
\hline $\mathrm{Pf}_{22}$ & - & - & - & - & - & - & 2.47 & 0.63 & 0.31 & - & - \\
\hline $\mathrm{Pf}_{23}$ & - & - & - & - & - & - & 4.64 & 0.66 & 0.41 & - & - \\
\hline $\mathrm{Pf}_{24}$ & - & - & - & - & - & - & 3.79 & 0.49 & 0.36 & - & - \\
\hline $\mathrm{Pf}_{25}$ & - & - & - & - & - & - & - & 0.70 & 0.32 & - & - \\
\hline $\mathrm{Pf}_{26}$ & - & - & - & - & - & - & - & - & 0.47 & - & - \\
\hline $\mathrm{Pf}_{27}$ & - & - & - & - & - & - & - & - & 0.49 & - & - \\
\hline
\end{tabular}

Tabla B.59: EW Lac - Tamaños de las regiones emisoras de las líneas de la serie de Pfund a partir del método $\Delta V$. Los valores indicados entre paréntesis fueron obtenidos a partir del tamaño relativo a $\mathrm{Pf}_{17}$.

\begin{tabular}{|c|c|c|c|c|c|c|c|c|c|}
\hline & \multicolumn{9}{|c|}{ Método $\Delta V$} \\
\hline & \multicolumn{3}{|c|}{2008} & \multicolumn{3}{|c|}{2010} & \multicolumn{3}{|c|}{2017} \\
\hline & $\Delta V_{\mathrm{n}}$ & $R_{\mathrm{n}}$ & $\frac{R\left(\mathrm{Pf}_{\mathrm{n}}\right)}{R\left(\mathrm{Pf}_{1,1)}\right.}$ & $\Delta V_{\mathrm{n}}$ & $R_{\mathrm{n}}$ & $\frac{R\left(\mathrm{Pf}_{\mathrm{n}}\right)}{R(\mathrm{Pf} \gamma)}$ & $\Delta V_{\mathrm{n}}$ & $R_{\mathrm{n}}$ & $\frac{R\left(\mathrm{Pf}_{\mathrm{n}}\right)}{R(\mathrm{Pf} \gamma)}$ \\
\hline Pf $\gamma$ & - & $(16.84)$ & - & 174 & 16.65 & 1.00 & 346 & 4.22 & 1.00 \\
\hline $\operatorname{Pf} \delta$ & - & $(6.47)$ & - & 228 & 9.69 & 0.58 & 420 & 2.85 & 0.68 \\
\hline $\mathrm{Pf}_{17}$ & 296 & 5.74 & 1.00 & 243 & 8.51 & 0.51 & - & - & - \\
\hline $\mathrm{Pf}_{18}$ & 273 & 6.76 & 1.18 & 292 & 5.91 & 0.36 & - & - & - \\
\hline $\mathrm{Pf}_{19}$ & 300 & 5.61 & 0.98 & 300 & 5.62 & 0.34 & - & - & - \\
\hline $\mathrm{Pf}_{20}$ & 365 & 3.79 & 0.66 & 299 & 5.63 & 0.34 & - & - & - \\
\hline $\mathrm{Pf}_{21}$ & 356 & 3.98 & 0.69 & 298 & 5.69 & 0.34 & - & - & - \\
\hline $\mathrm{Pf}_{22}$ & 362 & 3.85 & 0.67 & 345 & 4.23 & 0.25 & - & - & - \\
\hline $\mathrm{Pf}_{23}$ & 349 & 4.13 & 0.72 & 305 & 5.41 & 0.32 & - & - & - \\
\hline $\mathrm{Pf}_{24}$ & 347 & 4.20 & 0.73 & 329 & 4.66 & 0.28 & - & - & - \\
\hline $\mathrm{Pf}_{25}$ & 297 & 5.73 & 1.00 & 333 & 4.55 & 0.27 & - & - & - \\
\hline $\mathrm{Pf}_{26}$ & - & - & - & 285 & 6.22 & 0.37 & - & - & - \\
\hline $\mathrm{Pf}_{27}$ & - & - & - & 281 & 6.38 & 0.38 & - & - & - \\
\hline
\end{tabular}


Tabla B.60: EW Lac - Densidades columnares y tamaños de las regiones emisoras de las líneas de la serie de Brackett a partir de los métodos $N$, FWHM y $\Delta V$. Los valores indicados entre paréntesis fueron obtenidos a partir del tamaño relativo a $\mathrm{Pf}_{17}$ en $2008, \mathrm{Hu}_{13}$ en 2010 y $\mathrm{Br} \gamma$ en 2017.

\begin{tabular}{|c|c|c|c|c|c|c|c|c|c|c|c|c|c|c|}
\hline & \multirow{2}{*}{\multicolumn{2}{|c|}{$\begin{array}{c}\text { Método } N \\
2010\end{array}$}} & \multicolumn{5}{|c|}{ Método FWHM } & \multicolumn{7}{|c|}{ Método $\Delta V$} \\
\hline & & & \multirow{2}{*}{$\begin{array}{l}2006 \\
\frac{R\left(\mathrm{Br}_{\mathrm{n}}\right)}{R(\mathrm{Br} \delta)}\end{array}$} & \multirow{2}{*}{$\begin{array}{l}2008 \\
\frac{R\left(\mathrm{Br}_{\mathrm{n}}\right)}{R(\mathrm{Br} \delta)}\end{array}$} & \multirow{2}{*}{$\begin{array}{l}2010 \\
\frac{R\left(\mathrm{Br} \mathrm{r}_{n}\right)}{R(\mathrm{Br} \delta)}\end{array}$} & \multirow{2}{*}{$\begin{array}{l}2016 \\
\frac{R\left(\mathrm{Br}_{\mathrm{n}}\right)}{R(\mathrm{Br} \delta)}\end{array}$} & \multirow{2}{*}{$\begin{array}{l}2017 \\
\frac{R\left(\mathrm{Br}_{\mathrm{n}}\right)}{R(\mathrm{Br} \delta)}\end{array}$} & \multirow{2}{*}{$\begin{array}{c}2008 \\
R_{\mathrm{n}}\end{array}$} & \multicolumn{3}{|c|}{2010} & \multicolumn{3}{|c|}{2017} \\
\hline & $N$ & $\frac{\Delta R\left(\mathrm{Br}_{\mathrm{n}}\right)}{\Delta R\left(\mathrm{Br}_{11}\right)}$ & & & & & & & $\Delta V_{\mathrm{n}}$ & $R_{\mathrm{n}}$ & $\frac{R\left(\mathrm{Br}_{\mathrm{n}}\right)}{R\left(\mathrm{Br}_{11}\right)}$ & $\Delta V_{\mathrm{n}}$ & $R_{\mathrm{n}}$ & $\frac{R\left(\mathrm{Br}_{\mathrm{n}}\right)}{R(\mathrm{Br} \gamma)}$ \\
\hline $\operatorname{Br} \alpha$ & - & - & 1.65 & 2.43 & 3.40 & 1.31 & 5.85 & (13.93) & - & $(30.02)$ & - & - & (9.09) & - \\
\hline $\mathrm{Br} \gamma$ & - & - & 1.81 & 4.16 & 2.30 & 1.99 & 0.99 & $(23.85)$ & - & (20.35) & - & 571 & 1.54 & 0.75 \\
\hline $\operatorname{Br} \delta$ & - & - & 1.00 & 1.00 & 1.00 & 1.00 & 1.00 & $(5.74)$ & - & $(8.83)$ & - & 494 & 2.06 & 1.00 \\
\hline $\mathrm{Br}_{9}$ & 4.25 & 0.46 & - & - & 0.83 & 1.25 & - & - & - & $(7.36)$ & - & - & - & - \\
\hline $\mathrm{Br}_{10}$ & 11.95 & 1.30 & - & - & 0.96 & - & - & - & 250 & 8.08 & 1.15 & - & - & - \\
\hline $\mathrm{Br}_{11}$ & 9.19 & 1.00 & - & - & 1.50 & - & - & - & 267 & 7.06 & 1.00 & - & - & - \\
\hline $\mathrm{Br}_{12}$ & 21.35 & 2.32 & - & - & 0.76 & - & - & - & 299 & 5.63 & 0.80 & - & - & - \\
\hline $\mathrm{Br}_{13}$ & 25.87 & 2.81 & - & - & 0.97 & - & - & - & 314 & 5.12 & 0.73 & - & - & - \\
\hline $\mathrm{Br}_{14}$ & - & - & - & - & 0.81 & - & - & - & 334 & 4.52 & 0.64 & - & - & - \\
\hline $\mathrm{Br}_{15}$ & - & - & - & - & 0.89 & - & - & - & 350 & 4.11 & 0.58 & - & - & - \\
\hline $\mathrm{Br}_{16}$ & - & - & - & - & 0.60 & - & - & - & 378 & 3.53 & 0.50 & - & - & - \\
\hline $\mathrm{Br}_{17}$ & - & - & - & - & 0.62 & - & - & - & 363 & 3.82 & 0.54 & - & - & - \\
\hline $\mathrm{Br}_{18}$ & - & - & - & - & 0.52 & - & - & - & 376 & 3.57 & 0.51 & - & - & - \\
\hline $\mathrm{Br}_{19}$ & - & - & - & - & 0.68 & - & - & - & 386 & 3.39 & 0.48 & - & - & - \\
\hline $\mathrm{Br}_{20}$ & - & - & - & - & 0.44 & - & - & - & 405 & 3.08 & 0.44 & - & - & - \\
\hline $\mathrm{Br}_{21}$ & - & - & - & - & 0.21 & - & - & - & 387 & 3.36 & 0.48 & - & - & - \\
\hline $\mathrm{Br}_{22}$ & - & - & - & - & 0.28 & - & - & - & 406 & 3.06 & 0.43 & - & - & - \\
\hline $\mathrm{Br}_{23}$ & - & - & - & - & 0.26 & - & - & - & - & - & - & - & - & - \\
\hline
\end{tabular}

Tabla B.61: EW Lac - Tamaños de las regiones emisoras de las líneas de la serie de Paschen a partir del método $\Delta V$.

\begin{tabular}{c||ccc}
\hline \hline \multicolumn{1}{c||}{} & \multicolumn{3}{c}{ Método $\Delta V$} \\
& \multicolumn{3}{c}{2017} \\
& $\Delta V_{\mathrm{n}}$ & $R_{\mathrm{n}}$ & $\frac{R\left(\mathrm{~Pa}_{\mathrm{n}}\right)}{R(\mathrm{~Pa} \alpha)}$ \\
\hline $\mathrm{Pa} \alpha$ & 292 & 5.92 & 1.00 \\
$\mathrm{~Pa} \beta$ & 570 & 1.55 & 0.26 \\
\hline \hline
\end{tabular}




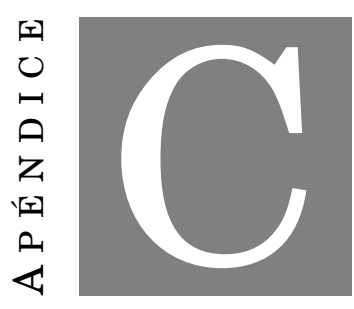

\section{CONCEPTOS BÁSICOS DE INTERFEROMETRÍA}

$\mathrm{L}$ os tamaños angulares de las estrellas más cercanas resultan menores a la mejor resolución alcanzada con un gran telescopio sobre la superficie terrestre. Para un telescopio de apertura $\mathrm{D}$, observando en una longitud de onda $\lambda$, existe un límite a la resolución angular dado por el límite de difracción, $1.22 \lambda / D$ (e.g. 10 mas si $D=10 \mathrm{~m}$ y $\lambda=500 \mathrm{~nm}$ ). Sin embargo, la atmósfera afecta esta resolución haciendo que el valor típico de la misma sea del orden de 1 ".

Una forma de mejorar la resolución sería construir telescopios más grandes, usar óptica adaptativa o poner telescopios en el espacio. Sin embargo, las limitaciones de resolución por difracción siguen presentes. La interferometría permite obtener mejor resolución espacial combinando la señal recibida por dos o más telescopios o aperturas. La resolución angular resulta dada por $\lambda / \mathrm{B}$, donde $\mathrm{B}$ es la mayor separación entre los telescopios, denominada línea de base. Así, tomando varios telescopios pequeños y separándolos en una distancia B, podemos obtener la misma resolución angular que con un gran telescopio de diámetro B.

A través de la interferometría es posible no sólo estimar los tamaños angulares de las estrella sino las dimensiones, geometría y cinemática de las estructuras que las rodean. En este apéndice presentaremos algunos conceptos básicos de la técnica.

\section{C.1 La experiencia de Young}

En 1803, Young describió una experiencia en el cual un haz de luz pasando a través de una pequeña abertura hasta llegar a una pantalla con dos pequeñas rendijas, genera un patrón de interferencia sobre una tercera pantalla (ver Fig. C.1). Tapando la luz de una de las rendijas, el patrón desaparecía, con lo cual llegó a la conclusión de que en la posición de las bandas oscuras la intensidad era reducida al agregarse la contribución del segundo haz, mientras que en la parte 
Figura C.1: Configuración de la experiencia de doble rendija de Young. Si $\theta$ es el ángulo que forman los haces de luz con la pantalla, ocurrirá interferencia constructiva (máximos) cuando la diferencia de trayectoria sea un número entero de longitudes de onda: $d \sin \theta=n \lambda$, y ocurrirá interferencia destructiva (mínimos) cuando la diferencia en trayectoria sea igual a un número semientero de longitudes de onda: $d \sin \theta=(n+1 / 2) \lambda$.

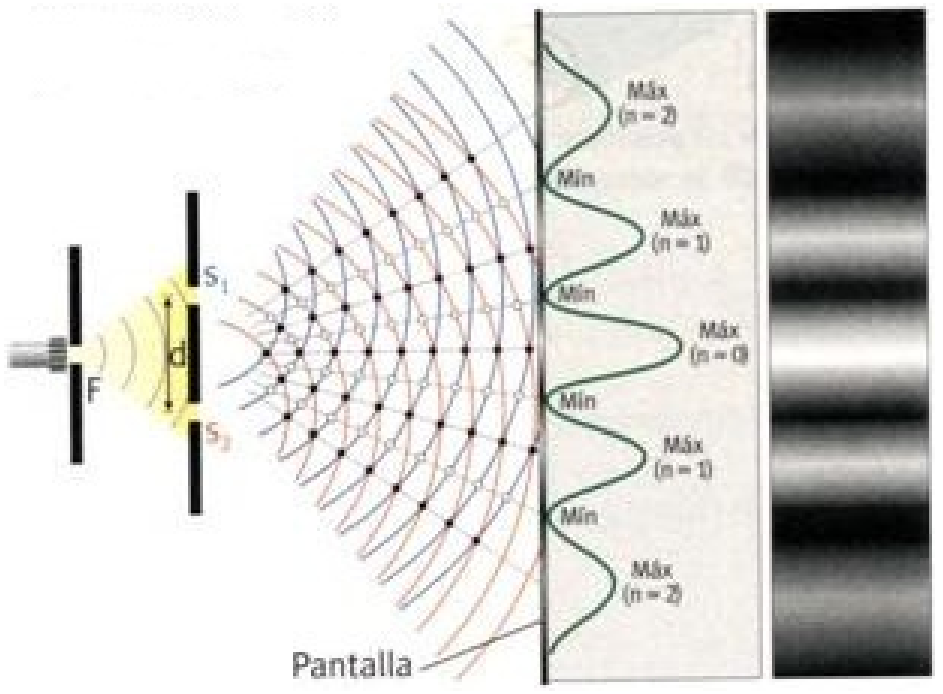

brillante se incrementaba. Además, al tapar parcialmente uno de los haces, el patrón permanecía, pero con menos contraste. Young obtuvo así evidencias de la naturaleza ondulatoria de la luz, y determinó el valor de la longitud de onda para luz de diferentes colores.

\section{C.2 Interferencia y difracción}

Cuando dos o más ondas llegan a un mismo punto desde diferentes fuentes o por diferentes caminos, se interfieren. En la mayoría de los casos, la interferencia ocurre cuando las ondas llegan de la misma fuente pero por diferentes caminos, y el patrón de interferencia ocurre como resultado de la superposición de ondas. En general, el término interferencia se utiliza para describir situaciones cuando los caminos estás separados, y difracción para el caso donde las diferentes rutas forman un continuo.

Los interferómetros son instrumentos creados para generar interferencia y usar este fenómeno para realizar alguna medida.

\section{C.3 Interferometría estelar}

La interferometría estelar se basa en los principios básicos de la interferencia de ondas, y fue propuesta por H. Fizeau en 1868, al decir que el patrón de interferencia producido por una fuente de dimensiones finitas tenía que estar relacionado con el tamaño de dicha fuente. El desafío lo tomó M. Stéphan, quien era director del observatorio de Marsella, cuyo telescopio de $80 \mathrm{~cm}$ era en 
Figura C.2: Interferómetro de Michelson: (a) esquema óptico; (b) típico patrón de interferencia (Labeyrie et al., 2006).
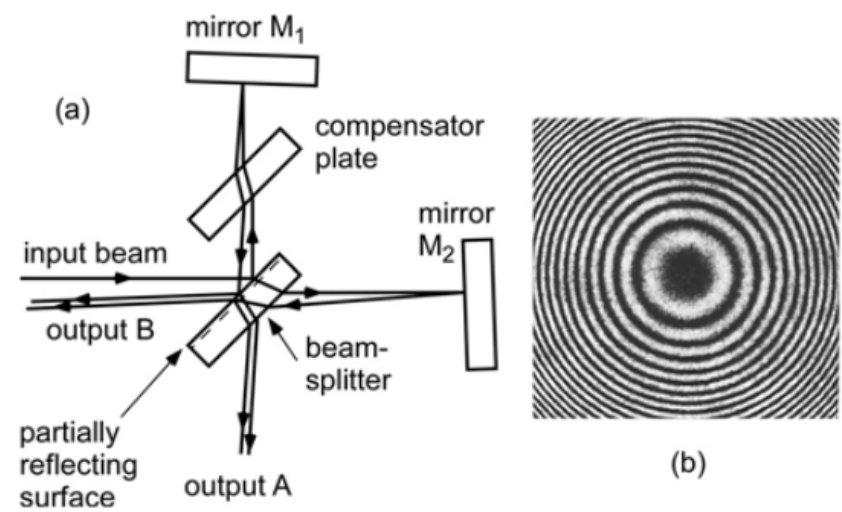

(b)

ese momento el más grande del mundo. Stéphan enmascaró el telescopio, dejando dos pequeñas aperturas con la mayor separación posible, y midiendo los patrones de interferencia para varias estrellas, obtuvo un valor 0.16 " como límite superior para sus diámetros.

Más de una década después, A. A. Michelson (1890) utilizó pequeñas aperturas en una máscara que cubría la apertura del telescopio para producir patrones de interferencia y medir el perfil de la estrella. Michelson no solo reinventó la idea de interferometría estelar, sino que describió en detalle cómo debe ser realizada la medida de la visibilidad del patrón, definida por primera vez en función de la separación de las aperturas. Además, describió los resultados esperados para un disco uniforme, un disco con oscurecimiento al limbo y una estrella binaria. En 1891 probó sus ideas midiendo los tamaños de los 4 mayores satélites de Júpiter, que ya habían sido medidos con otras técnicas, con excelente acuerdo.

\section{C.3.1 El interferómetro de Michelson}

El interferómetro de Michelson consiste básicamente en dos espejos M1 y M2, un espejo divisor de haces que separa el haz de luz en dos (reflejando el 50\% y transmitiendo el 50\%) y un vidrio compensador de igual material y profundidad que el espejo (para que las dos ondas que interfieren hayan pasado a través de la misma cantidad de vidrio). Cada uno de los haces formados es reflejado nuevamente y recombinado en el divisor de haces. De los haces recombinados, uno sale hacia la dirección A fuera del interferómetro y otro vuelve en la dirección B que es cercana a la dirección a la fuente. Puede verse un esquema del interferómetro de Michelson en la Fig. C.2.

Los patrones de interferencia de las salidas A y B están en antifase, es decir que cuando cambiamos la diferencia de caminos, la intensidad en uno aumenta y en el otro disminuye. Además, la salida A tiene mejor contraste que la salida B. El contraste en el patrón, o visibilidad, se define el términos de los máximos y mínimos de intensidades.

$$
V=\frac{I_{\max }-I_{\min }}{I_{\max }+I_{\min }} .
$$


En A, la visibilidad vale 1, independientemente de los valores de los coeficientes de transmisión $T$ y de reflexión $R$ en el divisor de haces. Pero en $\mathrm{B}$, la visibilidad es menor. Reemplazando las expresiones para $I_{\max }$ e $I_{\min }$ se obtiene

$$
V=\frac{2 R^{2} T^{2}}{R^{4}+T^{4}}
$$

En general se usa la salida A ya que la visibilidad es mayor, además de encontrarse en una posición más cómoda.

\section{C.3.2 El patrón de difracción de Fraunhofer}

Todos los problemas de propagación de ondas electromagnéticas pueden ser expresados como soluciones de las ecuaciones de Maxwell para los campos eléctricos y magnéticos sujetos a ciertas condiciones de borde. Una aproximación a esto es representar los campos por una variable escalar que puede ser la amplitud de una de las componentes de ese campo.

Consideremos un caso en dos dimensiones, donde una onda incidente plana propagándose en el eje $Z$ es limitada por una apertura en la región $-H<x<H$, simétrica alrededor del origen $O$. Fuera de la región $2 H$ no hay señal transmitida. El frente de onda saliente tiene una amplitud $f(x)=|f(x)| \exp [i \phi(x)]$. Si observamos la luz que llega a un punto cualquiera $Q$ a una distancia $L>>H$ ( $\phi$ pequeño), cada punto $P$ del frente saliente actuará como una nueva fuente que generará una onda esférica (principio de Huygens, ver Fig. C.3). La distancia de propagación será $P Q$, la variación de fase $k_{0} P Q$ ( $k_{0}$ número de onda) y la amplitud de la onda esférica será proporcional a $P Q^{-1} f\left(x_{P}\right) \exp \left[\mathrm{i} k_{0} P Q\right]$. Si $|x|<H<<L$, tendremos que $P Q=O Q-O T \approx O Q-x \sin \theta$ y, para ángulos pequeños, $P Q \approx L$. Integrando para todos los puntos $x$, obtenemos la amplitud $A(\theta)$,

$$
A(\theta)=\frac{1}{L} \exp \left(\mathrm{i} k_{0} O Q\right) \int_{-H}^{H} f(x) \exp \left(-\mathrm{i} k_{0} x \sin \theta\right) \mathrm{d} x .
$$

Como $f(x)$ vale 0 fuera de $(-H, H)$, los límites de esa integral pueden extenderse a $\pm \infty$. La integral toma la forma de una transformada de Fourier,

$$
F(u)=\int_{-\infty}^{\infty} f(x) \exp (-\mathrm{i} u x) \mathrm{d} x .
$$

$A(\theta)$ es proporcional a $F(u)$, donde $u=k_{0} \sin \theta$ es la frecuencia espacial. El patrón de difracción calculado es conocido como difracción de Fraunhofer.

En su generalización en dos dimensiones, la transformada de Fourier se define como

$$
F(u, v)=\int_{-\infty}^{\infty} \int_{-\infty}^{\infty} f(x, y) \exp [-\mathrm{i}(u x+v y)] \mathrm{d} x \mathrm{~d} y
$$

donde $(u, v)$ son las frecuencias espaciales (proyección del vector línea de base en el plano normal a la dirección a la fuente, medido en unidades de la longitud de onda) relacionadas con $k_{0}$, $(u, v)=(x, y) k_{0} / 2 \pi=(x, y) / \lambda$.

Cuando observamos el patrón de difracción, observamos la intensidad $I(u, v)=|F(u, v)|^{2}$. 
Figura C.3: Formación del patrón de difracción de Fraunhofer a partir del principio de Huygens (Labeyrie et al., 2006).

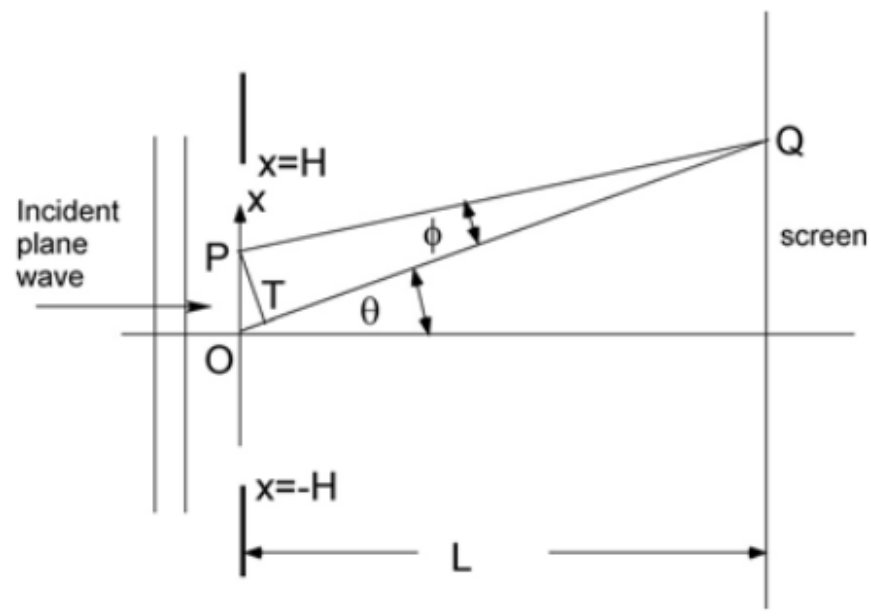

\section{C.3.3 La función de dispersión de punto (PSF)}

Supongamos que estamos observando una fuente puntual. La misma producirá un patrón de difracción que dependerá de la apertura, representada por una función de transmisión compleja en dos dimensiones $f(\mathbf{r})$.

Para una apertura circular $f(\mathbf{r})=\operatorname{circ}(r / R), F\left(k_{r}\right)=2 \pi R^{2} J_{1}(\rho R) / \rho R$ será su transformada de Fourier, donde $J_{1}$ es la función de Bessel de orden 1 y $\rho=k_{0} \sin \theta=\sqrt{u^{2}+v^{2}}$. La intensidad estará dada por $4 \pi^{2} R^{4}\left[J_{1}(\rho R) / \rho R\right]^{2}$. En astronomía, donde los ángulos son pequeños, $\sin \theta \approx \theta$, y la intensidad queda expresada como $4 \pi^{2} R^{4}\left[J_{1}\left(k_{0} \theta R\right) / k_{0} \theta R\right]^{2}$.

Este patrón es denominado función de dispersión de punto (Point Spread Function - PSF). Tiene la forma de un pico central, rodeado de circunferencias concéntricas, donde el pico central determina la resolución del sistema óptico. La función $J_{1}$ tiene su primer cero en $x=3.83$ (ver Fig. C.4), por lo que el ancho del primer pico es $\rho R \approx k_{0} \theta R=3.83$, y la resolución angular será $\theta=3.83 / k_{0} R=3.83 \lambda / 2 \pi R=1.22 \lambda / 2 R=1.22 \lambda / D$ que es el límite de resolución de Rayleigh de un telescopio.

Conociendo la PSF de un sistema óptico, podemos crear la imagen de cualquier objeto, convolucionando la PSF con cada punto de la imagen.

\section{C.3.4 Relación entre la función de coherencia y el patrón de interferencia}

Si tenemos una fuente extendida en la cual todos los puntos de la región oscilan con la misma frecuencia y fase, diremos que la fuente tiene coherencia espacial. Si además, esa variación se mantiene constante a lo largo del tiempo, hablaremos de coherencia temporal. En todo el análisis anterior, consideramos ondas incidentes que eran coherentes espacial y temporalmente. 
Figura C.4: Función de Bessel de orden 1. El primer cero de la misma se ubica en $x=3.83$.

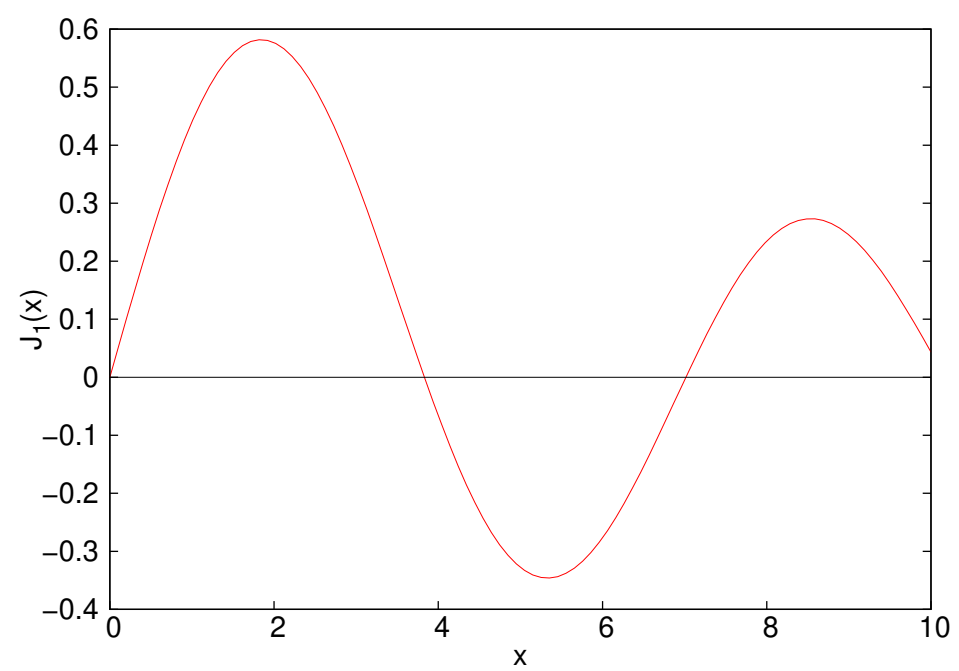

La función de coherencia $\gamma$, que relaciona las ondas recibidas de dos puntos vecinos, es una correlación normalizada entre las ondas $f(\mathbf{r}, t)$ para $\mathbf{r}_{1} \mathrm{y} \mathbf{r}_{2}$ :

$$
\gamma\left(\mathbf{r}_{1}, \mathbf{r}_{2}\right)=\frac{<f\left(\mathbf{r}_{1}, t\right) f^{*}\left(\mathbf{r}_{2}, t\right)>}{\left[<\left|f\left(\mathbf{r}_{1}, t\right)\right|^{2}><\left|f\left(\mathbf{r}_{2}, t\right)\right|^{2}>\right]^{1 / 2}}
$$

donde $\langle\ldots\rangle$ indica promedios temporales. Si el vector $\mathbf{r}_{1}-\mathbf{r}_{2}$ es paralelo a la dirección de propagación, las dos ondas son la misma pero en diferente tiempo $\tau=\left|\mathbf{r}_{1}-\mathbf{r}_{2}\right| / c$, y hablaremos de $\gamma(\tau)$.

La función de coherencia nos indica cuán bueno será el patrón de interferencia que podremos obtener para las ondas provenientes de esas fuentes. Consideremos que las dos ondas tienen intensidades medias $A_{1}^{2}$ y $A_{2}^{2}$, y que $f\left(\mathbf{r}_{1}\right)$ y $f\left(\mathbf{r}_{2}\right)$ están normalizadas. Las ondas serán $A_{1} f\left(\mathbf{r}_{1}\right)$ y $A_{2} f\left(\mathbf{r}_{2}\right)$, por lo que

$$
\gamma=\frac{<A 1 f\left(\mathbf{r}_{1}\right) \cdot A 2 f^{*}\left(\mathbf{r}_{2}\right)>}{A 1 A 2}=<f\left(\mathbf{r}_{1}\right) f^{*}\left(\mathbf{r}_{2}\right)>.
$$

El patrón de interferencia será la superposición de dos campos con diferencia de fase $\phi$ variable en cada punto, $g(\phi)=A_{1} f\left(\mathbf{r}_{1}\right)+A_{2} f\left(\mathbf{r}_{2}\right) \exp (\mathrm{i} \phi)$. La intensidad instantánea será

$$
\begin{aligned}
I(\phi) & =|g(\phi)|^{2}=\left|A_{1} f\left(\mathbf{r}_{1}\right)+A_{2} f\left(\mathbf{r}_{2}\right) \exp (\mathrm{i} \phi)\right|^{2} \\
& =A_{1}^{2}\left|f\left(\mathbf{r}_{1}\right)\right|^{2}+A_{2}^{2}\left|f\left(\mathbf{r}_{2}\right)\right|^{2}+A 1 A 2\left[\left|f\left(\mathbf{r}_{1}\right) f^{*}\left(\mathbf{r}_{2}\right)\right| \exp (\mathrm{i} \phi)+\left|f^{*}\left(\mathbf{r}_{1}\right) f\left(\mathbf{r}_{2}\right)\right| \exp (-\mathrm{i} \phi)\right]
\end{aligned}
$$

y tomando promedios temporales podrá escribirse como

$$
\begin{aligned}
I(\phi) & =A_{1}^{2}+A_{2}^{2}+A_{1} A_{2}\left[\gamma \exp (\mathrm{i} \phi)+\gamma^{*} \exp (-\mathrm{i} \phi)\right] \\
& =A_{1}^{2}+A_{2}^{2}+2 A_{1} A_{2}\left|\gamma\left(\mathbf{r}_{1}, \mathbf{r}_{2}\right)\right| \cos (\phi+\Delta)
\end{aligned}
$$

donde $\gamma=|\gamma| \exp (\mathrm{i} \Delta)$. La diferencia de fase $\phi$ varía de un punto al otro y $\Delta$ indica la posición central del patrón, por lo que esto representa el patrón de interferencia. Reemplazando los valores 
máximos y mínimos de $I(\phi)$ en la expresión de la visibilidad, se obtiene la visibilidad del patrón:

$$
V=\frac{2 A_{1} A_{2}}{A_{1}^{2}+A_{2}^{2}}\left|\gamma\left(\mathbf{r}_{1}, \mathbf{r}_{2}\right)\right|
$$

En general, si las intensidades $A_{1}^{2}$ y $A_{2}^{2}$ son medidas simultáneamente con el patrón, el valor complejo de $\gamma$ puede obtenerse midiendo $V$ y $\Delta$.

\section{C.3.5 Teorema de Van Cittert-Zernike}

La relación exacta cuantitativa entre la visibilidad y la estructura de la fuente está dada por el Teorema de Van Cittert-Zernike. Definamos el eje óptico del telescopio como el eje $Z$, y supongamos que hay una fuente estelar situada cerca al eje $Z$, que se podrá describir por su intensidad $I\left(\theta_{x}, \theta_{y}\right)$ como una función del ángulo respecto al eje. Consideremos solo la longitud de onda $\lambda=2 \pi / k_{0} \mathrm{y}$ utilicemos cosenos directores $\vec{l} \equiv(l, m, n)$ para indicar una posición en el espacio. Como la fuente está cerca al eje $\mathrm{z}, l=\sin \theta_{x} \approx \theta_{x}, l=\sin \theta_{y} \approx \theta_{y} \mathrm{y} n \approx 1$.

La onda que llega al observatorio y es medida en el plano $(x, y)$ será una superposición de ondas originadas en cada punto de la fuente y viajando en la dirección $-\vec{l}$. Si podemos escribir la intensidad $I(l, m)$ en términos de la amplitud $a(l, m)$, cada elemento de fuente tendrá una contribución en el plano $z=0$ dada por

$$
\mathrm{d}^{2} f\left(k_{0} x, k_{0} y\right)=a(l, m) \exp \left[\mathrm{i}\left(\omega t+k_{0} \vec{l} \cdot \mathbf{r}\right)\right] \mathrm{d} l \mathrm{~d} m
$$

que al integrarlo queda

$$
\begin{aligned}
f\left(k_{0} x, k_{0} y\right) & =\iint a(l, m) \exp \left[\mathrm{i}\left(\omega t+k_{0} \vec{l} \cdot \mathbf{r}\right)\right] \mathrm{d} l \mathrm{~d} m \\
& =\exp [\mathrm{i} \omega t] \iint a(l, m) \exp \left[\mathrm{i}\left(k_{0} l x+k_{0} l y\right)\right] \mathrm{d} l \mathrm{~d} m .
\end{aligned}
$$

Si extendemos los límites de las integrales a $\pm \infty$, tenemos la transformada de Fourier en dos dimensiones, y la ecuación anterior toma la forma

$$
f\left(k_{0} x, k_{0} y\right)=\exp [\mathrm{i} \omega t] A\left(-k_{0} x,-k_{0} y\right) .
$$

Consideremos la función de coherencia espacial $\gamma$ en el plano $(x, y)$. Si $<|f(\mathbf{r})|^{2}>=1, \gamma(\mathbf{r})$ puede escribirse como una autocorrelación

$$
\gamma(\mathbf{r})=<f(\mathbf{r}) \star f^{*}(-\mathbf{r})>
$$

que en términos de $k_{o} \mathbf{r}$ quedará

$$
\begin{aligned}
\gamma\left(k_{0} \mathbf{r}\right) & =<f\left(k_{0} \mathbf{r}\right) \star f^{*}\left(-k_{0} \mathbf{r}\right)> \\
& =<A\left(-k_{0} \mathbf{r}\right) \star A^{*}\left(k_{0} \mathbf{r}\right)>.
\end{aligned}
$$


La transformada de Fourier bidimensional de esta ecuación será

$$
\begin{aligned}
\Gamma(l, m) & =<a(l, m) \cdot a^{*}(l, m)> \\
& =<|a(l, m)|^{2}> \\
& =I(l, m)
\end{aligned}
$$

Este es el Teorema de Van Cittert-Zernike, que establece que para una fuente monocromática incoherente, la transformada de Fourier de la función de coherencia espacial es la distribución angular de intensidades de la fuente $I(l, m)$.

\section{C.3.6 Modelos geométricos}

Veamos como se aplica el Teorema de Van Cittert-Zernike considerando diferentes modelos geométricos para la fuente. En la Fig. C.5 pueden verse ejemplos de las funciones de visibilidad para cada uno de los casos propuestos.

\section{Fuente puntual}

La PSF de una fuente puntual tendrá forma de delta de Dirac. Si la fuente está en la posición $(x, y)$ entonces la visibilidad será:

$$
V=F(u, v)=\exp [-2 \pi i(u x+v y)] .
$$

Tendremos que para cualquier línea de base $|V|=1$, por lo que el objeto no puede ser resuelto. Esto se observa en la Fig. C.5 (panel superior izquierdo).

\section{Disco uniforme}

Supongamos que la estrella es un disco uniformemente iluminado de diámetro angular $\alpha$. La visibilidad absoluta será

$$
|V|=|F(u, v)|=\left|2 \frac{J_{1}\left(\pi \alpha B_{p} / \lambda\right)}{\pi \alpha B_{p} / \lambda}\right|
$$

donde $J_{1}$ es la función de Bessel de orden 1 y $B_{p}$ es la proyección de la línea de base. Esta función se anula para la línea de base B que verifique $\pi \alpha B / \lambda=3.83 \rightarrow \alpha=1.22 \lambda / B$. Obtener el valor de $B$ para el cual la visibilidad se anula nos permite obtener un valor de $\alpha$. Un ejemplo se muestra en la Fig. C.5 (panel superior central).

\section{Distribución Gaussiana}

Para estimar la extensión de una envoltura, suele utilizarse un modelo de disco Gaussiano. La transformada de Fourier de una distribución Gaussiana es una distribución Gaussiana, y la 
visibilidad absoluta tomará la expresión

$$
|V|=|F(u, v)|=\exp \left[-\frac{\left(\pi \sigma B_{p} / \lambda\right)^{2}}{4 \ln 2}\right]
$$

donde $\sigma$ puede ser reemplazada por el FWHM de la distribución según FWHM $\sim 2.35 \sigma$. Obtener el FWHM de la visibilidad nos permite estimar el FWHM de la fuente. La Fig. C.5 (panel superior derecha) presenta un ejemplo de este modelo.

\section{Sistema binario}

La función de visibilidad dependerá de como modelemos a las estrellas individuales (puntuales o como un disco uniforme) y de la orientación de la línea de base proyectada. Si cada componente se ubica en las posiciones $\left(x_{1}, y_{1}\right)$ y $\left(x_{2}, y_{2}\right)$, separadas según el vector $\mathbf{r}$, la visibilidad toma la expresión

$$
|V|=|F(u, v)|=\frac{V_{1}^{2}+F V_{2}^{2}+2 F\left|V_{1}\right|\left|V_{2}\right| \cos [(2 \pi / \lambda) \mathbf{B} \cdot \mathbf{r}]}{(1+F)^{2}}
$$

donde $V_{1}$ y $V_{2}$ son las visibilidades de cada componente, y $F$ es el cociente de sus flujos.

- Fuentes puntuales: si ambas componentes tienen el mismo flujo, $F=V=1$. La función de visibilidad queda entonces

$$
|V|=|F(u, v)|=\frac{1+\cos [(2 \pi / \lambda) \mathbf{B} \cdot \mathbf{r}]}{2} .
$$

La visibilidad absoluta dependerá de las orientaciones de los vectores $\mathbf{B}$ y $\mathbf{r}$. Si B y $\mathbf{r}$ son perpendiculares, $\mathbf{B} \cdot \mathbf{r}=0 \mathrm{y}|V|=1$. En cambio, si la proyección de la línea de base en la orientación del sistema binario no es nula, la visibilidad dependerá del coseno y la separación de las franjas solo dependerá de la separación del sistema (Fig. C.5, inferior izquierda). Ejemplos para ambos casos se muestran en la Fig. C.5 (panel inferior izquierdo).

- Discos uniformes: bajo las mismas condiciones $F=V=1$, la función de visibilidad queda

$$
|V|=|F(u, v)|=\left|2 \frac{J_{1}\left(\pi \alpha B_{p} / \lambda\right)}{\pi \alpha B_{p} / \lambda}\right|^{2} \frac{(1+\cos [(2 \pi / \lambda) \mathbf{B} \cdot \mathbf{r}])}{2} .
$$

De manera similar al caso anterior, si la línea de base y la orientación del sistema son perpendiculares, $\mathbf{B} \cdot \mathbf{r}=0$ y la visibilidad será igual a la de un disco uniforme elevada al cuadrado. En caso de que no sean perpendiculares, la visibilidad dependerá del coseno, pero decrecerá con el aumento de la línea de base por el término $J_{1}$. La Fig. C.5 (panel inferior central) muestra un ejemplo para cada caso. 
Figura C.5: Visibilidad para diferentes modelos geométricos. De izquierda a derecha, superior: fuente puntual, disco uniforme, distribución Gaussiana; inferior: binarias puntuales y con disco uniforme (B y $\mathbf{r}$ paralelos en negro, B y $\mathbf{r}$ perpendiculares en gris), anillo. Fuente: Arcos (2017).
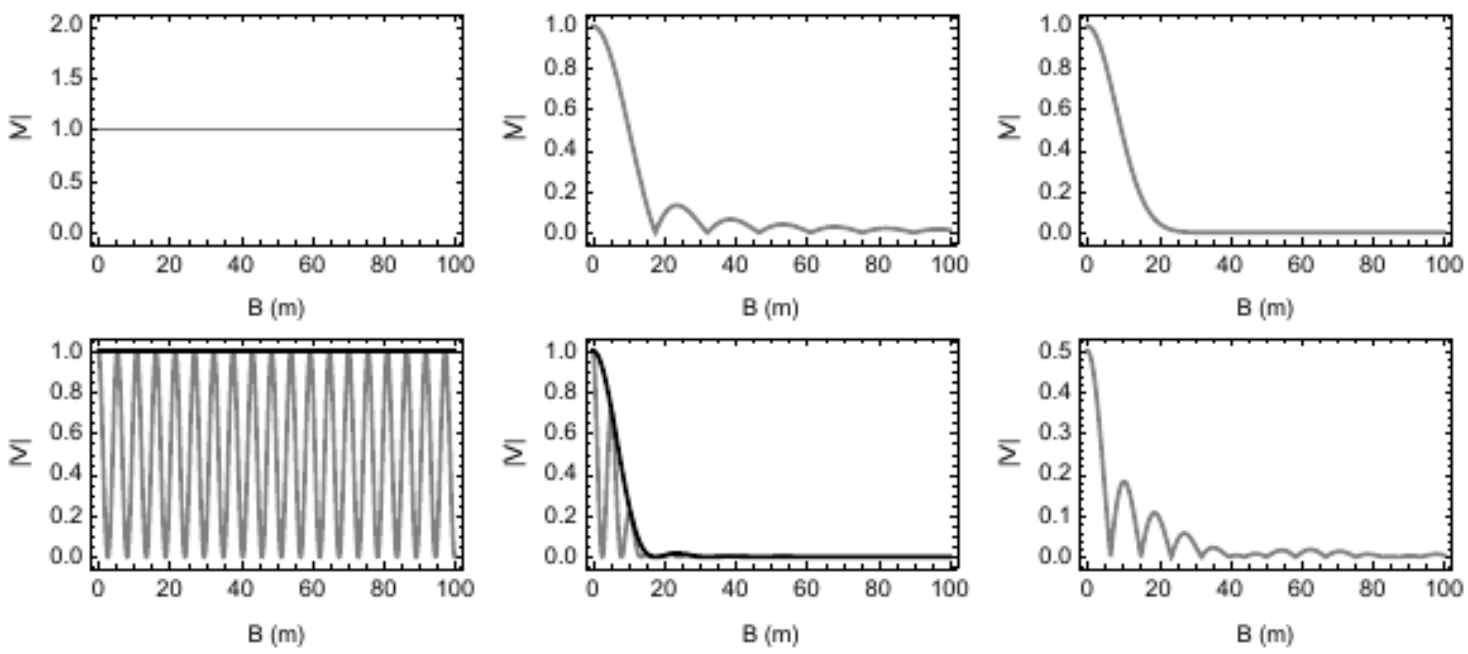

\section{Distribución en forma de anillo}

Para armar la distribución en forma de anillo consideramos dos discos uniformes de diámetros $\alpha_{1}$ y $\alpha_{2}$, con $\alpha_{1}>\alpha_{2}$, y restamos el disco más pequeño al disco más grande. La función de visibilidad será

$$
|V|=|F(u, v)|=\frac{1}{\alpha_{1}^{2}-\alpha_{2}^{2}}\left|\alpha_{2}^{2} \frac{J_{1}\left(\pi \alpha_{2} B / \lambda\right)}{\pi \alpha_{2} B / \lambda}-\alpha_{1}^{2} \frac{J_{1}\left(\pi \alpha_{1} B / \lambda\right)}{\pi \alpha_{1} B / \lambda}\right| .
$$

Esta aproximación suele usarse para estructuras de polvo, donde el radio interno corresponde a la distancia donde comienza a formarse el polvo (cuando la temperatura cae a $\sim 1500 \mathrm{~K}$, temperatura de sublimación de los silicatos). Este valor puede ser determinado a partir del modelado de la distribución espectral de energía (SED). Un ejemplo de la visibilidad para este caso se muestra en la Fig. C.5 (panel inferior derecho).

\section{C.3.7 Síntesis de apertura}

La síntesis de apertura es la manera en la que el Teorema de Van Cittert-Zernike es utilizado en la práctica para obtener imágenes de mayor resolución que las obtenidas con una apertura simple.

Supongamos que estamos midiendo la función de coherencia espacial compleja de una fuente distante usando dos receptores separados por un vector $\mathbf{r}$ en un plano normal a la dirección al objeto. Luego de esa observación podemos cambiar el vector $\mathbf{r}$ y hacer otra medición, las veces necesarias para obtener una gran cantidad de datos de $\gamma(\mathbf{r})$. A partir de esos datos, la transformada de Fourier puede ser calculada para obtener la imagen del objeto. Debido a que 
Figura C.6: Separación de los receptores B y su proyección $\mathbf{r}$ en el plano $(u, v)$ (Labeyrie et al., 2006).

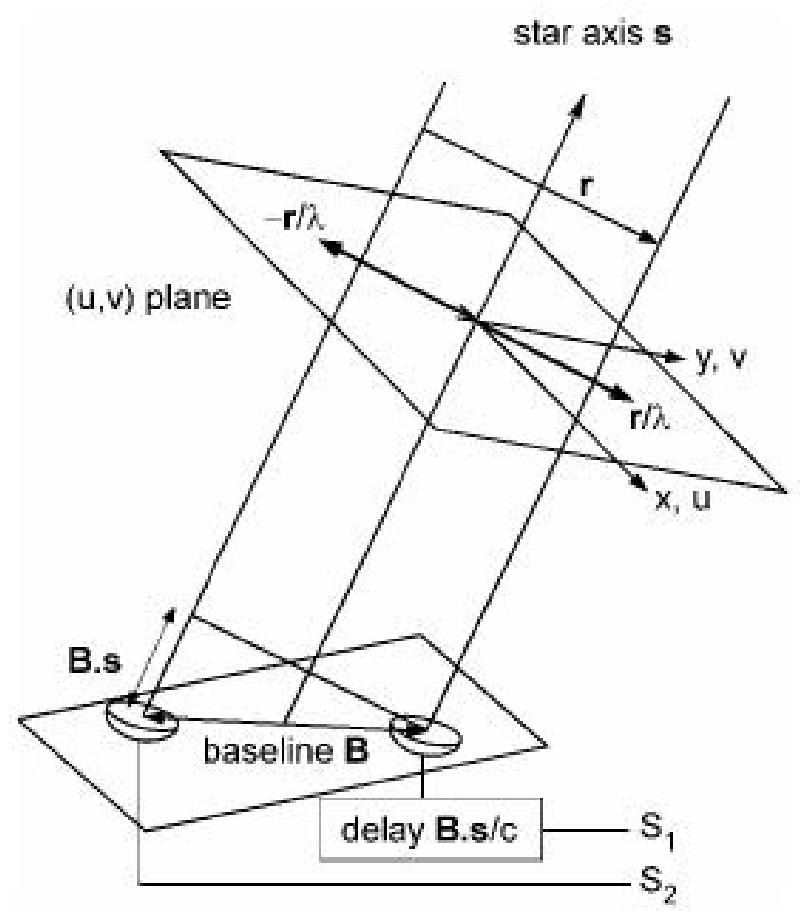

$\gamma(\mathbf{r})$ está muestreada en una cantidad finita de puntos, la imagen perderá detalles, pero pueden utilizarse distintos algoritmos para completar los detalles faltantes.

Lo ideal sería cubrir el plano $(u, v)$ completo de manera uniformemente, pero eso requiere un número infinito de observaciones. Por lo tanto, lo que se hace es tratar de obtener una cobertura suficientemente buena con un arreglo apropiado formado por una cantidad finita de receptores.

Supongamos que tenemos dos receptores separados por $\mathbf{B}$ en la Tierra, donde no es necesario que el vector $\mathbf{B}$ que une los receptores esté en el plano normal a la dirección de objeto, ya que la diferencia de camino desde la estrella a los receptores puede ser compensada introduciendo el retraso apropiado al receptor más próximo. El vector $\mathbf{r}$ es la proyección de $\mathbf{B}$ en el plano $(u, v)$, que está fijo al ser normal a la dirección al objeto, y para cada separación entre receptores obtenemos dos vectores $\pm \mathbf{r} / \lambda$ en el plano ( $u, v)$ (ver Fig. C.6). El plano $(u, v)$ será muestreado por dos procesos: cambios en $\mathbf{r}$ por la rotación de la Tierra o cambios en $\mathbf{B}$. Además, como $I(\vec{l})$ es una función real, su transformada de Fourier satisface $\gamma\left(-k_{0} \mathbf{r}\right)=\gamma^{*}\left(k_{0} \mathbf{r}\right)$, por lo que solo es necesario muestrear medio plano $(u, v)$. Por lo tanto, al construir un interferómetro, es necesario decidir cuál será la disposición geométrica de los telescopios, fijas o movibles, para obtener la mejor cobertura posible del plano $(u, v)$ y una mejor PSF.

Como la función de coherencia $\gamma(u, v)$ puede ser medida a partir de la visibilidad, la imagen puede ser calculada por la transformada de Fourier. Sin embargo, para hacer eso es necesario 
conocer el módulo y la fase de $\gamma(u, v)$. El patrón de interferencia nos da $|\gamma|$, y encontrar la fase es, como veremos a continuación, un problema más complicado.

\section{C.3.8 Closure phase}

Usando solamente la visibilidad obtenida, ésta puede ser relacionada con la esperada para un modelo dado y puede permitir definir valores para los parámetros libres del modelo. Este es el método aceptado para determinar diámetros estelares y separación de binarias.

Si se realizan tres o más observaciones simultáneas, se puede obtener información de la diferencia de fase relativa de $\gamma$ para los vectores formados entre pares de estos receptores, mediante una técnica denominada phase closure, ya que las diferencias de fase originadas por turbulencias atmosféricas se cancelan. Como la información proviene de la diferencia entre las fases observadas en los diferentes pares de receptores, podemos considerar que $\gamma=0$ para dos pares de receptores.

Supongamos entonces que estamos observando con tres fases a la vez, y que la amplitud y la fase recibidas por un receptor contiene un pequeño cambio de fase de valor desconocido $\phi_{i 0}$ producido por la atmósfera u otro factor. La señal recibida en el receptor $i$ será entonces $\left|f_{i}(t)\right| \exp \left[\mathrm{i}\left(\phi_{i}(t)-\phi_{i 0}\right)\right]$, donde $\left|f_{i}(t)\right| \exp \left[\mathrm{i} \phi_{i}(t)\right]$ es el valor verdadero. $\mathrm{Si}<\left|f_{i}\right|^{2}>$ está normalizado, mediremos tres valores aparentes para la función de coherencia, uno para cada línea de base:

$$
\begin{aligned}
\gamma_{i j}^{a} & =<\left|f_{i}\right| \exp \left[\mathrm{i}\left(\phi_{i}-\phi_{i 0}\right)\right]\left|f_{j}\right| \exp \left[\mathrm{i}\left(\phi_{j}-\phi_{j 0}\right)\right]> \\
& =\gamma_{i j} \exp \left[\mathrm{i}\left(\phi_{i 0}-\phi_{j 0}\right)\right]
\end{aligned}
$$

donde $\phi_{i 0}$ y $\phi_{j 0}$ son independientes del tiempo durante la medición. En consecuencia, el producto de las funciones de coherencia para un arreglo cerrado de tres bases será

$$
\begin{aligned}
\gamma_{12}^{a} \gamma_{23}^{a} \gamma_{31}^{a} & =\gamma_{12} \exp \left[\mathrm{i}\left(\phi_{10}-\phi_{20}\right)\right] \gamma_{23} \exp \left[\mathrm{i}\left(\phi_{20}-\phi_{30}\right)\right] \gamma_{31} \exp \left[\mathrm{i}\left(\phi_{30}-\phi_{10}\right)\right] \\
& =\gamma_{12} \gamma_{23} \gamma_{31} \exp \left[\mathrm{i}\left(\phi_{10}-\phi_{20}+\phi_{20}-\phi_{30}+\phi_{30}-\phi_{10}\right)\right] \\
& =\gamma_{12} \gamma_{23} \gamma_{31} \\
& =\left|\gamma_{12}\left\|\gamma_{23}\right\| \gamma_{31}\right| \exp \left[\mathrm{i}\left(\phi_{c}\right)\right]
\end{aligned}
$$

donde $\phi_{c}$ es la closure phase. En términos de $\Delta$ y las líneas de base $\mathbf{r}$, tendremos que $\mathbf{r}_{12}+\mathbf{r}_{23}+\mathbf{r}_{31}=0$ y que $\Delta_{12}+\Delta_{23}+\Delta_{31}=\phi_{c}$. 
Abt, H. A. \& Cardona, O.: 1984, ApJ 285, 190

Abt, H. A., Levato, H., \& Grosso, M.: 2002, ApJ 573, 359

Ahumada, J. A. \& Lapasset, E.: 2007, A\&A 463, 789

Aidelman, Y., Cidale, L. S., Zorec, J., \& Panei, J. A.: 2018, A\&A 610, A30

Albert, E. \& Huang, S.-S.: 1974, ApJ 189, 479

Andrillat, A., Jaschek, M., \& Jaschek, C.: 1990, A\&AS 84, 11

Andrillat, Y. \& Fehrenbach, C.: 1982, $A \& A S$ 48, 93

Antonello, E., Fracassini, M., Pasinetti, L. E., \& Pastori, L.: 1982, Ap\&SS 83, 381

Arcos, C.: 2017, Ph.D. thesis, Instituto de Física y Astronomía, Facultad de Ciencias, Universidad de Valparaíso

Arcos, C., Jones, C. E., Sigut, T. A. A., Kanaan, S., \& Curé, M.: 2017, ApJ 842, 48

Arcos, C., Kanaan, S., Chávez, J., Vanzi, L., Araya, I., \& Curé, M.: 2018, MNRAS 474, 5287

Arias, M. L., Cidale, L. S., \& Ringuelet, A. E.: 2004, A\&A 417, 679

Astraatmadja, T. L. \& Bailer-Jones, C. A. L.: 2016, ApJ 833, 119

Baade, D.: 1982, A\&A 105, 65

Baade, D.: 1989, A\&AS 79, 423

Baade, D.: 2000, in M. A. Smith, H. F. Henrichs, \& J. Fabregat (eds.), IAU Colloq. 175: The Be Phenomenon in Early-Type Stars, Vol. 214 of Astronomical Society of the Pacific Conference Series, p. 178

Baker, J. G. \& Menzel, D. H.: 1938, ApJ 88, 52

Baldwin, R. B.: 1939, ApJ 89, 255 
Baldwin, R. B.: 1940, ApJ 92, 82

Baldwin, R. B.: 1941, ApJ 94, 283

Ballereau, D., Alvarez, M., Chauville, J., \& Michel, R.: 1987, Rev. Mexicana Astron. Astrofis. 15, 29

Balona, L. A.: 1990, MNRAS 245, 92

Balona, L. A.: 1995, MNRAS 277, 1547

Balona, L. A., Cuypers, J., \& Marang, F.: 1992, A\&AS 92, 533

Balona, L. A., Handler, G., Aerts, C., Bozic, H., Guinan, E. F., James, D. J., Kaye, A. B., \& Shobbrook, R. R.: 2002, in C. Sterken \& D. W. Kurtz (eds.), Observational Aspects of Pulsating $B$ - and A Stars, Vol. 256 of Astronomical Society of the Pacific Conference Series, p. 9

Balona, L. A., Marang, F., Monderen, P., Reitermann, A., \& Zickgraf, F.-J.: 1987, A\&AS 71, 11

Barker, P. K.: 1982, ApJS 49, 89

Barnsley, R. M. \& Steele, I. A.: 2013, A\&A 556, A81

Bergin, E. A., Burns, J. F., Guinan, E. F., \& McCook, G. P.: 1989, Information Bulletin on Variable Stars 3358

Bhat, S. S., Paul, K. T., Subramaniam, A., \& Mathew, B.: 2016, Research in Astronomy and Astrophysics 16(5), 007

Bjorkman, J. E.: 1997, in J. P. De Greve, R. Blomme, \& H. Hensberge (eds.), Stellar Atmospheres: Theory and Observations, Vol. 497 of Lecture Notes in Physics, Berlin Springer Verlag, p. 239

Bjorkman, K. S.: 2000, in M. A. Smith, H. F. Henrichs, \& J. Fabregat (eds.), IAU Colloq. 175: The Be Phenomenon in Early-Type Stars, Vol. 214 of Astronomical Society of the Pacific Conference Series, p. 384

Bjorkman, K. S., Miroshnichenko, A. S., McDavid, D., \& Pogrosheva, T. M.: 2002, ApJ 573, 812

Borges Fernandes, M., Meilland, A., Bendjoya, P., Domiciano de Souza, A., Niccolini, G., Chesneau, O., Millour, F., Spang, A., Stee, P., \& Kraus, M.: 2011, A\&A 528, A20

Bossi, M., Guerrero, G., \& Bianchini, G.: 1989, Information Bulletin on Variable Stars 3348

Bouret, J.-C., Lanz, T., Hillier, D. J., Heap, S. R., Hubeny, I., Lennon, D. J., Smith, L. J., \& Evans, C. J.: 2003, ApJ 595, 1182

Brown, J. C. \& McLean, I. S.: 1977, A\&A 57, 141 
Carciofi, A. C., Bjorkman, J. E., Otero, S. A., Okazaki, A. T., Štefl, S., Rivinius, T., Baade, D., \& Haubois, X.: 2012, ApJ 744, L15

Carciofi, A. C., Miroshnichenko, A. S., Kusakin, A. V., Bjorkman, J. E., Bjorkman, K. S., Marang, F., Kuratov, K. S., García-Lario, P., Calderón, J. V. P., Fabregat, J., \& Magalhães, A. M.: 2006, ApJ 652, 1617

Cardelli, J. A., Clayton, G. C., \& Mathis, J. S.: 1989, ApJ 345, 245

Carrier, F., Burki, G., \& Burnet, M.: 2002, A\&A 385, 488

Castle, K. G.: 1977, PASP 89, 862

Castor, J. I., Abbott, D. C., \& Klein, R. I.: 1975, ApJ 195, 157

Catanzaro, G.: 2013, A\&A 550, A79

Chauville, J., Zorec, J., Ballereau, D., Morrell, N., Cidale, L., \& Garcia, A.: 2001, A\&A 378, 861

Che, X., Monnier, J. D., Tycner, C., Kraus, S., Zavala, R. T., Baron, F., Pedretti, E., ten Brummelaar, T., McAlister, H., Ridgway, S. T., Sturmann, J., Sturmann, L., \& Turner, N.: 2012, ApJ 757, 29

Chelli, A., Utrera, O. H., \& Duvert, G.: 2009, A\&A 502, 705

Chesneau, O., Meilland, A., Rivinius, T., Stee, P., Jankov, S., Domiciano de Souza, A., Graser, U., Herbst, T., Janot-Pacheco, E., Koehler, R., Leinert, C., Morel, S., Paresce, F., Richichi, A., \& Robbe-Dubois, S.: 2005, A\&A 435, 275

Chojnowski, S. D., Whelan, D. G., d, J. P., Majewski, S. R., Hall, M., Shetrone, M., Beaton, R., Burton, A., Damke, G., Eikenberry, S., Hasselquist, S., Holtzman, J. A., Mészáros, S., Nidever, D., Schneider, D. P., Wilson, J., Zasowski, G., Bizyaev, D., Brewington, H., Brinkmann, J., Ebelke, G., Frinchaboy, P. M., Kinemuchi, K., Malanushenko, E., Malanushenko, V., Marchante, M., Oravetz, D., Pan, K., \& Simmons, A.: 2015, AJ 149, 7

Clark, J. S. \& Steele, I. A.: 2000, A\&AS 141, 65

Cochetti, Y. R., Arcos, C., Kanaan, S., Meilland, A., Cidale, L. S., \& Curé, M.: 2019, A\&A 621, A123

Cochetti, Y. R., Zorec, J., Cidale, L. S., Arias, M. L., Aidelman, Y., Torres, A. F., Frémat, Y., \& Granada, A.: 2020, A\&A 634, A18

Coe, M. J.: 2000, in M. A. Smith, H. F. Henrichs, \& J. Fabregat (eds.), IAU Colloq. 175: The Be Phenomenon in Early-Type Stars, Vol. 214 of Astronomical Society of the Pacific Conference Series, p. 656 
Coe, M. J., Haigh, N. J., \& Reig, P.: 2000, MNRAS 314, 290

Cohen, D. H.: 2000, in M. A. Smith, H. F. Henrichs, \& J. Fabregat (eds.), IAU Colloq. 175: The Be Phenomenon in Early-Type Stars, Vol. 214 of Astronomical Society of the Pacific Conference Series, p. 156

Cohen, D. H., Cassinelli, J. P., \& Waldron, W. L.: 1997, ApJ 488, 397

Collins, II, G. W.: 1987, in A. Slettebak \& T. P. Snow (eds.), IAU Colloq. 92: Physics of Be Stars, pp 3-19

Collins, II, G. W. \& Truax, R. J.: 1995, ApJ 439, 860

Cote, J. \& Waters, L. B. F. M.: 1987, A\&A 176, 93

Cowley, A., Cowley, C., Jaschek, M., \& Jaschek, C.: 1969, AJ 74, 375

Cox, A. N. \& Pilachowski, C. A.: 2000, Physics Today 53(10), 77

Cranmer, S. R.: 2005, ApJ 634, 585

Curé, M., Rial, D. F., Christen, A., \& Cassetti, J.: 2014, A\&A 565, A85

Cyr, R. P., Jones, C. E., \& Tycner, C.: 2015, ApJ 799, 33

Delaa, O., Stee, P., Meilland, A., Zorec, J., Mourard, D., Bério, P., Bonneau, D., Chesneau, O., Clausse, J. M., Cruzalebes, P., Perraut, K., Marcotto, A., Roussel, A., Spang, A., McAlister, H., ten Brummelaar, T., Sturmann, J., Sturmann, L., Turner, N., Farrington, C., \& Goldfinger, P. J.: 2011, A\&A 529, A87

Doazan, V., Harmanec, P., Koubsky, P., Krpata, J., \& Zdarsky, F.: 1982, A\&A 115, 138

Doazan, V., Marlborough, J. M., Morossi, C., Peters, G. J., Rusconi, L., Sedmak, G., Stalio, R., Thomas, R. N., \& Willis, A.: 1986, A\&A 158, 1

Doazan, V., Sedmak, G., Barylak, M., Rusconi, L., \& Battrick, B. (eds.): 1991, A Be star atlas of far UV and optical high-resolution spectra, Vol. 1147 of ESA Special Publication

Domiciano de Souza, A., Kervella, P., Jankov, S., Abe, L., Vakili, F., di Folco, E., \& Paresce, F.: 2003, A\&A 407, L47

Dougherty, S. M. \& Taylor, A. R.: 1992, Nature 359(6398), 808

Ekström, S., Meynet, G., Maeder, A., \& Barblan, F.: 2008, A\&A 478, 467

Fekel, F. C.: 1997, PASP 109, 514 
Floquet, M., Neiner, C., Janot-Pacheco, E., Hubert, A. M., Jankov, S., Zorec, J., Briot, D., Chauville, J., Leister, N. V., Percy, J. R., Ballereau, D., \& Bakos, A. G.: 2002, A\&A 394, 137

Frémat, Y., Zorec, J., Hubert, A.-M., \& Floquet, M.: 2005, A\&A 440, 305

Friend, D. B. \& Abbott, D. C.: 1986, ApJ 311, 701

Fukuda, I.: 1982, PASP 94, 271

Gayley, K. G., Owocki, S. P., \& Cranmer, S. R.: 1999, ApJ 513, 442

Gies, D. R., Bagnuolo, Jr., W. G., Baines, E. K., ten Brummelaar, T. A., Farrington, C. D., Goldfinger, P. J., Grundstrom, E. D., Huang, W., McAlister, H. A., Mérand, A., Sturmann, J., Sturmann, L., Touhami, Y., Turner, N. H., Wingert, D. W., Berger, D. H., McSwain, M. V., Aufdenberg, J. P., Ridgway, S. T., Cochran, A. L., Lester, D. F., Sterling, N. C., Bjorkman, J. E., Bjorkman, K. S., \& Koubský, P.: 2007, ApJ 654, 527

González, J. F. \& Lapasset, E.: 2000, AJ 119, 2296

Grady, C. A., Bjorkman, K. S., Snow, T. P., Sonneborn, G., Shore, S. N., \& Barker, P. K.: 1989, ApJ 339, 403

Granada, A., Arias, M. L., \& Cidale, L. S.: 2010, AJ 139, 1983

Gray, R. O. \& Corbally, J., C.: 2009, Stellar Spectral Classification

Grundstrom, E. D. \& Gies, D. R.: 2006, ApJ 651, L53

Gulliver, A. F.: 1977, ApJS 35, 441

Gulliver, A. F., Bolton, C. T., \& Poeckert, R.: 1980, PASP 92, 774

Hanson, M. M., Conti, P. S., \& Rieke, M. J.: 1996, ApJS 107, 281

Hanson, M. M., Rieke, G. H., \& Luhman, K. L.: 1998, AJ 116, 1915

Hanuschik, R. W.: 1996, A\&A 308, 170

Hanuschik, R. W., Hummel, W., Dietle, O., \& Sutorius, E.: 1995, A\&A 300, 163

Hanuschik, R. W., Kozok, J. R., \& Kaiser, D.: 1988, A\&A 189, 147

Harmanec, P.: 1984, Bulletin of the Astronomical Institutes of Czechoslovakia 35, 164

Harmanec, P., Koubský, P., \& Krpata, J.: 1972, Bulletin of the Astronomical Institutes of Czechoslovakia 23, 218

Harper, W. E.: 1937, Publications of the Dominion Astrophysical Observatory Victoria 7, 1 
Hesselbach, E. N.: 2009, Ph.D. thesis, The University of Toledo

Hirata, R.: 2007, in A. T. Okazaki, S. P. Owocki, \& S. Stefl (eds.), Active OB-Stars: Laboratories for Stellare and Circumstellar Physics, Vol. 361 of Astronomical Society of the Pacific Conference Series, p. 267

Hony, S., Waters, L. B. F. M., Zaal, P. A., de Koter, A., Marlborough, J. M., Millar, C. E., Trams, N. R., Morris, P. W., \& de Graauw, T.: 2000, A\&A 355, 187

Huang, S.-S.: 1972, ApJ 171, 549

Huang, S.-S.: 1973, ApJ 183, 541

Hubeny, I.: 1988, Computer Physics Communications 52, 103

Hubeny, I. \& Lanz, T.: 1995, ApJ 439, 875

Hubeny, I. \& Lanz, T.: 2011, Synspec: General Spectrum Synthesis Program, Astrophysics Source Code Library

Hubeny, I. \& Lanz, T.: 2017, arXiv e-prints

Hubert, A. M. \& Floquet, M.: 1998, A\&A 335, 565

Hubert, A. M., Floquet, M., \& Zorec, J.: 2000, in M. A. Smith, H. F. Henrichs, \& J. Fabregat (eds.), IAU Colloq. 175: The Be Phenomenon in Early-Type Stars, Vol. 214 of Astronomical Society of the Pacific Conference Series, p. 348

Hummel, W.: 1994, A\&A 289, 458

Hummel, W.: 1998, A\&A 330, 243

Hummer, D. G. \& Storey, P. J.: 1987, MNRAS 224, 801

Hunter, I., Brott, I., Lennon, D. J., Langer, N., Dufton, P. L., Trundle, C., Smartt, S. J., de Koter, A., Evans, C. J., \& Ryans, R. S. I.: 2008, ApJ 676, L29

Hutchings, J. B.: 1970, MNRAS 150, 55

Jaschek, M., Slettebak, A., \& Jaschek, C.: 1981, Be star terminology., Be Star Newsletter

Jeong, J. H., Suh, C. W., \& Nha, I.-S.: 1986, Ap\&SS 119, 73

Jones, C. E., Sigut, T. A. A., \& Porter, J. M.: 2008, MNRAS 386, 1922

Jones, C. E., Tycner, C., \& Smith, A. D.: 2011, AJ 141, 150 
Kanaan, S., Meilland, A., Stee, P., Zorec, J., Domiciano de Souza, A., Frémat, Y., \& Briot, D.: 2008, $A \& A$ 486, 785

Katahira, J.-I., Hirata, R., Ito, M., Katoh, M., Ballereau, D., \& Chauville, J.: 1996a, in V. Niemela, N. Morrell, P. Pismis, \& S. Torres-Peimbert (eds.), Revista Mexicana de Astronomia y Astrofisica Conference Series, Vol. 5 of Revista Mexicana de Astronomia y Astrofisica Conference Series, p. 114

Katahira, J.-I., Hirata, R., Ito, M., Katoh, M., Ballereau, D., \& Chauville, J.: 1996b, PASJ 48, 317

Kee, N. D., Owocki, S., \& Sundqvist, J. O.: 2016, MNRAS 458, 2323

Keller, S. C.: 2004, PASA 21, 310

Kervella, P. \& Domiciano de Souza, A.: 2006, A\&A 453, 1059

Koubský, P., Gulliver, A. F., Harmanec, P., Ballereau, D., Chauville, J., Gráf, T., Horn, J., Iliev, L. H., \& Lyons, R. W.: 1989, Bulletin of the Astronomical Institutes of Czechoslovakia 40, 31

Kraus, S., Monnier, J. D., Che, X., Schaefer, G., Touhami, Y., Gies, D. R., Aufdenberg, J. P., Baron, F., Thureau, N., ten Brummelaar, T. A., McAlister, H. A., Turner, N. H., Sturmann, J., \& Sturmann, L.: 2012, ApJ 744, 19

Krtička, J., Owocki, S. P., \& Meynet, G.: 2011, A\&A 527, A84

Kurucz, R. L.: 1979, ApJS 40, 1

Labadie-Bartz, J., Pepper, J., McSwain, M. V., Bjorkman, J. E., Bjorkman, K. S., Lund, M. B., Rodriguez, J. E., Stassun, K. G., Stevens, D. J., James, D. J., Kuhn, R. B., Siverd, R. J., \& Beatty, T. G.: 2017, AJ 153, 252

Labeyrie, A., Lipson, S. G., \& Nisenson, P.: 2006, An Introduction to Optical Stellar Interferometry, Cambridge University Press

Lee, U., Osaki, Y., \& Saio, H.: 1991, MNRAS 250, 432

Lefèvre, L., Marchenko, S. V., Moffat, A. F. J., \& Acker, A.: 2009, A\&A 507, 1141

Lenorzer, A., de Koter, A., \& Waters, L. B. F. M.: 2002a, A\&A 386, L5

Lenorzer, A., Vandenbussche, B., Morris, P., de Koter, A., Geballe, T. R., Waters, L. B. F. M., Hony, S., \& Kaper, L.: 2002b, $A \& A$ 384, 473

Lynch, D. K., Rudy, R. J., Mazuk, S., \& Puetter, R. C.: 2000, ApJ 541, 791

Lynds, C. R.: 1960, ApJ 131, 390 
Maeder, A., Grebel, E. K., \& Mermilliod, J.-C.: 1999, A\&A 346, 459

Martayan, C., Baade, D., \& Fabregat, J.: 2010, A\&A 509, A11

Martayan, C., Frémat, Y., Hubert, A.-M., Floquet, M., Zorec, J., \& Neiner, C.: 2006, A\&A 452, 273

Martayan, C., Frémat, Y., Hubert, A.-M., Floquet, M., Zorec, J., \& Neiner, C.: 2007, A\&A 462, 683

Mason, B. D., Hartkopf, W. I., Gies, D. R., Henry, T. J., \& Helsel, J. W.: 2009, The Astronomical Journal 137(2), 3358

Mathew, B., Banerjee, D. P. K., Naik, S., \& Ashok, N. M.: 2012a, MNRAS 423, 2486

Mathew, B., Banerjee, D. P. K., Subramaniam, A., \& Ashok, N. M.: 2012b, ApJ 753, 13

McDavid, D., Hirata, R., Guinan, E. F., Bjorkman, K. S., \& Babler, B. L.: 1996, in American Astronomical Society Meeting Abstracts \#188, Vol. 28 of Bulletin of the American Astronomical Society, p. 913

McLaughlin, D. B.: 1963, ApJ 137, 1085

Meilland, A., Delaa, O., Stee, P., Kanaan, S., Millour, F., Mourard, D., Bonneau, D., Petrov, R., Nardetto, N., Marcotto, A., Roussel, A., Clausse, J. M., Perraut, K., McAlister, H., ten Brummelaar, T., Sturmann, J., Sturmann, L., Turner, N., Ridgway, S. T., Farrington, C., \& Goldfinger, P. J.: 2011, A\&A 532, A80

Meilland, A., Kanaan, S., Borges Fernandes, M., Chesneau, O., Millour, F., Stee, P., \& Lopez, B.: 2010, A\&A 512, A73

Meilland, A., Millour, F., Kanaan, S., Stee, P., Petrov, R., Hofmann, K.-H., Natta, A., \& Perraut, K.: 2012, A\&A 538, A110

Meilland, A., Millour, F., Stee, P., Domiciano de Souza, A., Petrov, R. G., Mourard, D., Jankov, S., Robbe-Dubois, S., Spang, A., Aristidi, E., Antonelli, P., Beckmann, U., Bresson, Y., Chelli, A., Dugué, M., Duvert, G., Gennari, S., Glück, L., Kern, P., Lagarde, S., Le Coarer, E., Lisi, F., Malbet, F., Perraut, K., Puget, P., Rantakyrö, F., Roussel, A., Tatulli, E., Weigelt, G., Zins, G., Accardo, M., Acke, B., Agabi, K., Altariba, E., Arezki, B., Baffa, C., Behrend, J., Blöcker, T., Bonhomme, S., Busoni, S., Cassaing, F., Clausse, J.-M., Colin, J., Connot, C., Delboulbé, A., Driebe, T., Feautrier, P., Ferruzzi, D., Forveille, T., Fossat, E., Foy, R., Fraix-Burnet, D., Gallardo, A., Giani, E., Gil, C., Glentzlin, A., Heiden, M., Heininger, M., Hernandez Utrera, O., Hofmann, K.-H., Kamm, D., Kiekebusch, M., Kraus, S., Le Contel, D., Le Contel, J.-M., Lesourd, T., Lopez, B., Lopez, M., Magnard, Y., Marconi, A., Mars, G., Martinot-Lagarde, G., Mathias, P., Mège, P., Monin, J.-L., Mouillet, D., Nussbaum, E., Ohnaka, K., Pacheco, J., Perrier, C., Rabbia, Y., Rebattu, S., Reynaud, F., Richichi, A., Robini, A., Sacchettini, M., Schertl, D., Schöller, M., 
Solscheid, W., Stefanini, P., Tallon, M., Tallon-Bosc, I., Tasso, D., Testi, L., Vakili, F., von der Lühe, O., Valtier, J.-C., Vannier, M., \& Ventura, N.: 2007a, A\&A 464, 73

Meilland, A., Millour, F., Stee, P., Spang, A., Petrov, R., Bonneau, D., Perraut, K., \& Massi, F.: 2008, A\&A 488, L67

Meilland, A., Stee, P., Chesneau, O., \& Jones, C.: 2009, A\&A 505, 687

Meilland, A., Stee, P., Vannier, M., Millour, F., Domiciano de Souza, A., Malbet, F., Martayan, C., Paresce, F., Petrov, R. G., Richichi, A., \& Spang, A.: 2007b, A\&A 464, 59

Mennickent, R. E., Sabogal, B., Granada, A., \& Cidale, L.: 2009, PASP 121, 125

Mennickent, R. E., Sterken, C., \& Vogt, N.: 1997, A\&A 326, 1167

Mennickent, R. E. \& Vogt, N.: 1988, A\&AS 74, 497

Merrill, P. W.: 1952, ApJ 116, 501

Merrill, P. W. \& Burwell, C. G.: 1933, ApJ 78, 87

Merrill, P. W., Humason, M. L., \& Burwell, C. G.: 1925, ApJ 61

Meyer, M. R., Edwards, S., Hinkle, K. H., \& Strom, S. E.: 1998, ApJ 508, 397

Millour, F., Chesneau, O., Borges Fernandes, M., Meilland, A., Mars, G., Benoist, C., Thiébaut, E., Stee, P., Hofmann, K.-H., Baron, F., Young, J., Bendjoya, P., Carciofi, A., Domiciano de Souza, A., Driebe, T., Jankov, S., Kervella, P., Petrov, R. G., Robbe-Dubois, S., Vakili, F., Waters, L. B. F. M., \& Weigelt, G.: 2009, A\&A 507, 317

Millour, F., Meilland, A., Chesneau, O., Stee, P., Kanaan, S., Petrov, R., Mourard, D., \& Kraus, S.: 2011, A\&A 526, A107

Millour, F., Petrov, R. G., Chesneau, O., Bonneau, D., Dessart, L., Bechet, C., Tallon-Bosc, I., Tallon, M., Thiébaut, E., Vakili, F., Malbet, F., Mourard, D., Antonelli, P., Beckmann, U., Bresson, Y., Chelli, A., Dugué, M., Duvert, G., Gennari, S., Glück, L., Kern, P., Lagarde, S., Le Coarer, E., Lisi, F., Perraut, K., Puget, P., Rantakyrö, F., Robbe-Dubois, S., Roussel, A., Tatulli, E., Weigelt, G., Zins, G., Accardo, M., Acke, B., Agabi, K., Altariba, E., Arezki, B., Aristidi, E., Baffa, C., Behrend, J., Blöcker, T., Bonhomme, S., Busoni, S., Cassaing, F., Clausse, J.-M., Colin, J., Connot, C., Delboulbé, A., Domiciano de Souza, A., Driebe, T., Feautrier, P., Ferruzzi, D., Forveille, T., Fossat, E., Foy, R., Fraix-Burnet, D., Gallardo, A., Giani, E., Gil, C., Glentzlin, A., Heiden, M., Heininger, M., Hernandez Utrera, O., Hofmann, K.-H., Kamm, D., Kiekebusch, M., Kraus, S., Le Contel, D., Le Contel, J.-M., Lesourd, T., Lopez, B., Lopez, M., Magnard, Y., Marconi, A., Mars, G., Martinot-Lagarde, G., Mathias, P., Mège, P., Monin, J.-L., Mouillet, D., Nussbaum, E., Ohnaka, K., Pacheco, J., Perrier, C., Rabbia, Y., Rebattu, S., Reynaud, F., 
Richichi, A., Robini, A., Sacchettini, M., Schertl, D., Schöller, M., Solscheid, W., Spang, A., Stee, P., Stefanini, P., Tasso, D., Testi, L., von der Lühe, O., Valtier, J.-C., Vannier, M., \& Ventura, N.: 2007, A\&A 464, 107

Miroshnichenko, A. S., Bjorkman, K. S., Morrison, N. D., d, J. P., Manset, N., Levato, H., Grosso, M., Pollmann, E., Buil, C., \& Knauth, D. C.: 2003, A\&A 408, 305

Mokiem, M. R., de Koter, A., Vink, J. S., Puls, J., Evans, C. J., Smartt, S. J., Crowther, P. A., Herrero, A., Langer, N., Lennon, D. J., Najarro, F., \& Villamariz, M. R.: 2007, A\&A 473, 603

Neiner, C.: 2010, in Revista Mexicana de Astronomia y Astrofisica Conference Series, Vol. 38 of Revista Mexicana de Astronomia y Astrofisica Conference Series, pp 109-112

Neiner, C., Grunhut, J. H., Petit, V., ud-Doula, A., Wade, G. A., Landstreet, J., de Batz, B., Cochard, F., Gutiérrez-Soto, J., \& Huat, A.-L.: 2012, MNRAS 426, 2738

Neiner, C., Hubert, A.-M., Floquet, M., Jankov, S., Henrichs, H. F., Foing, B., Oliveira, J., Orlando, S., Abbott, J., Baldry, I. K., Bedding, T. R., Cami, J., Cao, H., Catala, C., Cheng, K. P., Domiciano de Souza, Jr., A., Janot-Pacheco, E., Hao, J. X., Kaper, L., Kaufer, A., Leister, N. V., Neff, J. E., O’Toole, S. J., Schäfer, D., Smartt, S. J., Stahl, O., Telting, J., Tubbesing, S., \& Zorec, J.: 2002, $A \& A$ 388, 899

Neiner, C., Hubert, A.-M., Frémat, Y., Floquet, M., Jankov, S., Preuss, O., Henrichs, H. F., \& Zorec, J.: 2003, A\&A 409, 275

Neiner, C., Mathis, S., Saio, H., \& Lee, U.: 2013, in H. Shibahashi \& A. E. Lynas-Gray (eds.), Progress in Physics of the Sun and Stars: A New Era in Helio- and Asteroseismology, Vol. 479 of Astronomical Society of the Pacific Conference Series, p. 319

Nemravová, J., Harmanec, P., Kubát, J., Koubský, P., Iliev, L., Yang, S., Ribeiro, J., Šlechta, M., Kotková, L., Wolf, M., \& Škoda, P.: 2010, A\&A 516, A80

Ochsenbein, F., Bauer, P., \& Marcout, J.: 2000, A\&AS 143, 23

Okazaki, A. T.: 1991, PASJ 43, 75

Oudmaijer, R. D. \& Drew, J. E.: 1997, A\&A 318, 198

Oudmaijer, R. D. \& Parr, A. M.: 2010, MNRAS 405, 2439

Owocki, S. P.: 2004, in A. Maeder \& P. Eenens (eds.), Stellar Rotation, Vol. 215 of IAU Symposium, p. 515

Papaloizou, J. C., Savonije, G. J., \& Henrichs, H. F.: 1992, A\&A 265, L45

Pauldrach, A., Puls, J., \& Kudritzki, R. P.: 1986, A\&A 164, 86 
Pavlovski, K., Ruzic, Z., Pavlovic, M., Jeong, J. H., \& Nha, I.-S.: 1993, Ap\&SS 200, 201

Percy, J. R., Desjardins, A., \& Yeung, D.: 1996, Journal of the American Association of Variable Star Observers (JAAVSO) 25, 14

Percy, J. R., Hussain, A., Gomez-Forrellad, J. M., \& Garcia-Melendo, E.: 2001, Information Bulletin on Variable Stars 5163

Persson, S. E. \& McGregor, P. J.: 1985, AJ 90, 1860

Peters, G. J.: 1972, PASP 84, 334

Peters, G. J.: 1983, PASP 95, 311

Peters, G. J. \& Penrod, G. D.: 1988, in ESA Special Publication, Vol. 281 of ESA Special Publication

Peters, G. J., Wang, L., Gies, D. R., \& Grundstrom, E. D.: 2016, ApJ 828, 47

Petrov, R. G.: 1989, in D. M. Alloin \& J.-M. Mariotti (eds.), NATO Advanced Science Institutes (ASI) Series C, Vol. 274 of NATO Advanced Science Institutes (ASI) Series C, p. 249

Petrov, R. G., Malbet, F., Weigelt, G., Antonelli, P., Beckmann, U., Bresson, Y., Chelli, A., Dugué, M., Duvert, G., Gennari, S., Glück, L., Kern, P., Lagarde, S., Le Coarer, E., Lisi, F., Millour, F., Perraut, K., Puget, P., Rantakyrö, F., Robbe-Dubois, S., Roussel, A., Salinari, P., Tatulli, E., Zins, G., Accardo, M., Acke, B., Agabi, K., Altariba, E., Arezki, B., Aristidi, E., Baffa, C., Behrend, J., Blöcker, T., Bonhomme, S., Busoni, S., Cassaing, F., Clausse, J.-M., Colin, J., Connot, C., Delboulbé, A., Domiciano de Souza, A., Driebe, T., Feautrier, P., Ferruzzi, D., Forveille, T., Fossat, E., Foy, R., Fraix-Burnet, D., Gallardo, A., Giani, E., Gil, C., Glentzlin, A., Heiden, M., Heininger, M., Hernandez Utrera, O., Hofmann, K.-H., Kamm, D., Kiekebusch, M., Kraus, S., Le Contel, D., Le Contel, J.-M., Lesourd, T., Lopez, B., Lopez, M., Magnard, Y., Marconi, A., Mars, G., Martinot-Lagarde, G., Mathias, P., Mège, P., Monin, J.-L., Mouillet, D., Mourard, D., Nussbaum, E., Ohnaka, K., Pacheco, J., Perrier, C., Rabbia, Y., Rebattu, S., Reynaud, F., Richichi, A., Robini, A., Sacchettini, M., Schertl, D., Schöller, M., Solscheid, W., Spang, A., Stee, P., Stefanini, P., Tallon, M., Tallon-Bosc, I., Tasso, D., Testi, L., Vakili, F., von der Lühe, O., Valtier, J.-C., Vannier, M., \& Ventura, N.: 2007, A\&A 464, 1

Poeckert, R. \& Marlborough, J. M.: 1978, ApJS 38, 229

Pollmann, E.: 2018, Information Bulletin on Variable Stars 6239

Porter, J. M.: 1996, MNRAS 280, L31

Porter, J. M.: 1997, A\&A 324, 597 
Porter, J. M. \& Rivinius, T.: 2003, PASP 115, 1153

Prinja, R. K.: 1989, MNRAS 241, 721

Puls, J., Vink, J. S., \& Najarro, F.: 2008, A\&A Rev. 16, 209

Página web FIRE, http://web.mit.edu/ rsimcoe/www/FIRE/ob_data.htm

Página web GEMINI, https://www.gemini.edu/

Quirrenbach, A.: 1993, in American Astronomical Society Meeting Abstracts \#182, Vol. 25 of Bulletin of the American Astronomical Society, p. 916

Quirrenbach, A., Bjorkman, K. S., Bjorkman, J. E., Hummel, C. A., Buscher, D. F., Armstrong, J. T., Mozurkewich, D., Elias, II, N. M., \& Babler, B. L.: 1997, ApJ 479, 477

Quirrenbach, A., Buscher, D. F., Mozurkewich, D., Hummel, C. A., \& Armstrong, J. T.: 1994, A\&A 283, L13

Rivinius, T., Baade, D., \& Štefl, S.: 2003, A\&A 411, 229

Rivinius, T., Carciofi, A. C., \& Martayan, C.: 2013, A\&A Rev. 21, 69

Rivinius, T., Štefl, S., \& Baade, D.: 1999, A\&A 348, 831

Rivinius, T., Štefl, S., \& Baade, D.: 2006, A\&A 459, 137

Sabogal, B. E., Ubaque, K. Y., García-Varela, A., Álvarez, M., \& Salas, L.: 2017, PASP 129(1), 014203

Sadakane, K., Hirata, R., \& Tsuji, T.: 2005, PASJ 57, 1

Sareyan, J. P., Chauville, J., Alvarez, M., Guerrero, G., Arellano Ferro, A., Mathias, P., \& Peña, J.: 2002, in C. Aerts, T. R. Bedding, \& J. Christensen-Dalsgaard (eds.), IAU Colloq. 185: Radial and Nonradial Pulsationsn as Probes of Stellar Physics, Vol. 259 of Astronomical Society of the Pacific Conference Series, p. 238

Schaefer, G. H., Gies, D. R., Monnier, J. D., Richardson, N. D., Touhami, Y., Zhao, M., Che, X., Pedretti, E., Thureau, N., ten Brummelaar, T., McAlister, H. A., Ridgway, S. T., Sturmann, J., Sturmann, L., Turner, N. H., Farrington, C. D., \& Goldfinger, P. J.: 2010, AJ 140, 1838

Schaller, G., Schaerer, D., Meynet, G., \& Maeder, A.: 1992, A\&AS 96, 269

Shimazaki, H. \& Shinomoto, S.: 2007, Neural Computation 19, 1503

Sigut, T. A. A., Tycner, C., Jansen, B., \& Zavala, R. T.: 2015, ApJ 814, 159 
Silaj, J., Jones, C. E., Carciofi, A. C., Escolano, C., Okazaki, A. T., Tycner, C., Rivinius, T., Klement, R., \& Bednarski, D.: 2016, ApJ 826, 81

Silaj, J., Jones, C. E., Sigut, T. A. A., \& Tycner, C.: 2014, ApJ 795, 82

Silaj, J., Jones, C. E., Tycner, C., Sigut, T. A. A., \& Smith, A. D.: 2010, ApJS 187, 228

Simon, M., Felli, M., Cassar, L., Fischer, J., \& Massi, M.: 1983, ApJ 266, 623

Slettebak, A.: 1951, ApJ 113, 436

Slettebak, A.: 1979, Space Sci. Rev. 23, 541

Slettebak, A.: 1982, ApJS 50, 55

Slettebak, A. \& Reynolds, R. C.: 1978, ApJS 38, 205

Slettebak, A. \& Snow, T. P. (eds.): 1987, Physics of Be stars; Proceedings of the Ninety-second IAU Colloquium, University of Colorado, Boulder, Aug. 18-22, 1986

Smith, M. A.: 2001, ApJ 562, 998

Snow, Jr., T. P.: 1979, in P. S. Conti \& C. W. H. De Loore (eds.), Mass Loss and Evolution of O-Type Stars, Vol. 83 of IAU Symposium, pp 65-77

Stee, P.: 1995, Ap\&SS 224, 561

Stee, P.: 2011, in C. Neiner, G. Wade, G. Meynet, \& G. Peters (eds.), Active OB Stars: Structure, Evolution, Mass Loss, and Critical Limits, Vol. 272 of IAU Symposium, pp 313-324

Steele, I. A. \& Clark, J. S.: 2001, $A \& A$ 371, 643

Steele, I. A., Negueruela, I., \& Clark, J. S.: 1999, A\&AS 137, 147

Stoeckley, T. R.: 1968, MNRAS 140, 121

Storey, P. J. \& Hummer, D. G.: 1995, MNRAS 272, 41

Struve, O.: 1925, ApJ 62

Struve, O.: 1931, ApJ 73, 94

Tanaka, K., Sadakane, K., Narusawa, S.-Y., Naito, H., Kambe, E., Katahira, J.-I., \& Hirata, R.: 2007, PASJ 59, L35

Taranova, O., Shenavrin, V., \& Nadjip, A. D.: 2008, Peremennye Zvezdy Prilozhenie 8 
Tatulli, E., Millour, F., Chelli, A., Duvert, G., Acke, B., Hernandez Utrera, O., Hofmann, K.-H., Kraus, S., Malbet, F., Mège, P., Petrov, R. G., Vannier, M., Zins, G., Antonelli, P., Beckmann, U., Bresson, Y., Dugué, M., Gennari, S., Glück, L., Kern, P., Lagarde, S., Le Coarer, E., Lisi, F., Perraut, K., Puget, P., Rantakyrö, F., Robbe-Dubois, S., Roussel, A., Weigelt, G., Accardo, M., Agabi, K., Altariba, E., Arezki, B., Aristidi, E., Baffa, C., Behrend, J., Blöcker, T., Bonhomme, S., Busoni, S., Cassaing, F., Clausse, J.-M., Colin, J., Connot, C., Delboulbé, A., Domiciano de Souza, A., Driebe, T., Feautrier, P., Ferruzzi, D., Forveille, T., Fossat, E., Foy, R., FraixBurnet, D., Gallardo, A., Giani, E., Gil, C., Glentzlin, A., Heiden, M., Heininger, M., Kamm, D., Kiekebusch, M., Le Contel, D., Le Contel, J.-M., Lesourd, T., Lopez, B., Lopez, M., Magnard, Y., Marconi, A., Mars, G., Martinot-Lagarde, G., Mathias, P., Monin, J.-L., Mouillet, D., Mourard, D., Nussbaum, E., Ohnaka, K., Pacheco, J., Perrier, C., Rabbia, Y., Rebattu, S., Reynaud, F., Richichi, A., Robini, A., Sacchettini, M., Schertl, D., Schöller, M., Solscheid, W., Spang, A., Stee, P., Stefanini, P., Tallon, M., Tallon-Bosc, I., Tasso, D., Testi, L., Vakili, F., von der Lühe, O., Valtier, J.-C., \& Ventura, N.: 2007, A\&A 464, 29

Thaller, M. L., Bagnuolo, Jr., W. G., Gies, D. R., \& Penny, L. R.: 1995, ApJ 448, 878

Thom, C., Granes, P., \& Vakili, F.: 1986, A\&A 165, L13

Touhami, Y., Gies, D. R., \& Schaefer, G. H.: 2011, ApJ 729, 17

Touhami, Y., Gies, D. R., Schaefer, G. H., McAlister, H. A., Ridgway, S. T., Richardson, N. D., Matson, R., Grundstrom, E. D., ten Brummelaar, T. A., Goldfinger, P. J., Sturmann, L., Sturmann, J., Turner, N. H., \& Farrington, C.: 2013, ApJ 768, 128

Touhami, Y., Richardson, N. D., Gies, D. R., Schaefer, G. H., Boyajian, T. S., Williams, S. J., Grundstrom, E. D., McSwain, M. V., Clemens, D. P., \& Taylor, B.: 2010, PASP 122, 379

Townsend, R. H. D., Owocki, S. P., \& Howarth, I. D.: 2004, MNRAS 350, 189

Tycner, C., Hajian, A. R., Armstrong, J. T., Benson, J. A., Gilbreath, G. C., Hutter, D. J., Lester, J. B., Mozurkewich, D., \& Pauls, T. A.: 2004, AJ 127, 1194

Tycner, C., Lester, J. B., Hajian, A. R., Armstrong, J. T., Benson, J. A., Gilbreath, G. C., Hutter, D. J., Pauls, T. A., \& White, N. M.: 2005, ApJ 624, 359

Uesugi, A. \& Fukuda, I.: 1981, Revised catalogue of stellar rotational velocities

Underhill, A. \& Doazan, V.: 1982, B Stars with and without emission lines, parts 1 and 2

Štefl, S., Hadrava, P., Baade, D., Rivinius, T., Maintz, M., \& Stahl, O.: 2004, in A. Maeder \& P. Eenens (eds.), Stellar Rotation, Vol. 215 of IAU Symposium, p. 166

Štefl, S., Le Bouquin, J.-B., Carciofi, A. C., Rivinius, T., Baade, D., \& Rantakyrö, F.: 2012, A\&A 540, A76 
Štefl, S., Rivinius, T., Carciofi, A. C., Le Bouquin, J.-B., Baade, D., Bjorkman, K. S., Hesselbach, E., Hummel, C. A., Okazaki, A. T., Pollmann, E., Rantakyrö, F., \& Wisniewski, J. P.: 2009, A\&A 504, 929

van Belle, G. T.: 2012, A\&A Rev. 20, 51

van Leeuwen, F. (ed.): 2007, Hipparcos, the New Reduction of the Raw Data, Vol. 350 of Astrophysics and Space Science Library

Vieira, R. G., Carciofi, A. C., Bjorkman, J. E., Rivinius, T., Baade, D., \& Rímulo, L. R.: 2017, MNRAS 464, 3071

Vinicius, M. M. F., Zorec, J., Leister, N. V., \& Levenhagen, R. S.: 2006, A\&A 446, 643

Vink, J. S., Davies, B., Harries, T. J., Oudmaijer, R. D., \& Walborn, N. R.: 2009, A\&A 505(2), 743

Walker, M. F.: 1958, AJ 63, 237

Wallace, L., Meyer, M. R., Hinkle, K., \& Edwards, S.: 2000, ApJ 535, 325

Waters, L. B. F. M.: 1986, $A \& A$ 162, 121

Waters, L. B. F. M., Cote, J., \& Lamers, H. J. G. L. M.: 1987, $A \& A$ 185, 206

Waters, L. B. F. M., Cote, J., \& Pols, O. R.: 1991, A\&A 250, 437

Weigelt, G., Kraus, S., Driebe, T., Petrov, R. G., Hofmann, K.-H., Millour, F., Chesneau, O., Schertl, D., Malbet, F., Hillier, J. D., Gull, T., Davidson, K., Domiciano de Souza, A., Antonelli, P., Beckmann, U., Bresson, Y., Chelli, A., Dugué, M., Duvert, G., Gennari, S., Glück, L., Kern, P., Lagarde, S., Le Coarer, E., Lisi, F., Perraut, K., Puget, P., Rantakyrö, F., Robbe-Dubois, S., Roussel, A., Tatulli, E., Zins, G., Accardo, M., Acke, B., Agabi, K., Altariba, E., Arezki, B., Aristidi, E., Baffa, C., Behrend, J., Blöcker, T., Bonhomme, S., Busoni, S., Cassaing, F., Clausse, J.-M., Colin, J., Connot, C., Delboulbé, A., Feautrier, P., Ferruzzi, D., Forveille, T., Fossat, E., Foy, R., Fraix-Burnet, D., Gallardo, A., Giani, E., Gil, C., Glentzlin, A., Heiden, M., Heininger, M., Hernandez Utrera, O., Kamm, D., Kiekebusch, M., Le Contel, D., Le Contel, J.-M., Lesourd, T., Lopez, B., Lopez, M., Magnard, Y., Marconi, A., Mars, G., Martinot-Lagarde, G., Mathias, P., Mège, P., Monin, J.-L., Mouillet, D., Mourard, D., Nussbaum, E., Ohnaka, K., Pacheco, J., Perrier, C., Rabbia, Y., Rebattu, S., Reynaud, F., Richichi, A., Robini, A., Sacchettini, M., Schöller, M., Solscheid, W., Spang, A., Stee, P., Stefanini, P., Tallon, M., Tallon-Bosc, I., Tasso, D., Testi, L., Vakili, F., von der Lühe, O., Valtier, J.-C., Vannier, M., Ventura, N., Weis, K., \& Wittkowski, M.: 2007, A\&A 464, 87

Westerlund, B. E.: 1997, The Magellanic Clouds 
Wheelwright, H. E., Bjorkman, J. E., Oudmaijer, R. D., Carciofi, A. C., Bjorkman, K. S., \& Porter, J. M.: 2012, MNRAS 423, L11

Wisniewski, J. P. \& Bjorkman, K. S.: 2006, ApJ 652, 458

Wisniewski, J. P., Draper, Z. H., Bjorkman, K. S., Meade, M. R., Bjorkman, J. E., \& Kowalski, A. F.: 2010, ApJ 709, 1306

Wood, K., Bjorkman, J. E., Whitney, B., \& Code, A.: 1996, ApJ 461, 847

Wood, K., Bjorkman, K. S., \& Bjorkman, J. E.: 1997, ApJ 477, 926

Woolf, N. J., Stein, W. A., \& Strittmatter, P. A.: 1970, $A \& A \mathbf{9 ,} 252$

Yudin, R. V.: 2001, A\&A 368, 912

Zorec, J.: 2004, in A. Maeder \& P. Eenens (eds.), Stellar Rotation, Vol. 215 of IAU Symposium, p. 73

Zorec, J., Arias, M. L., Cidale, L., \& Ringuelet, A. E.: 2007, A\&A 470, 239

Zorec, J. \& Briot, D.: 1997, A\&A 318, 443

Zorec, J., Frémat, Y., \& Cidale, L.: 2005, A\&A 441, 235

Zorec, J., Frémat, Y., Domiciano de Souza, A., Royer, F., Cidale, L., Hubert, A.-M., Semaan, T., Martayan, C., Cochetti, Y. R., Arias, M. L., Aidelman, Y., \& Stee, P.: 2016, A\&A 595, A132

Zorec, J., Frémat, Y., \& Hubert, A. M.: 2000, in M. A. Smith, H. F. Henrichs, \& J. Fabregat (eds.), IAU Colloq. 175: The Be Phenomenon in Early-Type Stars, Vol. 214 of Astronomical Society of the Pacific Conference Series, p. 330 